\title{
Electrochemical Borylation of Alkyl Halides: Fast, Scalable Access to Alkyl Boronic Esters
}

\author{
Bingbing Wang $\ddagger{ }^{a}$ Pan Peng $\ddagger{ }^{a}$ Wan Ma,${ }^{a}$ Zhao Liu,${ }^{a}$ Cheng Huang, ${ }^{a}$ Yangmin Cao,${ }^{a}$ Ping Hu, ${ }^{a}$ \\ Xiaotian $\mathrm{Qi}^{b *}$ and Qingquan $\mathrm{Lu}^{a, c *}$ \\ ${ }^{a}$ The Institute for Advanced Studies (IAS), ${ }^{b}$ College of Chemistry and Molecular Sciences, Wuhan \\ University, Wuhan 430072, P. R. China. ${ }^{c}$ Ministry of Education Key Laboratory for the Synthesis \\ and Application of Organic Functional Molecules, Hubei University, Wuhan 430062, P. R. China. \\ $\$$ These authors contributed equally to this work \\ qi7xiaotian@whu.edu.cn; gci2011@whu.edu.cn
}

\section{Supporting Information}




\section{Table of Contents}

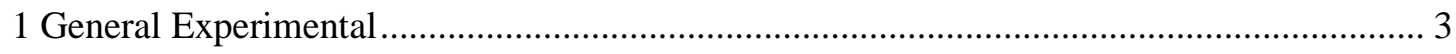

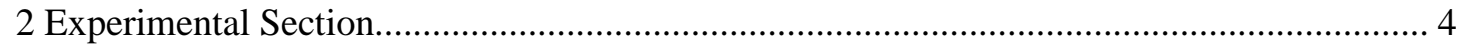

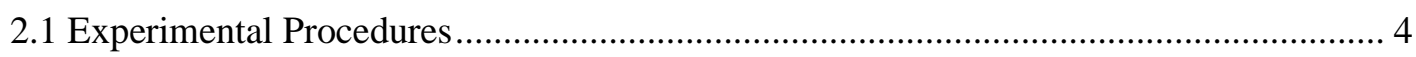

2.2 Graphical Guide for Borylation of Unactivated Alkyl Halides ..................................... 7

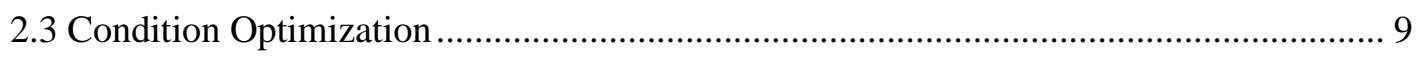

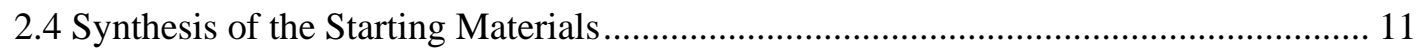

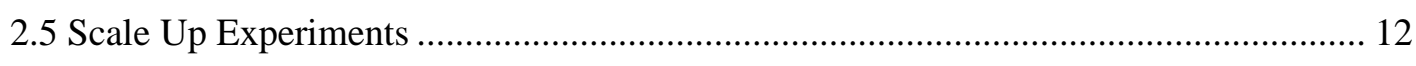

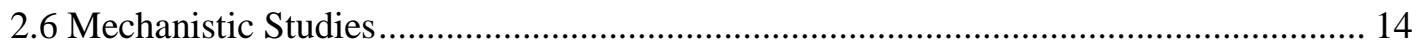

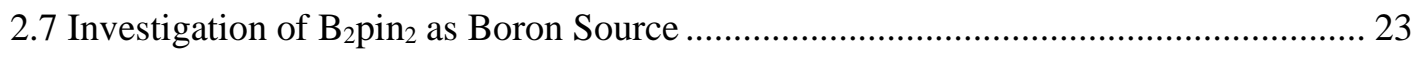

2.8 Expand this Electrochemical Borylation to Sacrificial Anode-Free Conditions ........... 24

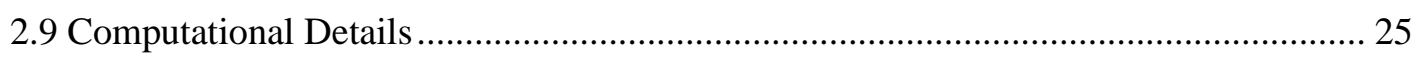

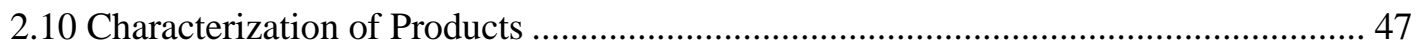

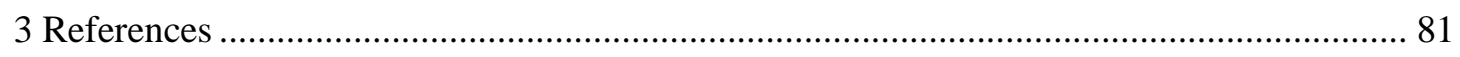

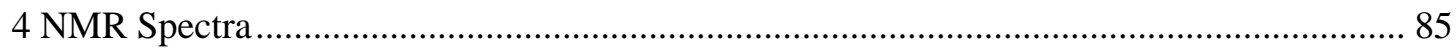




\section{General Experimental}

All reactions were conducted under an argon atmosphere unless otherwise noted. All reagents were directly used as received from commercial suppliers (Alfa, Tci, Innochem, Aladdin, Energy Chemical, etc.) without further purification unless otherwise stated. N,N-Dimethylacetamide (DMAc) from Aladdin (D108098, 99.8\%, around $280 \mathrm{ppm}$ of water), N,N-Dimethylformamide (DMF) from Aladdin (D112000, 99.9\%, around 700 ppm of water), Dry N,N-Dimethylacetamide (DMAc) from Energy Chemical (W610492-0010, 99.8\%, Water $\leqslant 50$ ppm) or Innochem (A93337, 99.8\%, Water $\leqslant$ $50 \mathrm{ppm}$ ). Thin layer chromatography (TLC) employed $0.25 \mathrm{~mm}$ glass silica gel plates. Visualization of spots on TLC plate was accomplished with short-wave UV light, phosphomolybdic acid, $\mathrm{KMnO}_{4}$ or staining over $\mathrm{I}_{2}$ chamber. Flash chromatography column was packed with 200-300 mesh silica gel in petroleum (bp. $60-90{ }^{\circ} \mathrm{C}$ ). Proton nuclear magnetic resonance $\left({ }^{1} \mathrm{H}\right.$ NMR) spectra and carbon nuclear magnetic resonance $\left({ }^{13} \mathrm{C}\right.$ NMR) spectra were recorded on JNM ECZ $400(400 \mathrm{MHz})$ or AVANCE NEO 600 $(600 \mathrm{MHz})$ spectrometers. All chemical shifts $(\delta)$ were reported in ppm and coupling constants $(J)$ were reported in Hz. All Chemical shifts are reported in parts per million downfield from tetramethylsilane and are referenced to residual undeuterated solvent $\left(\mathrm{CHCl}_{3}\right.$ at $7.26 \mathrm{ppm}$ for ${ }^{1} \mathrm{H} \mathrm{NMR}, 77.16 \mathrm{ppm}$ for $\left.{ }^{13} \mathrm{C} \mathrm{NMR}\right)$. Data are represented as follows: chemical shift, multiplicity $(\mathrm{br}=$ broad, $\mathrm{s}=$ singlet, $\mathrm{d}=$ doublet, $\mathrm{t}=$ triplet, $\mathrm{q}=$ quartet, $\mathrm{m}=$ multiplet). Cyclic voltammetry studies were carried out on a CHI660E potentiostat. All compounds were characterized by high resolution mass spectra (HRMS) on Bruker UltiMate3000 \& Compact. Gas chromatography-mass spectrometry (GC-MS) were recorded on an Agilent 5977B MSD Series spectrometer. Gas chromatography (GC) were recorded on an Agilent 8890 MSD Series spectrometer. Electrolysis was conducted on dual display potentiostat (HYELEC $®$ HY3005B, made in China). 


\section{Experimental Section}

\subsection{Experimental Procedures}

\section{General Procedure A:}

$$
\mathrm{R}-\mathrm{Br}+\mathrm{B}_{2} \mathrm{cat}_{2} \stackrel{\mathrm{Mg}(+) \mid \mathrm{CC}(-), 150 \mathrm{~mA}, \mathrm{Ar}, 3.0 \mathrm{~F} / \mathrm{mol}}{{ }^{n} \mathrm{Bu}_{4} \mathrm{NBF}_{4}, \mathrm{DMAc}, \mathrm{r.t} .}
$$

$\mathrm{B}_{2} \mathrm{cat}_{2}(2.4 \mathrm{mmol}, 4.0$ equiv., $571.0 \mathrm{mg})$ and ${ }^{n} \mathrm{Bu}_{4} \mathrm{NBF}_{4}(0.3 \mathrm{mmol}, 99.0 \mathrm{mg})$ were added into an oven-dried undivided three-necked cell $(20 \mathrm{~mL})$ equipped with a Teflon-coated magnetic stir bar. The undivided cell was equipped with $\mathrm{Mg}$ plate $(15 \mathrm{~mm} \times 15 \mathrm{~mm} \times 0.5$ $\mathrm{mm})$ as the anode and carbon cloth electrode $(15 \mathrm{~mm} \times 15 \mathrm{~mm})$ as the cathode and then charged with argon to replace air atmosphere. Alkyl bromide $(0.6 \mathrm{mmol}$, solid bromide was added into electrolyzer cell in advance) and DMAc $(6.0 \mathrm{~mL})$ were added to the tube through a syringe. Pre-stirring the resulting mixture until it is completely dissolved, and then the reaction mixture was electrolyzed at a constant current of $150 \mathrm{~mA}$ until passing $3.0 \mathrm{~F} / \mathrm{mol}$ of charge (around $19 \mathrm{~min}$ ) at room temperature (around $25{ }^{\circ} \mathrm{C}$ ). Afterwards, a solution of pinacol ( $4.8 \mathrm{mmol}, 8.0$ equiv., $567.2 \mathrm{mg})$ in triethylamine (1.5 $\mathrm{mL}$ ) was added to the electrolyzer cell and the reaction mixture kept stirring at room temperature for $1 \mathrm{~h}$. Then, saturated brine water was added into the reaction mixture and the aqueous layer was extracted with ethyl acetate $(3 \times 15 \mathrm{~mL})$. The organic layer was dried over anhydrous $\mathrm{Na}_{2} \mathrm{SO}_{4}$ and concentrated in vacuum. The concentrated reaction mixture was filtered through silica gel with copious washings to remove the partial impurity (ethyl acetate:petroleum ether $=1: 10$ ), concentrated, and purified by column chromatography (eluted with ethyl acetate/petroleum ether) to afford the pure desired product.

\section{General Procedure B:}

$$
\mathrm{R}-\mathrm{I}+\mathrm{B}_{2} \mathrm{Cat}_{2} \underset{{ }^{n} \mathrm{Bu}_{4} \mathrm{NBF}_{4}, \mathrm{DMF}, \mathrm{M.t.}}{\text { then pinacol, } \mathrm{Et}_{3} \mathrm{~N}, 1 \mathrm{~h}}
$$

$\mathrm{B}_{2} \mathrm{cat}_{2}(2.4 \mathrm{mmol}, 4.0$ equiv., $571.0 \mathrm{mg})$ and ${ }^{n} \mathrm{Bu}_{4} \mathrm{NBF}_{4}(0.3 \mathrm{mmol}, 99.0 \mathrm{mg})$ were added 
into an oven-dried three-necked the undivided cell $(20 \mathrm{~mL})$ equipped with a Tefloncoated magnetic stir bar. The undivided cell was equipped with $\mathrm{Mg}$ plate $(15 \mathrm{~mm} \times 15$ $\mathrm{mm} \times 0.5 \mathrm{~mm})$ as the anode and carbon cloth electrode $(15 \mathrm{~mm} \times 15 \mathrm{~mm})$ as the cathode and then charged with argon to replace air atmosphere. Alkyl iodide $(0.6 \mathrm{mmol}$, solid iodide was added into electrolyzer cell in advance) and DMF $(6.0 \mathrm{~mL})$ were added to the tube through a syringe. Pre-stirring the resulting mixture until it is completely dissolved, and then the reaction mixture was electrolyzed at a constant current of 200 $\mathrm{mA}$ until passing $2.5 \mathrm{~F} / \mathrm{mol}$ of charge (around $12 \mathrm{~min}$ ) at room temperature (around 25 $\left.{ }^{\circ} \mathrm{C}\right)$. Afterwards, a solution of pinacol (4.8 mmol, 8.0 equiv., $\left.567.2 \mathrm{mg}\right)$ in triethylamine $(1.5 \mathrm{~mL})$ was added to the electrolyzer cell and the reaction mixture kept stirring at room temperature for $1 \mathrm{~h}$. Then, saturated brine water was added into the reaction mixture and the aqueous layer was extracted with ethyl acetate $(3 \times 15 \mathrm{~mL})$. The organic layer was dried over anhydrous $\mathrm{Na}_{2} \mathrm{SO}_{4}$ and concentrated in vacuum. The concentrated reaction mixture was filtered through silica gel with copious washings to remove the partial impurity (ethyl acetate:petroleum ether $=1: 10$ ), concentrated, and purified by column chromatography (eluted with ethyl acetate/petroleum ether) to afford the pure desired product.

\section{General Procedure C:}

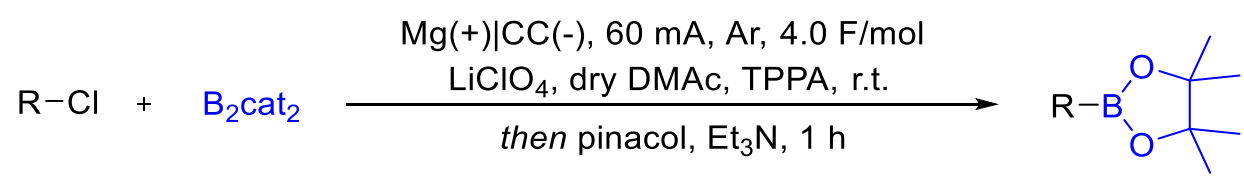

$\mathrm{B}_{2}$ cat 2 (2.4 mmol, 4.0 equiv., $\left.571.0 \mathrm{mg}\right)$ and $\mathrm{LiClO}_{4}(0.3 \mathrm{mmol}, 32.0 \mathrm{mg})$ were added into an oven-dried three-necked undivided cell $(20 \mathrm{~mL})$ equipped with a Teflon-coated magnetic stir bar. The undivided cell was equipped with $\mathrm{Mg}$ plate $(15 \mathrm{~mm} \times 15 \mathrm{~mm} \times 0.5$ $\mathrm{mm})$ as the anode and carbon cloth electrode $(15 \mathrm{~mm} \times 15 \mathrm{~mm})$ as the cathode and then charged with argon to replace air atmosphere. Alkyl chloride $(0.6 \mathrm{mmol}$, solid chloride was added into electrolyzer cell in advance), Dry DMAc (4.5 mL) and TPPA (1.5 mL) were added to the tube through a syringe. Pre-stirring the resulting mixture until it is completely dissolved, and then the reaction mixture was electrolyzed at a constant 
current of $60 \mathrm{~mA}$ until passing $4.0 \mathrm{~F} / \mathrm{mol}$ of charge (around $64 \mathrm{~min}$ ) at $25^{\circ} \mathrm{C}$. Afterwards, a solution of pinacol (4.8 mmol, 8.0 equiv., $567.2 \mathrm{mg})$ in triethylamine $(1.5 \mathrm{~mL})$ was added to the electrolyzer cell and the reaction mixture kept stirring at room temperature for $1 \mathrm{~h}$. Then, saturated brine water was added into the reaction mixture and the aqueous layer was extracted with ethyl acetate $(3 \times 15 \mathrm{~mL})$. The organic layer was dried over anhydrous $\mathrm{Na}_{2} \mathrm{SO}_{4}$ and concentrated in vacuum. The concentrated reaction mixture was filtered through silica gel with copious washings to remove the partial impurity (ethyl acetate:petroleum ether $=1: 10$ ), concentrated, and purified by column chromatography (eluted with ethyl acetate/petroleum ether) to afford the pure desired product.

\section{General Procedure D:}

$$
\mathrm{R}-\mathrm{I}+\mathrm{B}_{2} \mathrm{cat}_{2} \underset{{ }^{2} \mathrm{Bu}_{4} \mathrm{NBF}_{4} \text {, DMAc, r.t. }}{\text { then pinacol, } \mathrm{Et}_{3} \mathrm{~N}, 1 \mathrm{~h}}
$$

$\mathrm{B}_{2} \mathrm{cat}_{2}(0.8 \mathrm{mmol}, 4.0$ equiv., $190.0 \mathrm{mg})$ and ${ }^{n} \mathrm{Bu}_{4} \mathrm{NBF}_{4}(0.3 \mathrm{mmol}, 99.0 \mathrm{mg})$ were added into an oven-dried three-necked undivided cell $(20 \mathrm{~mL})$ equipped with a Teflon-coated magnetic stir bar. The undivided cell was equipped with $\mathrm{Mg}$ plate $(15 \mathrm{~mm} \times 15 \mathrm{~mm} \times 0.5$ $\mathrm{mm})$ as the anode and carbon cloth electrode $(15 \mathrm{~mm} \times 15 \mathrm{~mm})$ as the cathode and then charged with argon to replace air atmosphere. Alkyl iodide $(0.2 \mathrm{mmol}$, solid iodide was added into electrolyzer cell in advance) and DMAc $(6.0 \mathrm{~mL})$ were added to the tube through a syringe. Pre-stirring the resulting mixture for 5 minutes, and then the reaction mixture was electrolyzed at a constant current of $10 \mathrm{~mA}$ until passing $2.5 \mathrm{~F} / \mathrm{mol}$ of charge (around $80 \mathrm{~min}$ ) at room temperature (around $25^{\circ} \mathrm{C}$ ). Afterwards, a solution of pinacol (1.6 mmol, 8.0 equiv., $189.1 \mathrm{mg})$ in triethylamine $(1.5 \mathrm{~mL})$ was added to the electrolyzer cell and the reaction mixture kept stirring at room temperature for $1 \mathrm{~h}$. Then, saturated brine water was added into the reaction mixture and the aqueous layer was extracted with ethyl acetate $(3 \times 15 \mathrm{~mL})$. The organic layer was dried over anhydrous $\mathrm{Na}_{2} \mathrm{SO}_{4}$ and concentrated in vacuum. The concentrated reaction mixture was filtered through silica gel with copious washings to remove the partial impurity (ethyl acetate:petroleum ether $=1: 10$ ), concentrated, and purified by column chromatography 
(eluted with ethyl acetate/petroleum ether) to afford the pure desired product.

\subsection{Graphical Guide for Borylation of Unactivated Alkyl Halides}

\section{Materials Used for Set-up:}

Mg Plate was purchased from Dongwan Hongdi Metal Materials Co., Ltd (0.5mm thick, 97\% pure). Carbon cloth was purchased from PHYCHEMi (http://www.phychemi.com, Product Model: W0S1009). Electrode holder was purchased from Wuhan Gaossunion Technology Co., Ltd (www.gaossunion.com, Product Model: pt-3). All undivided cells were custom made by the Wuhan Ruiboer Technology Co., Ltd.

1. Materials used for three-necked cell set-up.

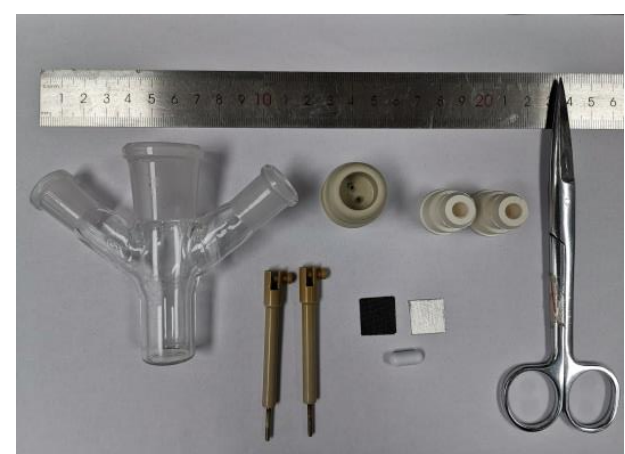

2. Reagents used for borylation: ${ }^{n} \mathrm{Bu}_{4} \mathrm{NBF}_{4}, \mathrm{~B}_{2} \mathrm{cat}_{2}, \mathrm{DMAc}$, Pinacol, Et ${ }_{3} \mathrm{~N}$ and the substrate (Such as bromocyclohexane).

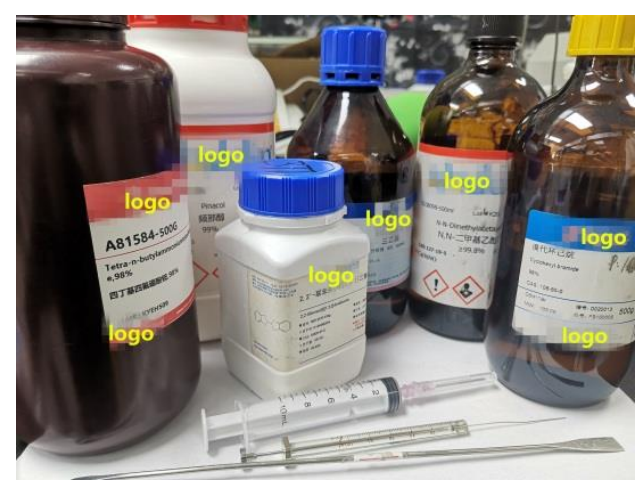

3. ${ }^{n} \mathrm{Bu}_{4} \mathrm{NBF}_{4}(0.3 \mathrm{mmol}, 99.0 \mathrm{mg})$ and $\mathrm{B}_{2} \mathrm{cat}_{2}(2.4 \mathrm{mmol}, 4$ equiv., $571.0 \mathrm{mg})$ were added to the oven-dried three-necked cell $(20 \mathrm{~mL})$ equipped with a Teflon-coated magnetic stir bar. 


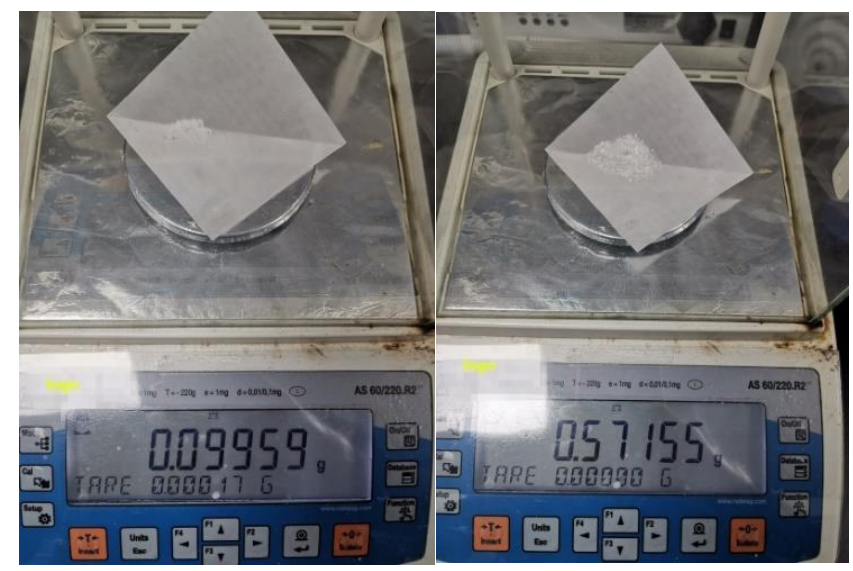

4. The three-necked cell was capped and then charged with argon to replace air atmosphere (Then the reaction vessel was evacuated and filled with argon for three times).

5. Bromocyclohexane ( $0.6 \mathrm{mmol}, 1.0$ equiv., $97.8 \mathrm{mg})$ was added to the three-necked cell by micro syringe.

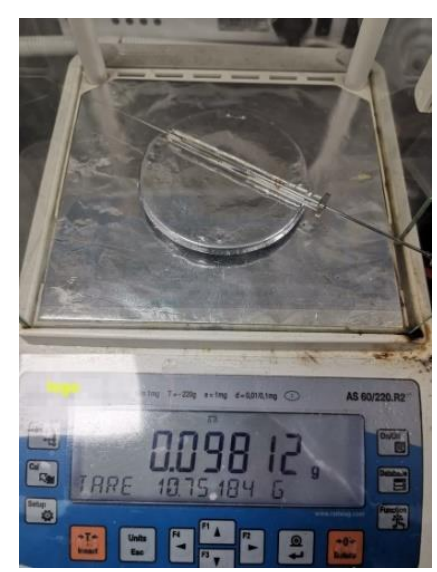

6. DMAc $(6.0 \mathrm{~mL})$ was added to the reaction mixture.

7. The reaction mixture was connected to a potentiostat with the current kept at $150 \mathrm{~mA}$. At the beginning, the cell voltage was $21.36 \mathrm{~V}$. After $19 \mathrm{~min}$ of electrolysis, the cell voltage decreased to $7.79 \mathrm{~V}(3.0 \mathrm{~F} / \mathrm{mol})$.

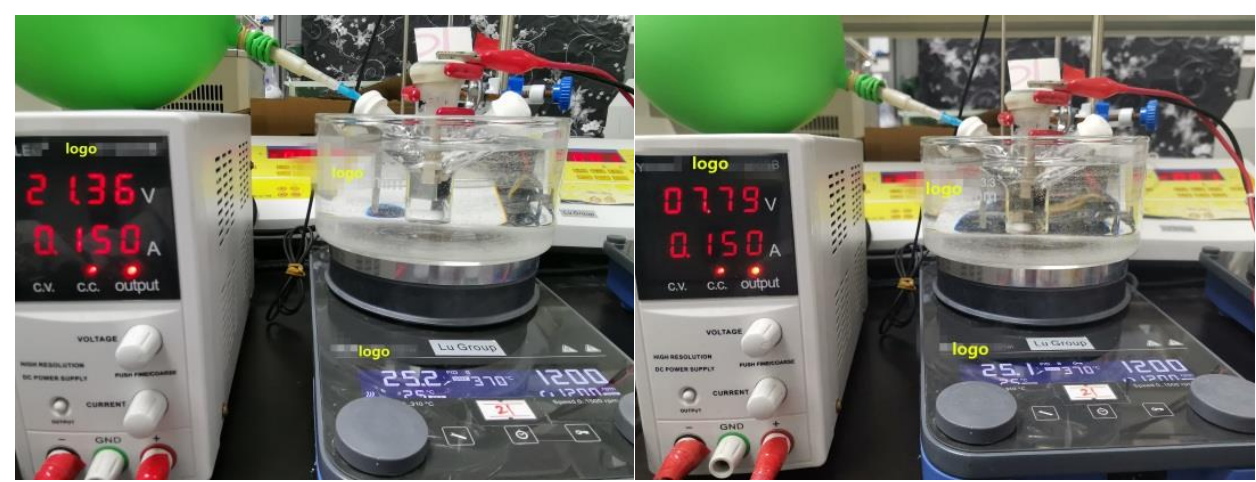


8. The color of reaction mixture turned from colorless (left) into gray (right) during the progress of the reaction.
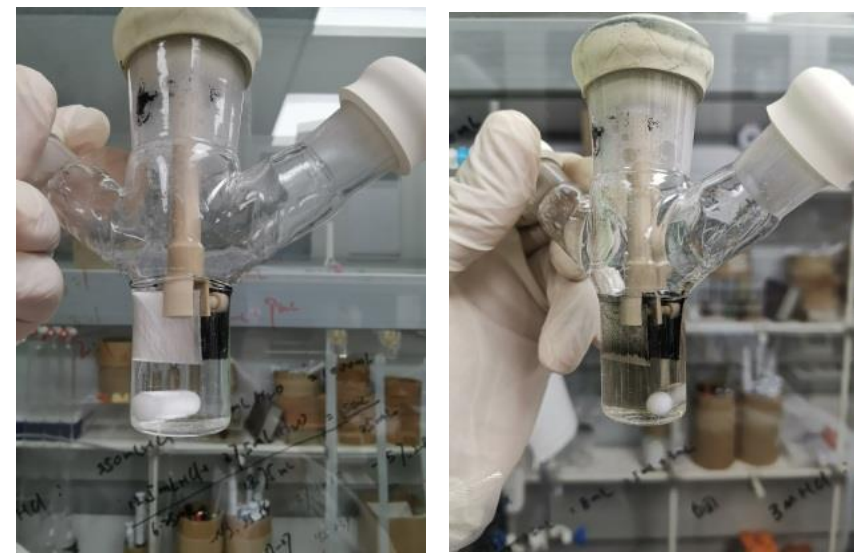

9. After electrolysis, a solution of pinacol ( $4.8 \mathrm{mmol}, 8.0$ equiv.) in triethylamine (1.5 $\mathrm{mL}$ ) was added to the electrolyzer cell and the reaction mixture kept stirring at room temperature for $1 \mathrm{~h}$.

10. When the reaction is completed, the reaction mixture was transferred to a separatory funnel. The electrodes were washed with $\mathrm{Et}_{2} \mathrm{O}$ or EtOAc (depending on solubility/volatility of product) to transfer residual product. Afterwards, saturated brine water $(100 \mathrm{~mL})$ was added into the reaction mixture and the aqueous layer was extracted with $\mathrm{Et}_{2} \mathrm{O}$ or EtOAc $(3 \times 20 \mathrm{~mL})$. The organic layer was dried over anhydrous $\mathrm{Na}_{2} \mathrm{SO}_{4}$ and concentrated in vacuum. The residue was purified by flash column chromatography on silica gel to furnish the desired product.

\subsection{Condition Optimization}

Table S1. Optimization of Reaction Conditions with Bromocyclohexane

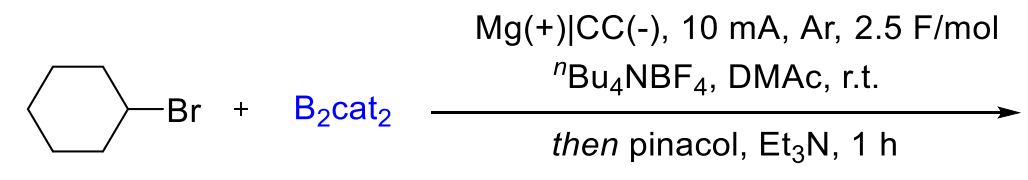

$1 \mathrm{a}$

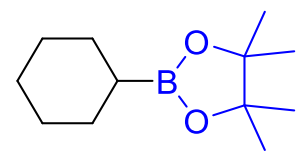

1

\begin{tabular}{|c|c|c|}
\hline Entry & Deviation from standard conditions & Yield (1) [\% $]^{\mathrm{a}}$ \\
\hline 1 & None & 86 \\
\hline 2 & $\mathrm{Zn}(+)$ & 4 \\
\hline 3 & Stainless Steel $(+)$ & 36 \\
\hline 4 & $\mathrm{LiClO}_{4}$ instead of ${ }^{n} \mathrm{Bu}_{4} \mathrm{NBF}_{4}$ & 87 \\
\hline 5 & DMF instead of DMAc & 63 \\
\hline
\end{tabular}


Reaction conditions: bromocyclohexane 1a ( $0.6 \mathrm{mmol}), \mathrm{B}_{2}$ cat2 2 (4.0 equiv.), ${ }^{n} \mathrm{Bu}_{4} \mathrm{NBF}_{4}(0.3 \mathrm{mmol}), \mathrm{DMAc}(6.0 \mathrm{~mL})$, $\mathrm{Mg}$ plate and carbon cloth, undivided cell, constant current, $2.5 \mathrm{~F} / \mathrm{mol}$, room temperature $\left(\operatorname{around} 25^{\circ} \mathrm{C}\right)$, n.d. $=$ not detected. Then pinacol (8.0 equiv.), $\mathrm{Et}_{3} \mathrm{~N}$ (1.5 mL), 1 h. ${ }^{a} \mathrm{GC}$ yields using biphenyl as internal standard.

Table S2. Optimization of Reaction Conditions with Iodocyclohexane.

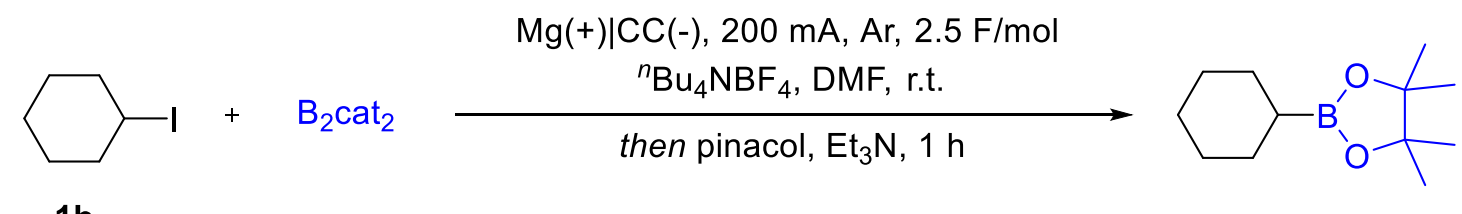

$1 \mathrm{~b}$

1

\begin{tabular}{|c|c|c|}
\hline Entry & Deviation from standard conditions & Yield (1) [\% $]^{\mathrm{a}}$ \\
\hline 1 & None & 86 \\
\hline 2 & $\mathrm{~B}_{2} \mathrm{cat}_{2}$ (3.0 equiv.) & 80 \\
\hline 3 & $\mathrm{~B}_{2} \mathrm{cat}_{2}(2.0$ equiv. $)$ & 69 \\
\hline 4 & ${ }^{n} \mathrm{Bu}_{4} \mathrm{NPF}_{6}$ instead of ${ }^{n} \mathrm{Bu}_{4} \mathrm{NBF}_{4}$ & 79 \\
\hline 5 & ${ }^{n} \mathrm{Bu}_{4} \mathrm{NI}$ instead of ${ }^{n} \mathrm{Bu}_{4} \mathrm{NBF}_{4}$ & 80 \\
\hline 6 & $\mathrm{Me}_{4} \mathrm{NBF}_{4}$ instead of ${ }^{n} \mathrm{Bu}_{4} \mathrm{NBF}_{4}$ & 76 \\
\hline 7 & ${ }^{n} \mathrm{Bu}_{4} \mathrm{NClO}_{4}$ instead of ${ }^{n} \mathrm{Bu}_{4} \mathrm{NBF}_{4}$ & 81 \\
\hline 8 & $\mathrm{LiClO}_{4}$ instead of ${ }^{n} \mathrm{Bu}_{4} \mathrm{NBF}_{4}$ & 82 \\
\hline 9 & Pt plate(-) instead of CC(-) & 52 \\
\hline 10 & Ni plate(-) instead of CC(-) & 53 \\
\hline 11 & $\mathrm{C}$ felt(-) instead of $\mathrm{CC}(-)$ & 79 \\
\hline 12 & Zn plate $(+)$ instead of $\mathrm{Mg}(+)$ & 35 \\
\hline 13 & $\mathrm{CC}(+)$ instead of $\mathrm{Mg}(+)$ & 56 \\
\hline 14 & Pt plate $(+)$ instead of $\mathrm{Mg}(+), 6.0 \mathrm{~F} / \mathrm{mol}$ & 80 \\
\hline 15 & NMP instead of DMF & 77 \\
\hline 17 & DMAc instead of DMF & 79 \\
\hline 18 & Under Air & 82 \\
\hline 19 & $\mathrm{H}_{2} \mathrm{O}$ (5.0 equiv.) & 80 \\
\hline 20 & Entry 1 without electricity, 12 min & trace \\
\hline
\end{tabular}

Reaction conditions: iodocyclohexane $\mathbf{1 b}(0.6 \mathrm{mmol}), \mathrm{B}_{2} \mathrm{cat} 2$ (4.0 equiv.), ${ }^{n} \mathrm{Bu}_{4} \mathrm{NBF}_{4}(0.3 \mathrm{mmol}), \mathrm{DMF}(6.0 \mathrm{~mL})$, $\mathrm{Mg}$ plate and carbon cloth, undivided cell, constant current, $2.5 \mathrm{~F} / \mathrm{mol}$, room temperature (around $25{ }^{\circ} \mathrm{C}$ ). Then pinacol (8.0 equiv.), Et $3 \mathrm{~N}(1.5 \mathrm{~mL}), 1$ h. ${ }^{a} \mathrm{GC}$ yields using biphenyl as internal standard. 
Table S3. Optimization of Reaction Conditions with 1-Chlorodecane.

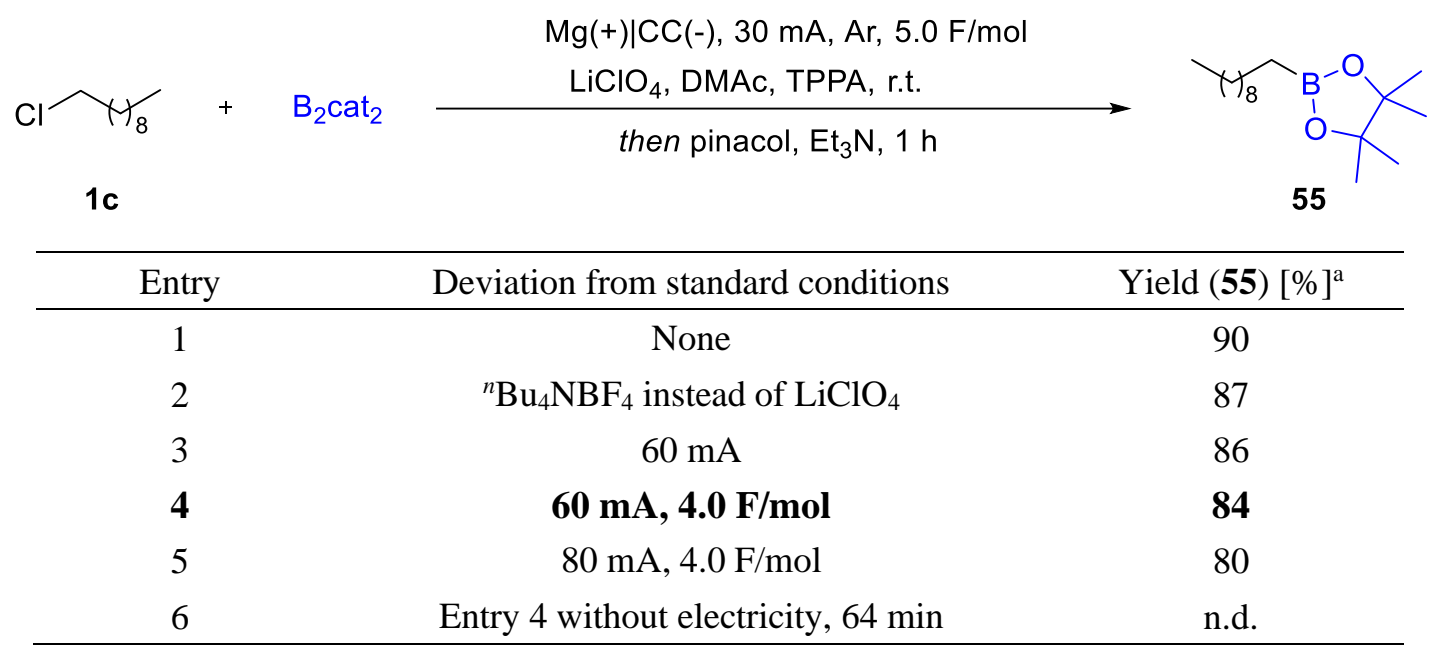

Reaction conditions: 1-chlorodecane $1 c$ ( $0.6 \mathrm{mmol}), \mathrm{B}_{2} \mathrm{cat}_{2}$ (4.0 equiv.), $\mathrm{LiClO}_{4}(0.3 \mathrm{mmol})$, dry DMAc:TPPA (3:1, $6.0 \mathrm{~mL}$ ), Mg plate and carbon cloth, undivided cell, constant current, $5.0 \mathrm{~F} / \mathrm{mol}$, room temperature (around $25{ }^{\circ} \mathrm{C}$ ), n.d. = not detected. Then pinacol $(8.0$ equiv. $), \mathrm{Et}_{3} \mathrm{~N}(1.5 \mathrm{~mL}), 1 \mathrm{~h} .{ }^{a} \mathrm{GC}$ yields using biphenyl as internal standard.

\subsection{Synthesis of Starting Materials}

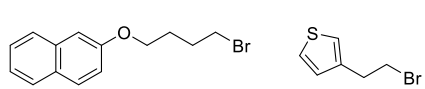

s1

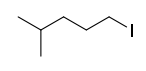

s7

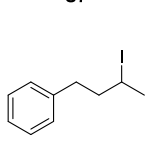

S13

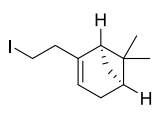

S19

S2

S8

S14

S20
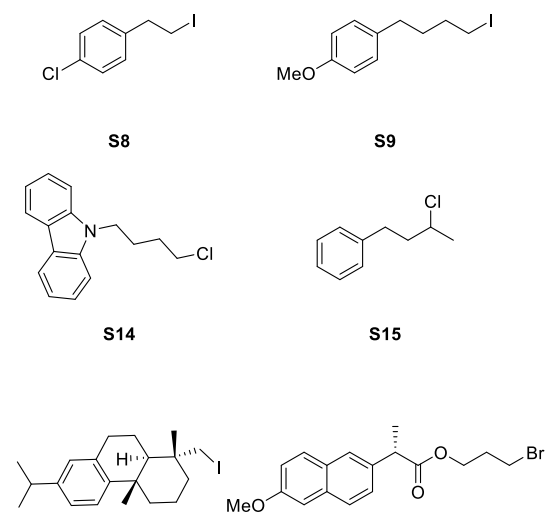

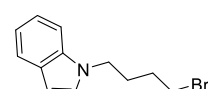

s3

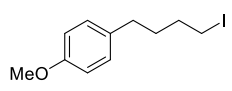

s9

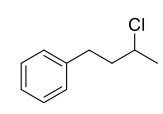

S15
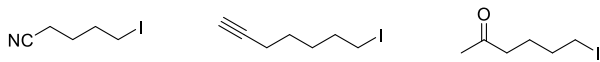

s4

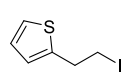

s10

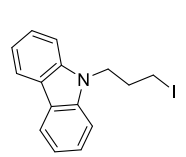

S11

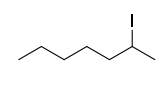

S12
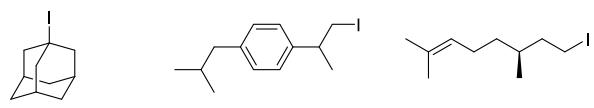

S16

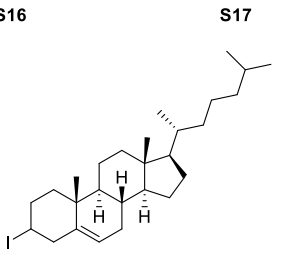

S22

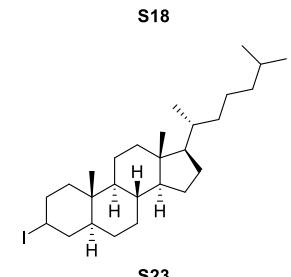

S23

Substrates $\mathbf{S} 1^{[1]}, \mathbf{S} 2^{[2]}, \mathbf{S} 3^{[3]}, \mathbf{S} 4^{[4]}, \mathbf{S 5 ^ { [ 4 ] }}, \mathbf{S} 6^{[4]}, \mathbf{S} 7^{[5]}, \mathbf{S} 8^{[5]}, \mathbf{S} 9^{[5]}, \mathbf{S 1 0} 0^{[5]}, \mathbf{S 1 1 ^ { [ 6 ] }}, \mathbf{S 1 2} 2^{[5]}$, $\mathbf{S} 13^{[5]}, \mathbf{S} 15^{[7]}, \mathbf{S} 16^{[8]}, \mathbf{S} 17^{[5,9]}, \mathbf{S} 18^{[5]}, \mathbf{S} 19^{[5]}, \mathbf{S} 20^{[5,9]}, \mathbf{S} 21^{[2]}, \mathbf{S} 22^{[5]}$ and $\mathbf{S} 23^{[5]}$ were prepared according to the literature. 


\section{General Procedure for the Synthesis of 9-(4-Chlorobutyl)-9H-Carbazole}
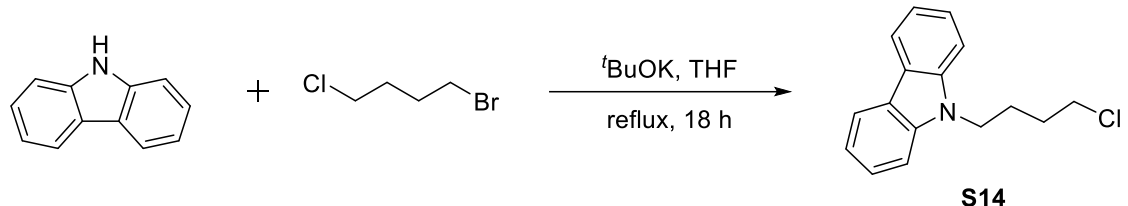

Potassium tert-butoxide (7.2 mmol, $0.808 \mathrm{~g})$ and 1-bromo-4-chlorobutane $(8.8 \mathrm{mmol}$, $1.5 \mathrm{~g})$ were added to a solution of carbazole $(7.2 \mathrm{mmol}, 1.2 \mathrm{~g})$ in tetrahydrofuran $(7.2$ $\mathrm{mL}$ ), afterwards, the reaction mixture was kept reflux for 18 hours, then the reaction mixture was evaporated in vacuum and purified by automated flash chromatography to give the title compound $\mathbf{S 1 4}$.

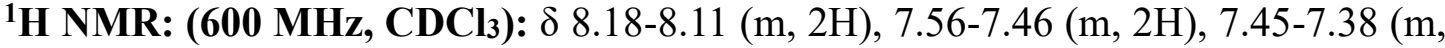
2H), 7.30-7.26 (m, 2H), $4.36(\mathrm{t}, J=7.0 \mathrm{~Hz}, 2 \mathrm{H}), 3.52(\mathrm{t}, J=6.4 \mathrm{~Hz}, 2 \mathrm{H}), 2.10-2.04(\mathrm{~m}$, 2H), $1.88-1.81(\mathrm{~m}, 2 \mathrm{H})$.

${ }^{13}$ C NMR: (151 MHz, CDCl3): $\delta$ 140.4, 125.8, 122.9, 120.5, 119.0, 108.7, 44.7, 42.4, $30.2,26.5$.

HRMS (ESI): calculated for $\mathrm{C}_{16} \mathrm{H}_{17} \mathrm{ClN}[\mathrm{M}+\mathrm{H}]^{+}:$258.1044, found: 258.1041 .

\subsection{Scale Up Experiments}

\subsubsection{Scale Up Experiments of Alkyl Bromide (4-Phenylbutyl Bromide)}

1. Materials used for scale up experiments.

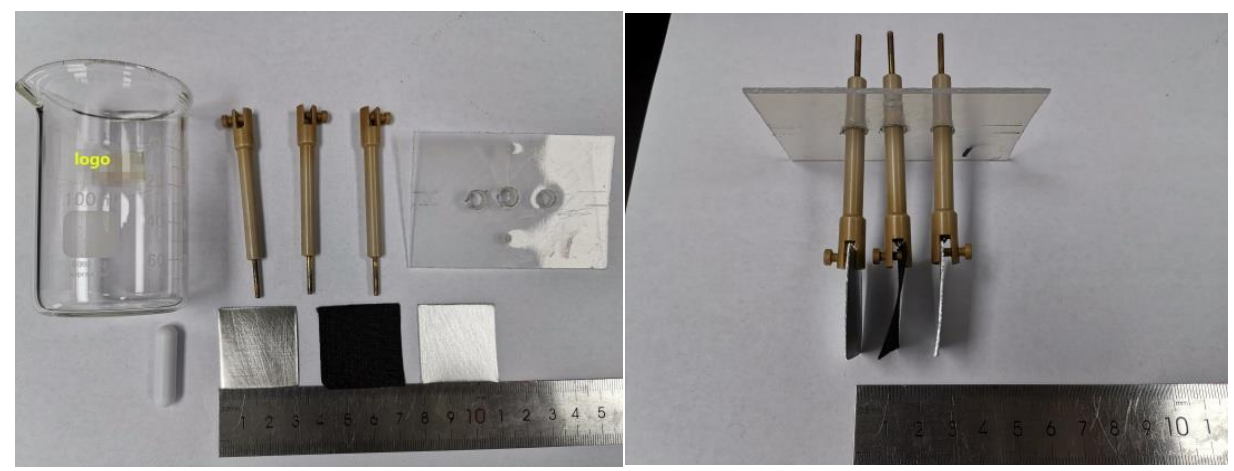

2. Reagents used in borylation: ${ }^{n} \mathrm{Bu}_{4} \mathrm{NBF}_{4}, \mathrm{~B}_{2}$ cat 2 , DMAc, Pinacol, Et ${ }_{3} \mathrm{~N}$ and the substrate. 


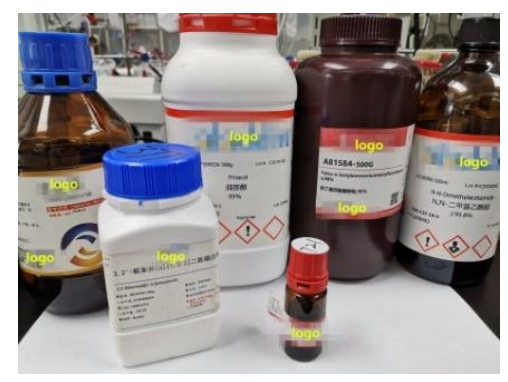

3. 4-Phenylbutyl bromide (7.2 mmol, 1.0 equiv.), $\mathrm{B}_{2} \mathrm{cat}_{2}(28.8 \mathrm{mmol}, 4$ equiv.) and ${ }^{n} \mathrm{Bu}_{4} \mathrm{NBF}_{4}$ (3.6 mmol, 0.5 equiv.) were added to the beaker $(100 \mathrm{~mL})$ equipped with a Teflon-coated magnetic stir bar.

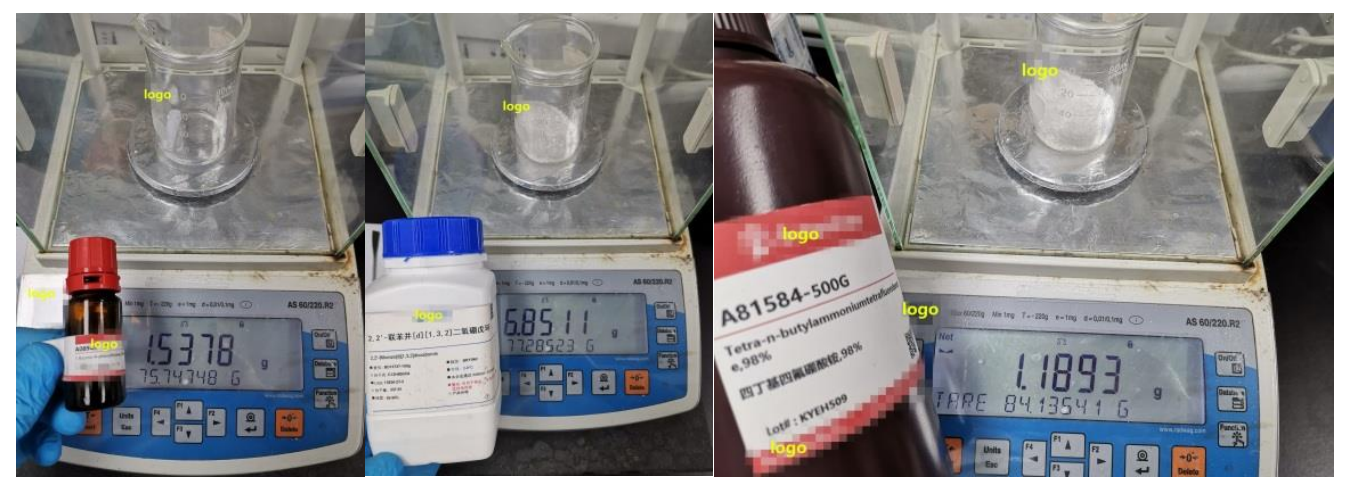

4. DMAc $(60.0 \mathrm{~mL})$ was added to the reaction mixture.

5. The reaction mixture was connected to a potentiostat with the current kept at $450 \mathrm{~mA}$ at room temperature $(3.0 \mathrm{~F} / \mathrm{mol}$, usually require $77 \mathrm{~min})$.

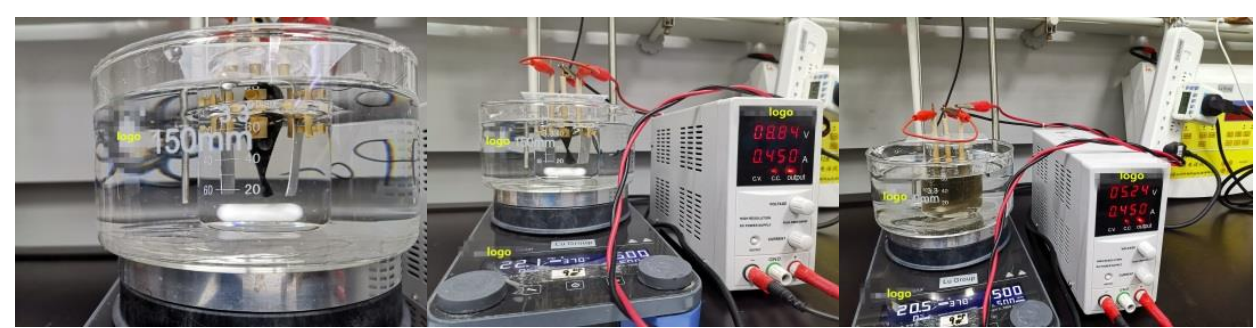

6. Initially, the current was set to $450 \mathrm{~mA}$ and the cell voltage was around $8.84 \mathrm{~V}$ (center picture). The cell voltage dropped to $5.24 \mathrm{~V}$ when the reaction was completed (right picture).

7. A solution of pinacol (57.6 mmol, 8.0 equiv.) in triethylamine $(18.0 \mathrm{~mL})$ was added to the electrolyzer cell and the reaction mixture kept stirring at room temperature for 1 h.

8. When the reaction is completed, the reaction mixture was transferred to a separatory funnel (the residual product on electrodes are washed with EtOAc). Saturated brine water $(500 \mathrm{~mL})$ was added and the aqueous layer was extracted with EtOAc (3 x 200 
$\mathrm{mL}$ ). The organic layer was dried over anhydrous $\mathrm{Na}_{2} \mathrm{SO}_{4}$ and concentrated in vacuum. The residue was purified by flash column chromatography on silica gel to furnish the desired product $(1.41 \mathrm{~g})$.

\subsubsection{Scale Up Experiments of Alkyl Iodides (Iodocyclohexane, $I=600 \mathrm{~mA}$ or 1000} $\mathbf{m A )}$

1. Iodocyclohexane (7.2 mmol, 1.0 equiv.), $\mathrm{B}_{2} \mathrm{cat}_{2}(28.8 \mathrm{mmol}, 4.0$ equiv.) and ${ }^{n} \mathrm{Bu}_{4} \mathrm{NBF}_{4}$ (3.6 mmol, 0.5 equiv.) was added to the beaker $(100 \mathrm{~mL})$ equipped with a Teflon-coated magnetic stir bar.

2. DMF $(60.0 \mathrm{~mL})$ was added to the reaction mixture.

3. The reaction mixture was connected to a potentiostat with the current kept at $600 \mathrm{~mA}$ at room temperature $(2.5 \mathrm{~F} / \mathrm{mol}$, usually require $48 \mathrm{~min})$.

4. The current was set to $600 \mathrm{~mA}$ and cell voltage was $10.2 \mathrm{~V}$. About $48 \mathrm{~min}$ of electrolysis and cell voltage dropped to $4.2 \mathrm{~V}$.

5. A solution of pinacol $(57.6 \mathrm{mmol}, 8.0$ equiv. $)$ in triethylamine $(18.0 \mathrm{~mL})$ was added to the electrolyzer cell and the reaction mixture kept stirring at room temperature for 1 h.

6. When the reaction is completed, the reaction mixture was transferred to a separatory funnel (The residual product on electrodes were washed with EtOAc). Saturated brine water $(500 \mathrm{~mL})$ was added and the aqueous layer was extracted with EtOAc $(3$ x 200 $\mathrm{mL}$ ). The organic layer was dried over anhydrous $\mathrm{Na}_{2} \mathrm{SO}_{4}$ and concentrated in vacuum. The residue was purified by flash column chromatography on silica gel to furnish the desired product $(1.14 \mathrm{~g})$.

\subsection{Mechanistic Studies}

\subsubsection{Cyclic Voltammetry Experiments}

General information: Cyclic voltammetry (CV) experiments were conducted in a 20 $\mathrm{mL}$ three-necked cell set-up fitted with a glassy carbon working electrode $(3 \mathrm{~mm}$ in diameter), an $\mathrm{Ag} / \mathrm{AgCl}$ reference electrode, and a platinum wire counter electrode. All measurements were carried out in dry DMAc with an electrolyte $\left({ }^{n} \mathrm{Bu}_{4} \mathrm{NBF}_{4}, 0.05\right.$ 
mmol), using a scan rate of $100 \mathrm{mV} / \mathrm{s}$.

\section{Blank:}

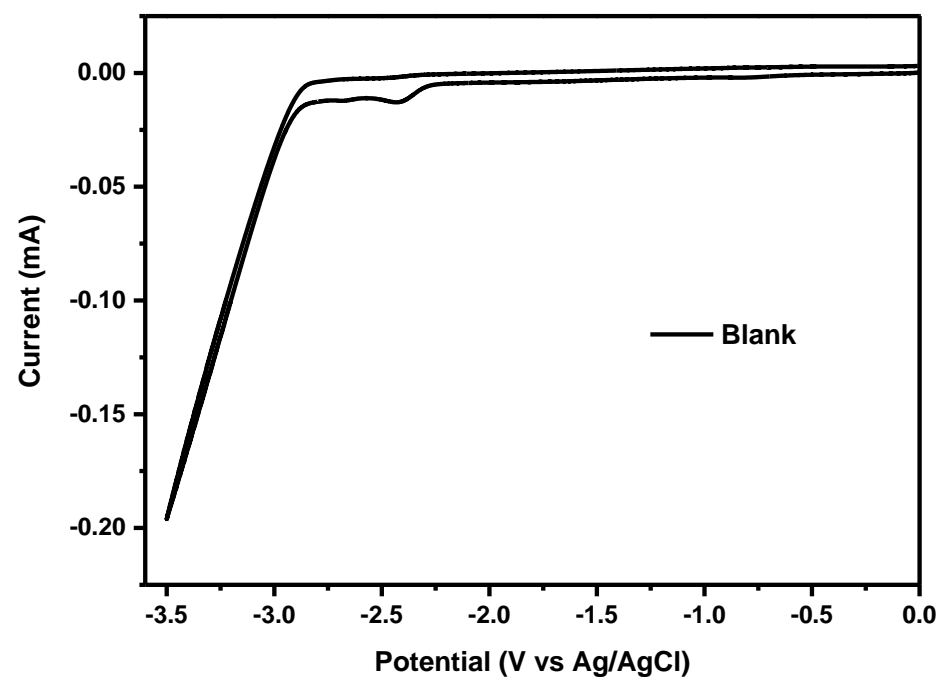

Figure S1. ${ }^{n} \mathrm{Bu}_{4} \mathrm{NBF}_{4}(0.05 \mathrm{mmol})$ in $6.0 \mathrm{~mL}$ DMAc.

\section{B2cat2:}

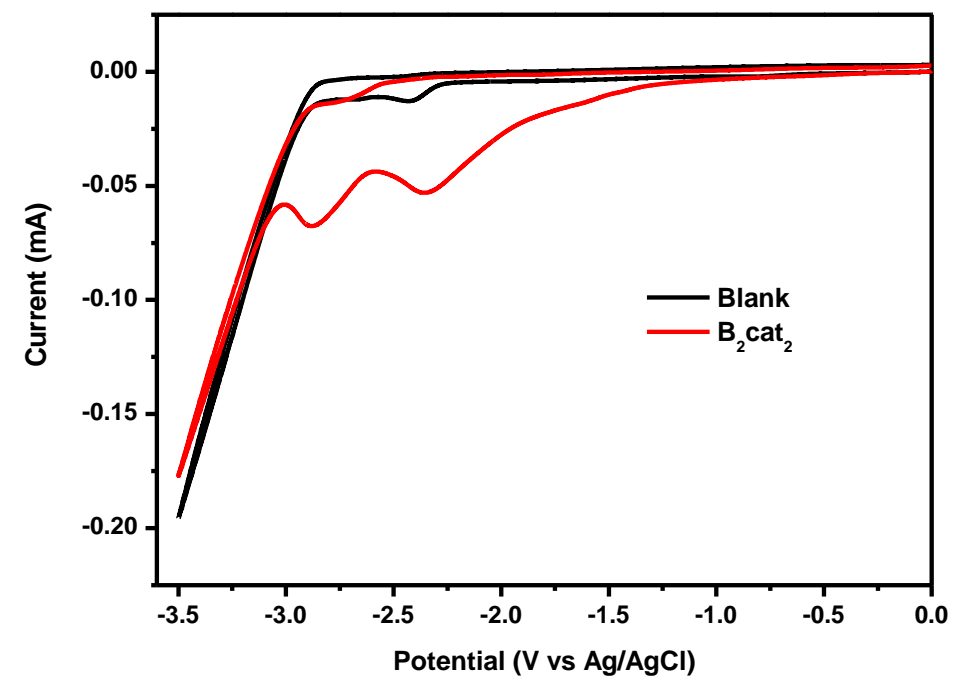

Figure S2. Blank line: ${ }^{n} \mathrm{Bu}_{4} \mathrm{NBF}_{4}(0.05 \mathrm{mmol})$ in $6.0 \mathrm{~mL}$ DMAc. Red line: $\mathrm{B}_{2} \mathrm{cat}_{2}(0.05$ mmol), ${ }^{n} \mathrm{Bu}_{4} \mathrm{NBF}_{4}(0.05 \mathrm{mmol})$ in $6.0 \mathrm{~mL}$ DMAc. 


\section{Cy-Br:}

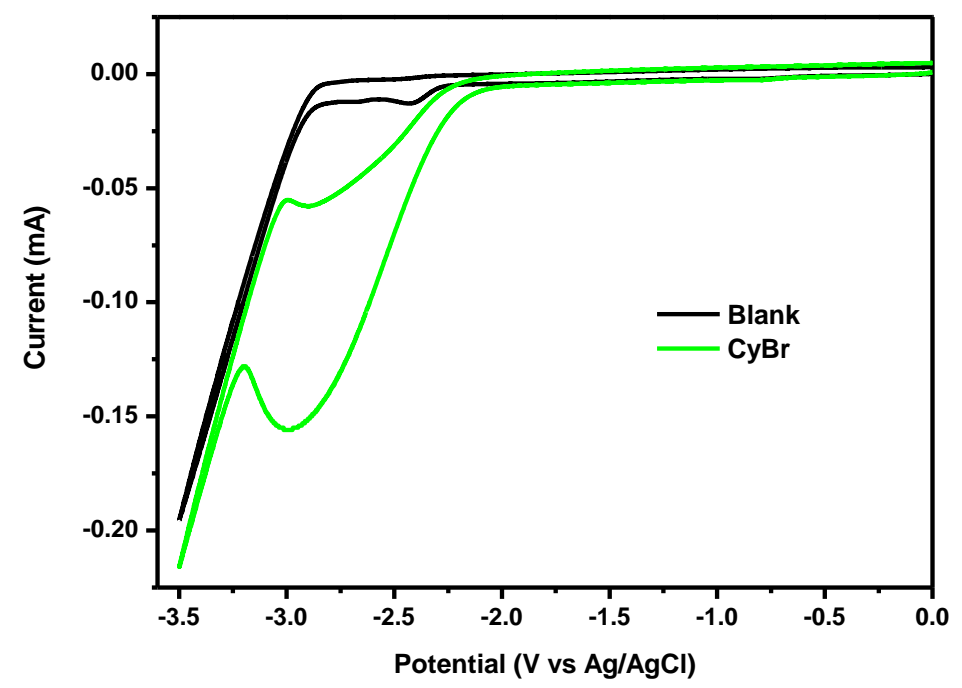

Figure S3. Blank line: ${ }^{n} \mathrm{Bu}_{4} \mathrm{NBF}_{4}(0.05 \mathrm{mmol})$ in $6.0 \mathrm{~mL}$ DMAc. Green line: $\mathrm{CyBr}$ (0.05 mmol), ${ }^{n} \mathrm{Bu}_{4} \mathrm{NBF}_{4}(0.05 \mathrm{mmol})$ in $6.0 \mathrm{~mL}$ DMAc.

\section{Cy-I:}

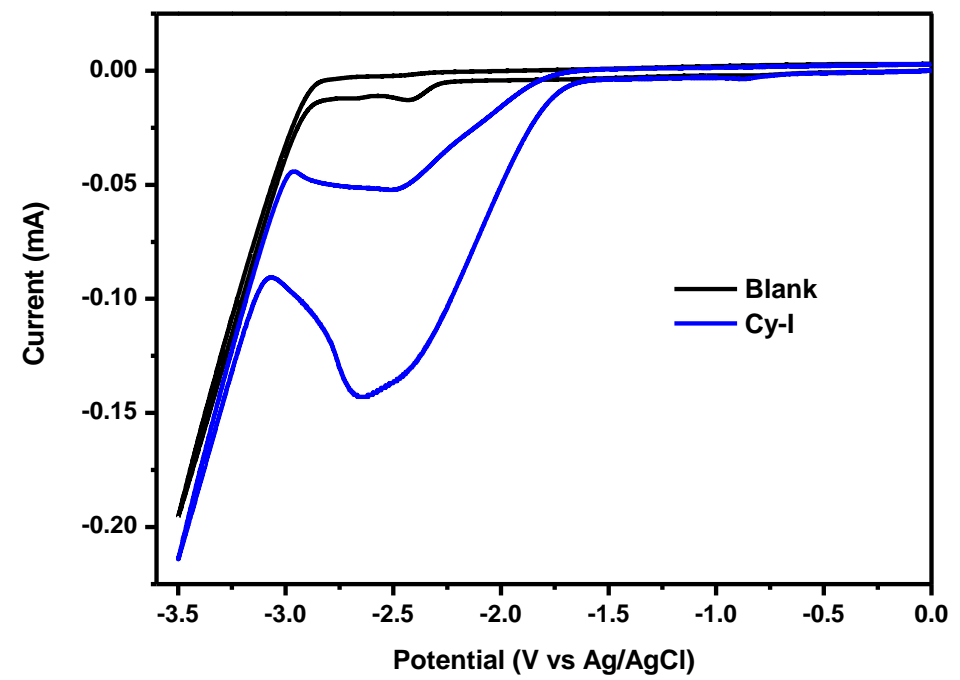

Figure S4. Blank line: ${ }^{n} \mathrm{Bu}_{4} \mathrm{NBF}_{4}(0.05 \mathrm{mmol})$ in $6.0 \mathrm{~mL}$ DMAc. Blue line: CyI $(0.05$ mmol), ${ }^{n} \mathrm{Bu}_{4} \mathrm{NBF}_{4}(0.05 \mathrm{mmol})$ in $6.0 \mathrm{~mL}$ DMAc. 


\section{Dec-Cl:}

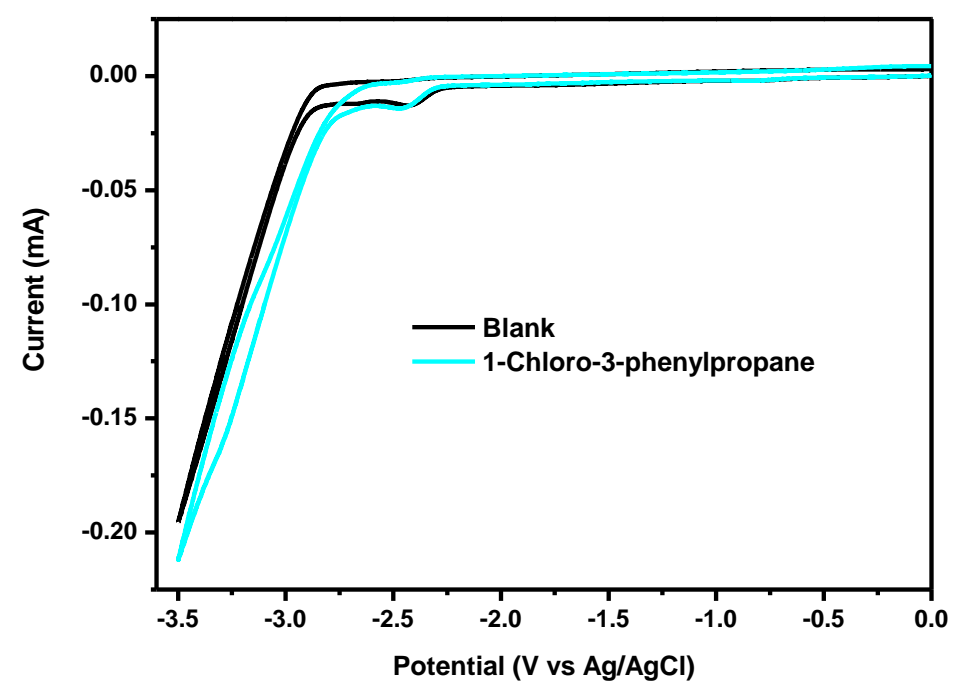

Figure S5. Blank line: ${ }^{n} \mathrm{Bu}_{4} \mathrm{NBF}_{4}(0.05 \mathrm{mmol})$ in $6.0 \mathrm{~mL}$ DMAc. Cyan line: 1-Chloro3-phenylpropane (0.05 mmol), ${ }^{n} \mathrm{Bu}_{4} \mathrm{NBF}_{4}(0.05 \mathrm{mmol})$ in $6.0 \mathrm{~mL}$ DMAc.

\section{Summary:}

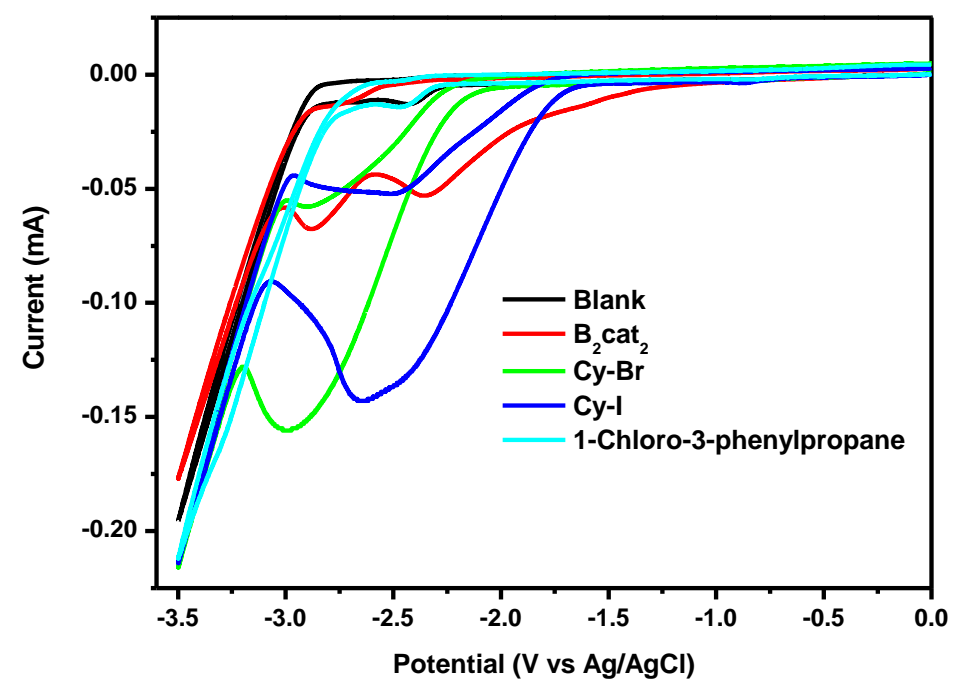

Figure S6. Blank line: ${ }^{n} \mathrm{Bu}_{4} \mathrm{NBF}_{4}(0.05 \mathrm{mmol})$ in $6.0 \mathrm{~mL}$ DMAc. Red line: $\mathrm{B}_{2} \mathrm{cat}_{2}(0.05$ mmol), ${ }^{n} \mathrm{Bu}_{4} \mathrm{NBF}_{4}(0.05 \mathrm{mmol})$ in $6.0 \mathrm{~mL}$ DMAc. Green line: $\mathrm{CyBr}(0.05 \mathrm{mmol})$, ${ }^{n} \mathrm{Bu}_{4} \mathrm{NBF}_{4}(0.05 \mathrm{mmol})$ in $6.0 \mathrm{~mL}$ DMAc. Blue line: $\mathrm{CyI}(0.05 \mathrm{mmol}),{ }^{n} \mathrm{Bu}_{4} \mathrm{NBF}_{4}(0.05$ mmol) in 6.0 mL DMAc. Cyan line: 1-Chloro-3-phenylpropane $(0.05 \mathrm{mmol}),{ }^{n} \mathrm{Bu}_{4} \mathrm{NBF}_{4}$ $(0.05 \mathrm{mmol})$ in $6.0 \mathrm{~mL}$ DMAc. 


\subsubsection{Constant Voltage Electrolysis}

\section{Constant Voltage Electrolysis of Bromocyclohexane:}

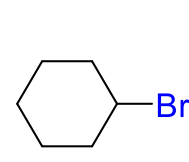

1a Cov. $66 \%$
$\mathrm{Mg}(+) \mid \mathrm{CC}(-),-1.7 \mathrm{~V}(\mathrm{vs} . \mathrm{Ag} / \mathrm{AgCl}), 8 \mathrm{~h}, \mathrm{Ar}$

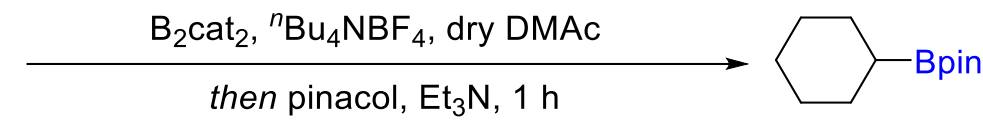

$1,38 \%$

$\mathrm{B}_{2} \mathrm{cat}_{2}(2.4 \mathrm{mmol}, 4.0$ equiv., $571.0 \mathrm{mg})$ and ${ }^{n} \mathrm{Bu}_{4} \mathrm{NBF}_{4}(0.3 \mathrm{mmol}, 99.0 \mathrm{mg})$ were added into an oven-dried three-necked undivided cell $(20 \mathrm{~mL})$ equipped with a Teflon-coated magnetic stir bar. The undivided cell was equipped with $\mathrm{Mg}$ plate $(15 \mathrm{~mm} \times 15 \mathrm{~mm} \times 0.5$ $\mathrm{mm})$ as the anode, carbon cloth electrode $(15 \mathrm{~mm} \times 15 \mathrm{~mm})$ as the cathode and $\mathrm{Ag} / \mathrm{AgCl}$ as reference electrode and then charged with argon to replace air atmosphere. Bromocyclohexane $(0.6 \mathrm{mmol}, 97.8 \mathrm{mg})$ and dry DMAc $(6.0 \mathrm{~mL})$ were added to the tube through a syringe firstly. Pre-stirring the resulting mixture until it is completely dissolved, and then the reaction mixture was electrolyzed at a constant potential of -1.7 $\mathrm{V}$ in dark at room temperature (around $25^{\circ} \mathrm{C}$ ) for $8 \mathrm{~h}$. Afterwards, a solution of pinacol (4.8 mmol, 8.0 equiv., $567.2 \mathrm{mg}$ ) in triethylamine $(1.5 \mathrm{~mL}$ ) was added to the electrolyzer cell and the reaction mixture kept stirring at room temperature for $1 \mathrm{~h}$. The conversion of 1a and yield of $\mathbf{1}$ were determined by GC analysis using dodecane as internal standard. For comparison, the same reaction was conducted unless no current was passed and $6 \%$ yield of 1 was obtained.

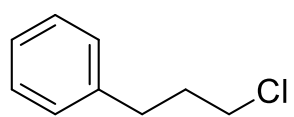

47a, Cov. $58 \%$
$\mathrm{Mg}(+) \mid \mathrm{CC}(-),-2.1 \mathrm{~V}(\mathrm{vs} . \mathrm{Ag} / \mathrm{AgCl}), 16 \mathrm{~h}, \mathrm{Ar}$

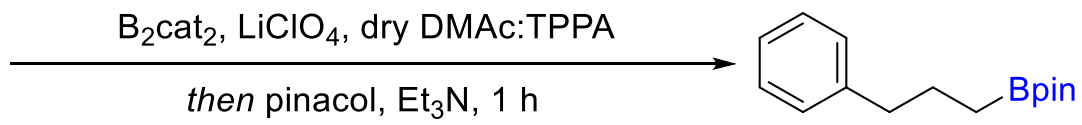

$47,36 \%$

\section{Constant Voltage Electrolysis of 1-Chloro-3-Phenylpropane:}

$\mathrm{B}_{2}$ cat 2 (2.4 mmol, 4.0 equiv., $\left.571.0 \mathrm{mg}\right)$ and $\mathrm{LiClO}_{4}(0.3 \mathrm{mmol}, 32.0 \mathrm{mg})$ were added into an oven-dried three-necked undivided cell $(20 \mathrm{~mL})$ equipped with a Teflon-coated magnetic stir bar. The undivided cell was equipped with $\mathrm{Mg}$ plate $(15 \mathrm{~mm} \times 15 \mathrm{~mm} \times 0.5$ $\mathrm{mm})$ as the anode, carbon cloth electrode $(15 \mathrm{~mm} \times 15 \mathrm{~mm})$ as the cathode and $\mathrm{Ag} / \mathrm{AgCl}$ as reference electrode and then charged with argon to replace air atmosphere. 1-Chloro- 
3-phenylpropane (0.6 mmol, 93.0 mg) and dry DMAc:TPPA (3:1, $6.0 \mathrm{~mL})$ were added to the tube through a syringe firstly. Pre-stirring the resulting mixture until it is completely dissolved, and then the reaction mixture was electrolyzed at a constant potential of $-2.1 \mathrm{~V}$ at room temperature (around $25^{\circ} \mathrm{C}$ ) for $16 \mathrm{~h}$. Afterwards, a solution of pinacol (4.8 mmol, 8.0 equiv., $567.2 \mathrm{mg})$ in triethylamine $(1.5 \mathrm{~mL})$ was added to the electrolyzer cell and the reaction mixture kept stirring at room temperature for $1 \mathrm{~h}$. The conversion of $\mathbf{4 7} \mathbf{a}$ and yield of $\mathbf{4 7}$ were determined by GC analysis using dodecane as internal standard. For comparison, the same reaction was conducted unless no current was passed and $\mathbf{4 7}$ was not detected.

\subsubsection{Radical Clock Experiments of (Bromomethyl)cyclopropane}

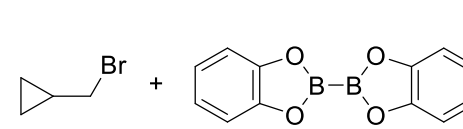

$68 \mathbf{a}$

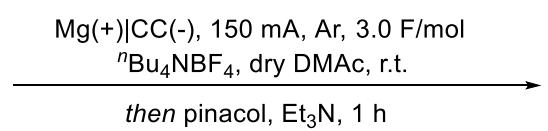

then pinacol, $\mathrm{Et}_{3} \mathrm{~N}, 1 \mathrm{~h}$

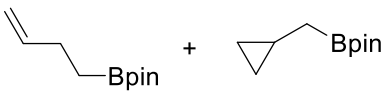

$68 \quad 69$

$(68+69)=57 \%, 68: 69=10: 1$

68a (0.6 mmol, $81.0 \mathrm{mg})$ was borylated according to General Procedure A. The crude reside was purified by flash column chromatography, affording $68+69(62.0 \mathrm{mg}, 57 \%)$ as a colorless oil. Note: the product is volatile! The ratio was determined by ${ }^{1} \mathrm{H}$ NMR spectrum.

68+69[10,11]: ${ }^{1} \mathrm{H}$ NMR: (600 MHz, CDCl3): $\delta 5.88$ (ddt, $\left.J=16.6,10.2,6.2 \mathrm{~Hz}, 1 \mathrm{H}\right)$, 5.02-4.95 (m, 1H), 4.91-4.83 (m, 1H), 2.19-2.12 (m, 2H), $1.23(\mathrm{~s}, 12 \mathrm{H}), 1.21(\mathrm{~s}, 1 \mathrm{H})$, $0.88(\mathrm{t}, J=7.8 \mathrm{~Hz}, 2 \mathrm{H})$ 0.78-0.74 (m, 0.3H), 0.43-0.40 (m, 0.2H), 0.01--0.01 (m, 0.2H).

${ }^{13}$ C NMR: (151 MHz, CDCl3): $\delta$ 140.8, 113.3, 83.1, 83.0, 28.1, 24.9, 24.9, 16.0, 6.1, 5.9.

${ }^{11}$ B NMR: (193 MHz, CDCl 3$): ~ \delta 34.00$. 


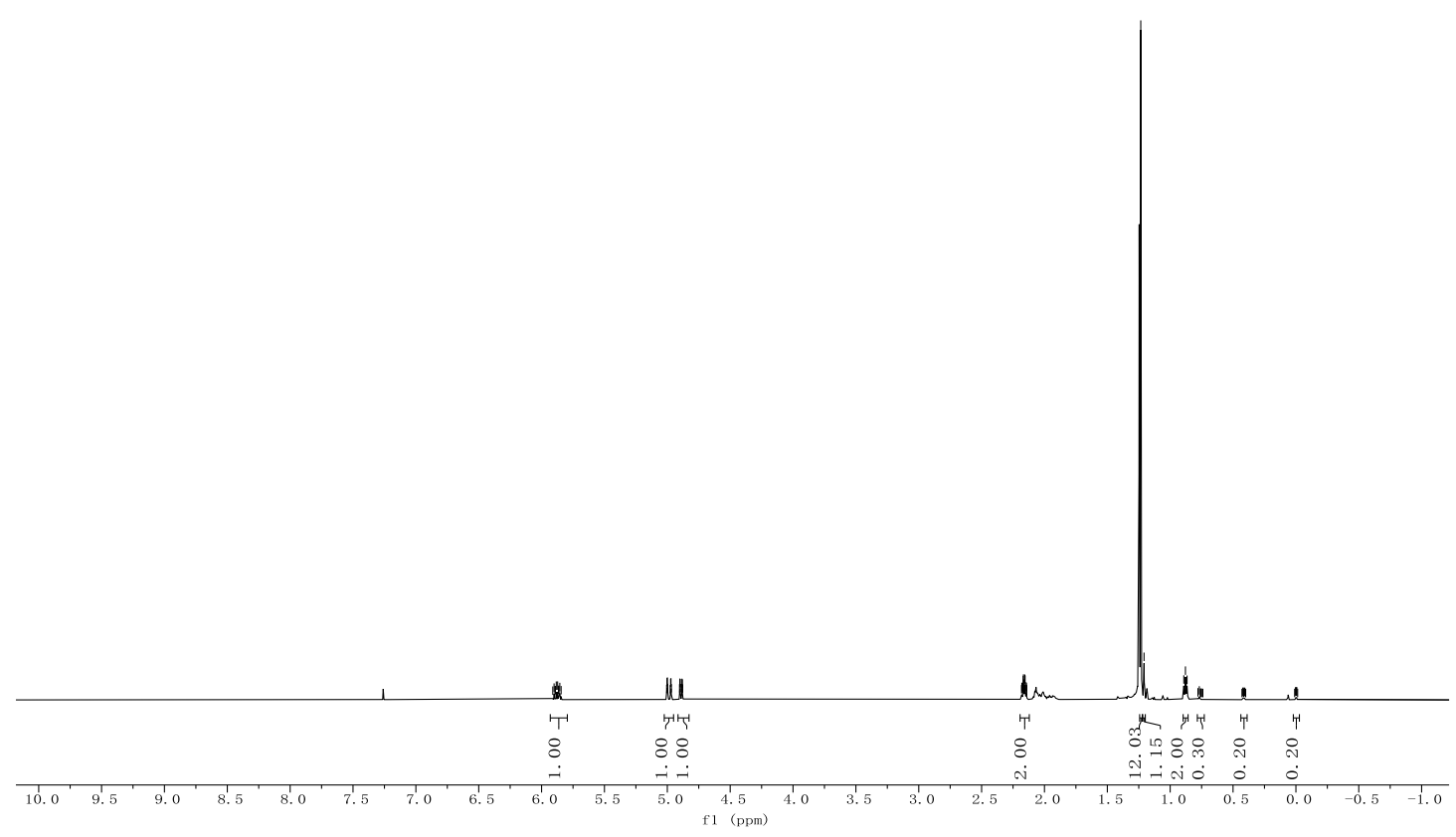

Figure S7. The ${ }^{1} \mathrm{H}$ NMR spectrum of 68 and 69.
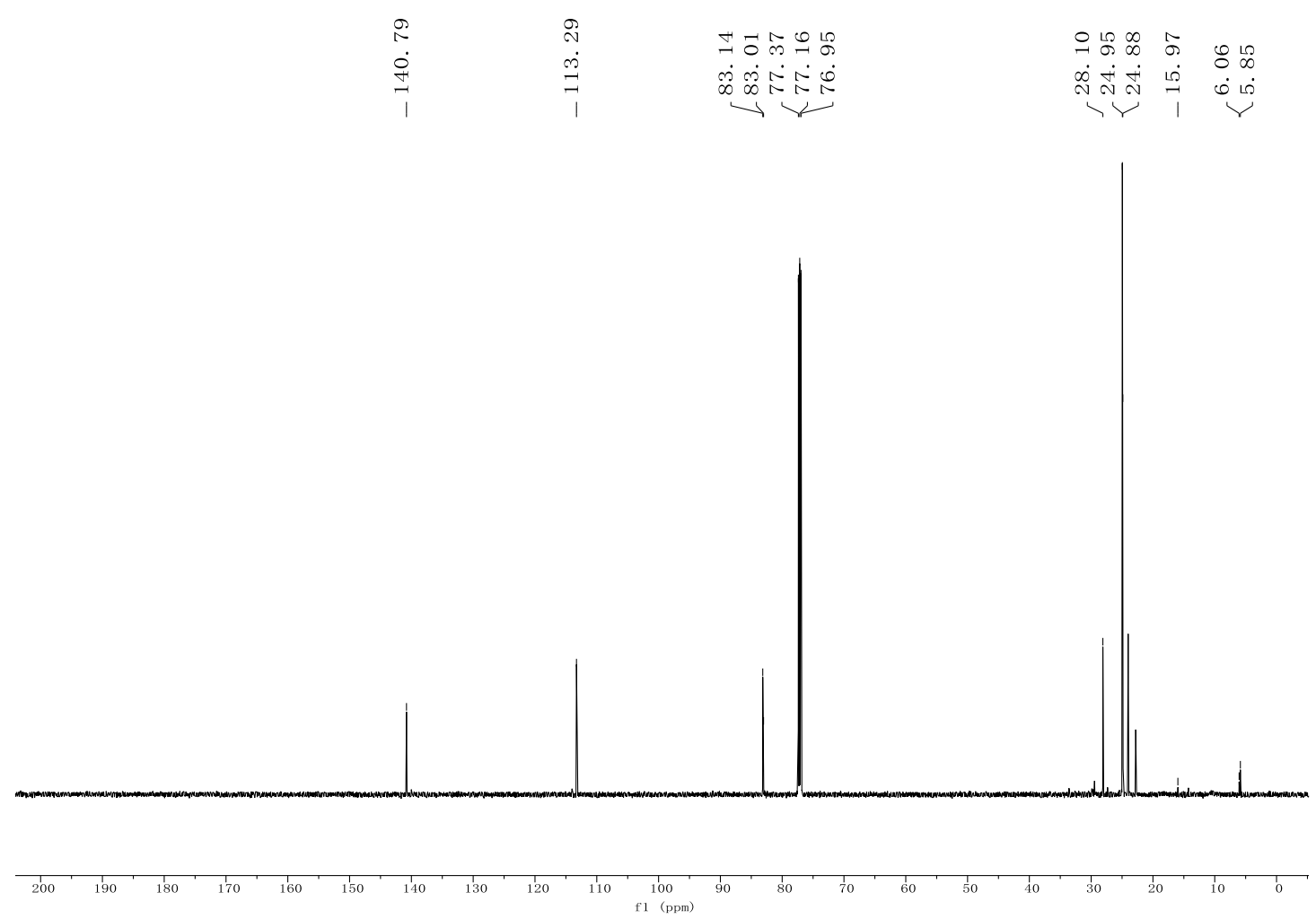

Figure S8. The ${ }^{13} \mathrm{C}$ NMR spectrum of 68 and 69. 

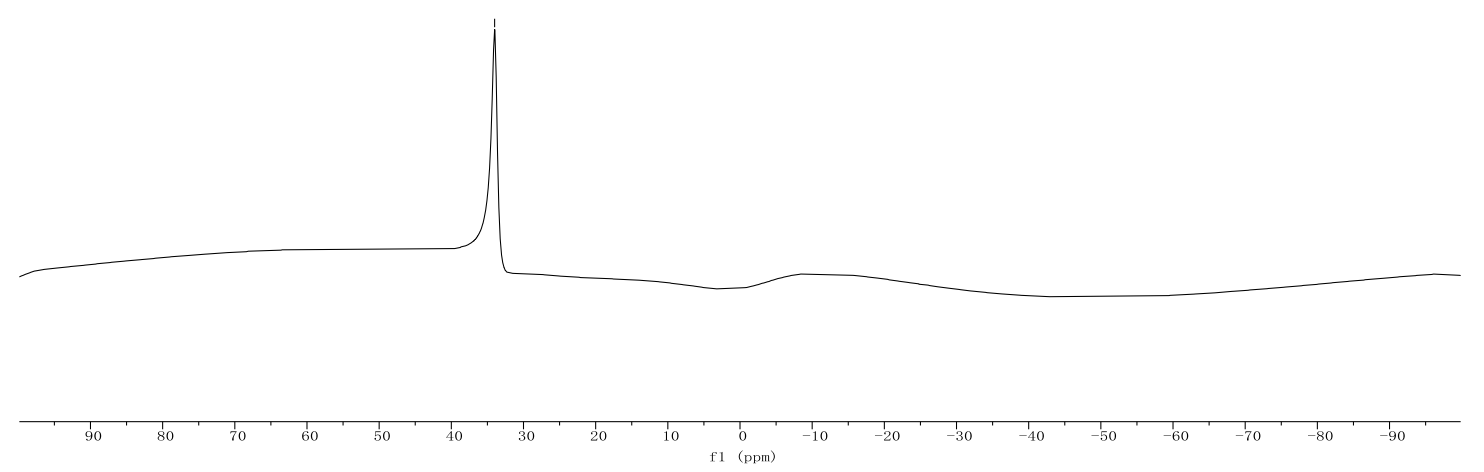

Figure S9. The ${ }^{11}$ B NMR spectrum of 68 and 69.

\subsection{4 ${ }^{11}$ B NMR Experiments}

$\infty$
$\infty$
$\infty$
$\infty$
$\infty$
1

1) $\mathrm{B}_{2} \mathrm{Cat}_{2}$ in $\mathrm{CDCl}_{3}$

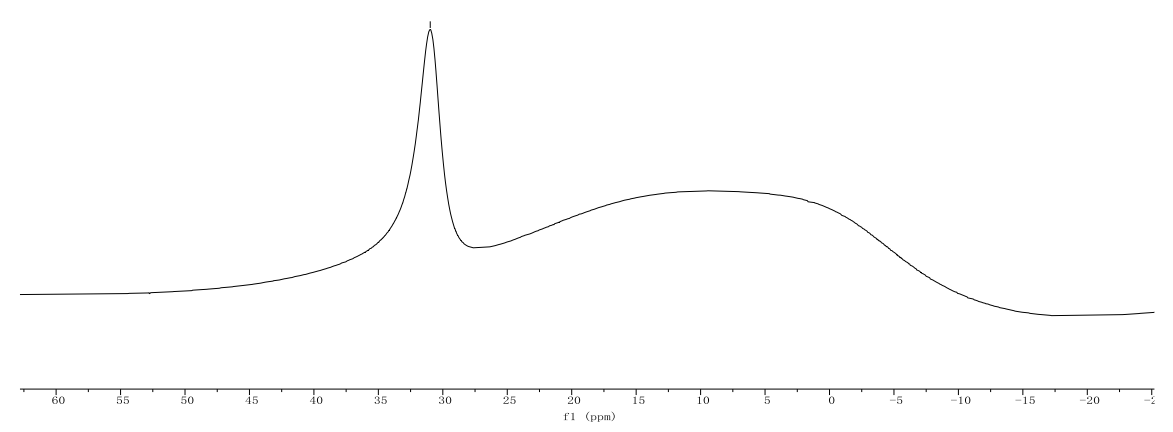

Figure S10. ${ }^{11} \mathrm{~B}$ NMR spectrum of $\mathrm{B}_{2} \mathrm{cat}_{2}(0.4 \mathrm{mmol}, 95.0 \mathrm{mg})$ and $\mathrm{CDCl}_{3}(0.5 \mathrm{~mL})$. 


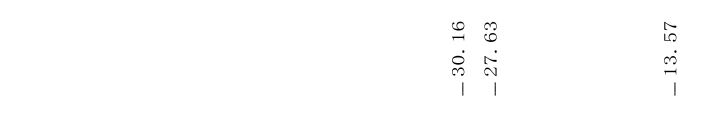

2) $\mathrm{B}_{2} \mathrm{Cat}_{2}$ in DMAc and $\mathrm{CDCl}_{3}$

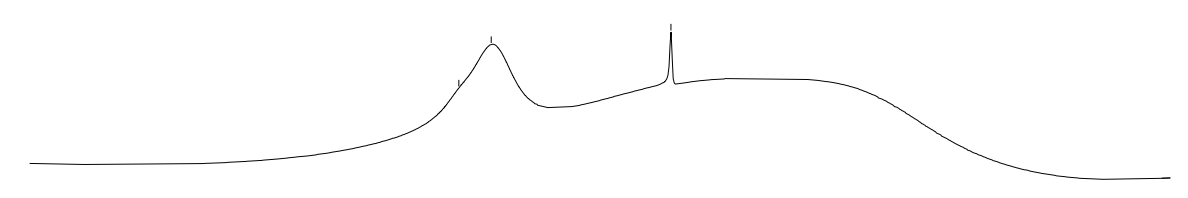

Figure S11. ${ }^{11} \mathrm{~B}$ NMR spectrum of solution which was prepared according to following procedure: $0.2 \mathrm{~mL}$ of mixed solution containing $\mathrm{B}_{2} \mathrm{cat}_{2}(0.4 \mathrm{mmol}, 95.0 \mathrm{mg})$ and DMAc $(1.0 \mathrm{~mL})$ was dissolved $\mathrm{CDCl}_{3}(0.5 \mathrm{~mL})$.
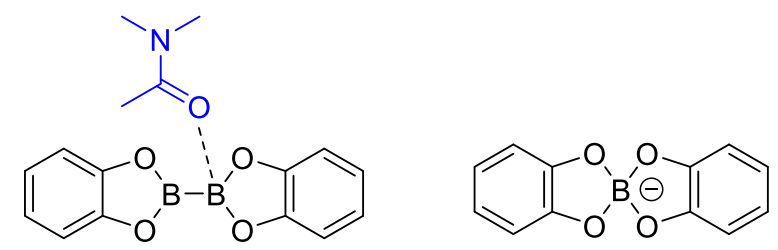

$31.0 \mathrm{ppm}$

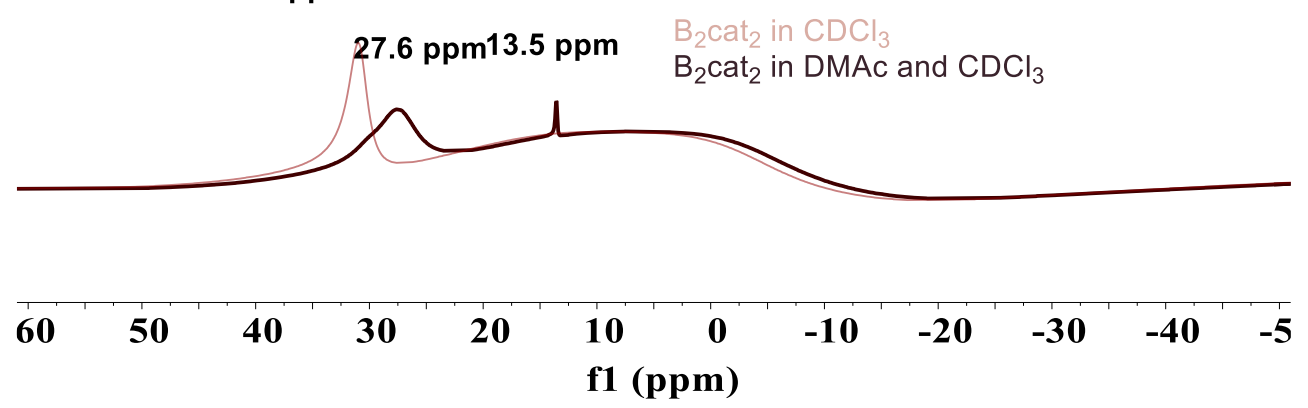

Figure S12. ${ }^{11} \mathrm{~B}$ NMR spectrum.

Comparing the ${ }^{11} \mathrm{~B}$ NMR spectrum of $\mathrm{B}_{2} \mathrm{Cat}_{2}$ in $\mathrm{CDCl}_{3}$ with that in a mixed-solvent system of DMAc and $\mathrm{CDCl}_{3}$ reveals that the presence of DMAc led to a chemical shift from $31.0 \mathrm{ppm}$ (a single signal) to $27.6 \mathrm{ppm}$ (a broad signal), illustrating the existence of ligated diboron. 


\subsection{Investigation of $B_{2}$ pin 2 as Boron Source}

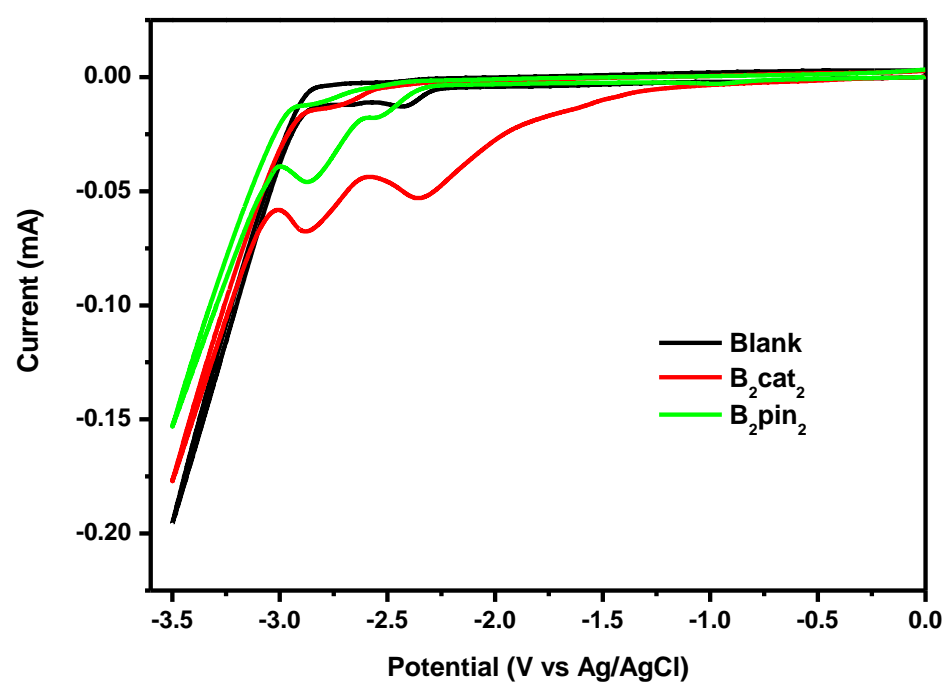

Figure S13. Comparison between $\mathrm{B}_{2} \mathrm{pin}_{2}$ and $\mathrm{B}_{2}$ cat 2 . Blank line: ${ }^{n} \mathrm{Bu}_{4} \mathrm{NBF}_{4}(0.05 \mathrm{mmol})$ in $6.0 \mathrm{~mL}$ dry DMAc. Green line: $\mathrm{B}_{2} \mathrm{pin}_{2}(0.05 \mathrm{mmol}),{ }^{n} \mathrm{Bu}_{4} \mathrm{NBF}_{4}(0.05 \mathrm{mmol})$ in 6.0 mL dry DMAc. Red line: $\mathrm{B}_{2} \mathrm{cat}_{2}(0.05 \mathrm{mmol}),{ }^{n} \mathrm{Bu}_{4} \mathrm{NBF}_{4}(0.05 \mathrm{mmol})$ in $6.0 \mathrm{~mL}$ dry DMAc.

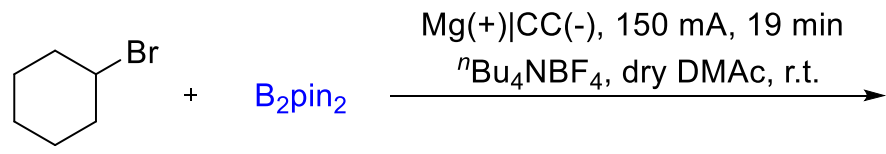

1a, Cov. $100 \%$

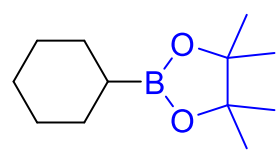

1, n.d.

$\mathrm{B}_{2} \operatorname{pin}_{2}$ (2.4 mmol, 4.0 equiv., 609.5 mg) and ${ }^{n} \mathrm{Bu}_{4} \mathrm{NBF}_{4}(0.3 \mathrm{mmol}, 99.0 \mathrm{mg})$ were added into an oven-dried undivided three-necked cell $(20 \mathrm{~mL})$ equipped with a Teflon-coated magnetic stir bar. The undivided cell was equipped with $\mathrm{Mg}$ plate $(15 \mathrm{~mm} \times 15 \mathrm{~mm} \times 0.5$ $\mathrm{mm})$ as the anode and carbon cloth electrode $(15 \mathrm{~mm} \times 15 \mathrm{~mm})$ as the cathode and then charged with argon to replace air atmosphere. Bromocyclohexane $(0.6 \mathrm{mmol}, 97.8 \mathrm{mg})$ and dry DMAc $(6.0 \mathrm{~mL})$ were added to the tube through a syringe. Pre-stirring the resulting mixture until it is completely dissolved, and then the reaction mixture was electrolyzed at a constant current of $150 \mathrm{~mA}$ until passing $3.0 \mathrm{~F} / \mathrm{mol}$ of charge (around $19 \mathrm{~min}$ ) at room temperature (around $25^{\circ} \mathrm{C}$ ). The conversion of $\mathbf{1 a}$ and yield of $\mathbf{1}$ were determined by GC analysis using biphenyl as internal standard. 


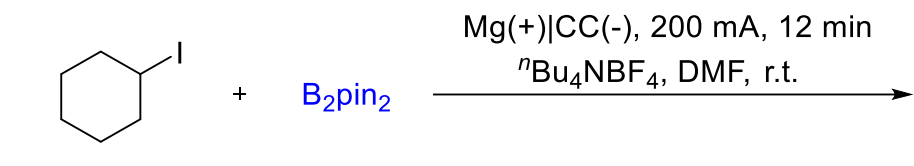

1b, Cov. $100 \%$

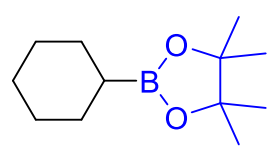

1, n.d.

$\mathrm{B}_{2} \operatorname{pin}_{2}(2.4 \mathrm{mmol}, 4.0$ equiv., $609.5 \mathrm{mg})$ and ${ }^{n} \mathrm{Bu}_{4} \mathrm{NBF}_{4}(0.3 \mathrm{mmol}, 99.0 \mathrm{mg})$ were added into an oven-dried undivided three-necked cell $(20 \mathrm{~mL})$ equipped with a Teflon-coated magnetic stir bar. The undivided cell was equipped with $\mathrm{Mg}$ plate $(15 \mathrm{~mm} \times 15 \mathrm{~mm} \times 0.5$ $\mathrm{mm})$ as the anode and carbon cloth electrode $(15 \mathrm{~mm} \times 15 \mathrm{~mm})$ as the cathode and then charged with argon to replace air atmosphere. Iodocyclohexane $(0.6 \mathrm{mmol}, 126.0 \mathrm{mg})$ and DMF $(6.0 \mathrm{~mL})$ were added to the tube through a syringe. Pre-stirring the resulting mixture until it is completely dissolved, and then the reaction mixture was electrolyzed at a constant current of $200 \mathrm{~mA}$ until passing $2.5 \mathrm{~F} / \mathrm{mol}$ of charge (around $12 \mathrm{~min}$ ) at room temperature (around $25{ }^{\circ} \mathrm{C}$ ). The conversion of $\mathbf{1 b}$ and yield of $\mathbf{1}$ were determined by GC analysis using biphenyl as internal standard.

\subsection{Expand this Electrochemical Borylation to Sacrificial Anode-Free Conditions}

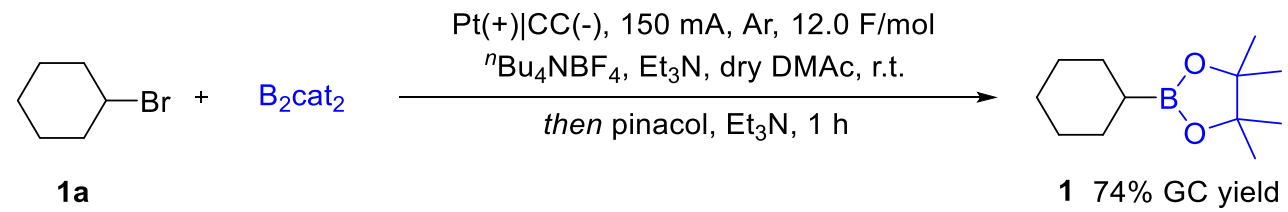

$\mathrm{B}_{2} \mathrm{cat}_{2}(2.4 \mathrm{mmol}, 4.0$ equiv., $571.0 \mathrm{mg})$ and ${ }^{n} \mathrm{Bu}_{4} \mathrm{NBF}_{4}(0.3 \mathrm{mmol}, 99.0 \mathrm{mg})$ were added into an oven-dried undivided three-necked cell $(20 \mathrm{~mL})$ equipped with a Teflon-coated magnetic stir bar. The undivided cell was equipped with Pt plate $(15 \mathrm{~mm} \times 15 \mathrm{~mm} \times 0.5$ $\mathrm{mm})$ as the anode and carbon cloth electrode $(15 \mathrm{~mm} \times 15 \mathrm{~mm})$ as the cathode and then charged with argon to replace air atmosphere. Bromocyclohexane (0.6 mmol, $97.8 \mathrm{mg})$, $\mathrm{Et}_{3} \mathrm{~N}$ (2.4 mmol, 4.0 equiv., $\left.242.8 \mathrm{mg}\right)$ and dry DMAc $(6.0 \mathrm{~mL})$ were added to the tube through a syringe. Pre-stirring the resulting mixture until it is completely dissolved, and then the reaction mixture was electrolyzed at a constant current of $150 \mathrm{~mA}$ until passing 12.0 F/mol of charge (around $72 \mathrm{~min}$ ) at room temperature (around $25^{\circ} \mathrm{C}$ ). Afterwards, a solution of pinacol ( $4.8 \mathrm{mmol}, 8.0$ equiv., $567.2 \mathrm{mg})$ in triethylamine $(1.5 \mathrm{~mL})$ was added to the electrolyzer cell and the reaction mixture kept stirring at room temperature for $1 \mathrm{~h}$. The yield of 1 was determined by GC analysis using biphenyl as internal 
standard.

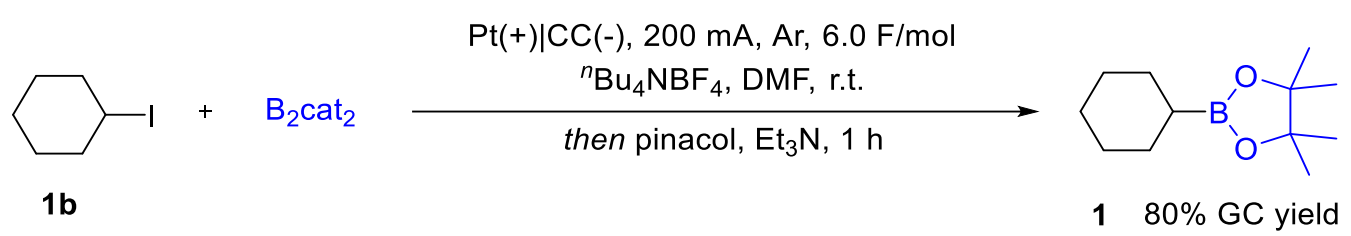

$\mathrm{B}_{2} \mathrm{cat}_{2}(2.4 \mathrm{mmol}, 4.0$ equiv., $571.0 \mathrm{mg})$ and ${ }^{n} \mathrm{Bu}_{4} \mathrm{NBF}_{4}(0.3 \mathrm{mmol}, 99.0 \mathrm{mg})$ were added into an oven-dried undivided three-necked cell $(20 \mathrm{~mL})$ equipped with a Teflon-coated magnetic stir bar. The undivided cell was equipped with Pt plate $(15 \mathrm{~mm} \times 15 \mathrm{~mm} \times 0.5$ $\mathrm{mm})$ as the anode and carbon cloth electrode $(15 \mathrm{~mm} \times 15 \mathrm{~mm})$ as the cathode and then charged with argon to replace air atmosphere. Iodocyclohexane $(0.6 \mathrm{mmol}, 126.0 \mathrm{mg})$ and DMF $(6.0 \mathrm{~mL})$ were added to the tube through a syringe. Pre-stirring the resulting mixture until it is completely dissolved, and then the reaction mixture was electrolyzed at a constant current of $200 \mathrm{~mA}$ until passing $12.0 \mathrm{~F} / \mathrm{mol}$ of charge (around $29 \mathrm{~min}$ ) at room temperature (around $25^{\circ} \mathrm{C}$ ). Afterwards, a solution of pinacol (4.8 mmol, 8.0 equiv., $567.2 \mathrm{mg})$ in triethylamine $(1.5 \mathrm{~mL})$ was added to the electrolyzer cell and the reaction mixture kept stirring at room temperature for $1 \mathrm{~h}$. The yield of $\mathbf{1}$ was determined by GC analysis using biphenyl as internal standard.

\subsection{Computational Details}

All density functional theory (DFT) calculations were carried out using the Gaussian 16 software package ${ }^{[12]}$. All geometries were optimized using the B3LYP hybrid functional ${ }^{[13,14]}$ with a basis set of $6-31 G(d)$ for all atoms and Grimme's D3(BJ) dispersion correction $(\mathrm{GD} 3 \mathrm{BJ})^{[15,16]}$. Frequencies were calculated for all the stationary points to confirm if each optimized structure is a local minimum on the respective potential energy surface or a transition state structure with only one imaginary frequency. Solvation energy correction was calculated in $\mathrm{N}, \mathrm{N}$-dimethylacetamide solvent with the SMD continuum solvation model ${ }^{[17]}$ based on the gas phase optimized geometries. The M06 functional ${ }^{[18]}$ with a basis set of $6-311+\mathrm{G}(\mathrm{d}, \mathrm{p})$ for all atoms was used for single point energy calculations. 
In our computational studies, the transition state of radical-radical cross-coupling in path $\mathrm{b}$ is not located. Computational results show that the formation of complex VI and R-Bcat (1) through the radical-radical cross-coupling between $\mathrm{Cy} \cdot$ and the $\mathrm{B}_{2} \mathrm{cat}_{2}$ radical anion II is exergonic by $55.8 \mathrm{kcal} / \mathrm{mol}$. Therefore, we surmise that this radicalradical cross-coupling step is most likely to be barrier-less.

\subsubsection{Free Energy Profile of Radical Substitution Pathway in Path a}

Our computational results show that the radical substitution pathway (path a) consists of the cyclohexyl radical addition to I (via TS-1) and the B-B bond cleavage (via TS2). The overall activation free energy is calculated to be $9.1 \mathrm{kcal} / \mathrm{mol}$ (Figure S14). The formation of DMAc-stabilized boryl radical III and the product $\mathbf{1}$ in this pathway is exergonic by $22.3 \mathrm{kcal} / \mathrm{mol}$.

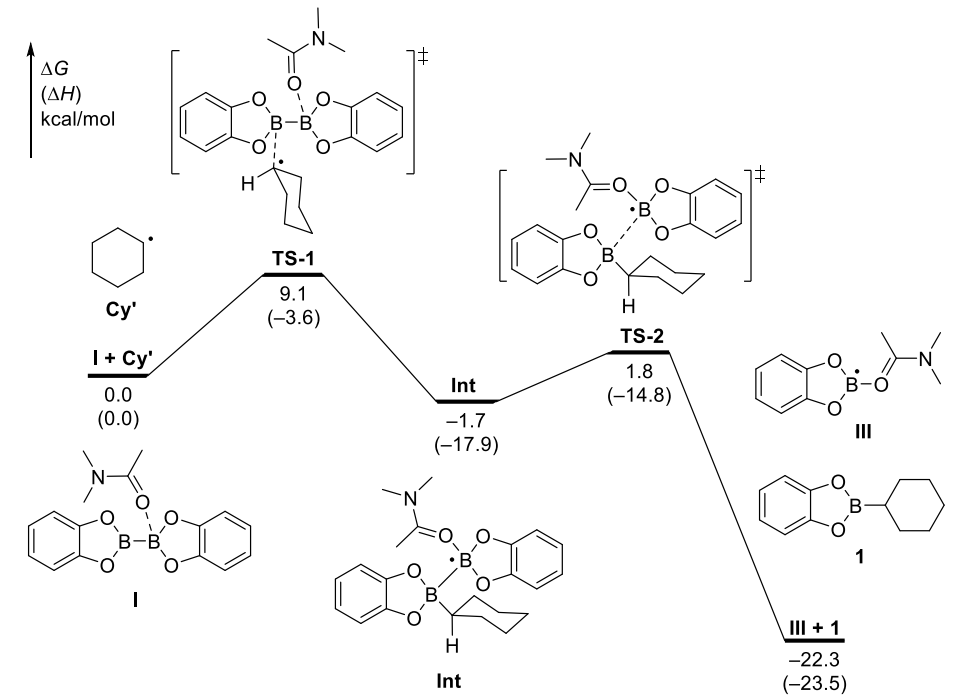

Figure S14. Free energy profile of radical substitution pathway in path a.

\subsubsection{Computational Results of the Transformation of Boryl Anion VI}

As shown in Figure S15a, the nucleophilic addition of VI towards cyclohexyl bromide may occur through $\mathrm{S}_{\mathrm{N}} 2$ mechanism, leading to the formation of product $\mathbf{1}$. However, the activation free energy of transition state TS-3 is $58.7 \mathrm{kcal} / \mathrm{mol}$, which indicates this pathway is kinetically impossible. Moreover, we speculated that boryl anion VI can be stabilized by combing with anode-generated $\mathrm{Mg}^{2+}$ (Figure S15b). The computational results also support that this process is highly exergonic. 

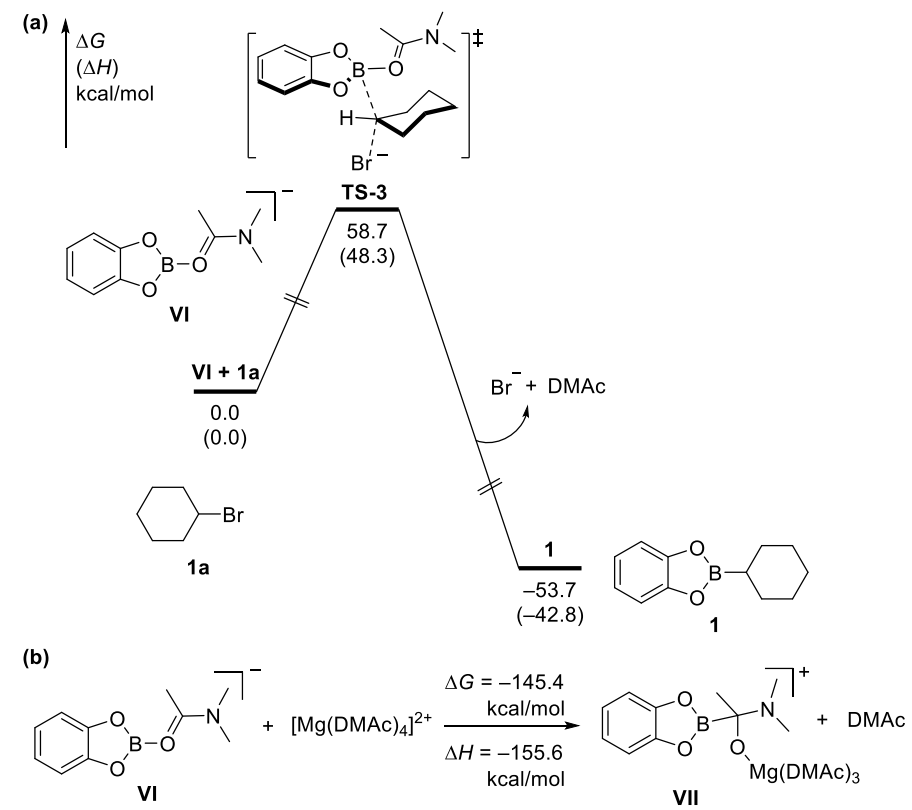

Figure S15. Computational results of the transformation of boryl anion VI.

\subsubsection{Computational Results of the Halogen Atom Transfer Mechanism for the} Formation of Alkyl Radical.

As shown in Figure S16, the cyclohexyl radical can be generated through the $\mathrm{Br}$ atom transfer transition state TS-4 with an activation free energy of $14.2 \mathrm{kcal} / \mathrm{mol}$. The formation of intermediate VIII and cyclohexyl radical is demonstrated to be exergonic by $18.5 \mathrm{kcal} / \mathrm{mol}$. These results suggest that the halogen atom transfer mechanism is kinetically and thermodynamically possible.

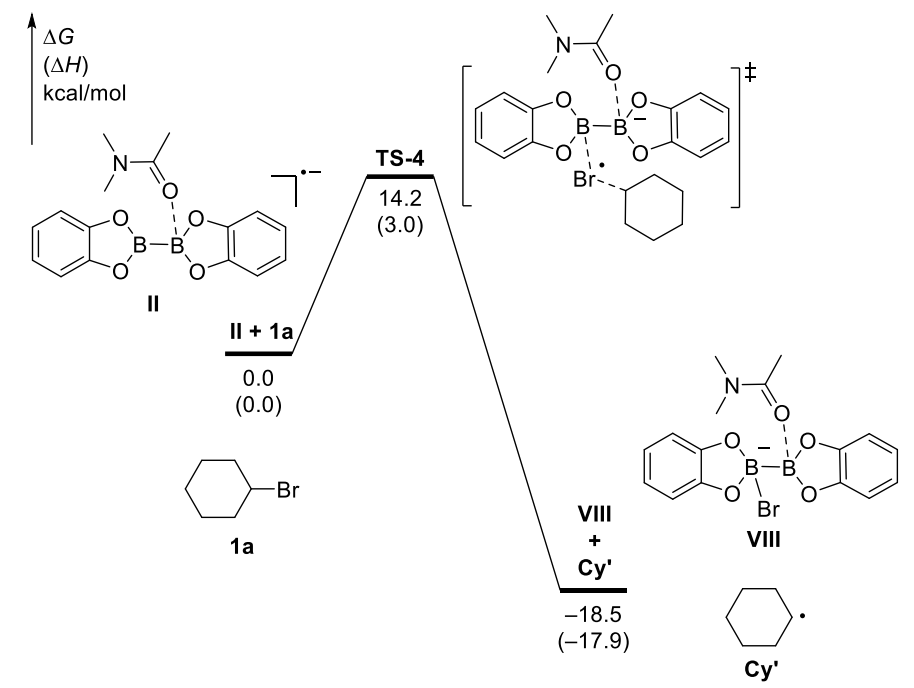

Figure S16. Computational results of the halogen atom transfer mechanism for the formation of alkyl radical. 


\subsubsection{Cartesian Coordinates and Energies of Optimized Structures}

\section{I}

B3LYP SCF energy: $\quad-1100.78566253$ a.u.

B3LYP enthalpy: $\quad-1100.441786$ a.u.

B3LYP free energy: $\quad-1100.518687$ a.u.

M06 SCF energy in solution: $\quad-1100.31334667$ a.u.

M06 enthalpy in solution: $\quad-1099.969470$ a.u.

M06 free energy in solution: $\quad-1100.046371$ a.u.

Cartesian coordinates

$\begin{array}{lccc}\text { ATOM } & \text { X } & \text { Y } & \text { Z } \\ \text { B } & 0.247939 & -1.047630 & 0.359986 \\ \text { B } & -1.406511 & -0.802686 & 0.264283 \\ \text { O } & 1.074955 & -0.432894 & 1.311267 \\ \text { O } & 1.010355 & -1.831916 & -0.505503 \\ \text { O } & -2.249800 & -1.374867 & -0.683309 \\ \text { O } & -2.126451 & 0.025800 & 1.129044 \\ \text { C } & 2.359769 & -0.782877 & 0.969140 \\ \text { C } & 2.319224 & -1.637448 & -0.135230 \\ \text { C } & 3.553788 & -0.389398 & 1.549056 \\ \text { C } & 3.472130 & -2.141741 & -0.712342 \\ \text { C } & 4.730439 & -0.889595 & 0.969055 \\ \text { H } & 5.690161 & -0.607378 & 1.391101 \\ \text { C } & 4.689853 & -1.747128 & -0.136524 \\ \text { H } & 5.619060 & -2.116871 & -0.559097 \\ \text { H } & 3.571450 & 0.269609 & 2.410607 \\ \text { H } & 3.428196 & -2.802320 & -1.571290 \\ \text { C } & -3.498976 & -0.854783 & -0.432523 \\ \text { C } & -3.424996 & -0.002239 & 0.670784 \\ \text { C } & -4.688338 & -1.083643 & -1.102670 \\ \text { H } & -4.733121 & -1.747357 & -1.959139 \\ \text { C } & -4.535459 & 0.664058 & 1.159328 \\ \text { H } & -4.465368 & 1.322793 & 2.018202 \\ \text { C } & -5.746551 & 0.441861 & 0.487517 \\ \text { H } & -6.644128 & 0.944728 & 0.834570 \\ \text { C } & -5.820996 & -0.412860 & -0.618649 \\ \text { H } & -6.775414 & -0.560863 & -1.114759 \\ \text { O } & -0.060950 & 1.084578 & -1.147975 \\ \text { C } & 0.658186 & 1.874770 & -0.522265 \\ \text { N } & 1.962110 & 2.075442 & -0.873273 \\ \text { C } & 0.113120 & 2.630516 & 0.675965 \\ \text { H } & 0.635589 & 2.331926 & 1.590399 \\ \text { H } & 0.205616 & 3.715496 & 0.558891 \\ \text { H } & -0.937773 & 2.364286 & 0.780200\end{array}$




$\begin{array}{llll}\mathrm{C} & 2.907050 & 2.884491 & -0.125368 \\ \mathrm{H} & 2.402077 & 3.486423 & 0.627753 \\ \mathrm{H} & 3.645707 & 2.242807 & 0.373861 \\ \mathrm{H} & 3.441159 & 3.559444 & -0.805629 \\ \mathrm{C} & 2.536576 & 1.312095 & -1.972767 \\ \mathrm{H} & 1.749241 & 0.710156 & -2.422416 \\ \mathrm{H} & 2.959114 & 1.993342 & -2.721713 \\ \mathrm{H} & 3.332748 & 0.655182 & -1.603211\end{array}$

\section{II}

B3LYP SCF energy: $\quad-1100.78723618$ a.u.

B3LYP enthalpy: $\quad-1100.446443$ a.u.

B3LYP free energy: $\quad-1100.524704$ a.u.

M06 SCF energy in solution: $\quad-1100.37978877$ a.u.

M06 enthalpy in solution: $\quad-1100.038996$ a.u.

M06 free energy in solution: $\quad-1100.117257$ a.u.

Cartesian coordinates

$\begin{array}{lccc}\text { ATOM } & \text { X } & \text { Y } & \text { Z } \\ \text { B } & -1.368529 & -0.992372 & 0.123000 \\ \text { B } & 0.212365 & -1.274545 & 0.179071 \\ \text { O } & -1.979808 & 0.295684 & 0.437043 \\ \text { O } & -2.452094 & -1.886706 & -0.227492 \\ \text { O } & 0.945890 & -2.480665 & -0.138464 \\ \text { O } & 1.194528 & -0.270394 & 0.545424 \\ \text { C } & -3.315166 & 0.144960 & 0.272316 \\ \text { C } & -3.598874 & -1.174928 & -0.129534 \\ \text { C } & -4.323173 & 1.082223 & 0.444062 \\ \text { C } & -4.901578 & -1.589337 & -0.369199 \\ \text { C } & -5.648613 & 0.664929 & 0.201844 \\ \text { H } & -6.460710 & 1.377083 & 0.328619 \\ \text { C } & -5.930613 & -0.640654 & -0.195426 \\ \text { H } & -6.961210 & -0.937592 & -0.375954 \\ \text { H } & -4.090273 & 2.096357 & 0.754743 \\ \text { H } & -5.110177 & -2.608729 & -0.679288 \\ \text { C } & 2.262305 & -2.183090 & 0.000314 \\ \text { C } & 2.414733 & -0.845374 & 0.410306 \\ \text { C } & 3.364874 & -2.997775 & -0.213482 \\ \text { H } & 3.240271 & -4.029051 & -0.529976 \\ \text { C } & 3.664540 & -0.277545 & 0.611132 \\ \text { H } & 3.755183 & 0.760988 & 0.912906 \\ \text { C } & 4.785140 & -1.104141 & 0.392406 \\ \text { H } & 5.781296 & -0.693189 & 0.539241 \\ \text { C } & 4.639885 & -2.432393 & -0.008129\end{array}$




$\begin{array}{lrrr}\mathrm{H} & 5.523425 & -3.046070 & -0.169198 \\ \mathrm{O} & 2.690161 & 3.051062 & 0.858120 \\ \mathrm{C} & 1.580254 & 2.781385 & 0.389751 \\ \mathrm{~N} & 1.411747 & 2.466686 & -0.933938 \\ \mathrm{C} & 0.336924 & 2.822681 & 1.261760 \\ \mathrm{H} & -0.420750 & 3.507576 & 0.863424 \\ \mathrm{H} & -0.116368 & 1.831012 & 1.342327 \\ \mathrm{H} & 0.646493 & 3.166548 & 2.249921 \\ \mathrm{C} & 0.183923 & 1.933937 & -1.501262 \\ \mathrm{H} & -0.036641 & 2.448398 & -2.447225 \\ \mathrm{H} & 0.272633 & 0.856718 & -1.690802 \\ \mathrm{H} & -0.656644 & 2.059622 & -0.824011 \\ \mathrm{C} & 2.594486 & 2.292099 & -1.757989 \\ \mathrm{H} & 3.429727 & 2.798735 & -1.276337 \\ \mathrm{H} & 2.841239 & 1.227348 & -1.867234 \\ \mathrm{H} & 2.417400 & 2.718311 & -2.754194\end{array}$

\section{$1 \mathbf{a}$}

B3LYP SCF energy: $\quad-2806.71528063$ a.u.

B3LYP enthalpy: $\quad-2806.545497$ a.u.

B3LYP free energy: $\quad-2806.585563$ a.u.

M06 SCF energy in solution: $\quad-2809.17622876$ a.u.

M06 enthalpy in solution: $\quad-2809.006445$ a.u.

M06 free energy in solution: $\quad-2809.046511$ a.u.

Cartesian coordinates

$\begin{array}{lccc}\text { ATOM } & \text { X } & \text { Y } & \text { Z } \\ \text { C } & 0.646172 & 1.267018 & -0.135043 \\ \text { C } & -0.006016 & 0.000000 & 0.406598 \\ \text { C } & 0.646172 & -1.267018 & -0.135042 \\ \text { C } & 2.150422 & -1.264975 & 0.192728 \\ \text { C } & 2.837156 & 0.000000 & -0.338970 \\ \text { C } & 2.150422 & 1.264975 & 0.192728 \\ \text { H } & 0.157394 & -2.152550 & 0.283428 \\ \text { H } & 0.006146 & 0.000000 & 1.499416 \\ \text { H } & 0.504063 & 1.302104 & -1.222579 \\ \text { H } & 0.157395 & 2.152551 & 0.283427 \\ \text { H } & 2.285288 & -1.321474 & 1.282428 \\ \text { H } & 2.616590 & -2.163928 & -0.227759 \\ \text { H } & 3.898860 & 0.000000 & -0.064520 \\ \text { H } & 2.793884 & 0.000000 & -1.437521 \\ \text { H } & 2.285288 & 1.321474 & 1.282428 \\ \text { H } & 2.616590 & 2.163928 & -0.227759 \\ \text { H } & 0.504062 & -1.302105 & -1.222578\end{array}$


$\mathrm{Br}$

$\mathbf{C y}^{\prime}$

B3LYP SCF energy: $\quad-235.23429288$ a.u.

B3LYP enthalpy: $\quad-235.070823$ a.u.

B3LYP free energy: $\quad-235.107545$ a.u.

M06 SCF energy in solution: $\quad-235.08089256$ a.u.

M06 enthalpy in solution: $\quad-234.917423$ a.u.

M06 free energy in solution: $\quad-234.954145$ a.u.

Cartesian coordinates

$\begin{array}{lccc}\text { ATOM } & \text { X } & \text { Y } & \text { Z } \\ \text { C } & -0.000012 & 1.408221 & 0.272879 \\ \text { C } & 1.265510 & 0.711905 & -0.245193 \\ \text { C } & 1.288490 & -0.776501 & 0.158938 \\ \text { C } & 0.000015 & -1.462159 & -0.169166 \\ \text { C } & -1.288475 & -0.776525 & 0.158934 \\ \text { C } & -1.265526 & 0.711884 & -0.245188 \\ \text { H } & 2.136173 & -1.289381 & -0.311970 \\ \text { H } & 1.291189 & 0.785914 & -1.341000 \\ \text { H } & 2.164097 & 1.216345 & 0.131158 \\ \text { H } & -0.000010 & 1.391162 & 1.373231 \\ \text { H } & -0.000022 & 2.464234 & -0.025050 \\ \text { H } & -2.136146 & -1.289420 & -0.311980 \\ \text { H } & -1.473213 & -0.828152 & 1.250634 \\ \text { H } & -1.291215 & 0.785900 & -1.340994 \\ \text { H } & -2.164119 & 1.216303 & 0.131174 \\ \text { H } & 1.473223 & -0.828119 & 1.250639 \\ \text { H } & 0.000024 & -2.525738 & -0.393070\end{array}$

$\mathrm{Br}^{-}$

B3LYP SCF energy: $\quad-2571.46438018$ a.u.

B3LYP enthalpy: $\quad-2571.462020$ a.u.

B3LYP free energy: $\quad-2571.480556$ a.u.

M06 SCF energy in solution: $\quad-2574.18270800$ a.u.

M06 enthalpy in solution: $\quad-2574.180348$ a.u.

M06 free energy in solution: $\quad-2574.198884$ a.u.

Cartesian coordinates

$\begin{array}{lccr}\text { ATOM } & \text { X } & \text { Y } & \text { Z } \\ \mathrm{Br} & 0.000000 & 0.000000 & 0.000000\end{array}$

1

B3LYP SCF energy: $\quad-641.77331678$ a.u. 
B3LYP enthalpy: $\quad-641.504912$ a.u.

B3LYP free energy: $\quad-641.558648$ a.u.

M06 SCF energy in solution: $\quad-641.44820425$ a.u.

M06 enthalpy in solution: $\quad-641.179799$ a.u.

M06 free energy in solution: $\quad-641.233535$ a.u.

Cartesian coordinates

$\begin{array}{lrrr}\text { ATOM } & \text { X } & \text { Y } & \text { Z } \\ \text { C } & 2.262620 & -1.271951 & 0.134456 \\ \text { C } & 1.600552 & 0.000093 & -0.439209 \\ \text { C } & 2.262565 & 1.271873 & 0.135134 \\ \text { C } & 3.780468 & 1.267630 & -0.088027 \\ \text { C } & 4.428924 & -0.000083 & 0.484955 \\ \text { C } & 3.780524 & -1.267521 & -0.088698 \\ \text { H } & 1.810771 & 2.165881 & -0.309755 \\ \text { H } & 1.789102 & 0.000405 & -1.528731 \\ \text { H } & 2.057350 & -1.327648 & 1.213686 \\ \text { H } & 1.810874 & -2.165749 & -0.310905 \\ \text { H } & 3.984449 & 1.320459 & -1.167800 \\ \text { H } & 4.231109 & 2.161934 & 0.360619 \\ \text { H } & 5.507720 & -0.000006 & 0.284895 \\ \text { H } & 4.312050 & -0.000375 & 1.578808 \\ \text { H } & 3.984512 & -1.319769 & -1.168499 \\ \text { H } & 4.231205 & -2.162042 & 0.359475 \\ \text { H } & 2.057302 & 1.326983 & 1.214397 \\ \text { B } & 0.047616 & 0.000048 & -0.263340 \\ \text { O } & -0.742077 & 1.146331 & -0.181886 \\ \text { O } & -0.741986 & -1.146271 & -0.181468 \\ \text { C } & -2.040427 & 0.698009 & -0.055277 \\ \text { C } & -2.040370 & -0.698007 & -0.055024 \\ \text { C } & -3.207761 & 1.431539 & 0.060424 \\ \text { C } & -3.207648 & -1.431586 & 0.060945 \\ \text { H } & -3.195119 & 2.515953 & 0.059305 \\ \text { C } & -4.399075 & 0.699615 & 0.178010 \\ \text { H } & -3.194922 & -2.515999 & 0.060221 \\ \text { C } & -4.399020 & -0.699713 & 0.178264 \\ \text { H } & -5.340091 & 1.233073 & 0.270826 \\ \text { H } & -5.339993 & -1.233212 & 0.271275\end{array}$

\section{TS-1}

B3LYP SCF energy: $\quad-1336.03474983$ a.u.

B3LYP enthalpy: $\quad-1335.525497$ a.u.

B3LYP free energy: $\quad-1335.618900$ a.u.

M06 SCF energy in solution: $\quad-1335.40180601$ a.u. 
M06 enthalpy in solution: $\quad-1334.892553$ a.u.

M06 free energy in solution: $\quad-1334.985956$ a.u. Imaginary frequency: $\quad-61.1217 \mathrm{~cm}^{-1}$

Cartesian coordinates

$\begin{array}{lccc}\text { ATOM } & \text { X } & \text { Y } & \text { Z } \\ \text { B } & 0.388623 & 0.156754 & -0.393576 \\ \text { B } & -1.208607 & -0.330531 & -0.326395 \\ \text { O } & 1.071774 & 0.541235 & -1.550760 \\ \text { O } & 1.203364 & 0.362517 & 0.735543 \\ \text { O } & -1.801271 & -0.951336 & 0.799584 \\ \text { O } & -2.022296 & -0.531211 & -1.460689 \\ \text { C } & 2.334953 & 0.892151 & -1.144063 \\ \text { C } & 2.416683 & 0.783428 & 0.247033 \\ \text { C } & 3.417873 & 1.289510 & -1.909047 \\ \text { C } & 3.582911 & 1.063623 & 0.937681 \\ \text { C } & 4.608287 & 1.574473 & -1.221069 \\ \text { H } & 5.482488 & 1.887455 & -1.783826 \\ \text { C } & 4.690154 & 1.463318 & 0.171454 \\ \text { H } & 5.625563 & 1.693917 & 0.672284 \\ \text { H } & 3.344087 & 1.367432 & -2.988253 \\ \text { H } & 3.630714 & 0.980652 & 2.018410 \\ \text { C } & -3.001300 & -1.444716 & 0.360415 \\ \text { C } & -3.134098 & -1.189538 & -1.010173 \\ \text { C } & -3.985745 & -2.101235 & 1.080946 \\ \text { H } & -3.870743 & -2.292766 & 2.142506 \\ \text { C } & -4.259205 & -1.580207 & -1.718633 \\ \text { H } & -4.350381 & -1.378362 & -2.780385 \\ \text { C } & -5.260908 & -2.246639 & -0.998373 \\ \text { H } & -6.158406 & -2.571130 & -1.516348 \\ \text { C } & -5.127387 & -2.501785 & 0.371804 \\ \text { H } & -5.923091 & -3.020274 & 0.898243 \\ \text { O } & 1.018671 & -2.344096 & -0.586640 \\ \text { C } & 1.709891 & -2.519446 & 0.422966 \\ \text { N } & 3.074479 & -2.568375 & 0.350057 \\ \text { C } & 1.061003 & -2.670601 & 1.787658 \\ \text { H } & -0.017090 & -2.607738 & 1.645038 \\ \text { H } & 1.314189 & -3.625986 & 2.260382 \\ \text { H } & 1.366402 & -1.859631 & 2.456257 \\ \text { C } & 3.961171 & -2.625781 & 1.496709 \\ \text { H } & 3.421411 & -2.906617 & 2.399371 \\ \text { H } & 4.747748 & -3.369995 & 1.321283 \\ \text { H } & 3.441901 & -1.651207 & 1.661349 \\ \text { H } & & -2.333324 & -0.928737\end{array}$




$\begin{array}{lrrr}\mathrm{H} & 2.965889 & -2.277812 & -1.701130 \\ \mathrm{H} & 4.291211 & -1.391321 & -0.902964 \\ \mathrm{H} & 4.425153 & -3.153394 & -1.148739 \\ \mathrm{C} & -1.880107 & 1.854947 & 0.170749 \\ \mathrm{C} & -1.480041 & 2.174161 & 1.580863 \\ \mathrm{C} & -1.387040 & 2.806694 & -0.879651 \\ \mathrm{C} & -1.947788 & 3.610903 & 1.929963 \\ \mathrm{H} & -0.387660 & 2.129336 & 1.679958 \\ \mathrm{H} & -1.898362 & 1.446073 & 2.282617 \\ \mathrm{C} & -1.864249 & 4.239973 & -0.529769 \\ \mathrm{H} & -1.729743 & 2.509436 & -1.875826 \\ \mathrm{H} & -0.288742 & 2.812421 & -0.904010 \\ \mathrm{C} & -1.437533 & 4.623163 & 0.894424 \\ \mathrm{H} & -3.045992 & 3.637369 & 1.954408 \\ \mathrm{H} & -1.599821 & 3.877949 & 2.935922 \\ \mathrm{H} & -1.460652 & 4.952284 & -1.260361 \\ \mathrm{H} & -1.801097 & 5.629061 & 1.139373 \\ \mathrm{H} & -0.339478 & 4.664027 & 0.941430 \\ \mathrm{H} & -2.897541 & 1.485080 & 0.035805 \\ \mathrm{H} & -2.959176 & 4.285952 & -0.608540\end{array}$

\section{Int}

B3LYP SCF energy: $\quad-1336.06054417$ a.u.

B3LYP enthalpy: $\quad-1335.548993$ a.u.

B3LYP free energy: $\quad-1335.636719$ a.u.

M06 SCF energy in solution: $\quad-1335.42703479$ a.u.

M06 enthalpy in solution: $\quad-1334.915484$ a.u.

M06 free energy in solution: $\quad-1335.003210$ a.u.

Cartesian coordinates

$\begin{array}{lccc}\text { ATOM } & \text { X } & \text { Y } & \text { Z } \\ \text { B } & -0.392861 & -1.124444 & -0.195708 \\ \text { B } & 0.041131 & 0.795287 & -0.203002 \\ \text { O } & -1.299003 & -1.468837 & 0.883835 \\ \text { O } & -1.037138 & -1.452932 & -1.437973 \\ \text { O } & 0.746060 & 0.987014 & -1.474267 \\ \text { O } & 1.066877 & 0.986686 & 0.843745 \\ \text { C } & -2.492011 & -1.752453 & 0.278344 \\ \text { C } & -2.328219 & -1.745584 & -1.115980 \\ \text { C } & -3.718679 & -1.998011 & 0.869526 \\ \text { C } & -3.392434 & -1.996155 & -1.966932 \\ \text { C } & -4.801017 & -2.256706 & 0.011721 \\ \text { H } & -5.779845 & -2.454432 & 0.438387 \\ \text { C } & -4.639858 & -2.256561 & -1.376207\end{array}$




\begin{tabular}{|c|c|c|c|}
\hline $\mathrm{H}$ & -5.495869 & -2.455567 & -2.014252 \\
\hline $\mathrm{H}$ & -3.831216 & -1.987858 & 1.948588 \\
\hline $\mathrm{H}$ & -3.258554 & -1.982932 & -3.043304 \\
\hline $\mathrm{C}$ & 2.050035 & 1.168897 & -1.187558 \\
\hline $\mathrm{C}$ & 2.246591 & 1.180927 & 0.206774 \\
\hline $\mathrm{C}$ & 3.115749 & 1.326130 & -2.064345 \\
\hline $\mathrm{H}$ & 2.953949 & 1.319590 & -3.137092 \\
\hline $\mathrm{C}$ & 3.507786 & 1.347190 & 0.760982 \\
\hline $\mathrm{H}$ & 3.642426 & 1.376522 & 1.838185 \\
\hline $\mathrm{C}$ & 4.589742 & 1.506397 & -0.123718 \\
\hline $\mathrm{H}$ & 5.587698 & 1.658429 & 0.277766 \\
\hline $\mathrm{C}$ & 4.395104 & 1.496245 & -1.509085 \\
\hline $\mathrm{H}$ & 5.246286 & 1.632097 & -2.170024 \\
\hline $\mathrm{O}$ & 0.953178 & -1.771548 & -0.116459 \\
\hline $\mathrm{C}$ & 1.781951 & -1.814663 & 0.862800 \\
\hline $\mathrm{N}$ & 3.061895 & -2.008096 & 0.555589 \\
\hline $\mathrm{C}$ & 1.340229 & -1.680456 & 2.292470 \\
\hline $\mathrm{H}$ & 0.259341 & -1.569304 & 2.325563 \\
\hline $\mathrm{H}$ & 1.625534 & -2.567763 & 2.867518 \\
\hline $\mathrm{H}$ & 1.800108 & -0.798671 & 2.746189 \\
\hline $\mathrm{C}$ & 4.133991 & -2.042971 & 1.543313 \\
\hline $\mathrm{H}$ & 3.740133 & -2.067312 & 2.556256 \\
\hline $\mathrm{H}$ & 4.746054 & -2.935670 & 1.378496 \\
\hline $\mathrm{H}$ & 4.758079 & -1.151863 & 1.421453 \\
\hline $\mathrm{C}$ & 3.518657 & -2.047309 & -0.836903 \\
\hline $\mathrm{H}$ & 2.664375 & -1.996339 & -1.505326 \\
\hline $\mathrm{H}$ & 4.174208 & -1.190773 & -1.017339 \\
\hline $\mathrm{H}$ & 4.070331 & -2.978352 & -1.003394 \\
\hline $\mathrm{C}$ & -1.366058 & 1.545297 & -0.002881 \\
\hline $\mathrm{C}$ & -1.293744 & 2.968128 & -0.606798 \\
\hline $\mathrm{C}$ & -1.793665 & 1.628087 & 1.474121 \\
\hline $\mathrm{C}$ & -2.624390 & 3.717123 & -0.445351 \\
\hline $\mathrm{H}$ & -1.014190 & 2.911722 & -1.664437 \\
\hline $\mathrm{H}$ & -0.495559 & 3.533241 & -0.101337 \\
\hline $\mathrm{C}$ & -3.130028 & 2.365382 & 1.635819 \\
\hline $\mathrm{H}$ & -1.862713 & 0.625127 & 1.905931 \\
\hline $\mathrm{H}$ & -1.014906 & 2.160257 & 2.039254 \\
\hline $\mathrm{C}$ & -3.069466 & 3.770569 & 1.022049 \\
\hline $\mathrm{H}$ & -3.395905 & 3.201516 & -1.036105 \\
\hline $\mathrm{H}$ & -2.540954 & 4.732837 & -0.855163 \\
\hline $\mathrm{H}$ & -3.920148 & 1.784207 & 1.137829 \\
\hline $\mathrm{H}$ & -3.406369 & 2.426931 & 2.697126 \\
\hline $\mathrm{H}$ & -2.351416 & 4.378902 & 1.592379 \\
\hline $\mathrm{H}$ & -4.043065 & 4.270736 & 1.108247 \\
\hline
\end{tabular}




\section{TS-2}

B3LYP SCF energy: $\quad-1336.05705411$ a.u.

B3LYP enthalpy: $\quad-1335.546983$ a.u.

B3LYP free energy: $\quad-1335.634283$ a.u.

M06 SCF energy in solution: $\quad-1335.42048544$ a.u.

M06 enthalpy in solution: $\quad-1334.910414$ a.u.

M06 free energy in solution: $\quad-1334.997714$ a.u.

Imaginary frequency: $\quad-437.9403 \mathrm{~cm}^{-1}$

Cartesian coordinates

\begin{tabular}{|c|c|c|c|}
\hline ATOM & $X$ & $\mathrm{Y}$ & Z \\
\hline B & -0.304872 & -1.333736 & -0.226360 \\
\hline B & 0.095580 & 0.979819 & -0.178961 \\
\hline $\mathrm{O}$ & -1.242220 & -1.604894 & 0.817839 \\
\hline $\mathrm{O}$ & -0.926439 & -1.528129 & -1.489378 \\
\hline $\mathrm{O}$ & 0.720546 & 1.170917 & -1.451107 \\
\hline $\mathrm{O}$ & 1.116477 & 1.063431 & 0.830285 \\
\hline $\mathrm{C}$ & -2.447119 & -1.767461 & 0.179405 \\
\hline $\mathrm{C}$ & -2.251394 & -1.724717 & -1.208589 \\
\hline $\mathrm{C}$ & -3.702160 & -1.919641 & 0.739238 \\
\hline $\mathrm{C}$ & -3.311994 & -1.842559 & -2.090563 \\
\hline $\mathrm{C}$ & -4.783750 & -2.043223 & -0.150082 \\
\hline $\mathrm{H}$ & -5.786093 & -2.164630 & 0.249271 \\
\hline $\mathrm{C}$ & -4.591417 & -2.006508 & -1.533140 \\
\hline $\mathrm{H}$ & -5.447390 & -2.100524 & -2.194687 \\
\hline $\mathrm{H}$ & -3.837508 & -1.937316 & 1.815334 \\
\hline $\mathrm{H}$ & -3.153429 & -1.800452 & -3.162659 \\
\hline $\mathrm{C}$ & 2.052634 & 1.301758 & -1.213772 \\
\hline $\mathrm{C}$ & 2.294757 & 1.252784 & 0.166937 \\
\hline $\mathrm{C}$ & 3.085189 & 1.473614 & -2.123614 \\
\hline $\mathrm{H}$ & 2.887568 & 1.512630 & -3.189402 \\
\hline $\mathrm{C}$ & 3.571511 & 1.378719 & 0.689032 \\
\hline $\mathrm{H}$ & 3.742260 & 1.355589 & 1.760503 \\
\hline $\mathrm{C}$ & 4.623612 & 1.559267 & -0.225895 \\
\hline $\mathrm{H}$ & 5.637028 & 1.678667 & 0.146483 \\
\hline $\mathrm{C}$ & 4.383571 & 1.603765 & -1.603602 \\
\hline $\mathrm{H}$ & 5.215148 & 1.750419 & -2.286624 \\
\hline $\mathrm{O}$ & 1.046621 & -1.716736 & -0.114584 \\
\hline $\mathrm{C}$ & 1.860799 & -1.815084 & 0.905463 \\
\hline $\mathrm{N}$ & 3.178510 & -1.823085 & 0.604315 \\
\hline $\mathrm{C}$ & 1.374860 & -1.779340 & 2.318509 \\
\hline $\mathrm{H}$ & 0.299499 & -1.945274 & 2.337184 \\
\hline
\end{tabular}




$\begin{array}{lrrr}\mathrm{H} & 1.861515 & -2.544656 & 2.931427 \\ \mathrm{H} & 1.568446 & -0.795743 & 2.768539 \\ \mathrm{C} & 4.213163 & -1.863856 & 1.622057 \\ \mathrm{H} & 3.836319 & -1.509275 & 2.580043 \\ \mathrm{H} & 4.611043 & -2.881284 & 1.747107 \\ \mathrm{H} & 5.030136 & -1.204756 & 1.313329 \\ \mathrm{C} & 3.657889 & -1.906072 & -0.770484 \\ \mathrm{H} & 2.819002 & -1.852483 & -1.458709 \\ \mathrm{H} & 4.334483 & -1.069257 & -0.967159 \\ \mathrm{H} & 4.195890 & -2.851755 & -0.920789 \\ \mathrm{C} & -1.389345 & 1.457627 & 0.093171 \\ \mathrm{C} & -1.589177 & 2.879804 & -0.493721 \\ \mathrm{C} & -1.765978 & 1.452989 & 1.586929 \\ \mathrm{C} & -3.026939 & 3.372617 & -0.278018 \\ \mathrm{H} & -1.340468 & 2.882021 & -1.560705 \\ \mathrm{H} & -0.889090 & 3.575157 & -0.006071 \\ \mathrm{C} & -3.206458 & 1.935659 & 1.801897 \\ \mathrm{H} & -1.636516 & 0.451234 & 2.006671 \\ \mathrm{H} & -1.074668 & 2.113293 & 2.130301 \\ \mathrm{C} & -3.422279 & 3.332596 & 1.204336 \\ \mathrm{H} & -3.711315 & 2.729348 & -0.850436 \\ \mathrm{H} & -3.143035 & 4.389559 & -0.675795 \\ \mathrm{H} & -3.894334 & 1.223511 & 1.323039 \\ \mathrm{H} & -3.451588 & 1.939968 & 2.872381 \\ \mathrm{H} & -2.807785 & 4.057698 & 1.758758 \\ \mathrm{H} & -4.466839 & 3.646510 & 1.328459 \\ \mathrm{H} & -2.101120 & 0.809829 & -0.436780\end{array}$

\section{III}

B3LYP SCF energy: $\quad-694.28589199$ a.u.

B3LYP enthalpy: $\quad-694.044626$ a.u.

B3LYP free energy: $\quad-694.102545$ a.u.

M06 SCF energy in solution: $\quad-693.98579428$ a.u.

M06 enthalpy in solution: $\quad-693.744528$ a.u.

M06 free energy in solution: $\quad-693.802447$ a.u.

Cartesian coordinates

$\begin{array}{lrrr}\text { ATOM } & \text { X } & \text { Y } & \text { Z } \\ \text { B } & 0.009080 & 0.357859 & -0.305964 \\ \text { O } & -0.555335 & -0.801051 & 0.234844 \\ \text { O } & -0.965327 & 1.301651 & -0.632399 \\ \text { C } & -1.915460 & -0.561013 & 0.242175 \\ \text { C } & -2.161306 & 0.711590 & -0.282021 \\ \text { C } & -2.936915 & -1.384439 & 0.677248\end{array}$




$\begin{array}{lrrr}\mathrm{H} & -2.732287 & -2.369463 & 1.082269 \\ \mathrm{C} & -3.442446 & 1.219490 & -0.394965 \\ \mathrm{H} & -3.621486 & 2.208142 & -0.803133 \\ \mathrm{C} & -4.489339 & 0.392758 & 0.043522 \\ \mathrm{H} & -5.511257 & 0.752496 & -0.027415 \\ \mathrm{C} & -4.242761 & -0.879646 & 0.567398 \\ \mathrm{H} & -5.075780 & -1.492839 & 0.897209 \\ \mathrm{O} & 1.317560 & 0.591702 & -0.513277 \\ \mathrm{C} & 2.315458 & -0.343112 & -0.174783 \\ \mathrm{~N} & 3.520530 & 0.318291 & 0.098389 \\ \mathrm{C} & 2.323664 & -1.573893 & -1.032336 \\ \mathrm{H} & 2.694742 & -1.372696 & -2.052837 \\ \mathrm{H} & 2.940386 & -2.364344 & -0.596016 \\ \mathrm{H} & 1.306123 & -1.964118 & -1.114447 \\ \mathrm{C} & 4.701408 & -0.512058 & 0.272643 \\ \mathrm{H} & 5.574193 & 0.140548 & 0.367093 \\ \mathrm{H} & 4.650409 & -1.148509 & 1.173452 \\ \mathrm{H} & 4.854194 & -1.152064 & -0.598684 \\ \mathrm{C} & 3.443203 & 1.413069 & 1.061931 \\ \mathrm{H} & 2.591115 & 2.049043 & 0.822638 \\ \mathrm{H} & 3.335679 & 1.049616 & 2.098109 \\ \mathrm{H} & 4.356651 & 2.011965 & 0.994634\end{array}$

\section{$\left[\mathrm{Mg}(\mathrm{DMAc})_{4}\right]^{2+}$}

B3LYP SCF energy: $\quad-1351.26243288$ a.u.

B3LYP enthalpy: $\quad-1350.689581$ a.u.

B3LYP free energy: $\quad-1350.807491$ a.u.

M06 SCF energy in solution: $\quad-1350.89000254$ a.u.

M06 enthalpy in solution: $\quad-1350.317151$ a.u.

M06 free energy in solution: $\quad-1350.435061$ a.u.

Cartesian coordinates

$\begin{array}{lccc}\text { ATOM } & \text { X } & \text { Y } & \text { Z } \\ \text { Mg } & 0.047561 & -0.007335 & 0.043354 \\ \text { O } & 1.274472 & -1.374938 & -0.615912 \\ \text { C } & 2.477068 & -1.582637 & -0.972011 \\ \text { N } & 2.914052 & -2.826784 & -1.144854 \\ \text { C } & 3.405165 & -0.413229 & -1.202295 \\ \text { C } & 4.264866 & -3.169875 & -1.602520 \\ \text { C } & 2.041648 & -3.976803 & -0.884497 \\ \text { H } & 3.692626 & -0.343747 & -2.257130 \\ \text { H } & 2.888288 & 0.505599 & -0.924096 \\ \text { H } & 4.321339 & -0.503007 & -0.611112 \\ \text { H } & 4.187120 & -3.889362 & -2.422438 \\ & & & \mathbf{S 3 8}\end{array}$




\begin{tabular}{|c|c|c|c|}
\hline $\mathrm{H}$ & 4.829578 & -3.628753 & -0.784575 \\
\hline $\mathrm{H}$ & 4.798547 & -2.293040 & -1.960640 \\
\hline $\mathrm{H}$ & 2.609844 & -4.731630 & -0.334934 \\
\hline $\mathrm{H}$ & 1.184573 & -3.660991 & -0.294401 \\
\hline $\mathrm{H}$ & 1.701900 & -4.411276 & -1.830684 \\
\hline $\mathrm{O}$ & -0.092211 & -0.183062 & 1.971017 \\
\hline $\mathrm{C}$ & -0.860863 & -0.265102 & 2.977532 \\
\hline $\mathrm{N}$ & -0.352817 & -0.380243 & 4.201610 \\
\hline $\mathrm{C}$ & -2.358771 & -0.229999 & 2.778172 \\
\hline $\mathrm{C}$ & -1.158390 & -0.463866 & 5.424012 \\
\hline $\mathrm{C}$ & 1.095973 & -0.440786 & 4.420086 \\
\hline $\mathrm{H}$ & -2.796839 & 0.662420 & 3.237859 \\
\hline $\mathrm{H}$ & -2.566739 & -0.210061 & 1.708004 \\
\hline $\mathrm{H}$ & -2.845031 & -1.106113 & 3.217505 \\
\hline $\mathrm{H}$ & -0.788365 & 0.272802 & 6.142791 \\
\hline $\mathrm{H}$ & -1.061948 & -1.461878 & 5.863667 \\
\hline $\mathrm{H}$ & -2.207487 & -0.259287 & 5.225820 \\
\hline $\mathrm{H}$ & 1.328083 & -1.318942 & 5.029545 \\
\hline $\mathrm{H}$ & 1.607316 & -0.508746 & 3.463508 \\
\hline $\mathrm{H}$ & 1.424849 & 0.456445 & 4.954035 \\
\hline $\mathrm{O}$ & 0.750865 & 1.763980 & -0.369550 \\
\hline $\mathrm{C}$ & 0.511674 & 2.886683 & -0.913975 \\
\hline $\mathrm{N}$ & 1.362404 & 3.897322 & -0.760674 \\
\hline $\mathrm{C}$ & -0.739930 & 3.067271 & -1.740482 \\
\hline $\mathrm{C}$ & 1.196577 & 5.214355 & -1.385224 \\
\hline $\mathrm{C}$ & 2.558514 & 3.758742 & 0.076958 \\
\hline $\mathrm{H}$ & -0.492793 & 3.212679 & -2.797950 \\
\hline $\mathrm{H}$ & -1.351719 & 2.169542 & -1.647545 \\
\hline $\mathrm{H}$ & -1.321803 & 3.932922 & -1.410929 \\
\hline $\mathrm{H}$ & 2.144750 & 5.506979 & -1.844442 \\
\hline $\mathrm{H}$ & 0.929676 & 5.957034 & -0.626314 \\
\hline $\mathrm{H}$ & 0.430647 & 5.195228 & -2.156746 \\
\hline $\mathrm{H}$ & 2.644411 & 4.640818 & 0.717004 \\
\hline $\mathrm{H}$ & 2.474301 & 2.865666 & 0.69148 \\
\hline $\mathrm{H}$ & 3.450913 & 3.693436 & -0.554365 \\
\hline $\mathrm{O}$ & -1.713801 & -0.241780 & -0.786812 \\
\hline $\mathrm{C}$ & -2.381515 & -0.999503 & -1.560356 \\
\hline $\mathrm{N}$ & -3.657607 & -0.727462 & -1.822885 \\
\hline $\mathrm{C}$ & -1.720057 & -2.208443 & -2.179324 \\
\hline $\mathrm{C}$ & -4.511541 & -1.536528 & -2.699120 \\
\hline $\mathrm{C}$ & -4.326643 & 0.436455 & -1.233313 \\
\hline $\mathrm{H}$ & -1.730317 & -2.150383 & -3.272546 \\
\hline $\mathrm{H}$ & -0.684995 & -2.254765 & -1.840388 \\
\hline $\mathrm{H}$ & -2.229889 & -3.132623 & -1.888653 \\
\hline
\end{tabular}




$\begin{array}{lrrr}\mathrm{H} & -4.895815 & -0.908105 & -3.508250 \\ \mathrm{H} & -5.358201 & -1.923856 & -2.123925 \\ \mathrm{H} & -3.965016 & -2.370986 & -3.130150 \\ \mathrm{H} & -5.193372 & 0.101694 & -0.655118 \\ \mathrm{H} & -3.634735 & 0.969109 & -0.586366 \\ \mathrm{H} & -4.673921 & 1.099360 & -2.031938\end{array}$

\section{DMAc}

B3LYP SCF energy: $\quad-287.84397943$ a.u.

B3LYP enthalpy: $\quad-287.704156$ a.u.

B3LYP free energy: $\quad-287.744832$ a.u.

M06 SCF energy in solution: $\quad-287.72555733$ a.u.

M06 enthalpy in solution: $\quad-287.585734$ a.u.

M06 free energy in solution: $\quad-287.626410$ a.u.

Cartesian coordinates

$\begin{array}{lccc}\text { ATOM } & \text { X } & \text { Y } & \text { Z } \\ \text { C } & -1.079808 & -1.449006 & -0.000125 \\ \text { H } & -1.698554 & -1.639697 & -0.888315 \\ \text { H } & -1.699932 & -1.639602 & 0.887095 \\ \text { H } & -0.256562 & -2.161128 & 0.000551 \\ \text { C } & -1.623001 & 0.944436 & 0.000000 \\ \text { H } & -2.260327 & 0.851685 & 0.889631 \\ \text { H } & -1.132662 & 1.916633 & 0.000429 \\ \text { H } & -2.259446 & 0.852126 & -0.890334 \\ \text { N } & -0.595214 & -0.083045 & 0.000322 \\ \text { C } & 0.728437 & 0.294466 & 0.000035 \\ \text { O } & 1.067055 & 1.473561 & -0.000125 \\ \text { C } & 1.774115 & -0.814268 & 0.000026 \\ \text { H } & 1.693513 & -1.454037 & -0.885938 \\ \text { H } & 2.752148 & -0.332794 & 0.000090 \\ \text { H } & 1.693422 & -1.454129 & 0.885919\end{array}$

\section{TS-3}

B3LYP SCF energy: $\quad-3500.98206847$ a.u.

B3LYP enthalpy: $\quad-3500.574959$ a.u.

B3LYP free energy: $\quad-3500.653979$ a.u.

M06 SCF energy in solution: $\quad-3503.20778868$ a.u.

M06 enthalpy in solution: $\quad-3502.800679$ a.u.

M06 free energy in solution: $\quad-3502.879699$ a.u.

Imaginary frequency: $\quad-687.0101 \mathrm{~cm}-1$

Cartesian coordinates

$\begin{array}{llll}\text { ATOM } & \mathrm{X} & \mathrm{Y} & \mathrm{Z}\end{array}$ 


\begin{tabular}{|c|c|c|c|}
\hline B & -1.059127 & -0.364103 & 0.332847 \\
\hline $\mathrm{O}$ & -1.085793 & 0.595973 & 1.442326 \\
\hline $\mathrm{O}$ & -1.274729 & 0.363701 & -0.928988 \\
\hline $\mathrm{C}$ & -1.408272 & 1.780813 & 0.857840 \\
\hline $\mathrm{C}$ & -1.518718 & 1.643277 & -0.541608 \\
\hline $\mathrm{C}$ & -1.620777 & 3.001639 & 1.475977 \\
\hline $\mathrm{H}$ & -1.523249 & 3.096443 & 2.552923 \\
\hline $\mathrm{C}$ & -1.848856 & 2.722143 & -1.344645 \\
\hline $\mathrm{H}$ & -1.921165 & 2.603892 & -2.421344 \\
\hline $\mathrm{C}$ & -2.068000 & 3.964163 & -0.719214 \\
\hline $\mathrm{H}$ & -2.321678 & 4.827635 & -1.328694 \\
\hline $\mathrm{C}$ & -1.955656 & 4.100981 & 0.662941 \\
\hline $\mathrm{H}$ & -2.122261 & 5.070689 & 1.124844 \\
\hline $\mathrm{O}$ & -0.954312 & -1.812745 & 0.474348 \\
\hline $\mathrm{C}$ & -2.311378 & -1.604555 & 0.510351 \\
\hline $\mathrm{N}$ & -3.046930 & -2.019003 & -0.632631 \\
\hline $\mathrm{C}$ & -2.970684 & -1.759555 & 1.856169 \\
\hline $\mathrm{H}$ & -2.925197 & -2.805752 & 2.200313 \\
\hline $\mathrm{H}$ & -2.468229 & -1.127718 & 2.592678 \\
\hline $\mathrm{H}$ & -4.026048 & -1.468237 & 1.817673 \\
\hline $\mathrm{C}$ & -4.098066 & -1.099507 & -1.056149 \\
\hline $\mathrm{H}$ & -4.797160 & -1.625255 & -1.718693 \\
\hline $\mathrm{H}$ & -4.654034 & -0.749844 & -0.181845 \\
\hline $\mathrm{H}$ & -3.699688 & -0.220041 & -1.582082 \\
\hline $\mathrm{C}$ & -2.281827 & -2.568632 & -1.738200 \\
\hline $\mathrm{H}$ & -1.617597 & -3.354841 & -1.374119 \\
\hline $\mathrm{H}$ & -2.981883 & -3.000966 & -2.463431 \\
\hline $\mathrm{H}$ & -1.665005 & -1.814460 & -2.253467 \\
\hline $\mathrm{C}$ & 1.194055 & -0.368436 & 0.120300 \\
\hline $\mathrm{C}$ & 1.699136 & -0.824047 & 1.451140 \\
\hline $\mathrm{C}$ & 1.957759 & -2.333269 & 1.560385 \\
\hline $\mathrm{C}$ & 2.644236 & -2.859536 & 0.293426 \\
\hline $\mathrm{C}$ & 1.733792 & -2.639302 & -0.921200 \\
\hline $\mathrm{C}$ & 1.444191 & -1.145624 & -1.129489 \\
\hline $\mathrm{H}$ & 2.579453 & -2.528157 & 2.444467 \\
\hline $\mathrm{H}$ & 2.648082 & -0.279849 & 1.574355 \\
\hline $\mathrm{H}$ & 1.029733 & -0.460963 & 2.242227 \\
\hline $\mathrm{H}$ & 1.084791 & 0.705772 & -0.003804 \\
\hline $\mathrm{H}$ & 3.588955 & -2.319585 & 0.141734 \\
\hline $\mathrm{H}$ & 2.883939 & -3.926708 & 0.402118 \\
\hline $\mathrm{H}$ & 2.198333 & -3.044846 & -1.829952 \\
\hline $\mathrm{H}$ & 0.795443 & -3.184200 & -0.766254 \\
\hline $\mathrm{H}$ & 2.325705 & -0.658483 & -1.573289 \\
\hline $\mathrm{H}$ & 0.617953 & -0.982901 & -1.837080 \\
\hline
\end{tabular}




$\begin{array}{llrr}\mathrm{H} & 1.010284 & -2.865113 & 1.705459 \\ \mathrm{Br} & 3.930221 & 1.223665 & -0.360863\end{array}$

\section{TS-4}

B3LYP SCF energy: $\quad-3907.51050893$ a.u.

B3LYP enthalpy: $\quad-3907.000037$ a.u.

B3LYP free energy: $\quad-3907.100486$ a.u.

M06 SCF energy in solution: $\quad-3909.55121014$ a.u.

M06 enthalpy in solution: $\quad-3909.040738$ a.u.

M06 free energy in solution: $\quad-3909.141187$ a.u.

Imaginary frequency: $\quad-295.7289 \mathrm{~cm}-1$

Cartesian coordinates

$\begin{array}{lrrr}\text { ATOM } & \text { X } & \text { Y } & \text { Z } \\ \text { B } & -1.706370 & 0.182025 & 1.199683 \\ \text { B } & -0.111130 & 0.400875 & 1.505271 \\ \text { O } & -2.481359 & 0.933534 & 0.271095 \\ \text { O } & -2.560046 & -0.745266 & 1.853045 \\ \text { O } & 0.664419 & -0.279685 & 2.488654 \\ \text { O } & 0.761579 & 1.244837 & 0.761065 \\ \text { C } & -3.740820 & 0.417276 & 0.318880 \\ \text { C } & -3.787923 & -0.601806 & 1.282059 \\ \text { C } & -4.864422 & 0.794568 & -0.398121 \\ \text { C } & -4.961678 & -1.284378 & 1.558577 \\ \text { C } & -6.059657 & 0.102344 & -0.127063 \\ \text { H } & -6.960339 & 0.365040 & -0.676188 \\ \text { C } & -6.106982 & -0.913694 & 0.828744 \\ \text { H } & -7.044214 & -1.432380 & 1.014179 \\ \text { H } & -4.813076 & 1.582104 & -1.143101 \\ \text { H } & -4.985279 & -2.073707 & 2.302847 \\ \text { C } & 1.959389 & 0.099268 & 2.287597 \\ \text { C } & 2.021503 & 1.024691 & 1.236977 \\ \text { C } & 3.103635 & -0.331167 & 2.942783 \\ \text { H } & 3.047215 & -1.057362 & 3.747203 \\ \text { C } & 3.222516 & 1.555244 & 0.790278 \\ \text { H } & 3.249935 & 2.221890 & -0.064580 \\ \text { C } & 4.384041 & 1.130386 & 1.460026 \\ \text { H } & 5.348151 & 1.513614 & 1.136184 \\ \text { C } & 4.328034 & 0.212071 & 2.511688 \\ \text { H } & 5.247261 & -0.102368 & 2.999312 \\ \text { O } & 2.140020 & 2.780621 & -2.102526 \\ \text { C } & 0.931745 & 2.799926 & -1.848401 \\ \text { N } & 0.398272 & 3.736214 & -0.995619 \\ \text { C } & -0.019072 & 1.816078 & -2.499344\end{array}$




$\begin{array}{lccc}\mathrm{H} & -0.355279 & 1.049521 & -1.792360 \\ \mathrm{H} & -0.907248 & 2.305030 & -2.914019 \\ \mathrm{H} & 0.525458 & 1.315220 & -3.300610 \\ \mathrm{C} & -0.921898 & 3.631616 & -0.399325 \\ \mathrm{H} & -1.509095 & 2.844191 & -0.862073 \\ \mathrm{H} & -0.839836 & 3.376161 & 0.664268 \\ \mathrm{H} & -1.455524 & 4.588326 & -0.492913 \\ \mathrm{C} & 1.308270 & 4.631259 & -0.306128 \\ \mathrm{H} & 2.215996 & 4.735592 & -0.899352 \\ \mathrm{H} & 0.829905 & 5.610075 & -0.174902 \\ \mathrm{H} & 1.576120 & 4.237341 & 0.685118 \\ \mathrm{Br} & -1.066047 & -1.722286 & -1.393150 \\ \mathrm{C} & 1.125098 & -2.088166 & -0.620543 \\ \mathrm{C} & 2.053927 & -1.273196 & -1.478585 \\ \mathrm{C} & 1.373344 & -3.570571 & -0.675434 \\ \mathrm{H} & 0.959333 & -1.701772 & 0.380901 \\ \mathrm{C} & 3.523576 & -1.577416 & -1.110267 \\ \mathrm{H} & 1.887712 & -1.534565 & -2.534531 \\ \mathrm{H} & 1.856054 & -0.203058 & -1.377153 \\ \mathrm{C} & 2.839969 & -3.881136 & -0.298368 \\ \mathrm{H} & 0.680260 & -4.104280 & -0.014987 \\ \mathrm{H} & 1.184531 & -3.930581 & -1.697757 \\ \mathrm{C} & 3.812882 & -3.083714 & -1.180095 \\ \mathrm{H} & 3.720855 & -1.215457 & -0.093877 \\ \mathrm{H} & 4.198836 & -1.023236 & -1.777726 \\ \mathrm{H} & 3.004781 & -3.609140 & 0.754058 \\ \mathrm{H} & 3.037513 & -4.960526 & -0.385348 \\ \mathrm{H} & 4.850849 & -3.288097 & -0.881982 \\ \mathrm{H} & 3.710591 & -3.424798 & -2.222076\end{array}$

\section{VI}

B3LYP SCF energy: $\quad-694.32717856$ a.u.

B3LYP enthalpy: $\quad-694.089195$ a.u.

B3LYP free energy: $\quad-694.144700$ a.u.

M06 SCF energy in solution: $\quad-694.10919432$ a.u.

M06 enthalpy in solution: $\quad-693.871211$ a.u.

M06 free energy in solution: $\quad-693.926716$ a.u.

Cartesian coordinates

$\begin{array}{lccc}\text { ATOM } & \text { X } & \text { Y } & \text { Z } \\ \text { B } & -0.505795 & -0.449194 & -0.367332 \\ \text { O } & 0.194252 & 0.877009 & -0.384321 \\ \text { O } & 0.583911 & -1.445369 & -0.128592 \\ \text { C } & 1.498715 & 0.634670 & -0.170433\end{array}$




$\begin{array}{lrrr}\mathrm{C} & 1.731300 & -0.754992 & -0.017146 \\ \mathrm{C} & 2.542791 & 1.544068 & -0.092303 \\ \mathrm{H} & 2.352031 & 2.607161 & -0.212821 \\ \mathrm{C} & 3.010307 & -1.239837 & 0.213390 \\ \mathrm{H} & 3.178208 & -2.307608 & 0.326577 \\ \mathrm{C} & 4.072543 & -0.314084 & 0.296872 \\ \mathrm{H} & 5.081689 & -0.677273 & 0.481689 \\ \mathrm{C} & 3.843488 & 1.050783 & 0.146969 \\ \mathrm{H} & 4.674006 & 1.750710 & 0.215752 \\ \mathrm{O} & -1.524069 & -0.730084 & -1.341959 \\ \mathrm{C} & -1.994148 & -0.638793 & 0.037909 \\ \mathrm{~N} & -2.938479 & 0.483734 & 0.285724 \\ \mathrm{C} & -2.596127 & -1.940793 & 0.526971 \\ \mathrm{H} & -2.877266 & -1.869334 & 1.587098 \\ \mathrm{H} & -3.507421 & -2.187558 & -0.039898 \\ \mathrm{H} & -1.878192 & -2.761102 & 0.420069 \\ \mathrm{C} & -2.568003 & 1.183941 & 1.505477 \\ \mathrm{H} & -1.602566 & 1.715839 & 1.424940 \\ \mathrm{H} & -3.348566 & 1.913293 & 1.771372 \\ \mathrm{H} & -2.486432 & 0.466516 & 2.330648 \\ \mathrm{C} & -3.068925 & 1.412204 & -0.825351 \\ \mathrm{H} & -3.319172 & 0.862106 & -1.734044 \\ \mathrm{H} & -3.881362 & 2.119082 & -0.599208 \\ \mathrm{H} & -2.151030 & 1.992543 & -1.030730\end{array}$

\section{VII}

B3LYP SCF energy: $\quad-1758.17001283$ a.u.

B3LYP enthalpy: $\quad-1757.503998$ a.u.

B3LYP free energy: $\quad-1757.620474$ a.u.

M06 SCF energy in solution: $\quad-1757.51655960$ a.u.

M06 enthalpy in solution: $\quad-1756.850545$ a.u.

M06 free energy in solution: $\quad-1756.967021$ a.u.

Cartesian coordinates

$\begin{array}{lccc}\text { ATOM } & \text { X } & \text { Y } & \text { Z } \\ \text { B } & 1.525822 & 0.519216 & -0.114894 \\ \text { O } & 1.708457 & 2.075424 & 0.111607 \\ \text { O } & 0.151343 & 0.551754 & -0.944665 \\ \text { C } & 0.801575 & 2.718699 & -0.625387 \\ \text { C } & -0.136586 & 1.839937 & -1.227952 \\ \text { C } & 0.694772 & 4.092681 & -0.821513 \\ \text { H } & 1.424345 & 4.759475 & -0.368622 \\ \text { C } & -1.190721 & 2.327461 & -1.983230\end{array}$




\begin{tabular}{|c|c|c|c|}
\hline $\mathrm{H}$ & -1.908966 & 1.639629 & -2.416693 \\
\hline $\mathrm{C}$ & -1.299645 & 3.722730 & -2.169819 \\
\hline $\mathrm{H}$ & -2.113844 & 4.117388 & -2.773654 \\
\hline $\mathrm{C}$ & -0.365376 & 4.588923 & -1.606391 \\
\hline $\mathrm{H}$ & -0.451568 & 5.661520 & -1.769806 \\
\hline $\mathrm{O}$ & 1.295858 & -0.229074 & 1.008609 \\
\hline $\mathrm{C}$ & 2.744445 & 0.069590 & -1.114552 \\
\hline $\mathrm{N}$ & 4.016560 & -0.085464 & -0.526934 \\
\hline $\mathrm{C}$ & 2.541746 & -0.179331 & -2.430485 \\
\hline $\mathrm{H}$ & 3.323163 & -0.445474 & -3.139726 \\
\hline $\mathrm{H}$ & -2.263744 & -2.823750 & -0.893480 \\
\hline $\mathrm{H}$ & 1.538839 & -0.084785 & -2.829483 \\
\hline $\mathrm{C}$ & 4.301084 & 0.670083 & 0.685810 \\
\hline $\mathrm{H}$ & 5.174592 & 0.230784 & 1.186832 \\
\hline $\mathrm{H}$ & 4.509614 & 1.734829 & 0.488901 \\
\hline $\mathrm{H}$ & 3.438211 & 0.602391 & 1.349066 \\
\hline $\mathrm{C}$ & 5.154562 & -0.273782 & -1.398642 \\
\hline $\mathrm{H}$ & 5.321995 & 0.579866 & -2.081224 \\
\hline $\mathrm{H}$ & 6.057724 & -0.418970 & -0.795064 \\
\hline $\mathrm{H}$ & 5.010346 & -1.166817 & -2.019138 \\
\hline $\mathrm{Mg}$ & -0.583912 & -0.749195 & 0.751426 \\
\hline $\mathrm{O}$ & -2.362466 & -1.016982 & 0.080413 \\
\hline $\mathrm{O}$ & -1.299713 & 0.367631 & 2.363785 \\
\hline $\mathrm{O}$ & -0.060480 & -2.794295 & 0.820253 \\
\hline $\mathrm{C}$ & -2.830793 & -1.854187 & -0.881463 \\
\hline $\mathrm{C}$ & -1.061796 & 1.597955 & 2.344058 \\
\hline $\mathrm{C}$ & 1.018164 & -3.094149 & 0.255410 \\
\hline $\mathrm{N}$ & -4.263349 & -2.201589 & -0.610596 \\
\hline $\mathrm{C}$ & -2.700131 & -1.219704 & -2.278328 \\
\hline $\mathrm{N}$ & -1.880784 & 2.446063 & 1.691056 \\
\hline $\mathrm{C}$ & 0.181553 & 2.129437 & 3.020318 \\
\hline $\mathrm{N}$ & 2.154137 & -3.247607 & 0.964741 \\
\hline $\mathrm{C}$ & 1.045658 & -3.279291 & -1.243119 \\
\hline $\mathrm{C}$ & -4.769548 & -3.216066 & -1.513697 \\
\hline $\mathrm{C}$ & -4.422779 & -2.639710 & 0.767657 \\
\hline $\mathrm{H}$ & -3.354305 & -0.341651 & -2.333417 \\
\hline $\mathrm{H}$ & -1.665244 & -0.889413 & -2.404215 \\
\hline $\mathrm{H}$ & -2.949289 & -1.900432 & -3.102636 \\
\hline $\mathrm{C}$ & -1.677626 & 3.885902 & 1.632043 \\
\hline $\mathrm{C}$ & -3.072886 & 1.942441 & 1.002735 \\
\hline $\mathrm{H}$ & -0.000992 & 3.030527 & 3.614368 \\
\hline $\mathrm{H}$ & 0.570215 & 1.334349 & 3.65538 \\
\hline $\mathrm{H}$ & 0.929992 & 2.333497 & 2.244206 \\
\hline $\mathrm{C}$ & 3.470215 & -3.245364 & 0.333923 \\
\hline
\end{tabular}




$\begin{array}{lrrr}\mathrm{C} & 2.134143 & -2.806074 & 2.361440 \\ \mathrm{H} & 1.575511 & -4.186351 & -1.552323 \\ \mathrm{H} & 0.012525 & -3.321302 & -1.591562 \\ \mathrm{H} & 1.542479 & -2.409673 & -1.699402 \\ \mathrm{H} & -5.800505 & -3.474011 & -1.238166 \\ \mathrm{H} & -4.175556 & -4.157257 & -1.494445 \\ \mathrm{H} & -4.783581 & -2.851741 & -2.545352 \\ \mathrm{H} & -3.924926 & -3.612722 & 0.971401 \\ \mathrm{H} & -3.979534 & -1.891417 & 1.423449 \\ \mathrm{H} & -5.491112 & -2.754579 & 0.997858 \\ \mathrm{H} & -2.368658 & 4.406717 & 2.312100 \\ \mathrm{H} & -1.854163 & 4.225503 & 0.609225 \\ \mathrm{H} & -0.652875 & 4.147246 & 1.884323 \\ \mathrm{H} & -3.168765 & 2.481255 & 0.056907 \\ \mathrm{H} & -2.978455 & 0.874676 & 0.785157 \\ \mathrm{H} & -3.966266 & 2.125872 & 1.616610 \\ \mathrm{H} & 4.164809 & -3.813600 & 0.962617 \\ \mathrm{H} & 3.826955 & -2.212927 & 0.211258 \\ \mathrm{H} & 3.429017 & -3.720555 & -0.646031 \\ \mathrm{H} & 2.132515 & -1.709709 & 2.371523 \\ \mathrm{H} & 1.225254 & -3.170826 & 2.840833 \\ \mathrm{H} & 3.011969 & -3.217870 & 2.868982\end{array}$

\section{VIII}

B3LYP SCF energy: $\quad-3672.29467214$ a.u.

B3LYP enthalpy: $\quad-3671.949843$ a.u.

B3LYP free energy: $\quad-3672.032319$ a.u.

M06 SCF energy in solution: $\quad-3674.50140427$ a.u.

M06 enthalpy in solution: $\quad-3674.156575$ a.u.

M06 free energy in solution: $\quad-3674.239051$ a.u.

Cartesian coordinates

$\begin{array}{lccc}\text { ATOM } & \text { X } & \text { Y } & \text { Z } \\ \text { B } & -0.515614 & -0.931757 & 0.014103 \\ \text { B } & 1.115806 & -0.522214 & 0.037241 \\ \text { O } & -1.318551 & -0.567509 & -1.163216 \\ \text { O } & -1.349560 & -0.621106 & 1.178699 \\ \text { O } & 1.924653 & -0.414421 & 1.185063 \\ \text { O } & 1.904523 & -0.284644 & -1.108506 \\ \text { C } & -2.568377 & -0.328828 & -0.707558 \\ \text { C } & -2.583167 & -0.354621 & 0.702506 \\ \text { C } & -3.711663 & -0.039647 & -1.433938 \\ \text { C } & -3.742813 & -0.081136 & 1.411349 \\ \text { C } & -4.892509 & 0.241697 & -0.717600\end{array}$




\begin{tabular}{|c|c|c|c|}
\hline $\mathrm{H}$ & -5.803655 & 0.473442 & -1.263972 \\
\hline $\mathrm{C}$ & -4.907459 & 0.222976 & 0.676487 \\
\hline $\mathrm{H}$ & -5.830513 & 0.438925 & 1.209279 \\
\hline $\mathrm{H}$ & -3.686597 & -0.026487 & -2.519663 \\
\hline $\mathrm{H}$ & -3.743724 & -0.106317 & 2.497064 \\
\hline $\mathrm{C}$ & 3.175926 & -0.096277 & 0.742607 \\
\hline $\mathrm{C}$ & 3.164350 & -0.017082 & -0.655128 \\
\hline $\mathrm{C}$ & 4.330535 & 0.124540 & 1.472894 \\
\hline $\mathrm{H}$ & 4.326090 & 0.061404 & 2.556258 \\
\hline $\mathrm{C}$ & 4.305611 & 0.285497 & -1.376728 \\
\hline $\mathrm{H}$ & 4.282374 & 0.342744 & -2.460342 \\
\hline $\mathrm{C}$ & 5.483125 & 0.513964 & -0.644918 \\
\hline $\mathrm{H}$ & 6.400049 & 0.756479 & -1.176134 \\
\hline $\mathrm{C}$ & 5.495025 & 0.435023 & 0.750637 \\
\hline $\mathrm{H}$ & 6.421171 & 0.617501 & 1.289811 \\
\hline $\mathrm{O}$ & 1.246054 & 2.474565 & 0.211968 \\
\hline $\mathrm{C}$ & 0.152348 & 2.532296 & -0.350656 \\
\hline $\mathrm{N}$ & -1.016751 & 2.634142 & 0.359654 \\
\hline $\mathrm{C}$ & 0.045436 & 2.432997 & -1.863793 \\
\hline $\mathrm{H}$ & -0.480510 & 1.511078 & -2.134226 \\
\hline $\mathrm{H}$ & -0.480265 & 3.286727 & -2.306370 \\
\hline $\mathrm{H}$ & 1.061151 & 2.386250 & -2.256139 \\
\hline $\mathrm{C}$ & -2.307628 & 2.942210 & -0.228865 \\
\hline $\mathrm{H}$ & -2.366374 & 2.580987 & -1.253589 \\
\hline $\mathrm{H}$ & -3.094096 & 2.435076 & 0.335174 \\
\hline $\mathrm{H}$ & -2.509741 & 4.026551 & -0.217845 \\
\hline $\mathrm{C}$ & -0.948505 & 2.624171 & 1.810946 \\
\hline $\mathrm{H}$ & -0.004697 & 2.168914 & 2.106325 \\
\hline $\mathrm{H}$ & -1.004680 & 3.642572 & 2.226554 \\
\hline $\mathrm{H}$ & -1.779960 & 2.031278 & 2.202484 \\
\hline $\mathrm{Br}$ & -0.279660 & -3.174800 & -0.044870 \\
\hline
\end{tabular}

\subsection{Characterization of Products}

\section{Compound 1}<smiles>CC1(C)OB(C2CCCCC2)OC1(C)C</smiles>

2-cyclohexyl-4,4,5,5-tetramethyl-1,3,2-dioxaborolane ${ }^{[19]}$ : Colorless oil was obtained with $67 \%$ isolated yield following Procedure A (from bromide, $0.6 \mathrm{mmol}$ scale, 84.8 mg). $75 \%$ yield was obtained following Procedure B (from iodide, $0.6 \mathrm{mmol} \mathrm{scale}, 95.0$ mg). $75 \%$ yield was obtained in gram scale experiment at $600 \mathrm{~mA}$ (from iodide, 7.2 
mmol scale, $48 \mathrm{~min}, 1.14 \mathrm{~g}$ ). $75 \%$ yield was obtained in gram scale experiment at 1000 $\mathrm{mA}$ (from iodide, $7.2 \mathrm{mmol}$ scale, $29 \mathrm{~min}, 1.14 \mathrm{~g}$ ).

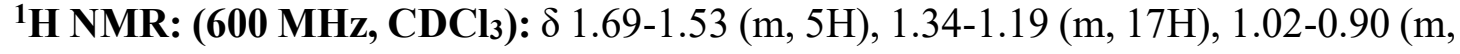
$1 \mathrm{H})$.

${ }^{13}$ C NMR: (151 MHz, $\left.\mathbf{C D C l}_{3}\right): \delta 82.8,28.1,27.3,26.9,24.9$. The signal of the $\alpha$-Bcarbon was not observed.

${ }^{11}$ B NMR: (193 MHz, CDCl 3$): ~ \delta 34.00$.

HRMS (ESI): calculated for $\mathrm{C}_{12} \mathrm{H}_{24} \mathrm{BO}_{2}[\mathrm{M}+\mathrm{H}]^{+}:$: 211.1864, found: 211.1857.

\section{Compound 2}

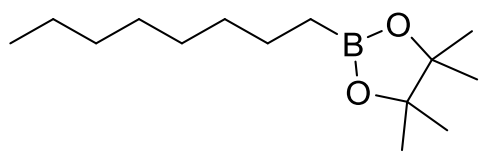

4,4,5,5-tetramethyl-2-octyl-1,3,2-dioxaborolane ${ }^{[19]}$ : colorless oil was obtained with 74\% isolated yield following Procedure A (from bromide, $0.6 \mathrm{mmol} \mathrm{scale,} 107.4 \mathrm{mg}$ ). $80 \%$ isolated yield was obtained following Procedure B (from iodide, 0.6 mmol scale, $116.0 \mathrm{mg}$ ). $58 \%$ isolated yield was obtained following Procedure C (from chloride, 0.6 mmol scale, $84.2 \mathrm{mg})$.

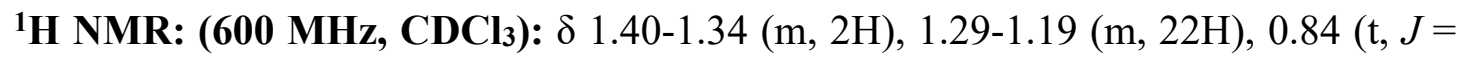
$6.8 \mathrm{~Hz}, 3 \mathrm{H}), 0.74(\mathrm{t}, J=7.8 \mathrm{~Hz}, 2 \mathrm{H})$.

${ }^{13}$ C NMR: (151 MHz, $\left.\mathbf{C D C l}_{3}\right): \delta$ 82.9, 32.6, 32.0, 29.5, 29.4, 24.9, 24.1, 22.8, 14.2, 11.3 (br, low intensity).

${ }^{11}$ B NMR: (193 MHz, CDCl3): $\delta 34.11$.

HRMS (ESI): calculated for $\mathrm{C}_{14} \mathrm{H}_{30} \mathrm{BO}_{2}[\mathrm{M}+\mathrm{H}]^{+}:$241.2333, found: 241.2333 .

\section{Compound 3}<smiles>CC(C)(C)CCB1OC(C)(C)C(C)(C)O1</smiles>

2-(3,3-dimethylbutyl)-4,4,5,5-tetramethyl-1,3,2-dioxaborolane ${ }^{[20]}:$ Colorless oil was obtained with 70\% isolated yield (Procedure A, $0.6 \mathrm{mmol} \mathrm{scale,} 89.6 \mathrm{mg}$ ). 


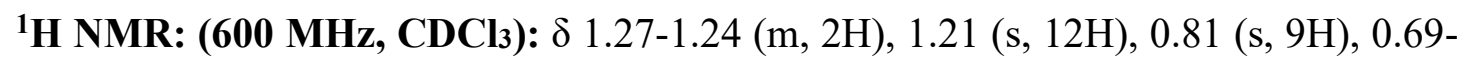
$0.65(\mathrm{~m}, 2 \mathrm{H})$.

${ }^{13}$ C NMR: (151 MHz, CDCl 3 ): $\delta$ 82.9, 37.8, 30.9, 28.9, 24.9, 5.8 (br, low intensity).

${ }^{11}$ B NMR: (193 MHz, CDCl3): $\delta 34.41$.

HRMS (ESI): calculated for $\mathrm{C}_{12} \mathrm{H}_{26} \mathrm{BO}_{2}[\mathrm{M}+\mathrm{H}]^{+}:$: 213.2020, found: 213.2014.

\section{Compound 4}<smiles>CC1(C)OB(CC2CCCCC2)OC1(C)C</smiles>

2-(cyclohexylmethyl)-4,4,5,5-tetramethyl-1,3,2-dioxaborolane ${ }^{[19]}$ : Colorless oil was obtained with 79\% isolated yield (Procedure A, $0.6 \mathrm{mmol} \mathrm{scale,} 106.2 \mathrm{mg}$ ).

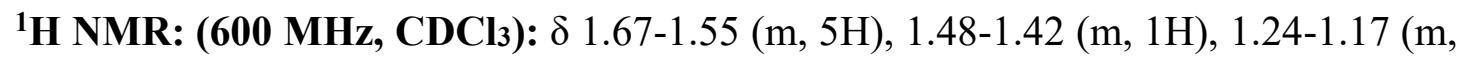
$14 \mathrm{H}), 1.10-1.03(\mathrm{~m}, 1 \mathrm{H}), 0.91-0.85(\mathrm{~m}, 2 \mathrm{H}), 0.67$ (d, $J=7.2 \mathrm{~Hz}, 2 \mathrm{H})$.

${ }^{13}$ C NMR: (151 MHz, CDCl 3$): \delta$ 82.9, 36.0, 34.3, 26.6, 26.4, 24.9, 20.3 (br, low intensity).

${ }^{11}$ B NMR: (193 MHz, CDCl 3$): ~ \delta 33.88$.

HRMS (ESI): calculated for $\mathrm{C}_{13} \mathrm{H}_{26} \mathrm{BO}_{2}[\mathrm{M}+\mathrm{H}]^{+}:$: 225.2020, found: 225.2013 .

\section{Compound 5}<smiles>CC1(C)OB(CCC2OCCCO2)OC1(C)C</smiles>

2-(2-(1,3-dioxan-2-yl)ethyl)-4,4,5,5-tetramethyl-1,3,2-dioxaborolane ${ }^{[19]}$ : Colorless oil was obtained with $63 \%$ isolated yield (Procedure A, $0.6 \mathrm{mmol} \mathrm{scale,} 92.0 \mathrm{mg}$ ).

1H NMR: (600 MHz, CDCl 3$): ~ \delta 4.46(t, J=5.2 \mathrm{~Hz}, 1 \mathrm{H}), 4.09-4.04(\mathrm{~m}, 2 \mathrm{H}), 3.75-3.69$ (m, 2H), 2.09-2.01 (m, 1H), 1.73-1.69 (m, 2H), 1.32-1.28 (m, 1H), $1.22(\mathrm{~s}, 12 \mathrm{H}), 0.82$ (t, $J=7.8 \mathrm{~Hz}, 2 \mathrm{H})$.

${ }^{13}$ C NMR: (151 MHz, $\left.\mathbf{C D C l}_{3}\right): \delta 103.3,83.0,66.9,29.6,25.9,24.9$. The signal of the $\alpha$-B-carbon was not observed. 
${ }^{11}$ B NMR: (193 MHz, CDCl 3$): ~ \delta 33.86$.

HRMS (ESI): calculated for $\mathrm{C}_{12} \mathrm{H}_{24} \mathrm{BO}_{4}[\mathrm{M}+\mathrm{H}]^{+}:$243.1762, found: 243.1757 .

\section{Compound 6}<smiles>CC1(C)OB(CCCB2OC(C)(C)C(C)(C)O2)OC1(C)C</smiles>

1,3-bis(4,4,5,5-tetramethyl-1,3,2-dioxaborolan-2-yl)propane ${ }^{[21]}$ : Colorless oil was obtained with 56\% isolated yield following Procedure A (from bromide, $0.6 \mathrm{mmol} \mathrm{scale}$, $99.6 \mathrm{mg}$ ). $40 \%$ isolated yield was obtained following Procedure B (from diiodide, 0.6 mmol scale, $72.0 \mathrm{mg})$.

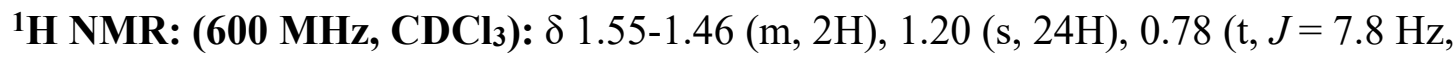
$4 \mathrm{H})$.

${ }^{13}$ C NMR: (151 MHz, $\left.\mathbf{C D C l}_{3}\right): \delta 82.9,24.9,18.7,14.2$ (br, low intensity).

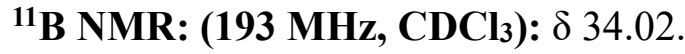

HRMS (ESI): calculated for $\mathrm{C}_{15} \mathrm{H}_{31} \mathrm{~B}_{2} \mathrm{O}_{4}[\mathrm{M}+\mathrm{H}]^{+}$: 297.2403, found: 297.2397.

\section{Compound 7}<smiles>CC1(C)OB(CCCCCF)OC1(C)C</smiles>

2-(5-fluoropentyl)-4,4,5,5-tetramethyl-1,3,2-dioxaborolane: Colorless oil was obtained with 74\% isolated yield (Procedure A, $0.6 \mathrm{mmol} \mathrm{scale,} 96.7 \mathrm{mg}$ ).

${ }^{1}$ H NMR: (600 MHz, CDCl3): $\delta 4.43(\mathrm{t}, J=6.2 \mathrm{~Hz}, 1 \mathrm{H}), 4.35(\mathrm{t}, J=6.2 \mathrm{~Hz}, 1 \mathrm{H}), 1.69$ $1.61(\mathrm{~m}, 2 \mathrm{H}), 1.45-1.35(\mathrm{~m}, 4 \mathrm{H}), 1.21(\mathrm{~s}, 12 \mathrm{H}), 0.76(\mathrm{t}, J=7.6 \mathrm{~Hz}, 2 \mathrm{H})$.

${ }^{13}$ C NMR: (151 MHz, CDCl 3$): ~ \delta 84.2(\mathrm{~d}, J=164.2 \mathrm{~Hz}), 83.0,30.3(\mathrm{~d}, J=19.4 \mathrm{~Hz})$, $27.9(\mathrm{~d}, J=5.6 \mathrm{~Hz}), 24.9,23.7,11.2$ (br, low intensity).

${ }^{19}$ F NMR (565 MHz, CDCl3): $\delta-217.96(t t, ~ J=47.4,24.7 \mathrm{~Hz})$.

${ }^{11}$ B NMR: (193 MHz, CDCl3): $\delta 34.10$.

HRMS (ESI): calculated for $\mathrm{C}_{11} \mathrm{H}_{23} \mathrm{BFO}_{2}[\mathrm{M}+\mathrm{H}]^{+}:$: 217.1770, found: 217.1771 . 


\section{Compound 8}<smiles>CC1(C)OB(CCCC(F)(F)F)OC1(C)C</smiles>

4,4,5,5-tetramethyl-2-(4,4,4-trifluorobutyl)-1,3,2-dioxaborolane: Colorless oil was obtained with $41 \%$ isolated yield (Procedure A, $0.6 \mathrm{mmol} \mathrm{scale,} 58.9 \mathrm{mg}$ ).

${ }^{1}$ H NMR: (600 MHz, $\left.\mathbf{C D C l}_{3}\right): \delta$ 2.13-2.02 (m, 2H), 1.69-1.62 (m, 2H), $1.24(\mathrm{~s}, 12 \mathrm{H})$, $0.84(\mathrm{t}, J=8.0 \mathrm{~Hz}, 2 \mathrm{H})$.

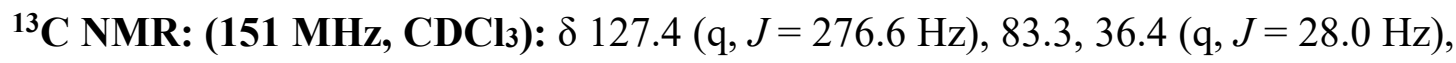
24.9, 16.7 (q, $J=3.0 \mathrm{~Hz}), 10.4$ (br, low intensity).

${ }^{19}$ F NMR (565 MHz, $\left.\mathbf{C D C l}_{3}\right): \delta-66.38(\mathrm{t}, J=11.0 \mathrm{~Hz})$.

${ }^{11}$ B NMR: (193 MHz, CDCl3): $\delta 33.67$.

HRMS (ESI): calculated for $\mathrm{C}_{10} \mathrm{H}_{19} \mathrm{BF}_{3} \mathrm{O}_{2}[\mathrm{M}+\mathrm{H}]^{+}:$: 239.1425, found: 239.1417 .

\section{Compound 9}<smiles>CC(=O)OCCCCCB1OC(C)(C)C(C)(C)O1</smiles>

5-(4,4,5,5-tetramethyl-1,3,2-dioxaborolan-2-yl)pentyl acetate ${ }^{[22]}$ : Colorless oil was obtained with 77\% isolated yield (Procedure A, $0.6 \mathrm{mmol} \mathrm{scale,} 119.0 \mathrm{mg}$ ).

${ }^{1}$ H NMR: (600 MHz, CDCl $): \delta 4.00(\mathrm{t}, J=6.8 \mathrm{~Hz}, 2 \mathrm{H}), 1.99(\mathrm{~s}, 3 \mathrm{H}), 1.60-1.55(\mathrm{~m}$, 2H), 1.42-1.37 (m, 2H), 1.33-1.29 (m, 2H), $1.20(\mathrm{~s}, 12 \mathrm{H}), 0.74(\mathrm{t}, J=7.8 \mathrm{~Hz}, 2 \mathrm{H})$.

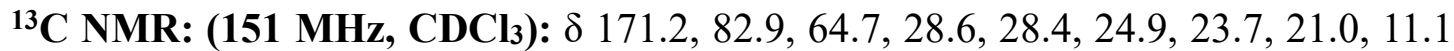
(br, low intensity).

${ }^{11}$ B NMR: (193 MHz, CDCl3): $\delta 34.12$.

HRMS (ESI): calculated for $\mathrm{C}_{13} \mathrm{H}_{26} \mathrm{BO}_{4}[\mathrm{M}+\mathrm{H}]^{+}: 257.1919$, found: 257.1917 .

\section{Compound 10}<smiles>CCOC(=O)CCCCB1OC(C)(C)C(C)(C)O1</smiles> 
ethyl 5-(4,4,5,5-tetramethyl-1,3,2-dioxaborolan-2-yl)pentanoate ${ }^{[21]}$ : Colorless oil was obtained with 45\% isolated yield (Procedure A, $0.6 \mathrm{mmol} \mathrm{scale,} 69.2 \mathrm{mg}$ ).

${ }^{1}$ H NMR: (600 MHz, CDCl 3$): \delta 4.10(\mathrm{q}, J=7.0 \mathrm{~Hz}, 2 \mathrm{H}), 2.27(\mathrm{t}, J=7.6 \mathrm{~Hz}, 2 \mathrm{H})$, 1.64-1.59 (m, 2H), 1.45-1.40 (m, 2H), 1.25-1.22 (m, 15H), $0.78(\mathrm{t}, J=8.0 \mathrm{~Hz}, 2 \mathrm{H})$.

${ }^{13}$ C NMR: (151 MHz, $\left.\mathbf{C D C l}_{3}\right): \delta 174.0,83.1,60.2,34.4,27.7,24.9,23.8,14.4$. The signal of the $\alpha$-B-carbon was not observed.

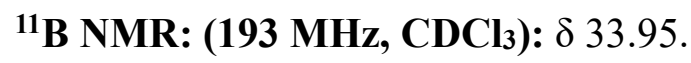

HRMS (ESI): calculated for $\mathrm{C}_{13} \mathrm{H}_{26} \mathrm{BO}_{4}[\mathrm{M}+\mathrm{H}]^{+}:$: 257.1919, found: 257.1923 .

\section{Compound 11}<smiles>CC1(C)OB(CCC(C#N)(c2ccccc2)c2ccccc2)OC1(C)C</smiles>

2,2-diphenyl-4-(4,4,5,5-tetramethyl-1,3,2-dioxaborolan-2-yl)butanenitrile: White solid was obtained with 65\% isolated yield (Procedure A, $0.6 \mathrm{mmol}$ scale, $136.0 \mathrm{mg}$ ).

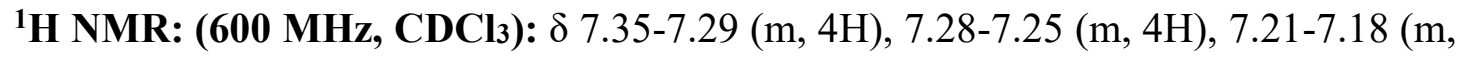
2H), 2.43-2.38 (m, 2H), $1.15(\mathrm{~s}, 12 \mathrm{H}), 0.86-0.82(\mathrm{~m}, 2 \mathrm{H})$.

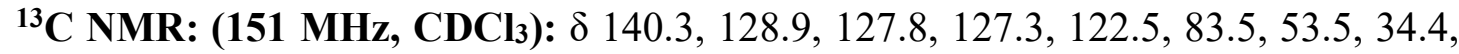
24.9. The signal of the $\alpha$-B-carbon was not observed.

${ }^{11}$ B NMR: (193 MHz, CDCl3): $\delta 34.21$.

HRMS (ESI): calculated for $\mathrm{C}_{22} \mathrm{H}_{27} \mathrm{BNO}_{2}[\mathrm{M}+\mathrm{H}]^{+}:$348.2129, found: 348.2125 .

\section{Compound 12}<smiles>CC1(C)OB(CCc2ccccc2)OC1(C)C</smiles>

4,4,5,5-tetramethyl-2-phenethyl-1,3,2-dioxaborolane ${ }^{[21]}$ : Colorless oil was obtained with $69 \%$ isolated yield (Procedure A, $0.6 \mathrm{mmol} \mathrm{scale,} 96.5 \mathrm{mg}$ ).

1H NMR: (600 MHz, CDCl $)$ ): $\delta$ 7.25-6.99 (m, 5H), 2.67 (t, $J=8.2 \mathrm{~Hz}, 2 \mathrm{H}), 1.13$ (s, 12H), $1.06(\mathrm{t}, J=8.4 \mathrm{~Hz}, 2 \mathrm{H})$. 
${ }^{13}$ C NMR: (151 MHz, CDCl 3$): \delta 144.5,128.3,128.1,125.6,83.2,30.0,24.9,13.1$ (br, low intensity).

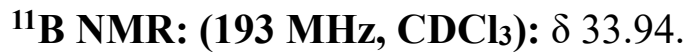

HRMS (ESI): calculated for $\mathrm{C}_{14} \mathrm{H}_{22} \mathrm{BO}_{2}[\mathrm{M}+\mathrm{H}]^{+}:$: 233.1707, found: 233.1704 .

\section{Compound 13}<smiles>CC1(C)OB(CCCCc2ccccc2)OC1(C)C</smiles>

4,4,5,5-tetramethyl-2-(4-phenylbutyl)-1,3,2-dioxaborolane ${ }^{[20]}$ : Colorless oil was obtained with $83 \%$ isolated yield (Procedure A, $0.6 \mathrm{mmol} \mathrm{scale,} 129.6 \mathrm{mg}$ ). 75\% isolated yield was obtained in gram scale experiment at $400 \mathrm{~mA}(77 \mathrm{~min}, 7.2 \mathrm{mmol}$ scale, $1.41 \mathrm{~g})$.

${ }^{1}$ H NMR: (600 MHz, CDCl 3$): \delta 7.19-7.13(\mathrm{~m}, 2 \mathrm{H}), 7.10-7.03(\mathrm{~m}, 3 \mathrm{H}), 2.51(\mathrm{t}, J=7.8$ $\mathrm{Hz}, 2 \mathrm{H}), 1.57-1.51(\mathrm{~m}, 2 \mathrm{H}), 1.42-1.36(\mathrm{~m}, 2 \mathrm{H}), 1.14(\mathrm{~s}, 12 \mathrm{H}), 0.73(\mathrm{t}, J=7.8 \mathrm{~Hz}, 2 \mathrm{H})$. ${ }^{13}$ C NMR: (151 MHz, CDCl $): \delta$ 142.9, 128.4, 128.2, 125.5, 82.9, 35.8, 34.3, 24.9, 23.8, 11.1 (br, low intensity).

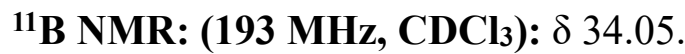

HRMS (ESI): calculated for $\mathrm{C}_{16} \mathrm{H}_{26} \mathrm{BO}_{2}[\mathrm{M}+\mathrm{H}]^{+}:$261.2020, found: 261.2019.

\section{Compound 14}<smiles>CC1(C)OB(CCC(c2ccccc2)c2ccccc2)OC1(C)C</smiles>

2-(3,3-diphenylpropyl)-4,4,5,5-tetramethyl-1,3,2-dioxaborolane ${ }^{[23,24]}$ : White solid was obtained with $81 \%$ isolated yield (Procedure A, $0.6 \mathrm{mmol} \mathrm{scale,} 156.9 \mathrm{mg}$ ).

${ }^{1}$ H NMR: (600 MHz, CDCl3): $\delta$ 7.16-7.12 (m, 8H), 7.06-7.01 (m, 2H), $3.75(\mathrm{t}, J=7.8$ $\mathrm{Hz}, 1 \mathrm{H}), 2.09-2.03(\mathrm{~m}, 2 \mathrm{H}), 1.11(\mathrm{~s}, 12 \mathrm{H}), 0.70-0.61(\mathrm{~m}, 2 \mathrm{H})$.

${ }^{13}$ C NMR: (151 MHz, CDCl $): \delta 145.1,128.4,128.1,126.0,82.9,53.7,30.1,24.9,9.9$ (br, low intensity). 
${ }^{11}$ B NMR: (193 MHz, CDCl 3$): ~ \delta 33.26$.

HRMS (ESI): calculated for $\mathrm{C}_{21} \mathrm{H}_{28} \mathrm{BO}_{2}[\mathrm{M}+\mathrm{H}]^{+}: 323.2177$, found: 323.2176 .

\section{Compound 15}<smiles>CC1(C)OB(CCc2cccc(Cl)c2)OC1(C)C</smiles>

2-(3-chlorophenethyl)-4,4,5,5-tetramethyl-1,3,2-dioxaborolane ${ }^{[25,26]}$ : Colorless oil was obtained with 56\% isolated yield (Procedure A, $0.6 \mathrm{mmol} \mathrm{scale,} 89.9 \mathrm{mg}$ ).

${ }^{1}$ H NMR: (600 MHz, CDCl $)$ ): $\delta$ 7.18-6.98 (m, 4H), $2.63(\mathrm{t}, J=8.0 \mathrm{~Hz}, 2 \mathrm{H}), 1.12(\mathrm{~s}$, 12H), $1.03(\mathrm{t}, J=8.0 \mathrm{~Hz}, 2 \mathrm{H})$.

${ }^{13}$ C NMR: (151 MHz, CDCl3): $\delta$ 146.5, 133.9, 129.5, 128.4, 126.4, 125.8, 83.3, 29.8, 24.9, 12.9 (br, low intensity).

11B NMR: (193 MHz, $\left.\mathrm{CDCl}_{3}\right): \delta 33.80$.

HRMS (ESI): calculated for $\mathrm{C}_{14} \mathrm{H}_{21} \mathrm{BClO}_{2}[\mathrm{M}+\mathrm{H}]^{+}:$: 267.1318, found: 267.1312 .

\section{Compound 16}<smiles>CC1(C)OB(CCCc2ccc(Cl)cc2)OC1(C)C</smiles>

2-(3-(4-chlorophenyl)propyl)-4,4,5,5-tetramethyl-1,3,2-dioxaborolane ${ }^{[27]}$ :

Colorless oil was obtained with $82 \%$ isolated yield (Procedure A, $0.6 \mathrm{mmol}$ scale, 137.3 $\mathrm{mg})$.

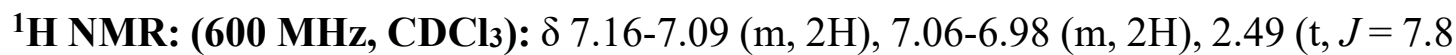
$\mathrm{Hz}, 2 \mathrm{H}), 1.65-1.59(\mathrm{~m}, 2 \mathrm{H}), 1.16(\mathrm{~s}, 12 \mathrm{H}), 0.72(\mathrm{t}, J=7.8 \mathrm{~Hz}, 2 \mathrm{H})$.

${ }^{13}$ C NMR: (151 MHz, CDCl $): \delta$ 141.2, 131.4, 130.0, 128.3, 83.1, 37.9, 26.2, 24.9, 10.9 (br, low intensity).

${ }^{11}$ B NMR: (193 MHz, CDCl 3$): ~ \delta 33.98$.

HRMS (ESI): calculated for $\mathrm{C}_{15} \mathrm{H}_{23} \mathrm{BClO}_{2}[\mathrm{M}+\mathrm{H}]^{+}:$: 281.1474, found: 281.1475 . 


\section{Compound 17}<smiles>CC1(C)OB(CCOc2ccccc2)OC1(C)C</smiles>

4,4,5,5-tetramethyl-2-(2-phenoxyethyl)-1,3,2-dioxaborolane ${ }^{[28]}$ : Colorless oil was obtained with 51\% isolated yield (Procedure A, $0.6 \mathrm{mmol} \mathrm{scale,} 76.0 \mathrm{mg}$ ).

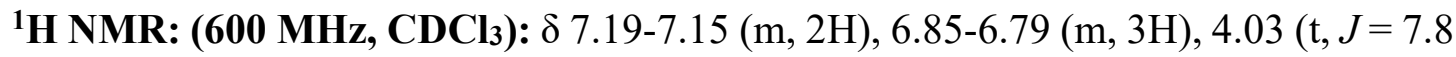
$\mathrm{Hz}, 2 \mathrm{H}), 1.29$ (t, $J=7.8 \mathrm{~Hz}, 2 \mathrm{H}), 1.18(\mathrm{~s}, 12 \mathrm{H})$.

${ }^{13}$ C NMR: (151 MHz, CDCl 3 ): $\delta$ 159.1, 129.4, 120.5, 114.7, 83.4, 64.9, 24.9, 12.8 (br, low intensity).

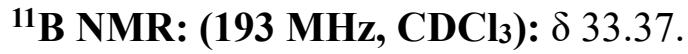

HRMS (ESI): calculated for $\mathrm{C}_{14} \mathrm{H}_{22} \mathrm{BO}_{3}[\mathrm{M}+\mathrm{H}]^{+}:$: 249.1657, found: 249.1652 .

\section{Compound 18}<smiles>CC1(C)OB(CCCCOc2ccccc2)OC1(C)C</smiles>

4,4,5,5-tetramethyl-2-(4-phenoxybutyl)-1,3,2-dioxaborolane ${ }^{[29]}$ : Colorless oil was obtained with $87 \%$ isolated yield (Procedure A, $0.6 \mathrm{mmol} \mathrm{scale,} 144.6 \mathrm{mg}$ ).

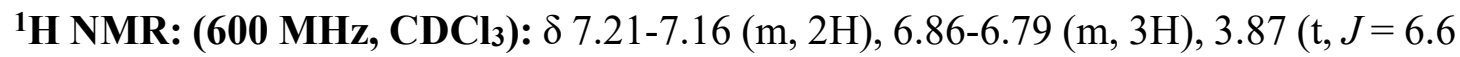
$\mathrm{Hz}, 2 \mathrm{H}), 1.74-1.69(\mathrm{~m}, 2 \mathrm{H}), 1.54-1.48(\mathrm{~m}, 2 \mathrm{H}), 1.17(\mathrm{~s}, 12 \mathrm{H}), 0.78(\mathrm{t}, J=8.0 \mathrm{~Hz}, 2 \mathrm{H})$.

${ }^{13}$ C NMR: (151 MHz, CDCl 3$): \delta$ 159.2, 129.5, 120.5, 114.6, 83.1, 67.7, 31.9, 24.9, 20.7, 11.0 (br, low intensity).

${ }^{11}$ B NMR: (193 MHz, CDCl3): $\delta 34.09$.

HRMS (ESI): calculated for $\mathrm{C}_{16} \mathrm{H}_{26} \mathrm{BO}_{3}[\mathrm{M}+\mathrm{H}]^{+}: 277.1970$, found: 277.1975 .

\section{Compound 19}<smiles>CC1(C)OB(CCCCOc2ccc3ccccc3c2)OC1(C)C</smiles>

4,4,5,5-tetramethyl-2-(4-(naphthalen-2-yloxy)butyl)-1,3,2-dioxaborolane ${ }^{[30]}$ :

White solid was obtained with 56\% isolated yield (Procedure A, $0.6 \mathrm{mmol}$ scale, 110.3 mg). 


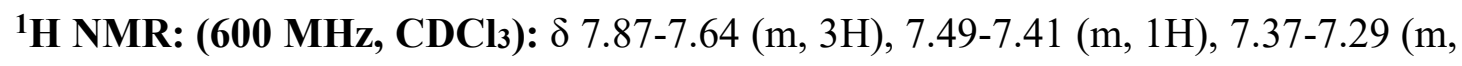
1H), 7.20-7.10 (m, 2H), 4.09 (t, $J=6.6 \mathrm{~Hz}, 2 \mathrm{H}), 1.93-1.86(\mathrm{~m}, 2 \mathrm{H}), 1.71-1.65(\mathrm{~m}, 2 \mathrm{H})$, $1.27(\mathrm{~s}, 12 \mathrm{H}), 0.92(\mathrm{t}, J=7.8 \mathrm{~Hz}, 2 \mathrm{H})$.

${ }^{13}$ C NMR: (151 MHz, CDCl3): $\delta$ 157.2, 134.7, 129.3, 128.9, 127.7, 126.8, 126.3, 123.5, 119.2, 106.6, 83.1, 67.8, 31.8, 24.9, 20.8, 11.1 (br, low intensity).

${ }^{11}$ B NMR: (193 MHz, CDCl 3$): ~ \delta 34.34$.

HRMS (ESI): calculated for $\mathrm{C}_{20} \mathrm{H}_{28} \mathrm{BO}_{3}[\mathrm{M}+\mathrm{H}]^{+}: 327.2126$, found: 327.2122 .

\section{Compound 20}<smiles>CC1(C)OB(CCc2ccc3c(c2)CCO3)OC1(C)C</smiles>

\section{2-(2-(2,3-dihydrobenzofuran-6-yl)ethyl)-4,4,5,5-tetramethyl-1,3,2-}

dioxaborolane $^{[19]}$ : Colorless oil was obtained with 75\% isolated yield (Procedure A, $0.6 \mathrm{mmol}$ scale, $123.4 \mathrm{mg})$.

${ }^{1}$ H NMR: (600 MHz, CDCl 3 ): $\delta 7.05$ (s, 1H), 6.97-6.91 (m, 1H), 6.67 (d, $J=8.0 \mathrm{~Hz}$, 1H), 4.52 (t, $J=8.6 \mathrm{~Hz}, 2 \mathrm{H}), 3.15(\mathrm{t}, J=8.6 \mathrm{~Hz}, 2 \mathrm{H}), 2.68$ (t, $J=8.2 \mathrm{~Hz}, 2 \mathrm{H}), 1.22$ (s, $12 \mathrm{H}), 1.10(\mathrm{t}, J=8.0 \mathrm{~Hz}, 2 \mathrm{H})$.

${ }^{13}$ C NMR: (151 MHz, CDCl3): $\delta$ 158.1, 136.6, 127.4, 126.8, 124.6, 108.8, 83.1, 71.1, 29.9, 29.5, 24.9. The signal of the $\alpha$-B-carbon was not observed.

${ }^{11}$ B NMR: (193 MHz, CDCl 3$): ~ \delta 34.23$.

HRMS (ESI): calculated for $\mathrm{C}_{16} \mathrm{H}_{24} \mathrm{BO}_{3}[\mathrm{M}+\mathrm{H}]^{+}:$: 275.1813, found: 275.1806 .

\section{Compound 21}<smiles>CC1(C)OB(CCc2ccsc2)OC1(C)C</smiles>

4,4,5,5-tetramethyl-2-(2-(thiophen-3-yl)ethyl)-1,3,2-dioxaborolane ${ }^{[19]}: \quad$ Colorless oil was obtained with 70\% isolated yield (Procedure A, $0.6 \mathrm{mmol} \mathrm{scale,} 100.2 \mathrm{mg}$ ). 
${ }^{1}$ H NMR: (600 MHz, CDCl3): $\delta 7.20(\mathrm{dd}, J=5.0,2.8 \mathrm{~Hz}, 1 \mathrm{H}), 6.97-6.93(\mathrm{~m}, 2 \mathrm{H})$, 2.79-2.75 (m, 2H), $1.22(\mathrm{~s}, 12 \mathrm{H}), 1.16(\mathrm{t}, J=8.0 \mathrm{~Hz}, 2 \mathrm{H})$.

${ }^{13}$ C NMR: (151 MHz, CDCl $)$ ): $\delta$ 144.9, 128.2, 125.0, 119.5, 83.1, 24.9, 24.6, 12.2 (br, low intensity).

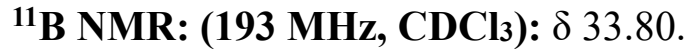

HRMS (ESI): calculated for $\mathrm{C}_{12} \mathrm{H}_{20} \mathrm{BO}_{2} \mathrm{~S}[\mathrm{M}+\mathrm{H}]^{+}:$: 239.1272, found: 239.1268.

\section{Compound 22}<smiles>CC1(C)OB(CCc2c[nH]c3ccccc23)OC1(C)C</smiles>

3-(2-(4,4,5,5-tetramethyl-1,3,2-dioxaborolan-2-yl)ethyl)-1H-indole ${ }^{[31]}$ : Colorless oil was obtained with $84 \%$ isolated yield (Procedure A, $0.6 \mathrm{mmol} \mathrm{scale,} 137.3 \mathrm{mg}$ ).

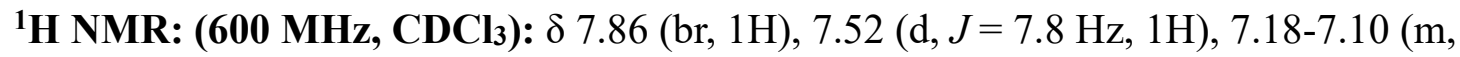
1H), 7.07-7.01 (m, 1H), 7.01-6.95 (m, 1H), 6.79 (t, $J=2.6 \mathrm{~Hz}, 1 \mathrm{H}), 2.79(\mathrm{t}, J=8.2 \mathrm{~Hz}$, $2 \mathrm{H}), 1.17(\mathrm{t}, J=8.0 \mathrm{~Hz}, 2 \mathrm{H}), 1.11(\mathrm{~s}, 12 \mathrm{H})$.

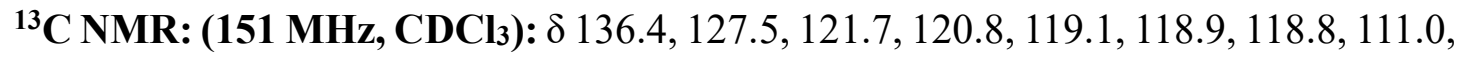
83.1, 24.8, 19.4, 11.7 (br, low intensity).

${ }^{11}$ B NMR: (193 MHz, CDCl3): $\delta 34.46$.

HRMS (ESI): calculated for $\mathrm{C}_{16} \mathrm{H}_{23} \mathrm{BNO}_{2}[\mathrm{M}+\mathrm{H}]^{+}:$272.1816, found: 272.1810 .

\section{Compound 23}

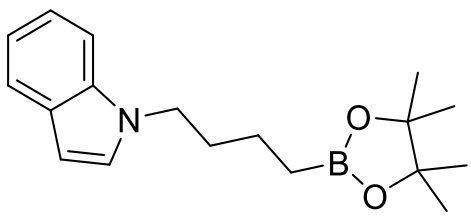

1-(4-(4,4,5,5-tetramethyl-1,3,2-dioxaborolan-2-yl)butyl)-1H-indole ${ }^{[32]}: \quad$ Colorless oil was obtained with 70\% isolated yield (Procedure A, $0.6 \mathrm{mmol}$ scale, $126.0 \mathrm{mg}$ ).

${ }^{1}$ H NMR: (600 MHz, $\left.\mathbf{C D C l}_{3}\right): \delta 7.52(\mathrm{~d}, J=6.8 \mathrm{~Hz}, 1 \mathrm{H}), 7.23(\mathrm{~d}, J=8.2 \mathrm{~Hz}, 1 \mathrm{H})$, $7.08(\mathrm{t}, J=7.6 \mathrm{~Hz}, 1 \mathrm{H}), 7.00-6.96(\mathrm{~m}, 2 \mathrm{H}), 6.38-6.35(\mathrm{~m}, 1 \mathrm{H}), 3.97(\mathrm{t}, J=7.8 \mathrm{~Hz}, 2 \mathrm{H})$, 1.76-1.71 (m, 2H), 1.39-1.34 (m, 2H), $1.11(\mathrm{~s}, 12 \mathrm{H}), 0.73(\mathrm{t}, J=7.8 \mathrm{~Hz}, 2 \mathrm{H})$. 
${ }^{13}$ C NMR: (151 MHz, CDCl $): \delta$ 135.9, 128.6, 127.8, 121.2, 120.9, 119.1, 109.5, 100.8, 83.0, 46.2, 32.6, 24.8, 21.5, 10.8 (br, low intensity).

${ }^{11}$ B NMR: (193 MHz, CDCl 3$): ~ \delta 33.90$.

HRMS (ESI): calculated for $\mathrm{C}_{18} \mathrm{H}_{27} \mathrm{BNO}_{2}[\mathrm{M}+\mathrm{H}]^{+}:$300.2129, found: 300.2127 .

\section{Compound 24}

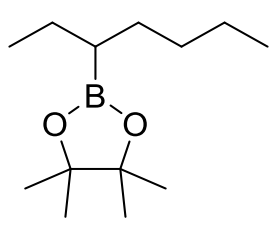

2-(heptan-3-yl)-4,4,5,5-tetramethyl-1,3,2-dioxaborolane ${ }^{[32]}$ : Colorless oil was obtained with $47 \%$ isolated yield (Procedure A, $0.6 \mathrm{mmol} \mathrm{scale}, 64.0 \mathrm{mg}$ ).

${ }^{1}$ H NMR: (400 MHz, CDCl3): $\delta$ 1.43-1.22 (m, 20H), 0.95-0.78 (m, 7H).

${ }^{13}$ C NMR: (101 MHz, CDCl 3$): \delta 82.9,31.7,30.9,24.9,24.4,23.1,14.2,13.9$. The signal of the $\alpha$-B-carbon was not observed.

${ }^{11}$ B NMR: (128 MHz, CDCl3): $\delta 33.91$.

HRMS (ESI): calculated for $\mathrm{C}_{13} \mathrm{H}_{28} \mathrm{BO}_{2}[\mathrm{M}+\mathrm{H}]^{+}:$227.2177, found: 227.2173.

\section{Compound 25}

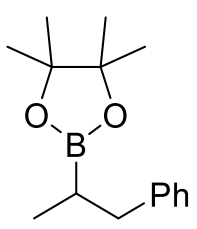

4,4,5,5-tetramethyl-2-(1-phenylpropan-2-yl)-1,3,2-dioxaborolane ${ }^{[28]}$ : Colorless oil was obtained with 60\% isolated yield (Procedure A, $0.6 \mathrm{mmol} \mathrm{scale}, 88.7 \mathrm{mg}$ ).

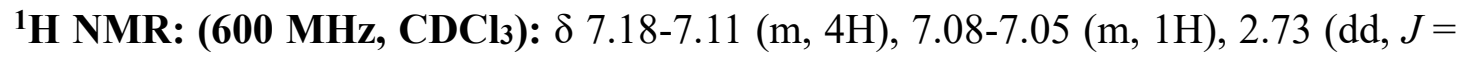
13.6, $7.6 \mathrm{~Hz}, 1 \mathrm{H}), 2.47(\mathrm{dd}, J=13.6,8.4 \mathrm{~Hz}, 1 \mathrm{H}), 1.33-1.26(\mathrm{~m}, 1 \mathrm{H}), 1.10(\mathrm{~d}, J=6.8$ $\mathrm{Hz}, 12 \mathrm{H}), 0.89$ (d, $J=7.4 \mathrm{~Hz}, 3 \mathrm{H})$.

${ }^{13}$ C NMR: (151 MHz, CDCl3): $\delta$ 142.4, 129.0, 128.1, 125.7, 83.1, 39.1, 24.8, 24.8, 19.2 (br, low intensity), 15.3.

${ }^{11}$ B NMR: (193 MHz, CDCl 3$): ~ \delta 34.27$.

HRMS (ESI): calculated for $\mathrm{C}_{15} \mathrm{H}_{24} \mathrm{BO}_{2}[\mathrm{M}+\mathrm{H}]^{+}:$: 247.1864, found: 247.1862 . 


\section{Compound 26}<smiles>CC1(C)OB(C2CCCC2)OC1(C)C</smiles>

2-cyclopentyl-4,4,5,5-tetramethyl-1,3,2-dioxaborolane ${ }^{[28]}$ : Colorless oil was obtained with 50\% isolated yield following Procedure A (from bromide, $0.6 \mathrm{mmol} \mathrm{scale}$, $59.5 \mathrm{mg}$ ). $61 \%$ isolated yield was obtained following Procedure B (from iodide, 0.6 mmol scale, $72.0 \mathrm{mg})$.

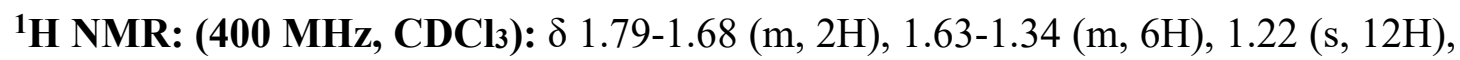
$1.18-1.10(\mathrm{~m}, 1 \mathrm{H})$.

${ }^{13}$ C NMR: (101 MHz, $\left.\mathbf{C D C l}_{3}\right): \delta 82.9,28.6,26.9,24.8$. The signal of the $\alpha$-B-carbon was not observed.

${ }^{11}$ B NMR: (128 MHz, CDCl 3$): \delta 33.66$.

HRMS (ESI): calculated for $\mathrm{C}_{11} \mathrm{H}_{22} \mathrm{BO}_{2}[\mathrm{M}+\mathrm{H}]^{+}$: 197.1707, found: 197.1707.

\section{Compound 27}<smiles>CC1(C)OB(C2CCCCCC2)OC1(C)C</smiles>

2-cycloheptyl-4,4,5,5-tetramethyl-1,3,2-dioxaborolane ${ }^{[28]}$ : Colorless oil was obtained with 57\% isolated yield (Procedure A, $0.6 \mathrm{mmol} \mathrm{scale,} 77.0 \mathrm{mg}$ ).

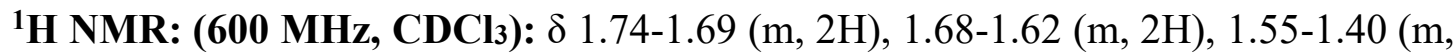
$8 \mathrm{H}), 1.21(\mathrm{~s}, 12 \mathrm{H}), 1.08-1.01(\mathrm{~m}, 1 \mathrm{H})$.

${ }^{13}$ C NMR: (151 MHz, CDCl3): $\delta$ 82.8, 29.7, 29.1, 28.5, 24.8, 23.5 (br, low intensity). ${ }^{11}$ B NMR: (193 MHz, CDCl3): $\delta 34.37$.

HRMS (ESI): calculated for $\mathrm{C}_{13} \mathrm{H}_{26} \mathrm{BO}_{2}[\mathrm{M}+\mathrm{H}]^{+}:$: 225.2020, found: 225.2019.

\section{Compound 28}

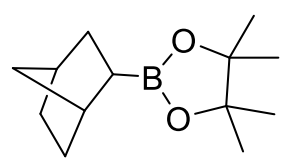

2-((1R,2S,4R)-bicyclo[2.2.1]heptan-2-yl)-4,4,5,5-tetramethyl-1,3,2-

dioxaborolane $^{[28]}$ : Colorless oil was obtained with $74 \%$ isolated yield (Procedure A, 
$0.6 \mathrm{mmol}$ scale, $99.5 \mathrm{mg})$.

${ }^{1}$ H NMR: (600 MHz, CDCl 3$): ~ \delta 2.27$ (br, 1H), 2.21 (br, 1H), 1.55-1.45 (m, 3H), 1.35$1.31(\mathrm{~m}, 1 \mathrm{H}), 1.21(\mathrm{~s}, 14 \mathrm{H}), 1.19-1.13(\mathrm{~m}, 2 \mathrm{H}), 0.89-0.84(\mathrm{~m}, 1 \mathrm{H})$.

${ }^{13}$ C NMR: (151 MHz, CDCl3): $\delta$ 82.9, 38.8, 38.3, 36.8, 32.3, 32.3, 29.4, 24.8. The signal of the $\alpha$-B-carbon was not observed.

${ }^{11}$ B NMR: (193 MHz, CDCl3): $\delta 34.14$.

HRMS (ESI): calculated for $\mathrm{C}_{13} \mathrm{H}_{24} \mathrm{BO}_{2}[\mathrm{M}+\mathrm{H}]^{+}:$223.1864, found: 223.1856 .

\section{Compound 29}

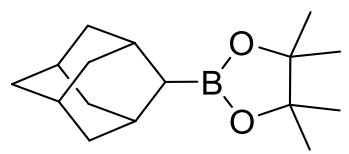

2-((1r,3r,5r,7r)-adamantan-2-yl)-4,4,5,5-tetramethyl-1,3,2-dioxaborolane ${ }^{[28]}$

Colorless oil was obtained with 93\% isolated yield (Procedure A, $0.6 \mathrm{mmol}$ scale, 146.7 $\mathrm{mg})$.

${ }^{1}$ H NMR: (600 MHz, CDCl3): $\delta 2.03$ (br, 2H), 1.87-1.65 (m, 12H), 1.34 (br, 1H), 1.22 $(\mathrm{s}, 12 \mathrm{H})$

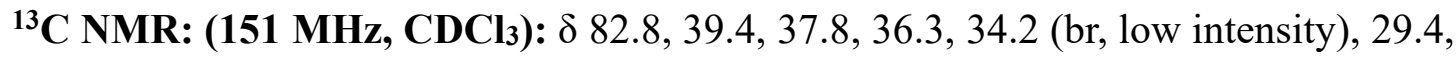
$28.3,28.2,24.9$.

${ }^{11}$ B NMR: (193 MHz, CDCl3): $\delta 33.96$.

HRMS (ESI): calculated for $\mathrm{C}_{16} \mathrm{H}_{28} \mathrm{BO}_{2}[\mathrm{M}+\mathrm{H}]^{+}:$: 263.2177, found: 263.2179.

\section{Compound 30}<smiles>CC(C)(C)OC(=O)N1CCC(B2OC(C)(C)C(C)(C)O2)C1</smiles>

tert-butyl 3-(4,4,5,5-tetramethyl-1,3,2-dioxaborolan-2-yl)pyrrolidine-1-carboxylate $^{[33]}$ : Colorless oil was obtained with $63 \%$ isolated yield (Procedure A, $0.6 \mathrm{mmol}$ scale, $113.0 \mathrm{mg})$.

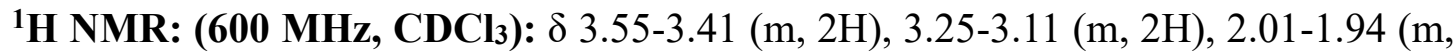
1H), 1.79-1.73 (m, 1H), 1.59-1.53 (m, 1H), $1.44(\mathrm{~s}, 9 \mathrm{H}), 1.23(\mathrm{~s}, 12 \mathrm{H})$.

${ }^{13}$ C NMR: (151 MHz, CDCl3): $\delta$ 154.7, 83.6, 78.9, 48.0, 46.7, 28.7, 24.9. The signal 
of the $\alpha$-B-carbon was not observed.

${ }^{11}$ B NMR: (193 MHz, CDCl 3$): ~ \delta 33.84$.

HRMS (ESI): calculated for $\mathrm{C}_{15} \mathrm{H}_{29} \mathrm{BNO}_{4}[\mathrm{M}+\mathrm{H}]^{+}:$: 298.2184, found: 298.2187 .

\section{Compound 31}

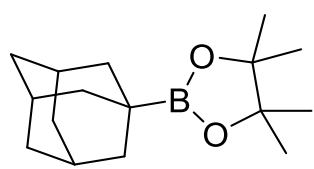

2-((3r,5r,7r)-adamantan-1-yl)-4,4,5,5-tetramethyl-1,3,2-dioxaborolane ${ }^{[28]}$ :

Colorless oil was obtained with $71 \%$ isolated yield following Procedure A $(0.6 \mathrm{mmol}$ scale, $112.2 \mathrm{mg}$ ) and $87 \%$ isolated yield following Procedure B (0.6 mmol scale, 137.0 mg).

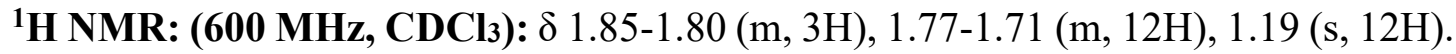

${ }^{13}$ C NMR: (151 MHz, $\left.\mathbf{C D C l}_{3}\right): \delta$ 82.7, 38.1, 37.6, 27.7, 24.7, 20.4 (br, low intensity).

${ }^{11}$ B NMR: (193 MHz, CDCl3): $\delta 33.88$.

HRMS (ESI): calculated for $\mathrm{C}_{16} \mathrm{H}_{28} \mathrm{BO}_{2}[\mathrm{M}+\mathrm{H}]^{+}: 263.2177$, found: 263.2179 .

\section{Compound 32}<smiles>CC1(C)OB(CCCCCl)OC1(C)C</smiles>

2-(4-chlorobutyl)-4,4,5,5-tetramethyl-1,3,2-dioxaborolane ${ }^{[34]}$ : Colorless oil was obtained with 64\% isolated yield (Procedure A, $0.6 \mathrm{mmol} \mathrm{scale,} 84.5 \mathrm{mg}$ ).

${ }^{1}$ H NMR: (600 MHz, CDCl3): $\delta 3.49(\mathrm{t}, J=6.8 \mathrm{~Hz}, 2 \mathrm{H}), 1.77-1.72(\mathrm{~m}, 2 \mathrm{H}), 1.54-1.48$ $(\mathrm{m}, 2 \mathrm{H}), 1.21(\mathrm{~s}, 12 \mathrm{H}), 0.76(\mathrm{t}, J=8.0 \mathrm{~Hz}, 2 \mathrm{H})$.

${ }^{13}$ C NMR: (151 MHz, CDCl3): $\delta$ 83.1, 44.9, 35.2, 24.9, 21.5, 10.5 (br, low intensity).

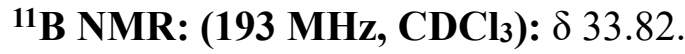

HRMS (ESI): calculated for $\mathrm{C}_{10} \mathrm{H}_{21} \mathrm{BClO}_{2}[\mathrm{M}+\mathrm{H}]^{+}:$219.1318, found: 219.1314 . 


\section{Compound 33}<smiles>CC1(C)OB(CCCCCB2OC(C)(C)C(C)(C)O2)OC1(C)C</smiles>

1,5-bis(4,4,5,5-tetramethyl-1,3,2-dioxaborolan-2-yl)pentane ${ }^{[34]}$ : Colorless oil was obtained with $37 \%$ isolated yield (Procedure A, 8.0 equiv. $\mathrm{B}_{2} \mathrm{cat}_{2}, 7.0 \mathrm{~F}, 0.6 \mathrm{mmol}$ scale, $73.1 \mathrm{mg})$.

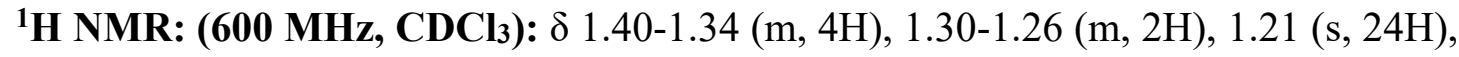
$0.73(\mathrm{t}, J=7.8 \mathrm{~Hz}, 4 \mathrm{H})$.

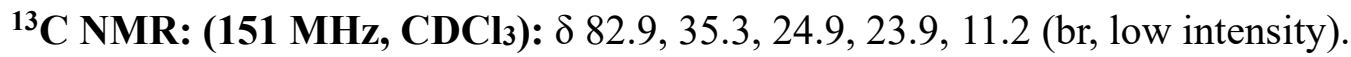

${ }^{11}$ B NMR: (193 MHz, CDCl 3$): ~ \delta 33.97$.

HRMS (ESI): calculated for $\mathrm{C}_{17} \mathrm{H}_{35} \mathrm{~B}_{2} \mathrm{O}_{4}[\mathrm{M}+\mathrm{H}]^{+}: 325.2716$, found: 325.2720 .

\section{Compound 34}

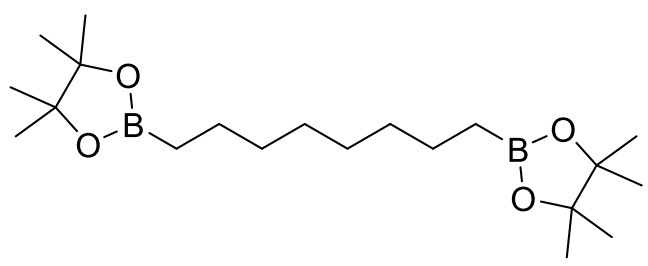

1,8-bis(4,4,5,5-tetramethyl-1,3,2-dioxaborolan-2-yl)octane ${ }^{[31]}$ : Colorless oil was obtained with 54\% isolated yield (Procedure A, 8.0 equiv. $\mathrm{B}_{2} \mathrm{cat}_{2}, 7.0 \mathrm{~F} / \mathrm{mol}, 0.6 \mathrm{mmol}$ scale, $119.7 \mathrm{mg})$.

${ }^{1}$ H NMR: (600 MHz, CDCl $)$ ): $\delta$ 1.39-1.34 (m, 4H), 1.24 (s, 8H), 1.22 (s, 24H), 0.73 (t, $J=7.8 \mathrm{~Hz}, 4 \mathrm{H})$.

${ }^{13}$ C NMR: (151 MHz, CDCl3): $\delta$ 82.9, 32.5, 29.5, 24.9, 24.1, 11.3 (br, low intensity). ${ }^{11}$ B NMR: (193 MHz, CDCl3): $\delta 34.14$.

HRMS (ESI): calculated for $\mathrm{C}_{20} \mathrm{H}_{41} \mathrm{~B}_{2} \mathrm{O}_{4}[\mathrm{M}+\mathrm{H}]^{+}: 367.3185$, found: 367.3185 . 


\section{Compound 35}<smiles>CC1(C)OB(C2CCCCC2B2OC(C)(C)C(C)(C)O2)OC1(C)C</smiles>

Cis-1,2-bis(4,4,5,5-tetramethyl-1,3,2-dioxaborolan-2-yl)cyclohexane ${ }^{[35]}$ : White solid was obtained with $84 \%$ isolated yield (Procedure A, $0.6 \mathrm{mmol}$ scale, $170.2 \mathrm{mg}$ ).

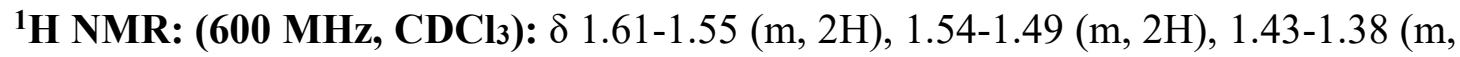
2H), 1.37-1.32 (m, 2H), 1.21-1.19 (m, 26H).

${ }^{13}$ C NMR: (151 MHz, CDCl3): $\delta$ 82.8, 28.1, 26.9, 24.9, 24.9, 23.3 (br, low intensity). ${ }^{11}$ B NMR: (193 MHz, CDCl3): $\delta 33.89$.

HRMS (ESI): calculated for $\mathrm{C}_{18} \mathrm{H}_{35} \mathrm{~B}_{2} \mathrm{O}_{4}[\mathrm{M}+\mathrm{H}]^{+}: 337.2716$, found: 337.2713 .

Note: Starting dibromo compound purchased from commercial available source is pure trans isomer. The product 35 were determined as pure cis isomer by following methods.

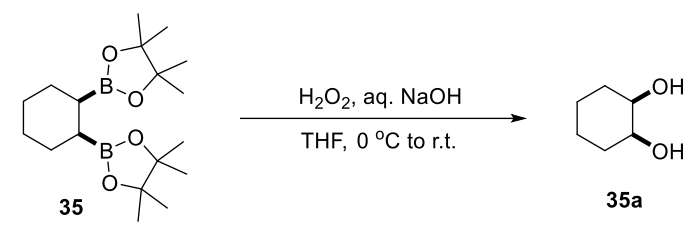

To a solution of $35(168.1 \mathrm{mg}, 0.5 \mathrm{mmol})$ in THF $(2.5 \mathrm{~mL})$, a premixed solution of $\mathrm{NaOH}(3.0 \mathrm{M}$ aqueous $) / \mathrm{H}_{2} \mathrm{O}_{2}(30 \%$ aqueous $)(2: 1,7.5 \mathrm{~mL})$ was added dropwise at 0 ${ }^{\circ} \mathrm{C}$. After addition the reaction was stirred at room temperature for a further $4 \mathrm{~h}$. The reaction was quenched by the addition of $10 \%$ aqueous $\mathrm{Na}_{2} \mathrm{~S}_{2} \mathrm{O}_{3}(15 \mathrm{~mL})$, diluted with water $(15 \mathrm{~mL})$, extracted with DCM $(2 \times 15 \mathrm{~mL})$, washed with brine and dried over $\mathrm{NaSO}_{4}$, concentrated, and the crude product 35a was afford. Only the cis-product was observed by ${ }^{1} \mathrm{H}$ NMR spectrum. ${ }^{[36,37]}$

1H NMR: (600 MHz, CDCl 3$): \delta 3.73-3.67$ (m, 2H), 1.76-1.64 (m, 2H), 1.61-1.43 (m, $4 \mathrm{H}), 1.27-1.22(\mathrm{~m}, 2 \mathrm{H})$. 


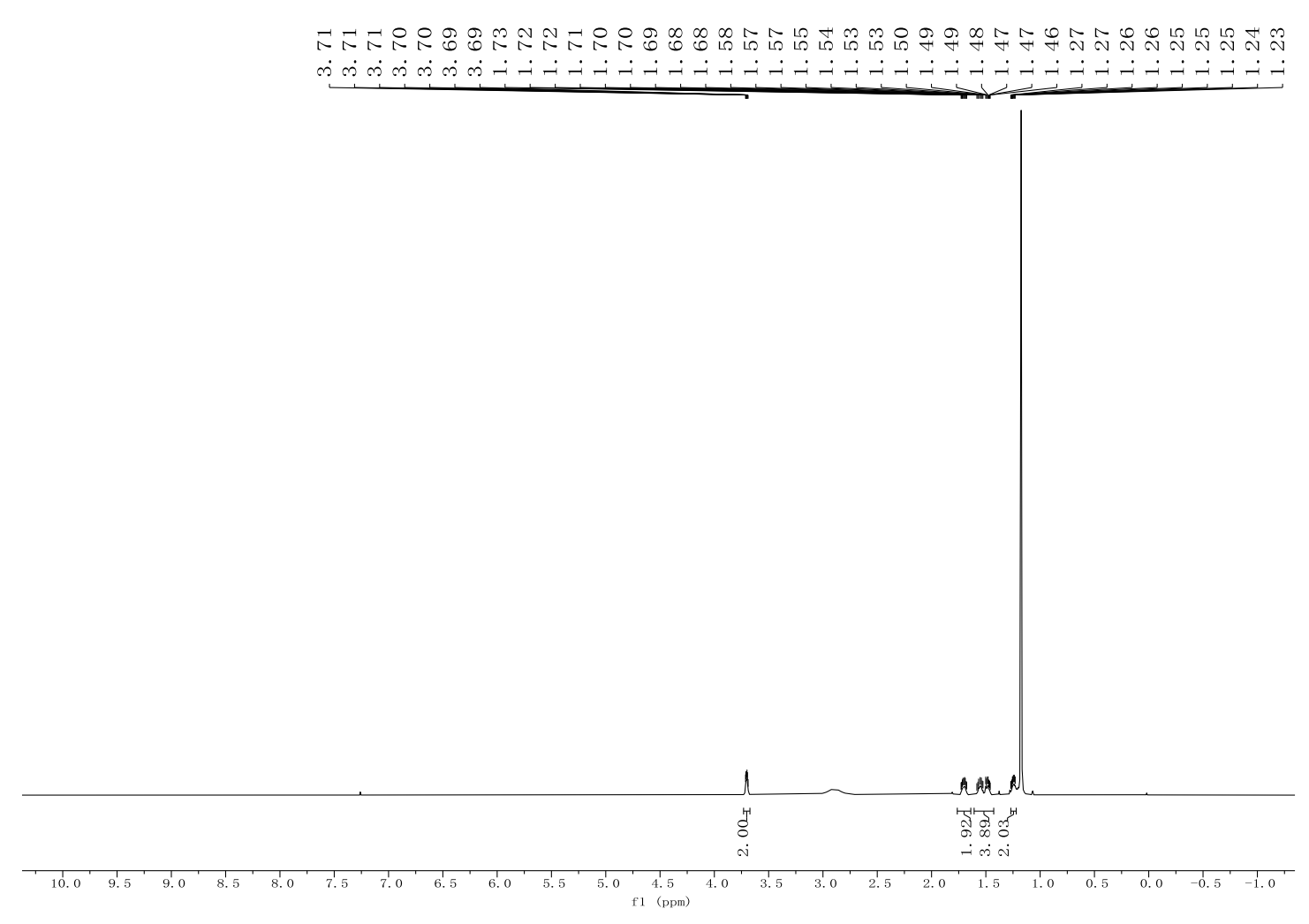

Figure S17. The ${ }^{1} \mathrm{H}$ NMR spectrum of crude product 35a.

\section{Compound 36}

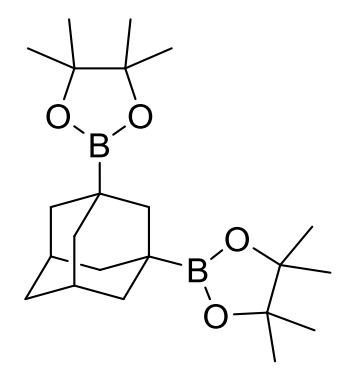

$(1 r, 3 r, 5 s, 7 s)-1,3-b i s(4,4,5,5-t e t r a m e t h y l-1,3,2-d i o x a b o r o l a n-2-y l) \operatorname{adamantine}^{[28]}$ :

White solid was obtained with $43 \%$ isolated yield (Procedure A, $\mathrm{B}_{2}$ cat 2 (8.0 eq.), 0.6 mmol scale, $100.2 \mathrm{mg}$ ).

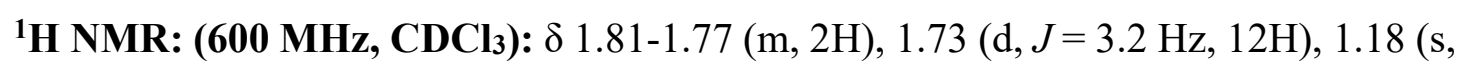
24H).

${ }^{13}$ C NMR: (151 MHz, CDCl 3$): \delta 82.7,37.9,37.8,37.3,26.8,24.8,19.3$ (br, low intensity).

${ }^{11}$ B NMR: (193 MHz, CDCl3): $\delta 33.76$.

HRMS (ESI): calculated for $\mathrm{C}_{22} \mathrm{H}_{39} \mathrm{~B}_{2} \mathrm{O}_{4}[\mathrm{M}+\mathrm{H}]^{+}: 389.3029$, found: 389.3028 . 


\section{Compound 37}<smiles>CC1(C)OB(CCCCC(B2OC(C)(C)C(C)(C)O2)B2OC(C)(C)C(C)(C)O2)OC1(C)C</smiles>

2,2',2'-(pentane-1,2,5-triyl)tris(4,4,5,5-tetramethyl-1,3,2-dioxaborolane):

Colorless oil was obtained with 44\% isolated yield (Procedure A, 0.6 mmol scale, 119.0 $\mathrm{mg})$.

${ }^{1}$ H NMR: (600 MHz, CDCl 3$): \delta 1.46-1.31(\mathrm{~m}, 3 \mathrm{H}), 1.29-1.25(\mathrm{~m}, 1 \mathrm{H}), 1.25-1.12(\mathrm{t}, J$ $=2.4 \mathrm{~Hz}, 36 \mathrm{H}), 1.09-1.05(\mathrm{~m}, 1 \mathrm{H}), 0.84-0.65(\mathrm{~m}, 4 \mathrm{H})$.

${ }^{13}$ C NMR: (151 MHz, CDCl3): $\delta$ 82.8, 82.8, 82.8, 36.7, 25.0, 24.9, 24.9, 24.9, 24.8, 23.4, 18.4 (br, low intensity), 12.6 (br, low intensity), 11.5 (br, low intensity).

${ }^{11}$ B NMR: (193 MHz, CDCl 3$): ~ \delta 34.41$.

HRMS (ESI): calculated for $\mathrm{C}_{23} \mathrm{H}_{46} \mathrm{~B}_{3} \mathrm{O}_{6}[\mathrm{M}+\mathrm{H}]^{+}:$: 451.3568, found: 451.3560 .

\section{Compound 38}<smiles>CC1(C)OB(CCCCC(CB2OC(C)(C)C(C)(C)O2)B2OC(C)(C)C(C)(C)O2)OC1(C)C</smiles>

2,2',2"'-(hexane-1,2,6-triyl)tris(4,4,5,5-tetramethyl-1,3,2-dioxaborolane): Colorless oil was obtained with 54\% isolated yield (Procedure A, $0.6 \mathrm{mmol} \mathrm{scale,} 151.0 \mathrm{mg}$ ).

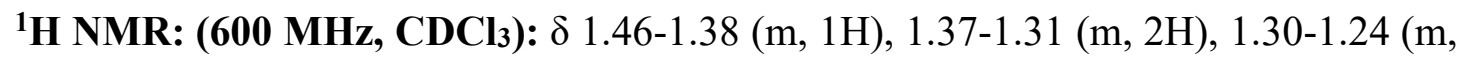
$3 \mathrm{H}), 1.18(\mathrm{t}, J=2.8 \mathrm{~Hz}, 36 \mathrm{H}), 1.08-1.03(\mathrm{~m}, 1 \mathrm{H}), 0.86-0.65(\mathrm{~m}, 4 \mathrm{H})$.

${ }^{13}$ C NMR: (151 MHz, $\left.\mathbf{C D C l}_{3}\right): \delta$ 82.8, 82.8, 33.7, 31.8, 25.1, 24.9, 24.9, 24.9, 24.9, 24.8, 24.4, 18.4 (br, low intensity), 12.8 (br, low intensity), 11.3 (br, low intensity).

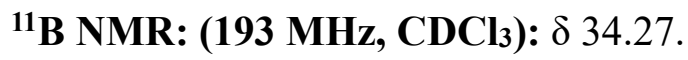

HRMS (ESI): calculated for $\mathrm{C}_{24} \mathrm{H}_{48} \mathrm{~B}_{3} \mathrm{O}_{6}[\mathrm{M}+\mathrm{H}]^{+}:$: 465.3725, found: 465.3720 . 


\section{Compound 39}

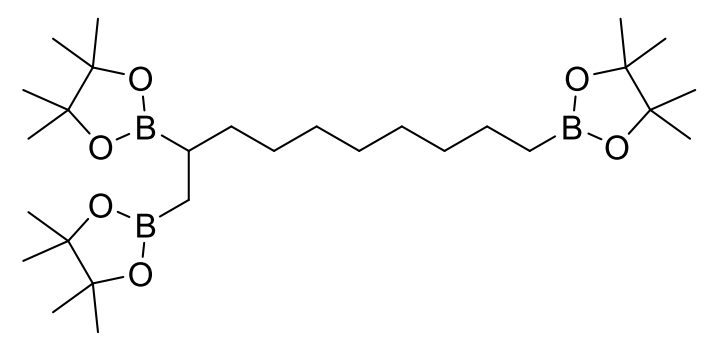

2,2',2'-(decane-1,2,10-triyl)tris(4,4,5,5-tetramethyl-1,3,2-dioxaborolane):

Colorless oil was obtained with 70\% isolated yield (Procedure A, 4.0 F/mol, $0.6 \mathrm{mmol}$ scale, $220.4 \mathrm{mg})$.

${ }^{1}$ H NMR: (600 MHz, CDCl 3$): ~ \delta 1.44-1.18(\mathrm{~m}, 50 \mathrm{H}), 1.11-1.06(\mathrm{~m}, 1 \mathrm{H}), 0.88-0.70(\mathrm{~m}$, $4 \mathrm{H})$.

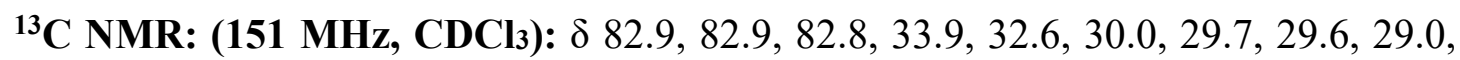
25.0, 24.9, 24.9, 24.9, 24.9, 24.1, 18.6 (br, low intensity), 12.8 (br, low intensity), 11.4 (br, low intensity).

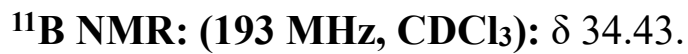

HRMS (ESI): calculated for $\mathrm{C}_{28} \mathrm{H}_{56} \mathrm{~B}_{3} \mathrm{O}_{6}[\mathrm{M}+\mathrm{H}]^{+}:$521.4351, found: 521.4338 .

\section{Compound 40}<smiles>CCCCCCB1OC(C)(C)C(C)(C)O1</smiles>

2-hexyl-4,4,5,5-tetramethyl-1,3,2-dioxaborolane ${ }^{[19]}$ : Colorless oil was obtained with $66 \%$ isolated yield (Procedure B, $0.6 \mathrm{mmol} \mathrm{scale,} 84.0 \mathrm{mg}$ ).

${ }^{1}$ H NMR: (400 MHz, CDCl3): $\delta$ 1.39-1.33 (m, 2H), 1.30-1.17 (m, 18H), 0.86-0.80 (m, $3 \mathrm{H}), 0.73(\mathrm{t}, J=7.8 \mathrm{~Hz}, 2 \mathrm{H})$.

${ }^{13}$ C NMR: (101 MHz, CDCl3): $\delta$ 82.9, 32.2, 31.8, 24.9, 24.1, 22.7, 14.2, 11.4 (br, low intensity).

${ }^{11}$ B NMR: (128 MHz, CDCl3): $\delta 33.10$.

HRMS (ESI): calculated for $\mathrm{C}_{12} \mathrm{H}_{26} \mathrm{BO}_{2}[\mathrm{M}+\mathrm{H}]^{+}:$: 213.2020, found: 213.2019 . 


\section{Compound 41}<smiles>CC(C)CCCB1OC(C)(C)C(C)(C)O1</smiles>

4,4,5,5-tetramethyl-2-(4-methylpentyl)-1,3,2-dioxaborolane ${ }^{[38]}$ : Colorless oil was obtained with $48 \%$ isolated yield (Procedure B, $0.6 \mathrm{mmol} \mathrm{scale,} 61.0 \mathrm{mg}$ ). $57 \%$ isolated yield was obtained in gram scale experiment at $600 \mathrm{~mA}$ (48 min, $7.2 \mathrm{mmol} \mathrm{scale,} 0.88$ g).

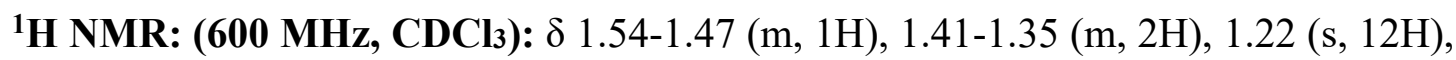
1.17-1.13 (m, 2H), $0.84(\mathrm{dd}, J=6.6,1.4 \mathrm{~Hz}, 6 \mathrm{H}), 0.75-0.70(\mathrm{~m}, 2 \mathrm{H})$.

${ }^{13}$ C NMR: (151 MHz, CDCl 3$): \delta$ 82.9, 42.1, 27.9, 24.9, 22.8, 21.9, 11.5 (br, low intensity).

${ }^{11}$ B NMR: (193 MHz, CDCl $\left.\mathbf{3}\right): \delta 34.10$.

HRMS (ESI): calculated for $\mathrm{C}_{12} \mathrm{H}_{26} \mathrm{BO}_{2}[\mathrm{M}+\mathrm{H}]^{+}:$: 213.2020, found: 213.2019.

\section{Compound 42}<smiles>CC1(C)OB(CCCCCCCl)OC1(C)C</smiles>

2-(6-chlorohexyl)-4,4,5,5-tetramethyl-1,3,2-dioxaborolane ${ }^{[6]}$ : Colorless oil was obtained with 89\% isolated yield (Procedure B, $0.6 \mathrm{mmol} \mathrm{scale,} 132.5 \mathrm{mg}$ ).

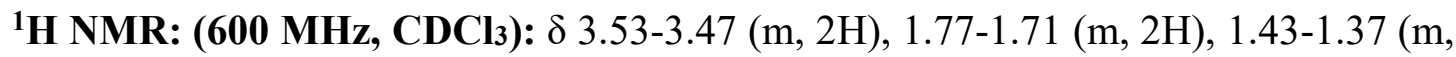
4H), 1.33-1.28 (m, 2H), $1.22(\mathrm{~s}, 12 \mathrm{H}), 0.75(\mathrm{t}, J=7.8 \mathrm{~Hz}, 2 \mathrm{H})$.

${ }^{13}$ C NMR: (151 MHz, CDCl3): $\delta$ 82.9, 45.1, 32.6, 31.6, 26.7, 24.9, 23.8, 11.2 (br, low intensity).

${ }^{11}$ B NMR: (193 MHz, CDCl 3$): ~ \delta 33.86$.

HRMS (ESI): calculated for $\mathrm{C}_{12} \mathrm{H}_{25} \mathrm{BClO}_{2}[\mathrm{M}+\mathrm{H}]^{+}:$: 247.1631, found: 247.1625 .

\section{Compound 43}<smiles>C#CCCCCCCB1OC(C)(C)C(C)(C)O1</smiles> 
4,4,5,5-tetramethyl-2-(oct-7-yn-1-yl)-1,3,2-dioxaborolane: Colorless oil was obtained with $41 \%$ isolated yield (Procedure B, $0.6 \mathrm{mmol} \mathrm{scale,} 58.3 \mathrm{mg}$ ).

${ }^{1}$ H NMR: (600 MHz, CDCl 3$): \delta 2.17-2.13$ (m, 2H), 1.91 (t, $\left.J=2.6 \mathrm{~Hz}, 1 \mathrm{H}\right), 1.53-1.48$ (m, 2H), 1.41-1.35 (m, 4H), 1.31-1.27 (m, 2H), $1.23(\mathrm{~s}, 12 \mathrm{H}), 0.75(\mathrm{t}, J=7.8 \mathrm{~Hz}, 2 \mathrm{H})$. ${ }^{13}$ C NMR: (151 MHz, CDCl $): \delta$ 84.8, 82.9, 68.1, 31.9, 28.6, 28.5, 24.9, 23.9, 18.4, 11.2 (br, low intensity).

${ }^{11}$ B NMR: (193 MHz, CDCl 3$): ~ \delta 34.06$.

HRMS (ESI): calculated for $\mathrm{C}_{14} \mathrm{H}_{26} \mathrm{BO}_{2}[\mathrm{M}+\mathrm{H}]^{+}:$: 237.2020, found: 237.2015.

\section{Compound 44}<smiles>CC(=O)CCCCB1OC(C)(C)C(C)(C)O1</smiles>

6-(4,4,5,5-tetramethyl-1,3,2-dioxaborolan-2-yl)hexan-2-one ${ }^{[39]}$ : Colorless oil was obtained with $48 \%$ isolated yield (Procedure B, $0.6 \mathrm{mmol}$ scale, $65.5 \mathrm{mg}$ ).

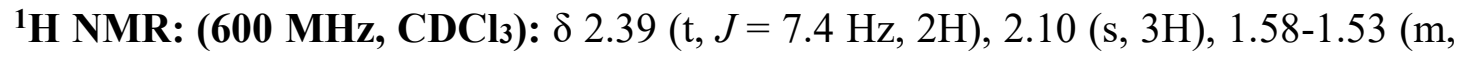
2H), 1.41-1.36 (m, 2H), $1.22(\mathrm{~s}, 12 \mathrm{H}), 0.76(\mathrm{t}, J=7.8 \mathrm{~Hz}, 2 \mathrm{H})$.

${ }^{13}$ C NMR: (151 MHz, CDCl $)$ ): $\delta$ 209.5, 83.1, 43.8, 29.9, 26.6, 24.9, 23.7, 11.2 (br, low intensity).

${ }^{11}$ B NMR: (193 MHz, CDCl3): $\delta 33.92$.

HRMS (ESI): calculated for $\mathrm{C}_{12} \mathrm{H}_{24} \mathrm{BO}_{3}[\mathrm{M}+\mathrm{H}]^{+}:$: 227.1813, found: 227.1809 .

\section{Compound 45}<smiles>CC(=O)OCCCCB1OC(C)(C)C(C)(C)O1</smiles>

4-(4,4,5,5-tetramethyl-1,3,2-dioxaborolan-2-yl)butyl acetate: Colorless oil was obtained with $61 \%$ isolated yield (Procedure B, $0.6 \mathrm{mmol} \mathrm{scale}, 89.0 \mathrm{mg}$ ).

${ }^{1}$ H NMR: (600 MHz, CDCl $): \delta 4.04(\mathrm{t}, J=6.6 \mathrm{~Hz}, 2 \mathrm{H}), 2.03(\mathrm{~s}, 3 \mathrm{H}), 1.65-1.60$ (m, 2H), 1.50-1.44 (m, 2H), $1.23(\mathrm{~s}, 12 \mathrm{H}), 0.79(\mathrm{t}, J=7.8 \mathrm{~Hz}, 2 \mathrm{H})$.

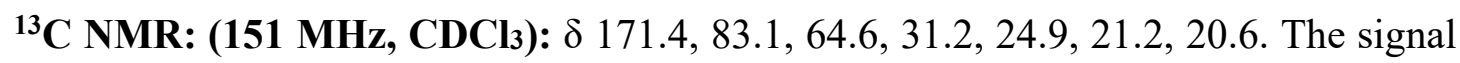


of the $\alpha$-B-carbon was not observed.

${ }^{11}$ B NMR: (193 MHz, CDCl3): $\delta 33.96$.

HRMS (ESI): calculated for $\mathrm{C}_{12} \mathrm{H}_{24} \mathrm{BO}_{4}[\mathrm{M}+\mathrm{H}]^{+}:$243.1762, found: 243.1762 .

\section{Compound 46}<smiles>CC1(C)OB(CCCCC#N)OC1(C)C</smiles>

5-(4,4,5,5-tetramethyl-1,3,2-dioxaborolan-2-yl)pentanenitrile: Colorless oil was obtained with 52\% isolated yield (Procedure B, $0.6 \mathrm{mmol} \mathrm{scale,} 65.4 \mathrm{mg}$ ).

${ }^{1}$ H NMR: (600 MHz, CDCl $): \delta 2.31(\mathrm{t}, J=7.2 \mathrm{~Hz}, 2 \mathrm{H}), 1.68-1.63(\mathrm{~m}, 2 \mathrm{H}), 1.57-1.52$ (m, 2H), $1.23(\mathrm{~s}, 12 \mathrm{H}), 0.79(\mathrm{t}, J=7.8 \mathrm{~Hz}, 2 \mathrm{H})$.

${ }^{13}$ C NMR: (151 MHz, $\left.\mathbf{C D C l}_{3}\right): \delta$ 120.0, 83.3, 27.9, 24.9, 23.3, 17.0. The signal of the $\alpha$-B-carbon was not observed.

${ }^{11}$ B NMR: (193 MHz, CDCl3): $\delta 33.85$.

HRMS (ESI): calculated for $\mathrm{C}_{11} \mathrm{H}_{21} \mathrm{BNO}_{2}[\mathrm{M}+\mathrm{H}]^{+}: 210.1660$, found: 210.1656 .

\section{Compound 47}<smiles>CC1(C)OB(CCCc2ccccc2)OC1(C)C</smiles>

4,4,5,5-tetramethyl-2-(3-phenylpropyl)-1,3,2-dioxaborolane ${ }^{[28]}$ : Colorless oil was obtained with $84 \%$ isolated yield following Procedure B (from iodide, 0.6 mmol scale, $125.0 \mathrm{mg}$ ). $62 \%$ isolated yield was obtained following Procedure $\mathrm{C}$ (from chloride, 0.6 mmol scale, $92.0 \mathrm{mg}$ ).

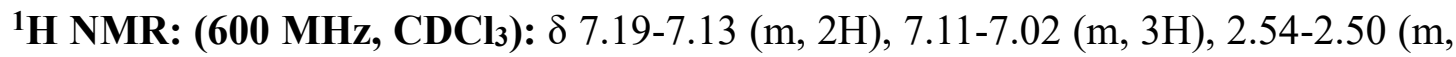
$2 \mathrm{H}), 1.68-1.62(\mathrm{~m}, 2 \mathrm{H}), 1.15(\mathrm{~s}, 12 \mathrm{H}), 0.74(\mathrm{t}, J=8.0 \mathrm{~Hz}, 2 \mathrm{H})$.

${ }^{13}$ C NMR: (151 MHz, CDCl3): $\delta$ 142.7, 128.6, 128.2, 125.6, 82.9, 38.7, 26.2, 24.9, 11.0 (br, low intensity).

${ }^{11}$ B NMR: (193 MHz, CDCl 3$): \delta 34.11$.

HRMS (ESI): calculated for $\mathrm{C}_{15} \mathrm{H}_{24} \mathrm{BO}_{2}[\mathrm{M}+\mathrm{H}]^{+}:$: 247.1864, found: 247.1869. 


\section{Compound 48}

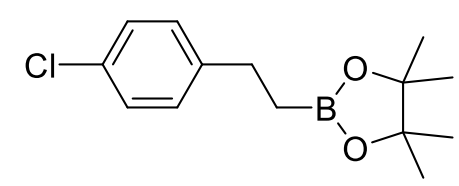

2-(4-chlorophenethyl)-4,4,5,5-tetramethyl-1,3,2-dioxaborolane ${ }^{[29]}$ : White solid was obtained with 75\% isolated yield (Procedure B, $0.6 \mathrm{mmol} \mathrm{scale,} 120.0 \mathrm{mg}$ ).

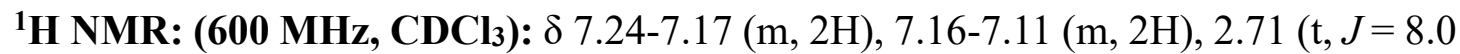
$\mathrm{Hz}, 2 \mathrm{H}), 1.21(\mathrm{~s}, 12 \mathrm{H}), 1.11(\mathrm{t}, J=8.2 \mathrm{~Hz}, 2 \mathrm{H})$.

${ }^{13}$ C NMR: (151 MHz, $\left.\mathbf{C D C l}_{3}\right): \delta 142.9,131.2,129.5,128.3,83.2,29.4,24.9,13.0 .(\mathrm{br}$, low intensity).

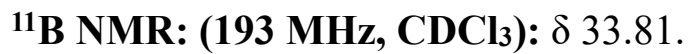

HRMS (ESI): calculated for $\mathrm{C}_{14} \mathrm{H}_{21} \mathrm{BClO}_{2}[\mathrm{M}+\mathrm{H}]^{+}:$267.1318, found: 267.1308.

\section{Compound 49}<smiles>COc1ccc(CCCCB2OC(C)(C)C(C)(C)O2)cc1</smiles>

2-(4-(4-methoxyphenyl)butyl)-4,4,5,5-tetramethyl-1,3,2-dioxaborolane ${ }^{[40]}$ :

Colorless oil was obtained with 78\% isolated yield (Procedure B, 0.6 mmol scale, 135.8 $\mathrm{mg})$.

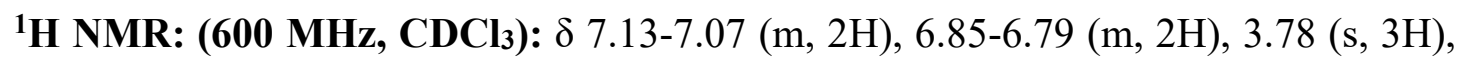
2.58-2.54 (m, 2H), 1.64-1.58 (m, 2H), 1.50-1.45 (m, 2H), $1.25(\mathrm{~s}, 12 \mathrm{H}), 0.82(\mathrm{t}, J=7.8$ $\mathrm{Hz}, 2 \mathrm{H})$.

${ }^{13}$ C NMR: (151 MHz, $\left.\mathbf{C D C l}_{3}\right): \delta$ 157.6, 135.0, 129.3, 113.7, 82.9, 55.2, 34.9, 34.5, 24.9, 23.7, 11.1 (br, low intensity).

${ }^{11}$ B NMR: (193 MHz, CDCl3): $\delta 34.16$.

HRMS (ESI): calculated for $\mathrm{C}_{17} \mathrm{H}_{28} \mathrm{BO}_{3}[\mathrm{M}+\mathrm{H}]^{+}$: 291.2126, found: 291.2121. 


\section{Compound 50}<smiles>CC1(C)OB(CCc2cccs2)OC1(C)C</smiles>

4,4,5,5-tetramethyl-2-(2-(thiophen-2-yl)ethyl)-1,3,2-dioxaborolane ${ }^{[31,41]}$ : Colorless oil was obtained with 76\% isolated yield (Procedure B, $0.6 \mathrm{mmol} \mathrm{scale,} 109.0 \mathrm{mg}$ ). 1H NMR: (600 MHz, CDCl $)$ ): $\delta$ 7.12-7.02 (m, 1H), 6.91-6.88 (m, 1H), 6.81-6.78 (m, 1H), 2.98-2.95 (m, 2H), 1.23 (s, 14H).

${ }^{13}$ C NMR: (151 MHz, CDCl 3 ): $\delta$ 147.8, 126.6, 123.5, 122.7, 83.3, 24.9, 24.4, 13.7 (br, low intensity).

${ }^{11}$ B NMR: (193 MHz, CDCl3): $\delta 33.74$.

HRMS (ESI): calculated for $\mathrm{C}_{12} \mathrm{H}_{20} \mathrm{BO}_{2} \mathrm{~S}[\mathrm{M}+\mathrm{H}]^{+}:$239.1272, found: 239.1268.

\section{Compound 51}<smiles>CC1(C)OB(CCCn2c3ccccc3c3ccccc32)OC1(C)C</smiles>

9-(3-(4,4,5,5-tetramethyl-1,3,2-dioxaborolan-2-yl)propyl)-9H-carbazole ${ }^{[29]}$ :

Colorless oil was obtained with 90\% isolated yield (Procedure B, 0.6 mmol scale, 181.0 $\mathrm{mg})$.

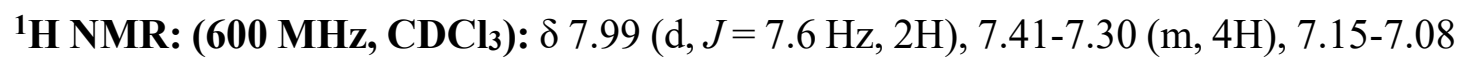
(m, 2H), 4.19 (t, $J=7.6 \mathrm{~Hz}, 2 \mathrm{H}), 1.92-1.84(\mathrm{~m}, 2 \mathrm{H}), 1.14(\mathrm{~s}, 12 \mathrm{H}), 0.78(\mathrm{t}, J=7.8 \mathrm{~Hz}$, $2 \mathrm{H})$.

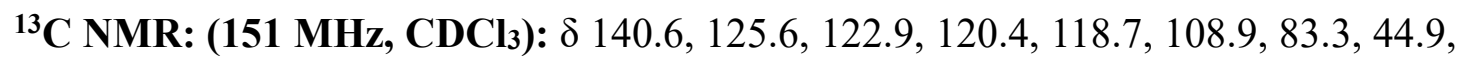
24.9, 23.6. The signal of the $\alpha$-B-carbon was not observed.

${ }^{11}$ B NMR: (193 MHz, CDCl3): $\delta 33.79$.

HRMS (ESI): calculated for $\mathrm{C}_{21} \mathrm{H}_{27} \mathrm{BNO}_{2}[\mathrm{M}+\mathrm{H}]^{+}: 336.2129$, found: 336.2128 . 


\section{Compound 52}

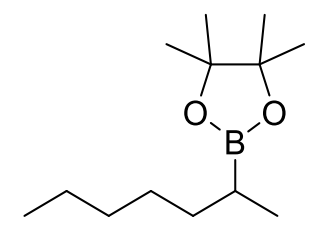

2-(heptan-2-yl)-4,4,5,5-tetramethyl-1,3,2-dioxaborolane ${ }^{[6,32]}$ : Colorless oil was obtained with $64 \%$ isolated yield (Procedure B, $0.6 \mathrm{mmol} \mathrm{scale,} 87.1 \mathrm{mg}$ ).

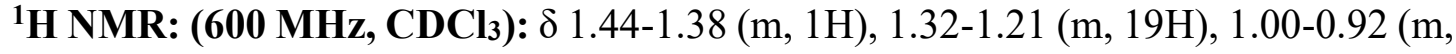
$4 \mathrm{H}), 0.85$ (t, $J=7.0 \mathrm{~Hz}, 3 \mathrm{H})$.

${ }^{13}$ C NMR: (151 MHz, $\left.\mathbf{C D C l}_{3}\right): \delta$ 82.9, 33.3, 32.2, 28.8, 24.9, 24.8, 22.8, 17.1 (br, low intensity), 15.6, 14.2.

${ }^{11}$ B NMR: (193 MHz, CDCl3): $\delta 34.51$.

HRMS (ESI): calculated for $\mathrm{C}_{13} \mathrm{H}_{28} \mathrm{BO}_{2}[\mathrm{M}+\mathrm{H}]^{+}:$: 227.2177, found: 227.2172 .

\section{Compound 53}<smiles>CC(CCc1ccccc1)B1OC(C)(C)C(C)(C)O1</smiles>

4,4,5,5-tetramethyl-2-(4-phenylbutan-2-yl)-1,3,2-dioxaborolane ${ }^{[31]}:$ Colorless oil was obtained with 78\% isolated yield following Procedure B (from iodide, $0.6 \mathrm{mmol}$ scale, $122.1 \mathrm{mg}$ ). $35 \%$ isolated yield was obtained following Procedure C (from chloride, $20 \mathrm{~mA}$, $4.0 \mathrm{~F} / \mathrm{mol}, 0.6 \mathrm{mmol} \mathrm{scale,} 54.9 \mathrm{mg}$ ).

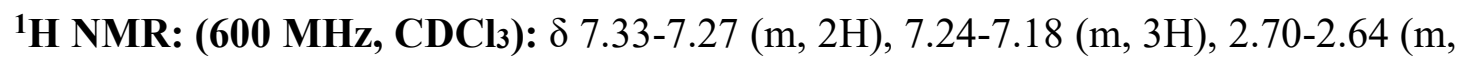
2H), 1.87-1.80 (m, 1H), 1.67-1.60 (m, 1H), $1.29(\mathrm{~s}, 12 \mathrm{H}), 1.15-1.10(\mathrm{~m}, 1 \mathrm{H}), 1.07(\mathrm{~d}$, $J=7.0 \mathrm{~Hz}, 3 \mathrm{H})$.

${ }^{13}$ C NMR: (151 MHz, CDCl $): \delta$ 143.2, 128.5, 128.3, 125.6, 82.9, 35.4, 35.4, 24.9, 24.8, 16.9 (br, low intensity), 15.5.

${ }^{11}$ B NMR: (193 MHz, CDCl 3$): ~ \delta 34.58$.

HRMS (ESI): calculated for $\mathrm{C}_{16} \mathrm{H}_{26} \mathrm{BO}_{2}[\mathrm{M}+\mathrm{H}]^{+}:$261.2020, found: 261.2016. 


\section{Compound 54}<smiles>CC1(C)OB(C2CCOCC2)OC1(C)C</smiles>

4,4,5,5-tetramethyl-2-(tetrahydro-2H-pyran-4-yl)-1,3,2-dioxaborolane ${ }^{[6]}$ : White solid was obtained with $45 \%$ isolated yield (Procedure B, $0.6 \mathrm{mmol} \mathrm{scale,} 57.8 \mathrm{mg}$ ).

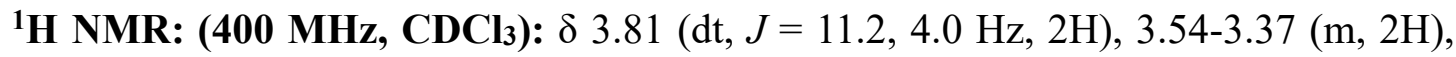
1.64-1.57 (m, 4H), 1.23-1.14 (m, 13H).

${ }^{13}$ C NMR: (101 MHz, $\left.\mathbf{C D C l}_{3}\right): \delta 83.2,68.9,27.7,24.9$. The signal of the $\alpha$-B-carbon was not observed.

11B NMR: (128 MHz, $\left.\mathbf{C D C l}_{3}\right): \delta 32.90$.

HRMS (ESI): calculated for $\mathrm{C}_{11} \mathrm{H}_{22} \mathrm{BO}_{3}[\mathrm{M}+\mathrm{H}]^{+}:$: 213.1657, found: 213.1653 .

\section{Compound 55}

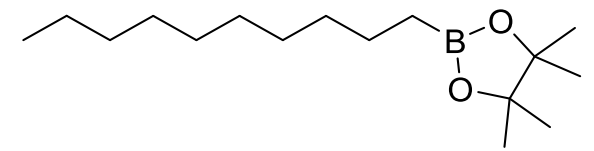

2-decyl-4,4,5,5-tetramethyl-1,3,2-dioxaborolane ${ }^{[20]}$ : Colorless oil was obtained with $62 \%$ isolated yield (Procedure C, $0.6 \mathrm{mmol}$ scale, $100.1 \mathrm{mg}$ ).

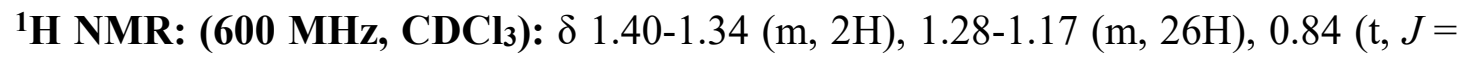
$7.0 \mathrm{~Hz}, 3 \mathrm{H}), 0.73(\mathrm{t}, J=7.8 \mathrm{~Hz}, 2 \mathrm{H})$.

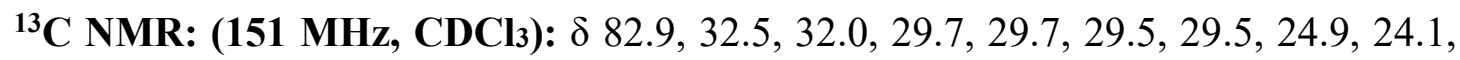
22.8, 14.2, 11.3 (br, low intensity).

${ }^{11}$ B NMR: (193 MHz, CDCl3): $\delta 34.03$.

HRMS (ESI): calculated for $\mathrm{C}_{16} \mathrm{H}_{34} \mathrm{BO}_{2}[\mathrm{M}+\mathrm{H}]^{+}:$269.2646, found: 269.2652.

\section{Compound 56}<smiles>CC1(C)OB(CCC[Si](C)(C)C)OC1(C)C</smiles>

trimethyl(3-(4,4,5,5-tetramethyl-1,3,2-dioxaborolan-2-yl)propyl)silane ${ }^{[20]}$ :

Colorless oil was obtained with 50\% isolated yield (Procedure C, $0.6 \mathrm{mmol}$ scale, 72.6 mg). 
${ }^{1}$ H NMR: (600 MHz, CDCl 3$): \delta 1.43-1.37(\mathrm{~m}, 2 \mathrm{H}), 1.21(\mathrm{~s}, 12 \mathrm{H}), 0.80(\mathrm{t}, J=7.6 \mathrm{~Hz}$, $2 \mathrm{H}), 0.52-0.47$ (m, 2H), -0.07 (s, 9H).

${ }^{13}$ C NMR: (151 MHz, $\left.\mathbf{C D C l}_{3}\right)$ : $\delta$ 82.9, 24.9, 20.2, 18.7, 15.7 (br, low intensity), -1.5.

${ }^{11}$ B NMR: (193 MHz, CDCl3): $\delta 33.87$.

HRMS (ESI): calculated for $\mathrm{C}_{12} \mathrm{H}_{28} \mathrm{BO}_{2} \mathrm{Si}[\mathrm{M}+\mathrm{H}]^{+}: 243.1946$, found: 243.1942 .

\section{Compound 57}<smiles>CC(C)(CB1OC(C)(C)C(C)(C)O1)c1ccccc1</smiles>

\section{4,4,5,5-tetramethyl-2-(2-methyl-2-phenylpropyl)-1,3,2-dioxaborolane ${ }^{[42]}$ :}

Colorless oil was obtained with $34 \%$ isolated yield (Procedure C, $0.6 \mathrm{mmol}$ scale, 53.7 mg).

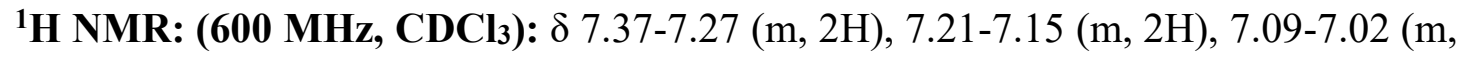
1H), $1.34(\mathrm{~s}, 6 \mathrm{H}), 1.17(\mathrm{~s}, 2 \mathrm{H}), 1.01(\mathrm{~s}, 12 \mathrm{H})$.

${ }^{13}$ C NMR: (151 MHz, $\left.\mathbf{C D C l}_{3}\right): \delta$ 151.5, 127.9, 125.6, 125.3, 82.8, 36.4, 31.4, 28.1 (br, low intensity), 24.8 .

${ }^{11}$ B NMR: (193 MHz, $\left.\mathrm{CDCl}_{3}\right): \delta 33.13$.

HRMS (ESI): calculated for $\mathrm{C}_{16} \mathrm{H}_{26} \mathrm{BO}_{2}[\mathrm{M}+\mathrm{H}]^{+}:$: 261.2020, found: 261.2026.

\section{Compound 58}<smiles>CC1(C)OB(CCc2ccc(F)cc2)OC1(C)C</smiles>

2-(4-fluorophenethyl)-4,4,5,5-tetramethyl-1,3,2-dioxaborolane ${ }^{[43]}:$ Colorless oil was obtained with 51\% isolated yield (Procedure C, $0.6 \mathrm{mmol} \mathrm{scale,} 76.8 \mathrm{mg}$ ).

${ }^{1}$ H NMR: (600 MHz, CDCl $)$ : $\delta$ 7.22-7.05 (m, 2H), 6.99-6.81 (m, 2H), $2.71(\mathrm{t}, J=8.0$ $\mathrm{Hz}, 2 \mathrm{H}), 1.20(\mathrm{~s}, 12 \mathrm{H}), 1.11(\mathrm{t}, J=8.0 \mathrm{~Hz}, 2 \mathrm{H})$.

${ }^{13}$ C NMR: (151 MHz, CDCl3): $\delta 161.2(\mathrm{~d}, J=242.8 \mathrm{~Hz}), 140.0(\mathrm{~d}, J=3.2 \mathrm{~Hz}), 129.4$ 
(d, $J=7.6 \mathrm{~Hz}), 114.9$ (d, $J=21.0 \mathrm{~Hz}), 83.2,29.2,24.9,13.2$ (br, low intensity).

${ }^{19}$ F NMR (565 MHz, $\left.\mathrm{CDCl}_{3}\right): \delta-118.34$.

${ }^{11}$ B NMR: (193 MHz, $\left.\mathrm{CDCl}_{3}\right): \delta 33.83$.

HRMS (ESI): calculated for $\mathrm{C}_{14} \mathrm{H}_{24} \mathrm{BFNO}_{2}[\mathrm{M}+\mathrm{H}]^{+}:$268.1879, found: 268.1889 .

\section{Compound 59}<smiles>COc1ccc(CCB2OC(C)(C)C(C)(C)O2)cc1</smiles>

2-(4-methoxyphenethyl)-4,4,5,5-tetramethyl-1,3,2-dioxaborolane ${ }^{[20]}$ : Colorless oil was obtained with 55\% isolated yield (Procedure C, $0.6 \mathrm{mmol} \mathrm{scale,} 86.8 \mathrm{mg}$ ).

${ }^{1}$ H NMR: (600 MHz, $\left.\mathbf{C D C l}_{3}\right): \delta 7.14(\mathrm{~d}, J=8.6 \mathrm{~Hz}, 2 \mathrm{H}), 6.81(\mathrm{~d}, J=8.6 \mathrm{~Hz}, 2 \mathrm{H})$, $3.77(\mathrm{~s}, 3 \mathrm{H}), 2.70(\mathrm{t}, J=8.2 \mathrm{~Hz}, 2 \mathrm{H}), 1.22(\mathrm{~s}, 12 \mathrm{H}), 1.12(\mathrm{t}, J=8.2 \mathrm{~Hz}, 2 \mathrm{H})$.

${ }^{13}$ C NMR: (151 MHz, CDCl $): \delta$ 157.6, 136.6, 128.9, 113.7, 83.1, 55.3, 29.1, 24.9, 13.4 (br, low intensity).

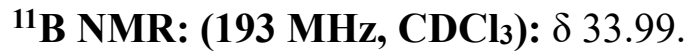

HRMS (ESI): calculated for $\mathrm{C}_{15} \mathrm{H}_{24} \mathrm{BO}_{3}[\mathrm{M}+\mathrm{H}]^{+}:$263.1813, found: 263.1811 .

\section{Compound 60}<smiles>CC1(C)OB(CCCCn2c3ccccc3c3ccccc32)OC1(C)C</smiles>

\section{9-(4-(4,4,5,5-tetramethyl-1,3,2-dioxaborolan-2-yl)butyl)-9H-carbazole ${ }^{[6]}$ :}

Colorless oil was obtained with 50\% isolated yield (Procedure C, $30 \mathrm{~mA}, 4.0 \mathrm{~F} / \mathrm{mol}$, $0.6 \mathrm{mmol}$ scale, $104.9 \mathrm{mg})$.

${ }^{1}$ H NMR: (600 MHz, CDCl3): $\delta 7.98$ (d, $\left.J=7.8 \mathrm{~Hz}, 2 \mathrm{H}\right), 7.35-7.28(\mathrm{~m}, 4 \mathrm{H}), 7.12-7.08$ (m, 2H), $4.16(\mathrm{t}, J=7.6 \mathrm{~Hz}, 2 \mathrm{H}), 1.79-1.74(\mathrm{~m}, 2 \mathrm{H}), 1.47-1.42(\mathrm{~m}, 2 \mathrm{H}), 1.10(\mathrm{~s}, 12 \mathrm{H})$, $0.75(\mathrm{t}, J=7.6 \mathrm{~Hz}, 2 \mathrm{H})$.

${ }^{13}$ C NMR: (151 MHz, CDCl3): $\delta$ 140.4, 125.6, 122.8, 120.4, 118.7, 108.7, 83.1, 42.9, 
31.3, 24.9, 21.8, 10.9 (br, low intensity).

${ }^{11}$ B NMR: (193 MHz, CDCl3): $\delta 34.00$.

HRMS (ESI): calculated for $\mathrm{C}_{22} \mathrm{H}_{29} \mathrm{BNO}_{2}[\mathrm{M}+\mathrm{H}]^{+}: 350.2286$, found: 350.2282 .

\section{Compound 61}<smiles>COc1ccc2cc([C@@H](C)C(=O)OCCCB3OC(C)(C)C(C)(C)O3)ccc2c1</smiles>

3-(4,4,5,5-tetramethyl-1,3,2-dioxaborolan-2-yl)propyl

(S)-2-(6-

methoxynaphthalen-2-yl)propanoate: Colorless oil was obtained with $61 \%$ isolated yield (Procedure A, $0.6 \mathrm{mmol}$ scale, $145.8 \mathrm{mg}$ ).

${ }^{1}$ H NMR: (600 MHz, CDCl3): $\delta$ 7.71-7.66 (m, 3H), $7.41(\mathrm{dd}, J=8.6,1.8 \mathrm{~Hz}, 1 \mathrm{H})$, 7.14-7.10 (m, 2H), 4.10-4.05 (m, 1H), 4.03-3.99 (m, 1H), 3.91 (s, 3H), 3.84 (q, $J=7.2$ $\mathrm{Hz}, 1 \mathrm{H}), 1.72-1.68(\mathrm{~m}, 2 \mathrm{H}), 1.57(\mathrm{~d}, J=7.2 \mathrm{~Hz}, 3 \mathrm{H}), 1.21(\mathrm{~s}, 12 \mathrm{H}), 0.75(\mathrm{t}, J=8.0 \mathrm{~Hz}$, $2 \mathrm{H})$.

${ }^{13}$ C NMR: (151 MHz, $\left.\mathbf{C D C l}_{3}\right): \delta 174.9,157.7,136.0,133.8,129.4,129.0,127.2,126.4$, $126.0,119.0,105.7,83.2,66.6,55.4,45.6,24.9,23.2,18.7$. The signal of the $\alpha$-Bcarbon was not observed.

${ }^{11}$ B NMR: (193 MHz, CDCl3): $\delta 33.75$.

HRMS (ESI): calculated for $\mathrm{C}_{23} \mathrm{H}_{32} \mathrm{BO}_{5}[\mathrm{M}+\mathrm{H}]^{+}: 399.2337$, found: 399.2337 .

\section{Compound 62}

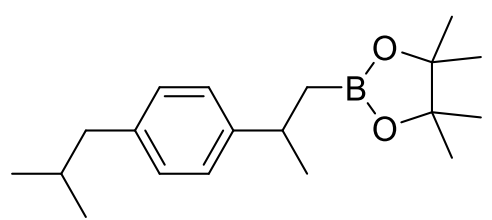

2-(2-(4-isobutylphenyl)propyl)-4,4,5,5-tetramethyl-1,3,2-dioxaborolane: Colorless oil was obtained with 73\% isolated yield (Procedure B, $0.6 \mathrm{mmol} \mathrm{scale,} 132.6 \mathrm{mg}$ ). ${ }^{1}$ H NMR: (600 MHz, $\left.\mathbf{C D C l}_{3}\right): \delta 7.16(\mathrm{~d}, J=8.0 \mathrm{~Hz}, 2 \mathrm{H}), 7.05(\mathrm{~d}, J=8.0 \mathrm{~Hz}, 2 \mathrm{H})$, 3.07-3.00 (m, 1H), $2.45(\mathrm{~d}, J=7.2 \mathrm{~Hz}, 2 \mathrm{H}), 1.89-1.81(\mathrm{~m}, 1 \mathrm{H}), 1.29(\mathrm{~d}, J=7.0 \mathrm{~Hz}, 3 \mathrm{H})$, $1.16(\mathrm{~s}, 14 \mathrm{H}), 0.91(\mathrm{dd}, J=6.6,2.0 \mathrm{~Hz}, 6 \mathrm{H})$. 
${ }^{13}$ C NMR: (151 MHz, CDCl 3$): \delta$ 146.4, 138.9, 128.9, 126.4, 82.9, 45.1, 35.5, 30.3, 25.2, 24.8, 24.8, 22.5, 22.4, 21.4 (br, low intensity).

${ }^{11}$ B NMR: (193 MHz, CDCl 3$): ~ \delta 33.72$.

HRMS (ESI): calculated for $\mathrm{C}_{19} \mathrm{H}_{32} \mathrm{BO}_{2}[\mathrm{M}+\mathrm{H}]^{+}: 303.2490$, found: 303.2482 .

\section{Compound 63}<smiles>CC(C)=CCC[C@H](C)CCB1OC(C)(C)C(C)(C)O1</smiles>

(R)-2-(3,7-dimethyloct-6-en-1-yl)-4,4,5,5-tetramethyl-1,3,2-dioxaborolane ${ }^{[44]}$ :

Colorless oil was obtained with 77\% isolated yield (Procedure B, 0.6 mmol scale, 124.0 mg).

${ }^{1}$ H NMR: (600 MHz, CDCl 3$): \delta$ 5.09-5.03 (m, 1H), 1.99-1.87 (m, 2H), $1.64(\mathrm{~s}, 3 \mathrm{H})$, $1.56(\mathrm{~s}, 3 \mathrm{H}), 1.51-1.23(\mathrm{~m}, 4 \mathrm{H}), 1.21(\mathrm{~s}, 12 \mathrm{H}), 1.11-1.04(\mathrm{~m}, 1 \mathrm{H}), 0.82(\mathrm{~d}, J=6.4 \mathrm{~Hz}$, $3 \mathrm{H}), 0.78-0.66(\mathrm{~m}, 2 \mathrm{H})$.

${ }^{13}$ C NMR: (151 MHz, CDCl3): $\delta$ 130.9, 125.2, 82.9, 36.8, 34.6, 31.0, 25.8, 25.7, 24.9, 24.9, 19.2, 17.7, 8.6 (br, low intensity).

${ }^{11}$ B NMR: (193 MHz, CDCl 3$): ~ \delta 34.25$.

HRMS (ESI): calculated for $\mathrm{C}_{16} \mathrm{H}_{32} \mathrm{BO}_{2}[\mathrm{M}+\mathrm{H}]^{+}:$: 267.2490, found: 267.2485.

\section{Compound 64}<smiles>CC1(C)CCC=C(CCB2OC(C)(C)C(C)(C)O2)C1</smiles>

2-(2-((1R,5S)-6,6-dimethylbicyclo[3.1.1] hept-2-en-2-yl)ethyl)-4,4,5,5-tetramethyl1,3,2-dioxaborolane: Colorless oil was obtained with $80 \%$ isolated yield (Procedure B, $0.6 \mathrm{mmol}$ scale, $132.5 \mathrm{mg}$ ).

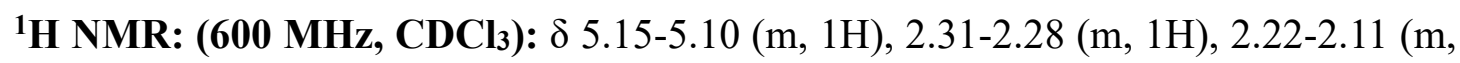
2H), 2.07-1.96 (m, 4H), $1.22(\mathrm{~s}, 3 \mathrm{H}), 1.21(\mathrm{~s}, 12 \mathrm{H}), 1.11(\mathrm{~d}, J=8.4 \mathrm{~Hz}, 1 \mathrm{H}), 0.89-0.78$ (m, 5H). 
${ }^{13}$ C NMR: (151 MHz, $\left.\mathbf{C D C l}_{3}\right): \delta$ 150.2, 114.3, 82.9, 46.0, 41.0, 38.0, 31.7, 31.3, 30.9, 26.4, 24.9, 24.9, 21.2, 8.8 (br, low intensity).

${ }^{11}$ B NMR: (193 MHz, CDCl 3$): ~ \delta 34.14$.

HRMS (ESI): calculated for $\mathrm{C}_{17} \mathrm{H}_{30} \mathrm{BO}_{2}[\mathrm{M}+\mathrm{H}]^{+}:$: 277.2333, found: 277.2331.

\section{Compound 65}

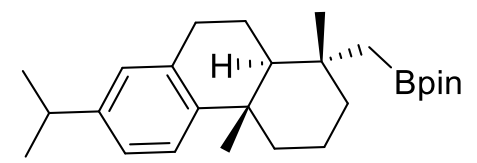

2-(((1R,4aS,10aS)-7-isopropyl-1,4a-dimethyl-1,2,3,4,4a,9,10,10aoctahydrophenanthren-1-yl)methyl)-4,4,5,5-tetramethyl-1,3,2-dioxaborolane ${ }^{[31]}$ : White solid was obtained with 44\% isolated yield (Procedure B, 0.6 mmol scale, 104.7 $\mathrm{mg}$ ).

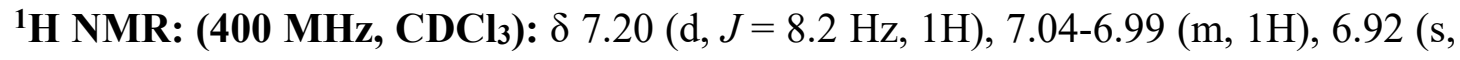
$1 \mathrm{H}), 2.97-2.82(\mathrm{~m}, 3 \mathrm{H}), 2.34-2.25(\mathrm{~m}, 1 \mathrm{H}), 2.00-1.93(\mathrm{~m}, 1 \mathrm{H}), 1.80-1.64(\mathrm{~m}, 4 \mathrm{H}), 1.45-$ 1.37 (m, 3H), 1.29-1.24 (m, 18H), 1.22 (s, 3H), 1.13 (d, J=14.2 Hz, 1H), 1.06 (s, 3H), $0.77(\mathrm{~d}, J=14.2 \mathrm{~Hz}, 1 \mathrm{H})$.

${ }^{13}$ C NMR: (101 MHz, $\left.\mathbf{C D C l}_{3}\right): \delta$ 147.8, 145.3, 135.1, 126.9, 124.4, 123.8, 82.8, 51.7, 40.4, 38.9, 37.9, 36.0, 33.5, 30.8, 28.9 (br, low intensity), 25.3, 25.1, 24.9, 24.1, 24.09, 21.9, 19.5, 19.2.

${ }^{11}$ B NMR: (128 MHz, CDCl 3$): ~ \delta 32.28$.

HRMS (ESI): calculated for $\mathrm{C}_{26} \mathrm{H}_{42} \mathrm{BO}_{2}[\mathrm{M}+\mathrm{H}]^{+}: 397.3272$, found: 397.3281 .

\section{Compound 66}

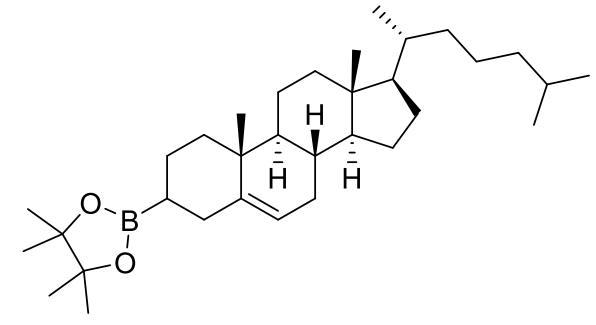

2-((3S,8S,9S,10R,13R,14S,17R)-10,13-dimethyl-17-((R)-6-methylheptan-2-yl)$2,3,4,7,8,9,10,11,12,13,14,15,16,17$-tetradecahydro-1 $H$-cyclopenta[a]phenanthren3-yl)-4,4,5,5-tetramethyl-1,3,2-dioxaborolane ${ }^{[19,45]}$ : White solid was obtained with S78 
74\% isolated yield (Procedure D, $0.2 \mathrm{mmol} \mathrm{scale,} 73.7 \mathrm{mg}$ ).

${ }^{1}$ H NMR: (400 MHz, CDCl 3$): ~ \delta$ (major+minor) 5.37-5.17 (m, 1H), 2.43-2.15 (m, 1H), 2.05-1.74 (m, 5H), 1.67-1.30 (m, 12H), $1.23(\mathrm{~s}, 12 \mathrm{H}), 1.16-0.94(\mathrm{~m}, 13 \mathrm{H}), 0.90(\mathrm{~d}, J=$ $6.4 \mathrm{~Hz}, 4 \mathrm{H}), 0.85$ (dd, $J=6.6,1.8 \mathrm{~Hz}, 6 \mathrm{H}), 0.66$ (s, 3H).

${ }^{13}$ C NMR: (101 MHz, CDCl 3 ): $\delta$ (major+minor) 143.9, 142.7, 119.9, 118.7, 82.9, 56.9, 56.3, 51.3, 50.7, 42.4, 41.0, 39.9, 39.6, 39.1, 37.4, 36.3, 35.9, 34.4, 33.9, 32.2, 32.0, $31.9,28.4,28.1,25.1,24.9,24.9,24.8,24.4,24.2,23.9,23.9,23.6,22.9,22.7,20.9$, $19.6,19.5,18.8,11.9$. The signal of the $\alpha$-B-carbon was not observed.

${ }^{11}$ B NMR: (128 MHz, CDCl 3$): \delta 32.74$.

HRMS (ESI): calculated for $\mathrm{C}_{33} \mathrm{H}_{58} \mathrm{BO}_{2}[\mathrm{M}+\mathrm{H}]^{+}:$497.4524, found: 497.4536 .

\section{Compound 67}

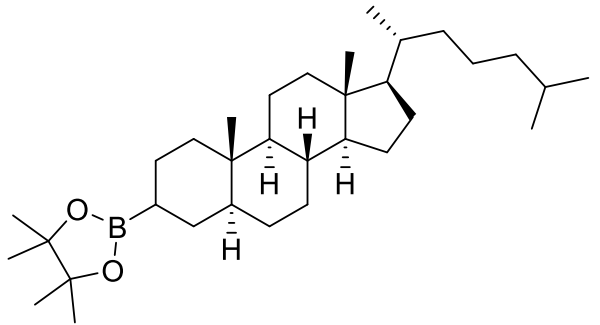

2-((3S,5R,8R,9S,10S,13R,14S,17R)-10,13-dimethyl-17-((R)-6-methylheptan-2yl)hexadecahydro-1 $H$-cyclopenta[a]phenanthren-3-yl)-4,4,5,5-tetramethyl-1,3,2dioxaborolane: White solid was obtained with $60 \%$ isolated yield (Procedure D, 0.2 mmol scale, $60.0 \mathrm{mg})$.

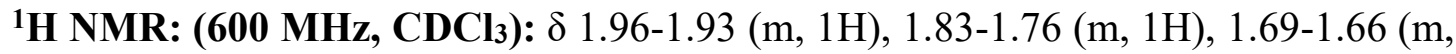
1H), 1.64-1.60 (m, 1H), 1.59-1.46 (m, 5H), 1.43-1.24 (m, 10H), $1.22(\mathrm{~s}, 12 \mathrm{H}), 1.21-$ $0.93(\mathrm{~m}, 13 \mathrm{H}), 0.89(\mathrm{~d}, J=6.6 \mathrm{~Hz}, 3 \mathrm{H}), 0.86(\mathrm{dd}, J=6.6,2.8 \mathrm{~Hz}, 6 \mathrm{H}), 0.77(\mathrm{~s}, 3 \mathrm{H})$, $0.63(\mathrm{~s}, 3 \mathrm{H})$.

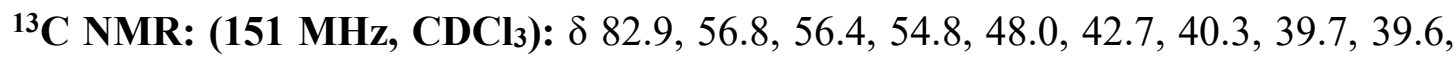
$36.3,36.2,35.9,35.7,32.3,30.3,29.1,28.4,28.2,24.9,24.9,24.3,24.0,23.6,22.9$, $22.7,20.9,18.8,12.5,12.2$. The signal of the $\alpha$-B-carbon was not observed.

${ }^{11}$ B NMR: (193 MHz, CDCl 3$): ~ \delta 33.78$.

HRMS (ESI): calculated for $\mathrm{C}_{30} \mathrm{H}_{60} \mathrm{BO}_{2}[\mathrm{M}+\mathrm{H}]^{+}:$499.4681, found: 499.4681 . 
In order to measure the $d . r$. value of 67,67 was converted to alcohol $67 \mathbf{a}$ and $67 \mathbf{b}$ by following procedure.
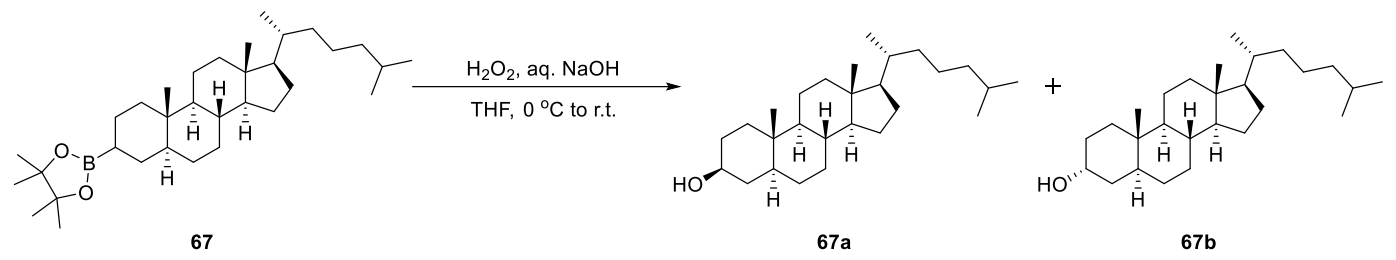

To a solution of $67(100.0 \mathrm{mg}, 0.2 \mathrm{mmol})$ in THF $(7.0 \mathrm{~mL})$, a premixed solution of $\mathrm{NaOH}(3.0 \mathrm{M}$ aqueous $) / \mathrm{H}_{2} \mathrm{O}_{2}(30 \%$ aqueous $)(2: 1,7.5 \mathrm{~mL})$ was added dropwise at 0 ${ }^{\circ} \mathrm{C}$. After addition the reaction was stirred at room temperature for a further $1 \mathrm{~h}$. The reaction was quenched by the addition of $10 \%$ aqueous $\mathrm{Na}_{2} \mathrm{~S}_{2} \mathrm{O}_{3}(15 \mathrm{~mL})$, diluted with water $(15 \mathrm{~mL})$, extracted with EtOAc $(3 \times 15 \mathrm{~mL})$, washed with brine and dried over $\mathrm{NaSO}_{4}$, concentrated, and purified by column chromatography (eluted with ethyl acetate/petroleum ether) to afford the pure desired product $67 \mathbf{a}(67.9 \mathrm{mg}, 87 \%)$ and $\mathbf{6 7} \mathbf{b}$ (7 $\mathrm{mg}, 9 \%$ ). The $d . r$. value of 67 is $9.7: 1$.

${ }^{1}$ H NMR: (600 MHz, CDCl $)^{[46]}$ : $\delta 3.57$ (tt, $\left.J=11.0,4.8 \mathrm{~Hz}, 1 \mathrm{H}\right), 1.95$ (dt, $J=12.6$, $3.4 \mathrm{~Hz}, 1 \mathrm{H}), 1.80-1.76(\mathrm{~m}, 2 \mathrm{H}), 1.72-1.62(\mathrm{~m}, 2 \mathrm{H}), 1.59-0.90(\mathrm{~m}, 27 \mathrm{H}), 0.88(\mathrm{~d}, J=6.6$ $\mathrm{Hz}, 3 \mathrm{H}), 0.85(\mathrm{dd}, J=6.6,2.8 \mathrm{~Hz}, 6 \mathrm{H}), 0.79$ (s, 3H), 0.63 (s, 3H).
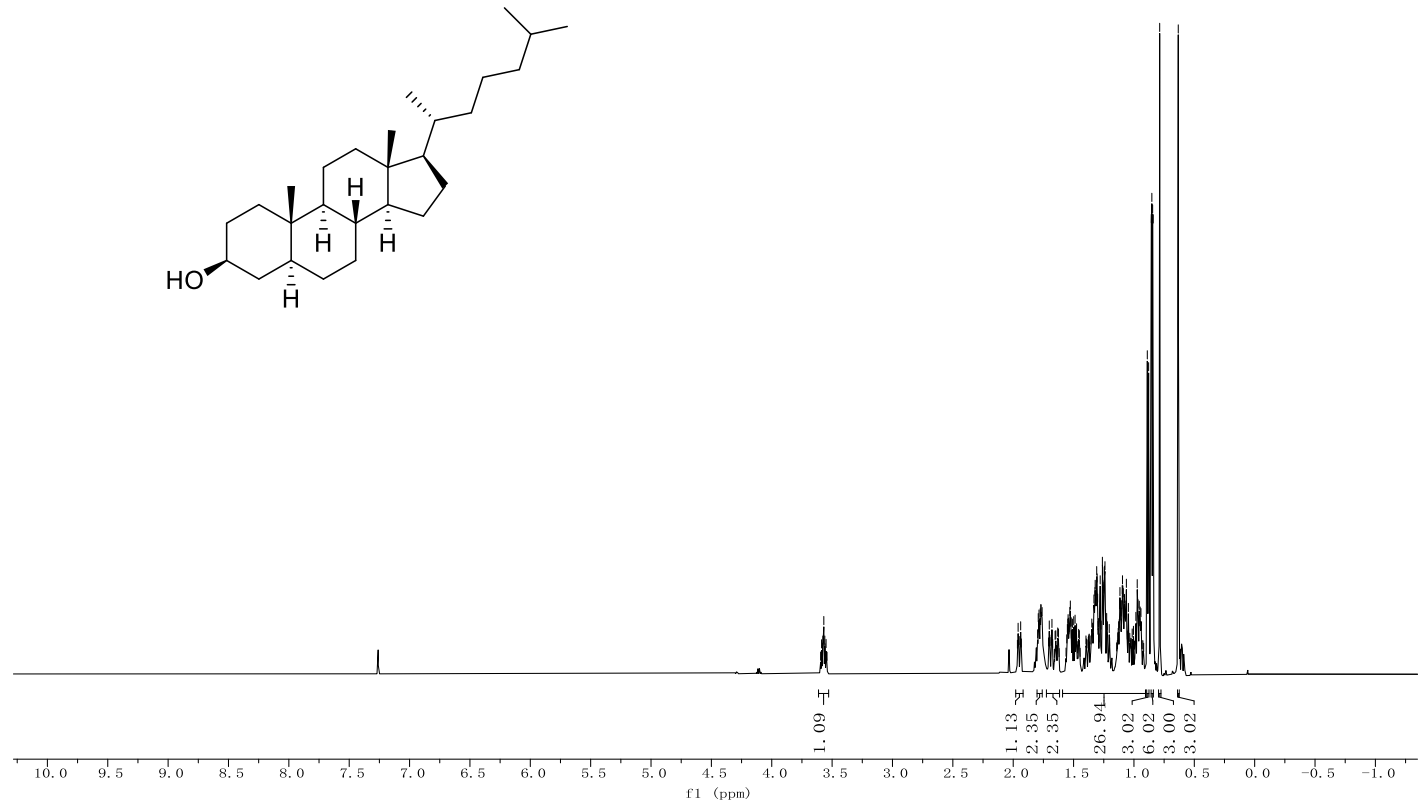

Figure S18. The ${ }^{1} \mathrm{H}$ NMR spectrum of $67 \mathbf{a}$. 
${ }^{1}$ H NMR: (600 MHz, $\left.\mathbf{C D C l}_{3}\right)^{[47]}$ : $\delta$ 4.05-4.02 (m, 1H), $1.96(\mathrm{dt}, J=12.6,3.4 \mathrm{~Hz}, 1 \mathrm{H})$, 1.83-1.77 (m, 1H), 1.71-1.61 (m, 3H), 1.52-0.95 (m, 26H), $0.89(\mathrm{~d}, J=6.4 \mathrm{~Hz}, 3 \mathrm{H})$, $0.86(\mathrm{dd}, J=6.6,3.0 \mathrm{~Hz}, 6 \mathrm{H}), 0.77-0.71(\mathrm{~m}, 4 \mathrm{H}), 0.64(\mathrm{~s}, 3 \mathrm{H})$.
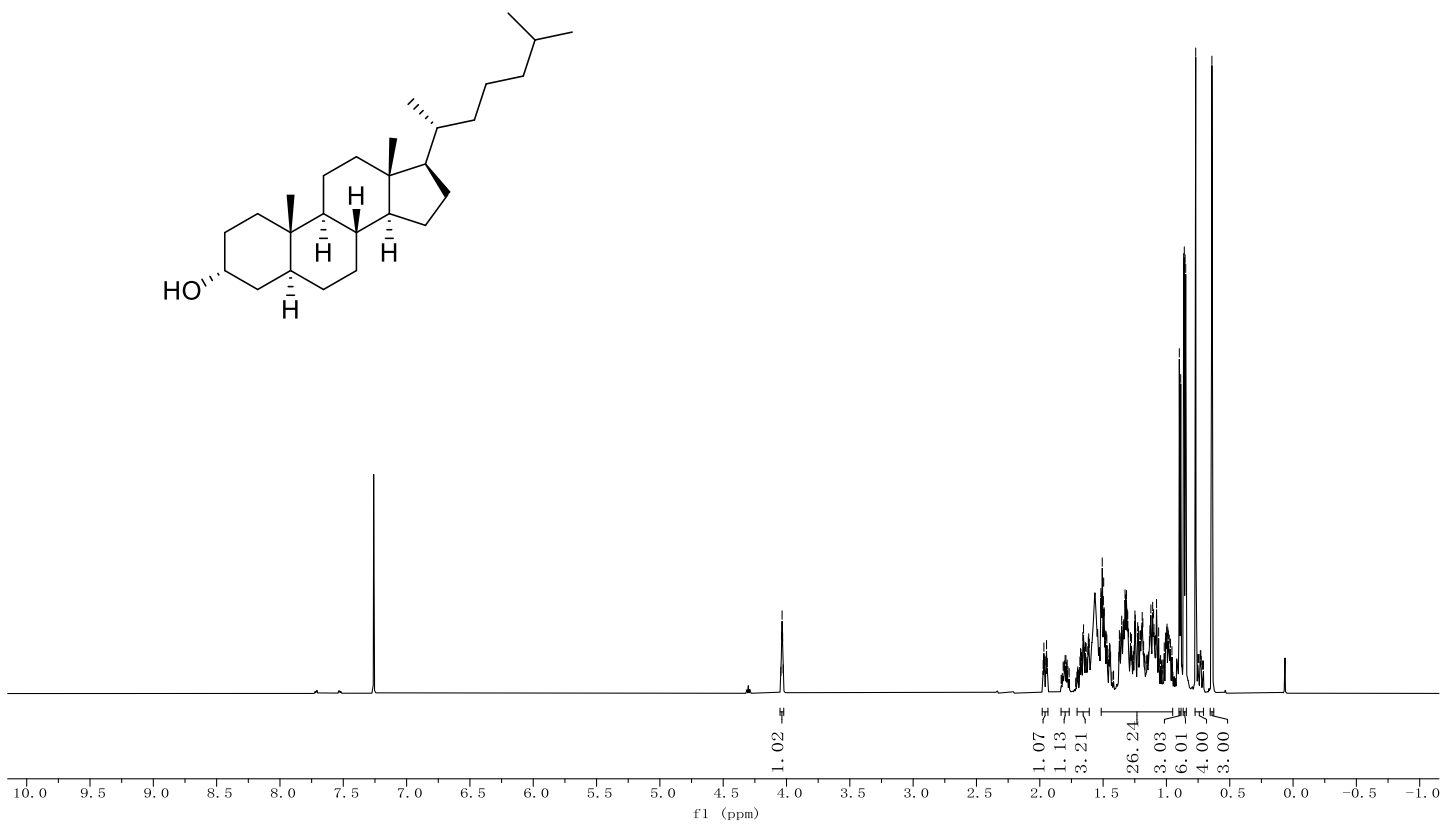

Figure S19. The ${ }^{1} \mathrm{H}$ NMR spectrum of $67 \mathbf{b}$.

\section{References}

[1] Huang, S.-X.; Li, H.-Y.; Liu, J.-Y.; Morisseau, C.; Hammock, B. D.; Long, Y.-Q. Incorporation of Piperazino Functionality into 1,3-Disubstituted Urea as the Tertiary Pharmacophore Affording Potent Inhibitors of Soluble Epoxide Hydrolase with Improved Pharmacokinetic Properties, J. Med. Chem. 2010, 53, 8376.

[2] Kim, D.; Lee, G. S.; Kim, D.; Hong, S. H. Direct $\mathrm{C}\left(\mathrm{sp}^{2}\right)-\mathrm{H}$ alkylation of unactivated arenes enabled by photoinduced Pd catalysis, Nat. Commun. 2020, 11, 5266. [3] Peng, D.-Y.; Sun, Q.; Zhu, X.-L.; Lin, H.-Y.; Chen, Q.; Yu, N.-X.; Yang, W.-C.; Yang, G.-F. Design, synthesis, and bioevaluation of benzamides: Novel acetylcholinesterase inhibitors with multi-functions on butylcholinesterase, A $\beta$ aggregation, and $\beta$-secretase, Bioorgan. Med. Chem. 2012, 20, 6739.

[4] Guisán-Ceinos, M.; Soler-Yanes, R.; Collado-Sanz, D.; Phapale, V. B.; Buñuel, E.; Cárdenas, D. J. Ni-Catalyzed Cascade Cyclization-Kumada Alkyl-Alkyl CrossCoupling, Chem. Eur. J. 2013, 19, 8405.

[5] Qian, D.; Hu, X. Ligand-Controlled Regiodivergent Hydroalkylation of Pyrrolines, Angew. Chem. In. Ed. 2019, 58, 18519.

[6] Cheng, Y.; Mück-Lichtenfeld, C.; Studer, A. Metal-Free Radical Borylation of 
Alkyl and Aryl Iodides, Angew. Chem. In. Ed. 2018, 57, 16832.

[7] Wu, X.; Hao, W.; Ye, K.-Y.; Jiang, B.; Pombar, G.; Song, Z.; Lin, S. Ti-Catalyzed Radical Alkylation of Secondary and Tertiary Alkyl Chlorides Using Michael Acceptors, J. Am. Chem. Soc. 2018, 140, 14836.

[8] Das, D.; H Anal, J. M.; Rokhum, L. A mild and highly chemoselective iodination of alcohol using polymer supported DMAP, J. Chem. Sci. 2016, 128, 1695.

[9] Lapuh, M. I.; Dana, A.; Di Chenna, P. H.; Darses, B.; Durán, F. J.; Dauban, P. Latestage C-H amination of abietane diterpenoids, Org. Biomol. Chem. 2019, 17, 4736.

[10]Kubota, K.; Yamamoto, E.; Ito, H. Copper(I)-Catalyzed Borylative exoCyclization of Alkenyl Halides Containing Unactivated Double Bond, J. Am. Chem. Soc. 2013, 135, 2635.

[11]Zhang, Q.; Li, X.; Zhang, W.; Ni, S.; Wang, Y.; Pan, Y. Decarboxylative Borylation of Stabilized and Activated Carbon Radicals, Angew. Chem. In. Ed. 2020, 59, 21875.

[12]Frisch, M. J.; Trucks, G. W.; Schlegel, H. B.; Scuseria, G. E.; Robb, M. A.; Cheeseman, J. R.; Scalmani, G.; Barone, V.; Petersson, G. A.; Nakatsuji, H.; Li, X.; Caricato, M.; Marenich, A. V.; Bloino, J.; Janesko, B. G.; Gomperts, R.; Mennucci, B.; Hratchian, H. P.; Ortiz, J. V.; Izmaylov, A. F.; Sonnenberg, J. L.; Williams; Ding, F.; Lipparini, F.; Egidi, F.; Goings, J.; Peng, B.; Petrone, A.; Henderson, T.; Ranasinghe, D.; Zakrzewski, V. G.; Gao, J.; Rega, N.; Zheng, G.; Liang, W.; Hada, M.; Ehara, M.; Toyota, K.; Fukuda, R.; Hasegawa, J.; Ishida, M.; Nakajima, T.; Honda, Y.; Kitao, O.; Nakai, H.; Vreven, T.; Throssell, K.; Montgomery Jr., J. A.; Peralta, J. E.; Ogliaro, F.; Bearpark, M. J.; Heyd, J. J.; Brothers, E. N.; Kudin, K. N.; Staroverov, V. N.; Keith, T. A.; Kobayashi, R.; Normand, J.; Raghavachari, K.; Rendell, A. P.; Burant, J. C.; Iyengar, S. S.; Tomasi, J.; Cossi, M.; Millam, J. M.; Klene, M.; Adamo, C.; Cammi, R.; Ochterski, J. W.; Martin, R. L.; Morokuma, K.; Farkas, O.; Foresman, J. B.; Fox, D. J. Wallingford, CT, 2016.

[13]Lee, C.; Yang, W.; Parr, R. G. Development of the Colle-Salvetti correlation-energy formula into a functional of the electron density, Phys. Rev. B 1988, 37, 785.

[14]Becke, A. D. Density - functional thermochemistry. III. The role of exact exchange, J. Chem. Phys. 1993, 98, 5648.

[15] Grimme, S.; Ehrlich, S.; Goerigk, L. Effect of the damping function in dispersion corrected density functional theory, J. Comput. Chem. 2011, 32, 1456.

[16] Grimme, S.; Antony, J.; Ehrlich, S.; Krieg, H. A consistent and accurate ab initio parametrization of density functional dispersion correction (DFT-D) for the 94 elements $\mathrm{H}-\mathrm{Pu}$, J. Chem. Phys. 2010, 132, 154104.

[17]Marenich, A. V.; Cramer, C. J.; Truhlar, D. G. Universal Solvation Model Based on Solute Electron Density and on a Continuum Model of the Solvent Defined by the Bulk Dielectric Constant and Atomic Surface Tensions, J. Phys. Chem. B 2009, 113, 6378.

[18]Zhao, Y.; Truhlar, D. G. The M06 suite of density functionals for main group thermochemistry, thermochemical kinetics, noncovalent interactions, excited states, and transition elements: two new functionals and systematic testing of four M06-class functionals and 12 other functionals, Theor. Chem. Acc. 2008, 120, 215.

[19]Wang, X.; Cui, P.; Xia, C.; Wu, L. Catalytic Boration of Alkyl Halides with Borane without Hydrodehalogenation Enabled by Titanium Catalyst, Angew. Chem. In. Ed. 
2021, 60, 12298.

[20]Kisan, S.; Krishnakumar, V.; Gunanathan, C. Ruthenium-Catalyzed AntiMarkovnikov Selective Hydroboration of Olefins, ACS Catal. 2017, 7, 5950.

[21]Panferova, L. I.; Dilman, A. D. Light-Mediated Sulfur-Boron Exchange, Org. Lett. 2021, 23, 3919.

[22]Bose, S. K.; Fucke, K.; Liu, L.; Steel, P. G.; Marder, T. B. Zinc-Catalyzed Borylation of Primary, Secondary and Tertiary Alkyl Halides with Alkoxy Diboron Reagents at Room Temperature, Angew. Chem. In. Ed. 2014, 53, 1799.

[23]Wang, Z.-C.; Shen, D.; Gao, J.; Jia, X.; Xu, Y.; Shi, S.-L. Base-catalysed reductive relay hydroboration of allylic alcohols with pinacolborane to form alkylboronic esters, Chem. Commun. 2019, 55, 8848.

[24]Wang, W.; Ding, C.; Pang, H.; Yin, G. Nickel-Catalyzed 1,2-Arylboration of Vinylarenes, Org. Lett. 2019, 21, 3968.

[25]Liu, Y.; Zhou, Y.; Wang, H.; Qu, J. FeCl2-catalyzed hydroboration of aryl alkenes with bis(pinacolato)diboron, RSC Adv. 2015, 5, 73705.

[26]Chen, X.; Cheng, Z.; Lu, Z. Cobalt-Catalyzed Asymmetric Markovnikov Hydroboration of Styrenes, ACS Catal. 2019, 9, 4025.

[27]Wang, D.; Xue, X.-S.; Houk, K. N.; Shi, Z. Mild Ring-Opening 1,3-Hydroborations of Non-Activated Cyclopropanes, Angew. Chem. In. Ed. 2018, 57, 16861.

[28]Bose, S. K.; Brand, S.; Omoregie, H. O.; Haehnel, M.; Maier, J.; Bringmann, G.; Marder, T. B. Highly Efficient Synthesis of Alkylboronate Esters via $\mathrm{Cu}$ (II)-Catalyzed Borylation of Unactivated Alkyl Bromides and Chlorides in Air, ACS Catal. 2016, 6, 8332.

[29]Zhang, L.; Wu, Z.-Q.; Jiao, L. Photoinduced Radical Borylation of Alkyl Bromides Catalyzed by 4-Phenylpyridine, Angew. Chem. In. Ed. 2020, 59, 2095.

[30]Yang, C.-T.; Zhang, Z.-Q.; Tajuddin, H.; Wu, C.-C.; Liang, J.; Liu, J.-H.; Fu, Y.; Czyzewska, M.; Steel, P. G.; Marder, T. B.; Liu, L. Alkylboronic Esters from CopperCatalyzed Borylation of Primary and Secondary Alkyl Halides and Pseudohalides, Angew. Chem. In. Ed. 2012, 51, 528.

[31]Wu, J.; He, L.; Noble, A.; Aggarwal, V. K. Photoinduced Deaminative Borylation of Alkylamines, J. Am. Chem. Soc. 2018, 140, 10700.

[32]Yi, J.; Liu, J.-H.; Liang, J.; Dai, J.-J.; Yang, C.-T.; Fu, Y.; Liu, L. Alkylboronic Esters from Palladium- and Nickel-Catalyzed Borylation of Primary and Secondary Alkyl Bromides, Adv. Synth. Catal. 2012, 354, 1685.

[33] Oeschger, R.; Su, B.; Yu, I.; Ehinger, C.; Romero, E.; He, S.; Hartwig, J. Diverse functionalization of strong alkyl $\mathrm{C}-\mathrm{H}$ bonds by undirected borylation, Science $\mathbf{2 0 2 0}$, 368,736 .

[34]Wei, D.; Liu, T.-M.; Zhou, B.; Han, B. Decarboxylative Borylation of mCPBAActivated Aliphatic Acids, Org. Lett. 2020, 22, 234.

[35]Bonet, A.; Pubill-Ulldemolins, C.; Bo, C.; Gulyás, H.; Fernández, E. TransitionMetal-Free Diboration Reaction by Activation of Diboron Compounds with Simple Lewis Bases, Angew. Chem. In. Ed. 2011, 50, 7158.

[36]Moriai, T.; Tsukamoto, T.; Tanabe, M.; Kambe, T.; Yamamoto, K. Selective Hydroperoxygenation of Olefins Realized by a Coinage Multimetallic 1-Nanometer 
Catalyst, Angew. Chem. In. Ed. 2020, 59, 23051.

[37]Emmanuvel, L.; Shaikh, T. M. A.; Sudalai, A. NaIO $4 / \mathrm{LiBr}_{-}$mediated Diastereoselective Dihydroxylation of Olefins: A Catalytic Approach to the Prevost-Woodward Reaction, Org. Lett. 2005, 7, 5071.

[38]Zhang, L.; Peng, D.; Leng, X.; Huang, Z. Iron-Catalyzed, Atom-Economical, Chemo- and Regioselective Alkene Hydroboration with Pinacolborane, Angew. Chem. In. Ed. 2013, 52, 3676.

[39]Ibrahim, A. D.; Entsminger, S. W.; Fout, A. R. Insights into a Chemoselective Cobalt Catalyst for the Hydroboration of Alkenes and Nitriles, ACS Catal. 2017, 7, 3730 .

[40]Zarate, C.; Nakajima, M.; Martin, R. A Mild and Ligand-Free Ni-Catalyzed Silylation via C-OMe Cleavage, J. Am. Chem. Soc. 2017, 139, 1191.

[41]Hu, J.; Wang, G.; Li, S.; Shi, Z. Selective C-N Borylation of Alkyl Amines Promoted by Lewis Base, Angew. Chem. In. Ed. 2018, 57, 15227.

[42]Zhang, G.; Li, M.-Y.; Ye, W.-B.; He, Z.-T.; Feng, C.-G.; Lin, G.-Q. Borylation of Unactivated C(sp3)-H Bonds with Bromide as a Traceless Directing Group, Org. Lett. 2021, 23, 2948.

[43]Ji, P.; Sawano, T.; Lin, Z.; Urban, A.; Boures, D.; Lin, W. Cerium-Hydride Secondary Building Units in a Porous Metal-Organic Framework for Catalytic Hydroboration and Hydrophosphination, J. Am. Chem. Soc. 2016, 138, 14860.

[44]Bergamaschi, E.; Beltran, F.; Teskey, C. J. Visible-Light Controlled Divergent Catalysis Using a Bench-Stable Cobalt(I) Hydride Complex, Chem. Eur. J. 2020, 26, 5180.

[45]Dudnik, A. S.; Fu, G. C. Nickel-Catalyzed Coupling Reactions of Alkyl Electrophiles, Including Unactivated Tertiary Halides, To Generate Carbon-Boron Bonds, J. Am. Chem. Soc. 2012, 134, 10693.

[46]Flinker, M.; Yin, H.; Juhl, R. W.; Eikeland, E. Z.; Overgaard, J.; Nielsen, D. U.; Skrydstrup, T. Efficient Water Reduction with $\mathrm{sp}^{3}-\mathrm{sp}^{3}$ Diboron(4) Compounds: Application to Hydrogenations, H-D Exchange Reactions, and Carbonyl Reductions, Angew. Chem. In. Ed. 2017, 56, 15910.

[47]Zhang, F.-H.; Zhang, F.-J.; Li, M.-L.; Xie, J.-H.; Zhou, Q.-L. Enantioselective hydrogenation of dialkyl ketones, Nat. Catal. 2020, 3, 621. 


\section{NMR Spectra}

\section{Compound 1}

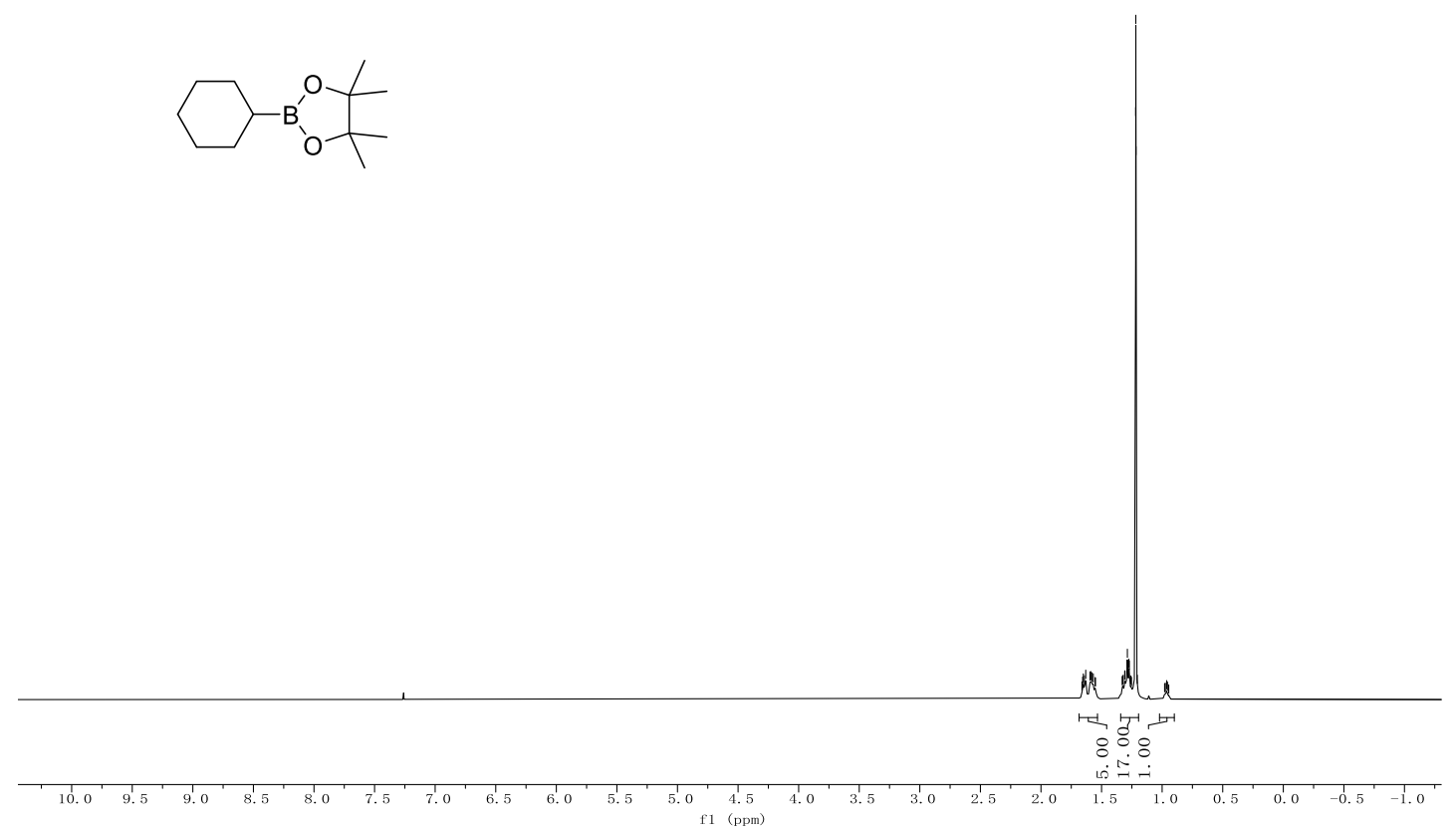

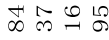

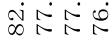

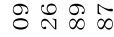

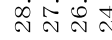

4

iri
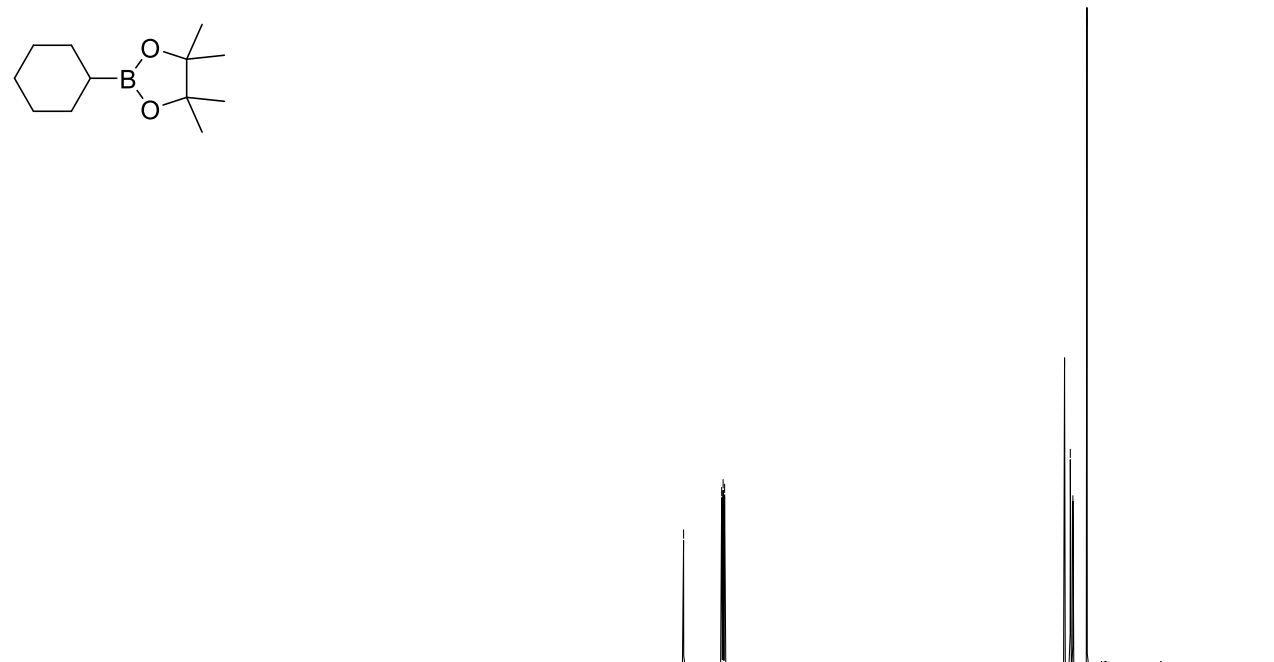

$\frac{1}{200}$
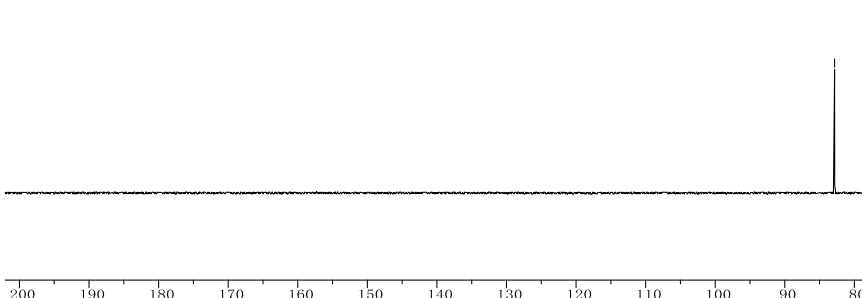

150

$140 \quad 130$

${ }_{120}^{1}{ }_{110}^{1}$

100
$\mathrm{f} 1 \quad \mathrm{ppm})$ 

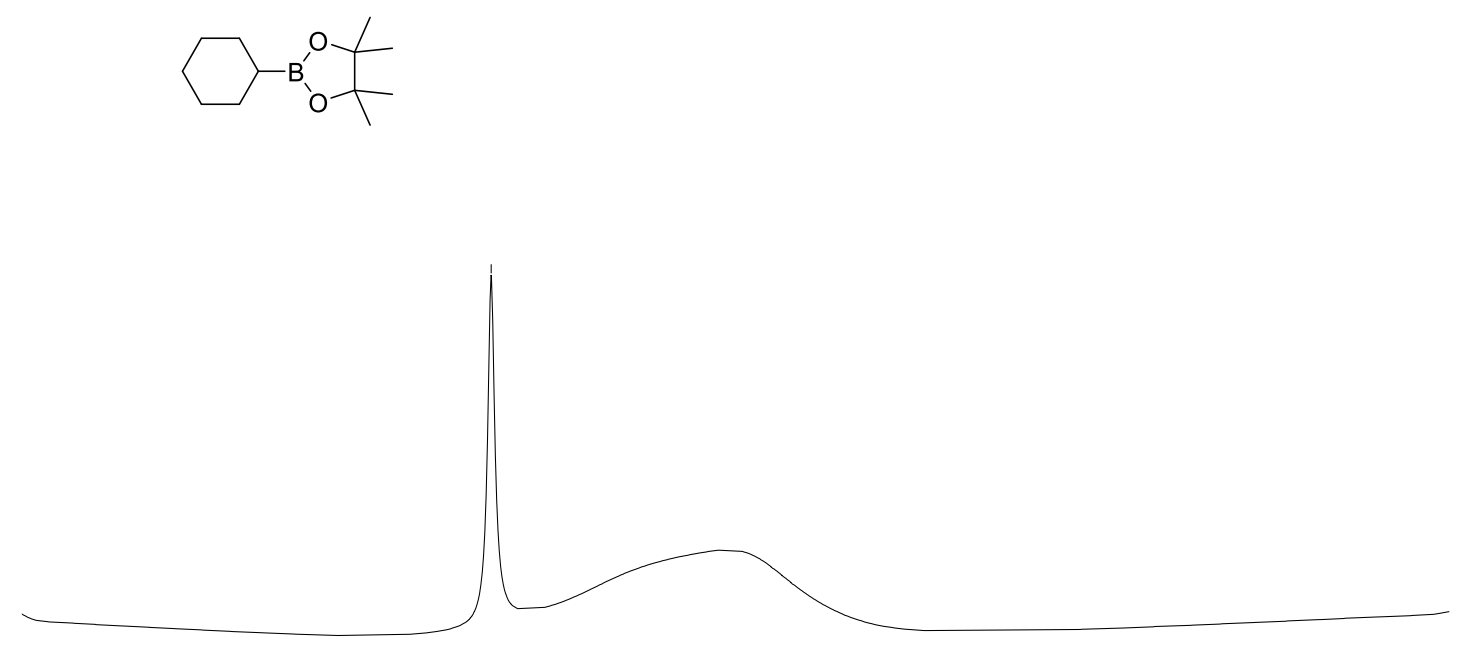

Compound 2

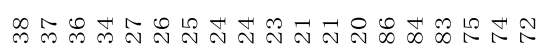

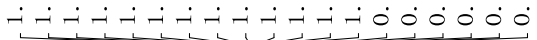

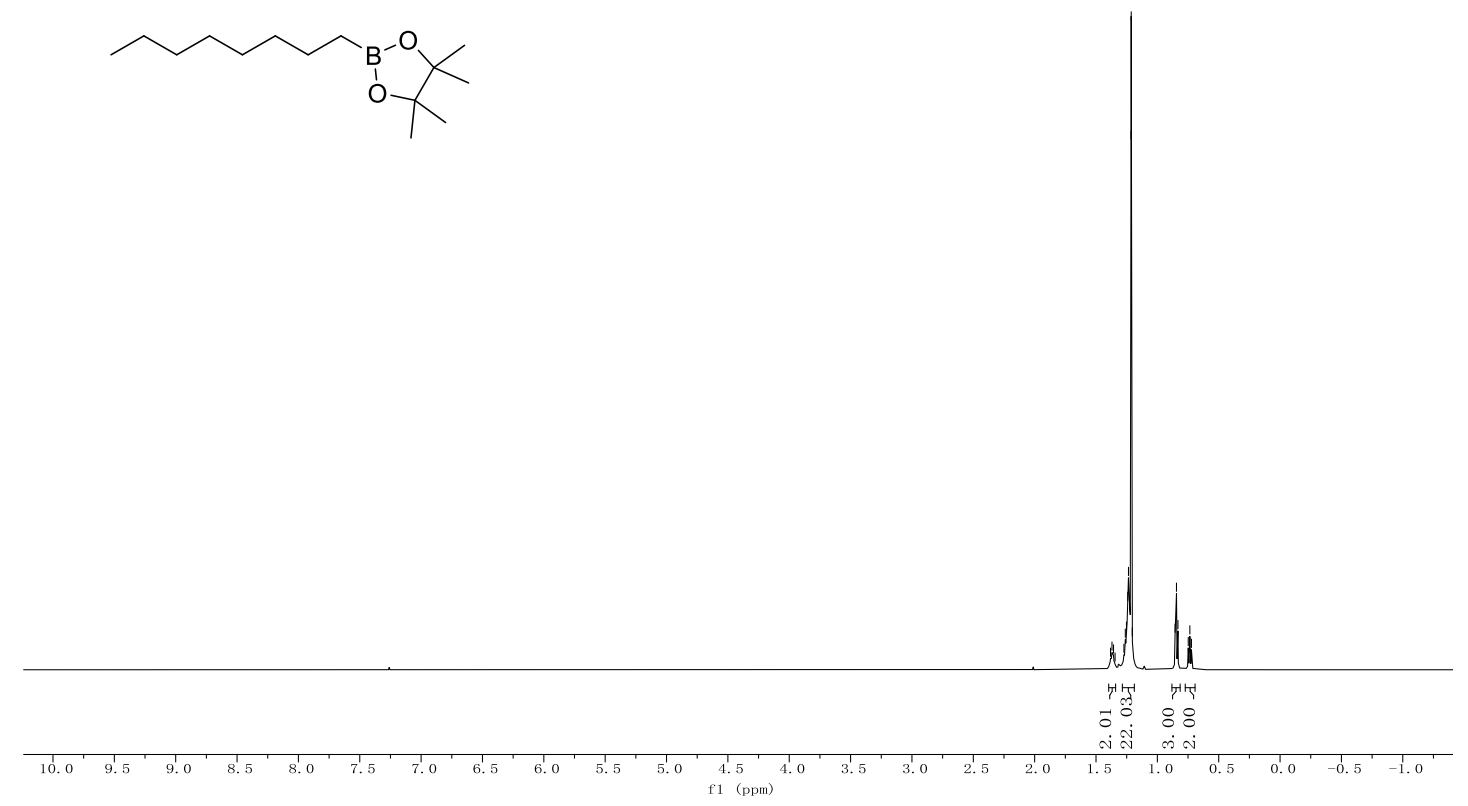


후용요

这소숭요

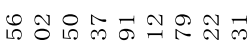

مَ
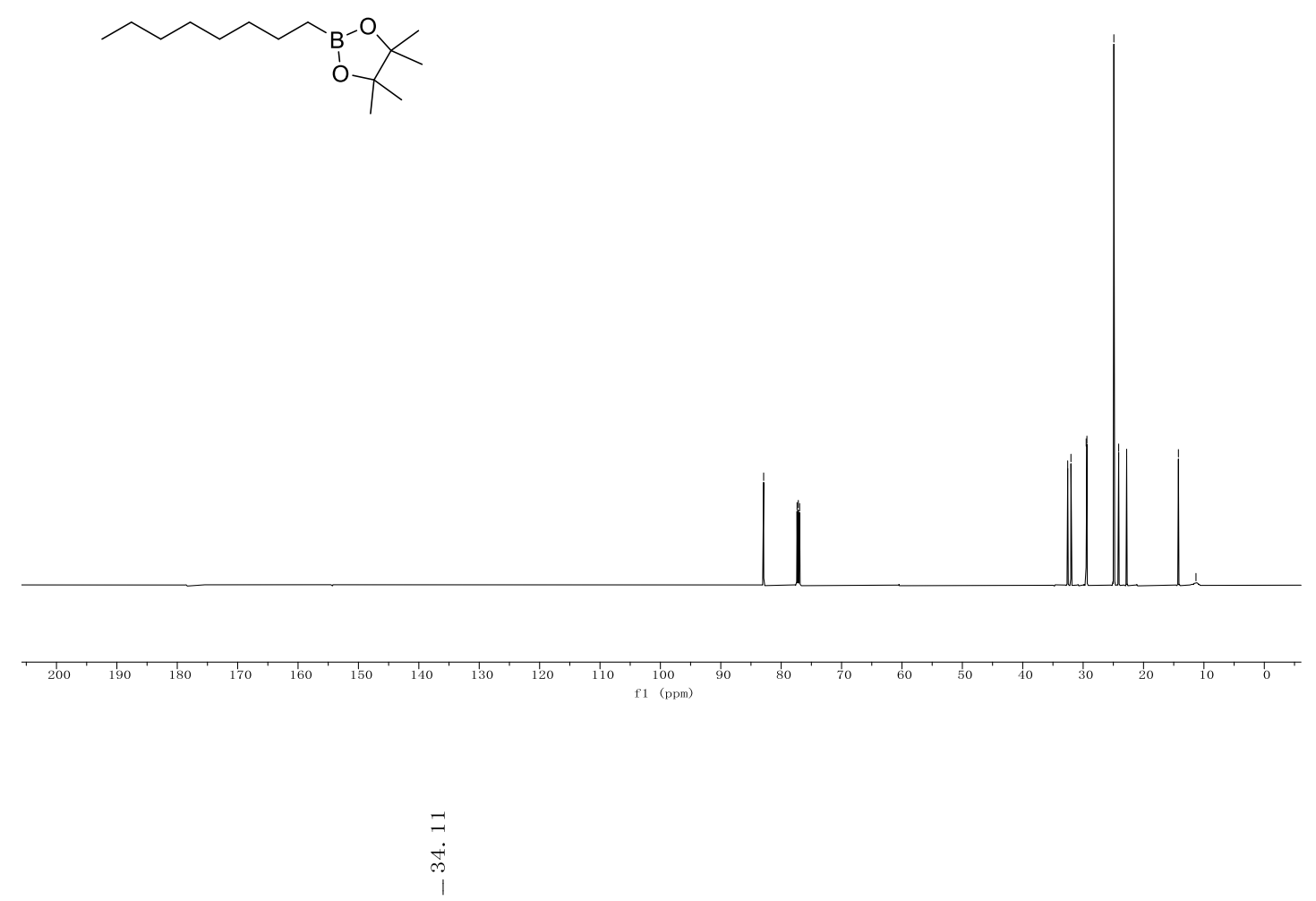

$\overbrace{}^{{ }^{B}-\mathrm{O}} \mathcal{1}$

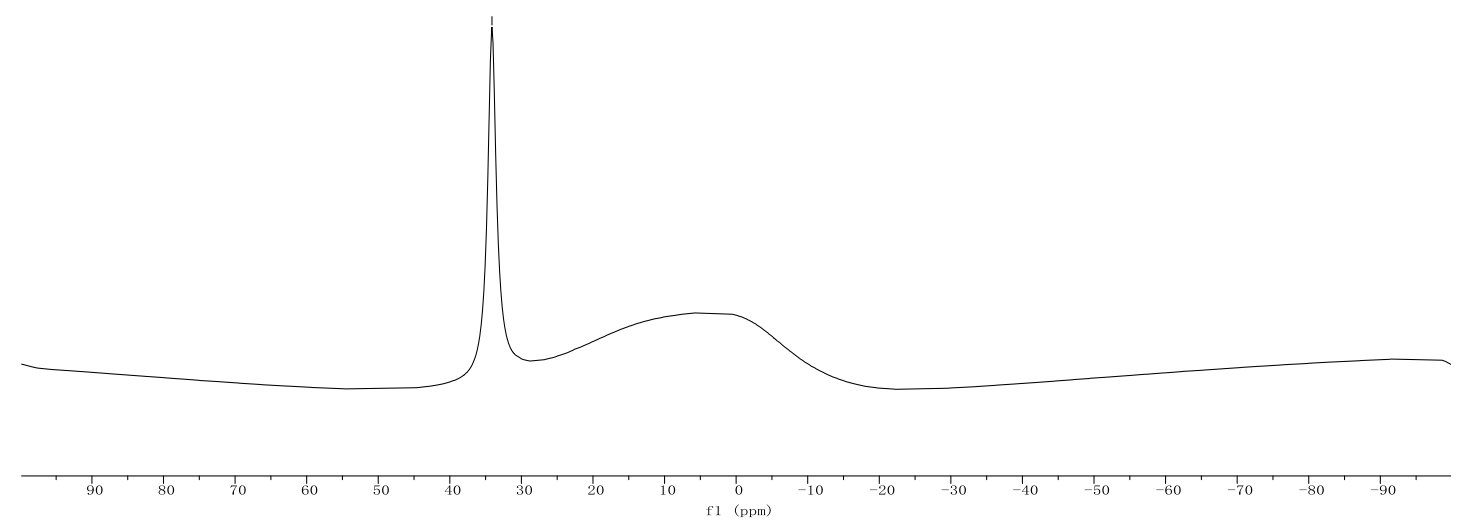




\section{Compound 3}

ล

-<smiles>CC(C)(C)CCB1OC(C)(C)C(C)(C)O1</smiles>

팔 4 is

$-708$

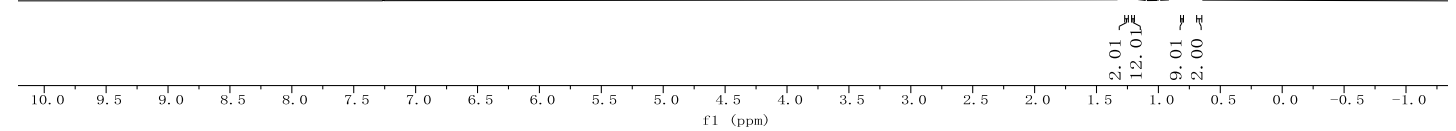

\begin{tabular}{|c|c|}
\hline 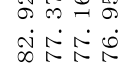 & 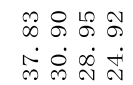 \\
\hline
\end{tabular}
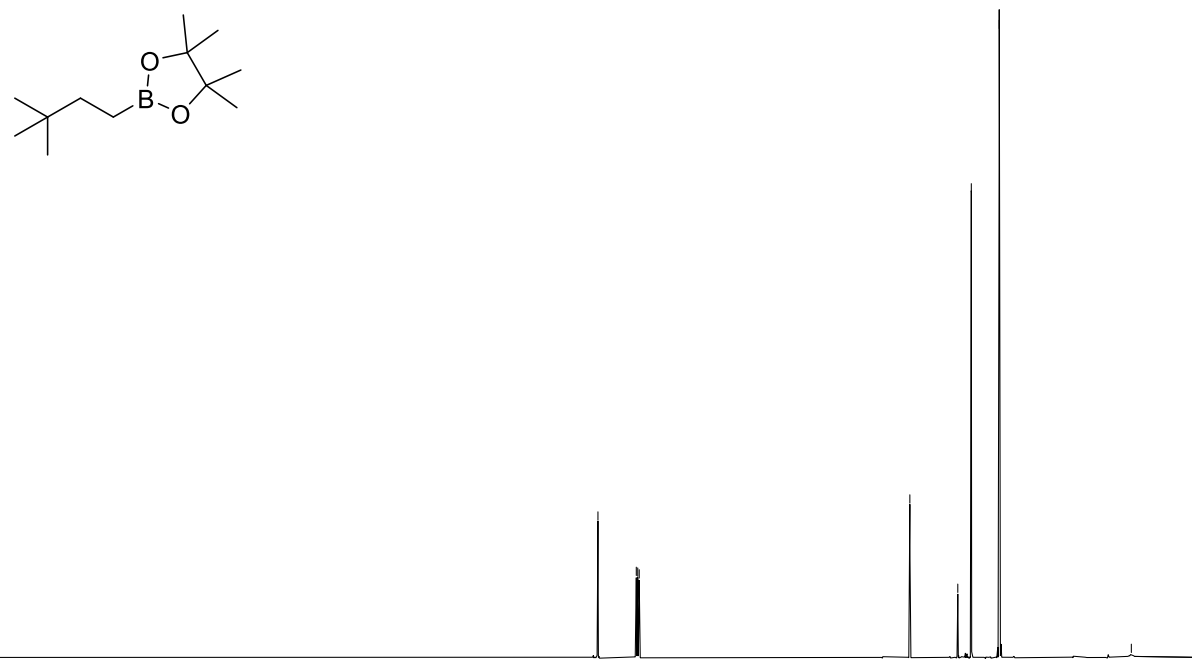

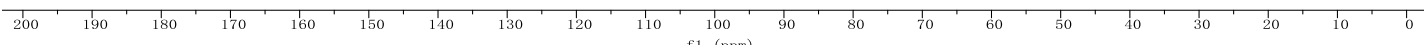



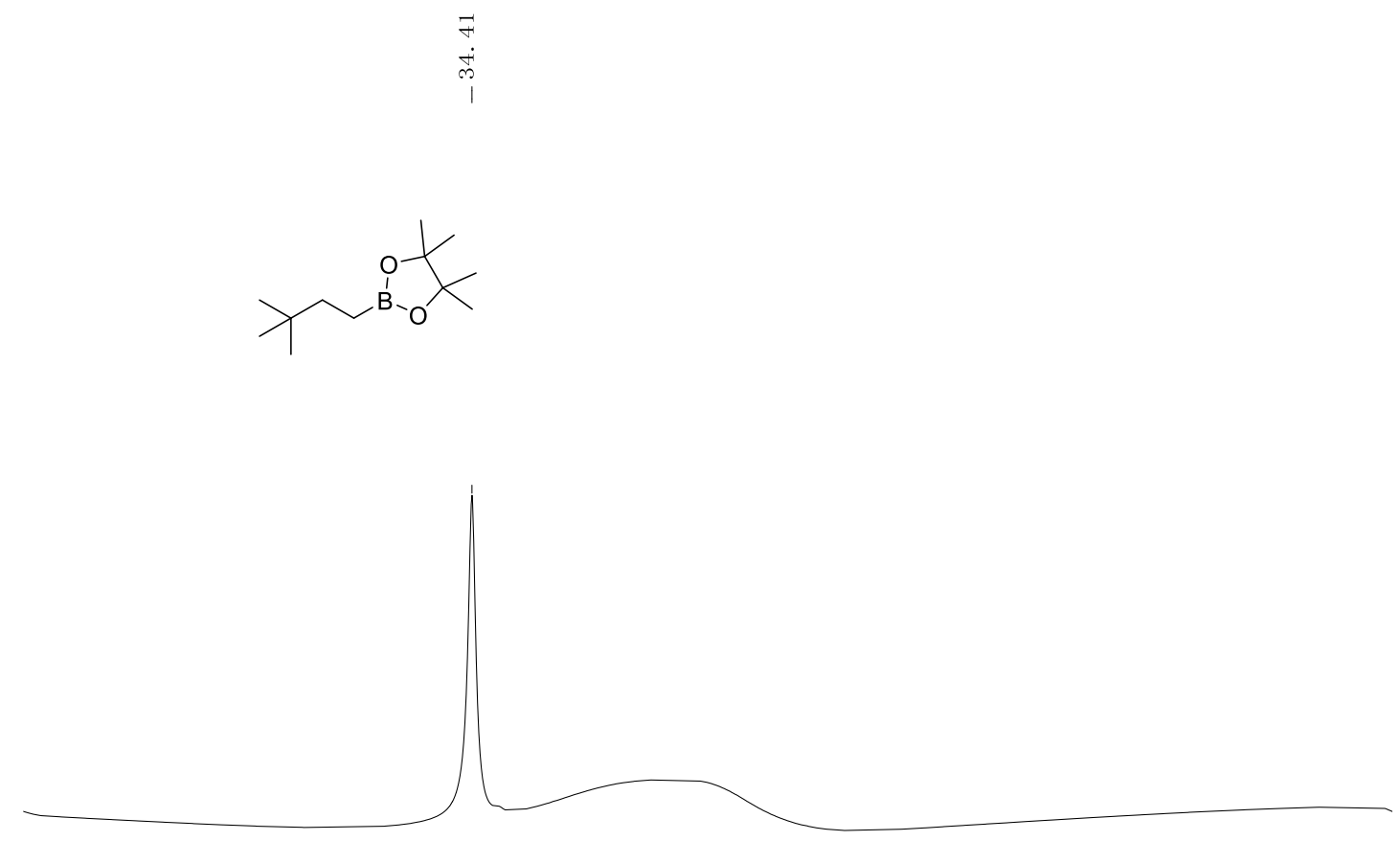

\section{Compound 4}

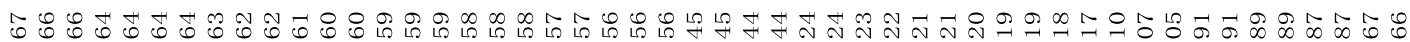
-

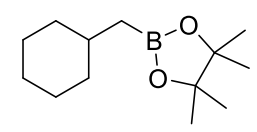




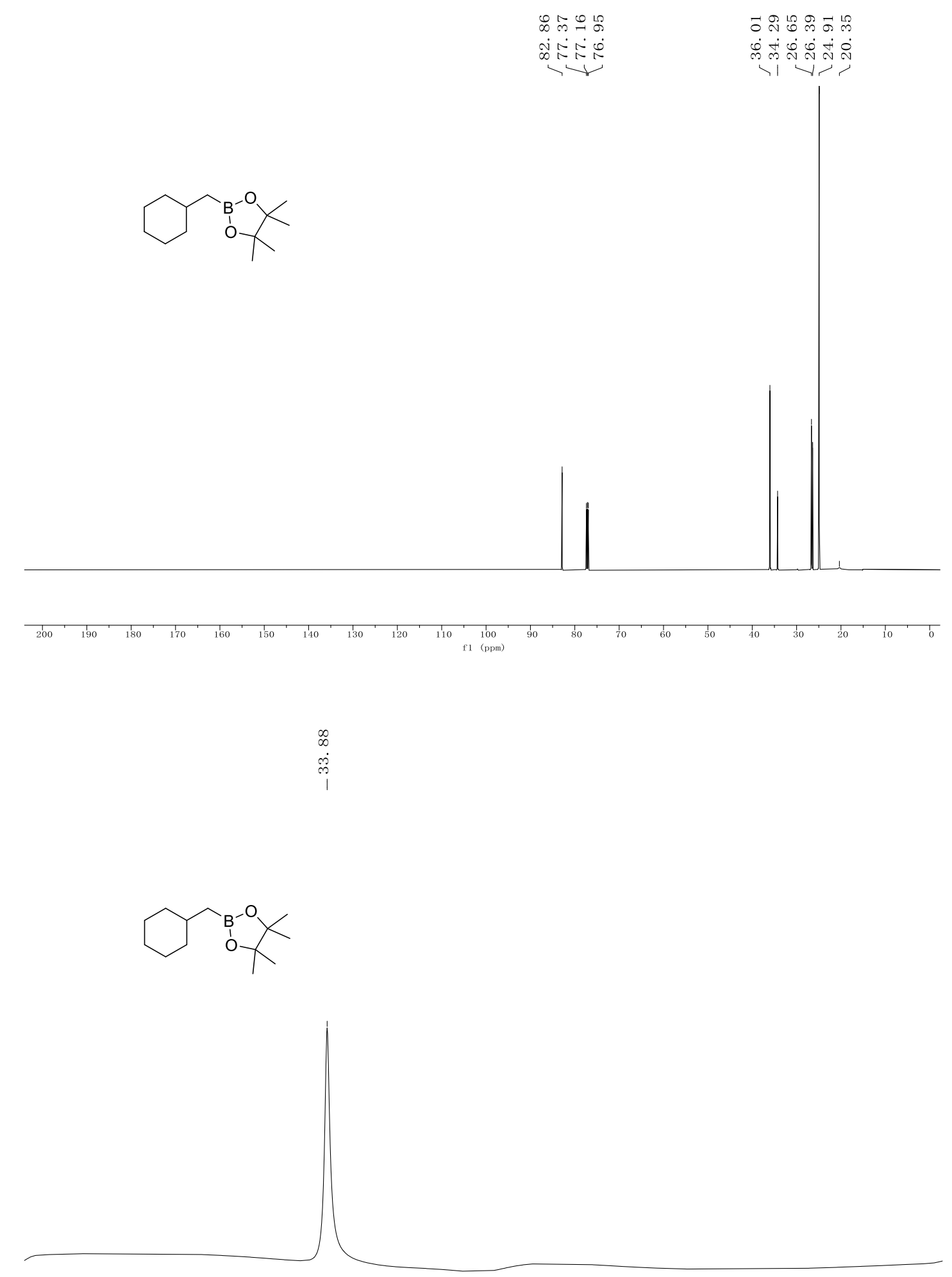

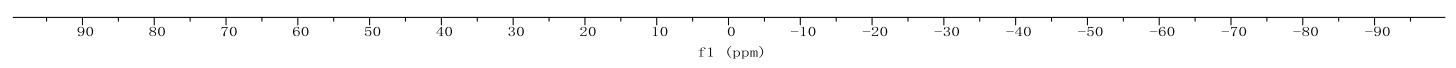




\section{Compound 5}

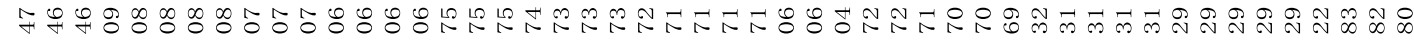

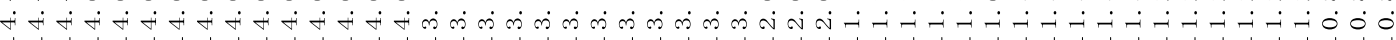

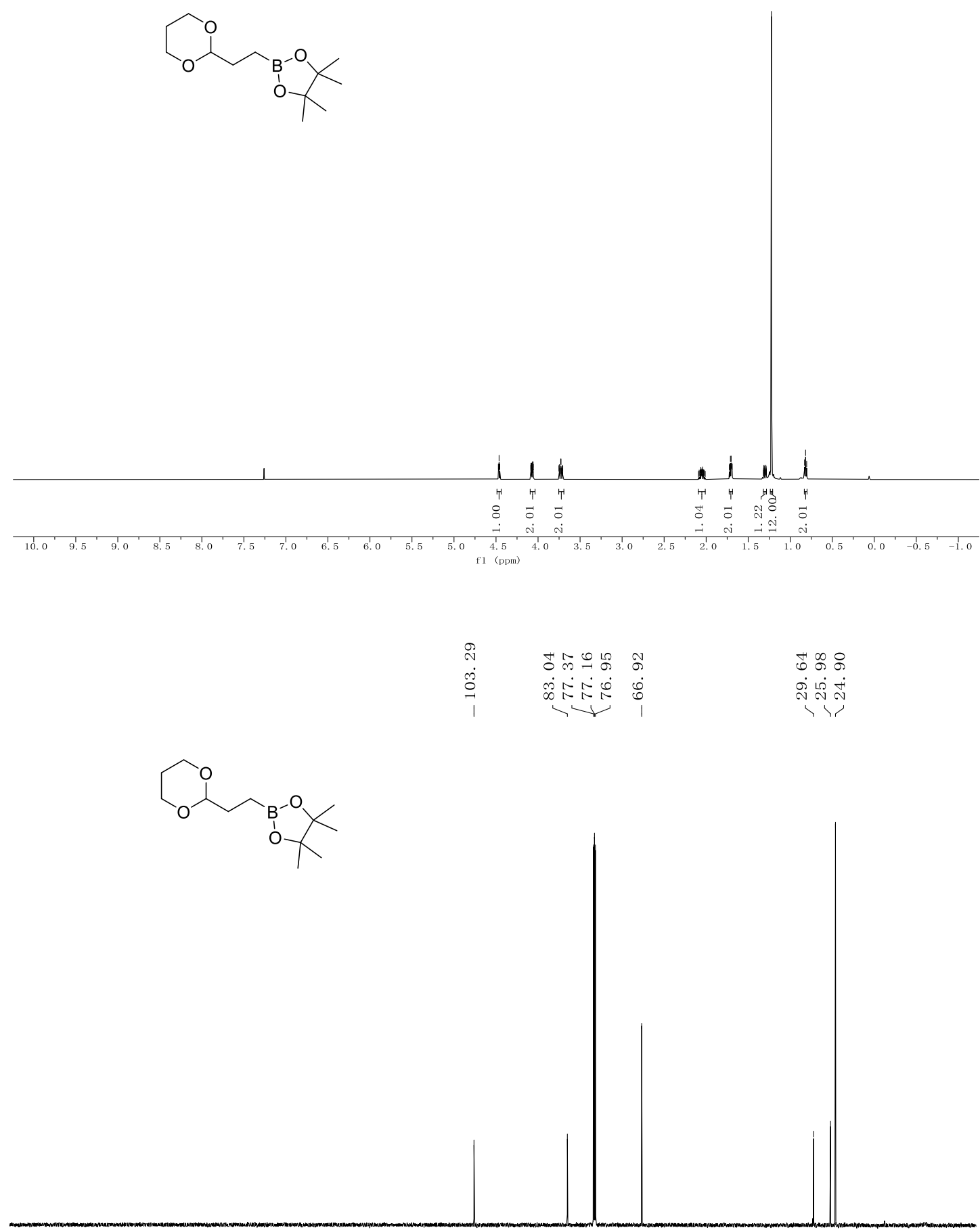

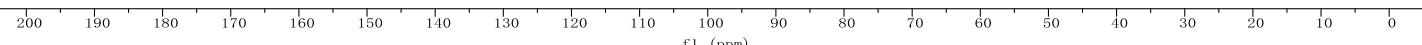



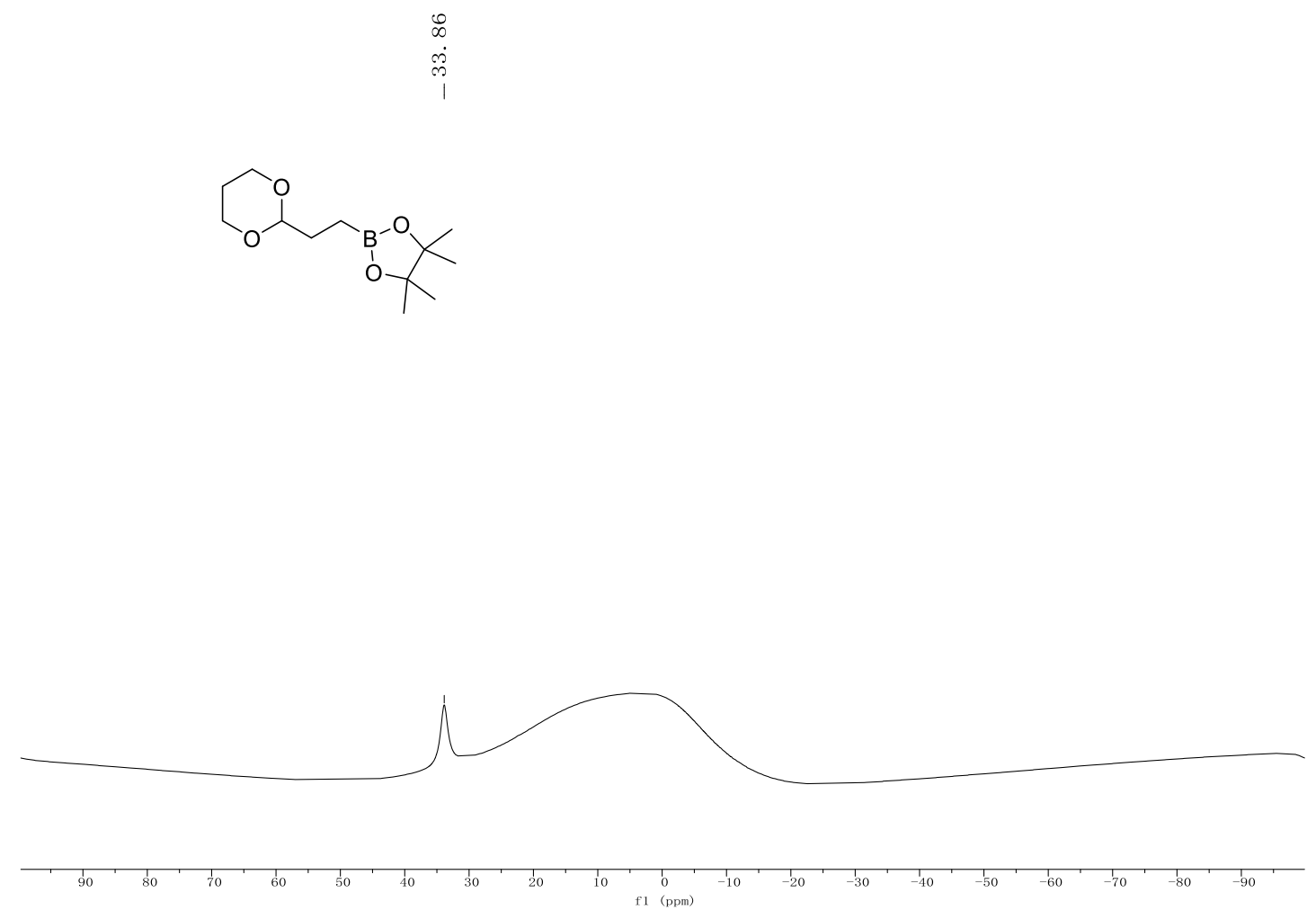

\section{Compound 6}

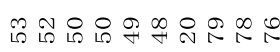

- ت-i

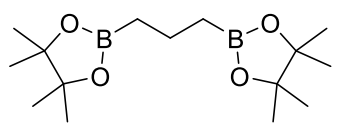


수용요 용

这쇼용

B̊N

要

$\overleftrightarrow{\sim} \stackrel{\infty}{\rightarrow}$

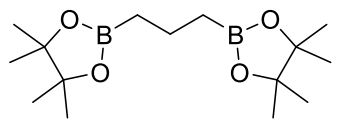

, 1

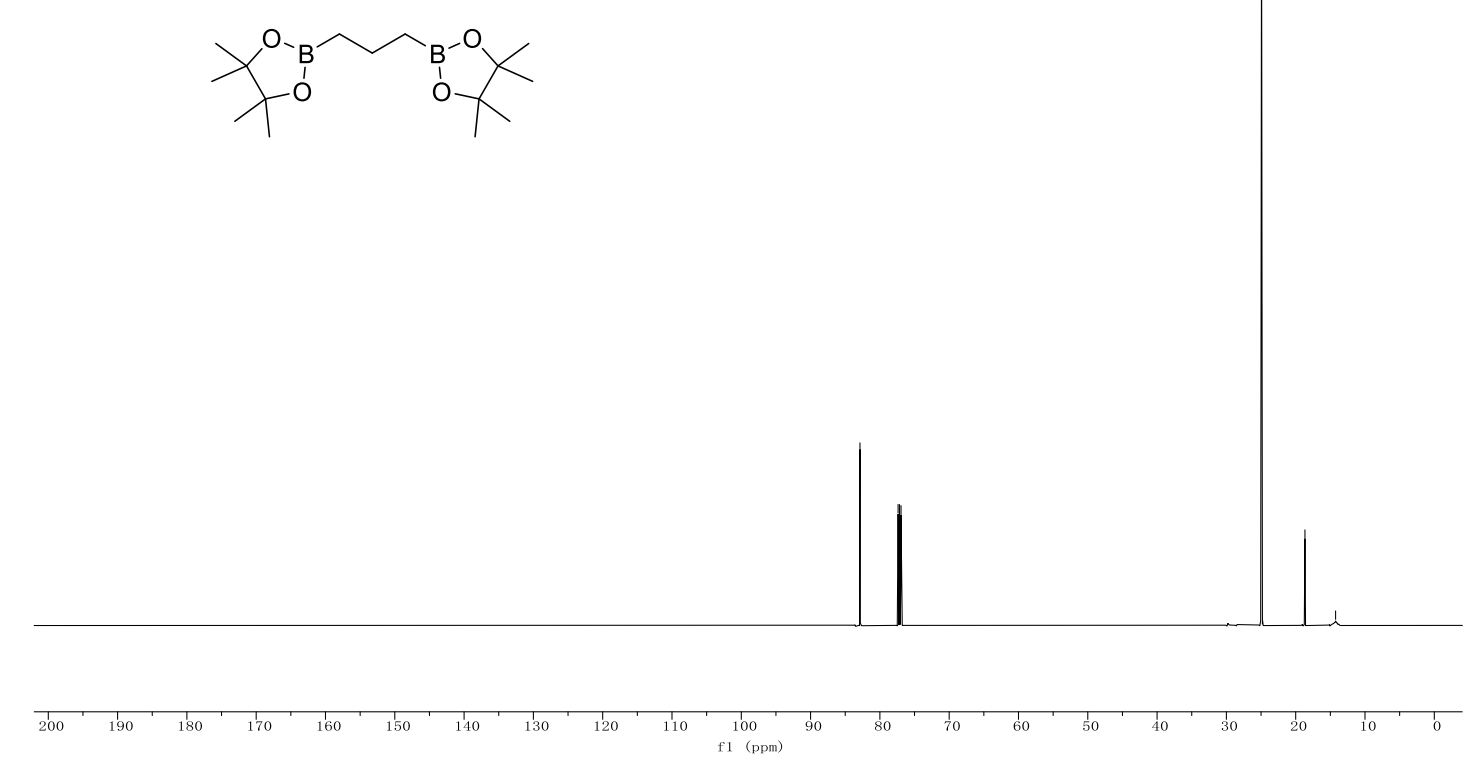

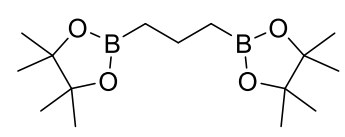

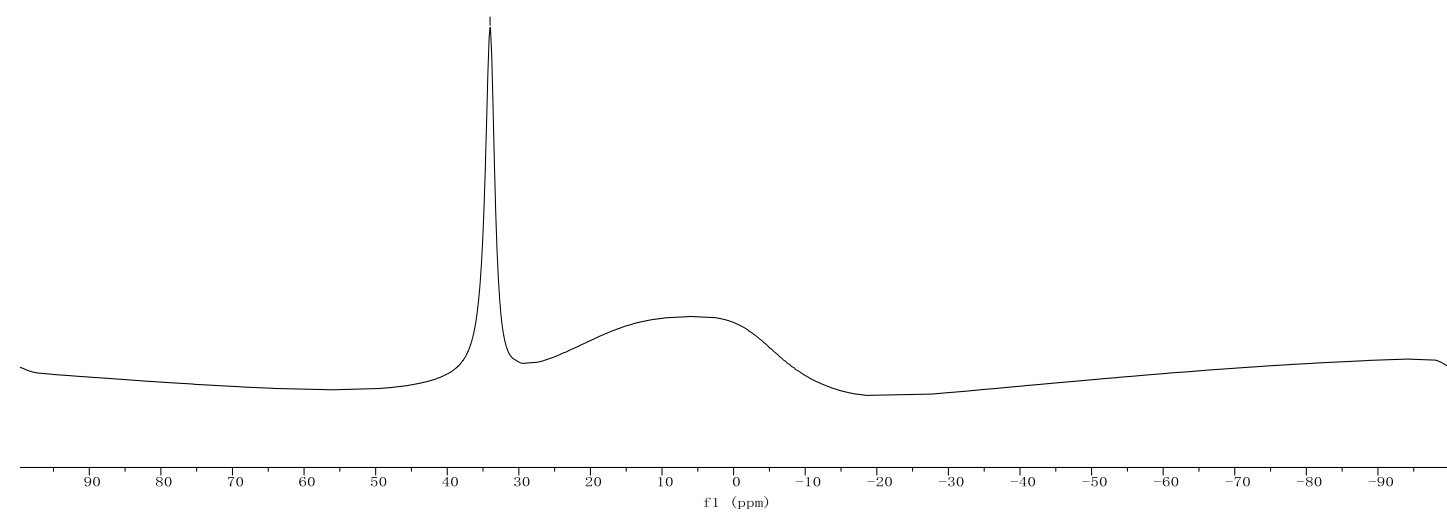




\section{Compound 7}

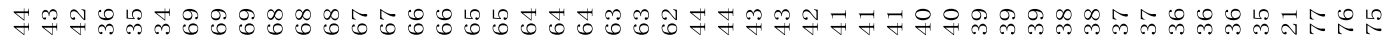

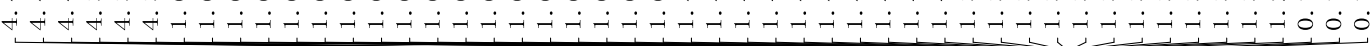

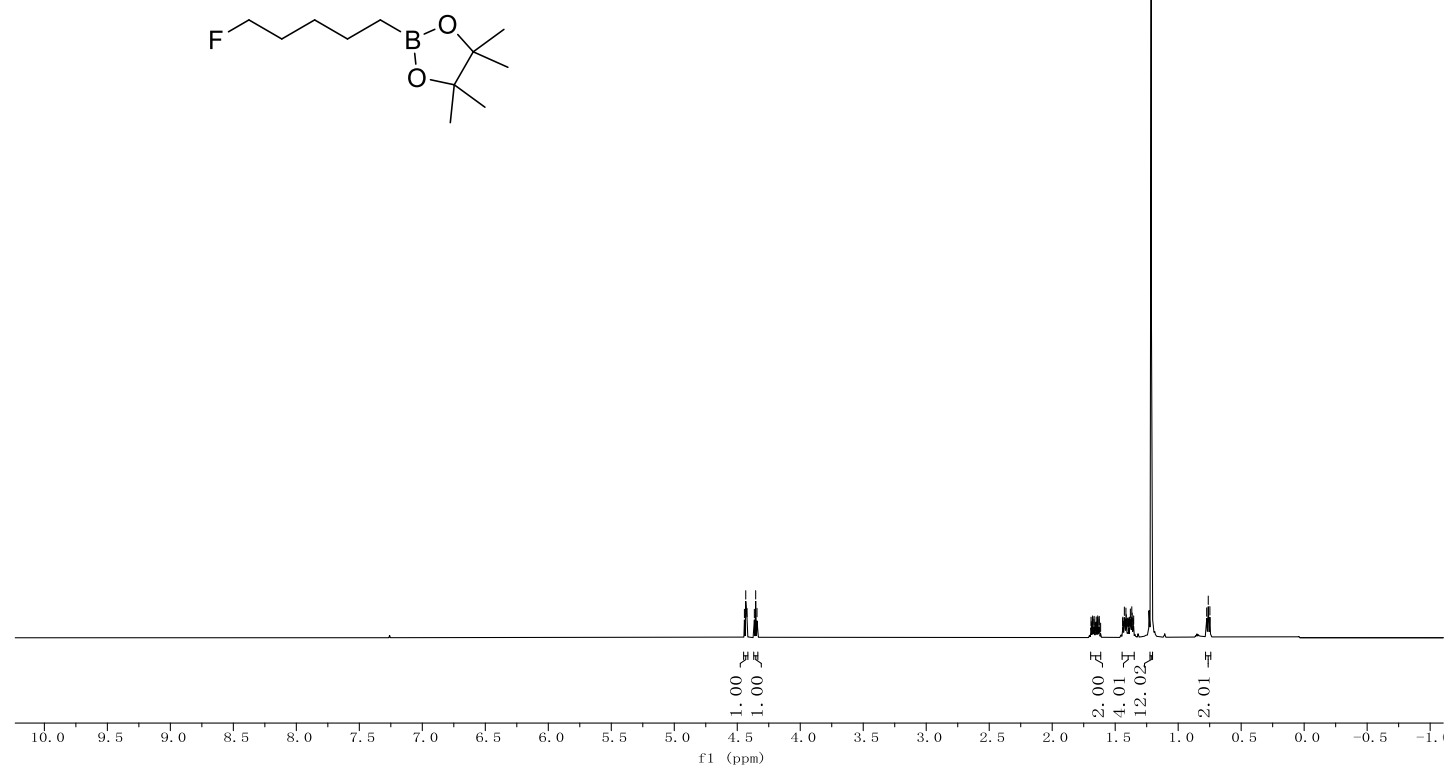

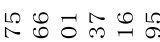

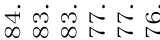

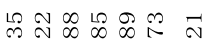

$\rightarrow 1$

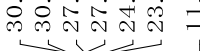
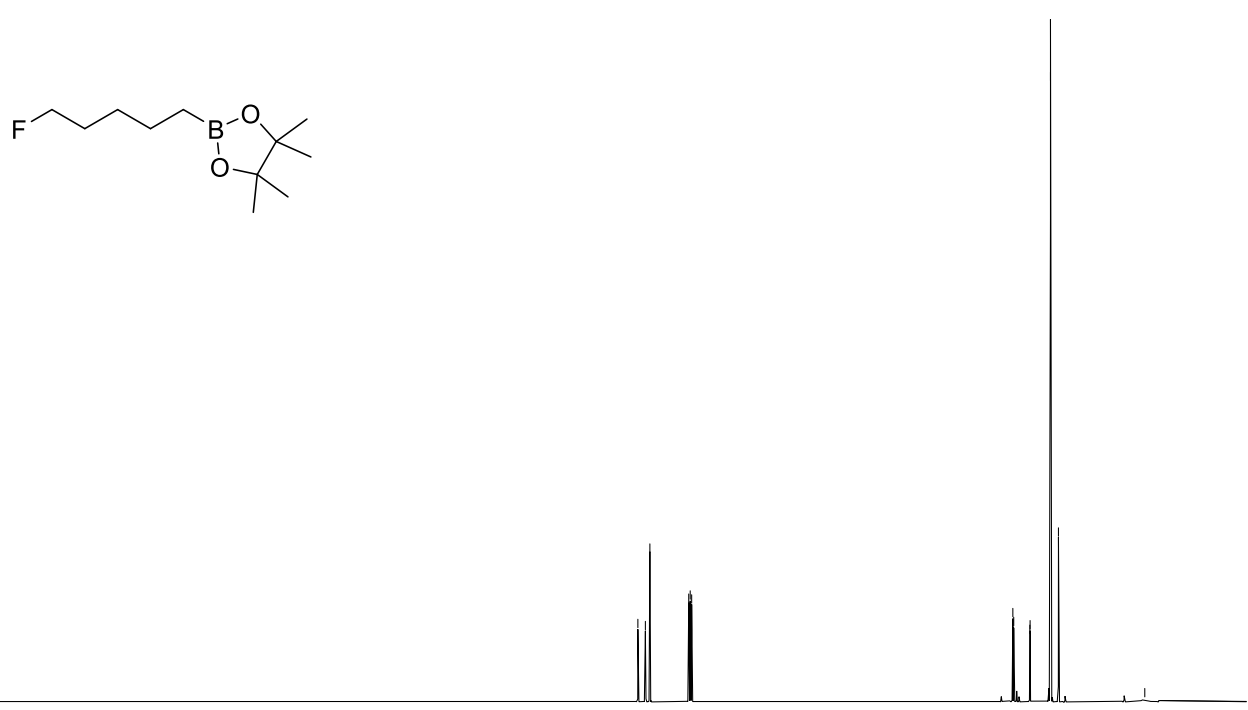

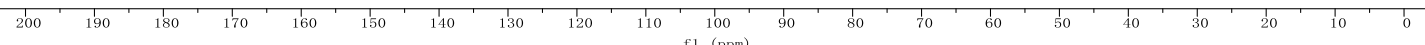


œ

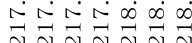

NN N N N
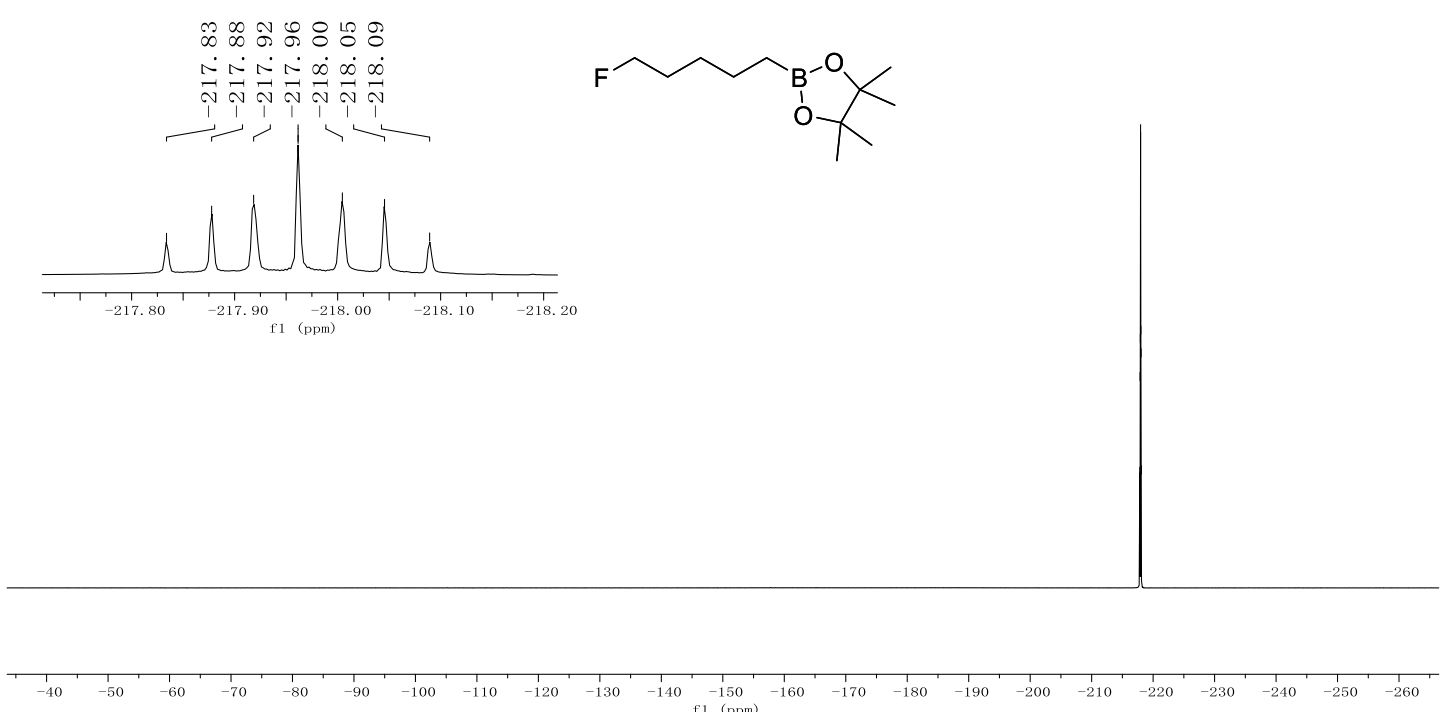

\section{웅}

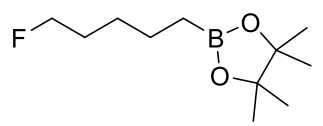
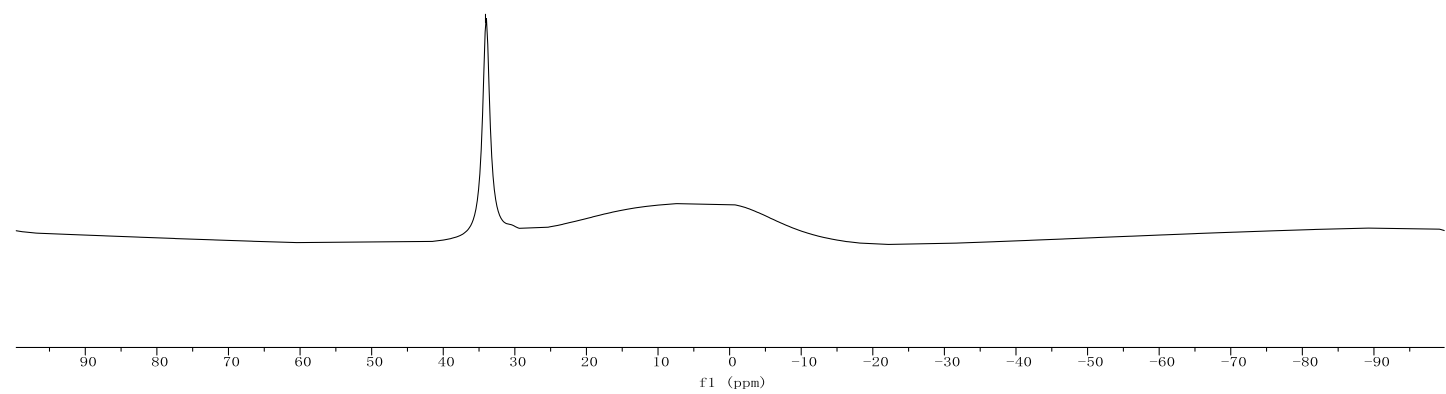


\section{Compound 8}

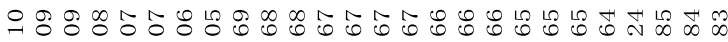
ن

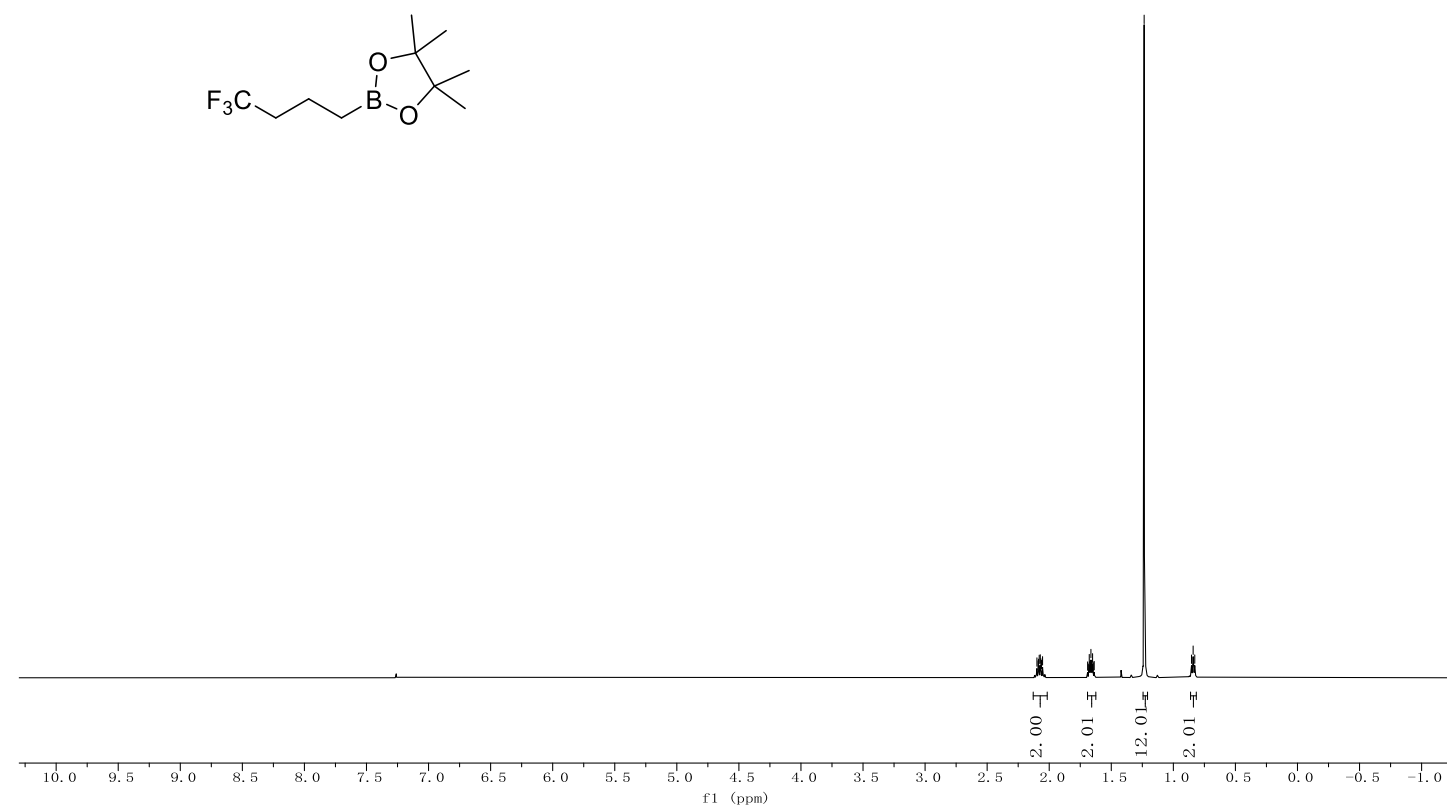

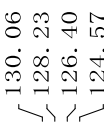

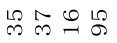

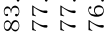

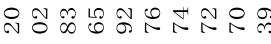

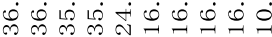

r

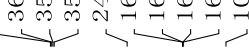

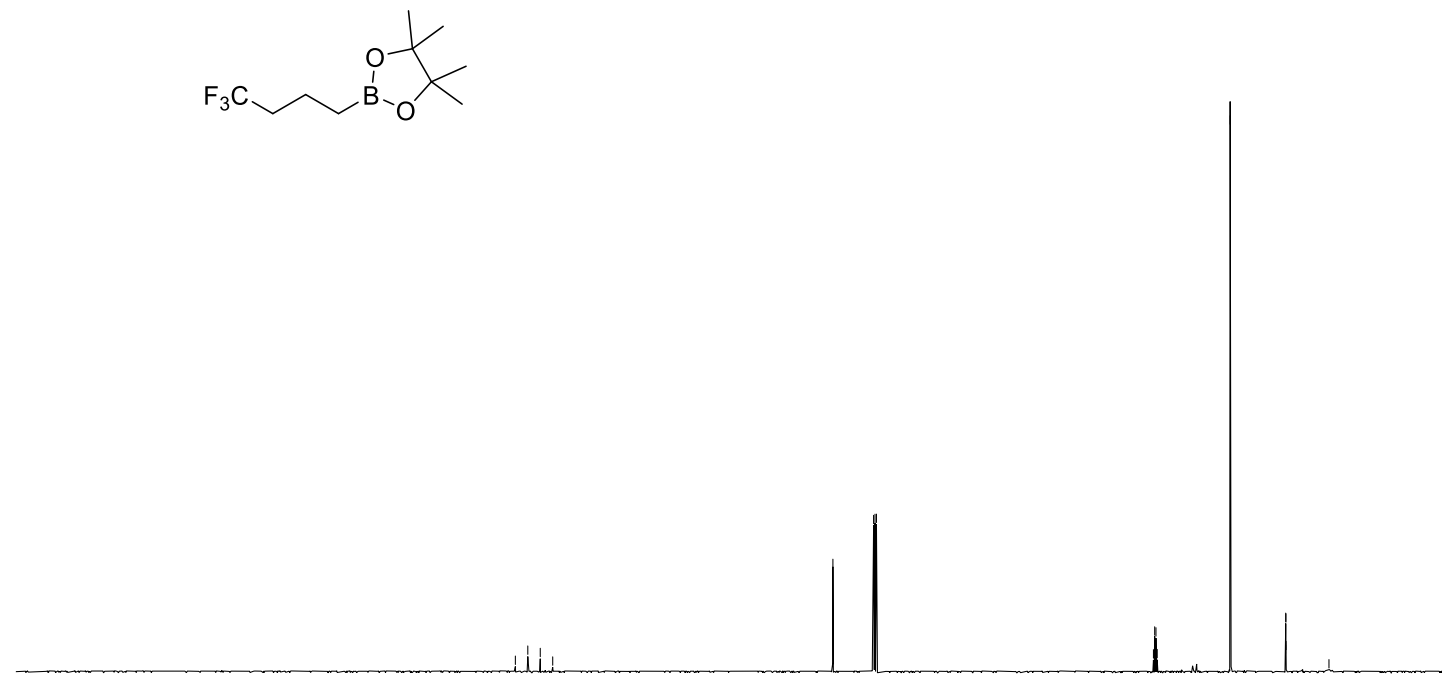

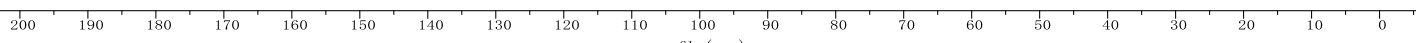


잉요아

:

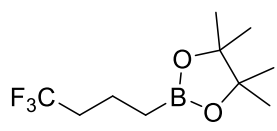
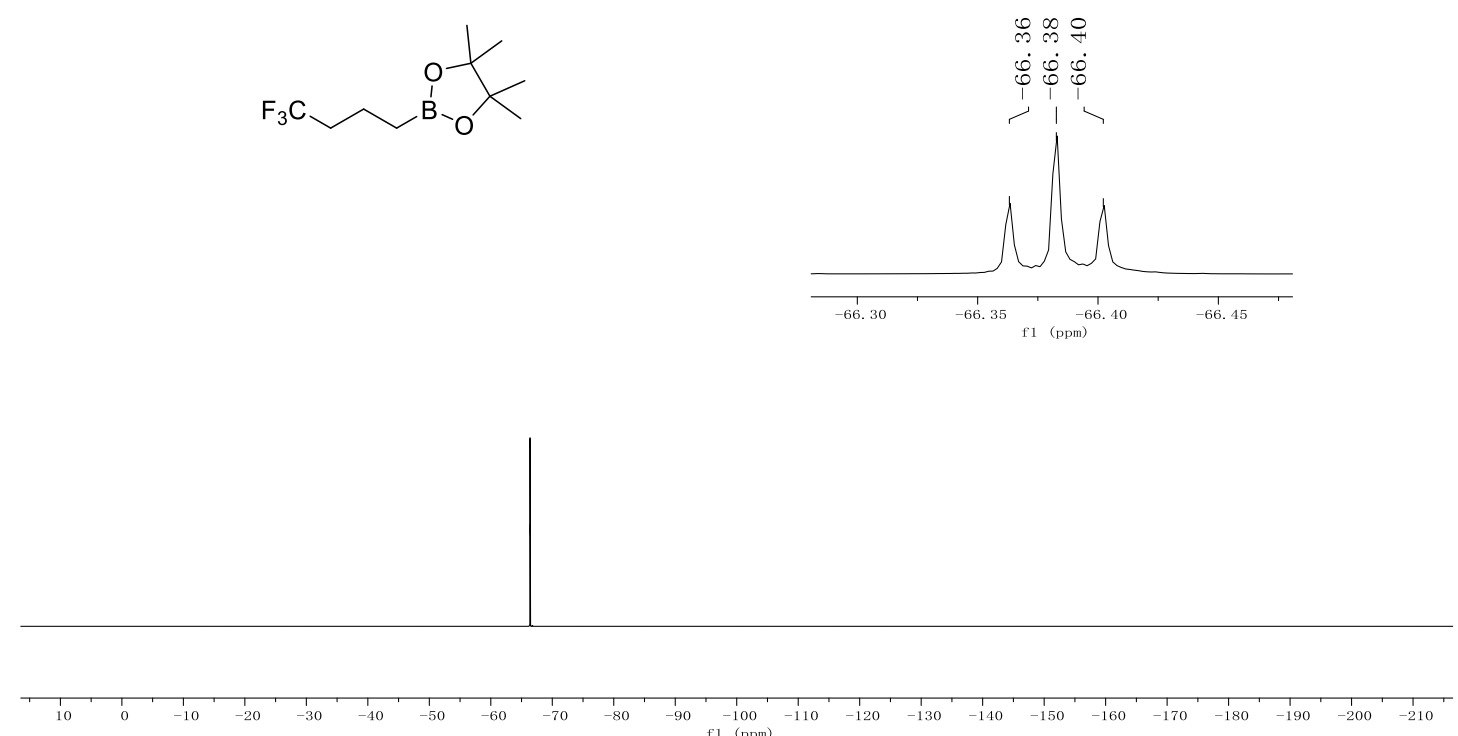

$\widehat{\circ}$

$\stackrel{\infty}{\infty}$

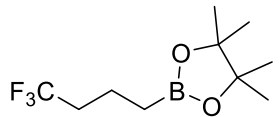

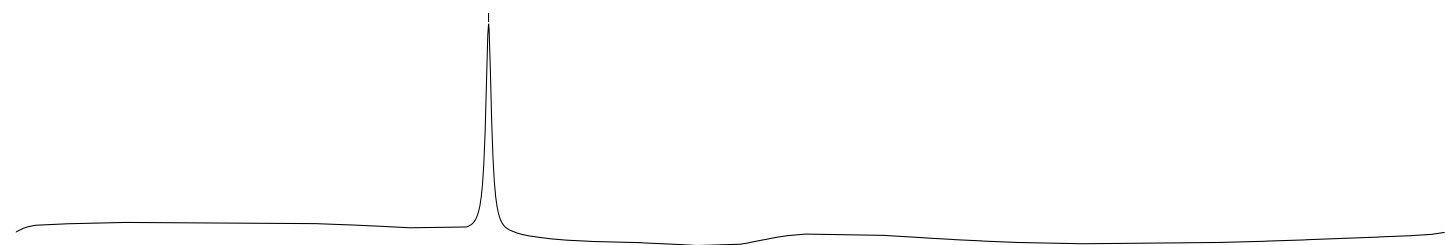




\section{Compound 9}

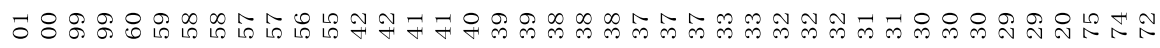

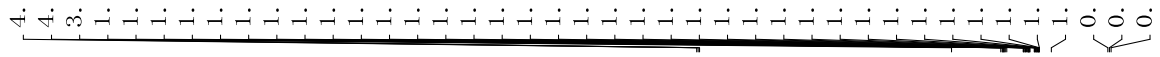

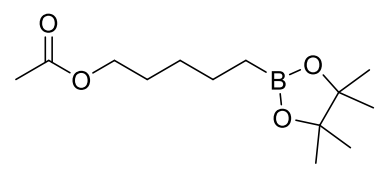

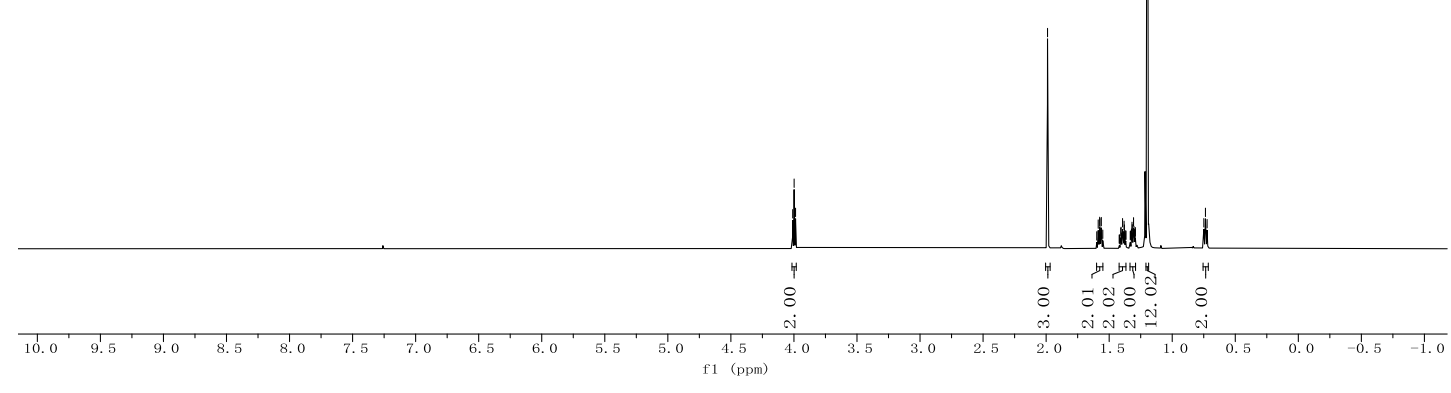

$\stackrel{\stackrel{\llcorner}{N}}{\stackrel{2}{ \pm}}$

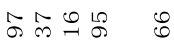

ชै

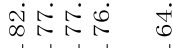

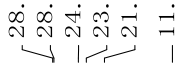

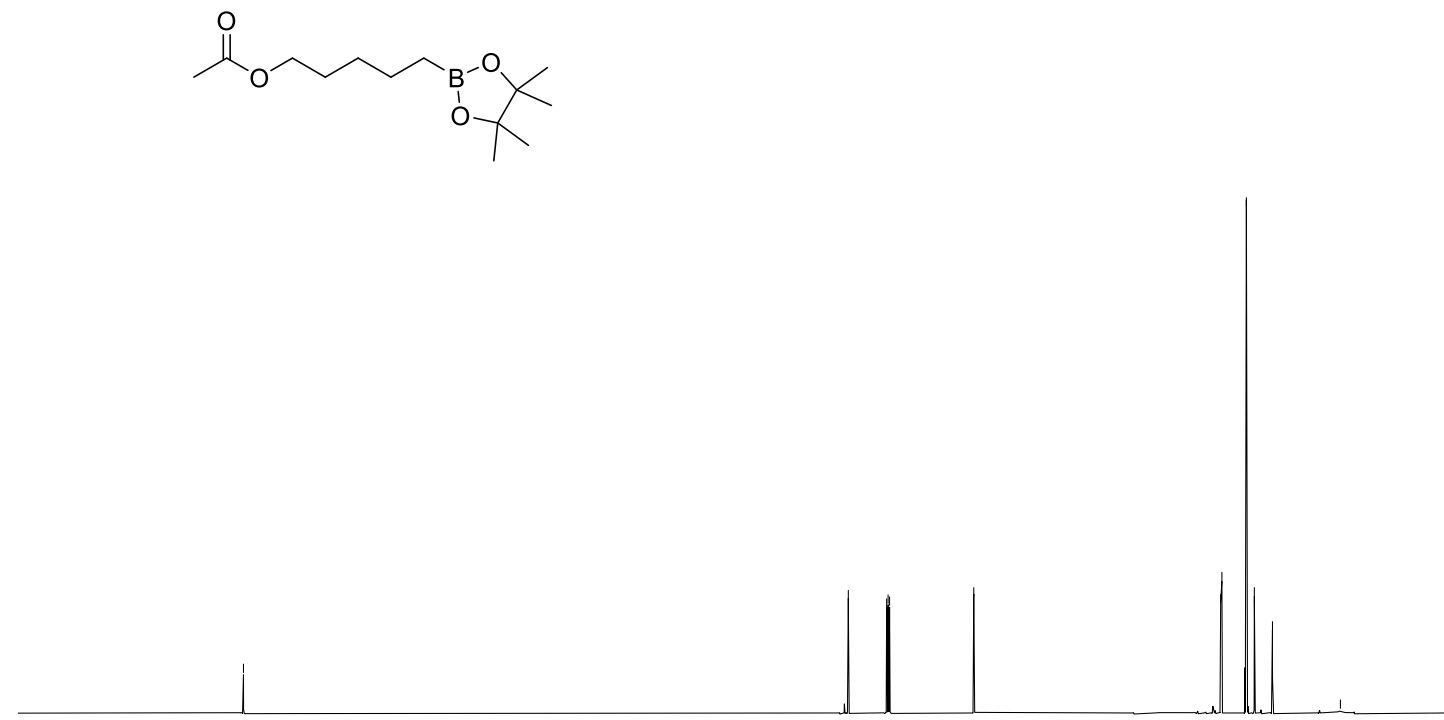

证

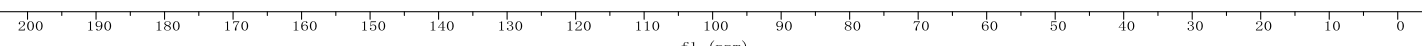



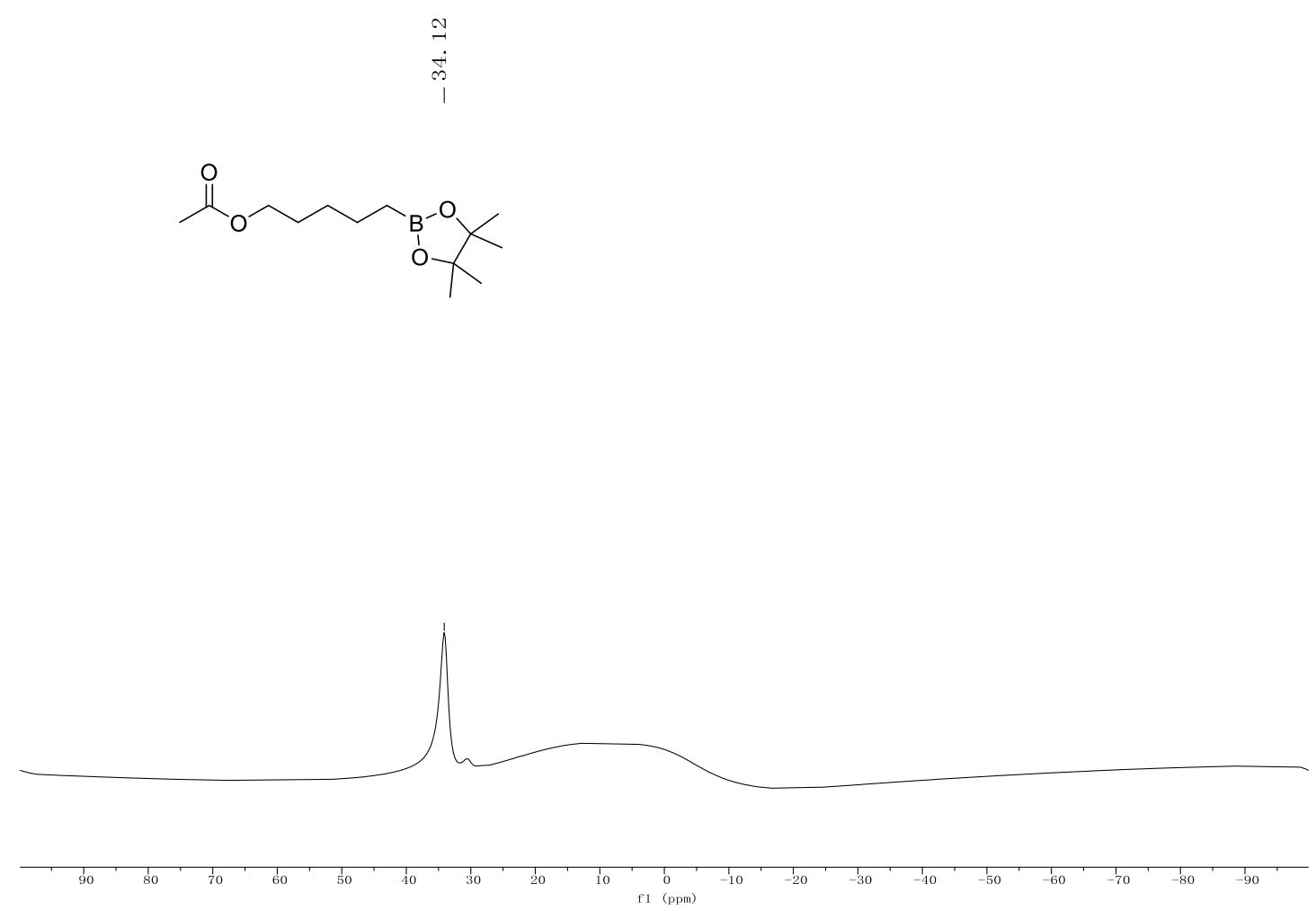

\section{Compound 10}

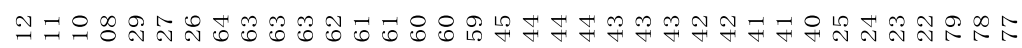

मં

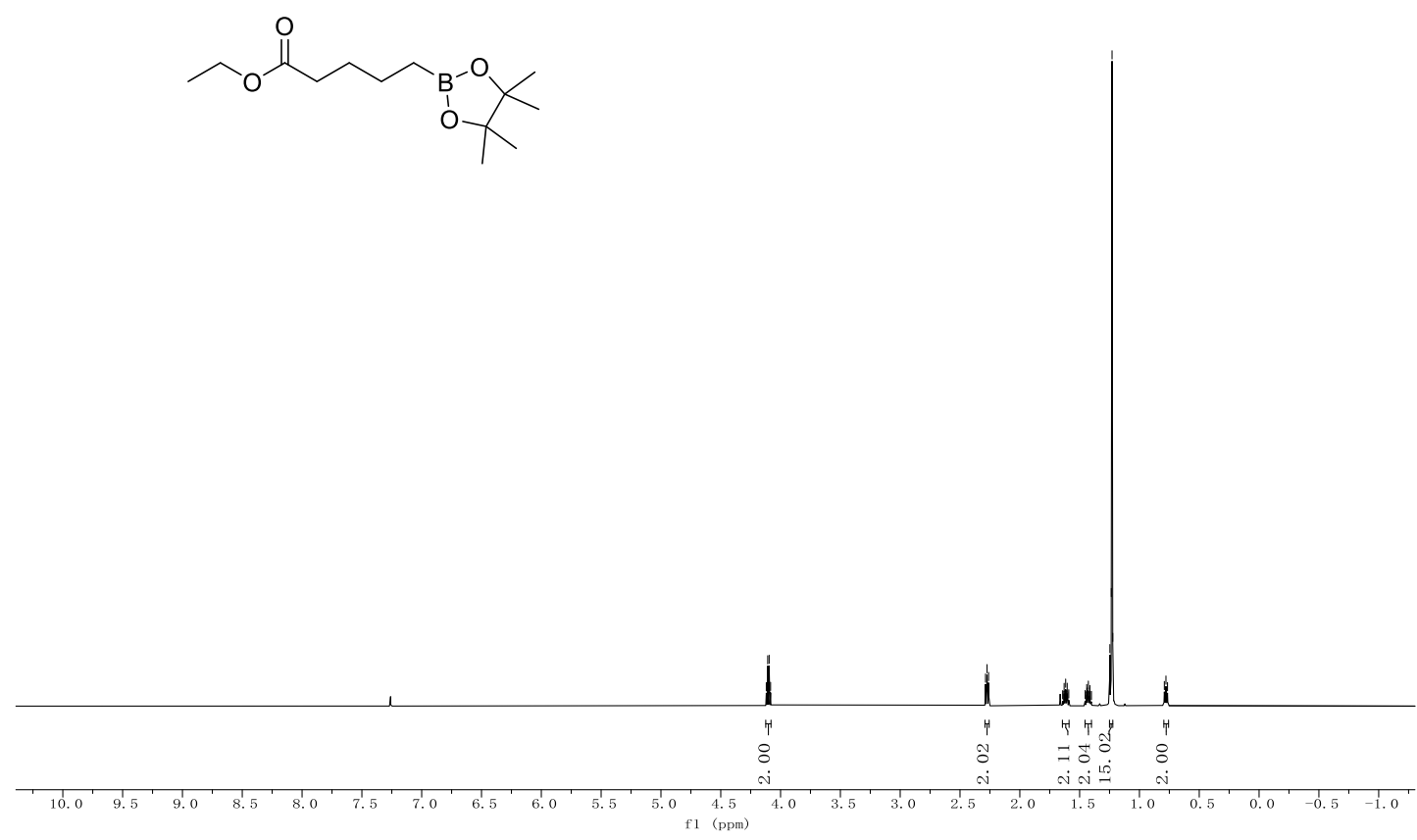



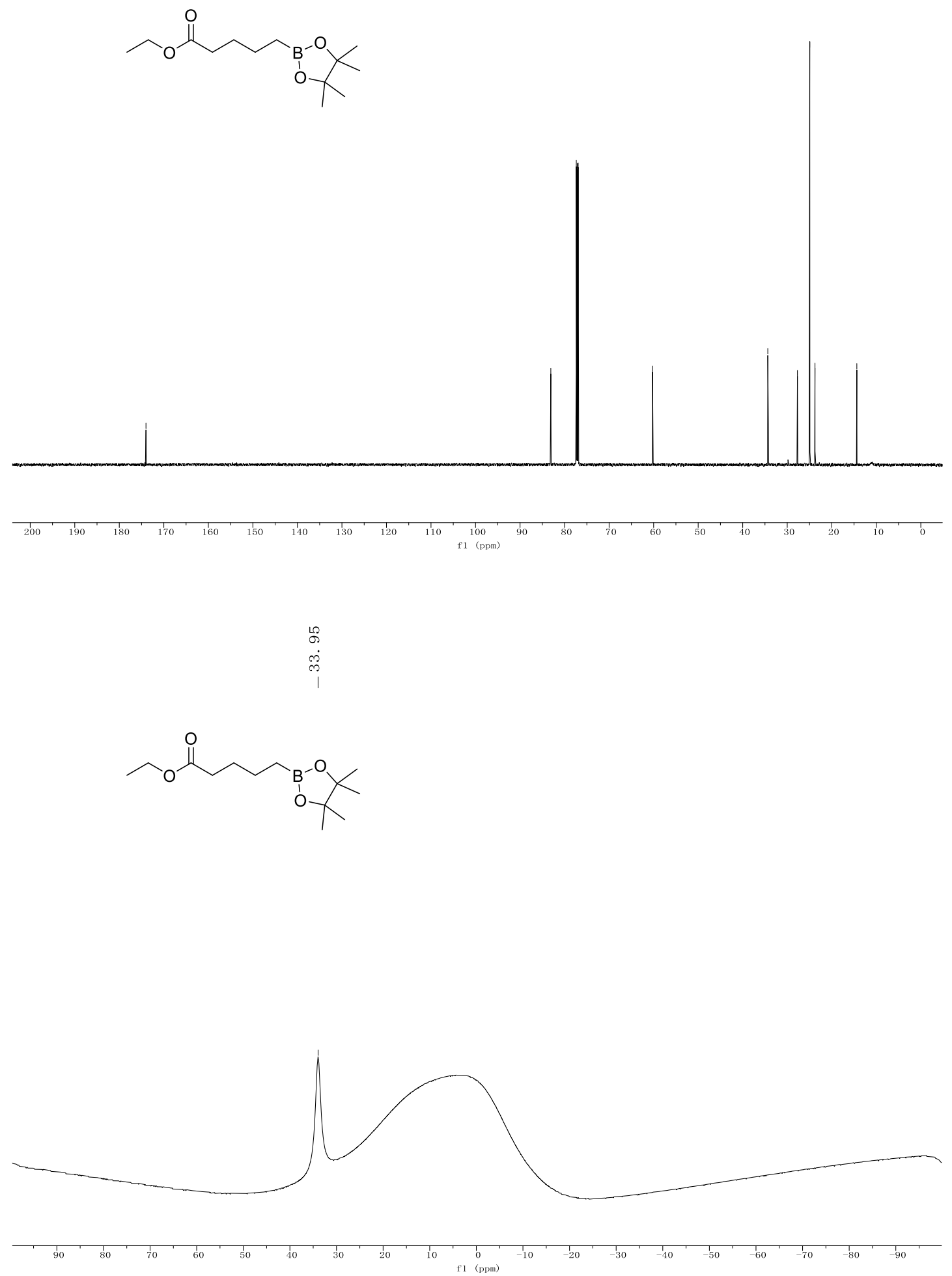


\section{Compound 11}

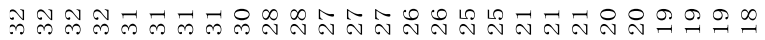

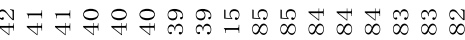

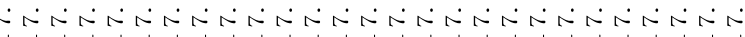

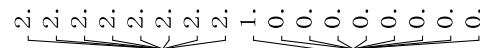

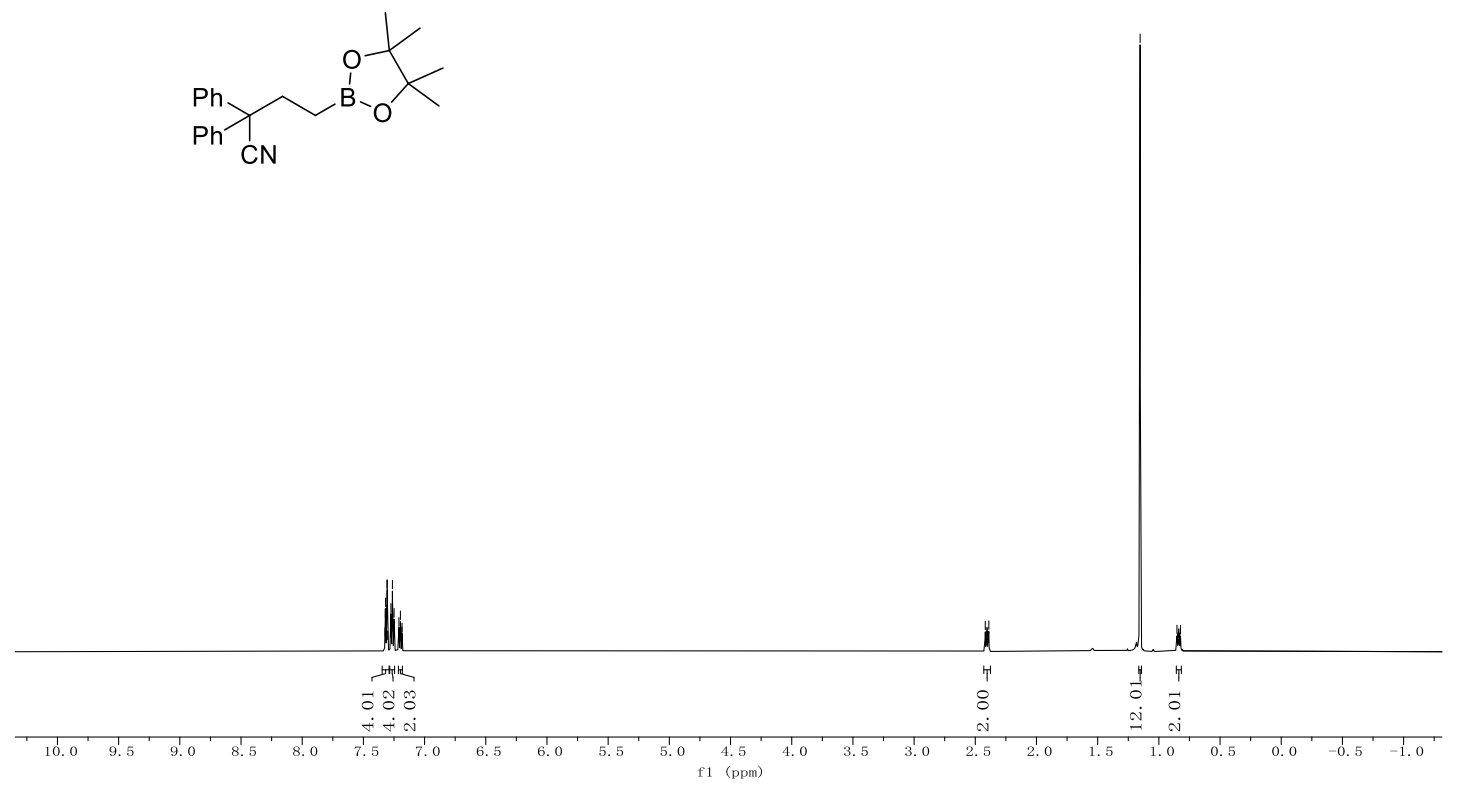

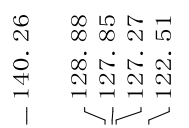

유으요요용

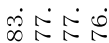

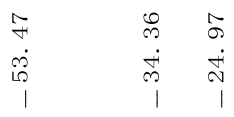
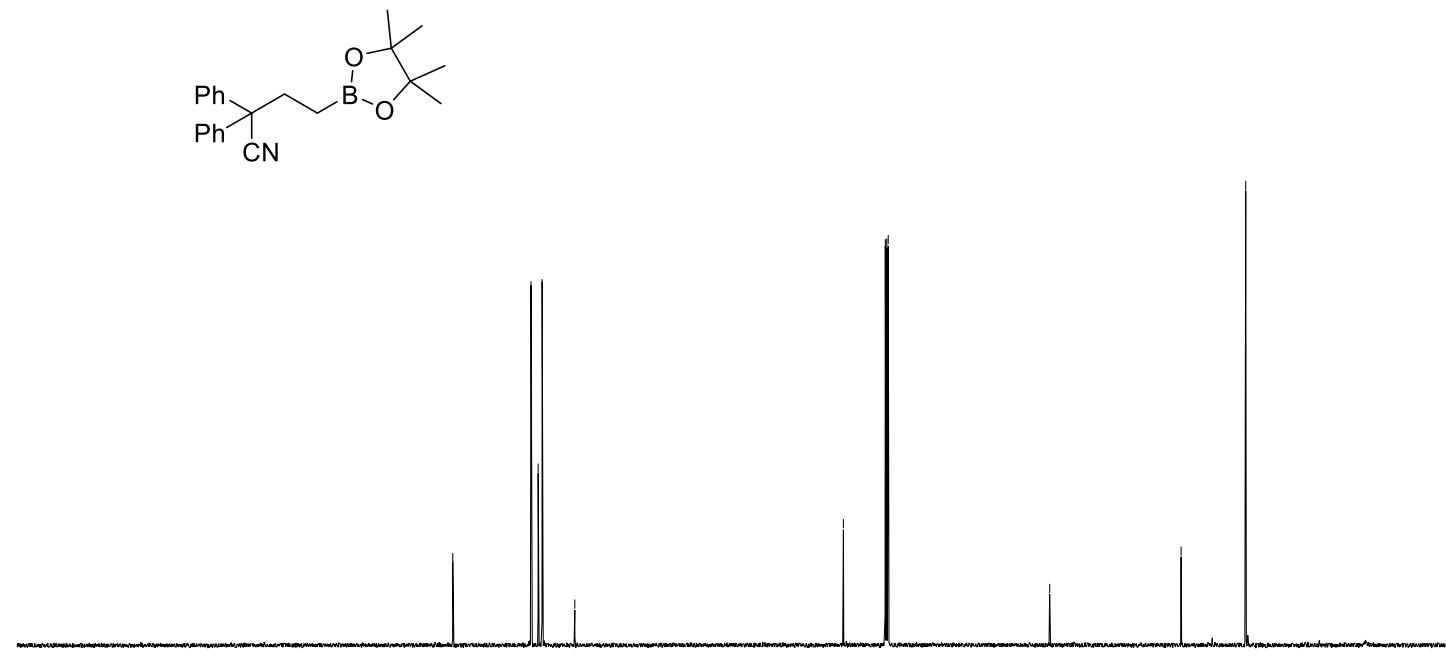

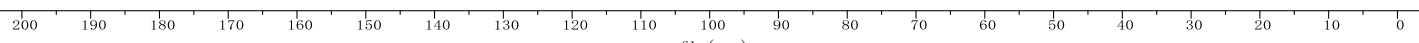



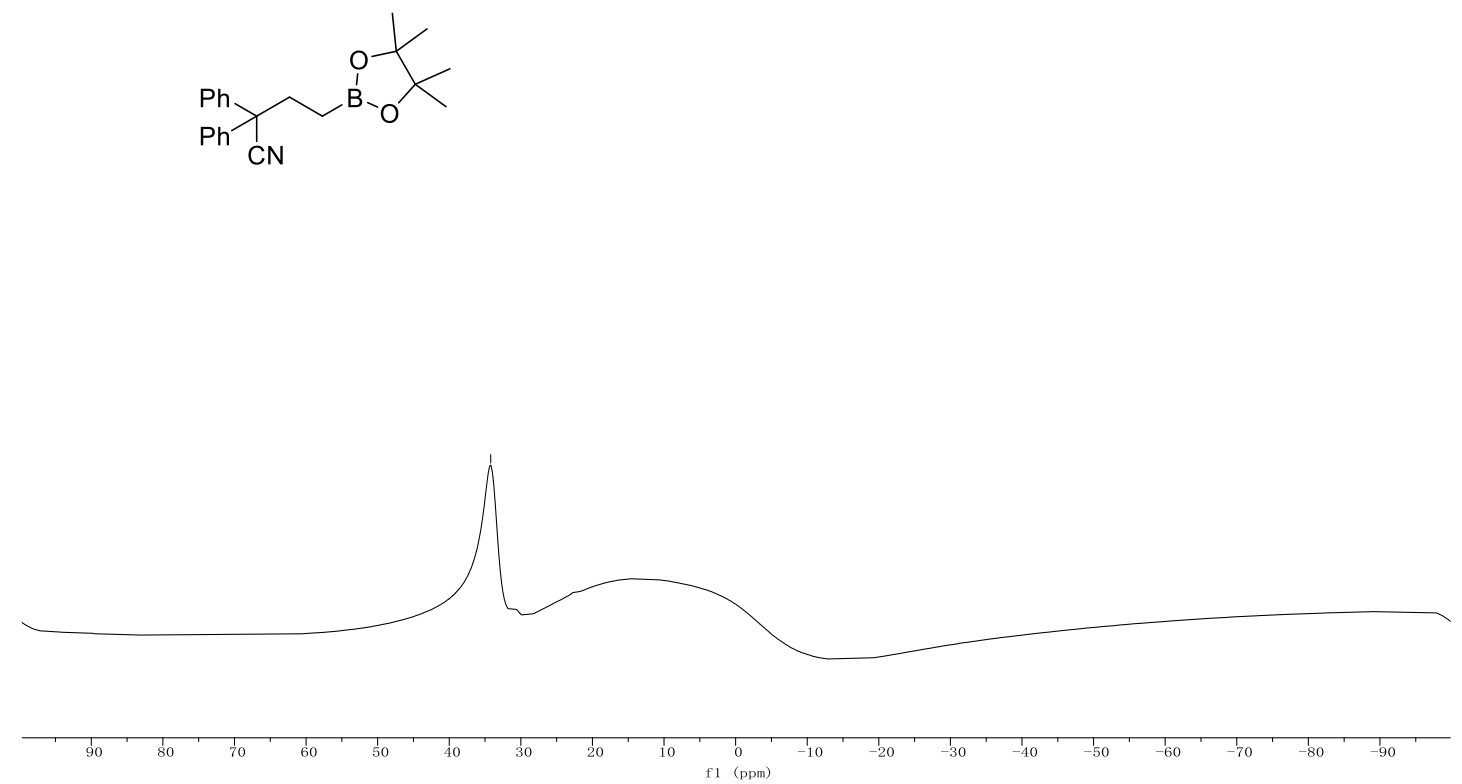

\section{Compound 12}

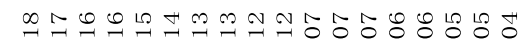

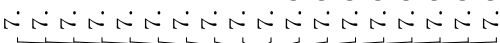

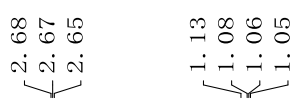<smiles>CC1(C)OB(CCc2ccccc2)OC1(C)C</smiles>

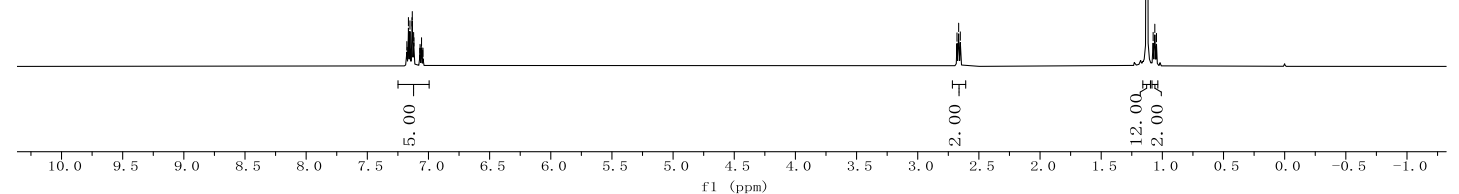




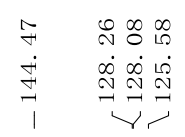

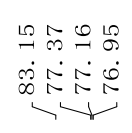

$\begin{array}{lll}+1 & 0 & 5 \\ 0 & 0 \\ \dot{0} & \dot{0} & \dot{0} \\ 0 & 1 & 1\end{array}$
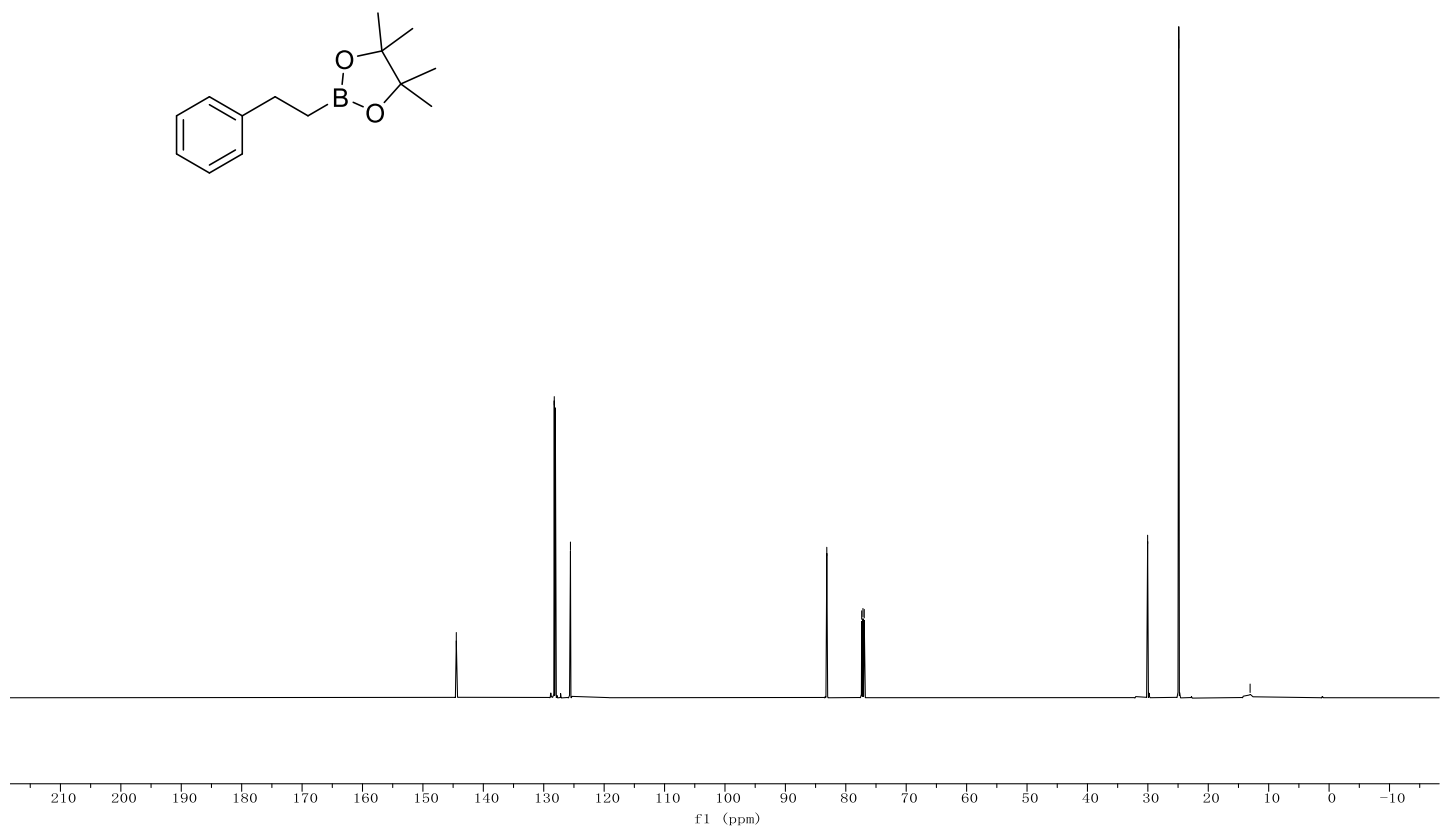

$\ddot{\sigma}$
$\dot{m}$
$\dot{1}$
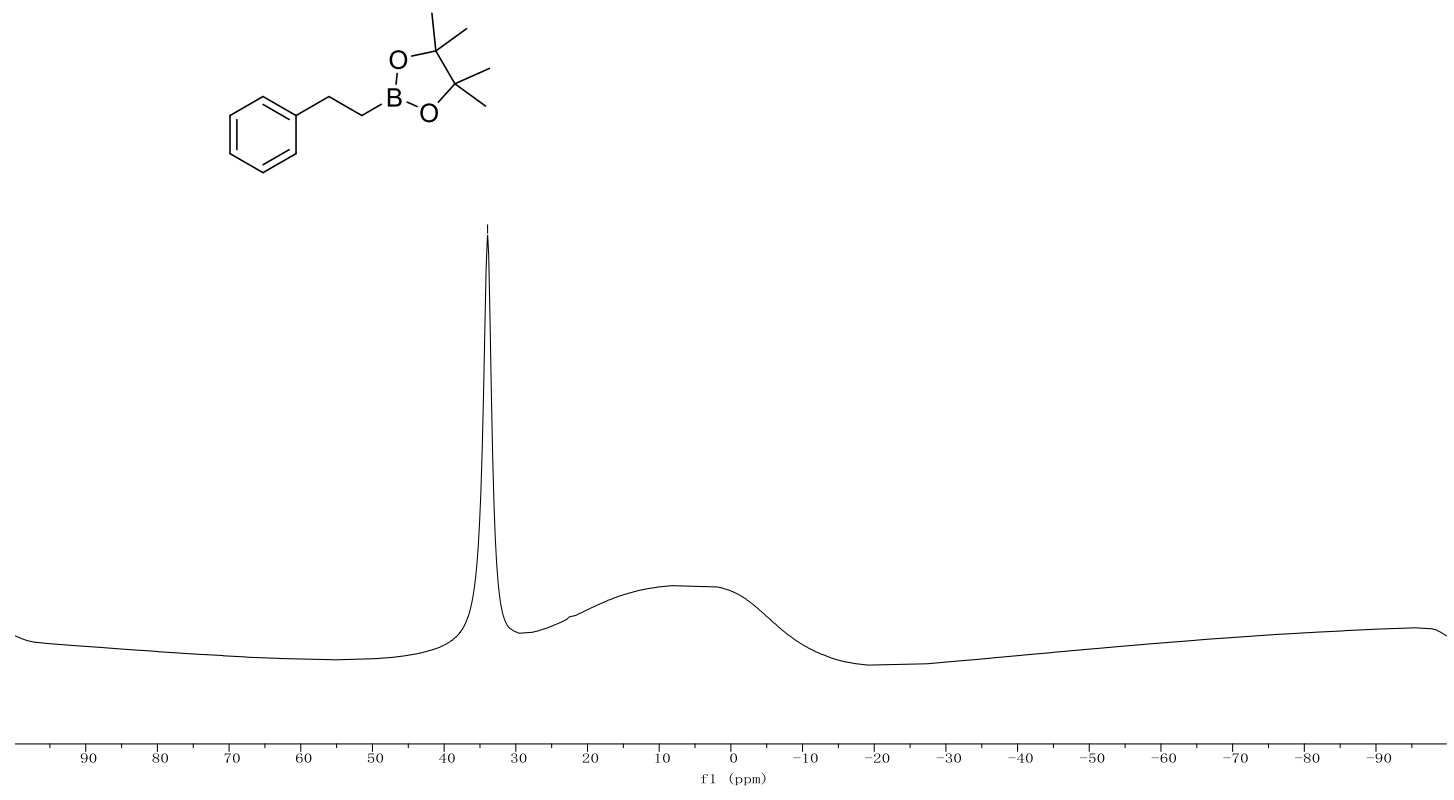

S103 


\section{Compound 13}

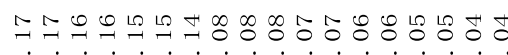

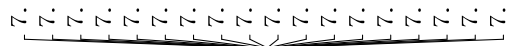

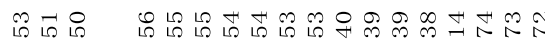

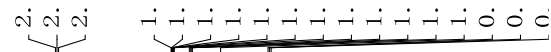<smiles>CC1(C)OB(CCCCc2ccccc2)OC1(C)C</smiles>

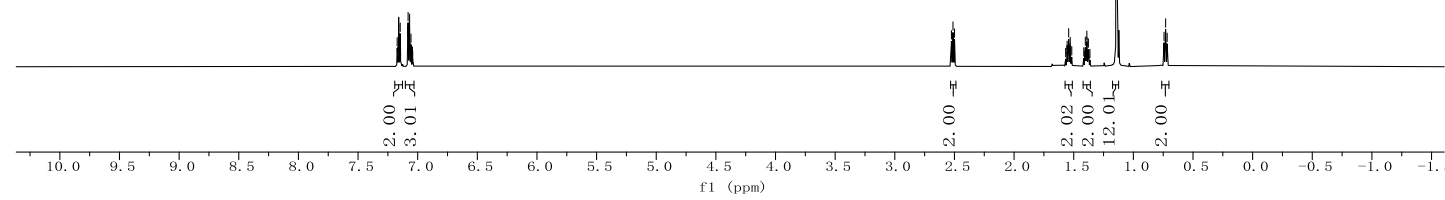<smiles>CC1(C)OB(CCCCc2ccccc2)OC1(C)C</smiles>

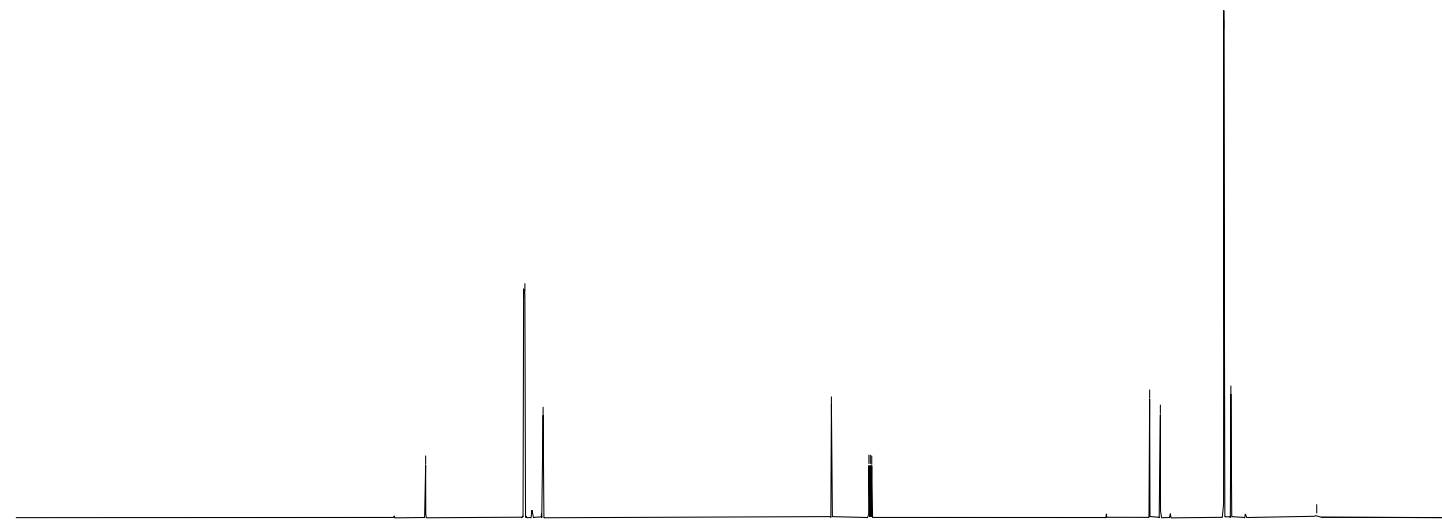

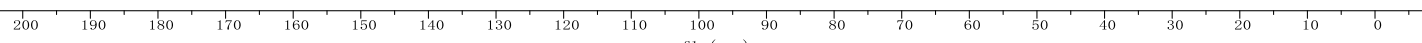




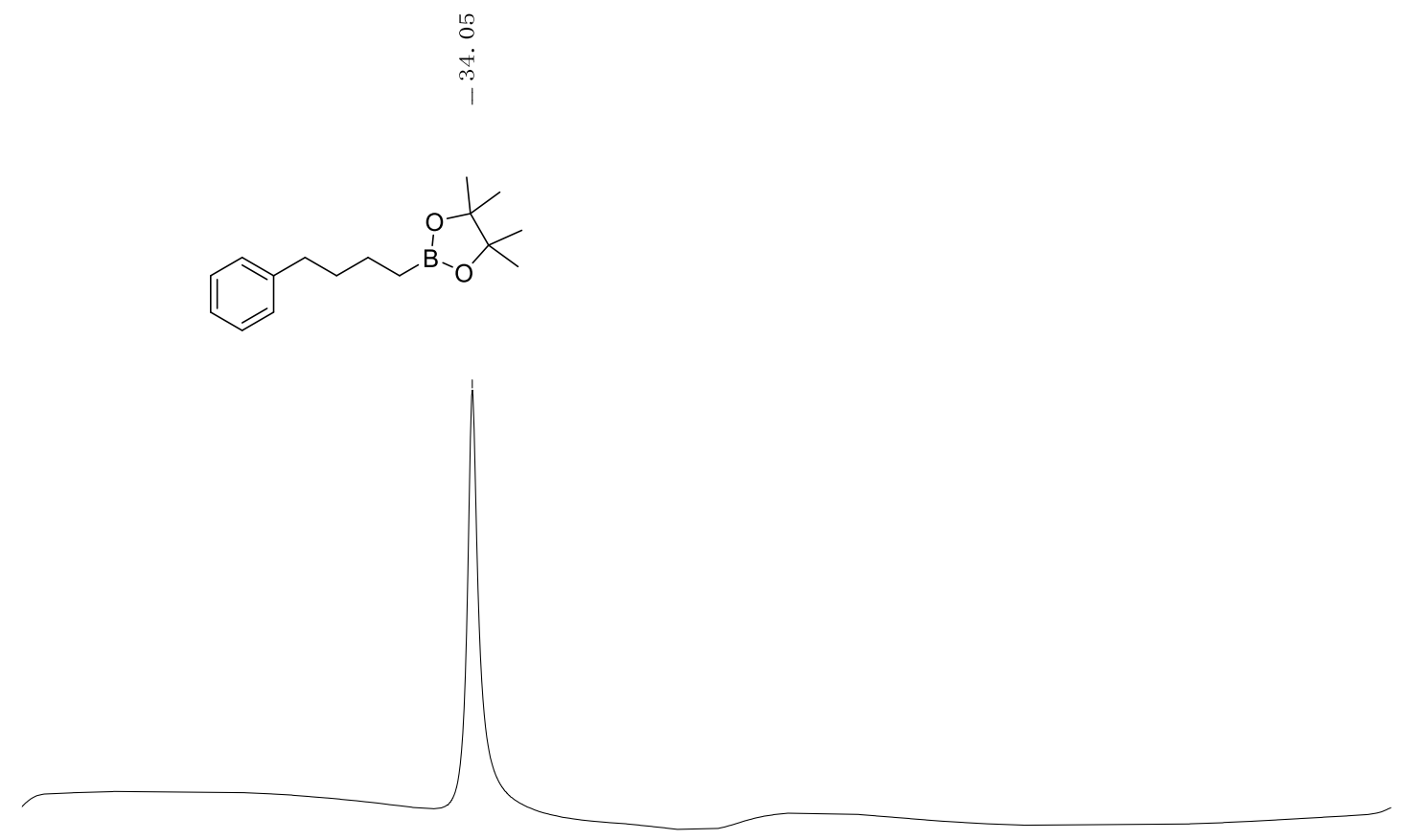

\section{Compound 14}

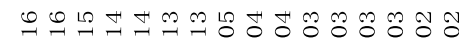

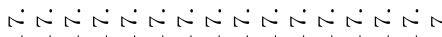

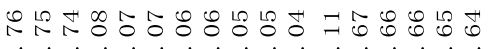

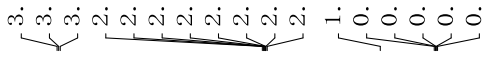

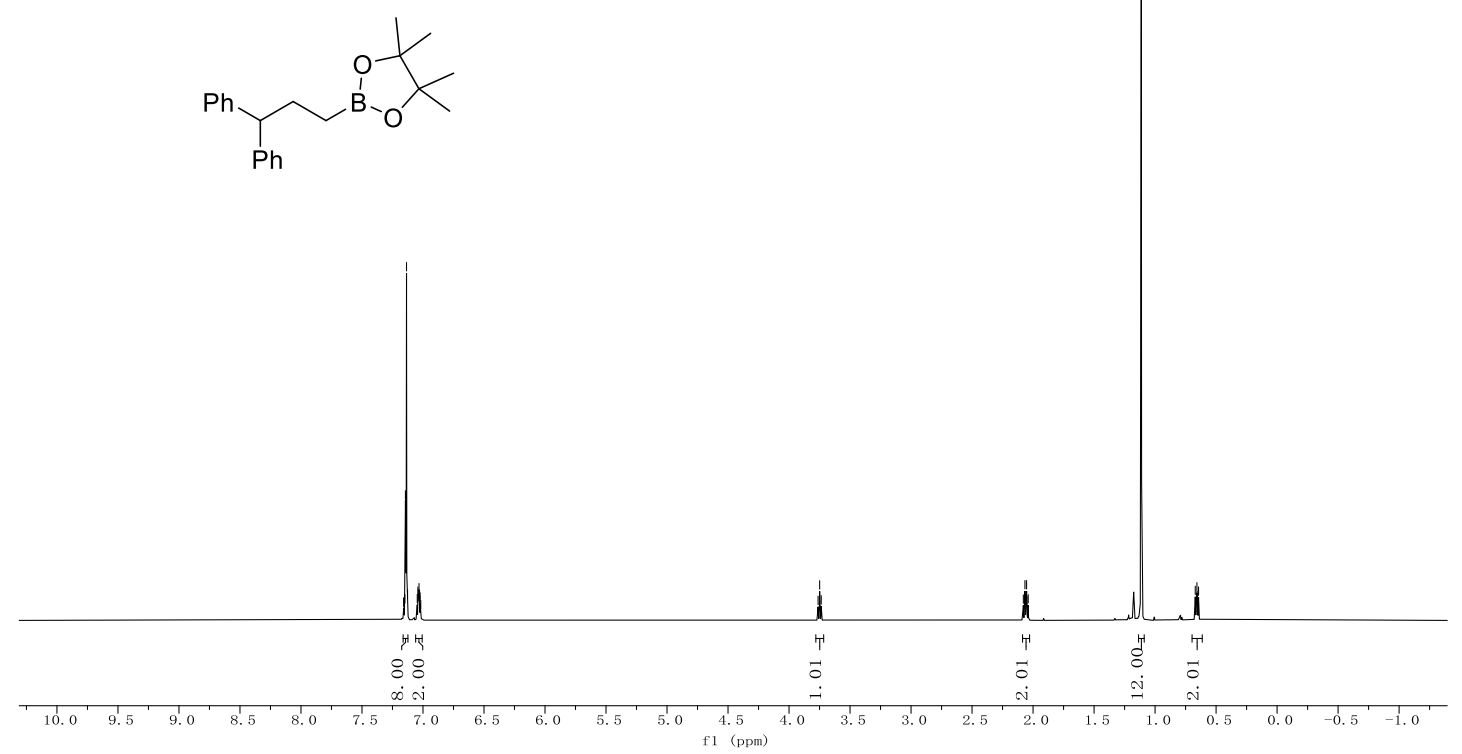




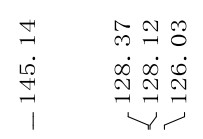

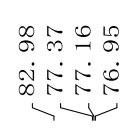

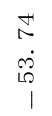

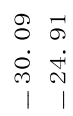

8
0
0
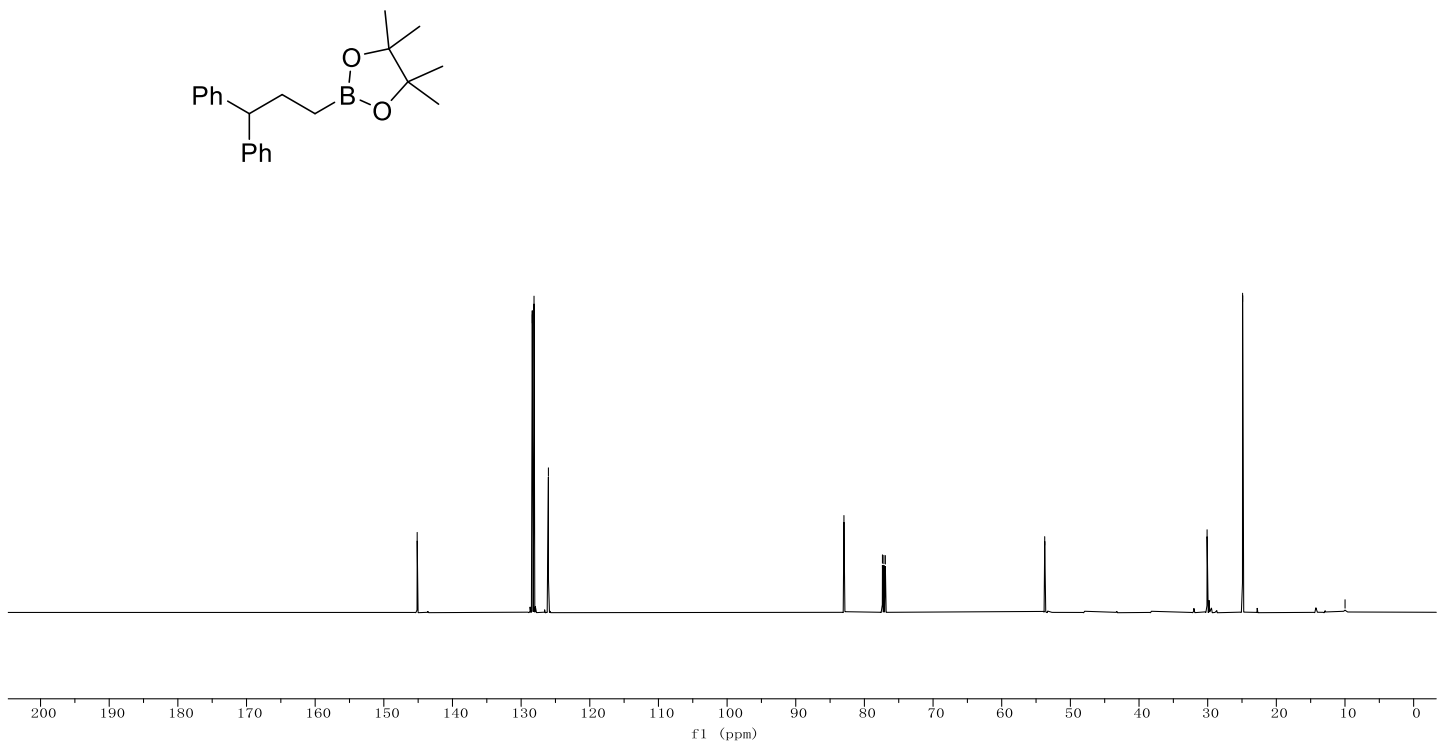

$\stackrel{\infty}{N}$
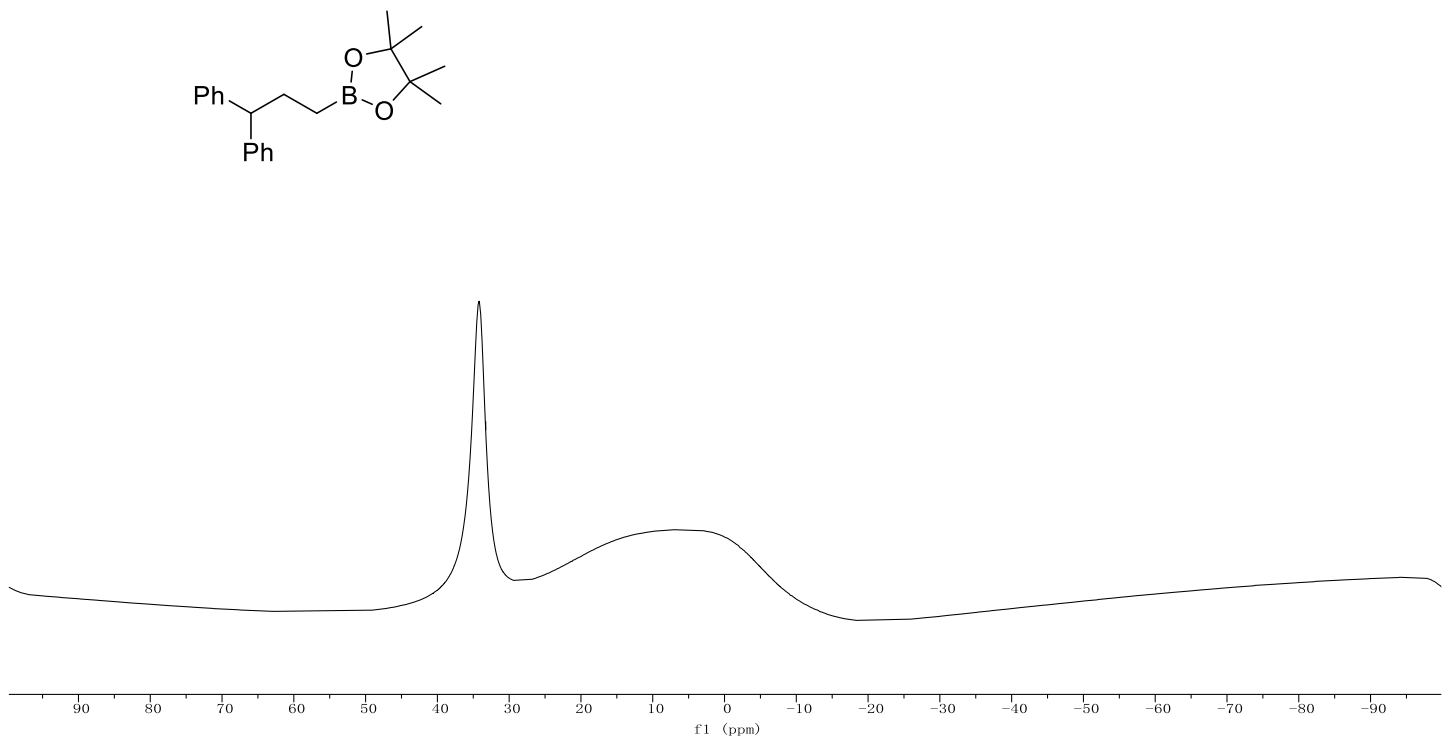

S106 


\section{Compound 15}

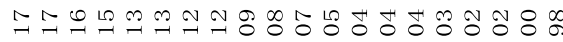

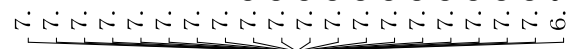

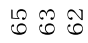

$\underbrace{i n j}$<smiles>CC1(C)OB(CCc2cccc(Cl)c2)OC1(C)C</smiles>

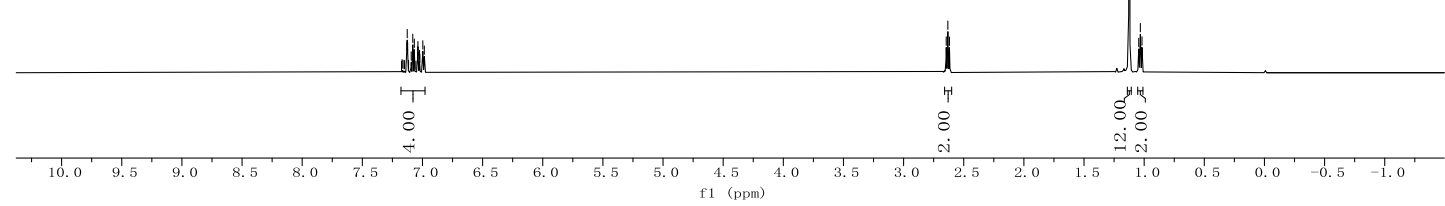

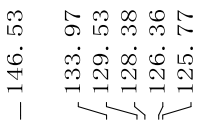

공ำ용

我穴远定

요

$\stackrel{i}{i} \stackrel{i}{\stackrel{1}{N}} \stackrel{i}{i}$<smiles>CC1(C)OB(CCc2cccc(Cl)c2)OC1(C)C</smiles>

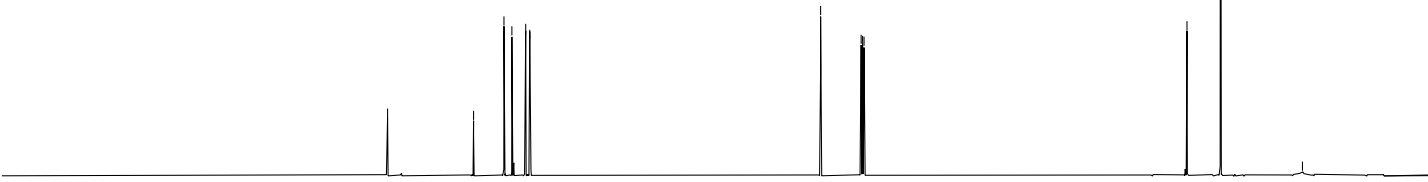

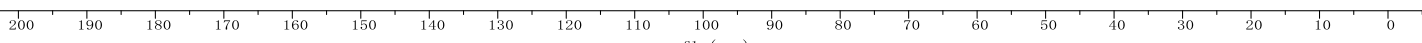



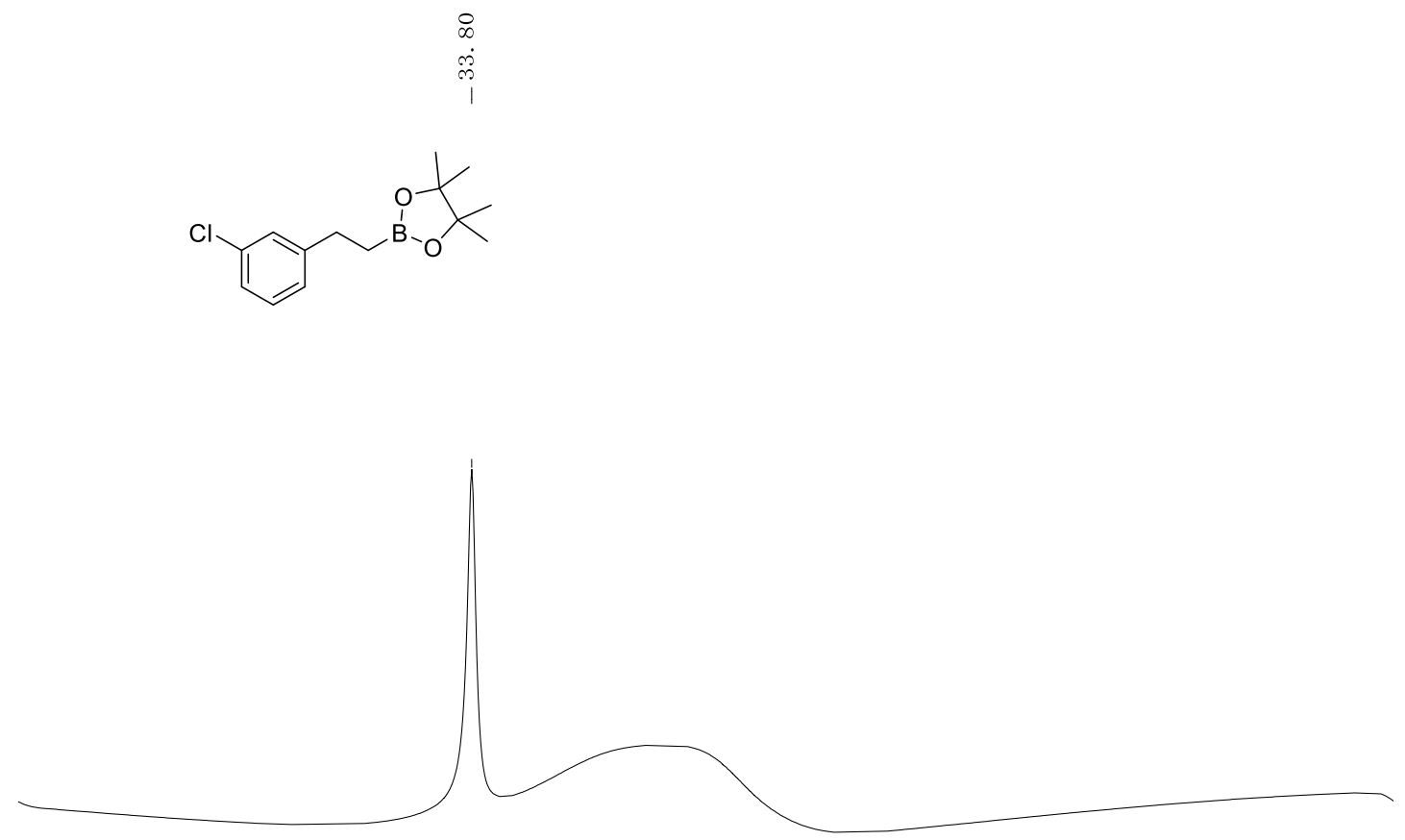

\section{Compound 16}

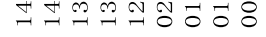

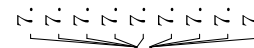

>

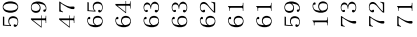

نูง ง - -

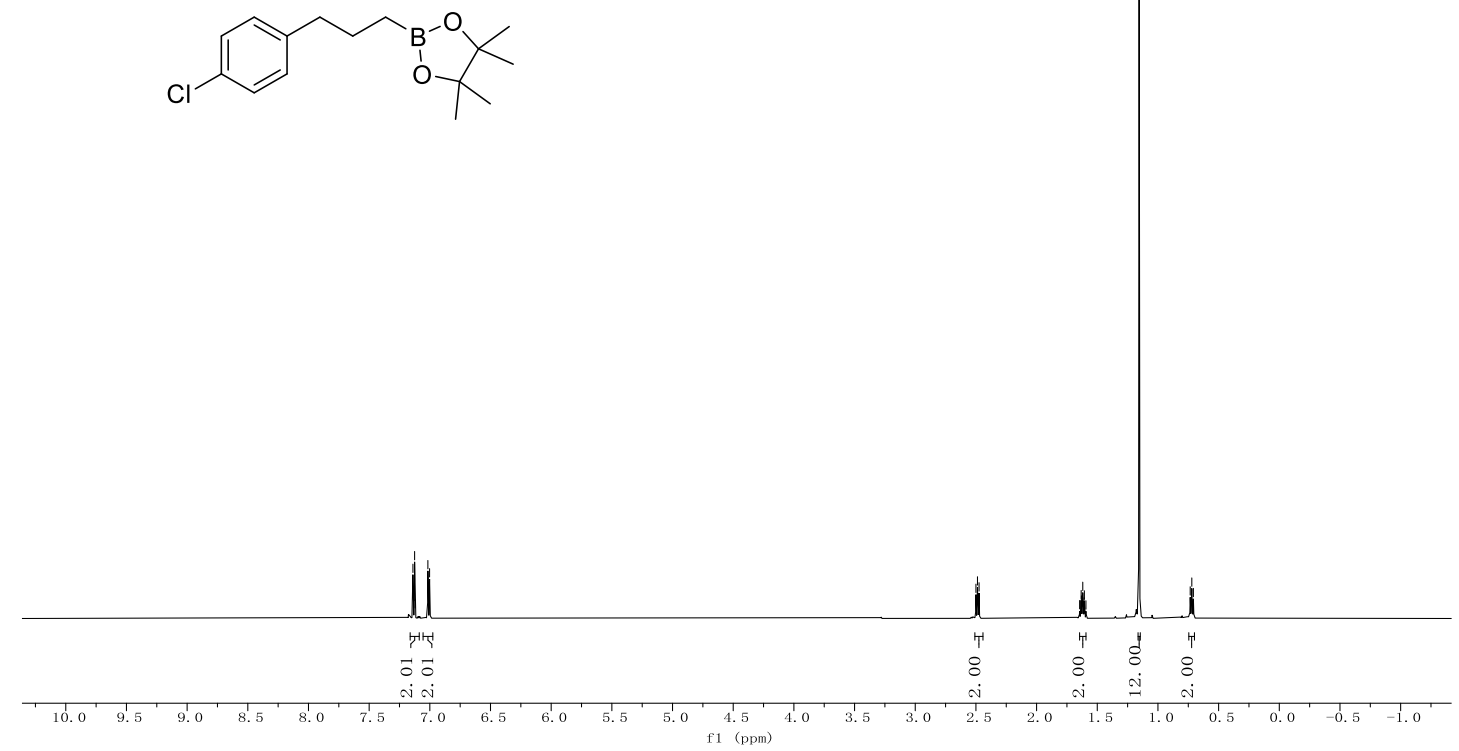




$$
\text { (1) }
$$

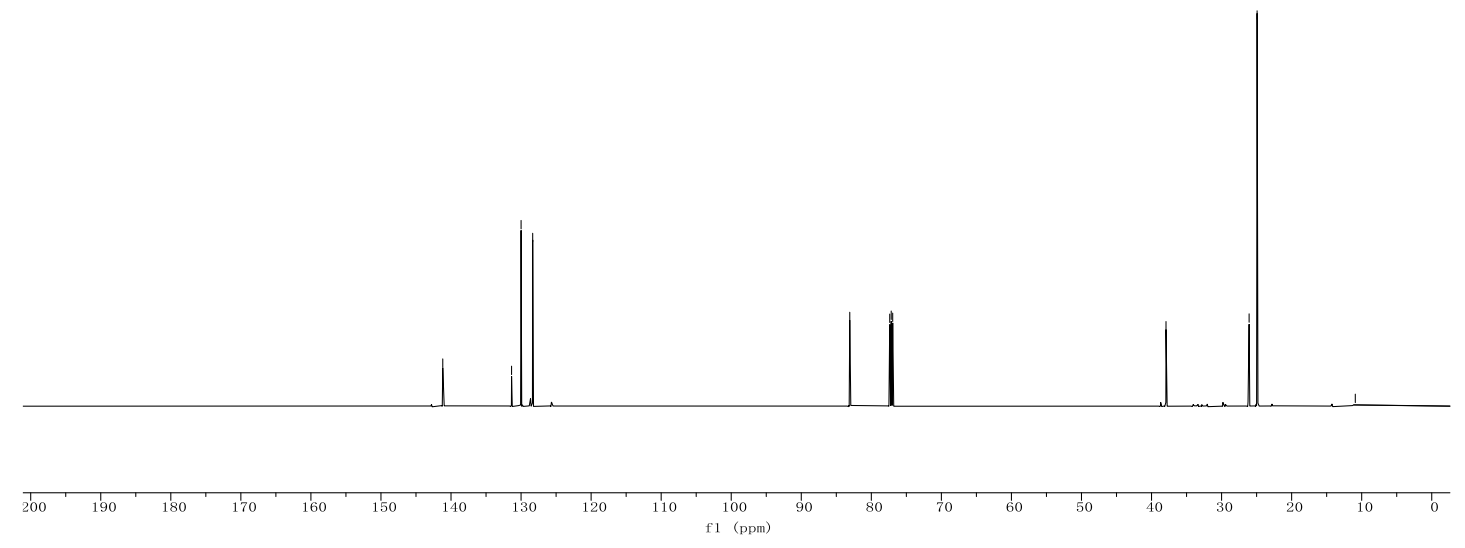

$$
\begin{aligned}
& \infty \\
& \infty \\
& \infty \\
& \infty \\
& 1
\end{aligned}
$$<smiles>CC1(C)OB(CCCc2ccc(Cl)cc2)OC1(C)C</smiles>

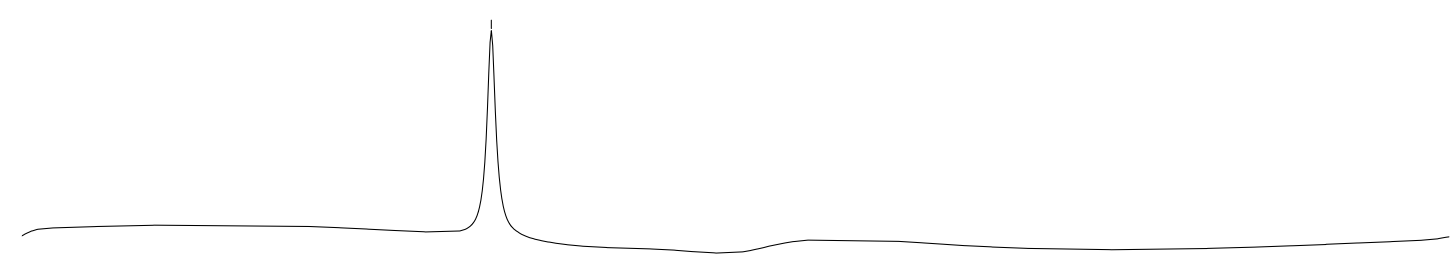




\section{Compound 17}

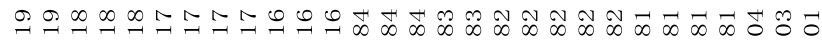

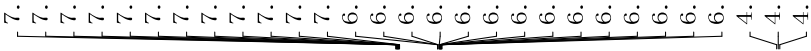

ㅇํำล ำ

ت-نت-<smiles>CC1(C)OB(CCOc2ccccc2)OC1(C)C</smiles>

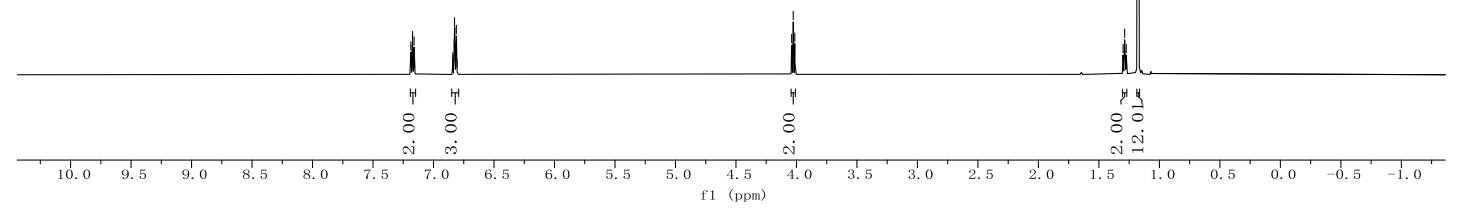

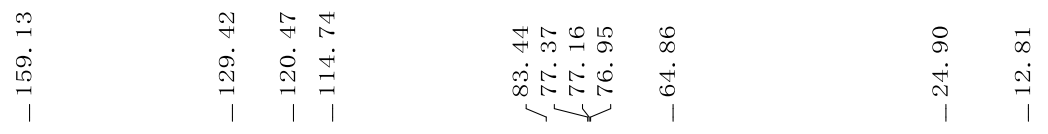<smiles>CC1(C)OB(CCOc2ccccc2)OC1(C)C</smiles>

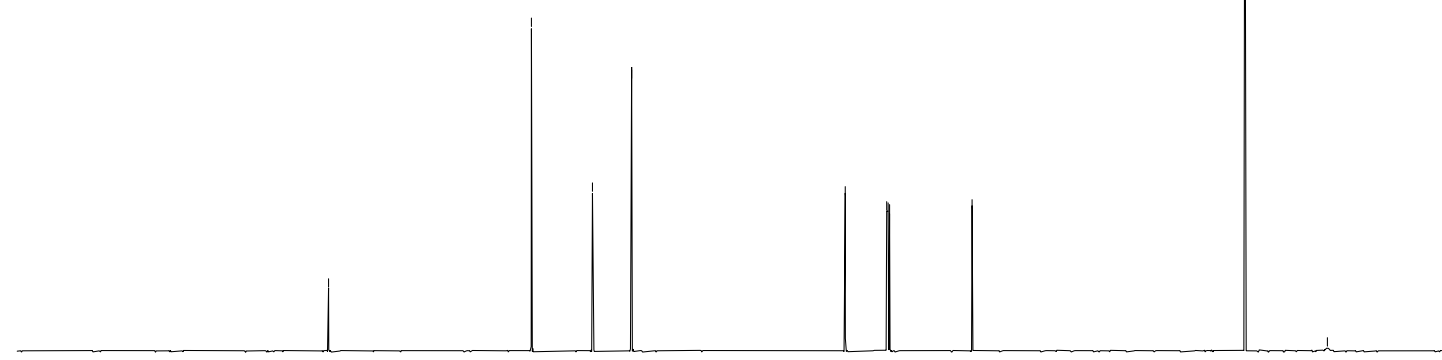

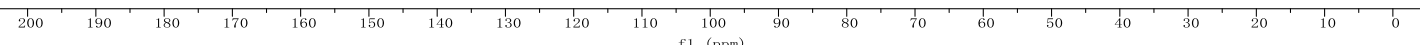



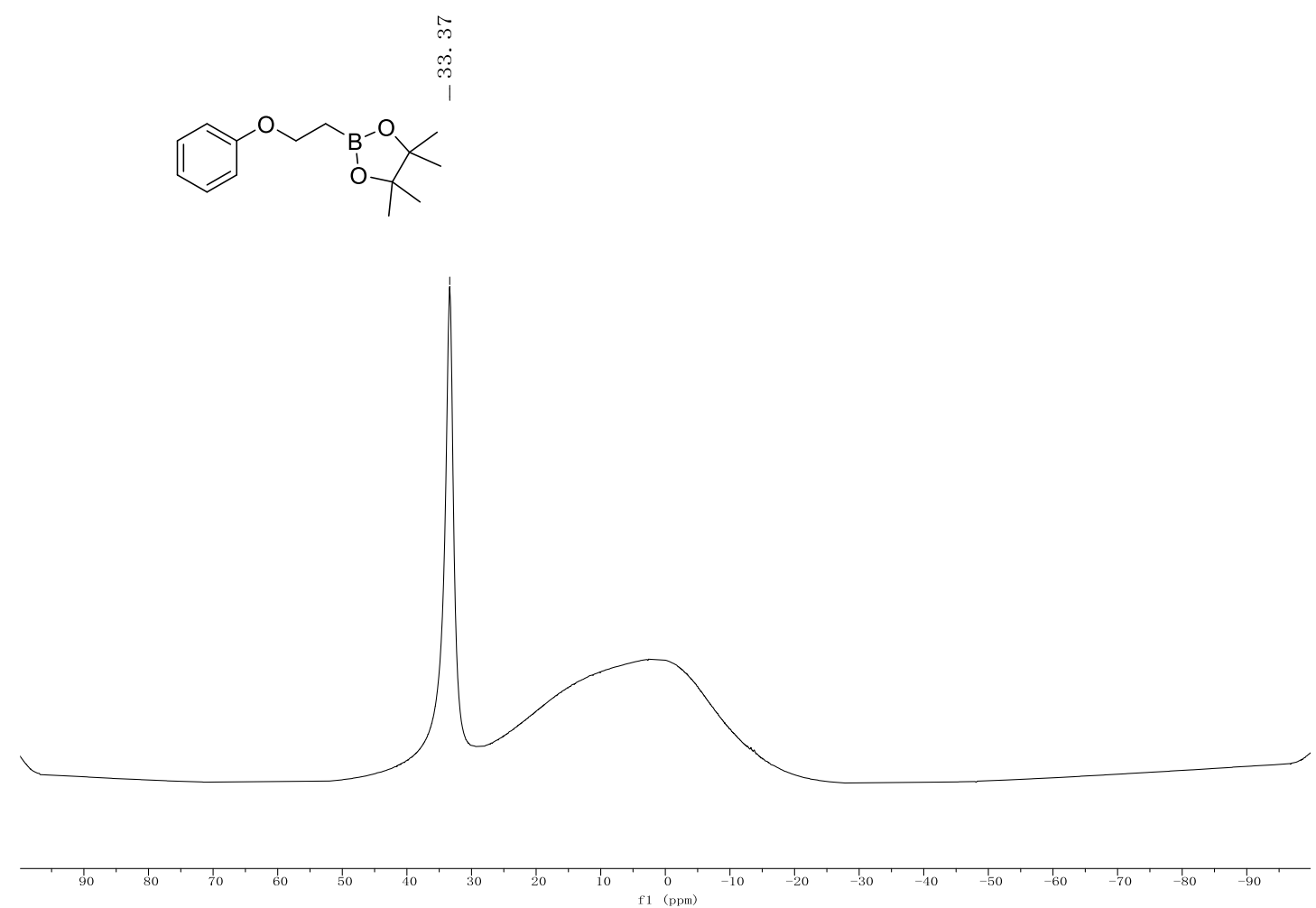

\section{Compound 18}

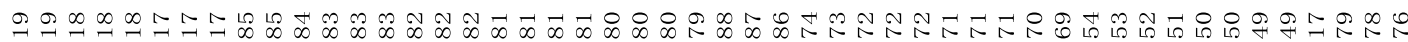

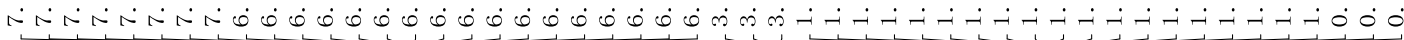<smiles>CC1(C)OB(CCCCOc2ccccc2)OC1(C)C</smiles>

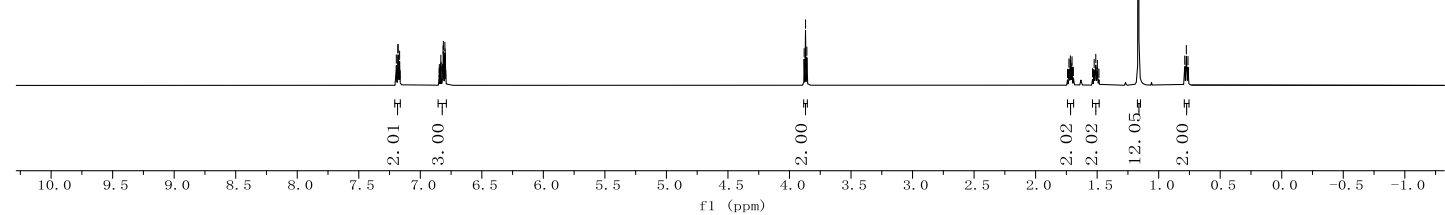



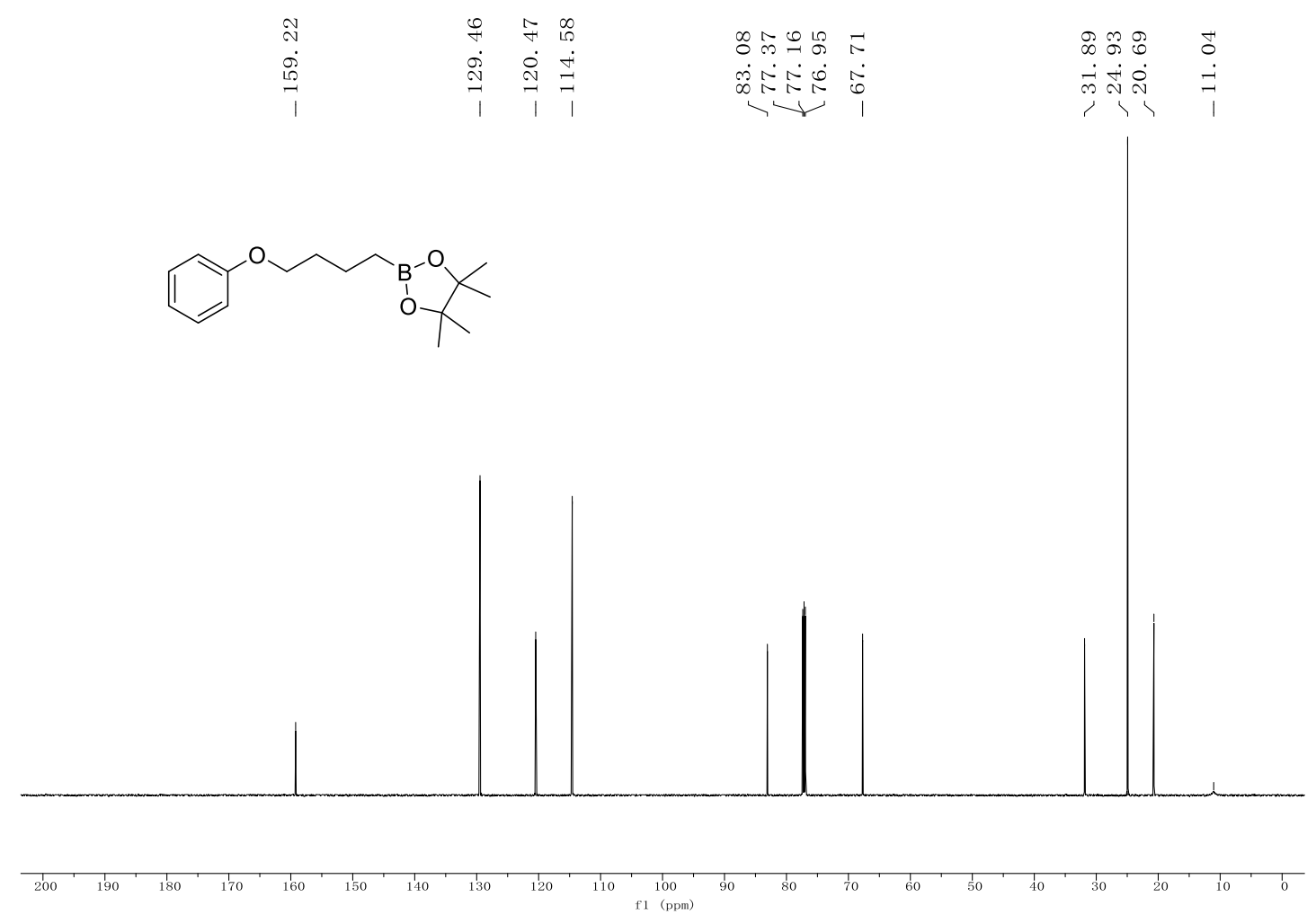

8
$\dot{0}$
0
1

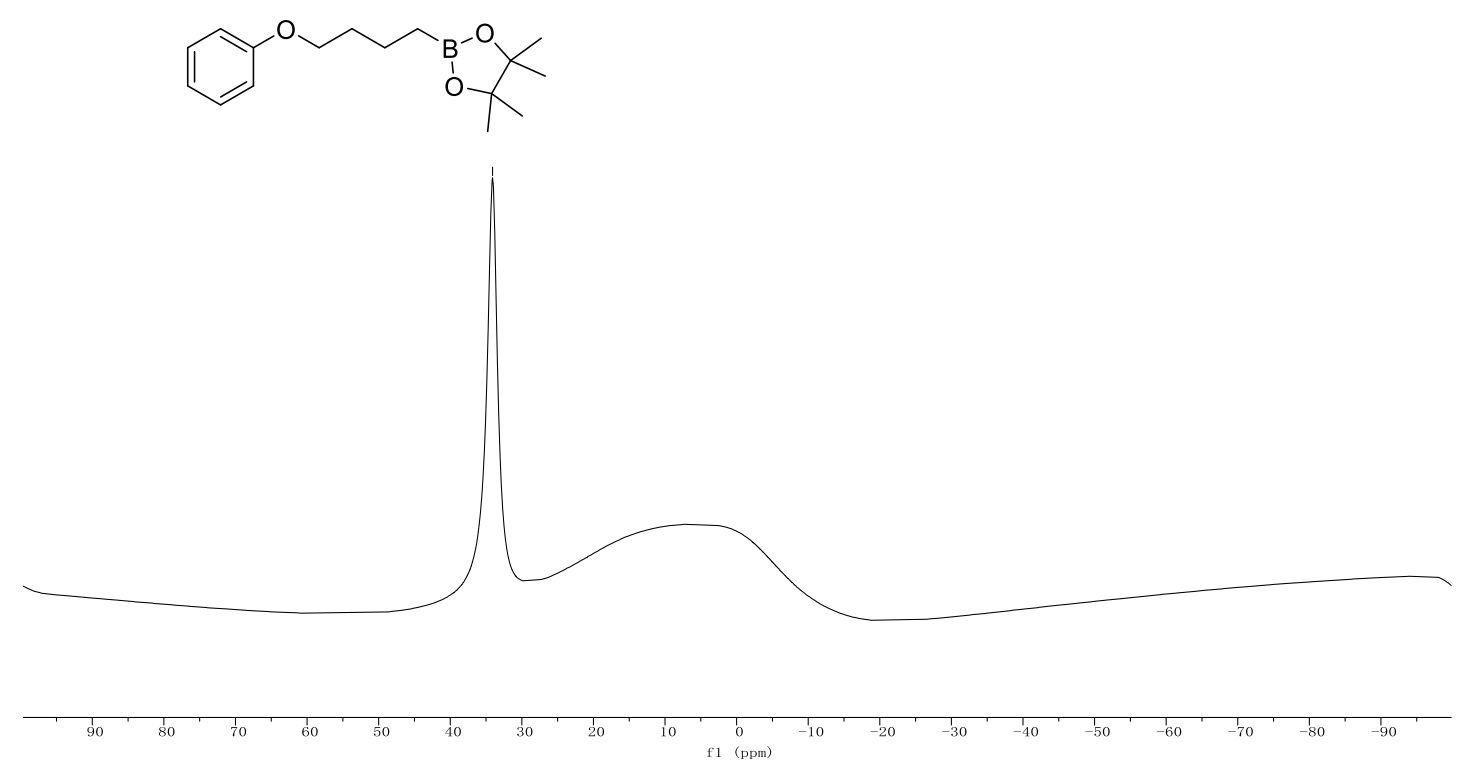




\section{Compound 19}

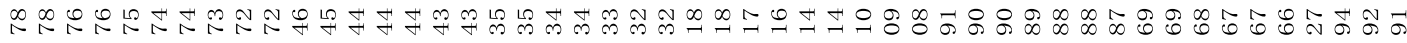

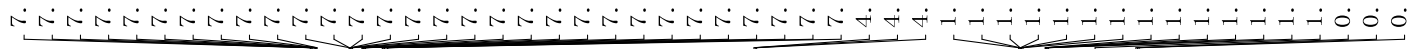<smiles>CC1(C)OB(CCCCOc2ccc3ccccc3c2)OC1(C)C</smiles>

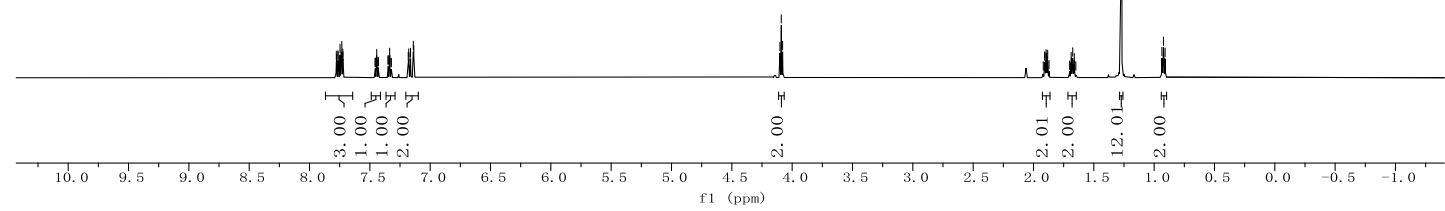

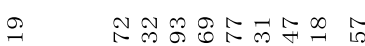

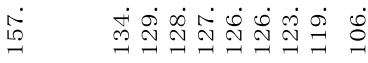

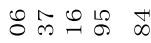

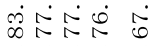

क హँ

证

$\checkmark$ ।

लं<smiles>CC1(C)OB(CCCCOc2ccc3ccccc3c2)OC1(C)C</smiles>

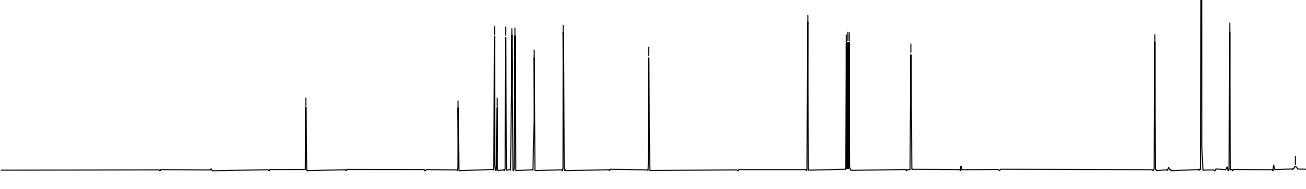

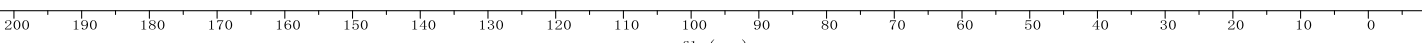



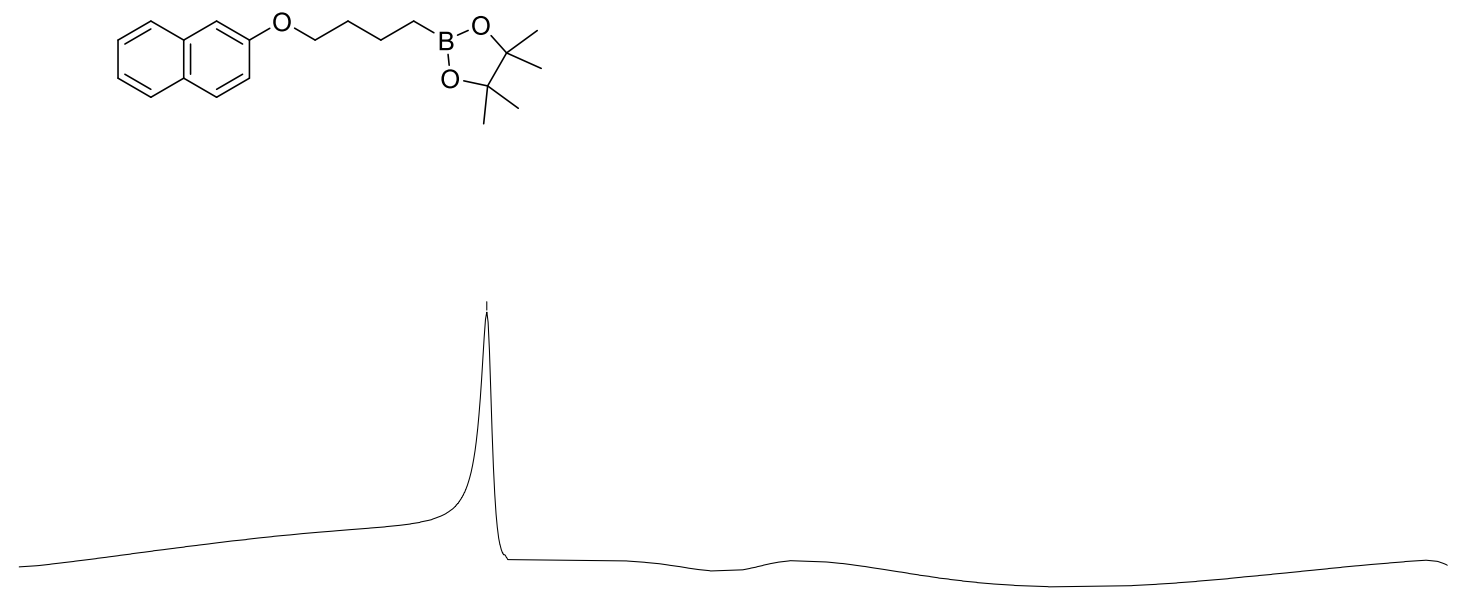

\section{Compound 20}

\begin{tabular}{|c|c|c|}
\hline 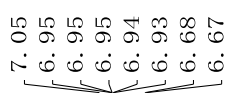 & 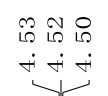 & 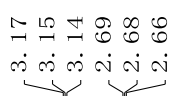 \\
\hline
\end{tabular}<smiles>CC1(C)OB(CCc2ccc3c(c2)CCO3)OC1(C)C</smiles>

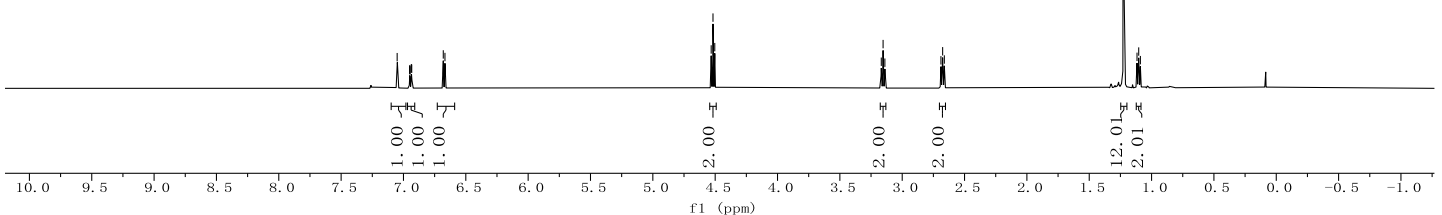




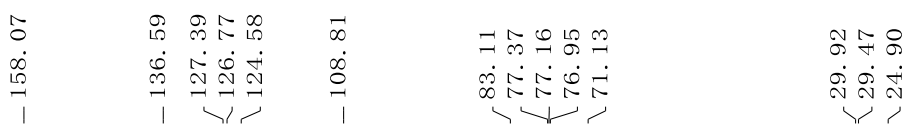<smiles>CC1(C)OB(CCc2ccc3c(c2)CCO3)OC1(C)C</smiles>
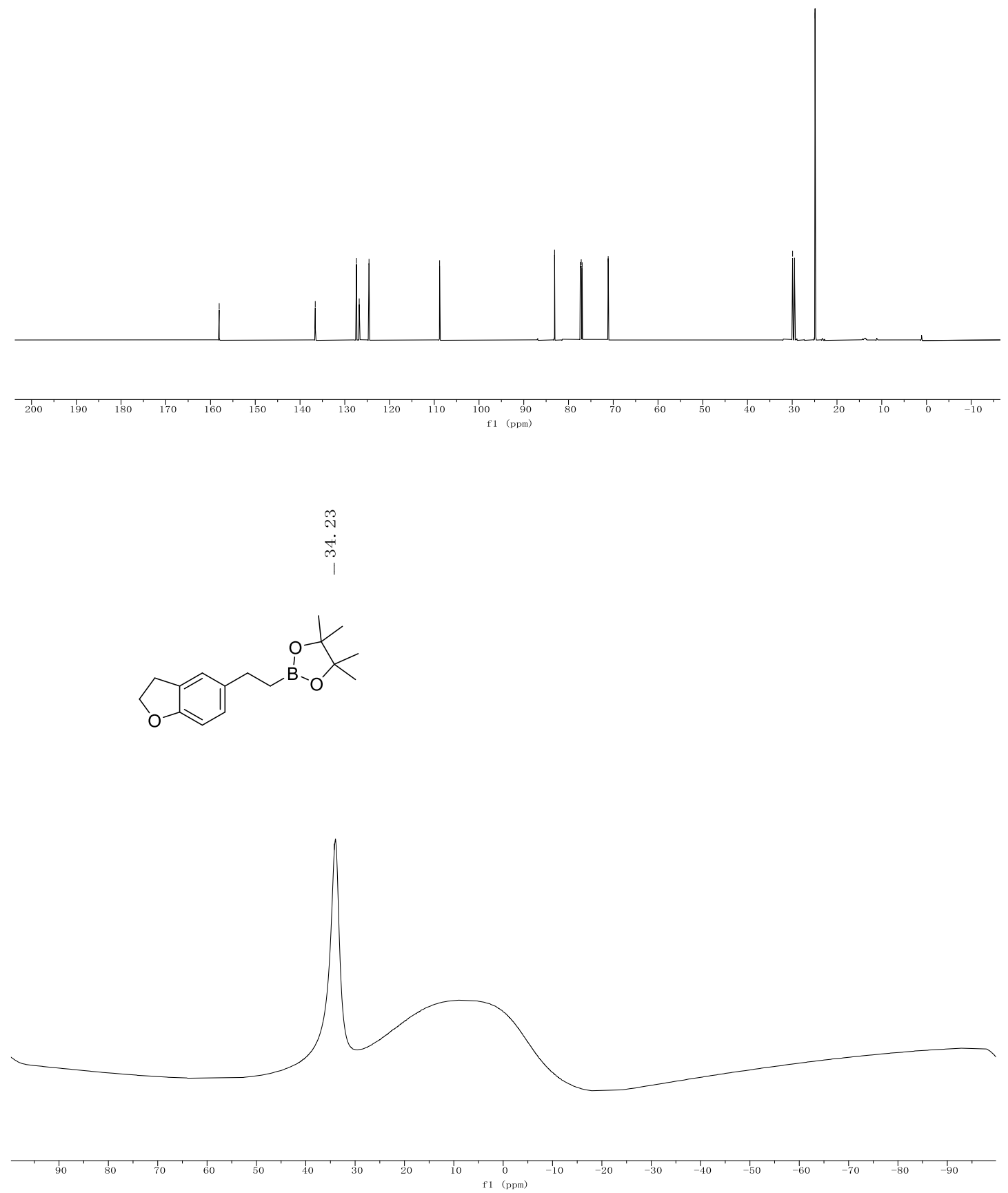


\section{Compound 21}

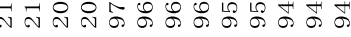

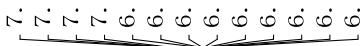

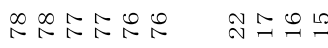

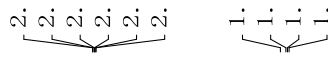<smiles>CC1(C)OB(CCc2ccsc2)OC1(C)C</smiles>

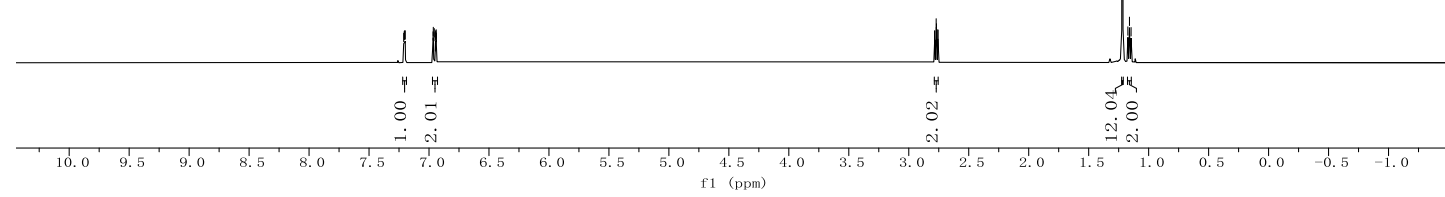

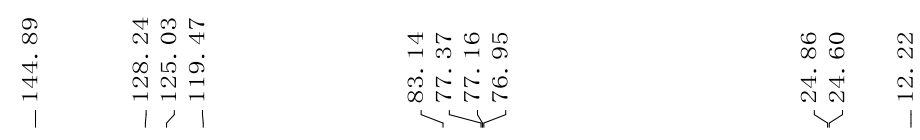
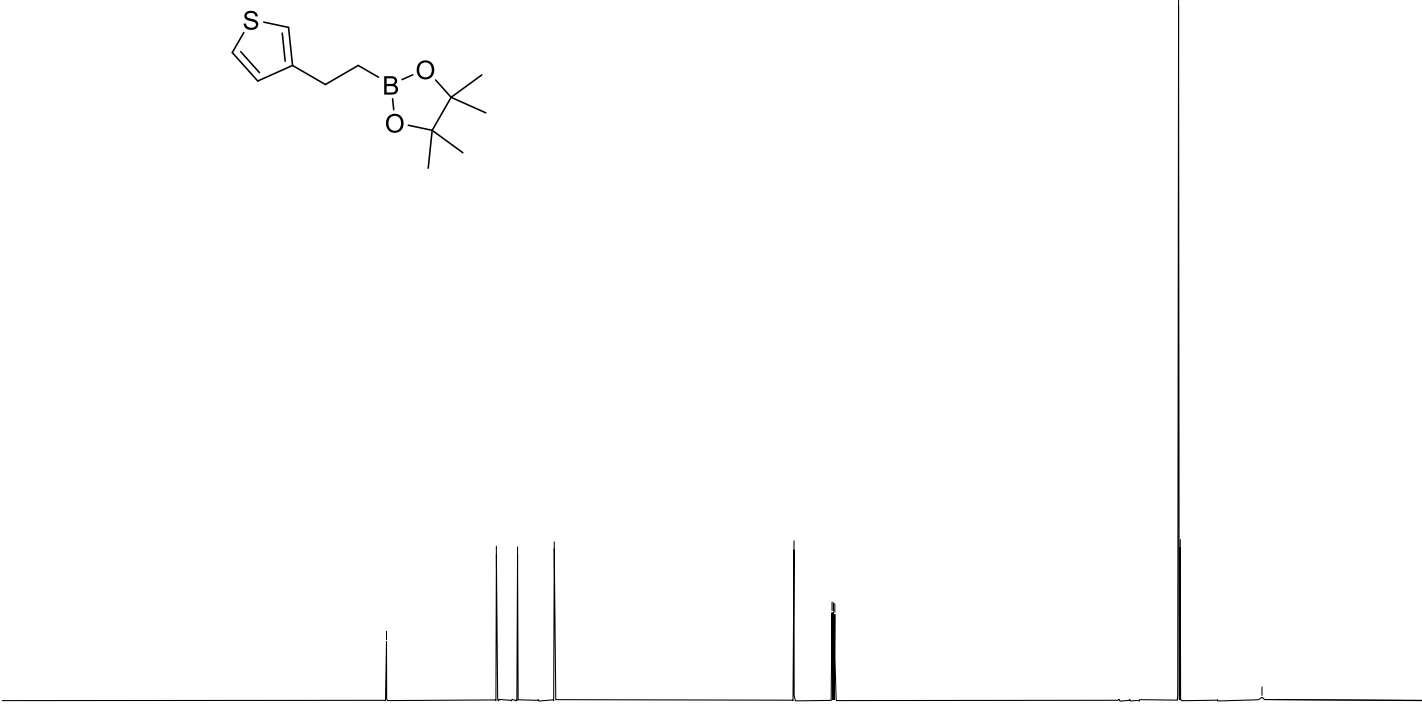

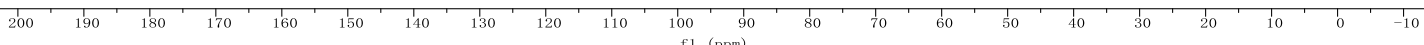



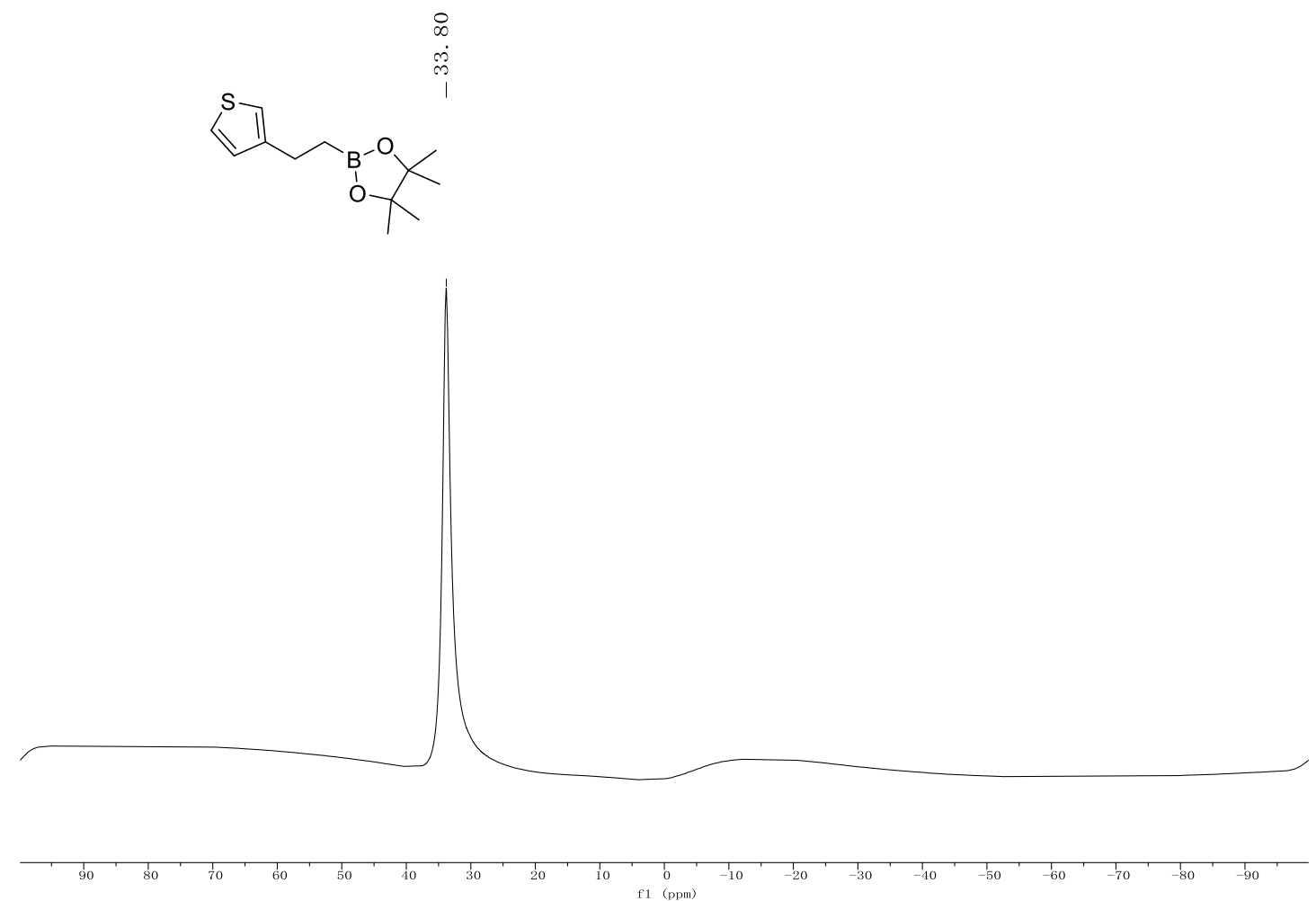

\section{Compound 22}

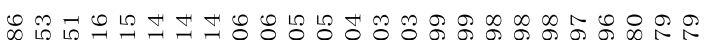

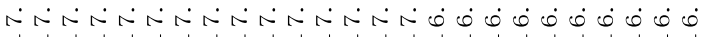

\begin{tabular}{|c|c|}
\hline$\vec{\infty} \stackrel{\infty}{\sim}$ & $\stackrel{\infty}{\sim}$ ニ \\
\hline ن v & $\dot{-}-\dot{-}-\dot{-}$ \\
\hline
\end{tabular}

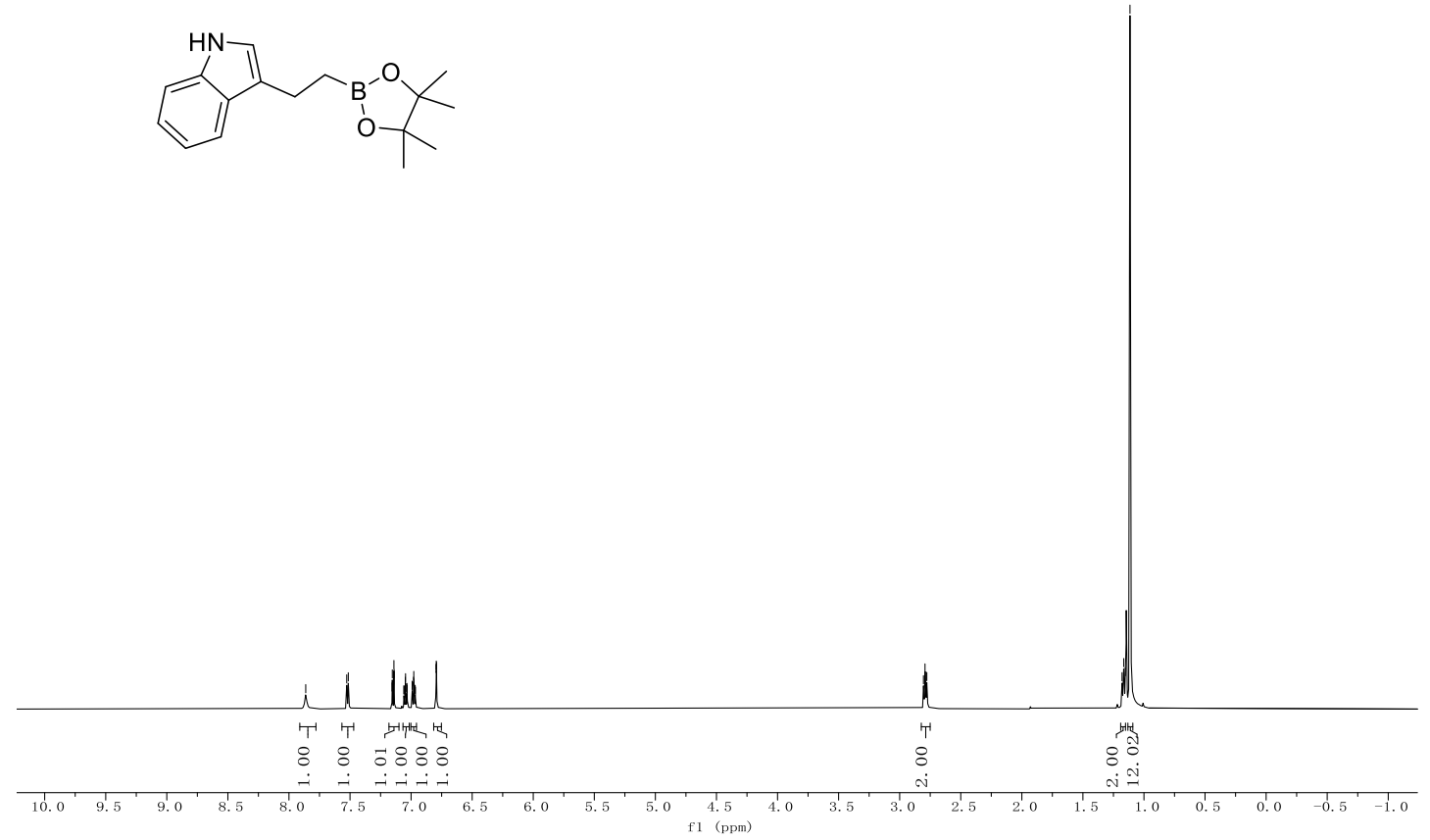




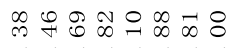

$\dot{\infty} \stackrel{\sim}{N} \dot{N} \dot{N} \stackrel{\infty}{=} \stackrel{\infty}{=}$

옹요용

舟新迹

死京

।

$\longrightarrow$

तें

$\overbrace{1}^{n+1}$
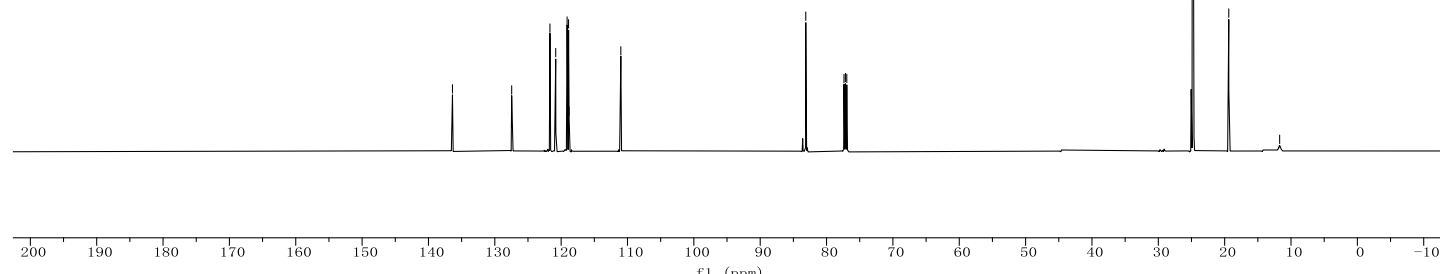

\section{0
$\stackrel{+}{+}$
$\dot{m}$}

$\overbrace{1}^{1}$

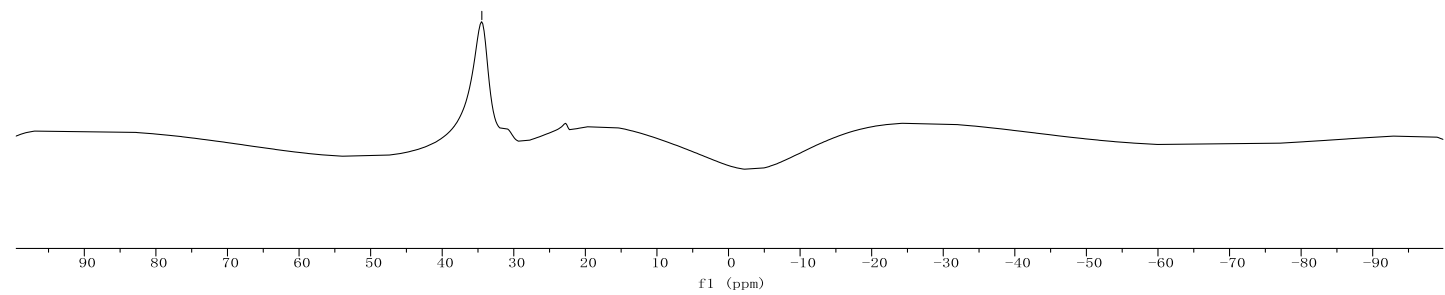




\section{Compound 23}

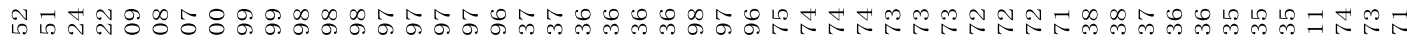

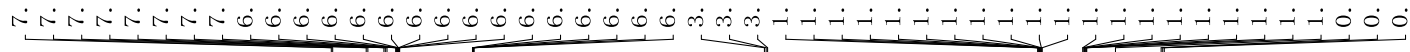
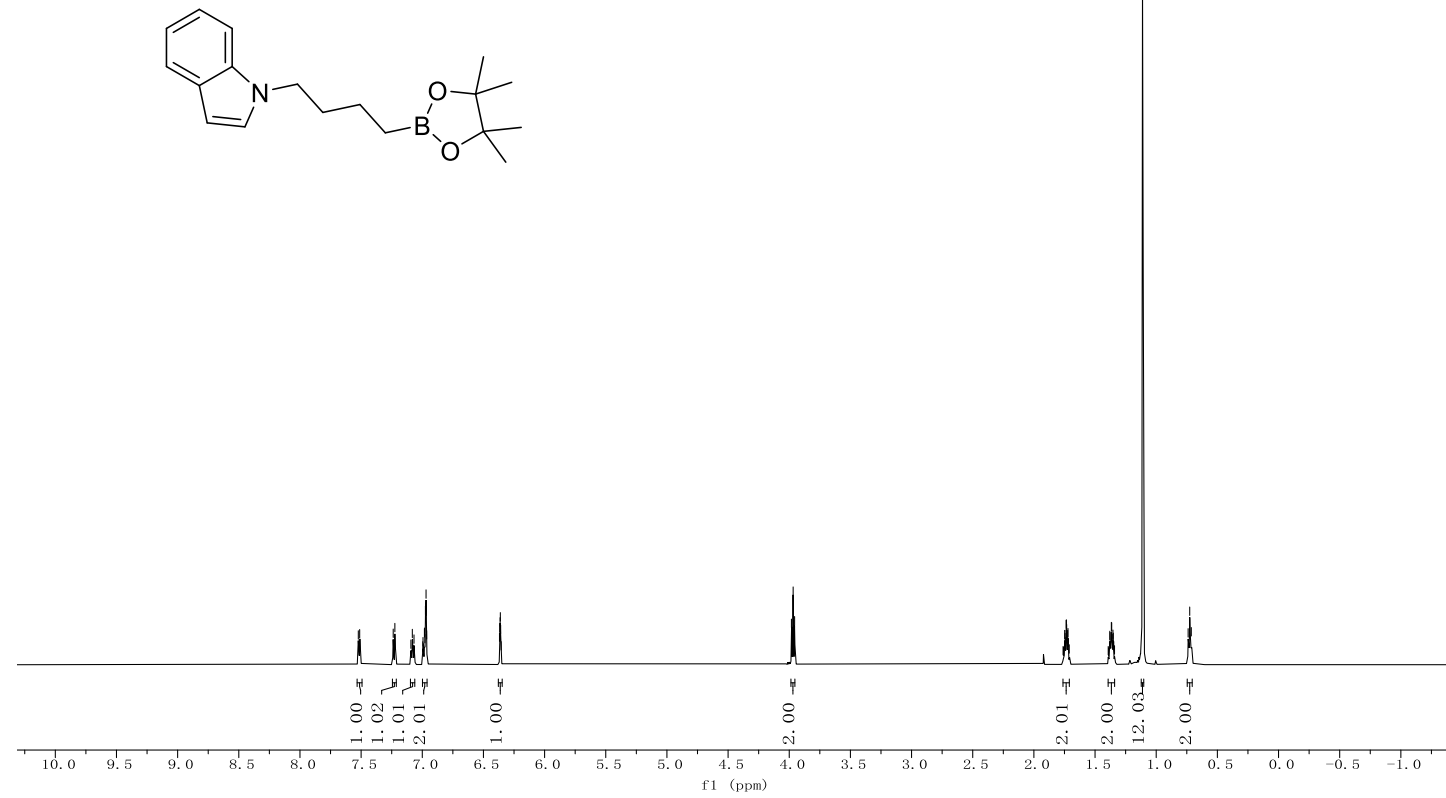

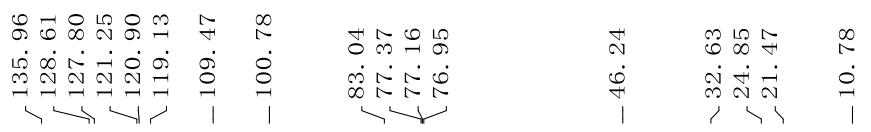
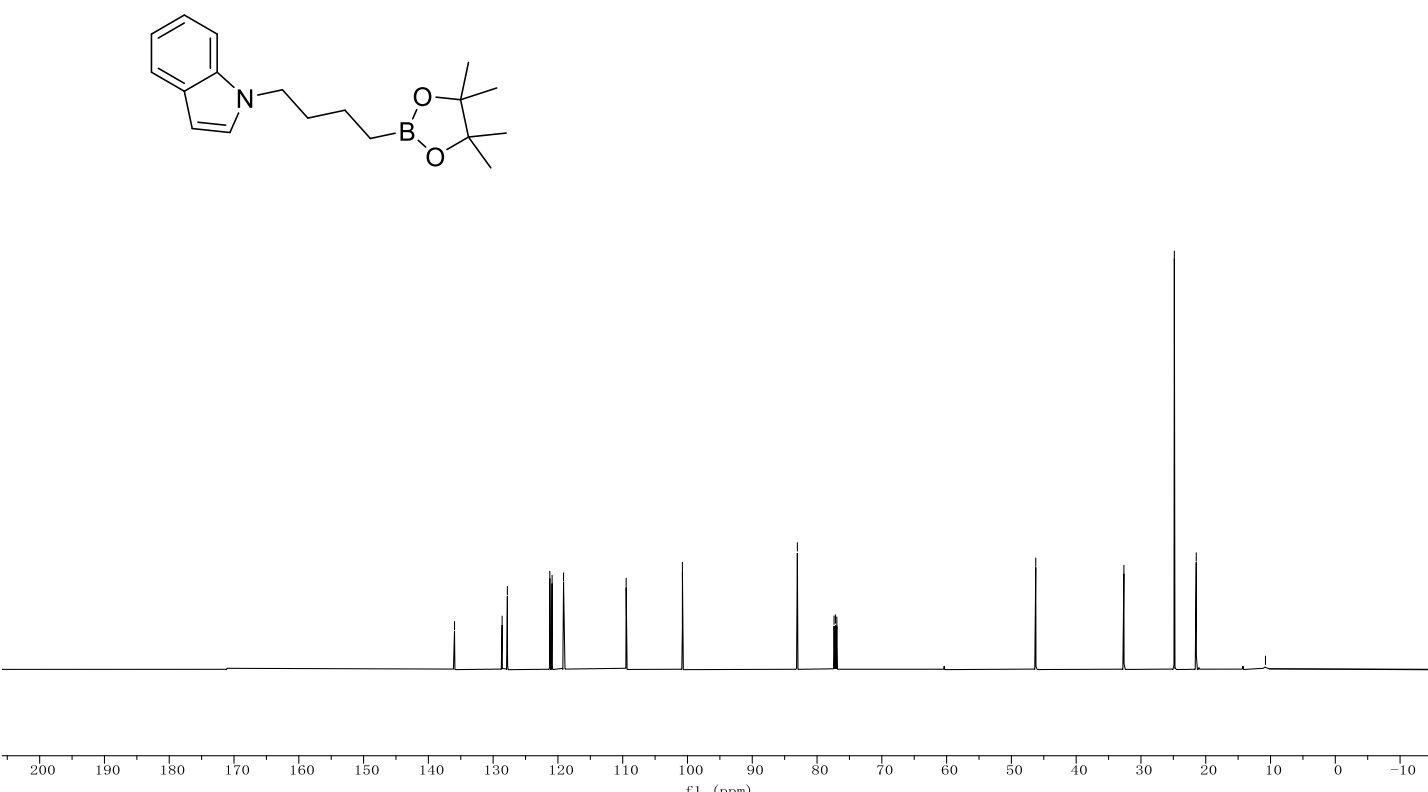

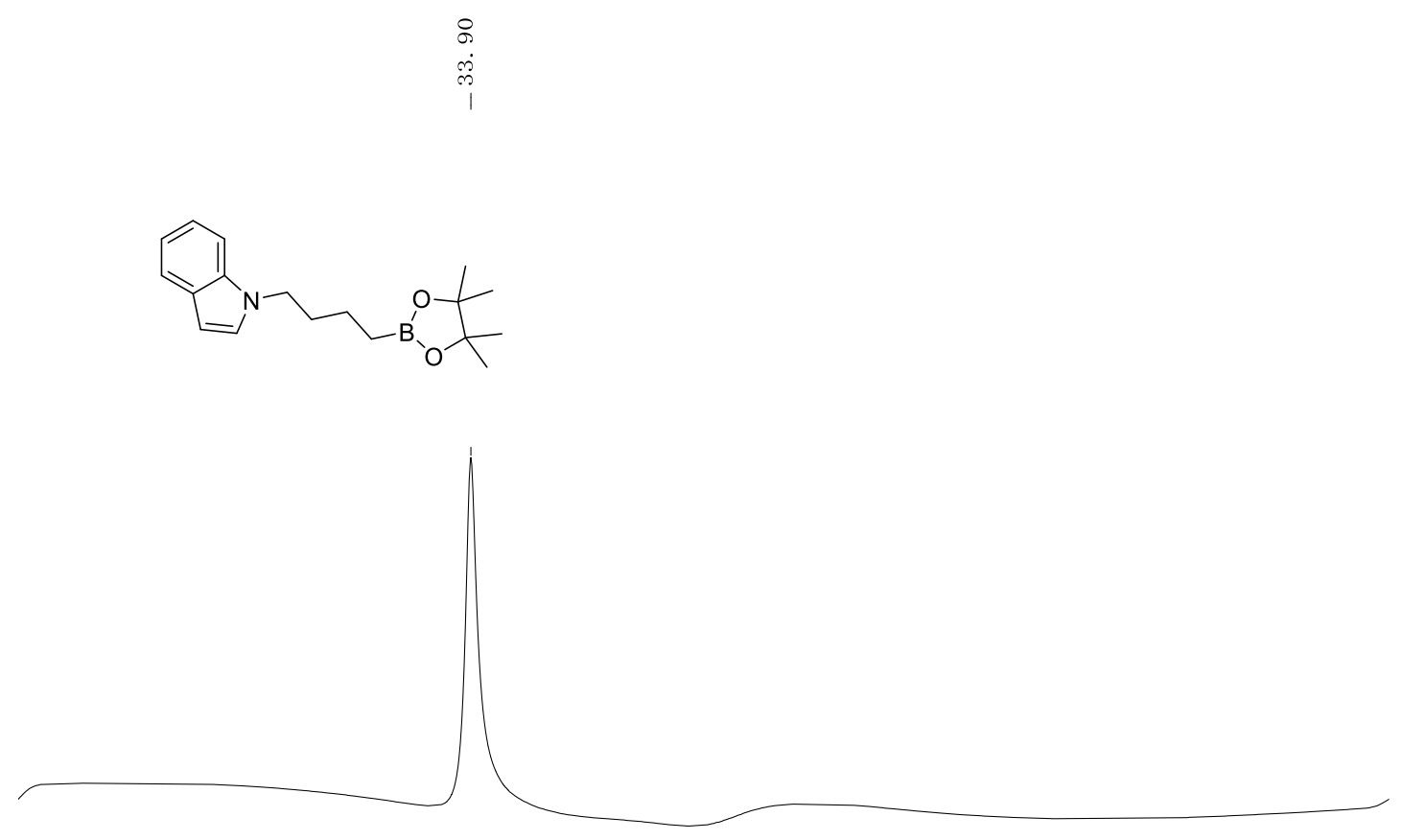

\begin{tabular}{l}
\hline \\
\hline
\end{tabular}

\section{Compound 24}

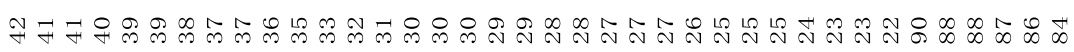

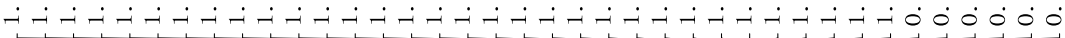

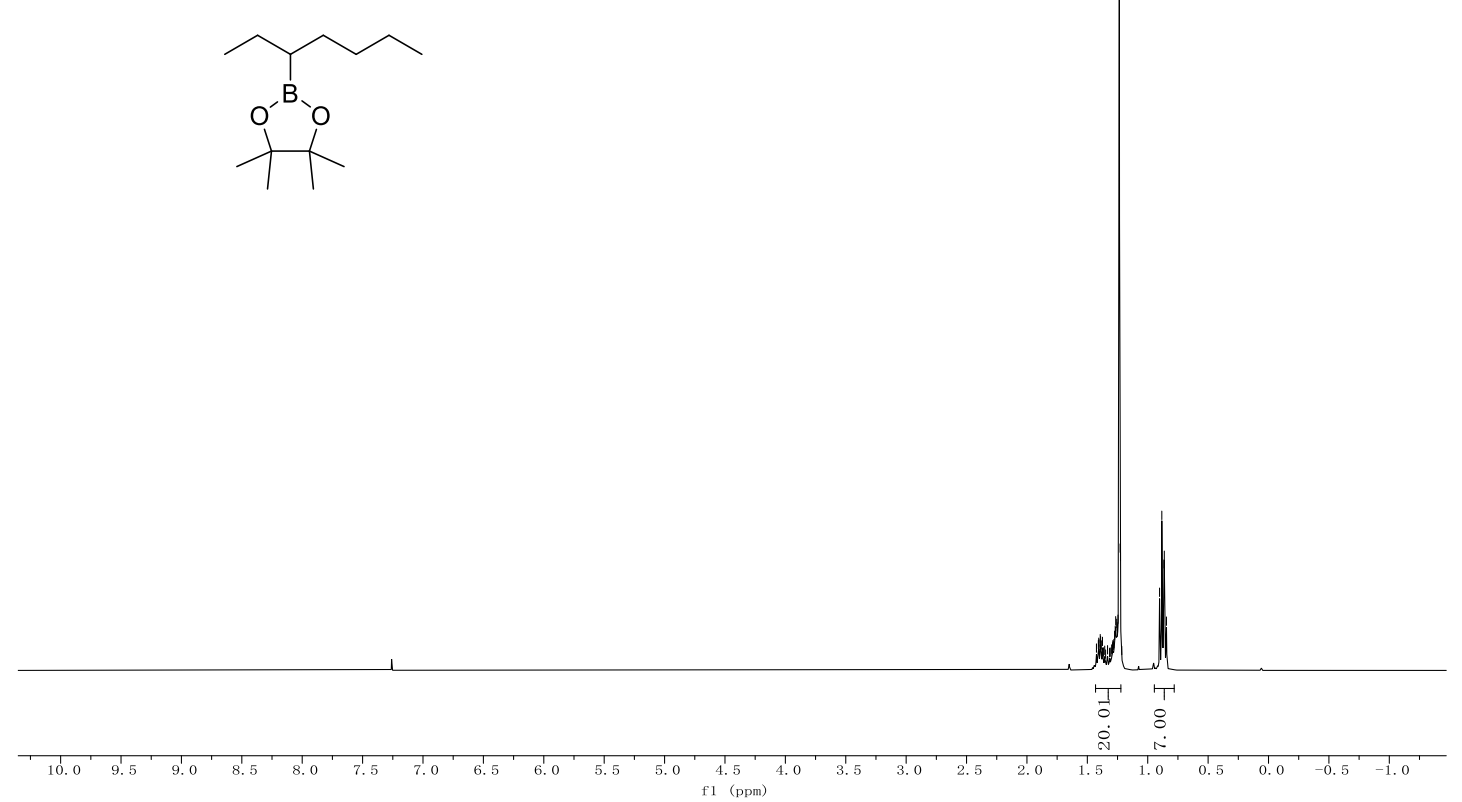




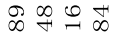

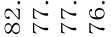

-

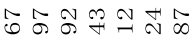

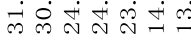

$\rightarrow>$<smiles>CCCCC(CC)B1OC(C)(C)C(C)(C)O1</smiles>

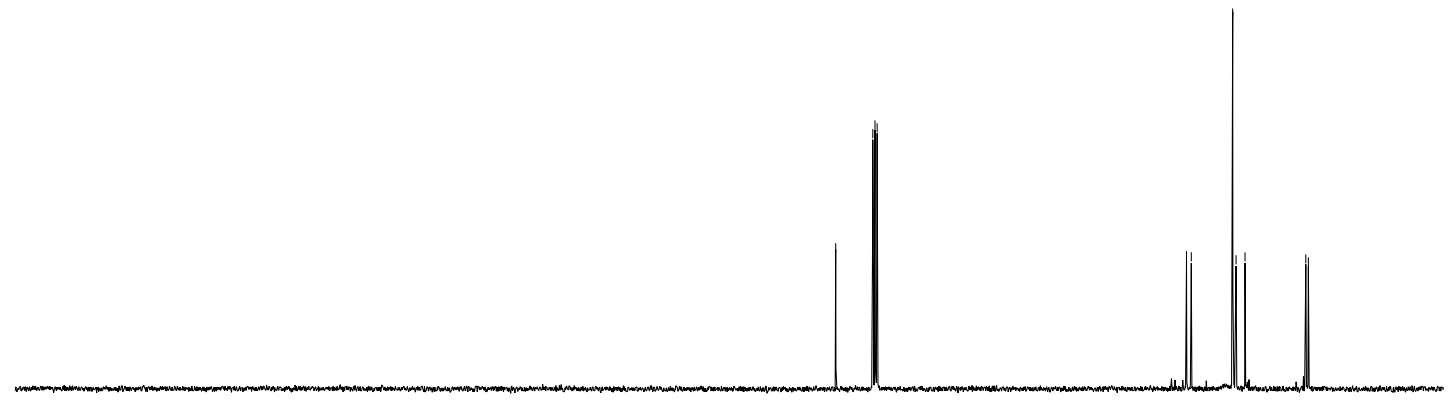

$\vec{\sigma}$
$\ddot{\sigma}$
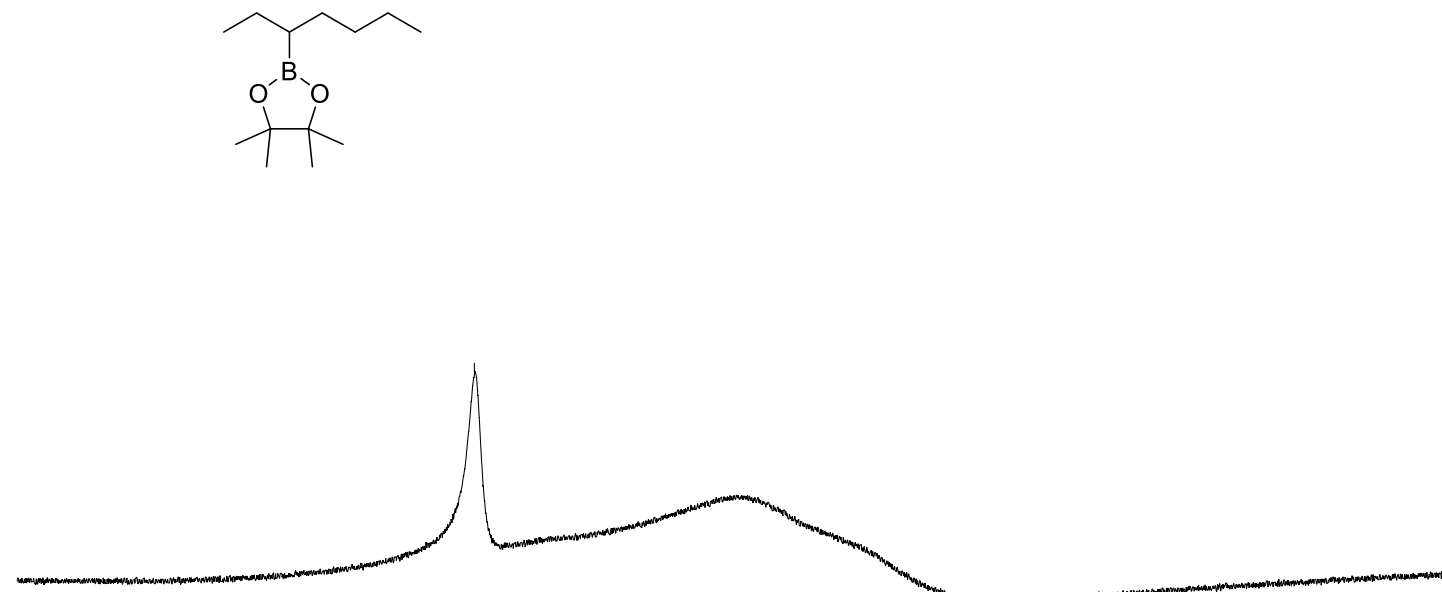

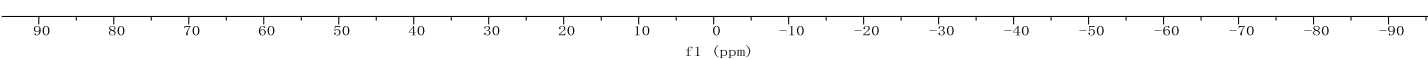




\section{Compound 25}

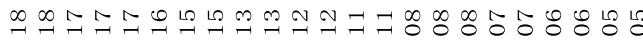

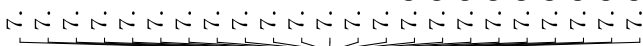

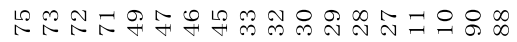

ง

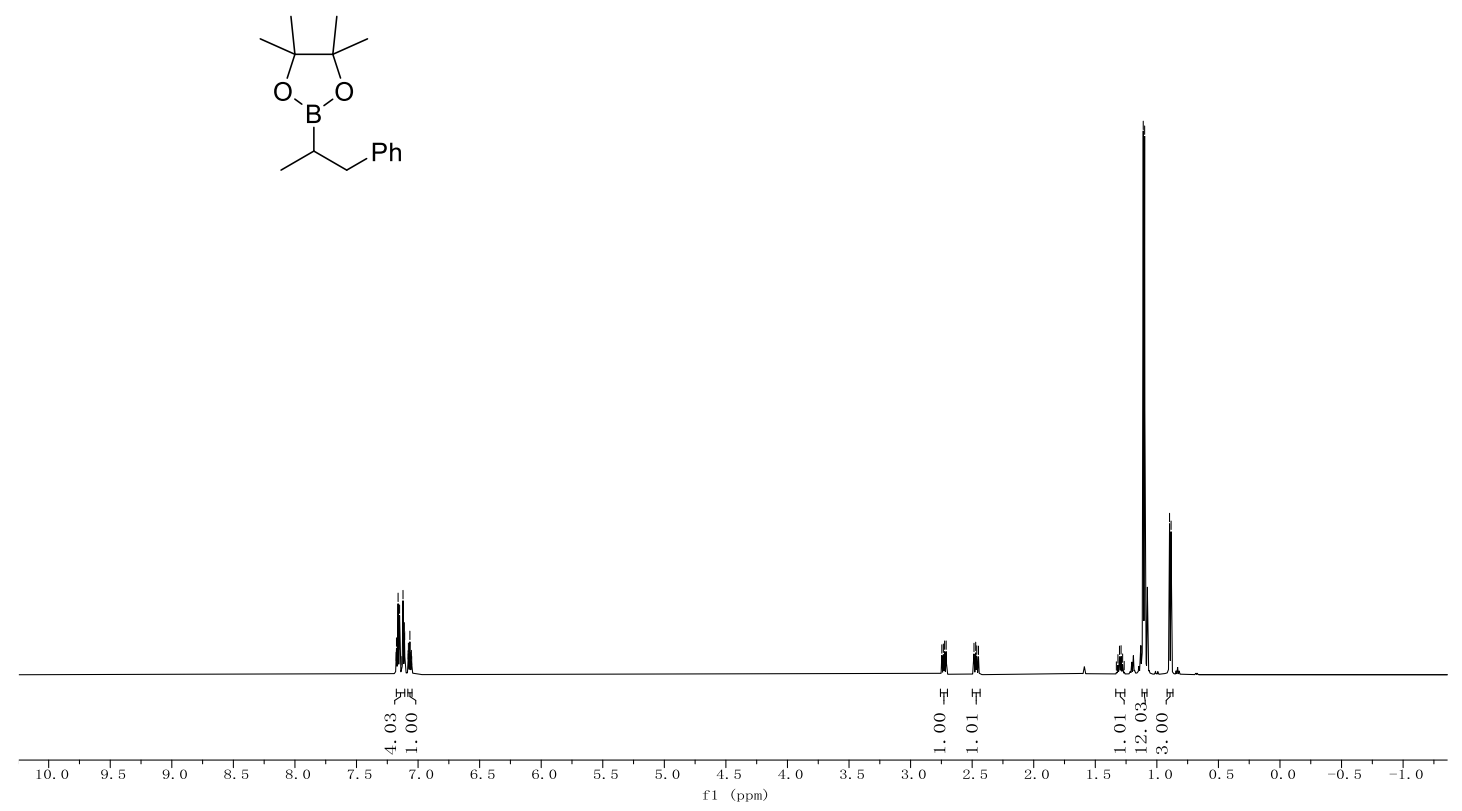

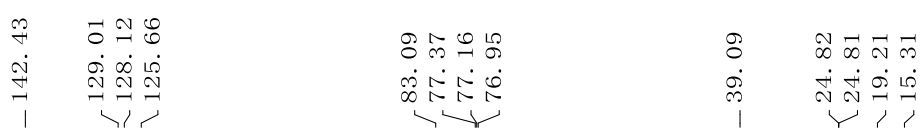

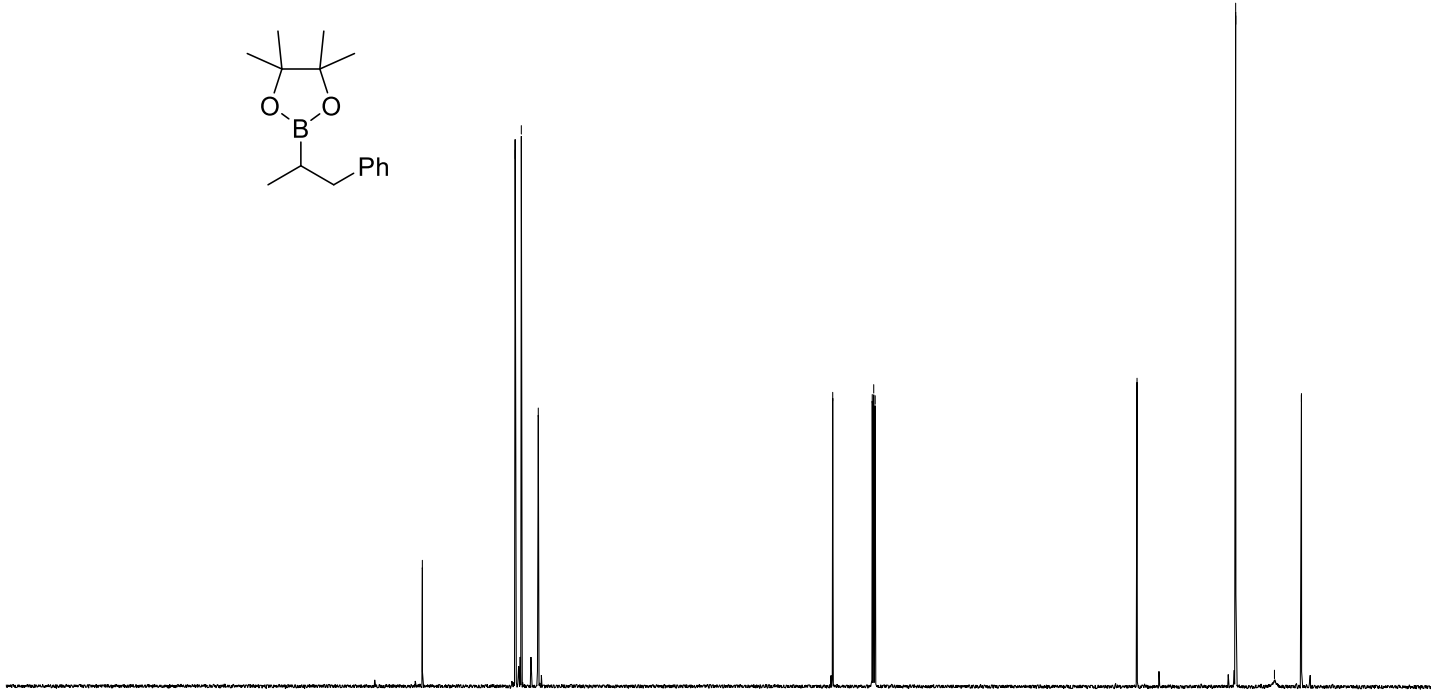

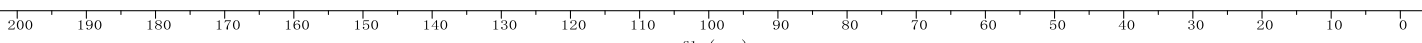



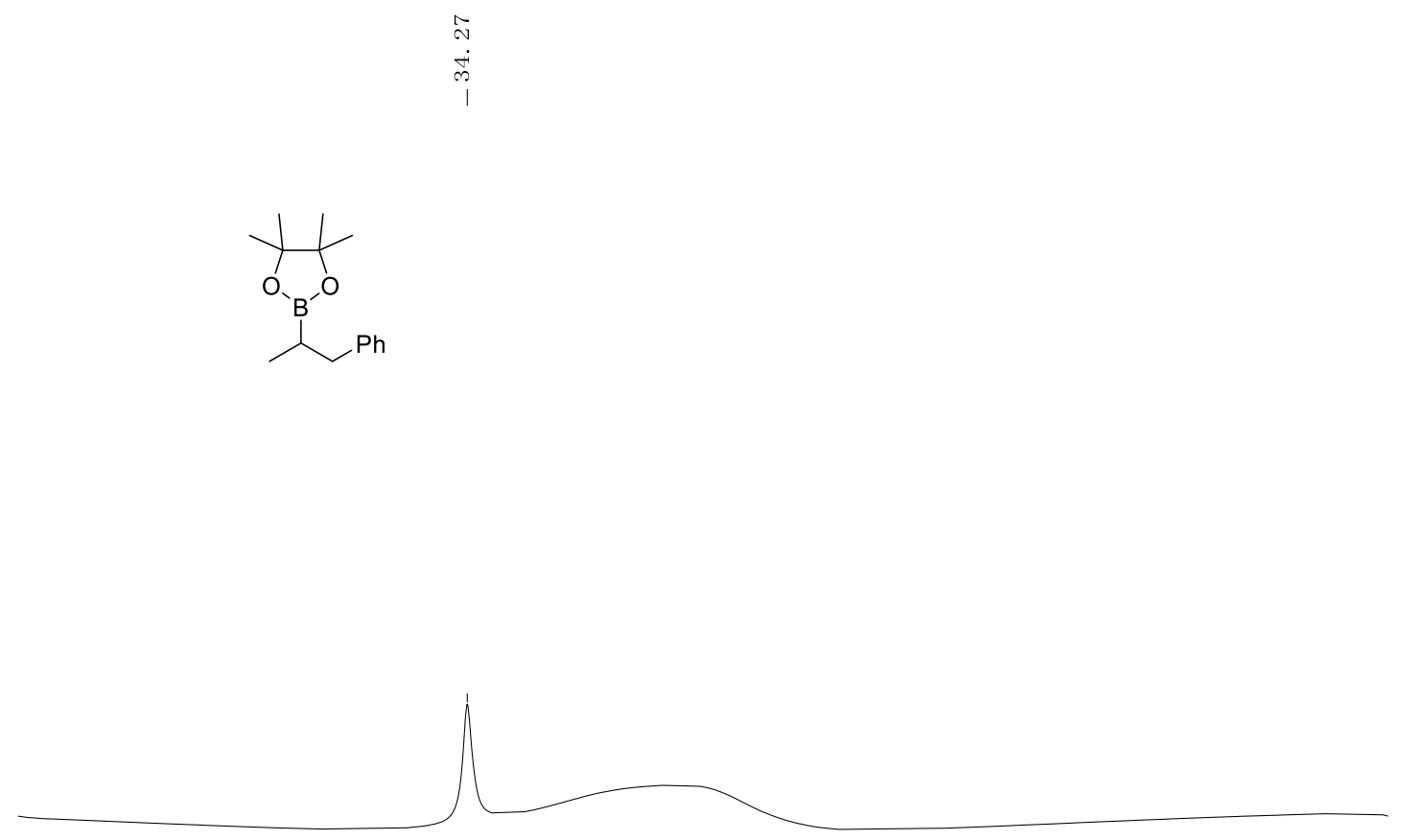

\section{Compound 26}

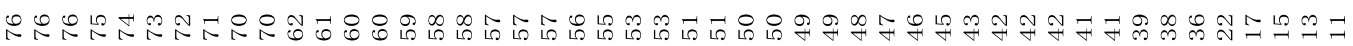

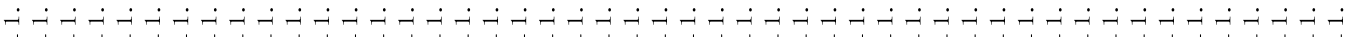

$$
\mathrm{V}_{\mathrm{O}}^{\mathrm{O}} \mathrm{B}^{\prime}
$$


$\infty \underset{\infty}{\infty} \stackrel{\infty}{\infty}$

这赴这是

పే゙

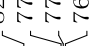

ஸे

1)

$\nabla-B_{i o f}^{\prime o}$

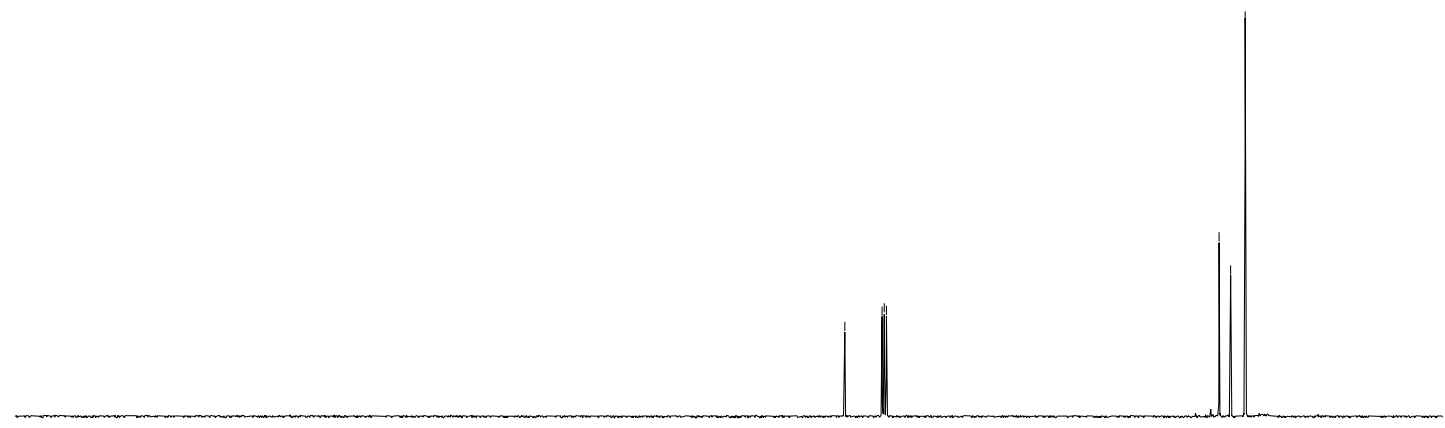

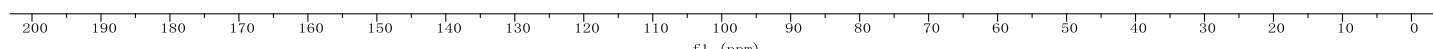

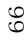

$\dot{m}$

$\nabla-B_{0}^{\prime o t}$

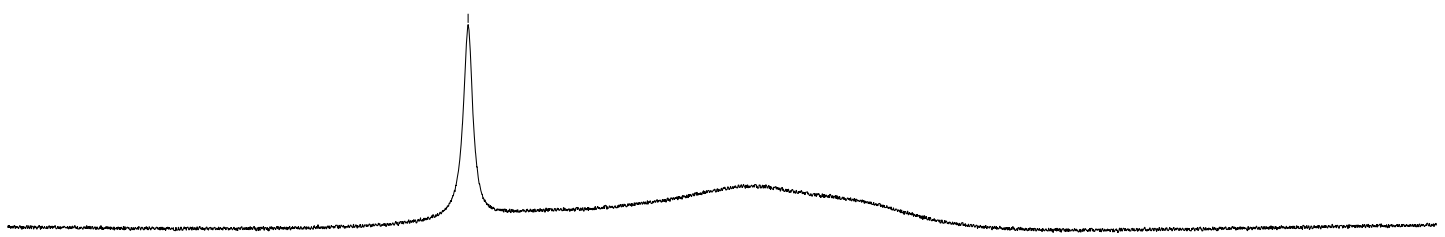

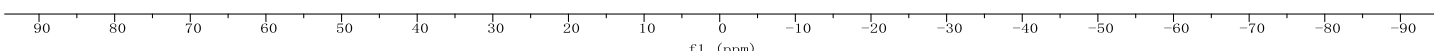




\section{Compound 27}

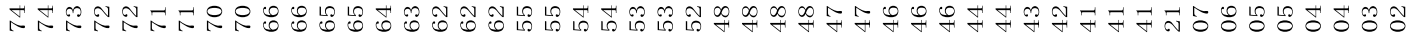

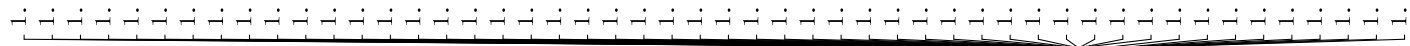

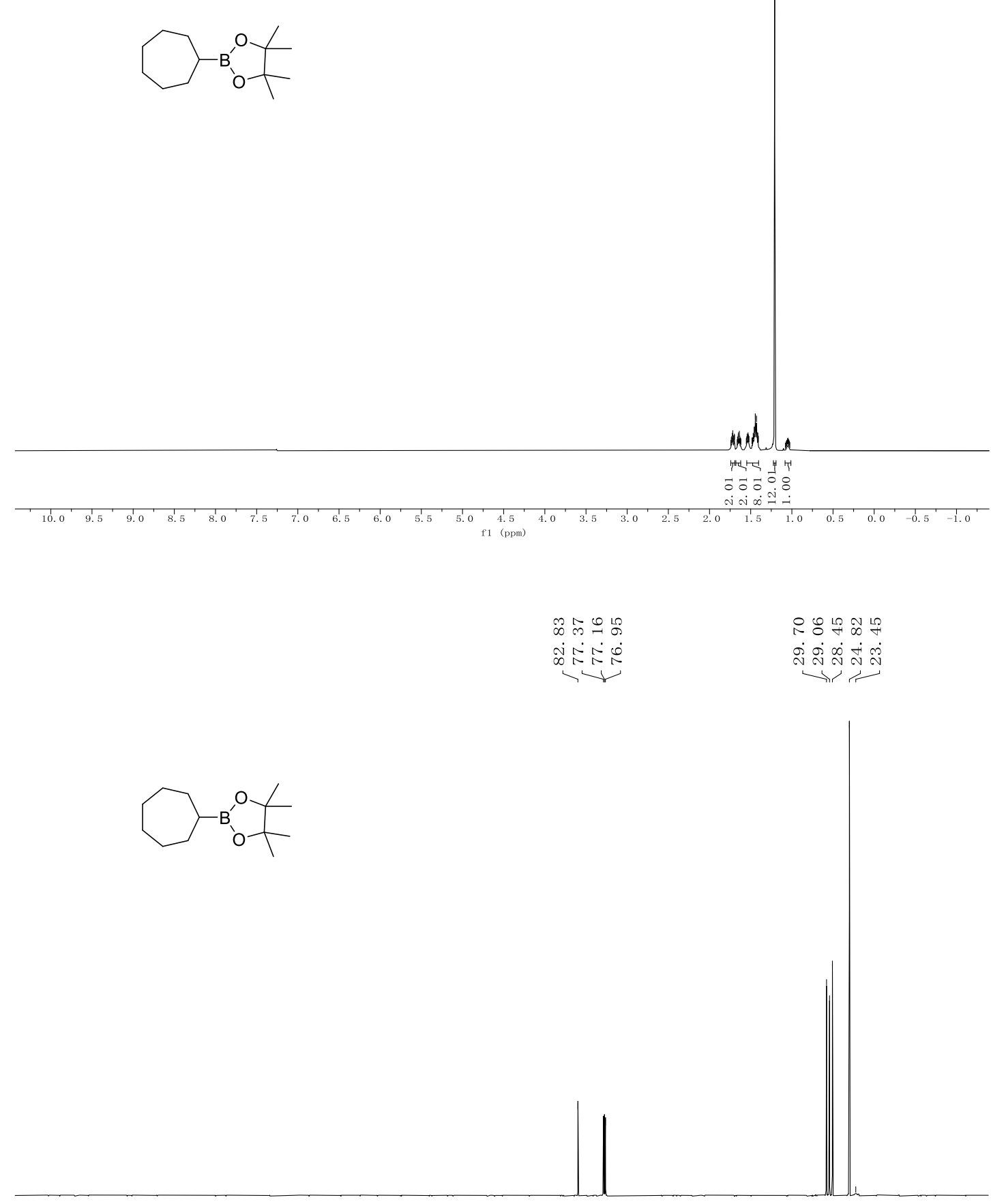

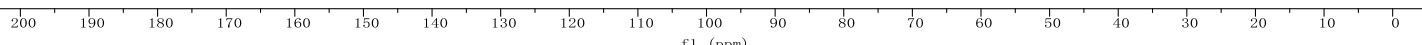



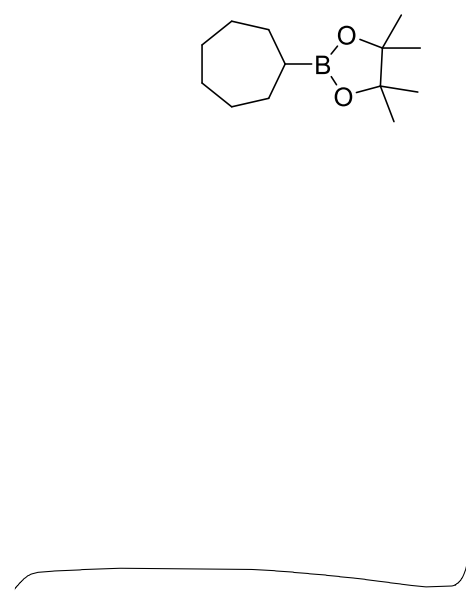

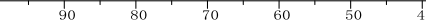

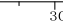

f1 $\stackrel{0}{(\mathrm{ppm})}$

\section{Compound 28}

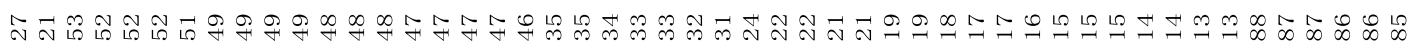

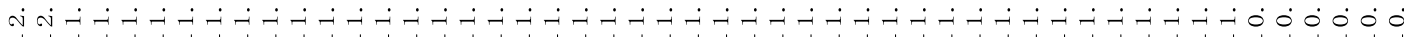

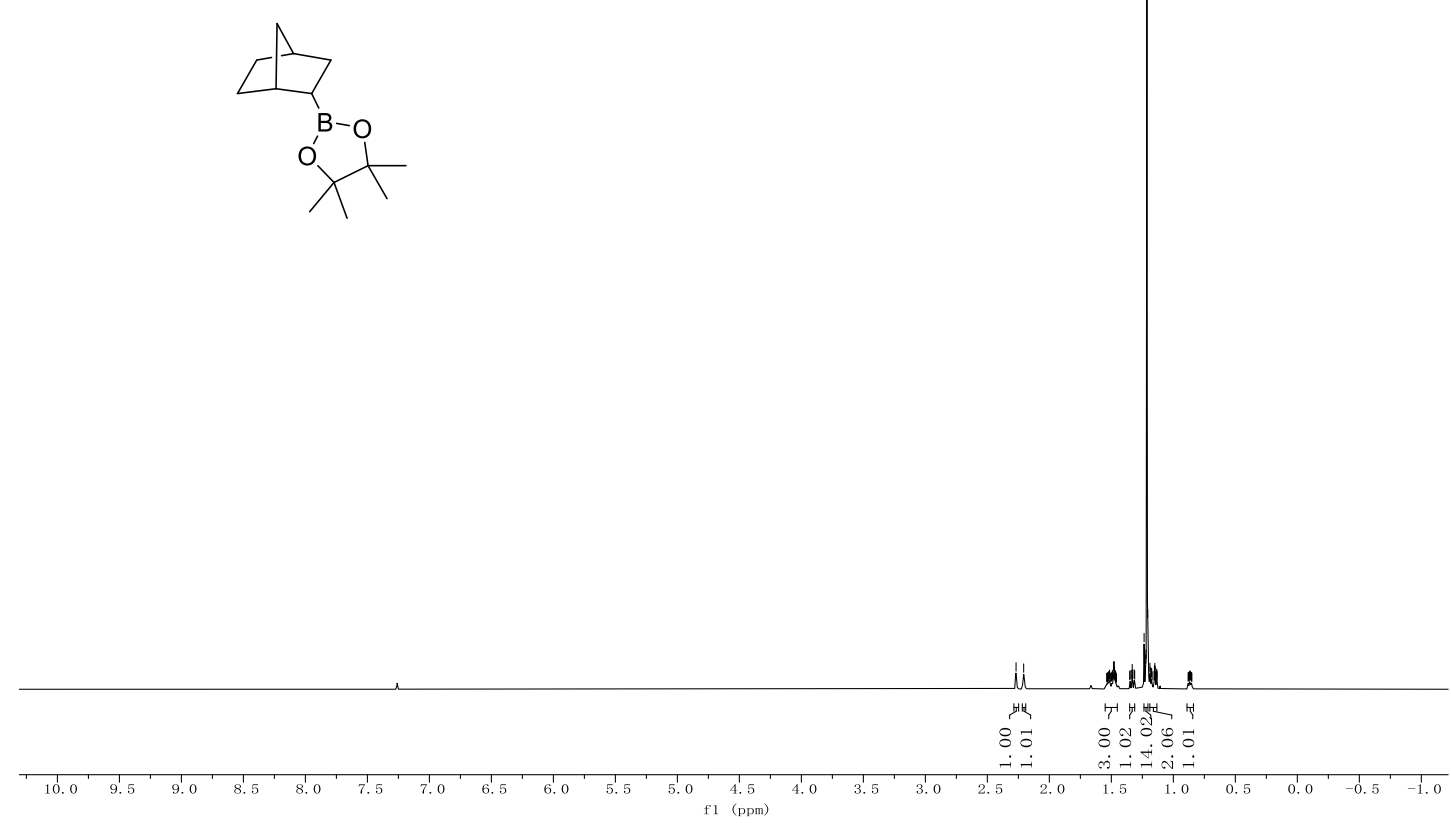




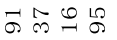

㐫令是

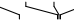

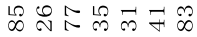

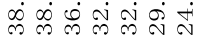
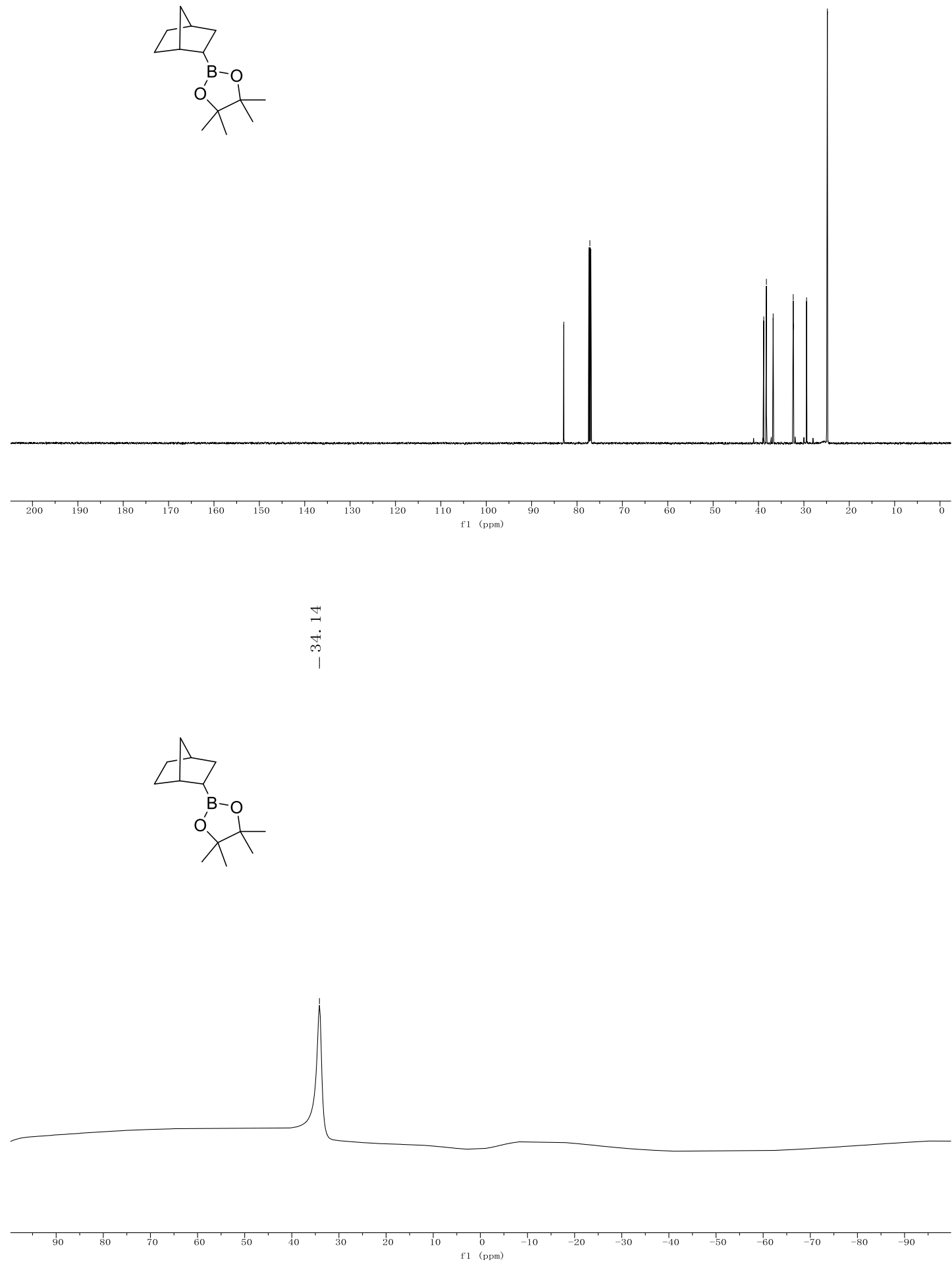


\section{Compound 29}

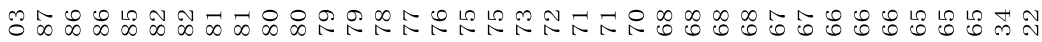

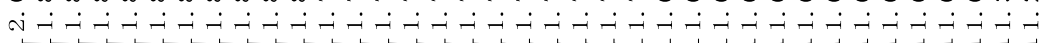

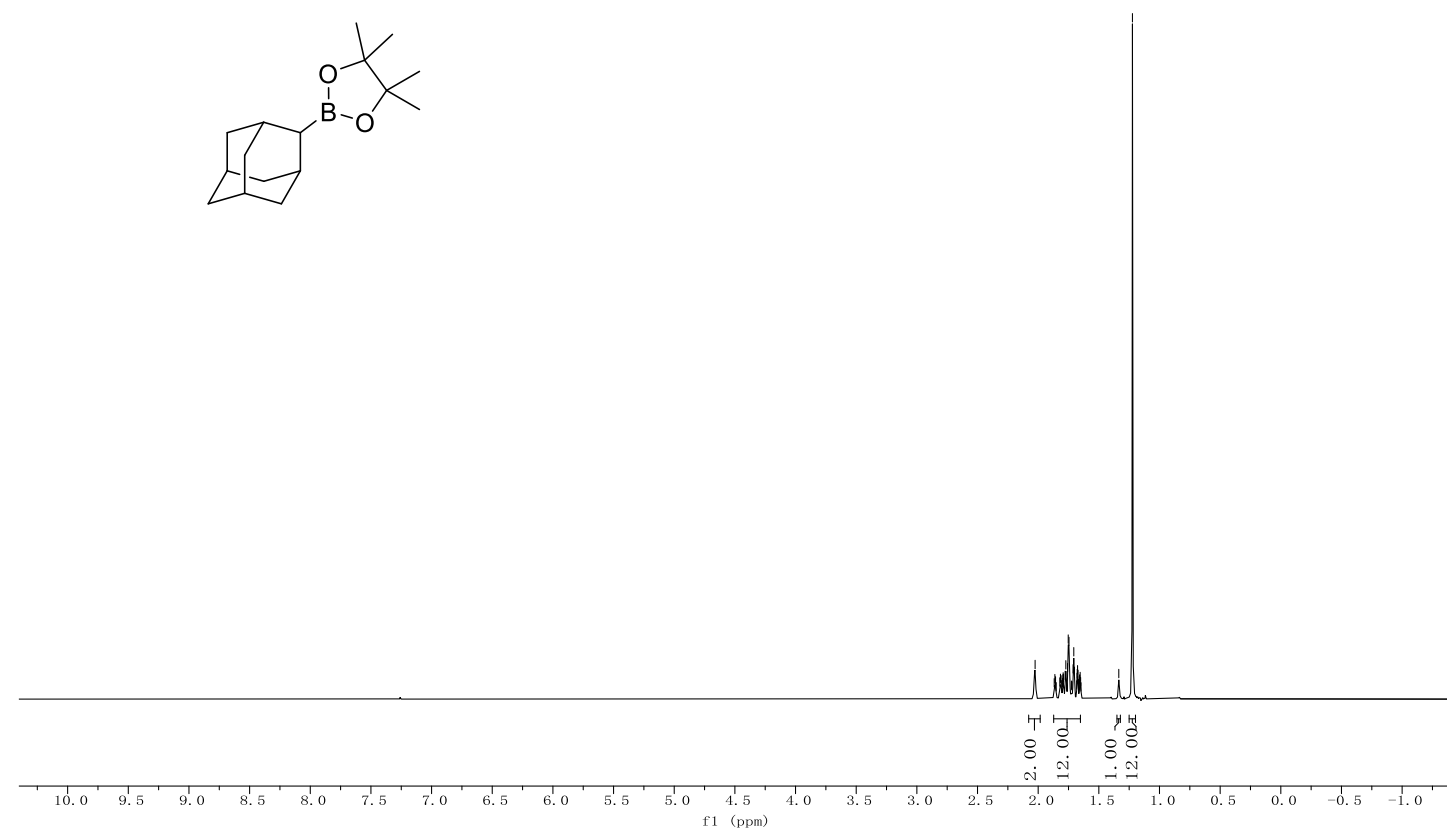

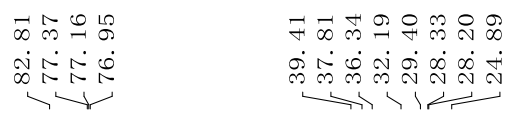<smiles>CC1(C)OB(C2C3CC4CC(C3)C2C4)OC1(C)C</smiles>

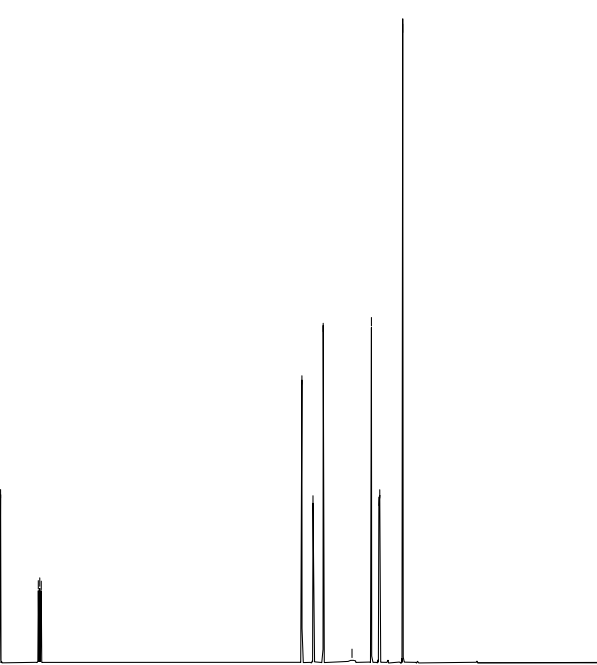

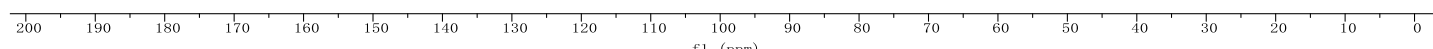



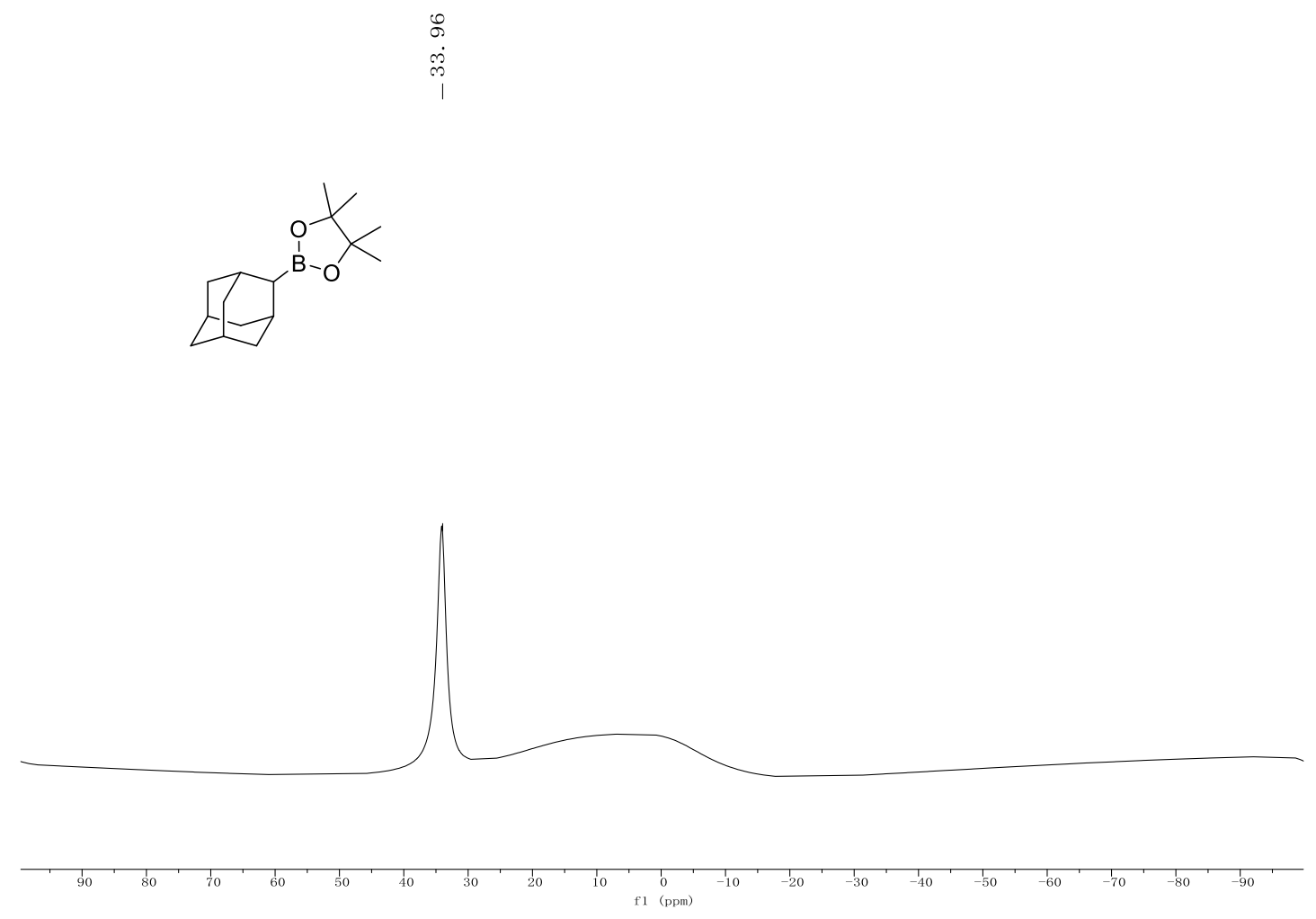

\section{Compound 30}

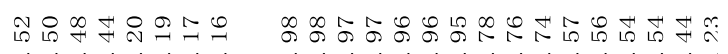

ம

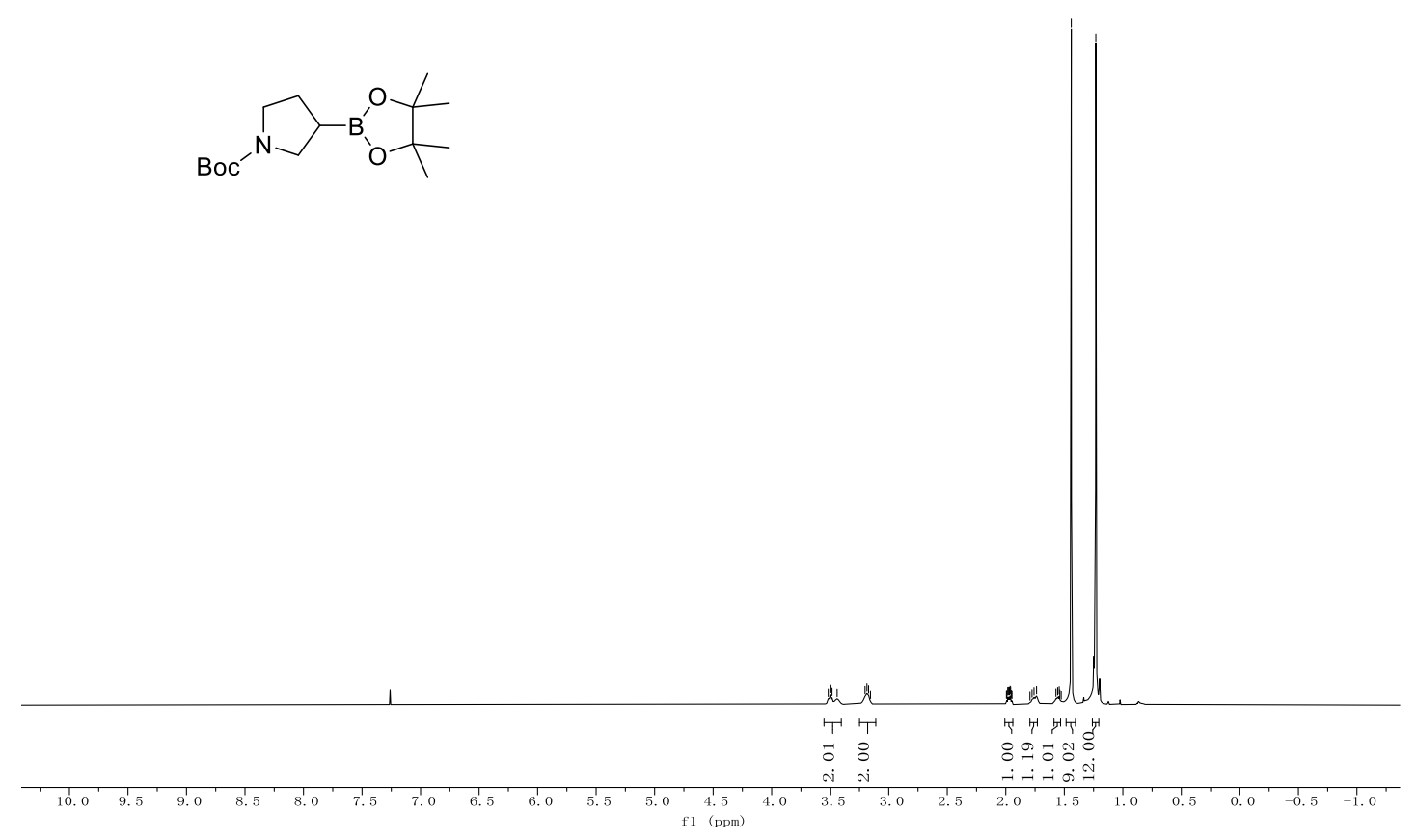


울

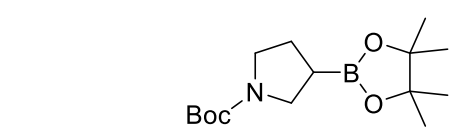

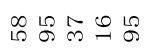

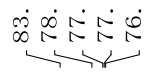

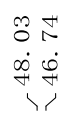

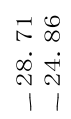

$\vec{\infty}$
$\infty$
$\infty$
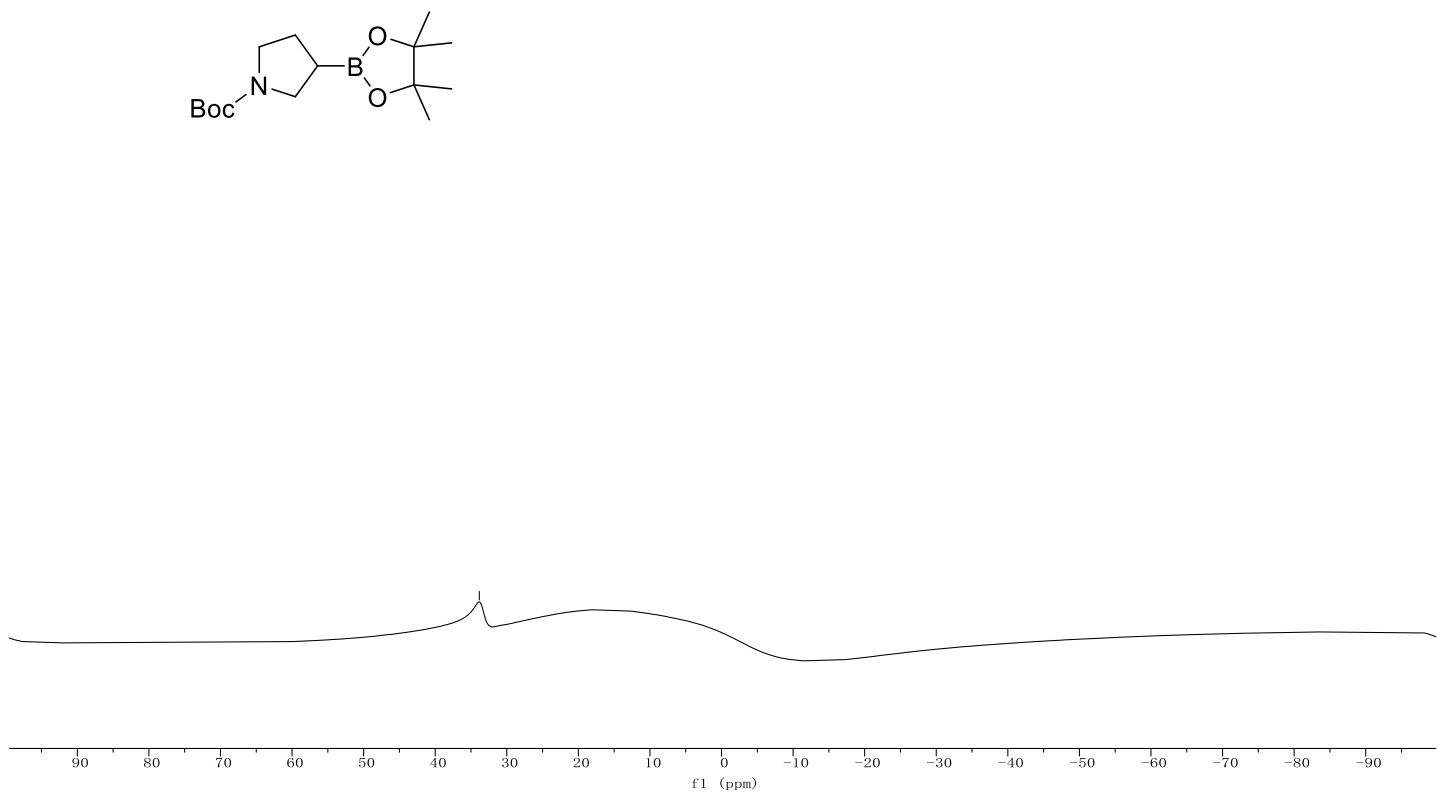

S130 


\section{Compound 31}

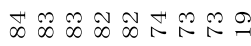

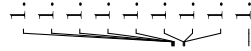

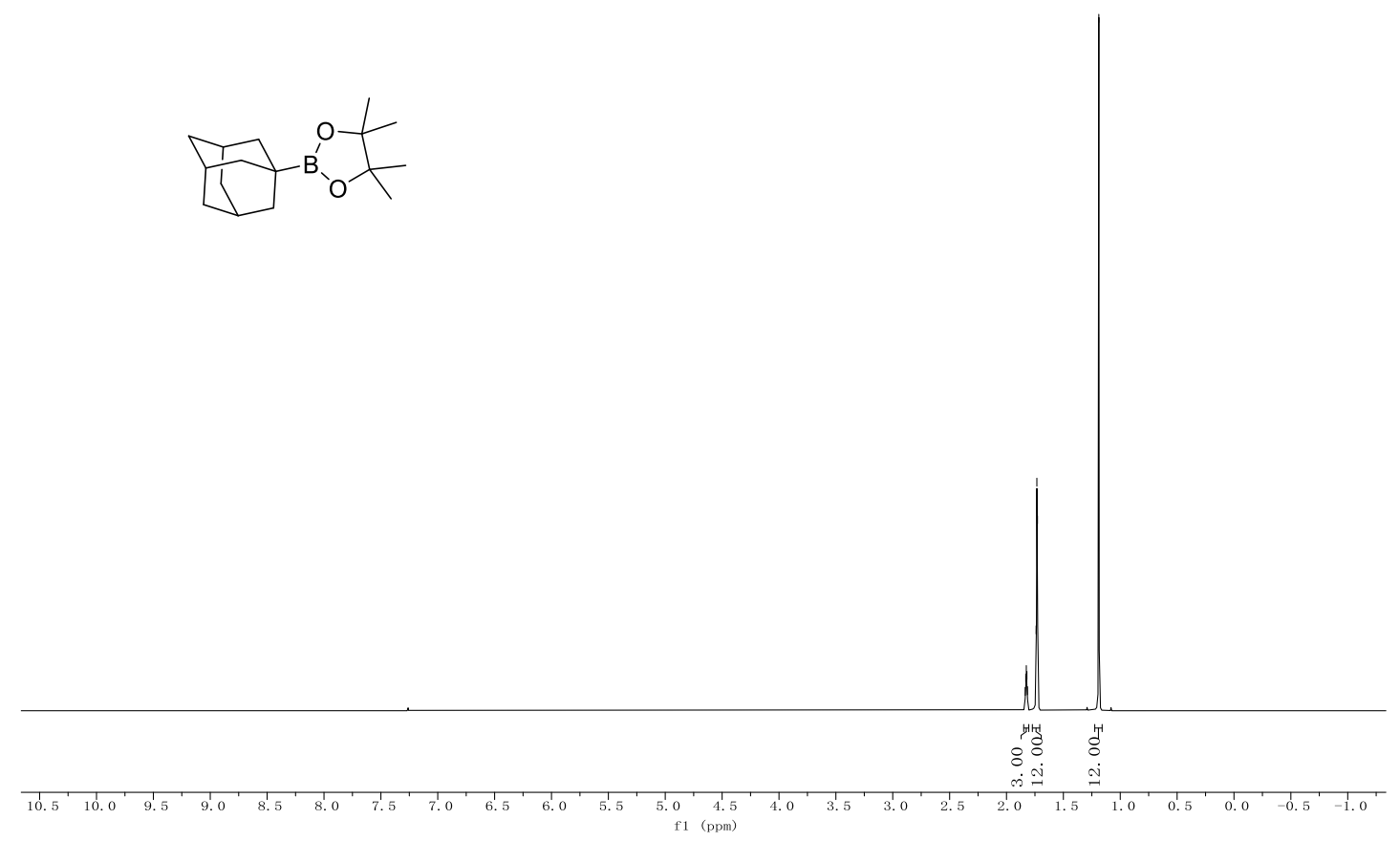

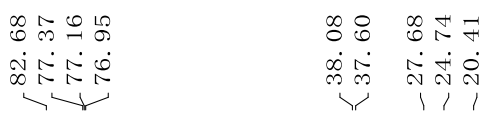

4 B.

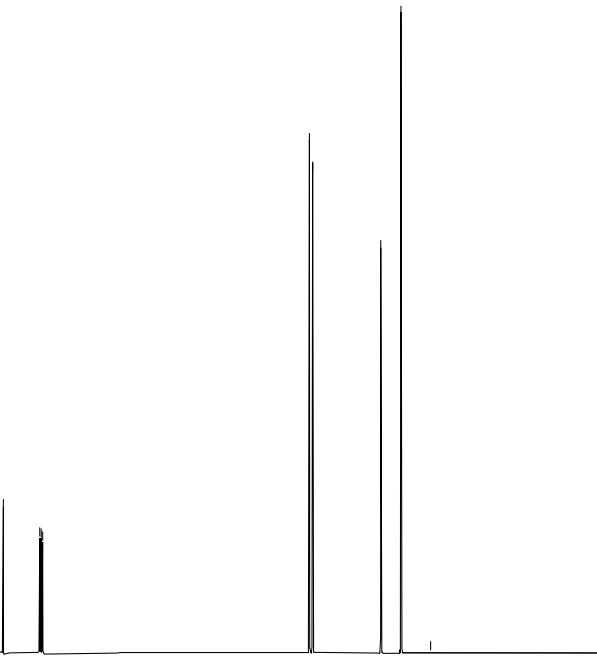

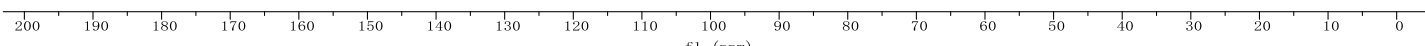




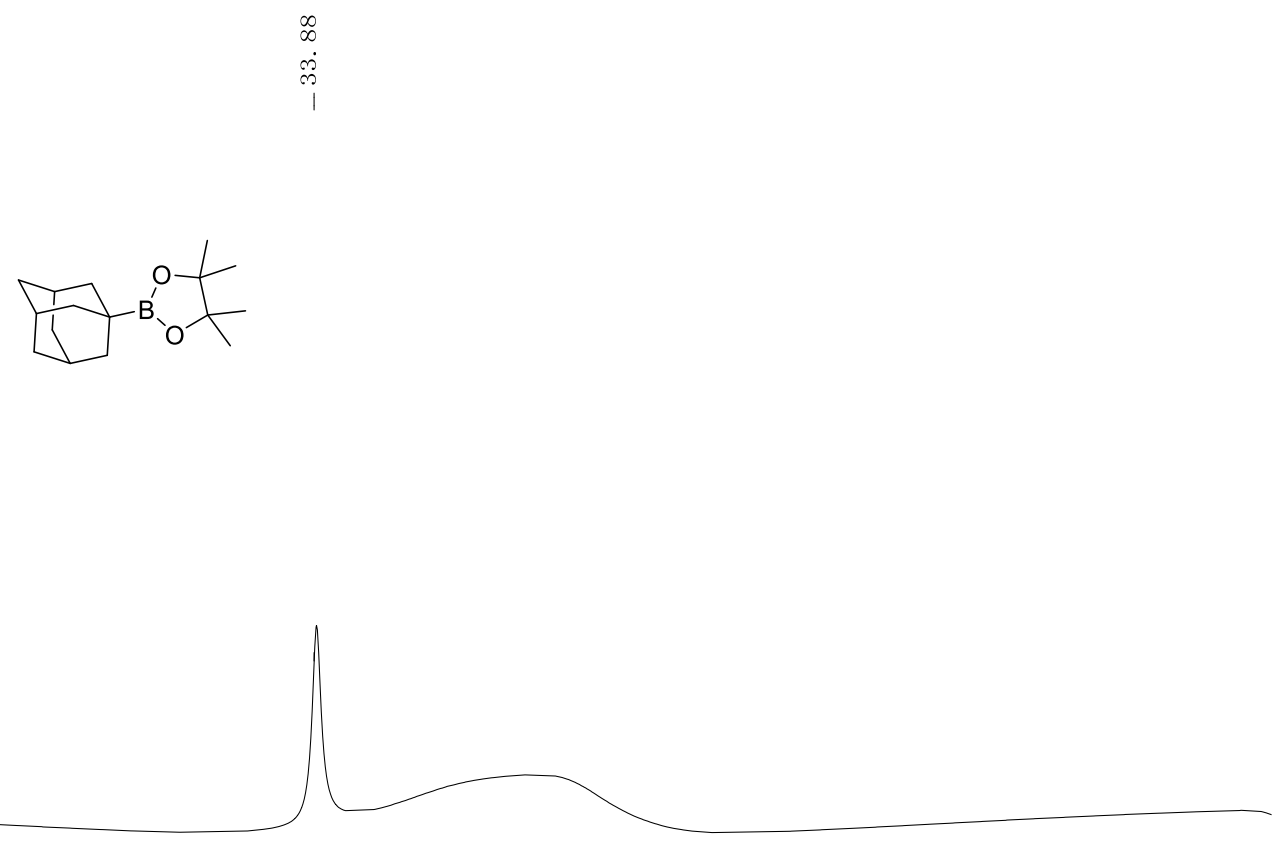

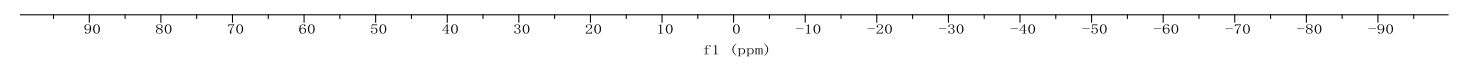

\section{Compound 32}

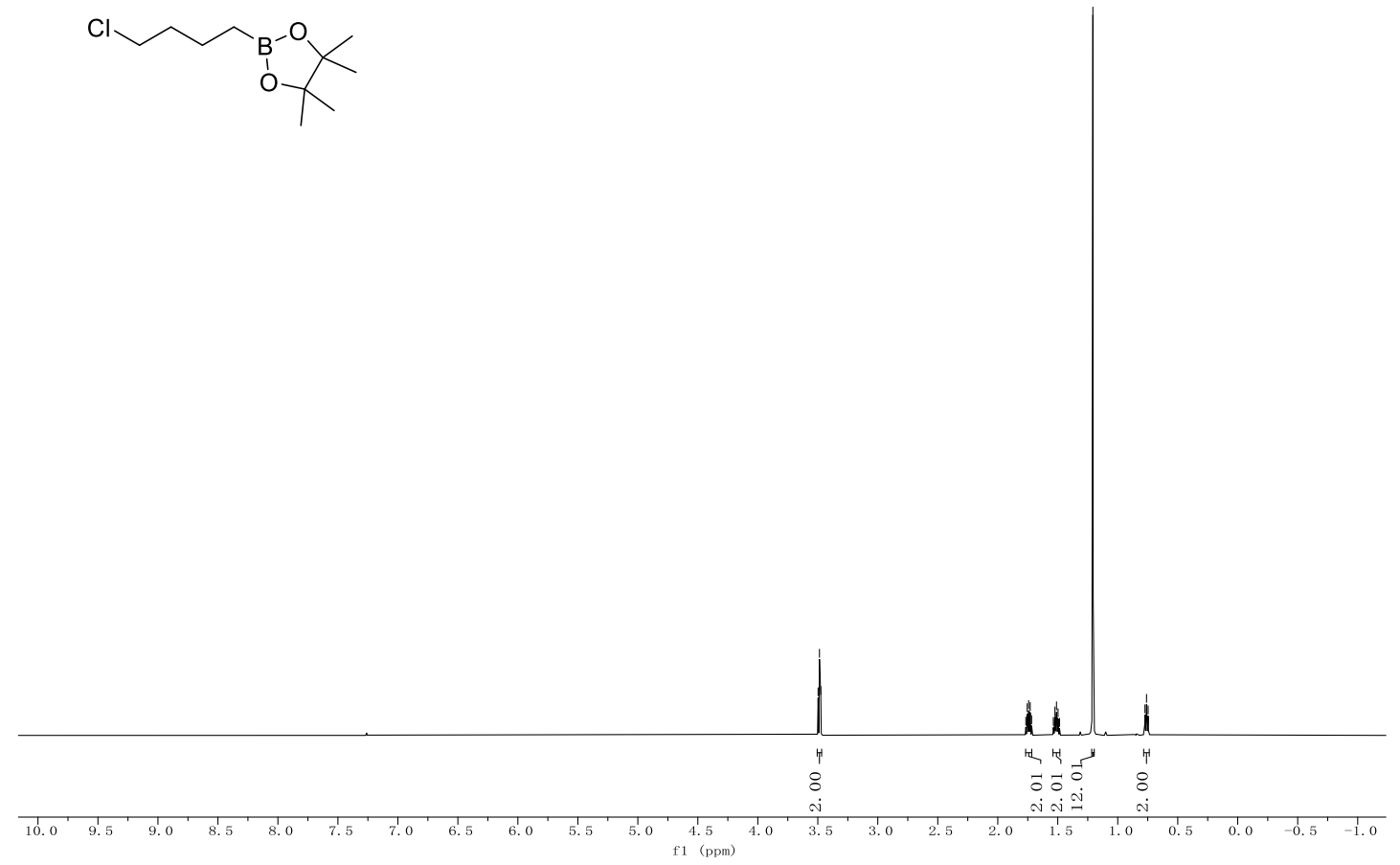




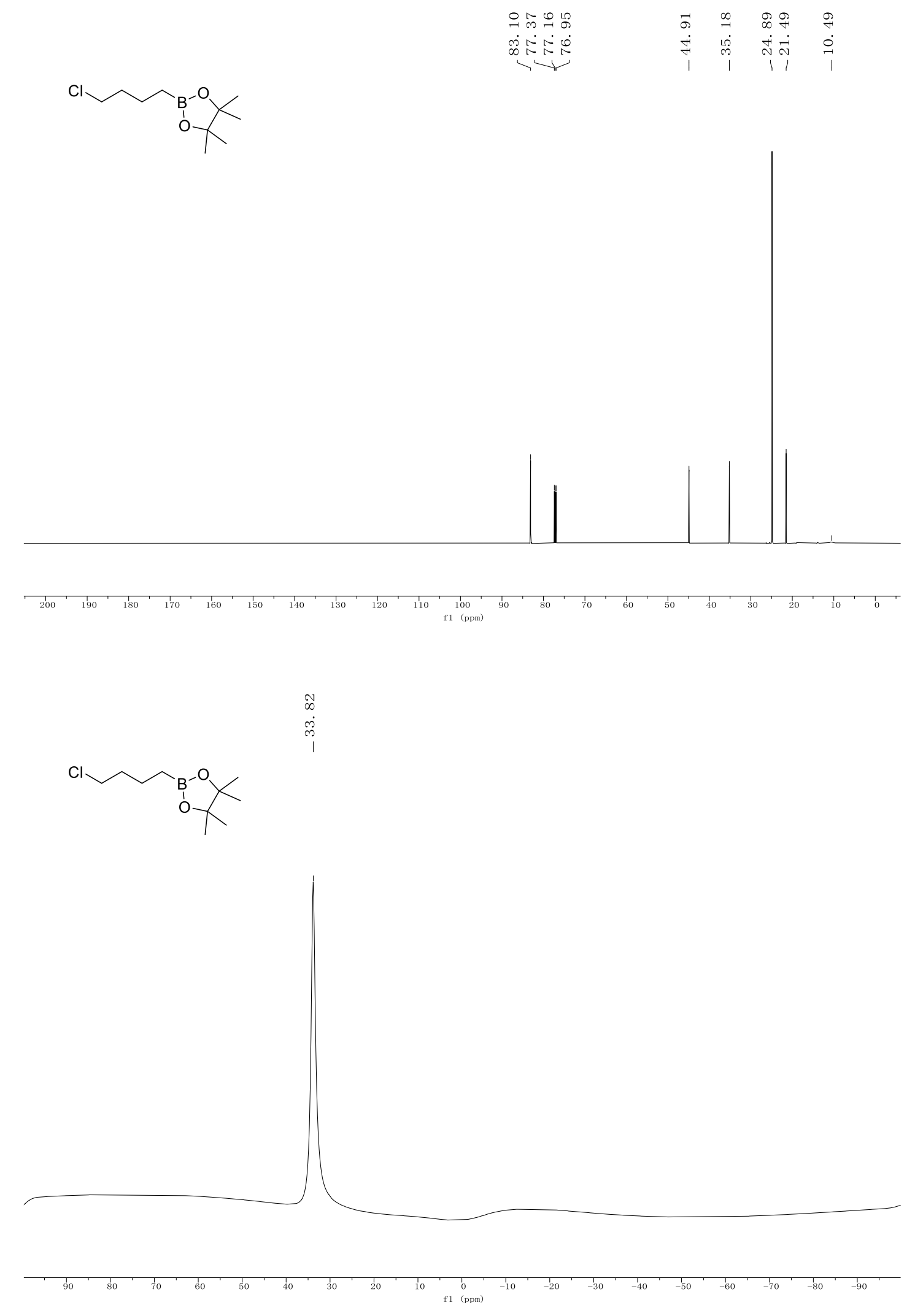




\section{Compound 33}

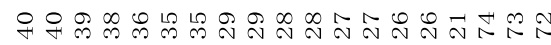

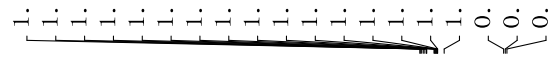<smiles>CC1(C)OB(CCCCCCB2OC(C)(C)C(C)(C)O2)OC1(C)C</smiles>

호요용

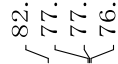

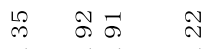

飠

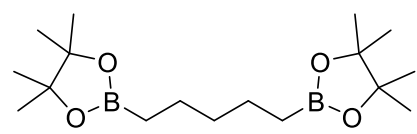

u.

50

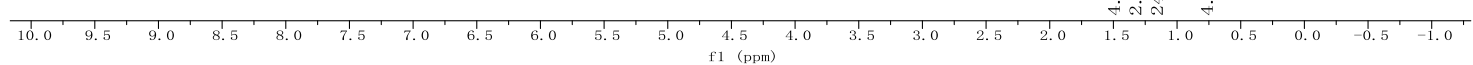

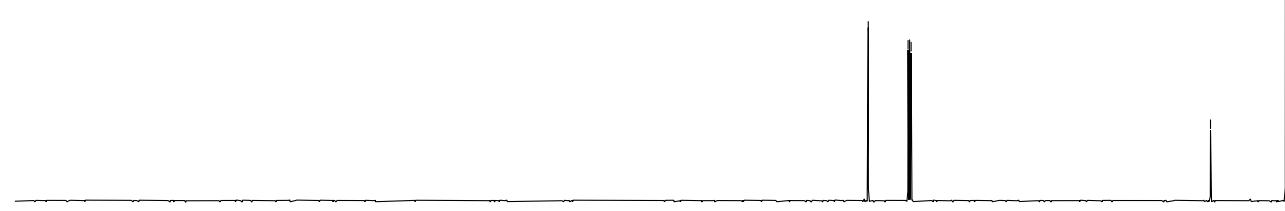

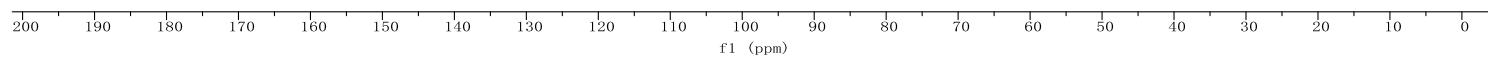



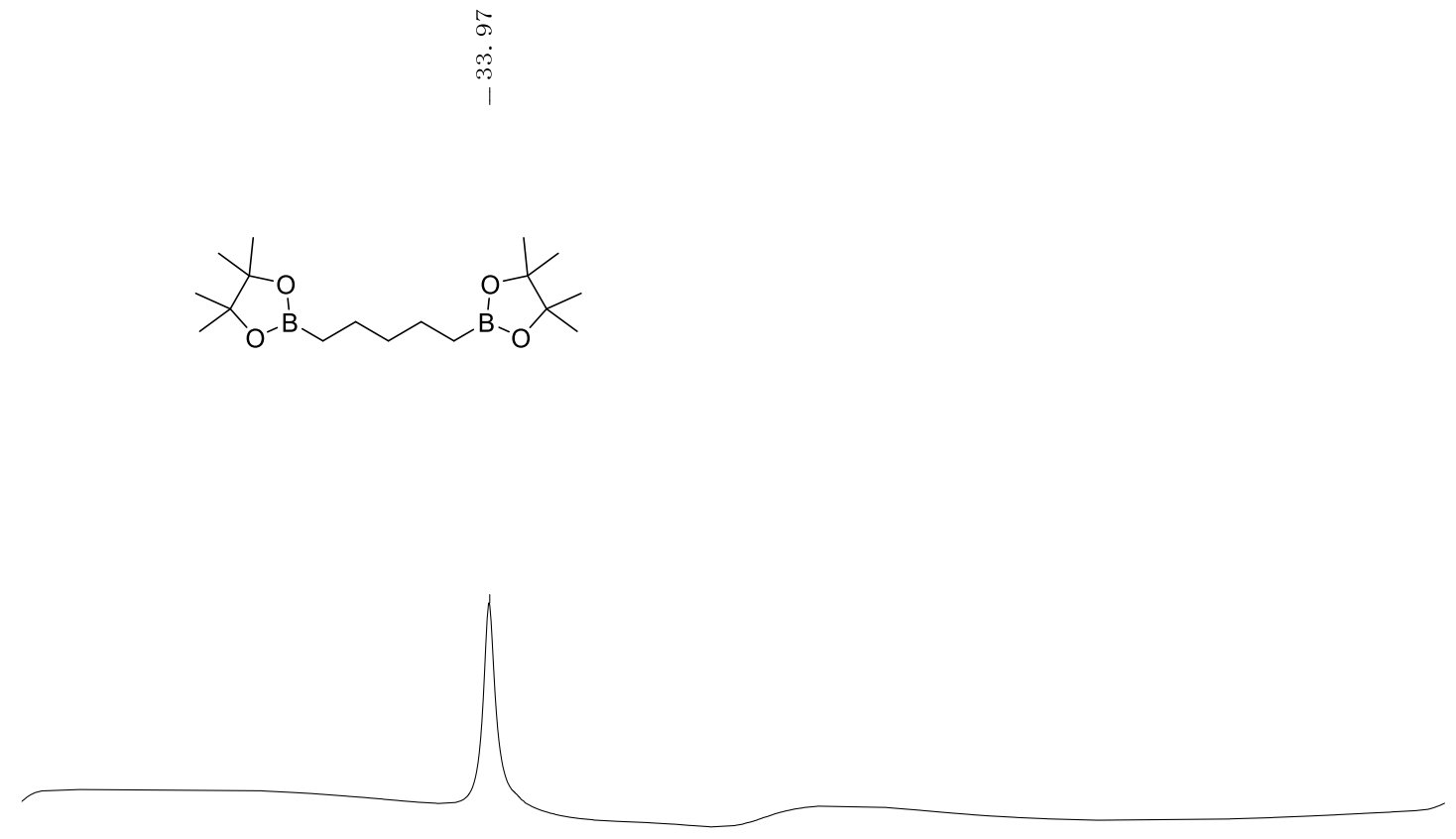

\section{Compound 34}

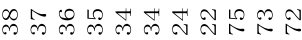

ت-ifi-ijio

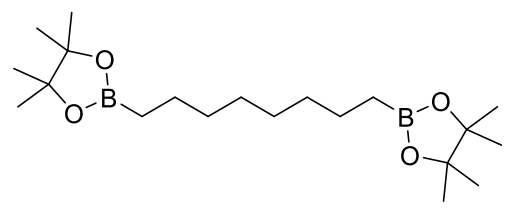


б승요용

这这定定

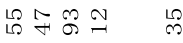

证

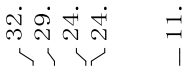

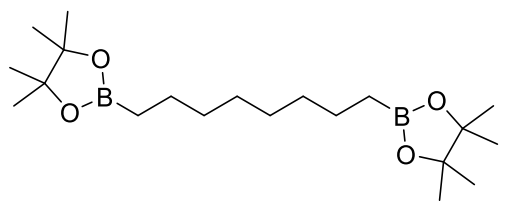
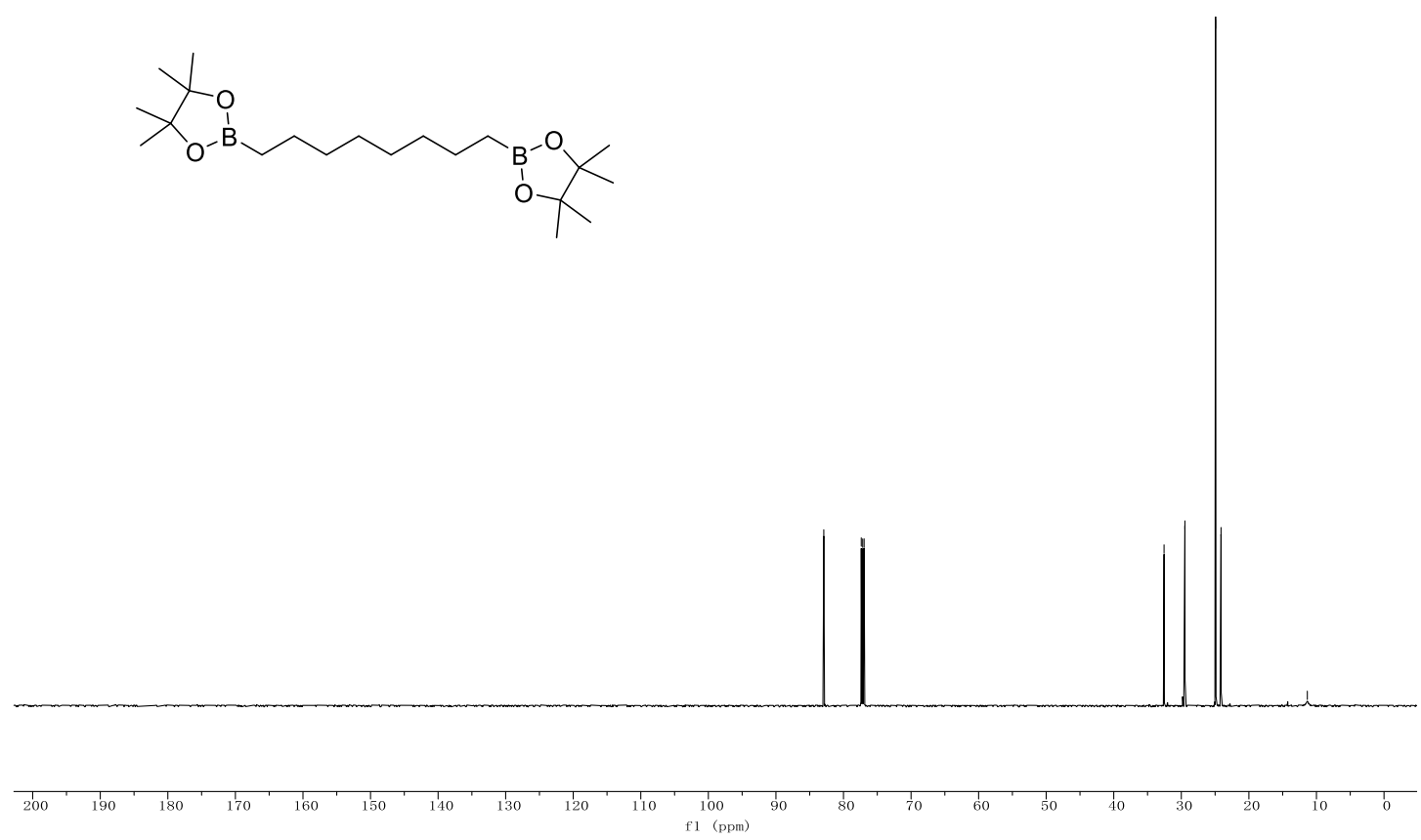

$\stackrel{\vec{H}}{\vec{m}}$

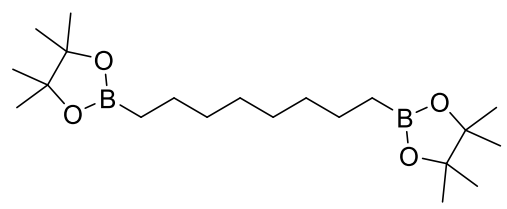

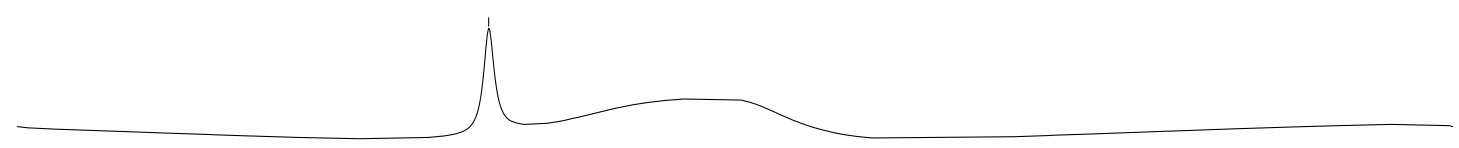




\section{Compound 35}

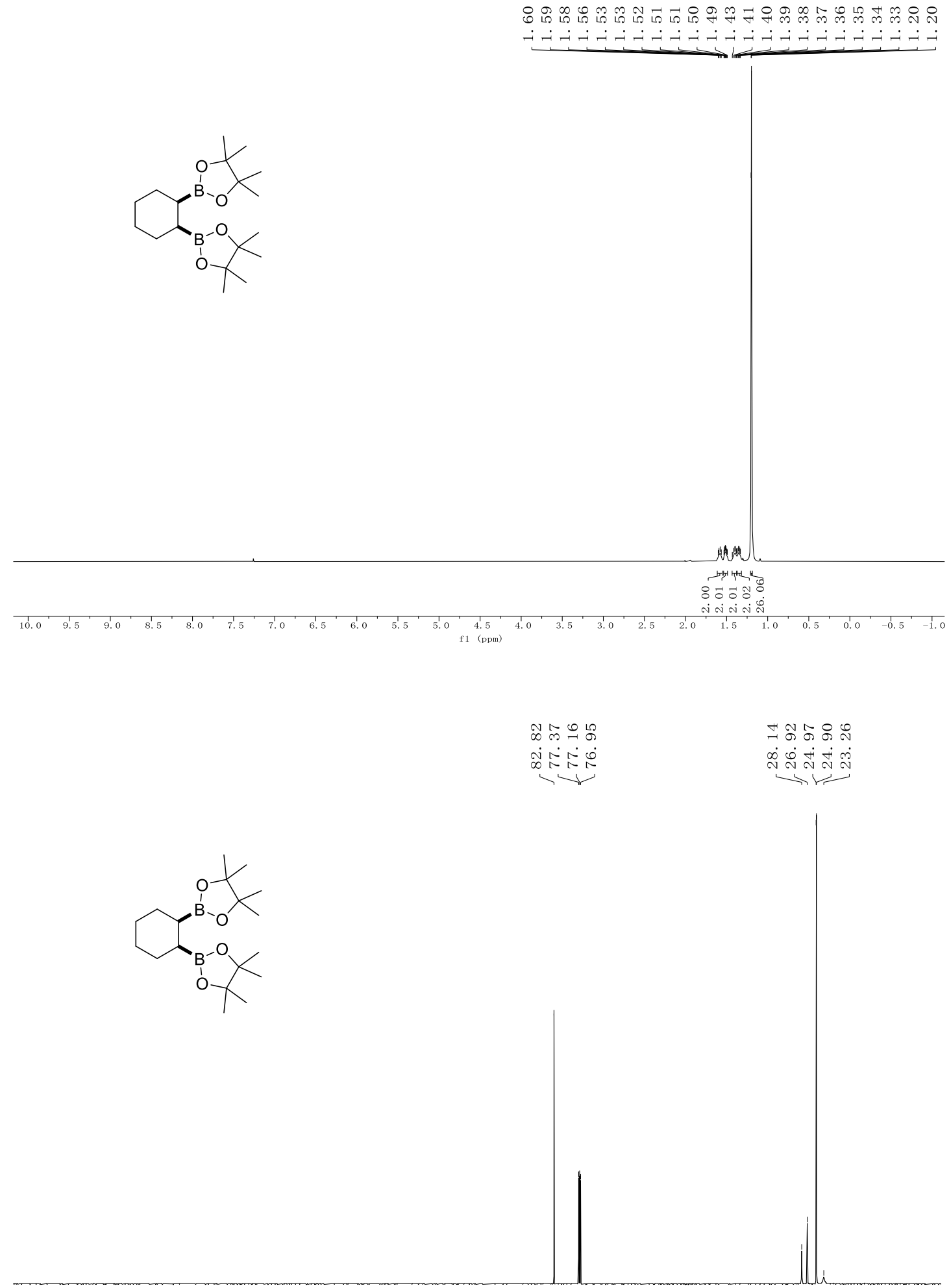

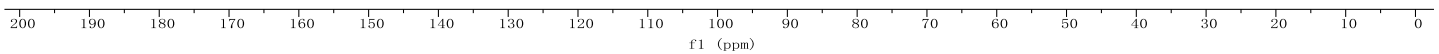




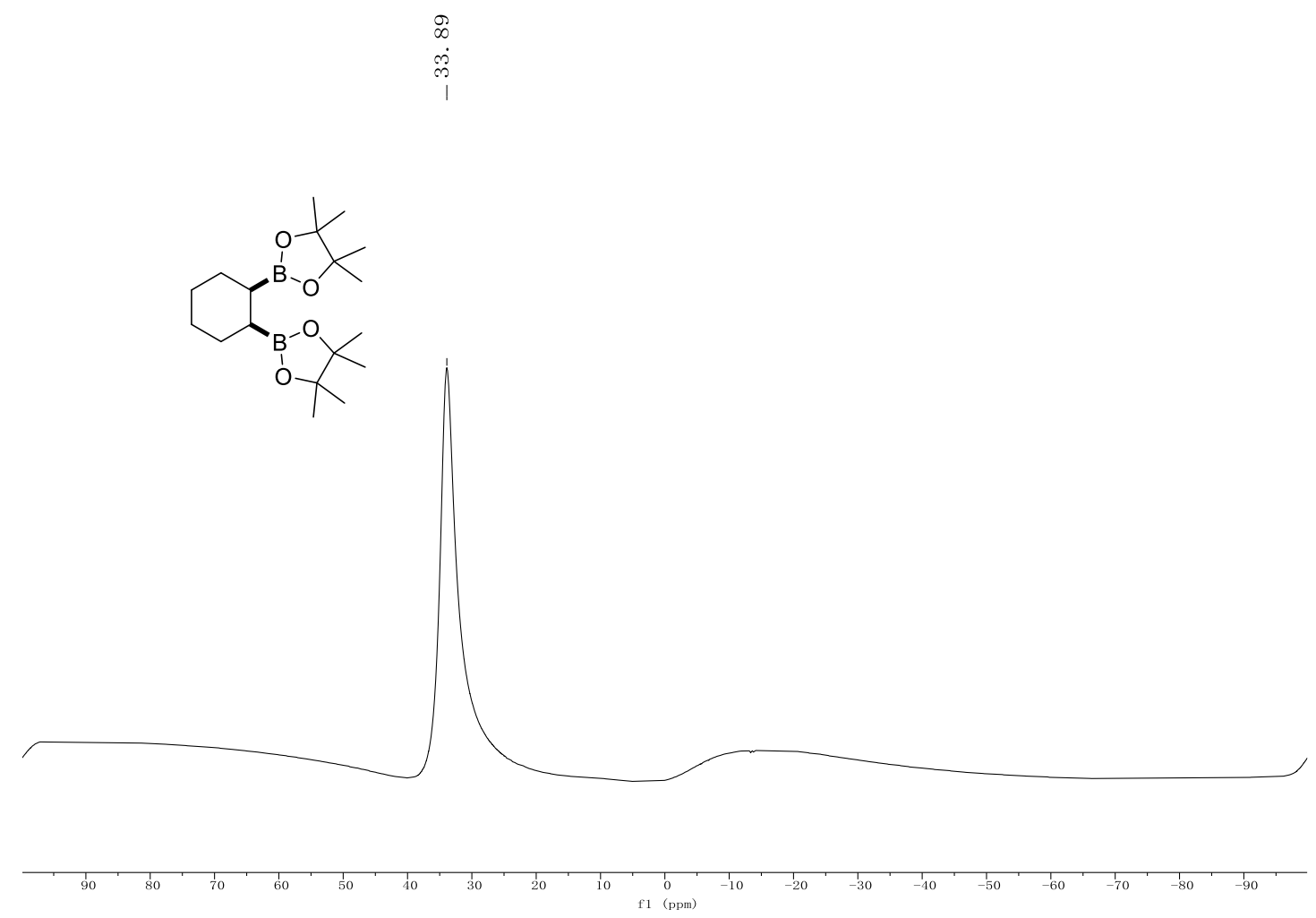

\section{Compound 36}

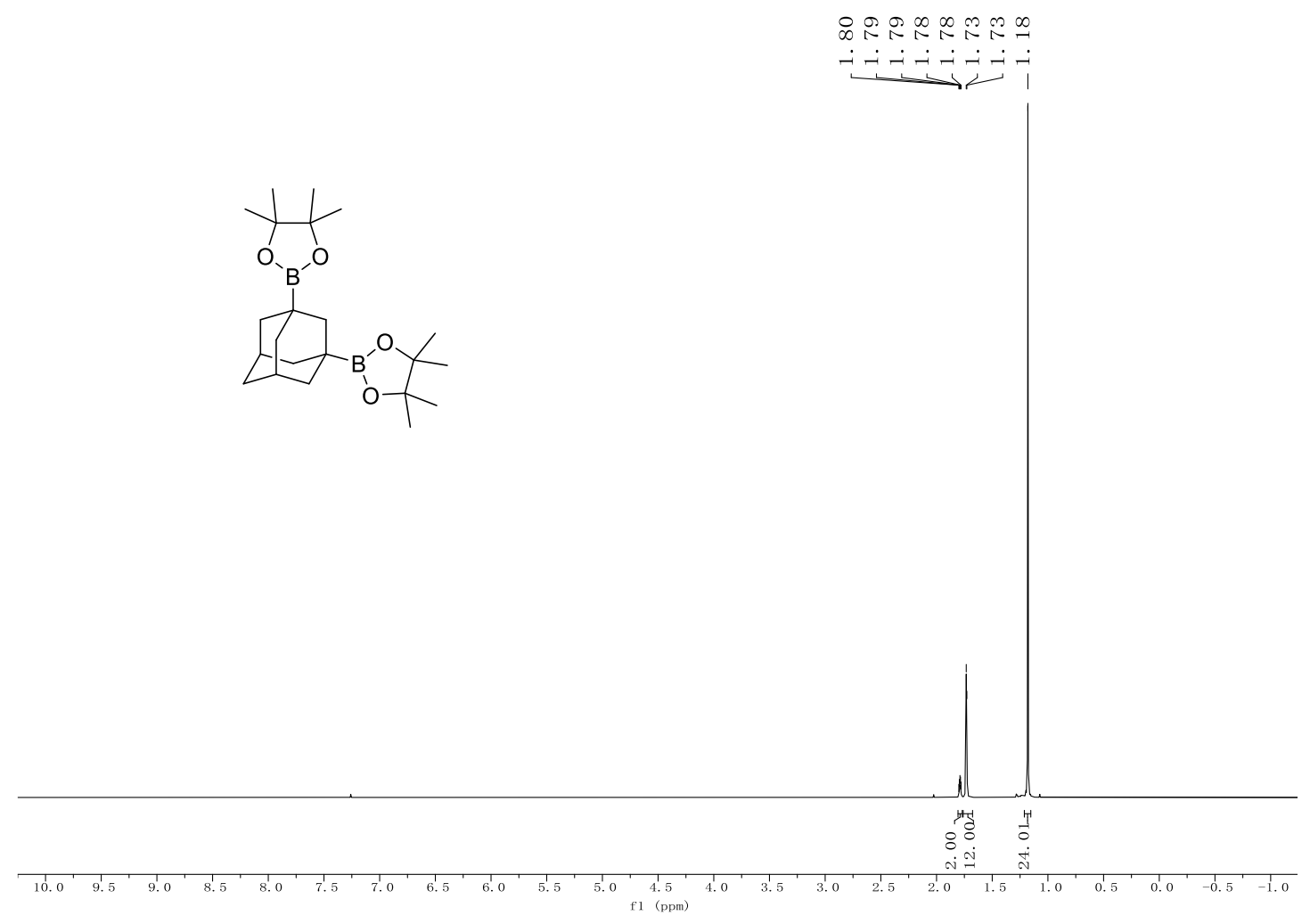


융숭으용

证余完

$\infty_{\infty}^{\infty} \underset{\infty}{\infty} \tilde{\sim}$

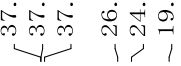
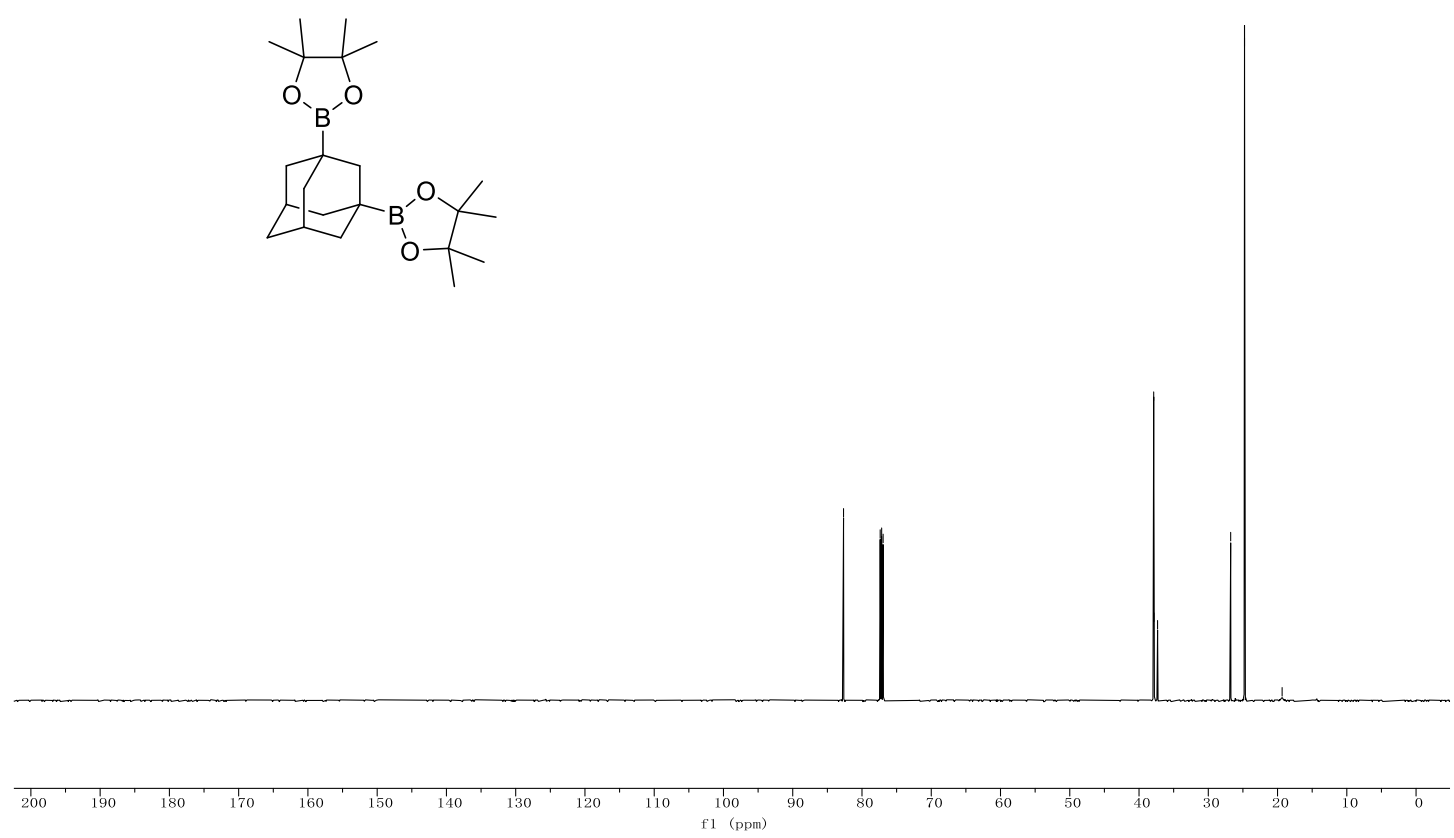

$\stackrel{0}{N}$
$\dot{n}$
1
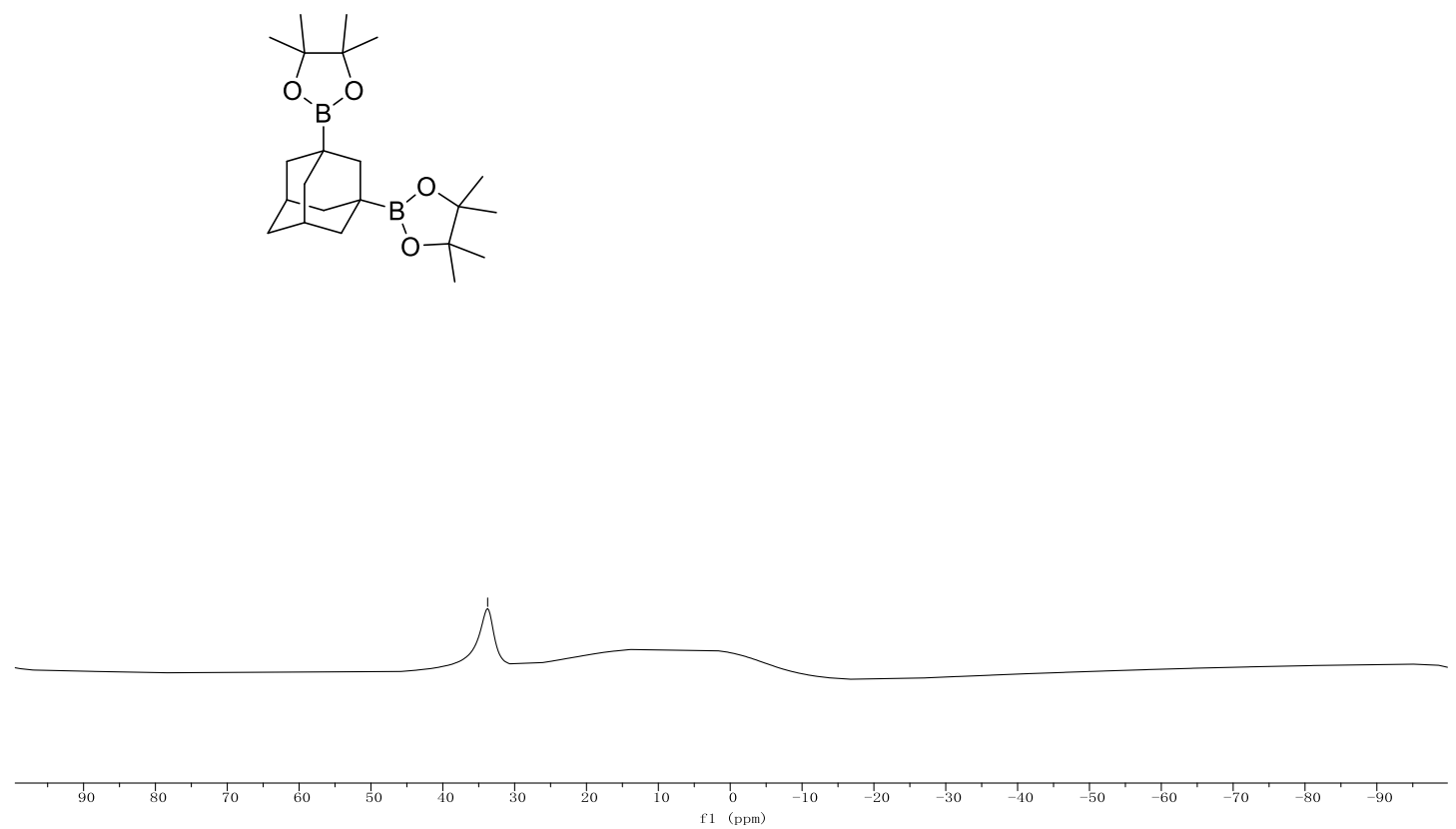
Compound 37

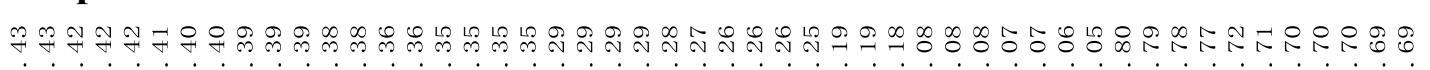

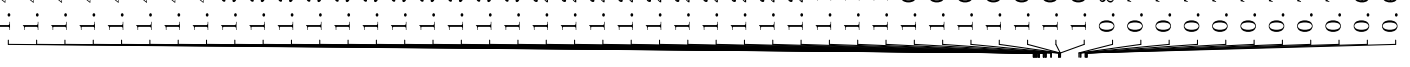

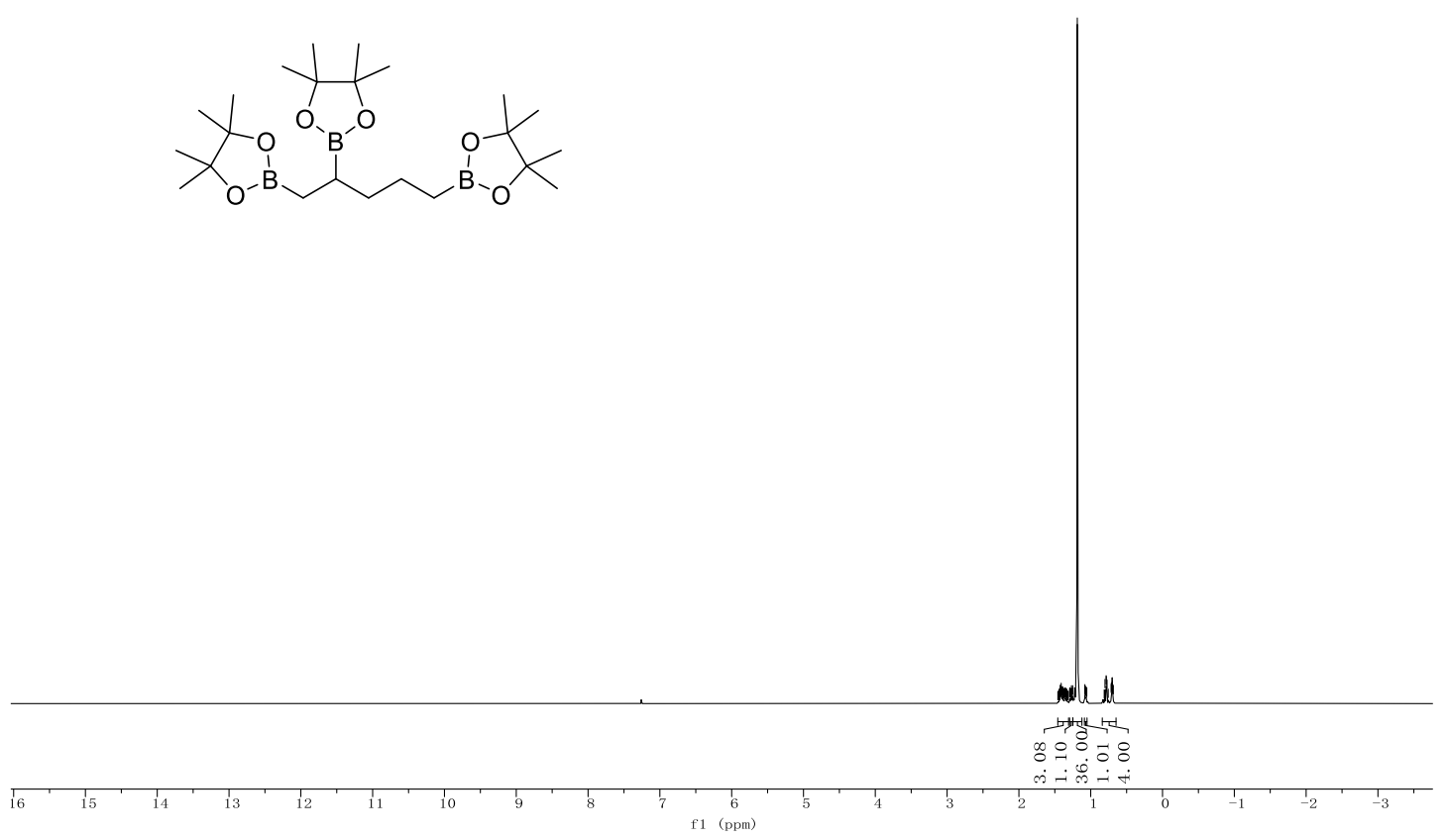

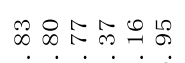

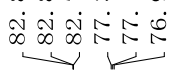

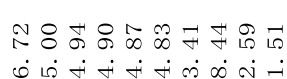

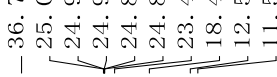

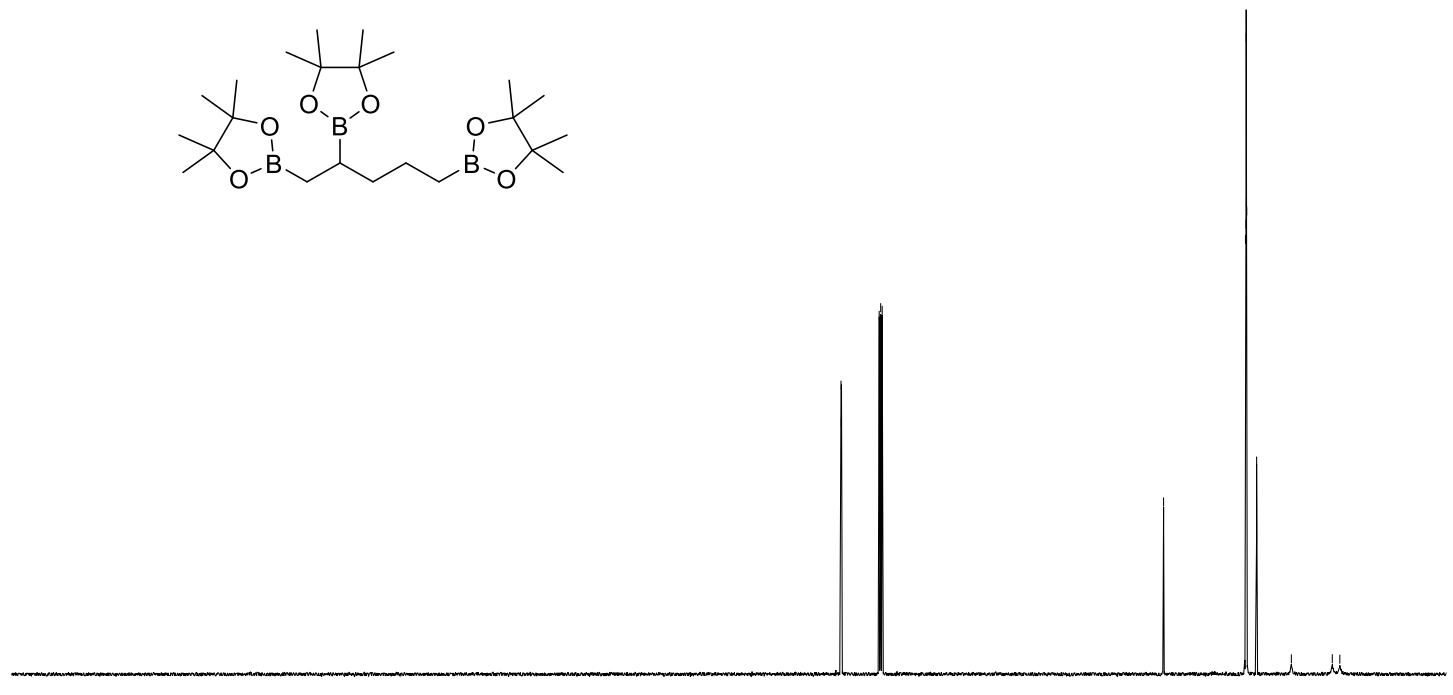

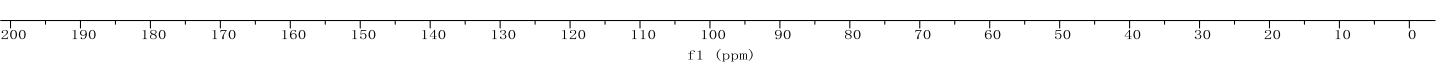

S140 

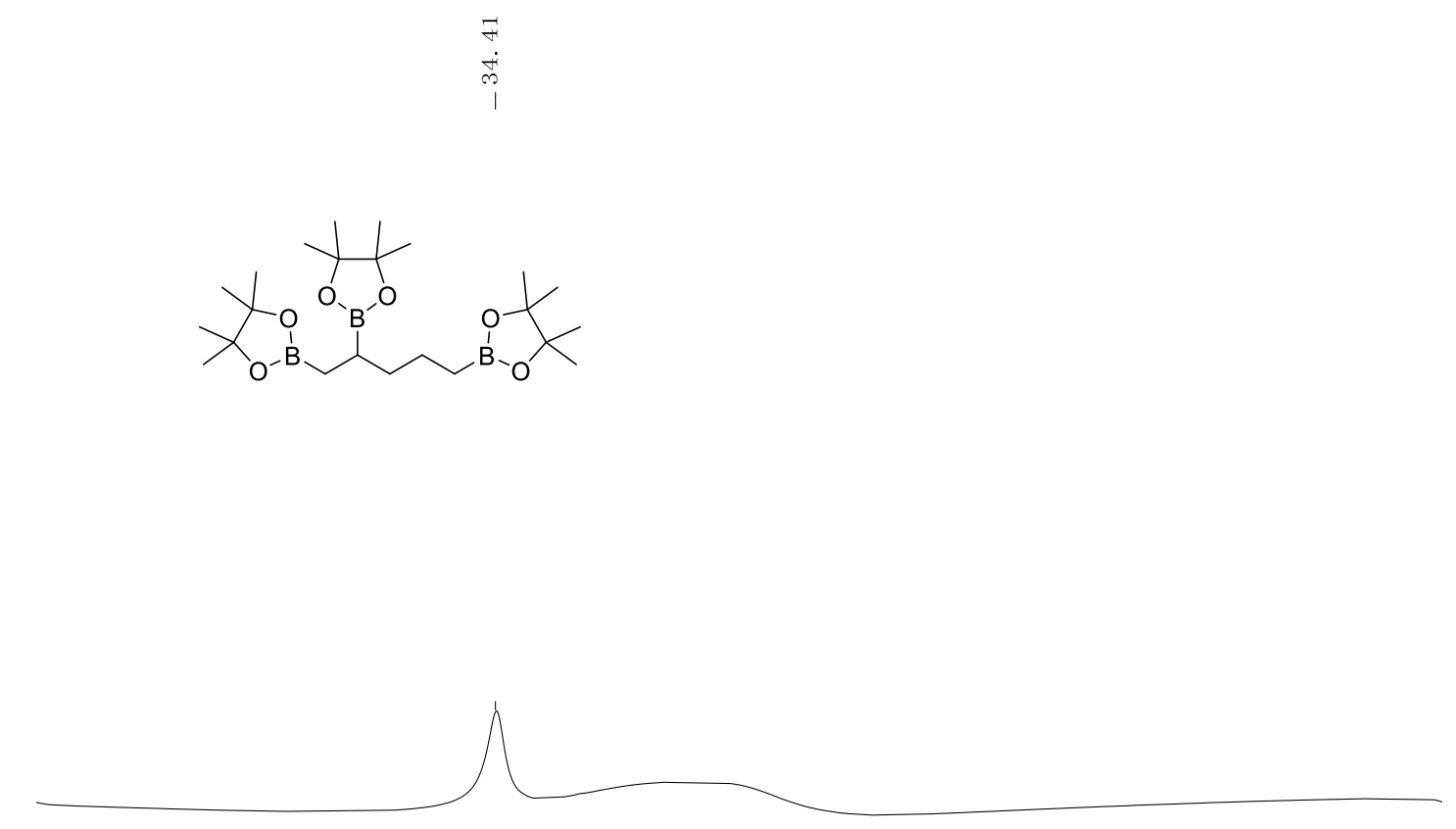

\section{Compound 38}

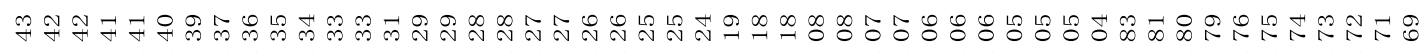

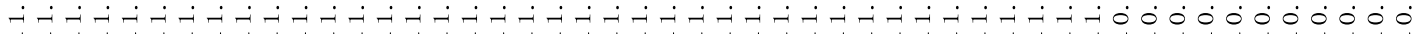

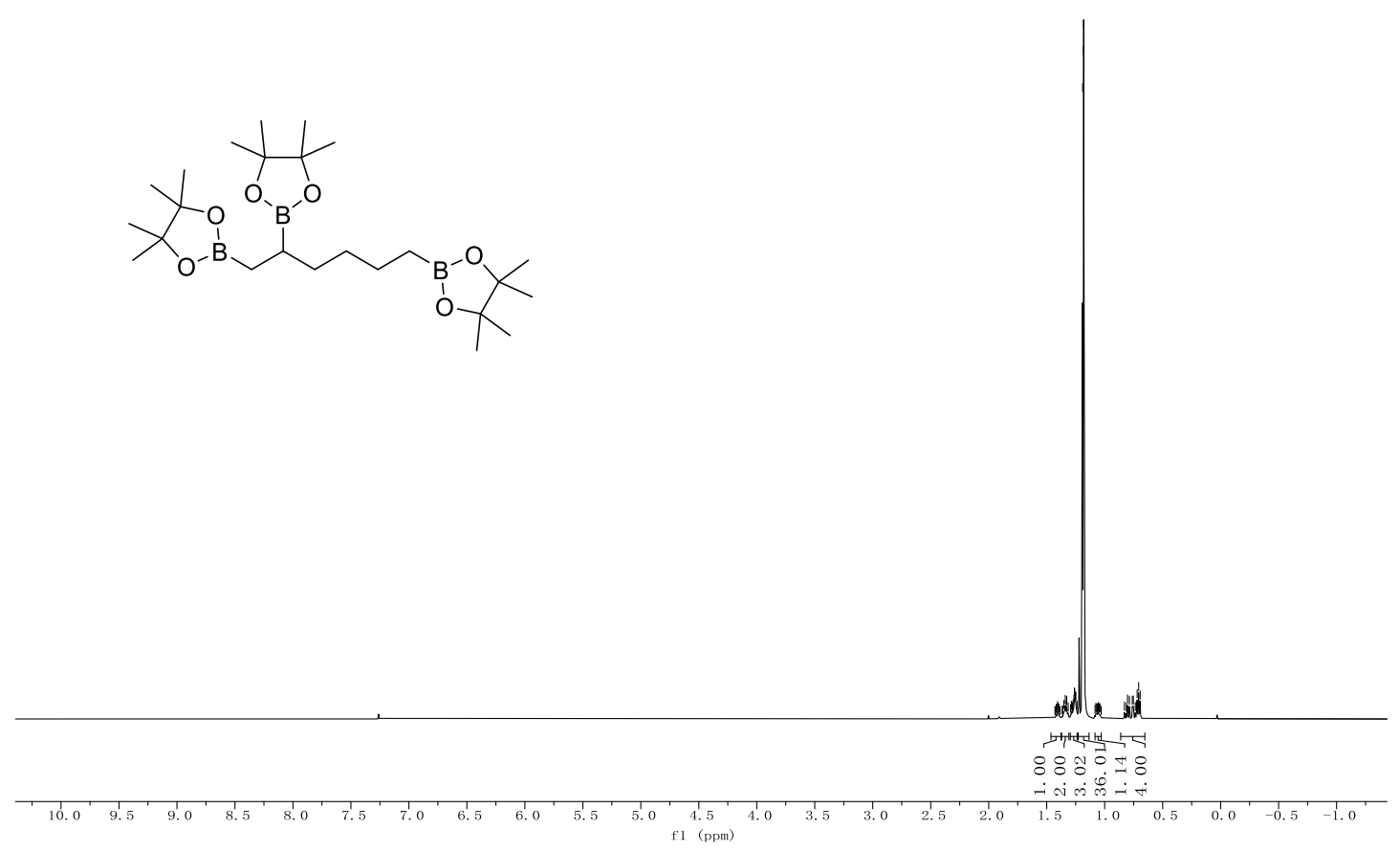




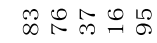

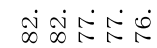

$\longrightarrow x$
총요용요 œ

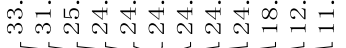

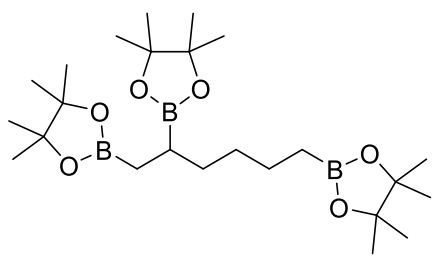

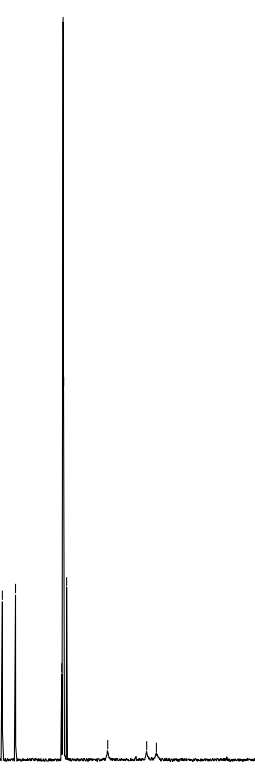

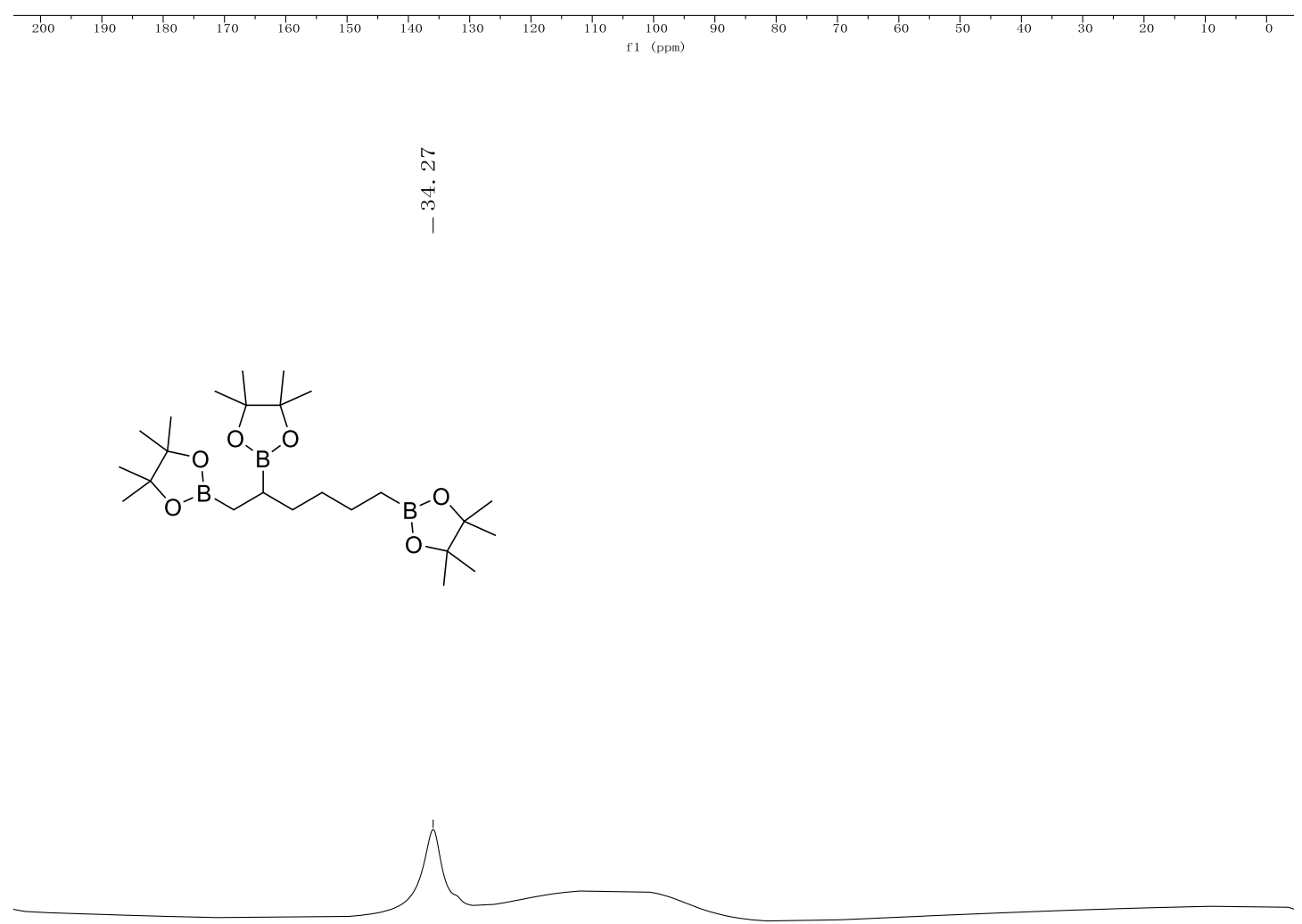




\section{Compound 39}

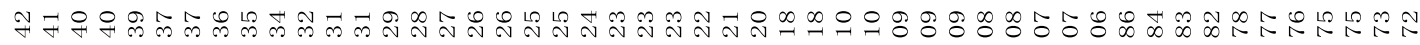

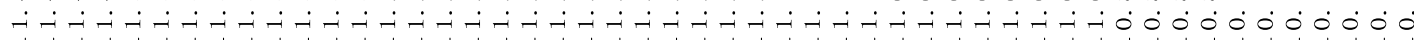

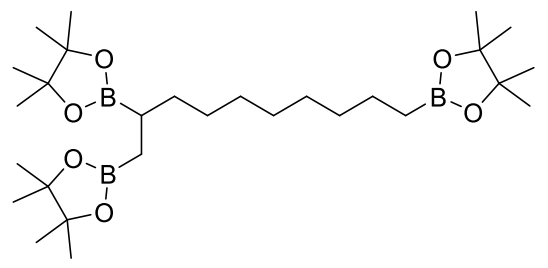

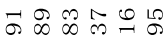

is

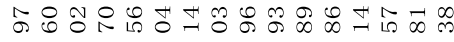
ळ்

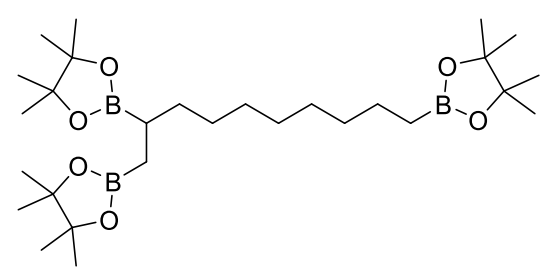

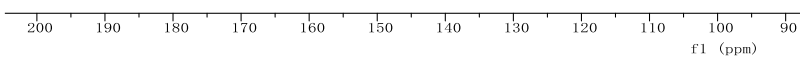



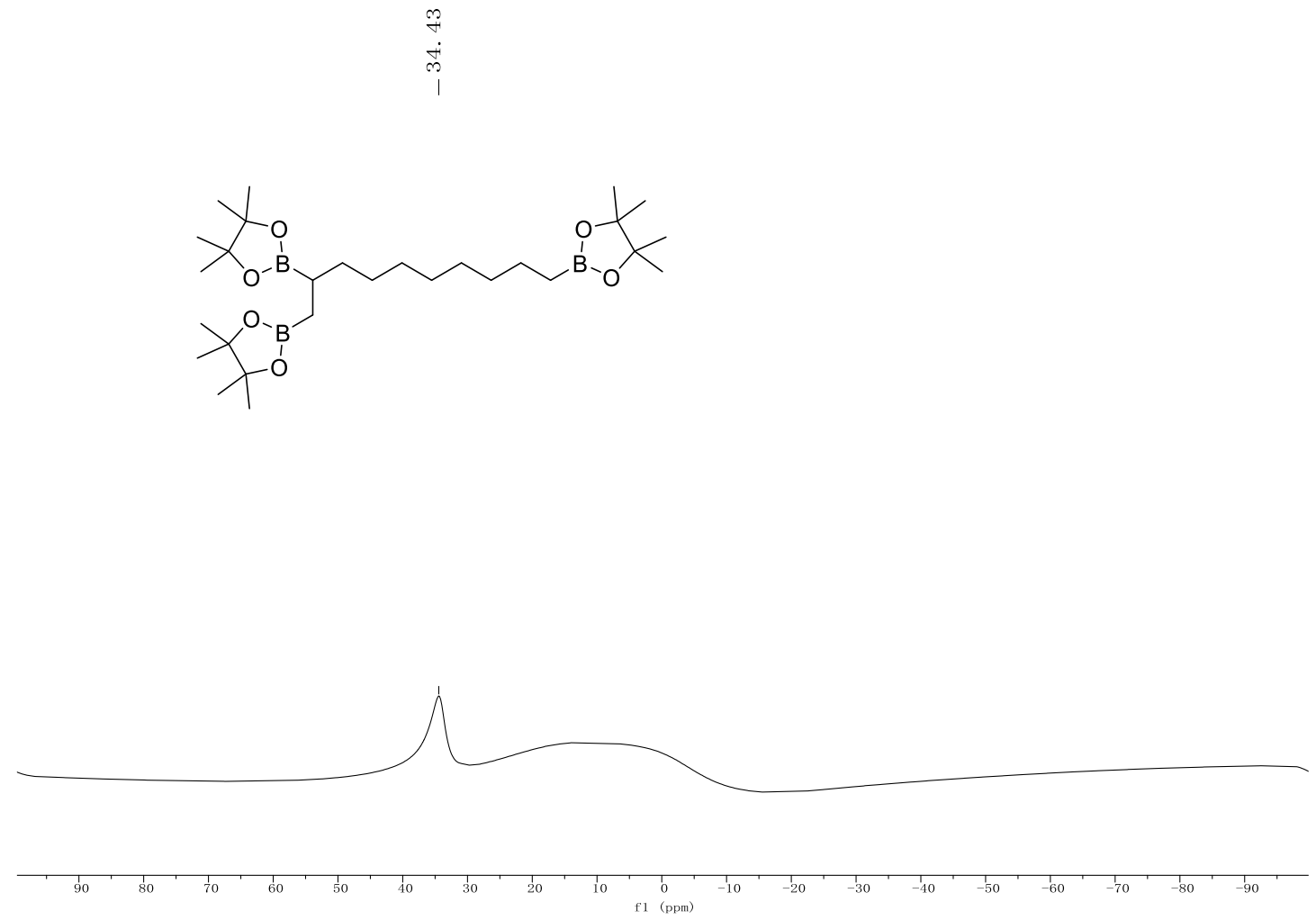

\section{Compound 40}
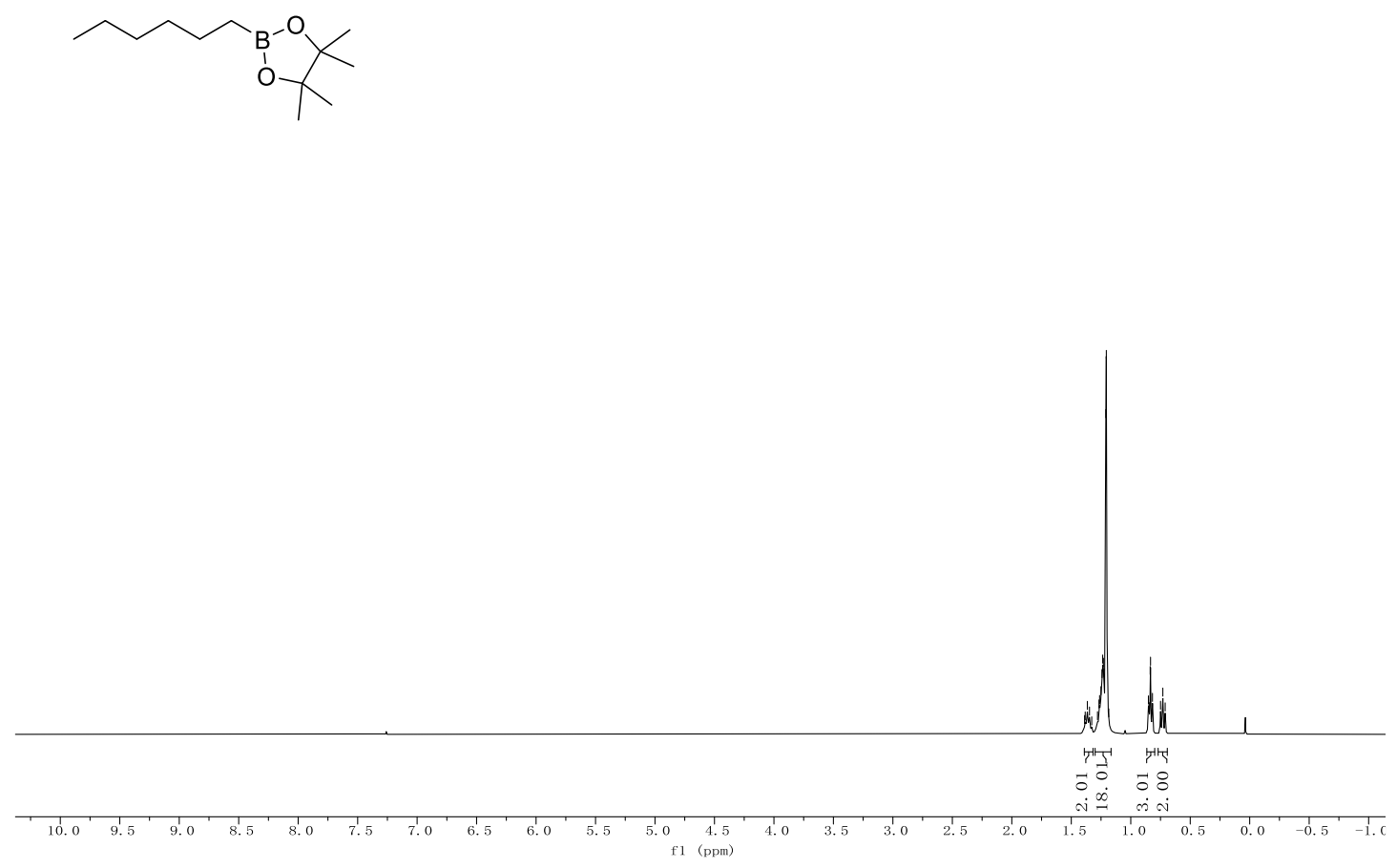

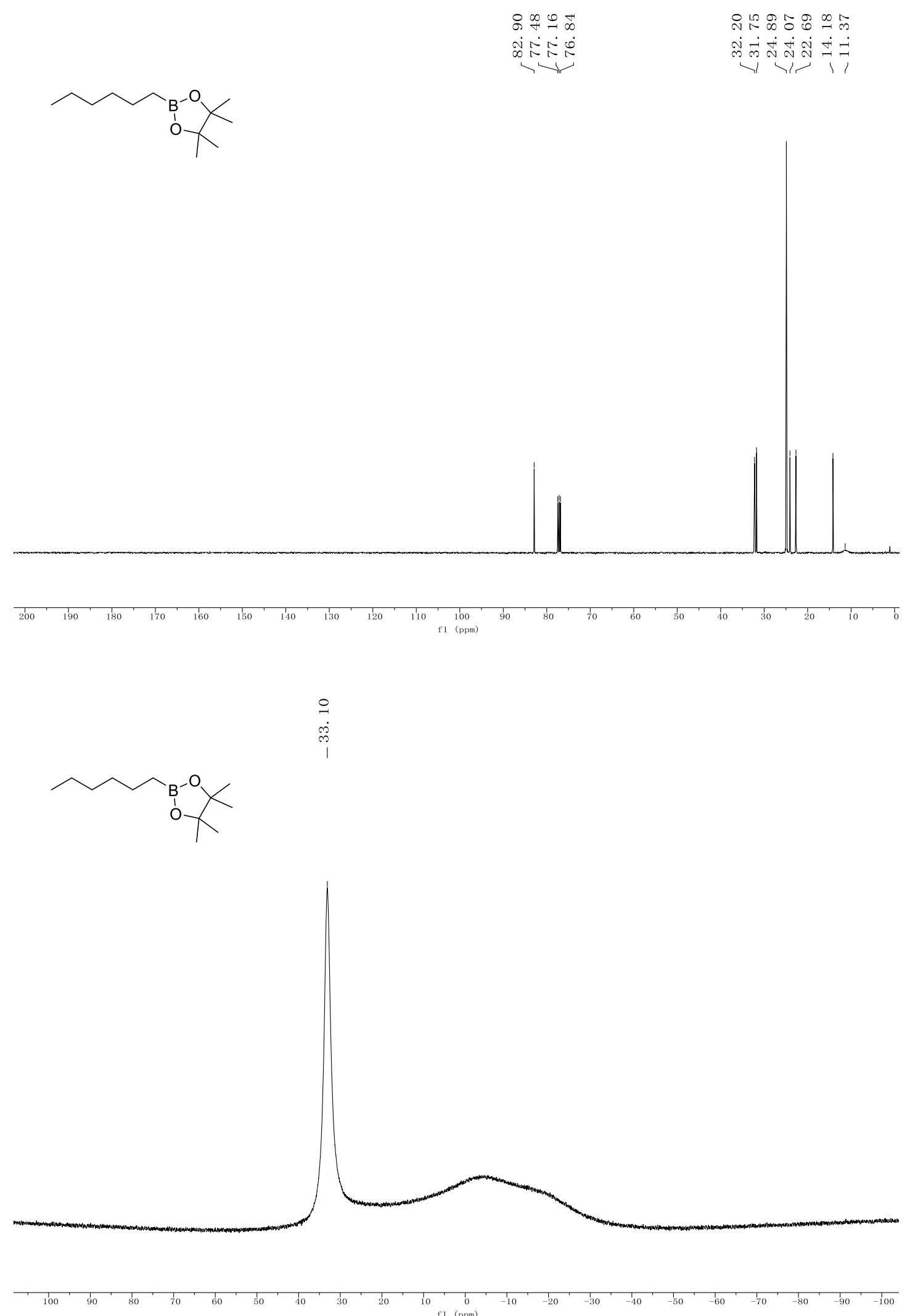


\section{Compound 41}

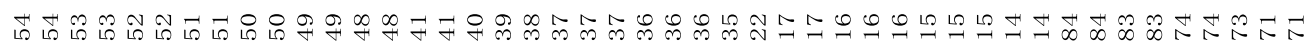

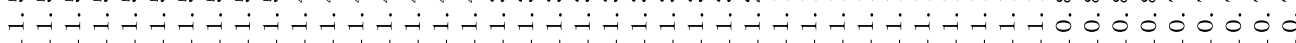

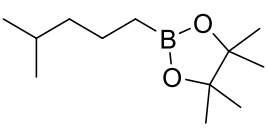

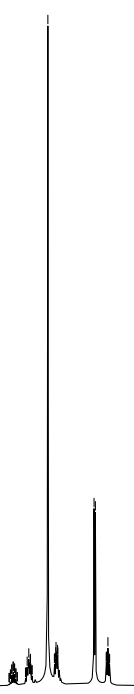

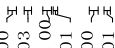

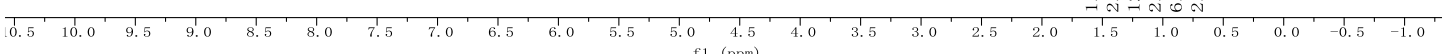

造的电造

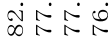

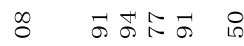

$\longrightarrow>$

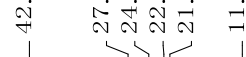
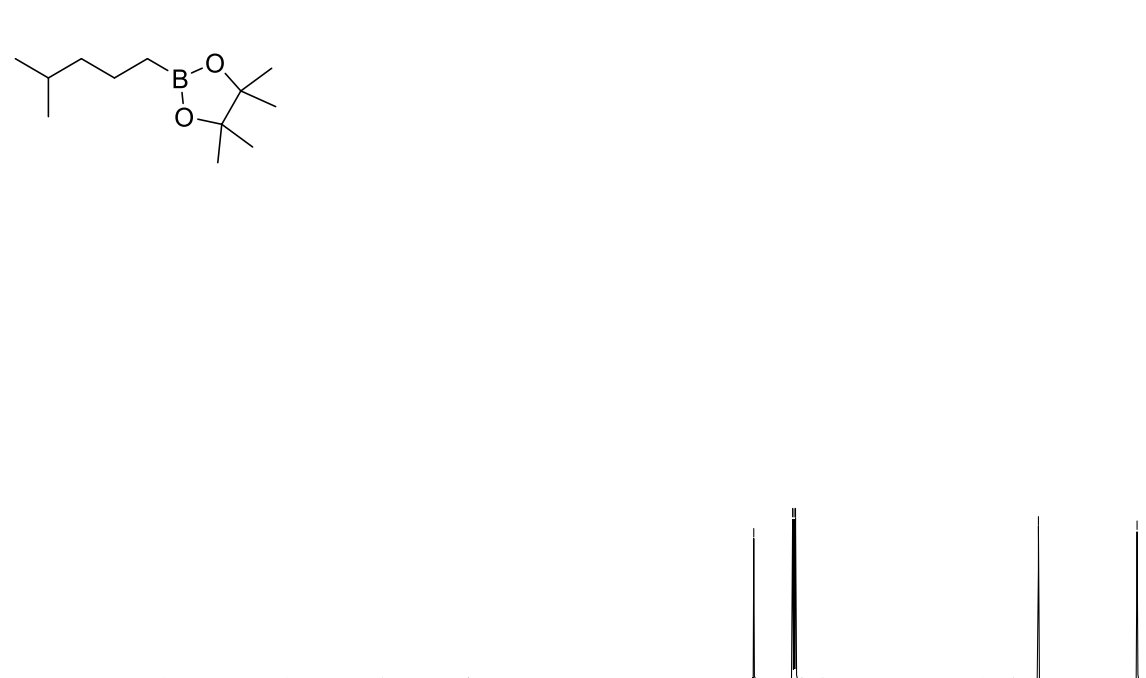

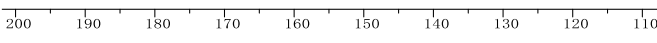
100
$f 1(\mathrm{ppm})$ 

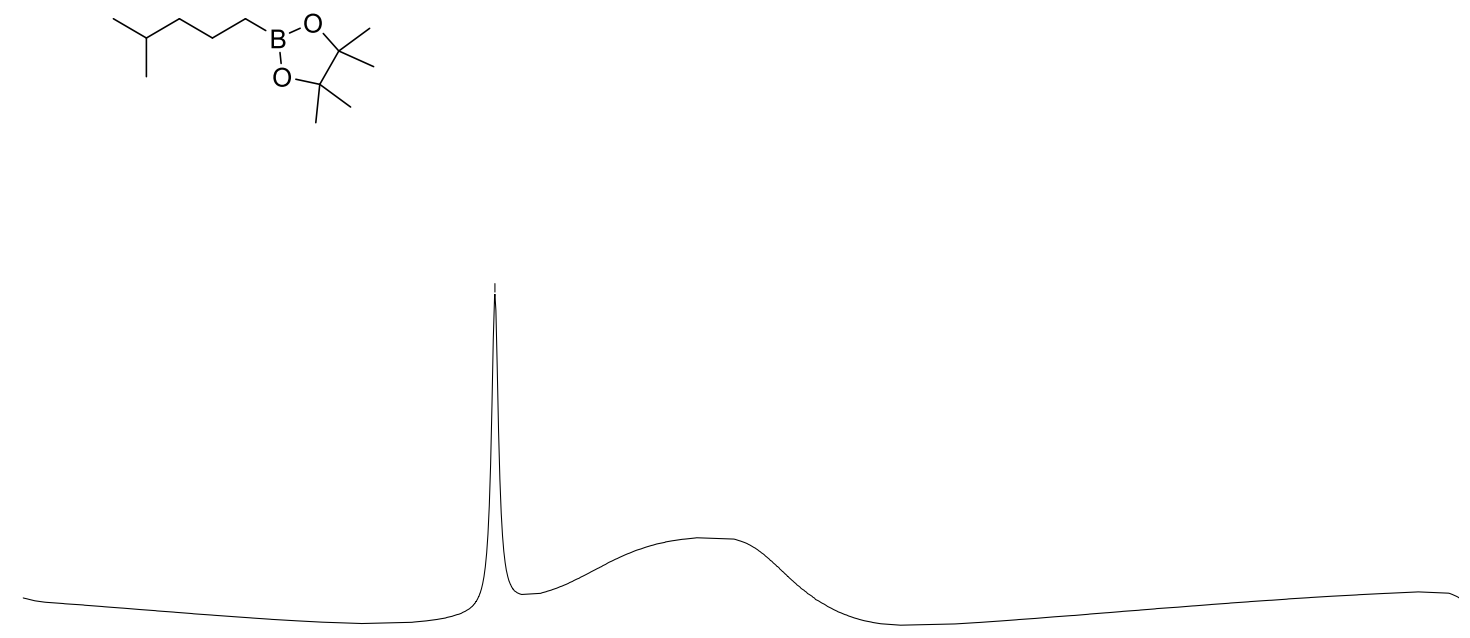

\section{Compound 42}

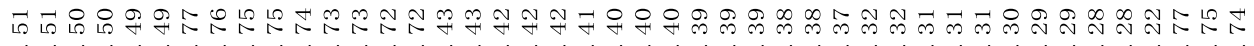
ம்<smiles>CC1(C)OB(CCCCCCCl)OC1(C)C</smiles>

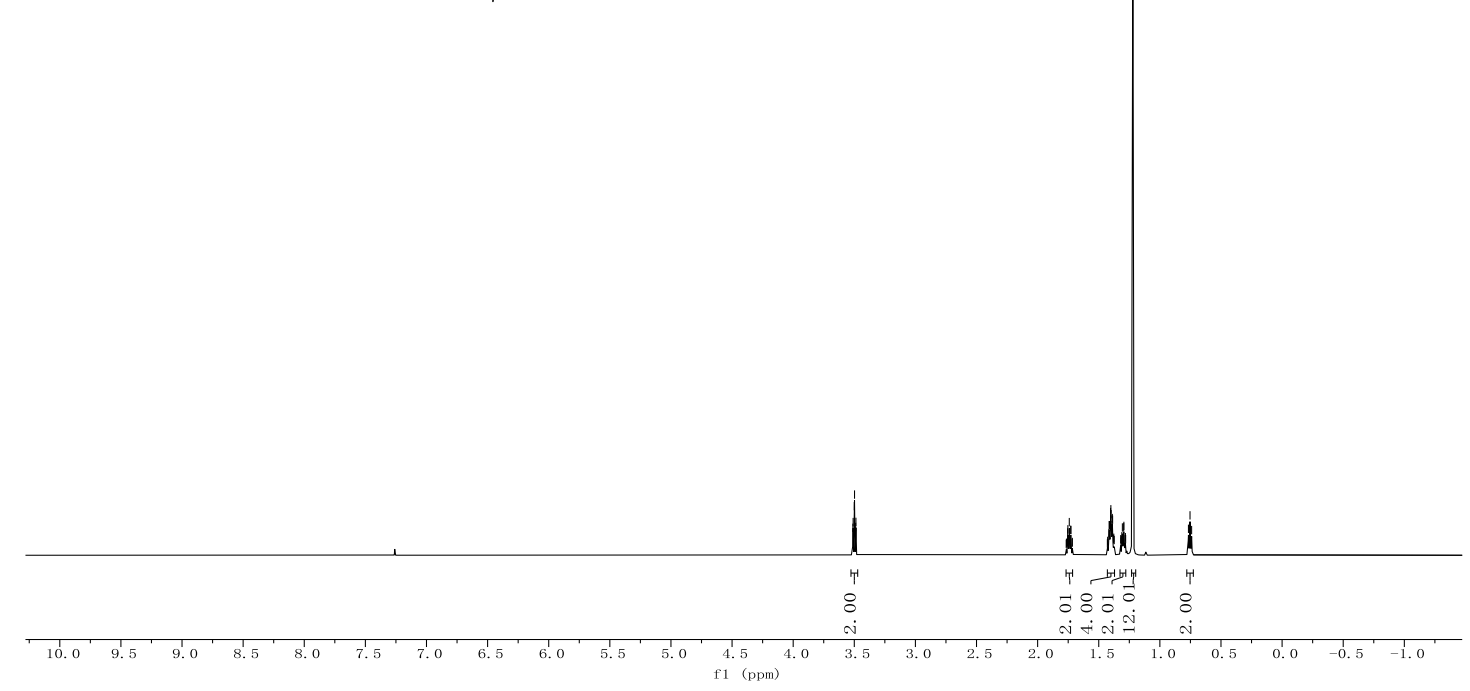




\section{Compound 43}

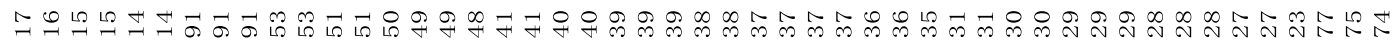

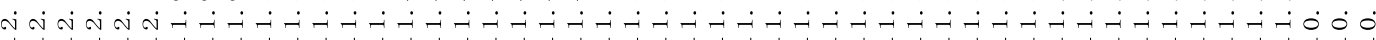<smiles>C#CCCCCCCB1OC(C)(C)C(C)(C)O1</smiles>

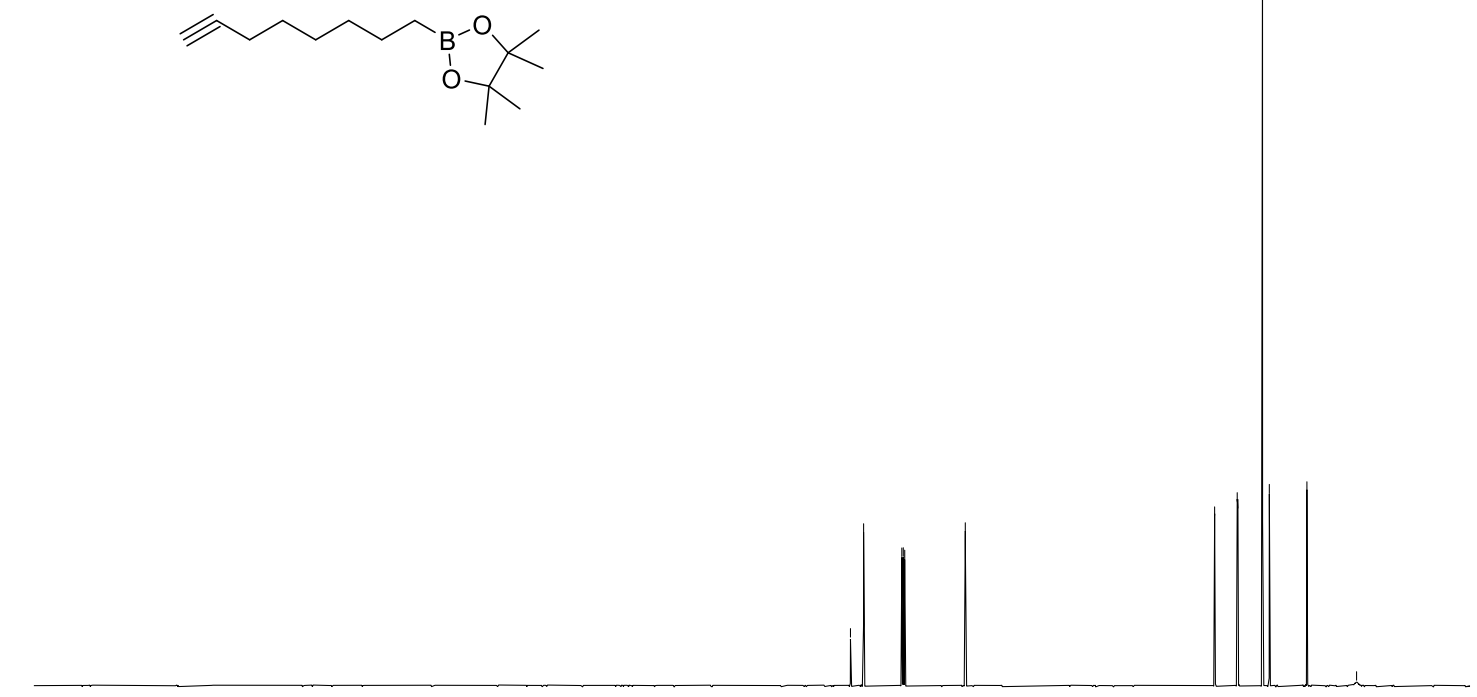




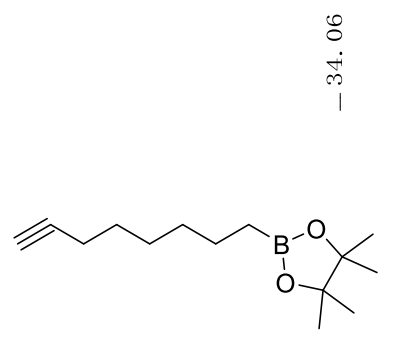

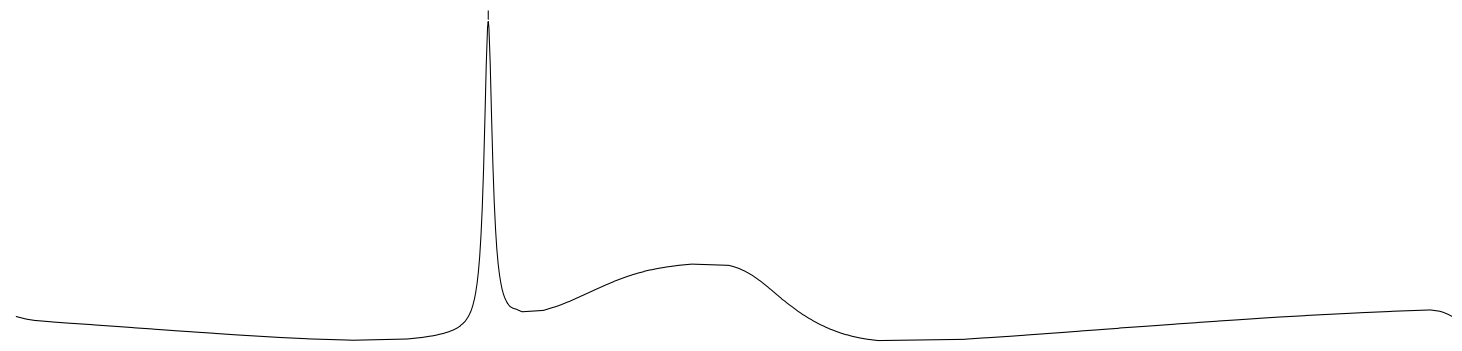

\section{Compound 44}

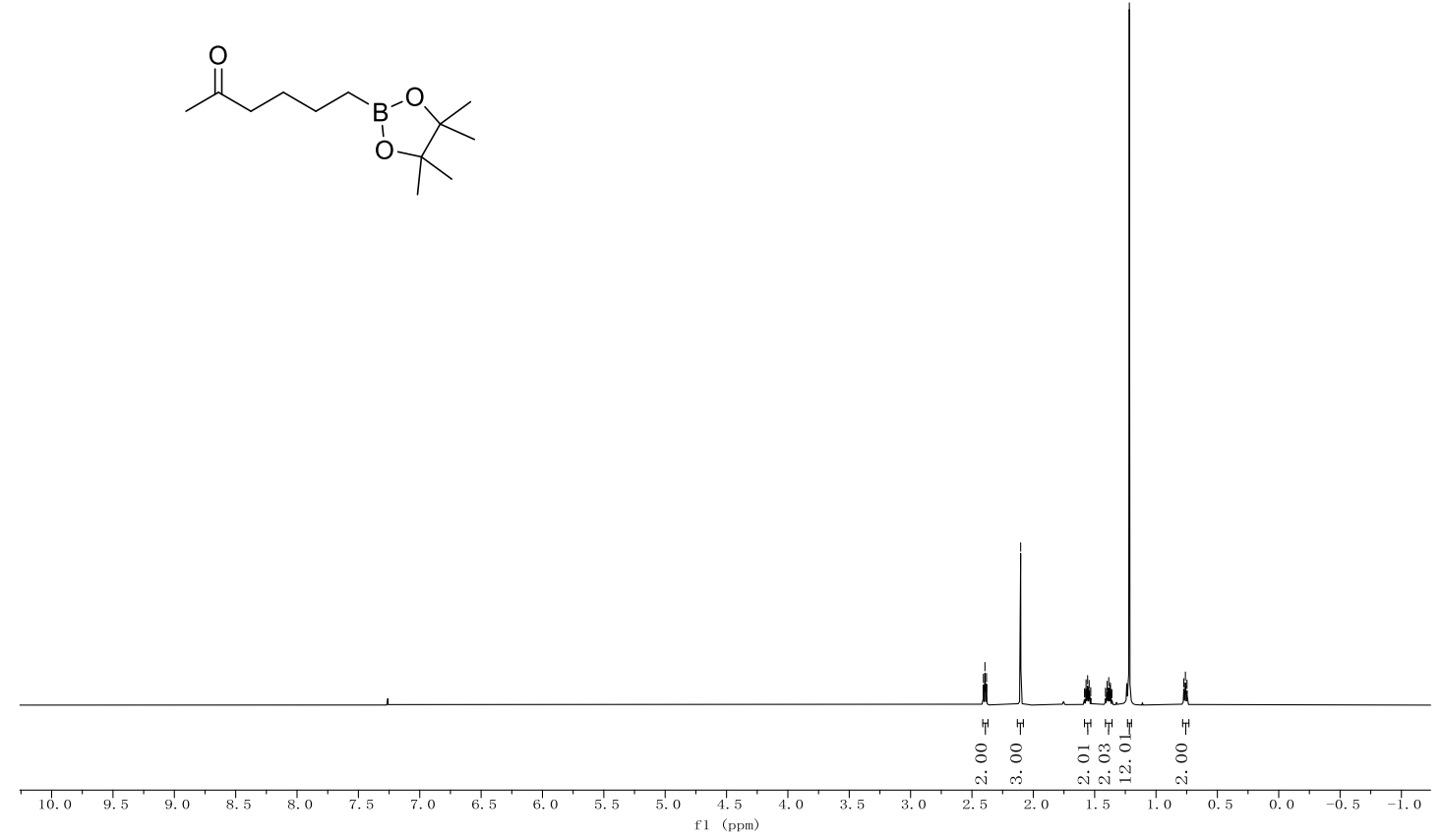



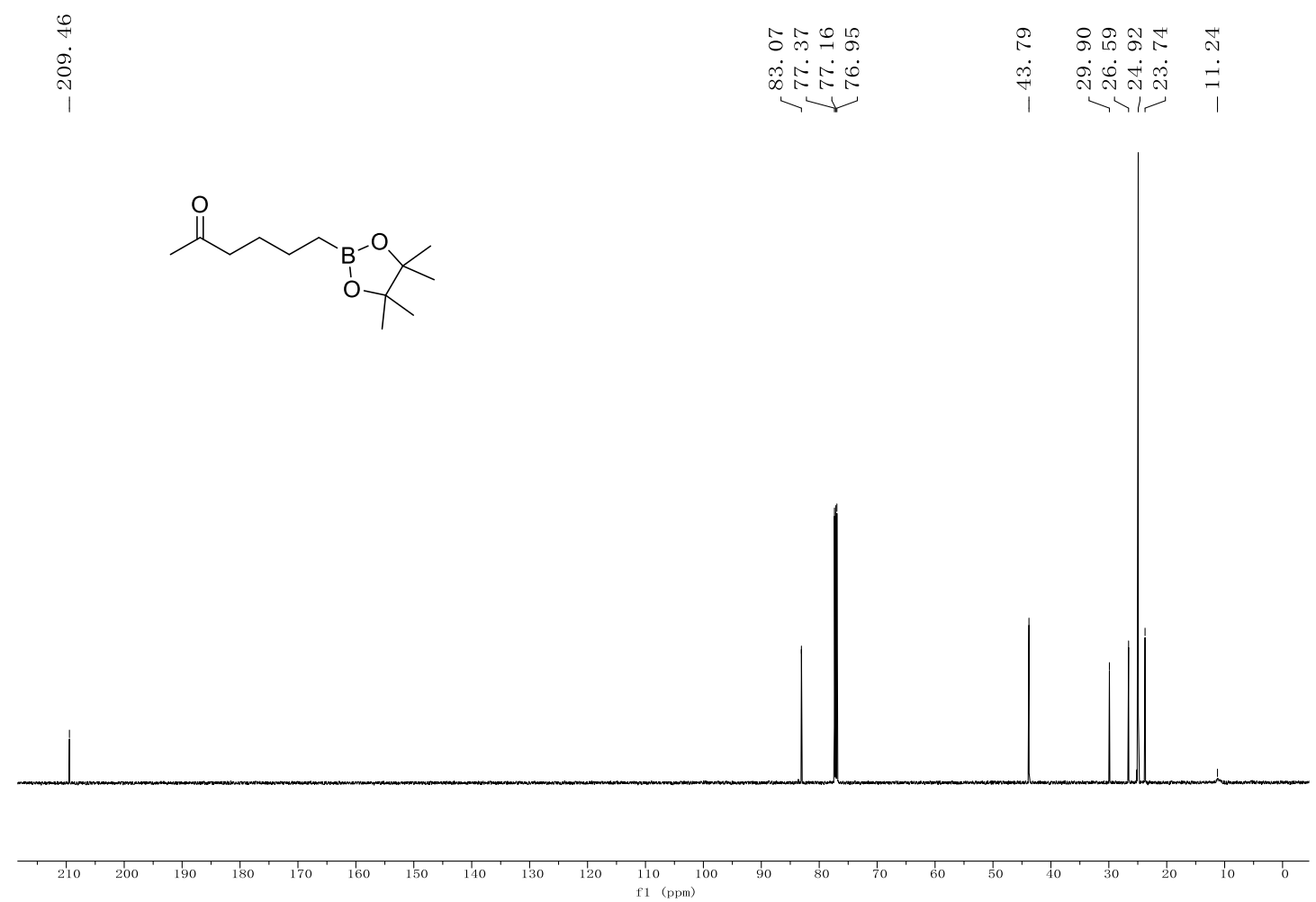

$$
\begin{aligned}
& \text { N } \\
& \text { ले }
\end{aligned}
$$<smiles>CC(=O)CCCCB1OC(C)(C)C(C)(C)O1</smiles>
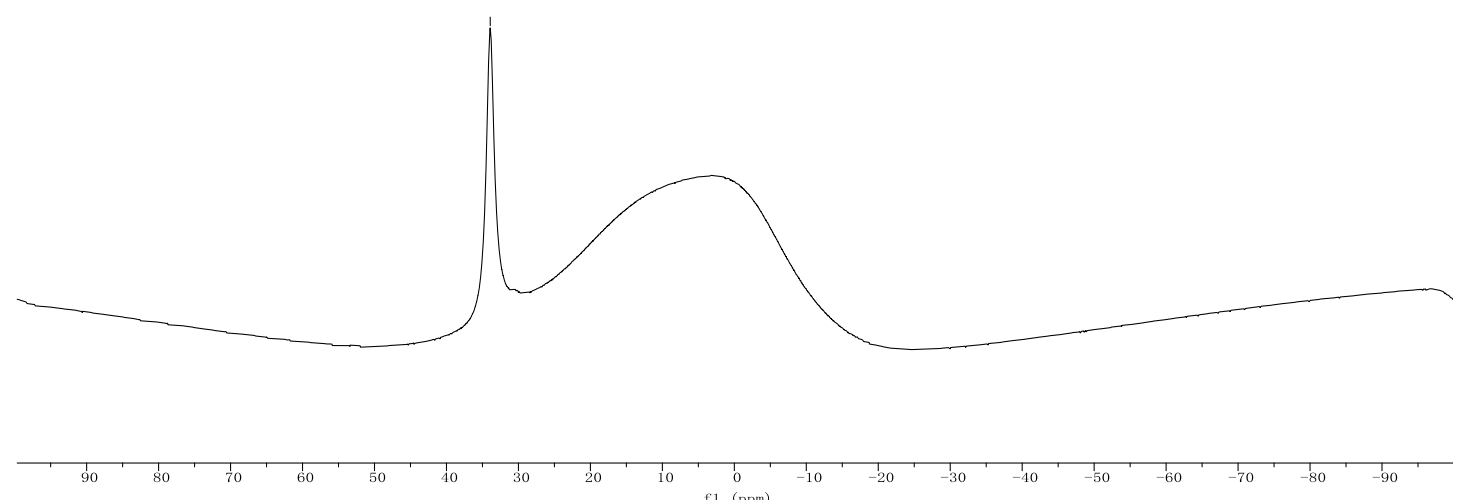


\section{Compound 45}

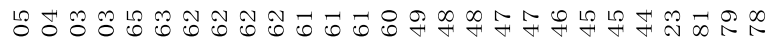

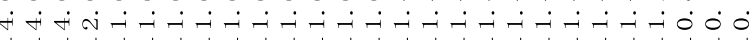<smiles>CC(=O)OCCCCB1OC(C)(C)C(C)(C)O1</smiles>

ul.
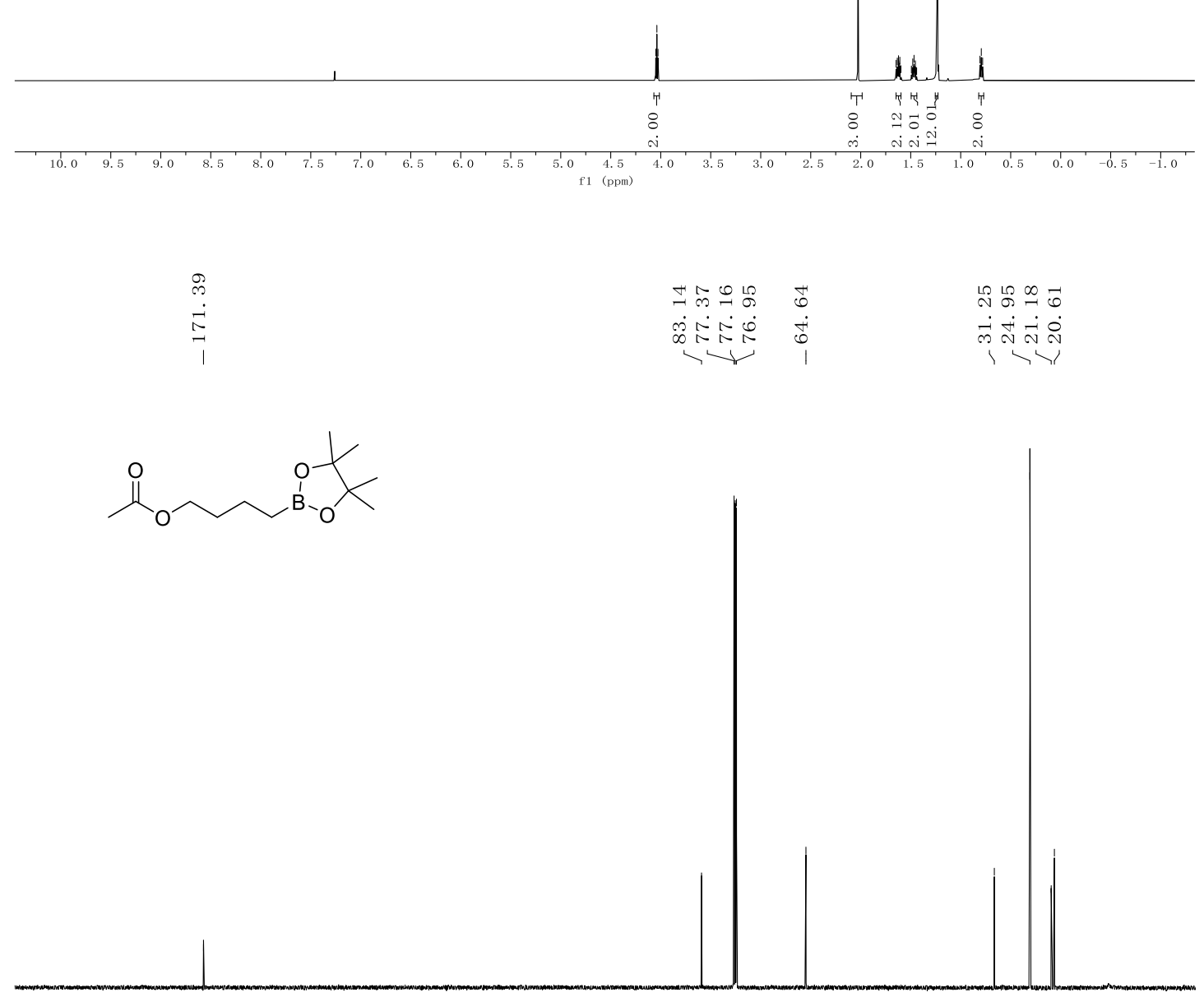

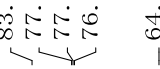

$\dot{m} \dot{\mathrm{N}} \dot{\mathrm{N}} \dot{\mathrm{N}}$

<प

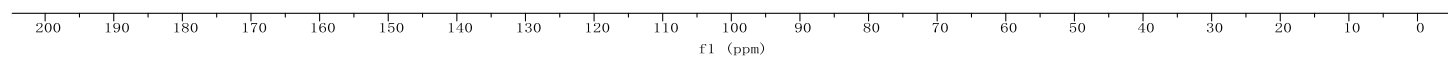



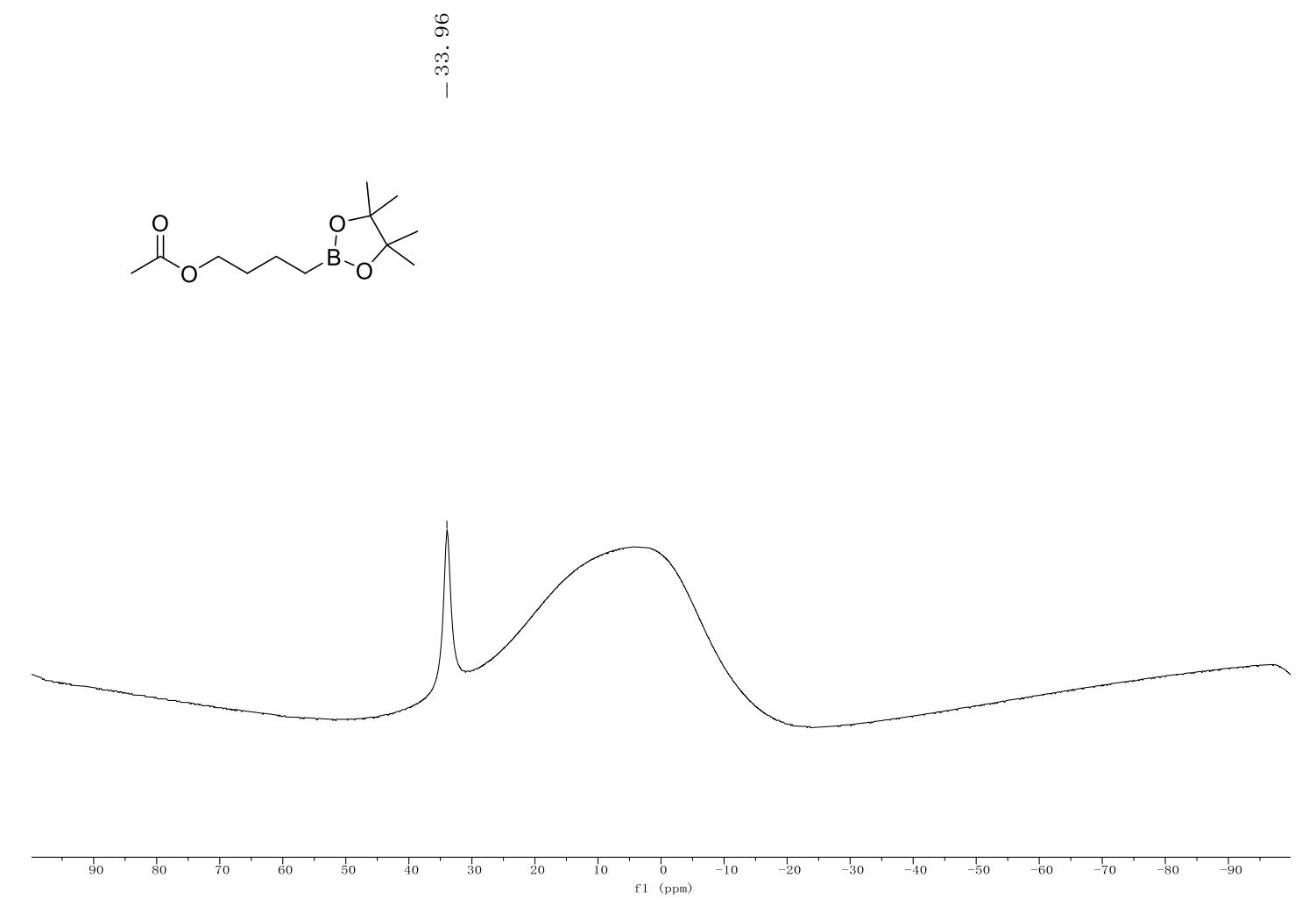

\section{Compound 46}

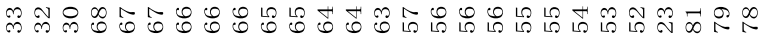

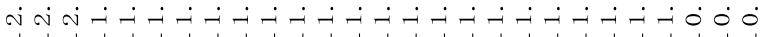

NC<smiles>CCCCB1OC(C)(C)C(C)(C)O1</smiles>

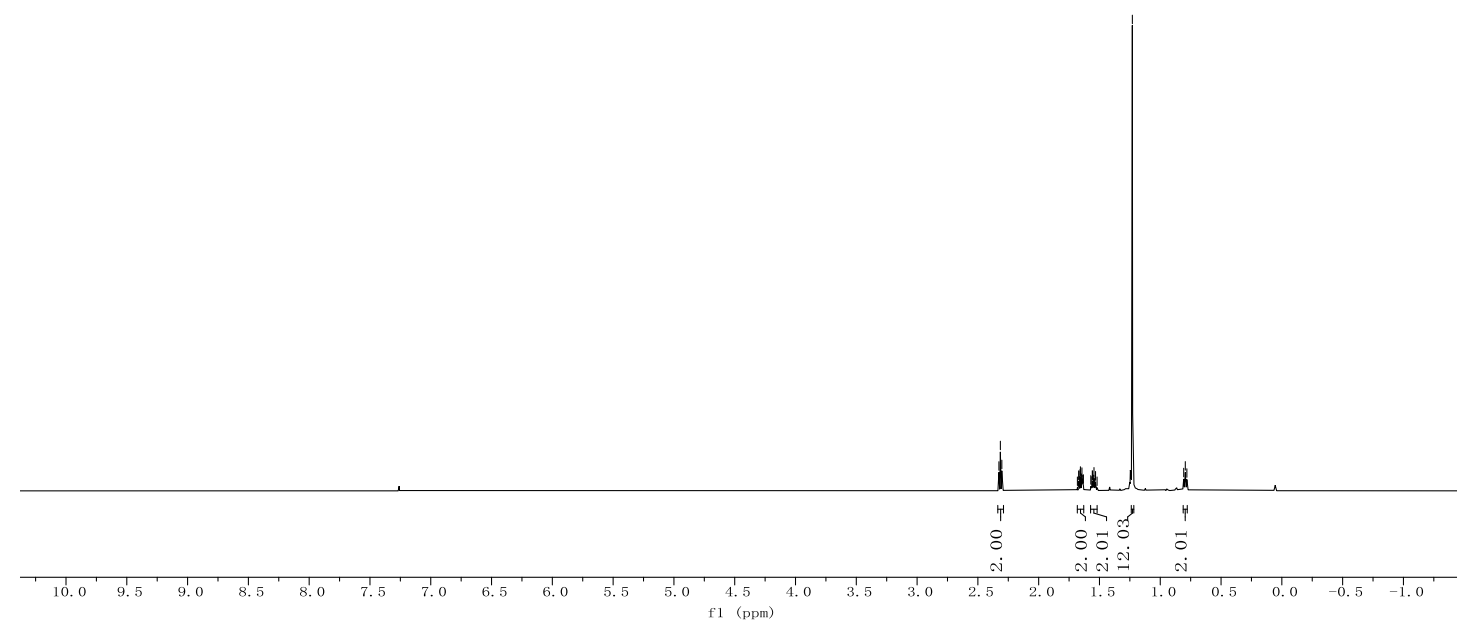




\begin{tabular}{|c|c|}
\hline & 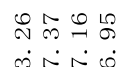 \\
\hline & 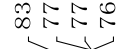 \\
\hline
\end{tabular}

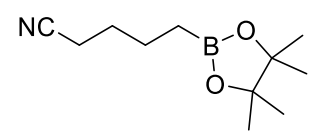

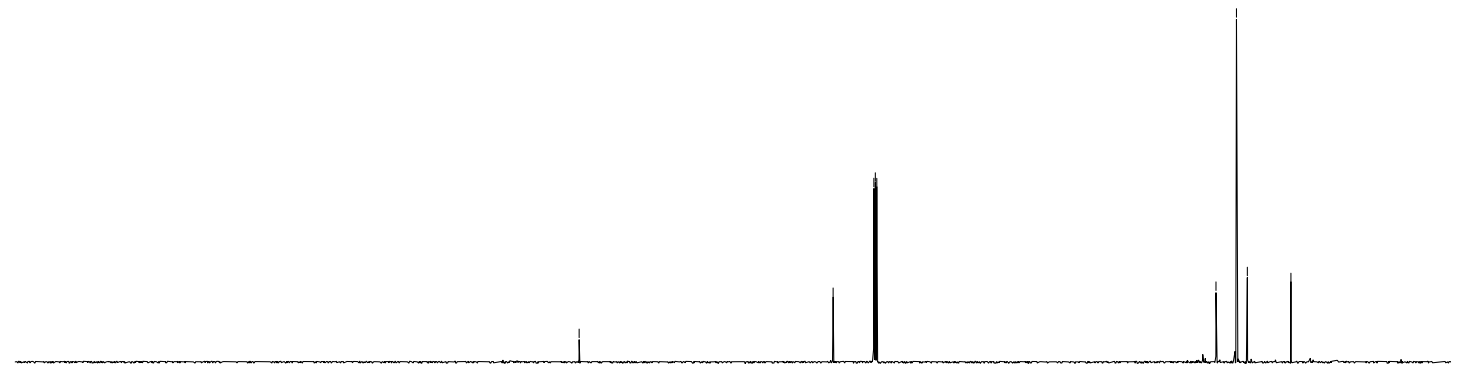

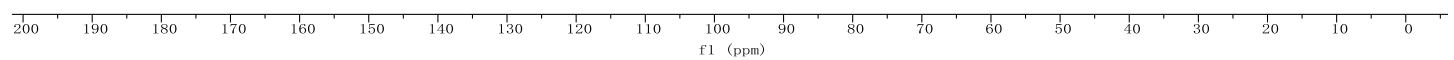

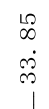

$\overbrace{}^{N C}{ }^{B_{1}^{-O}} \mathcal{C}$

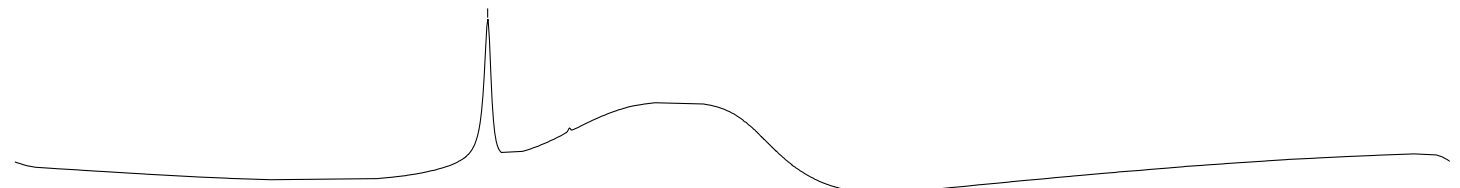




\section{Compound 47}

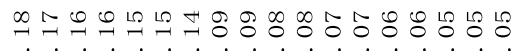

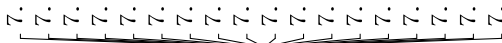

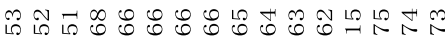

ง ผ<smiles>CC1(C)OB(CCCc2ccccc2)OC1(C)C</smiles>

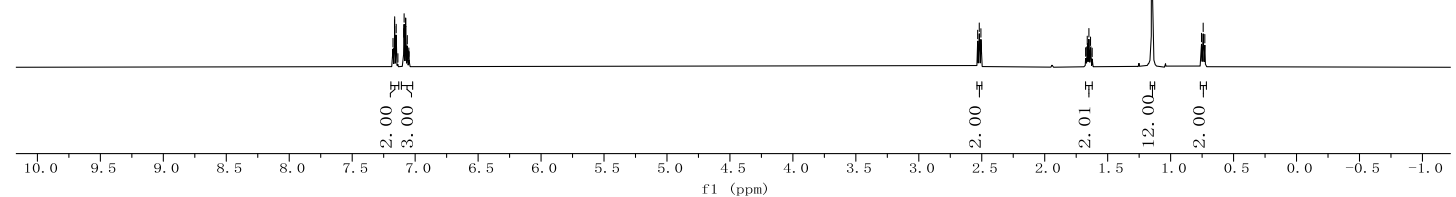

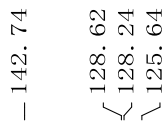

$\infty \stackrel{\infty}{\infty} \stackrel{0}{\sigma}$

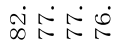

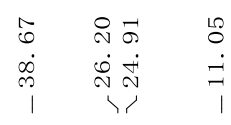

13

$\checkmark \longrightarrow$

$\stackrel{\circ}{\dot{j}}$

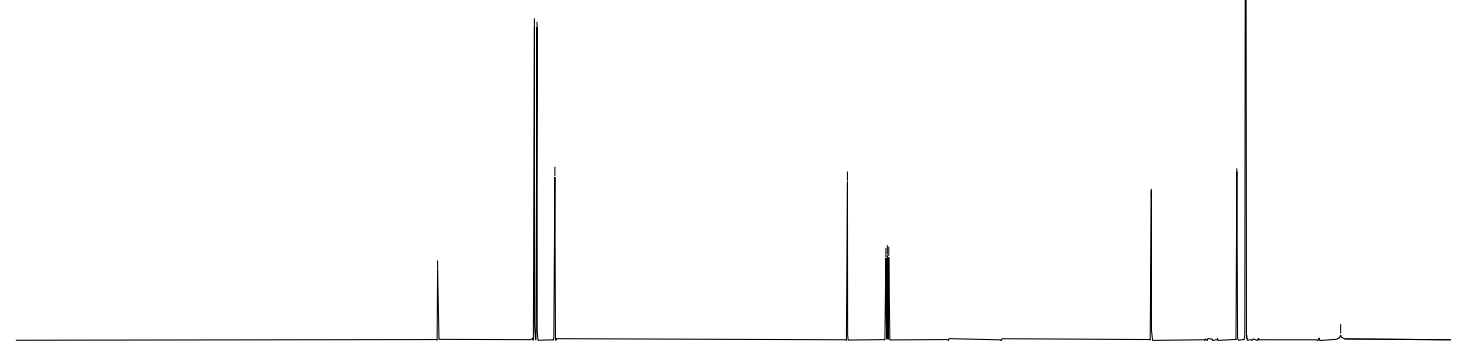

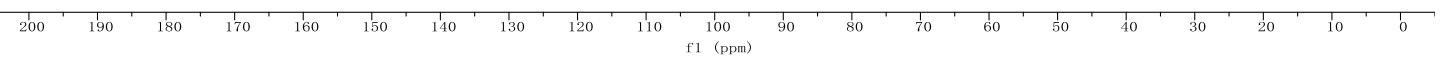




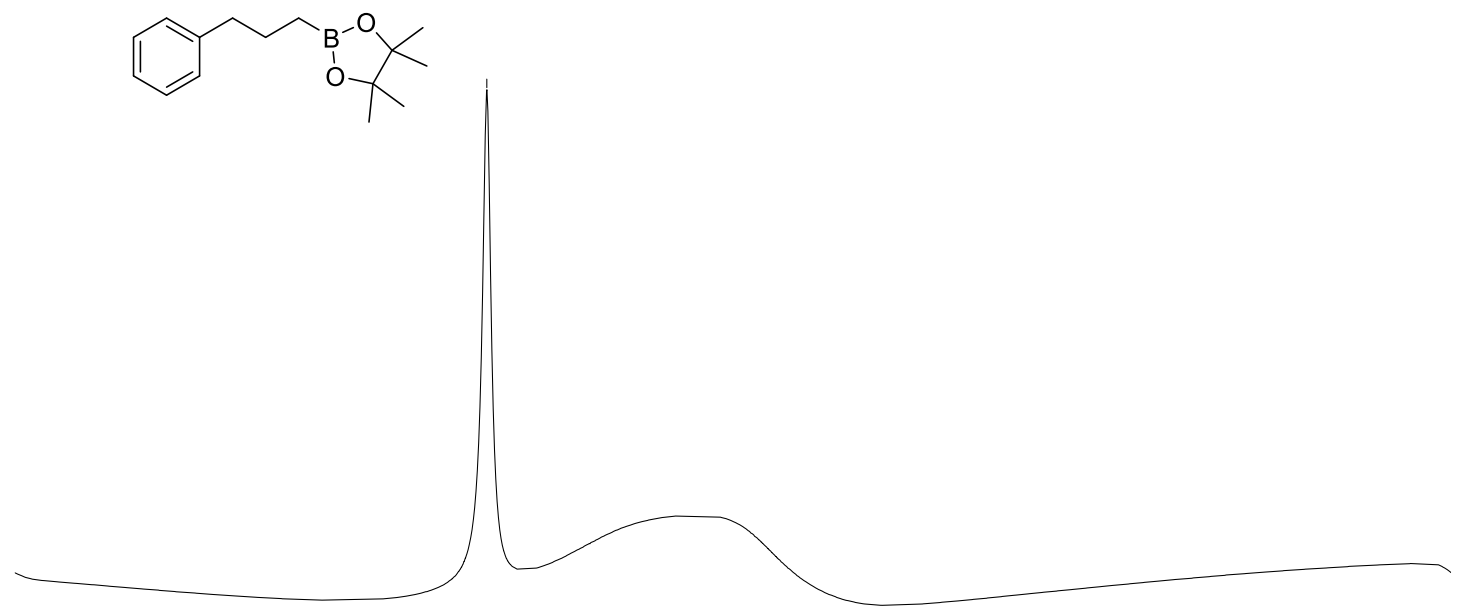

\section{Compound 48}

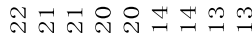

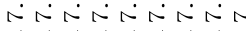

츄용

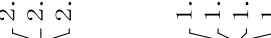

L

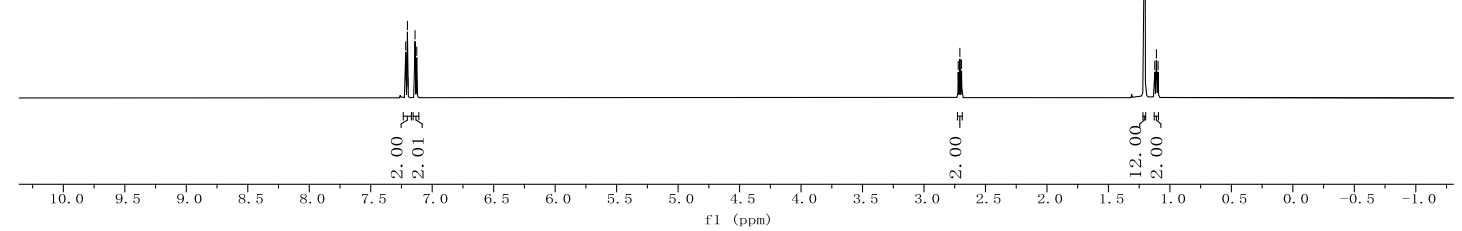




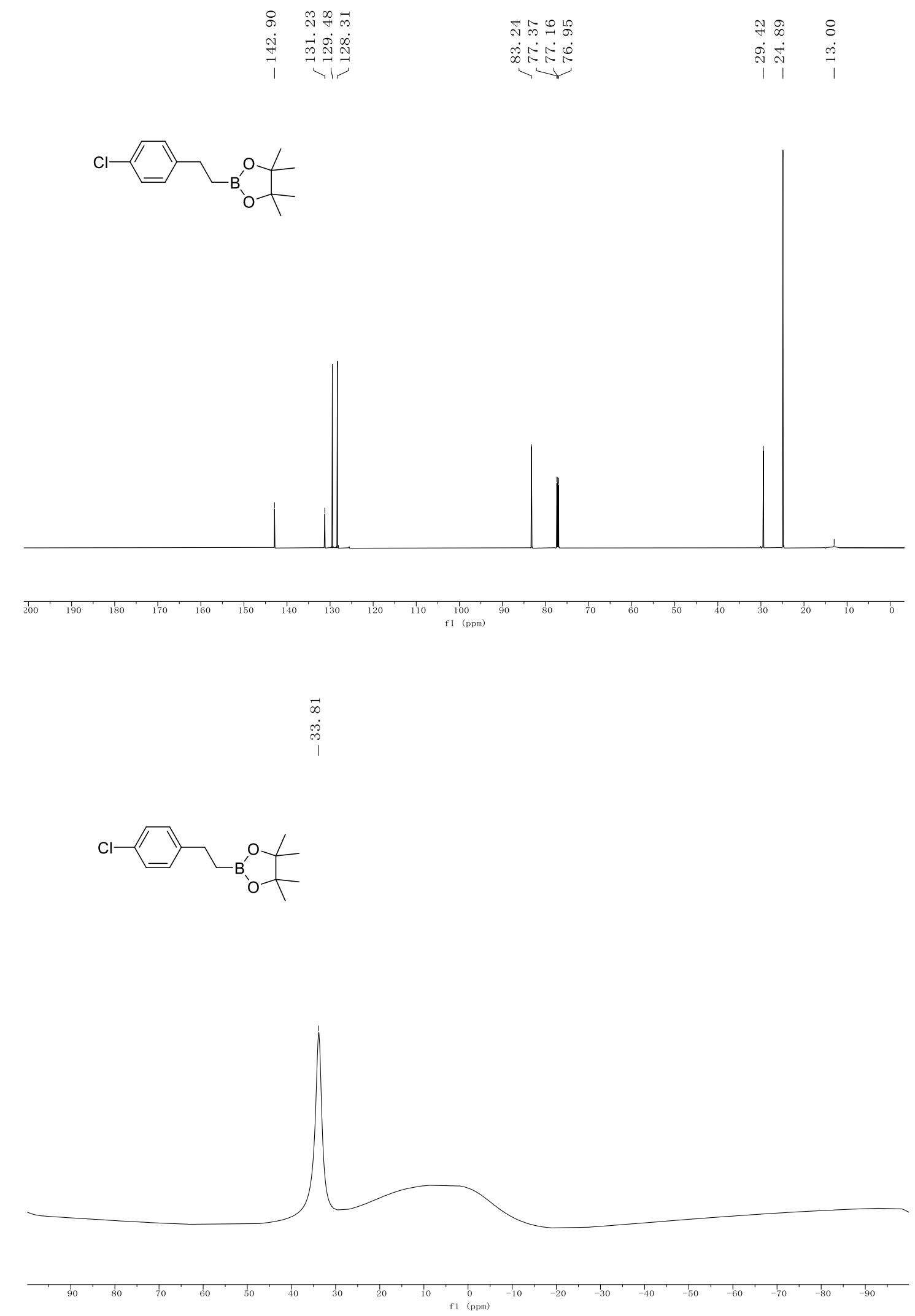




\section{Compound 49}

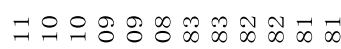

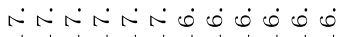

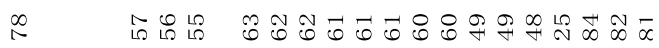

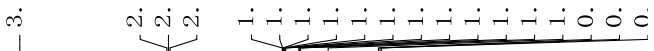

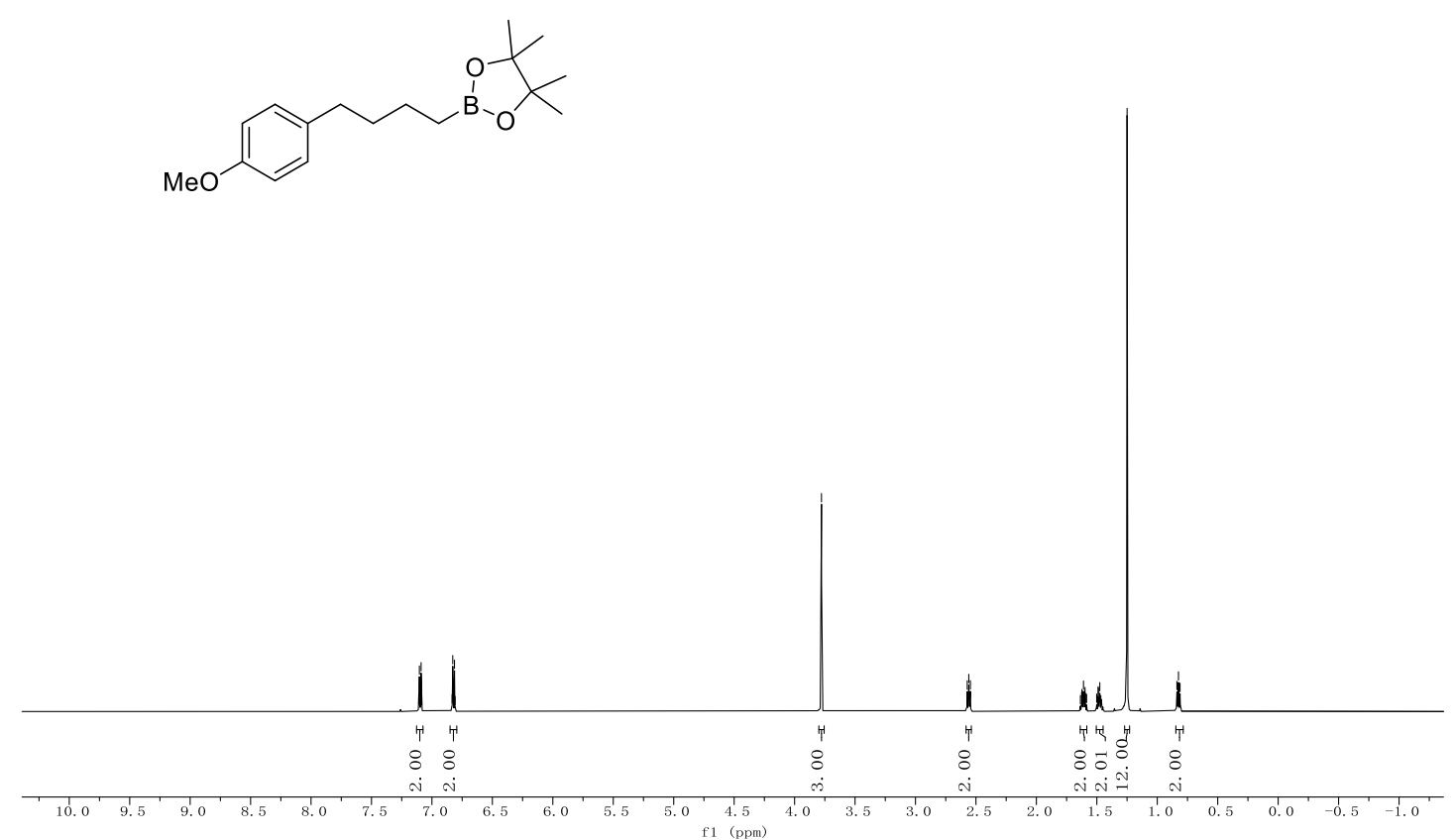

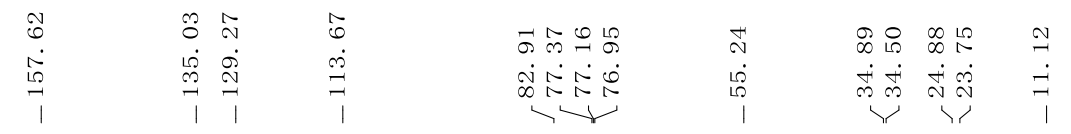<smiles></smiles>
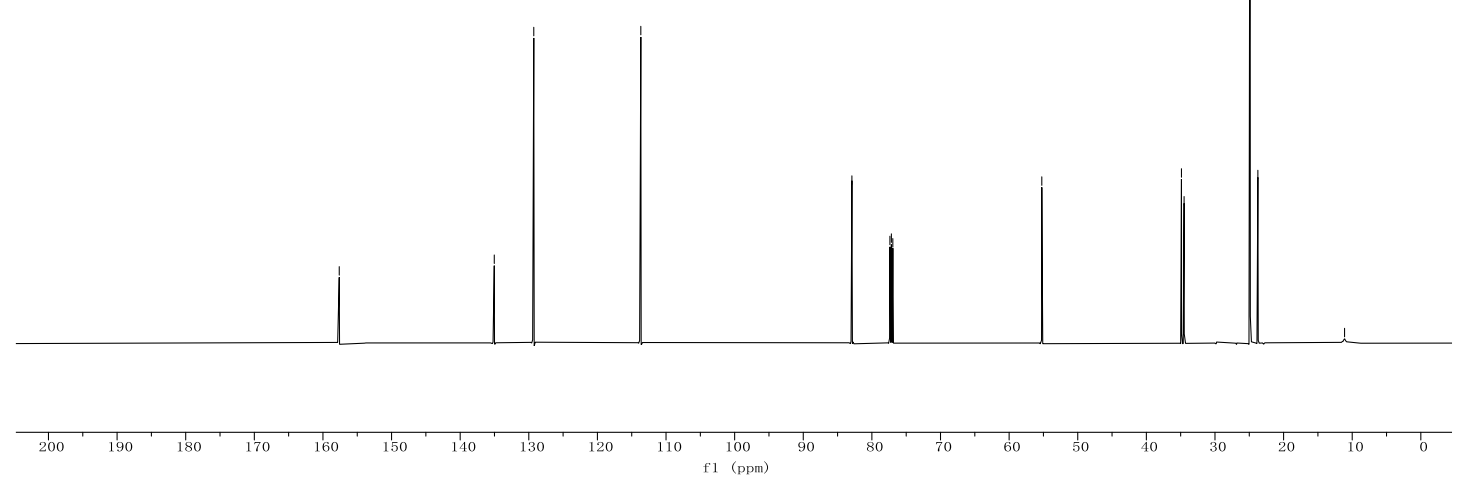

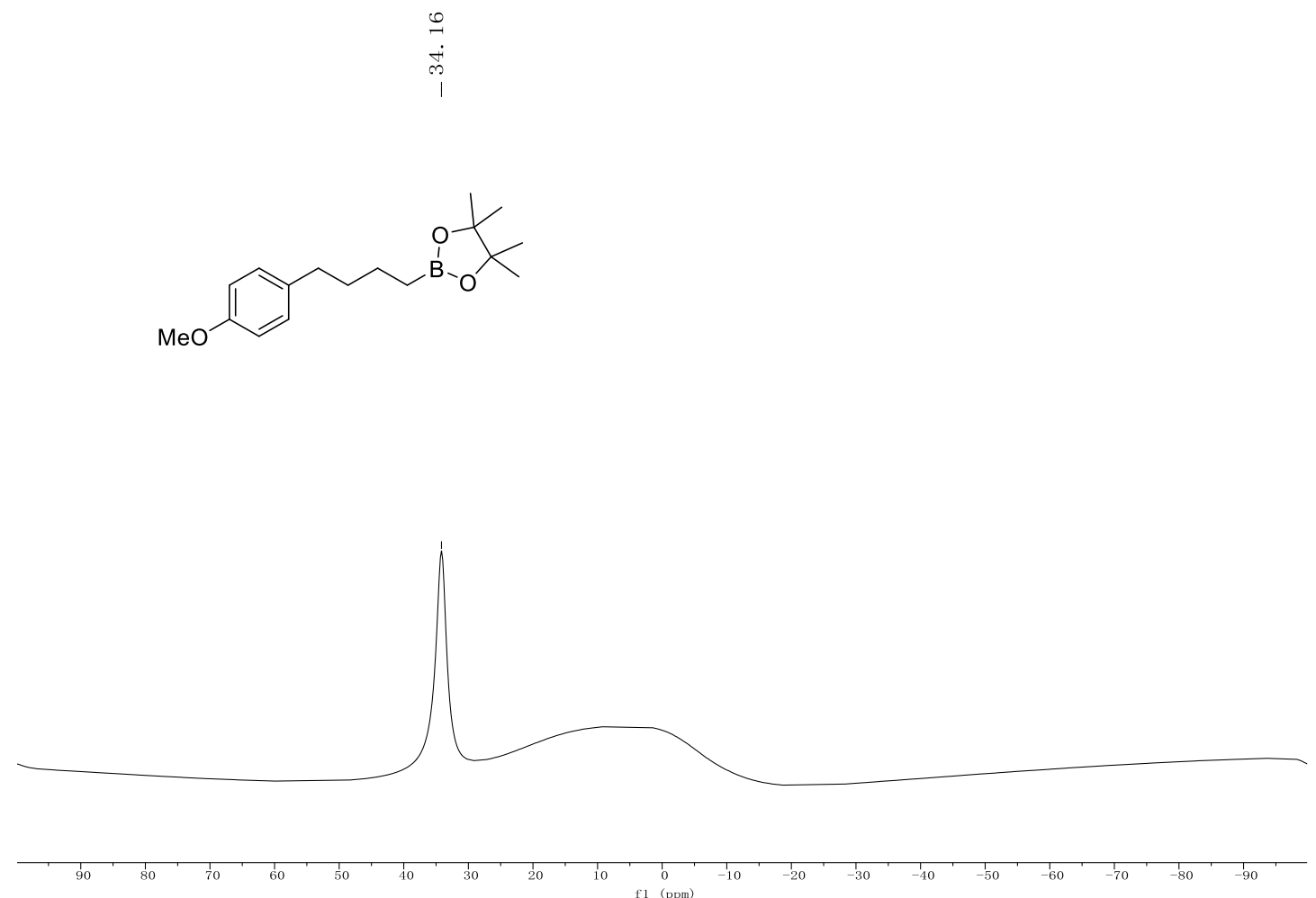

\section{Compound 50}

\begin{tabular}{|c|c|}
\hline 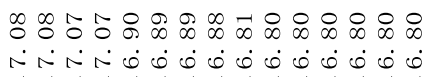 & 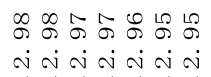 \\
\hline
\end{tabular}<smiles>CC1(C)OB(CCc2cccs2)OC1(C)C</smiles>

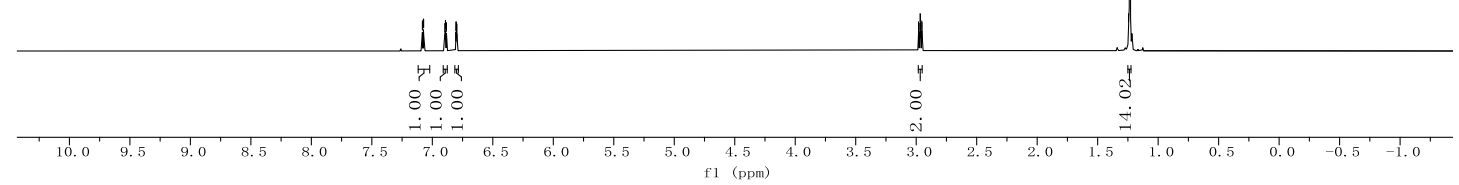




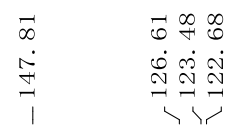

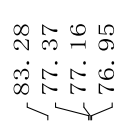

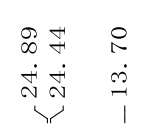
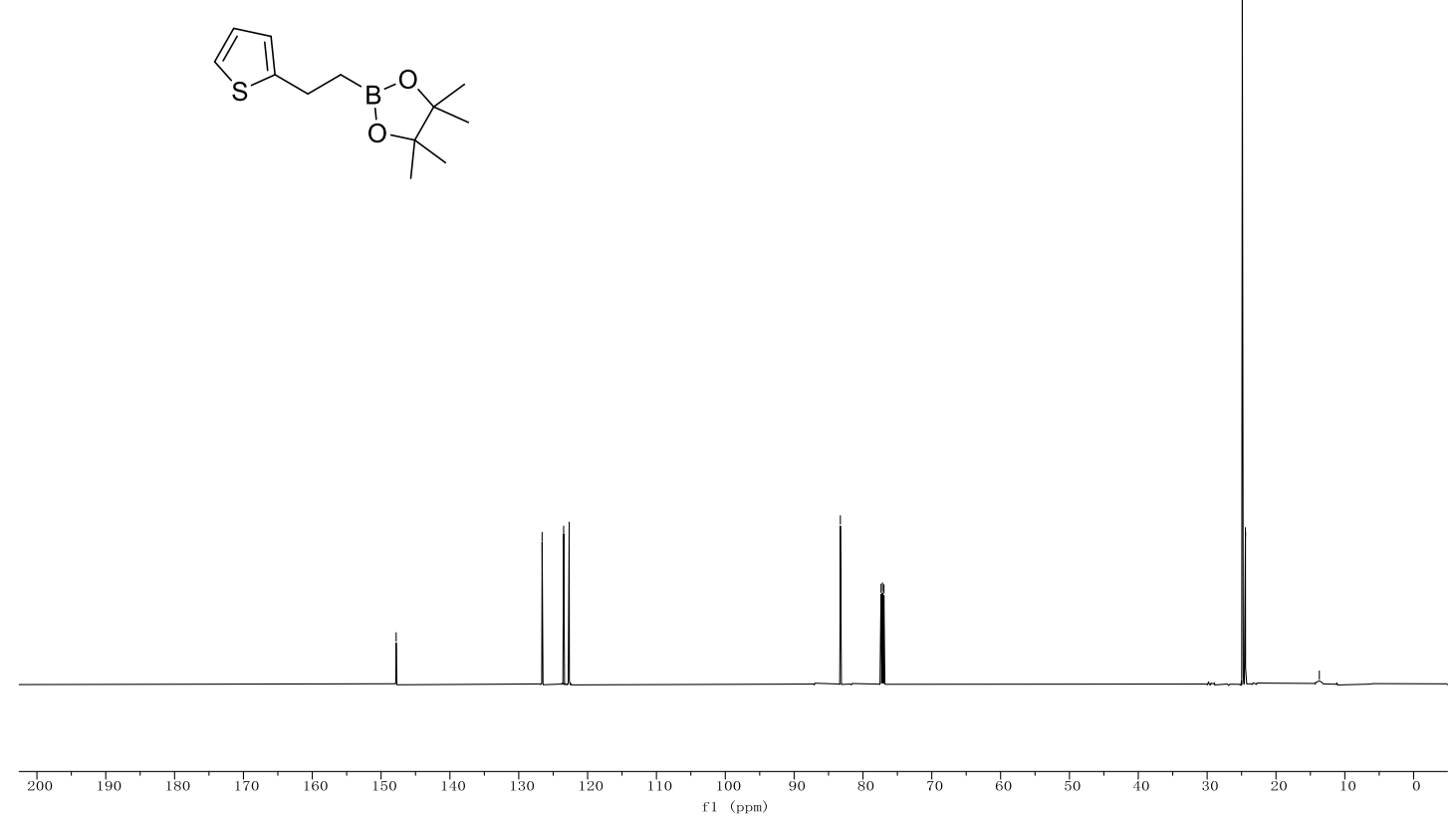

$\stackrel{⿱ 亠 䒑}{\infty}$
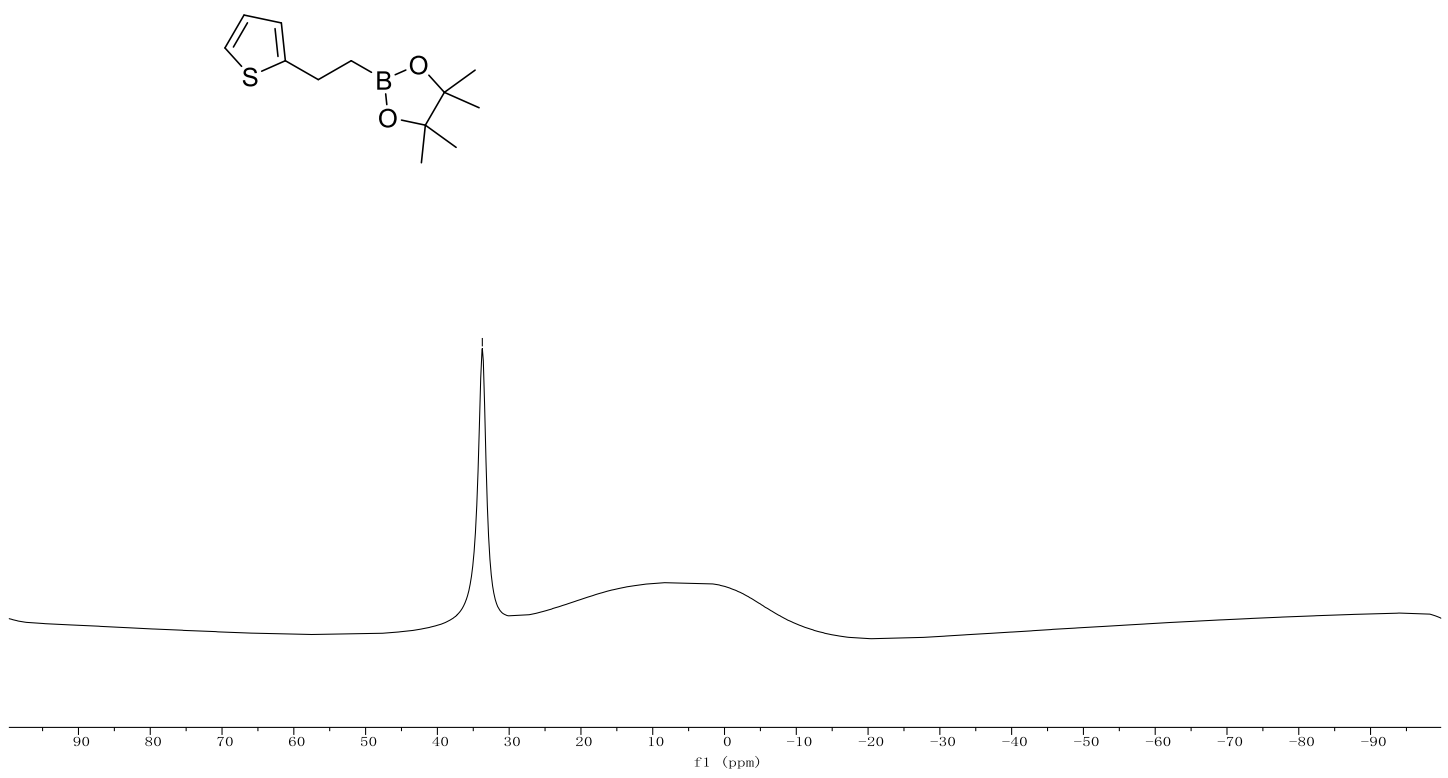

S160 


\section{Compound 51}

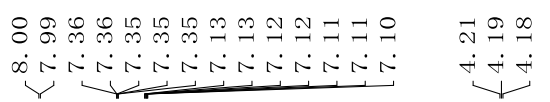

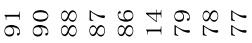

-
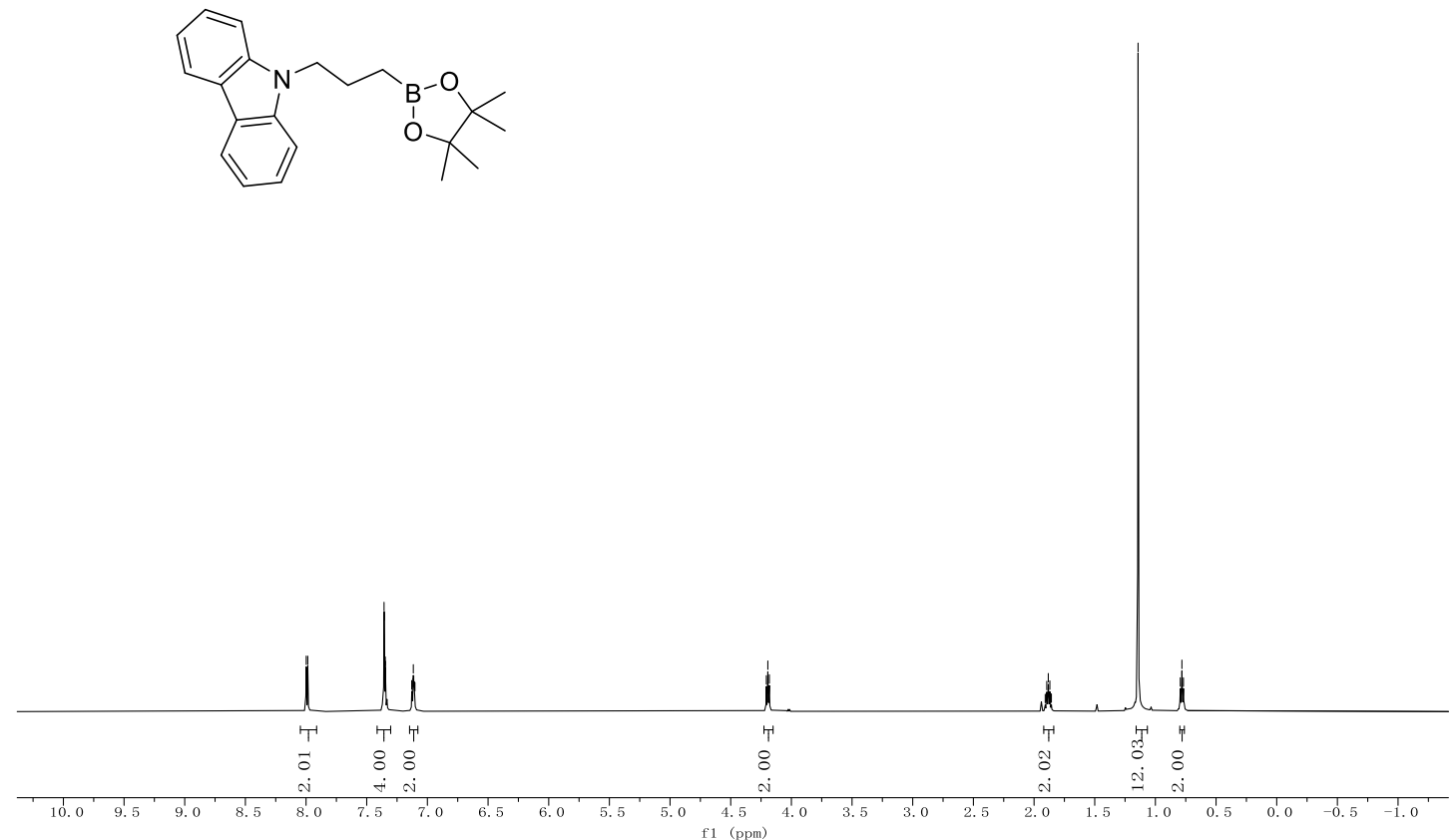

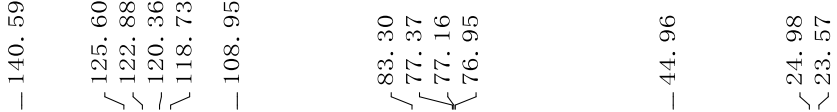
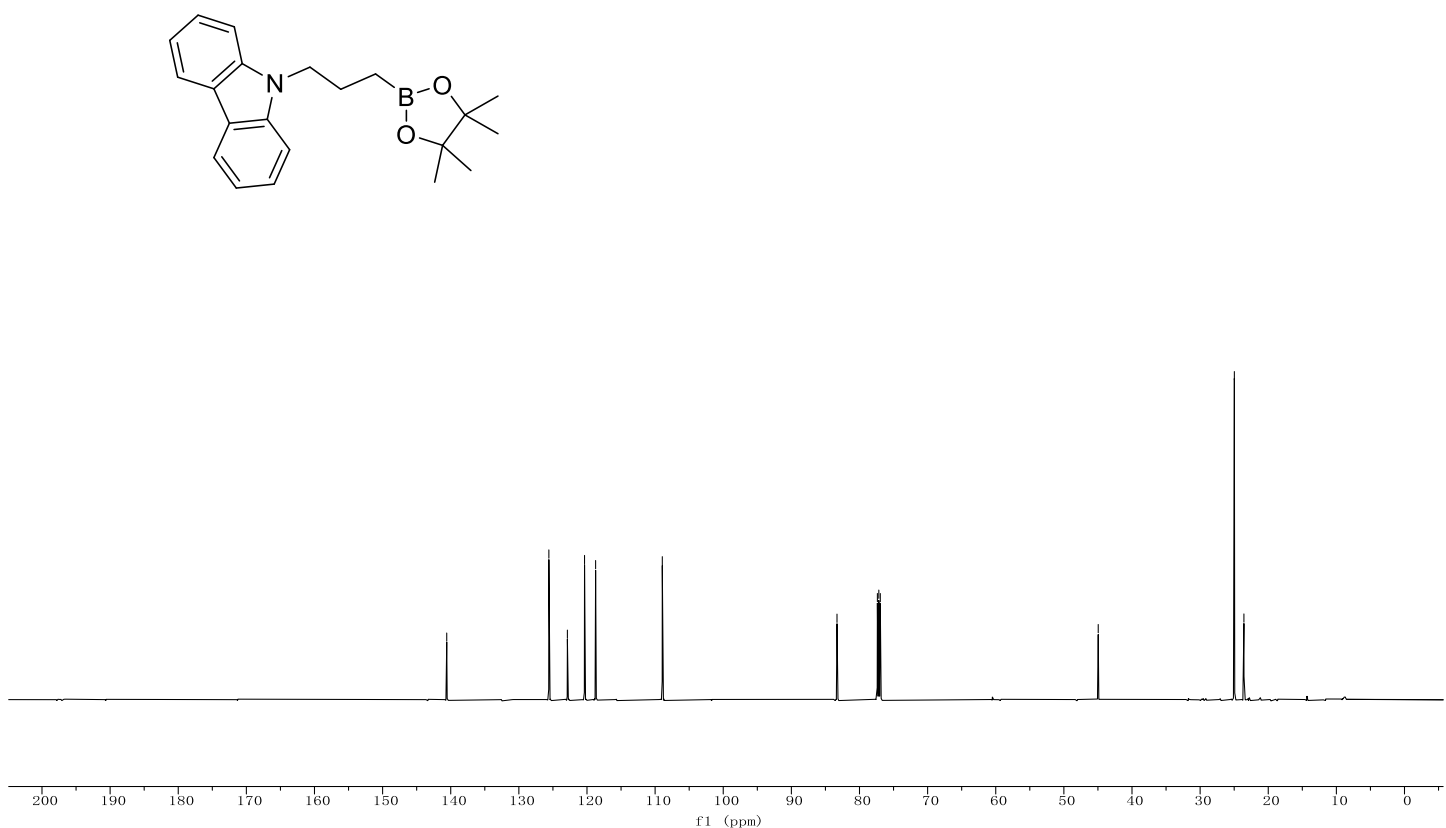


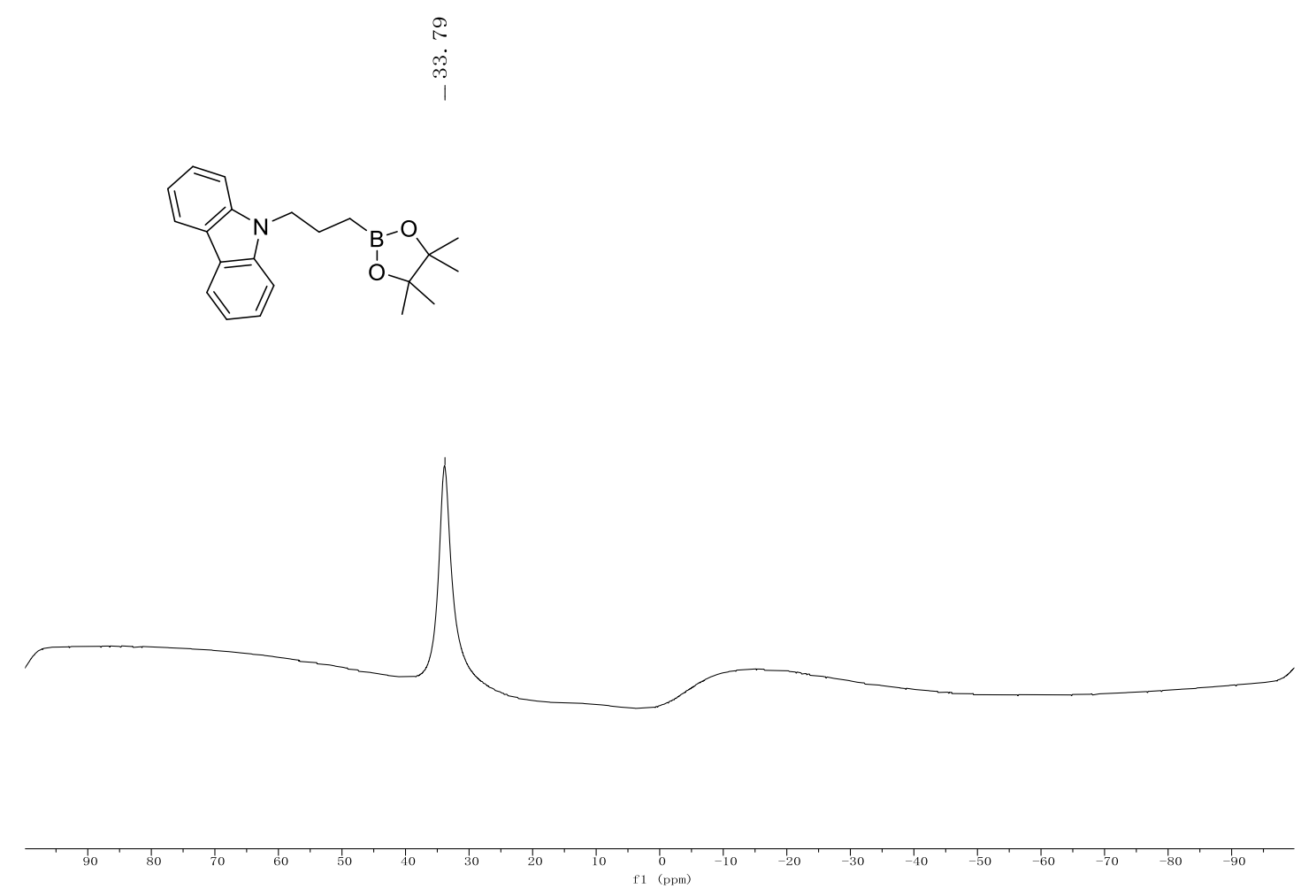

\section{Compound 52}

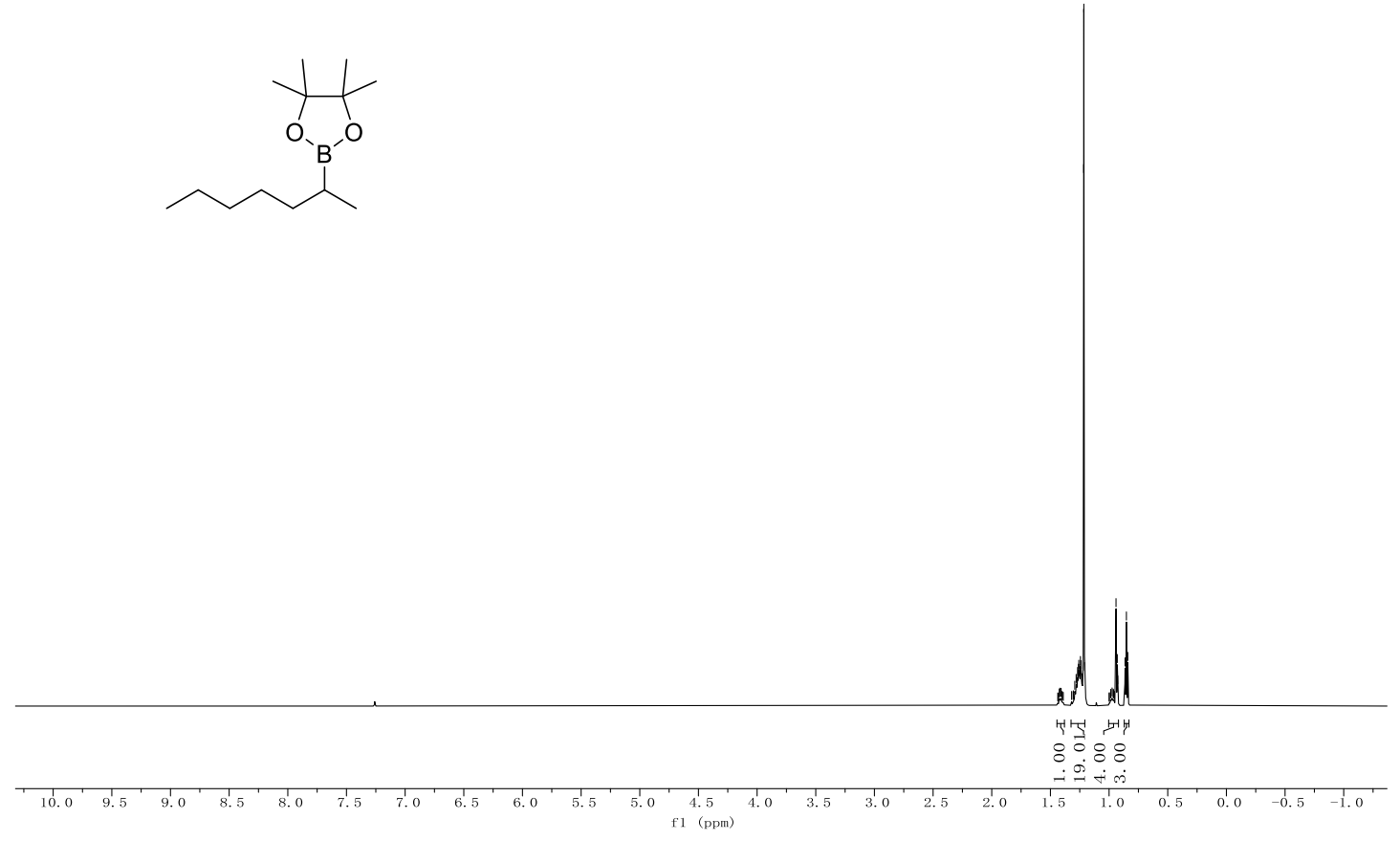


용요용

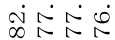

पर
冈ি $\vec{\sim}$

ฌ่

<证
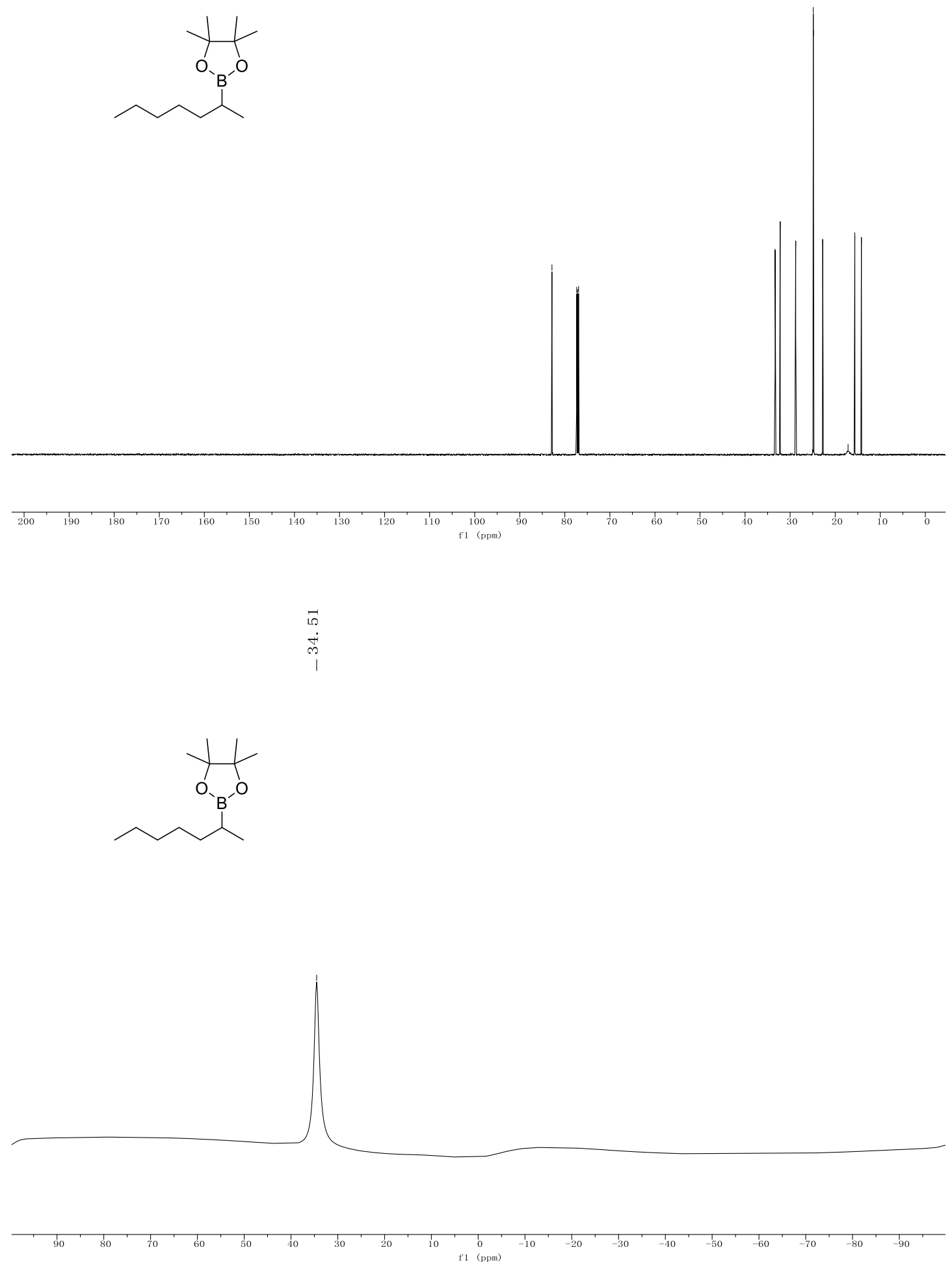


\section{Compound 53}

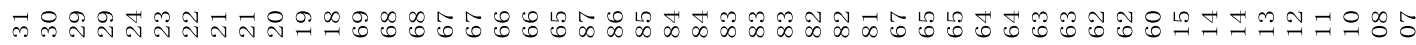

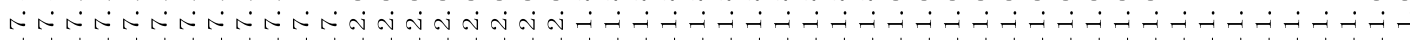

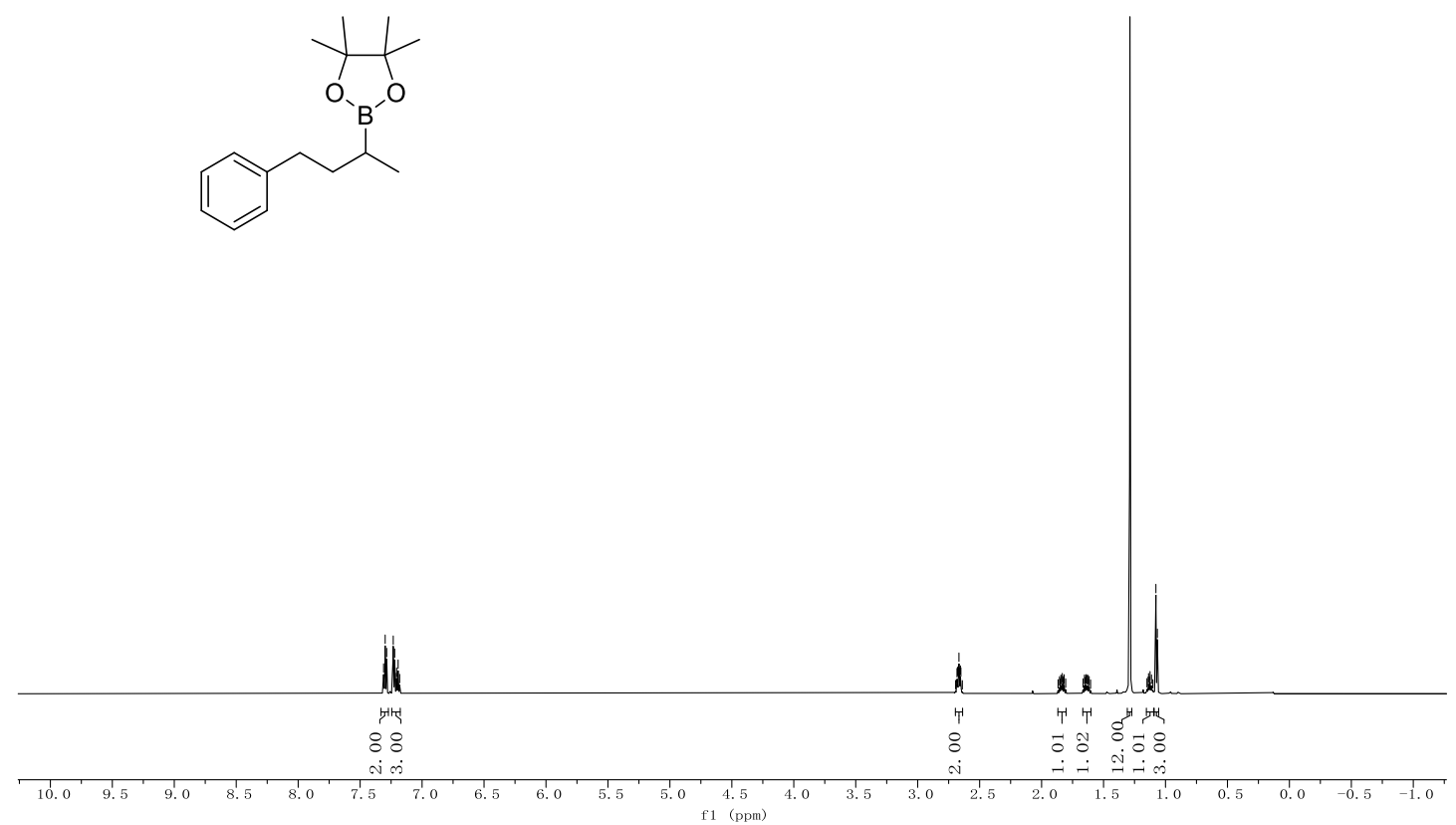

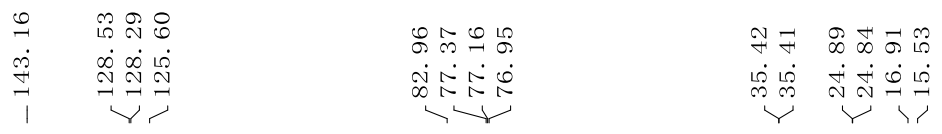
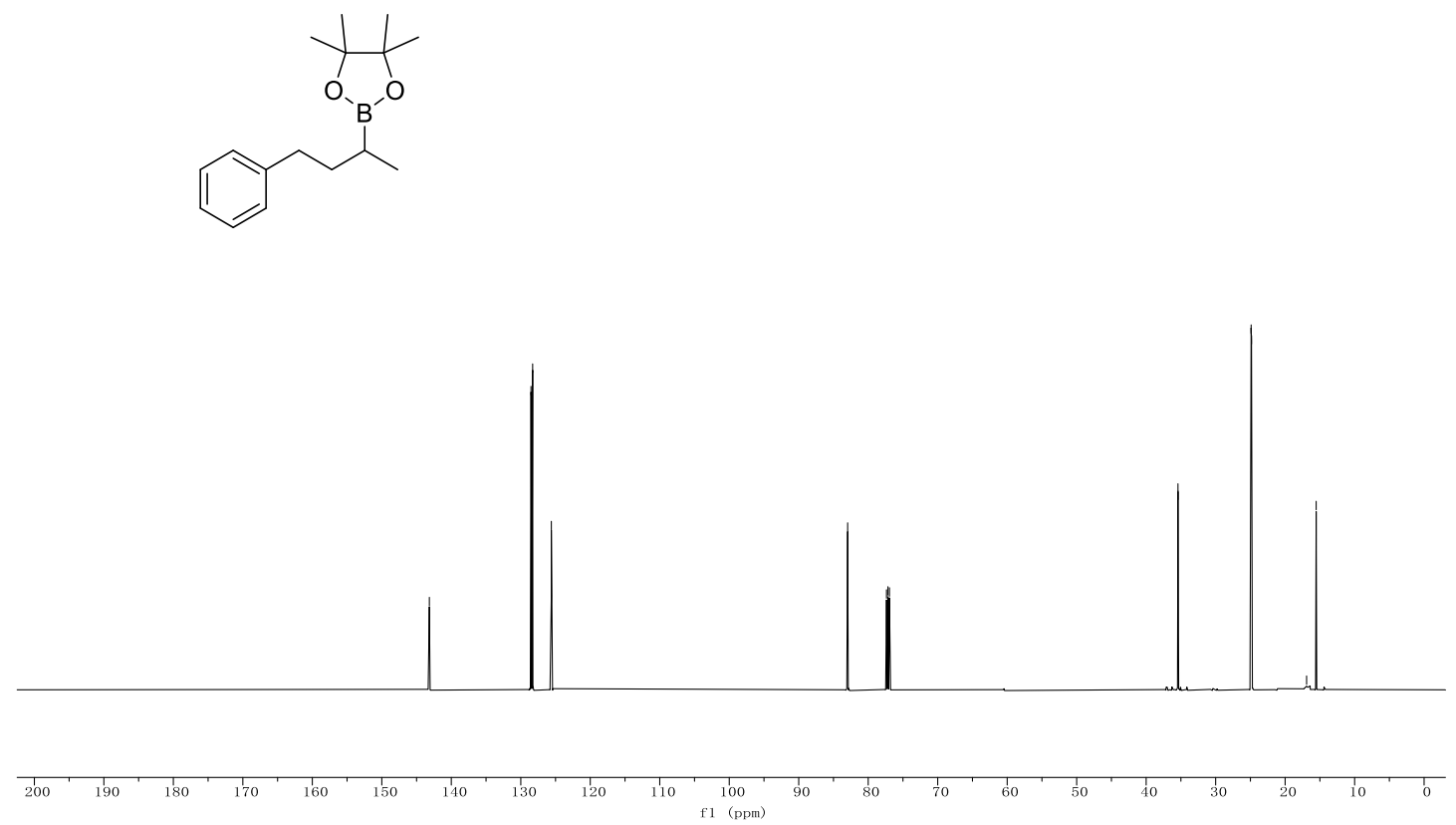


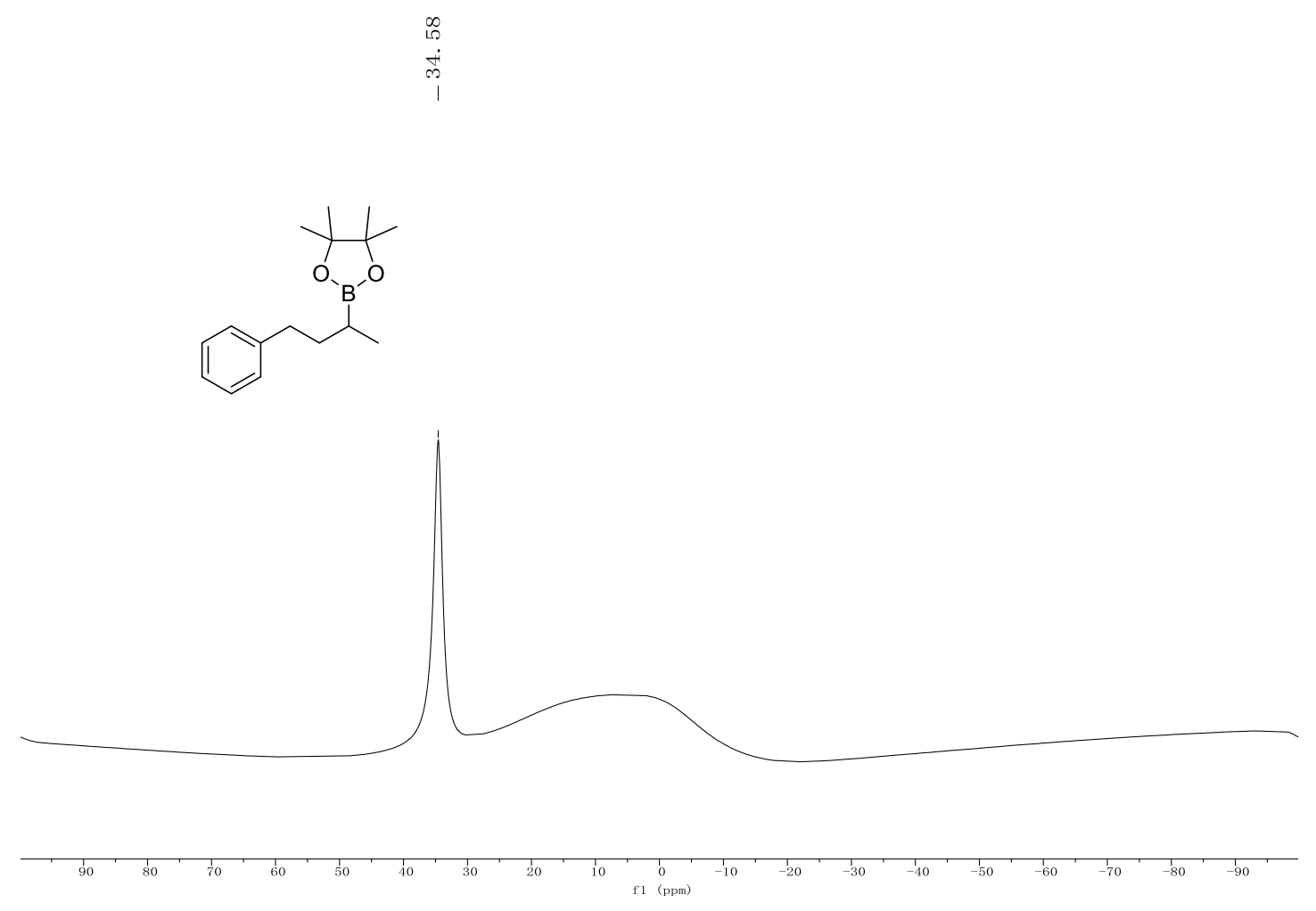

\section{Compound 54}

m

ம்

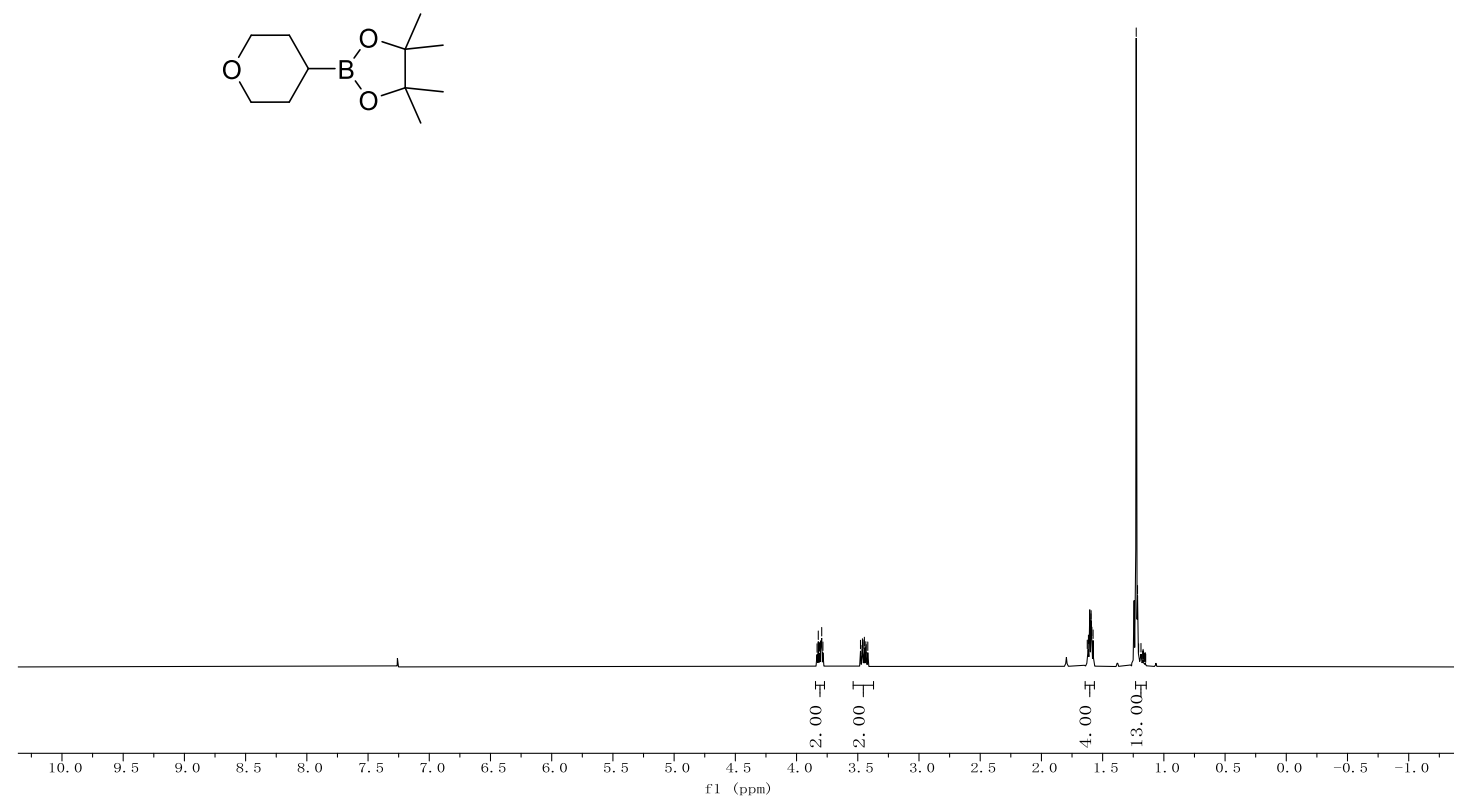


ปั

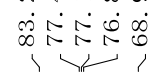

$\vec{N}$
$\stackrel{N}{N} \dot{N}^{-}$
1
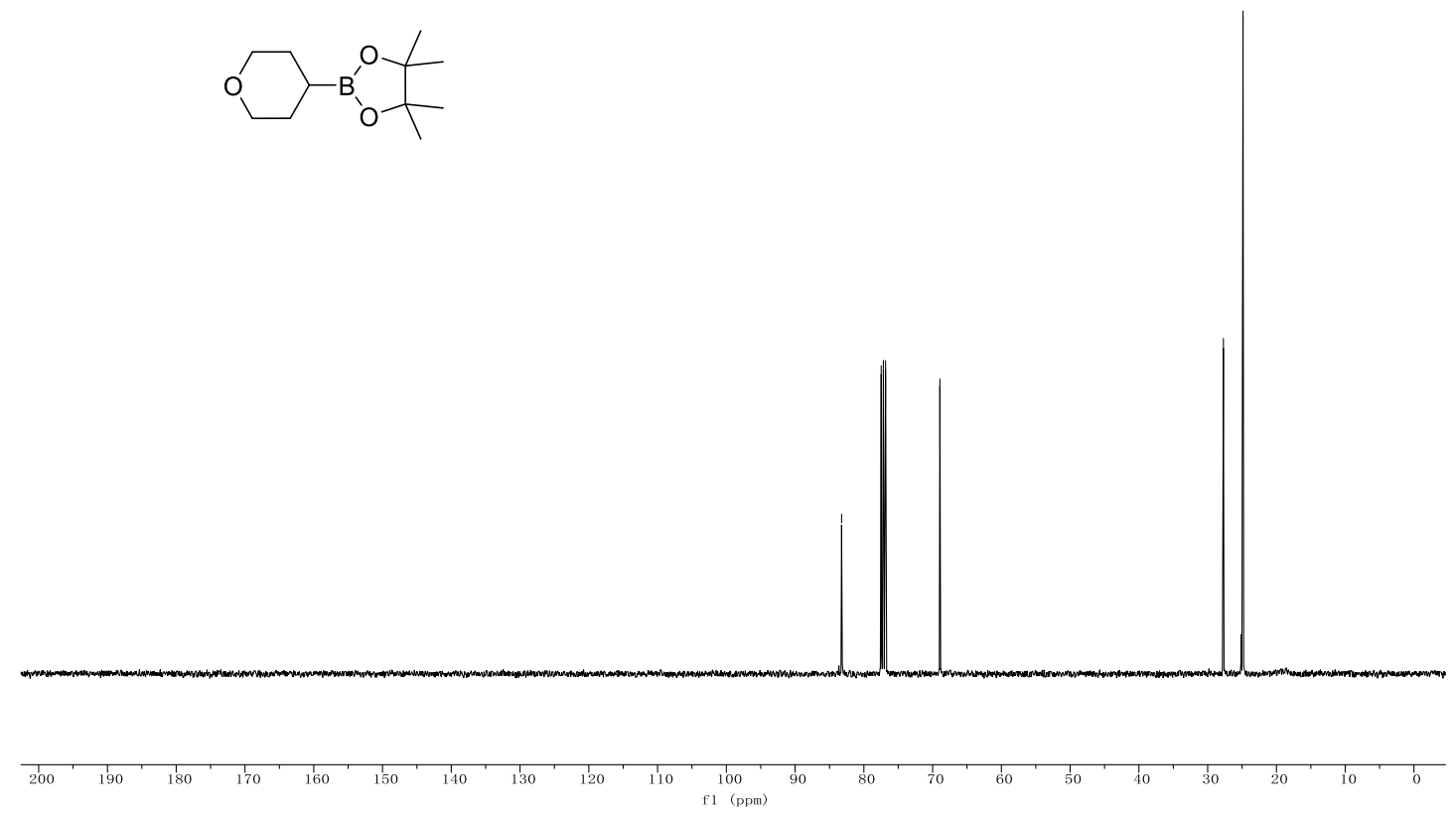

8
ले
ॠ
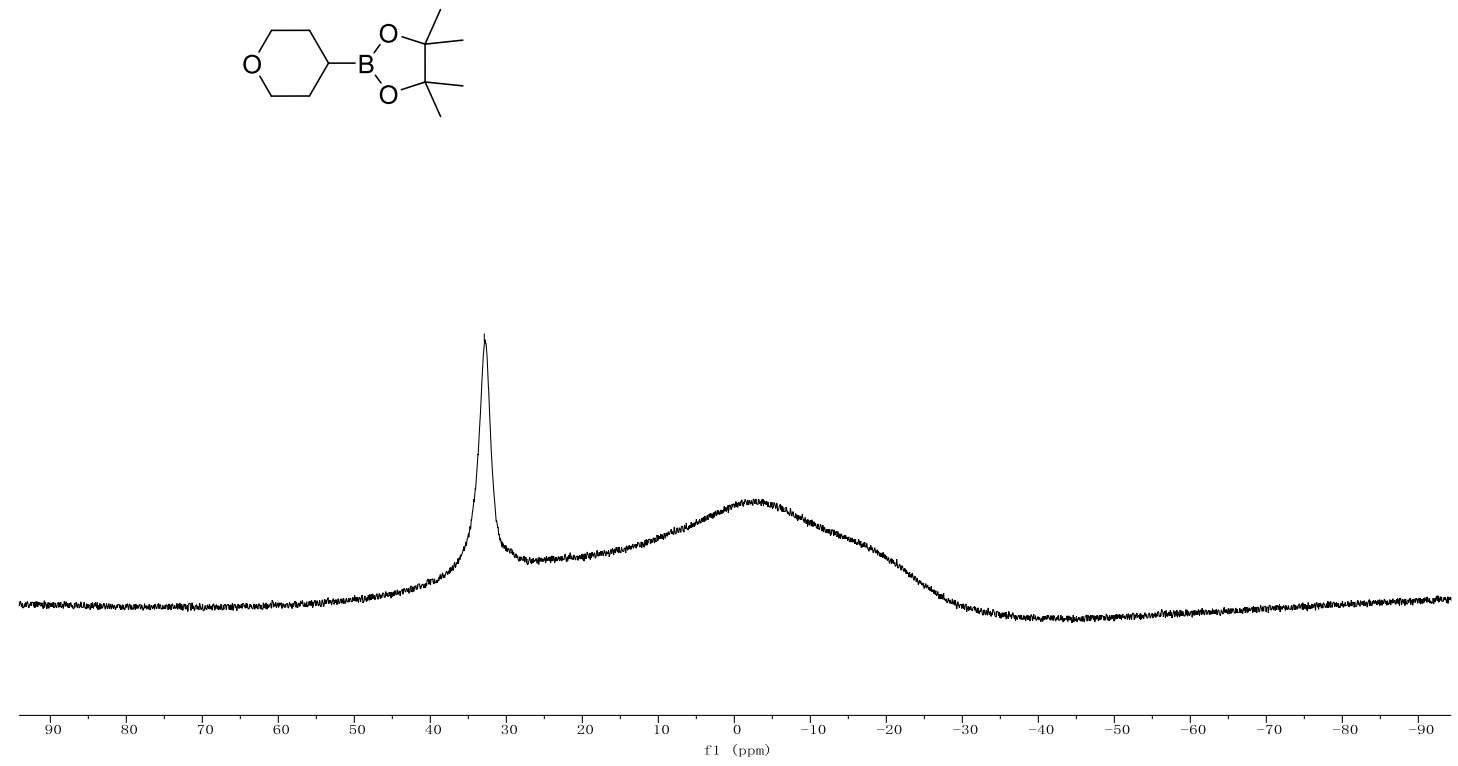

S166 


\section{Compound 55}
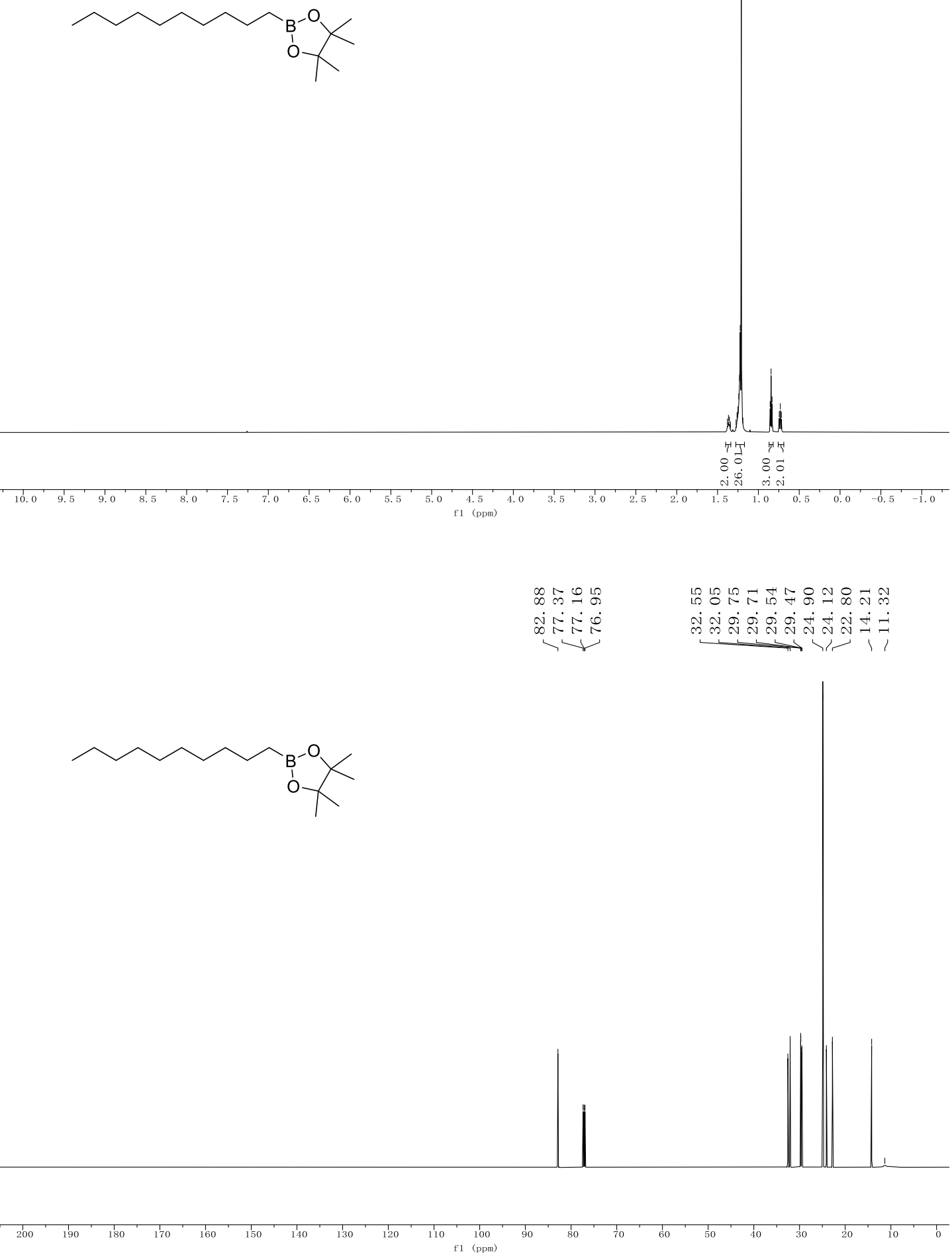

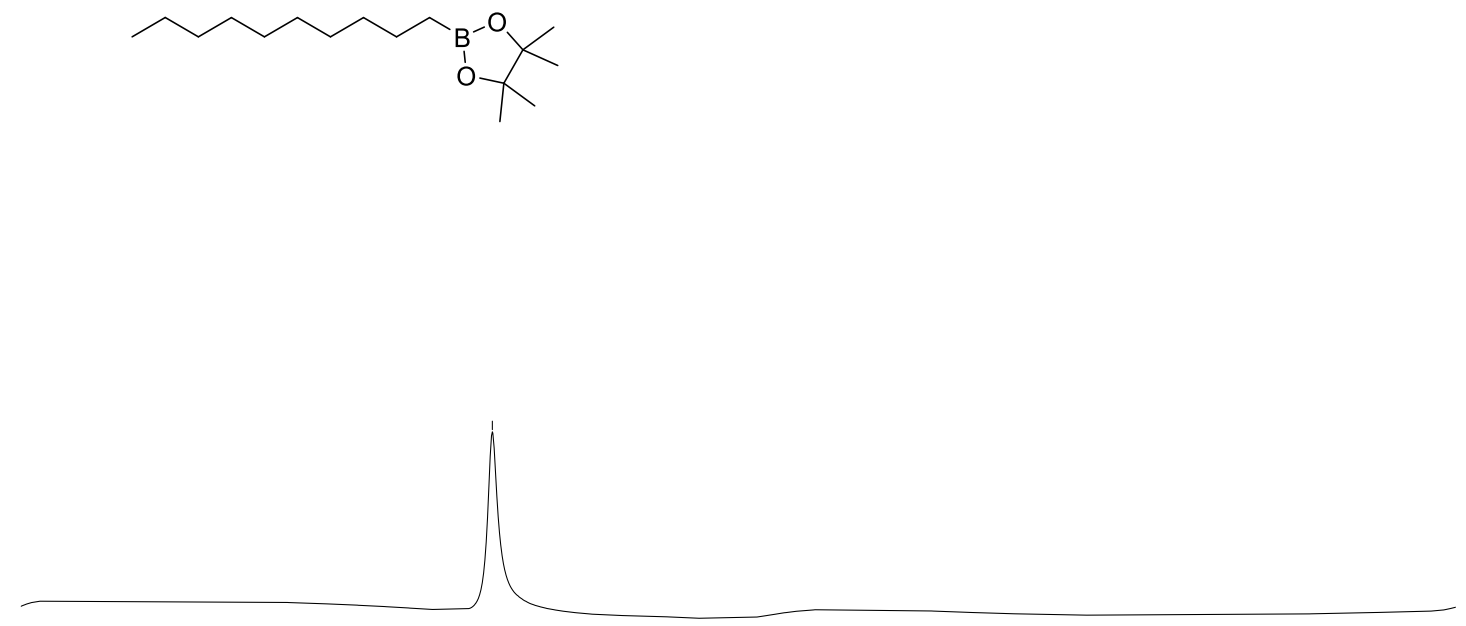

\section{Compound 56}

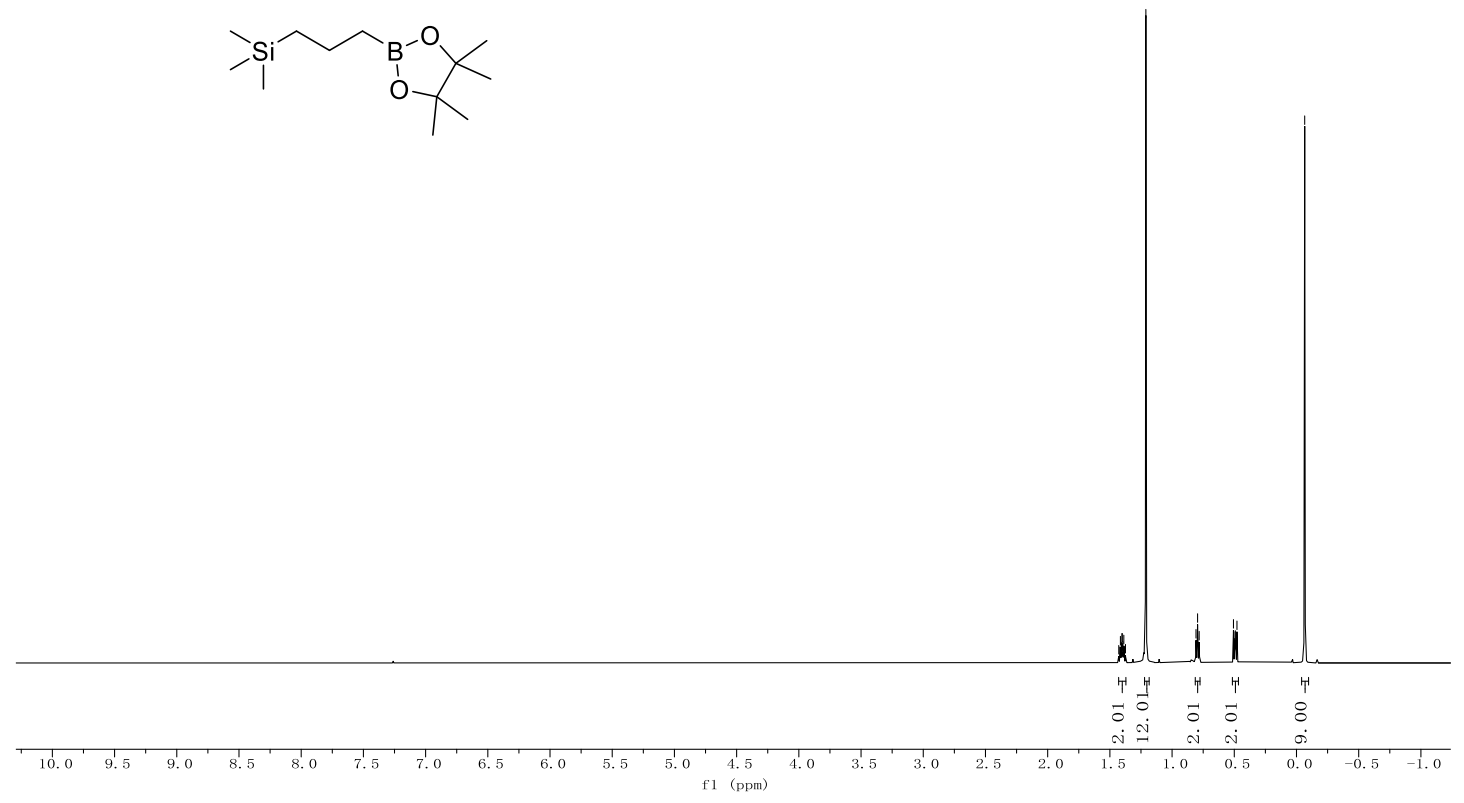




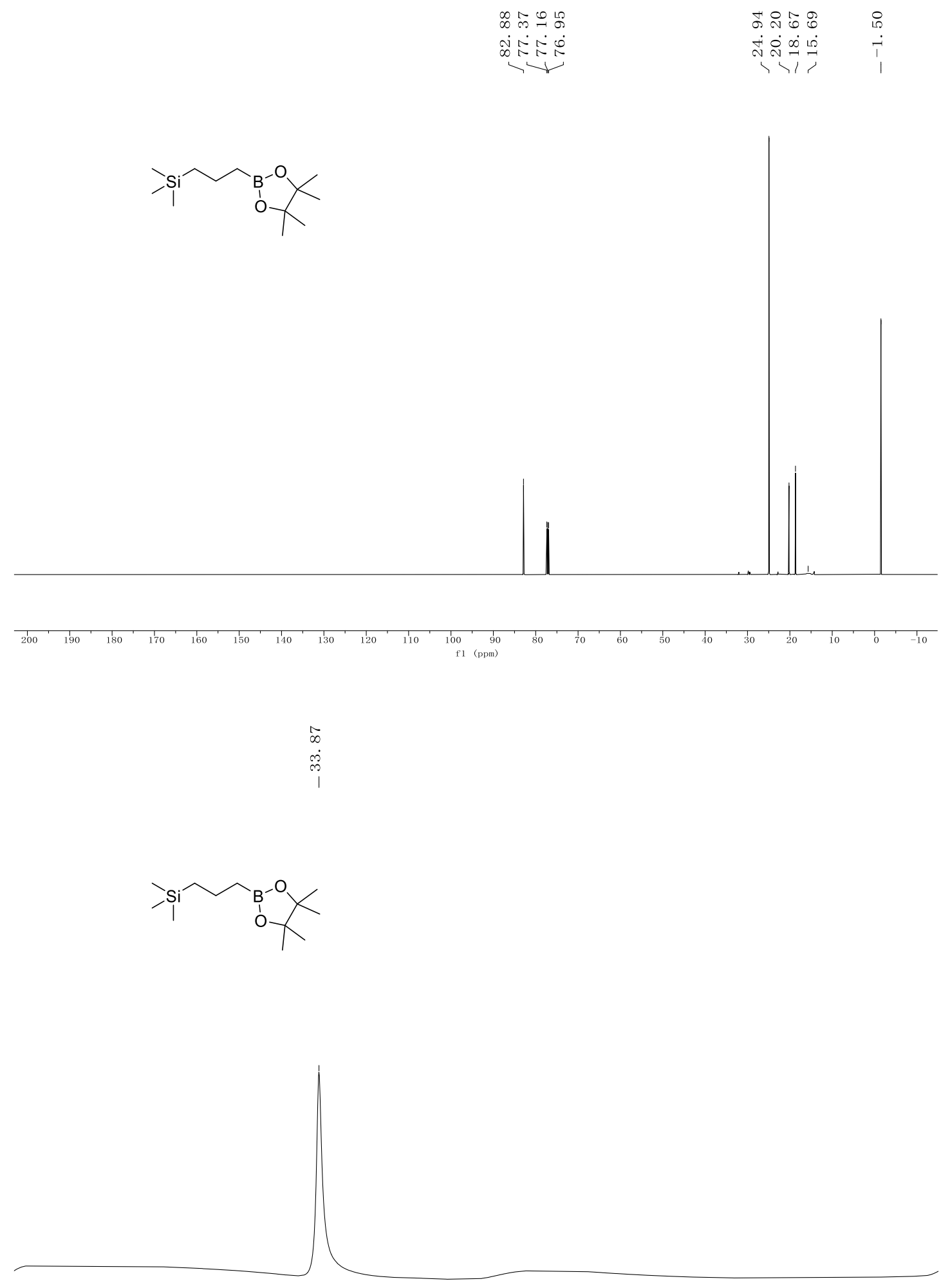

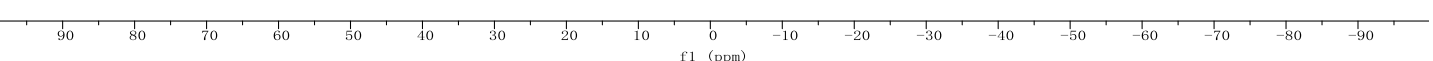




\section{Compound 57}

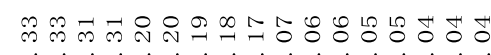

荄ニ゙

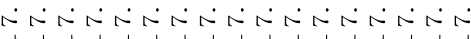

$\overbrace{\substack{1 \\ 1 \\ 1}}^{10}<$

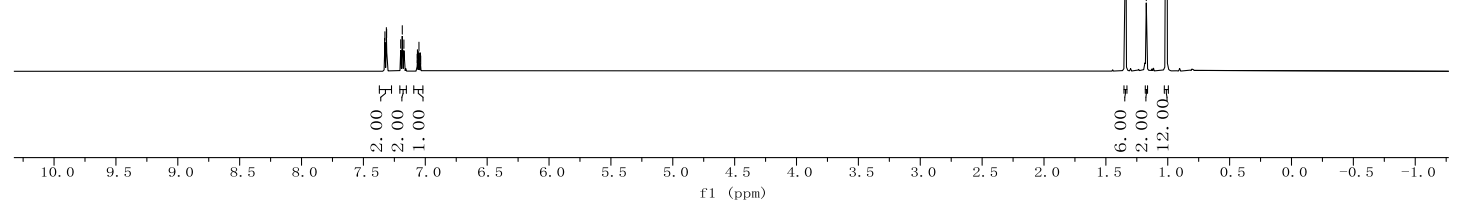

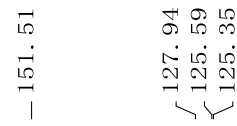

옹요용

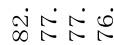

$\widehat{m} \forall \vec{\forall}$

ल
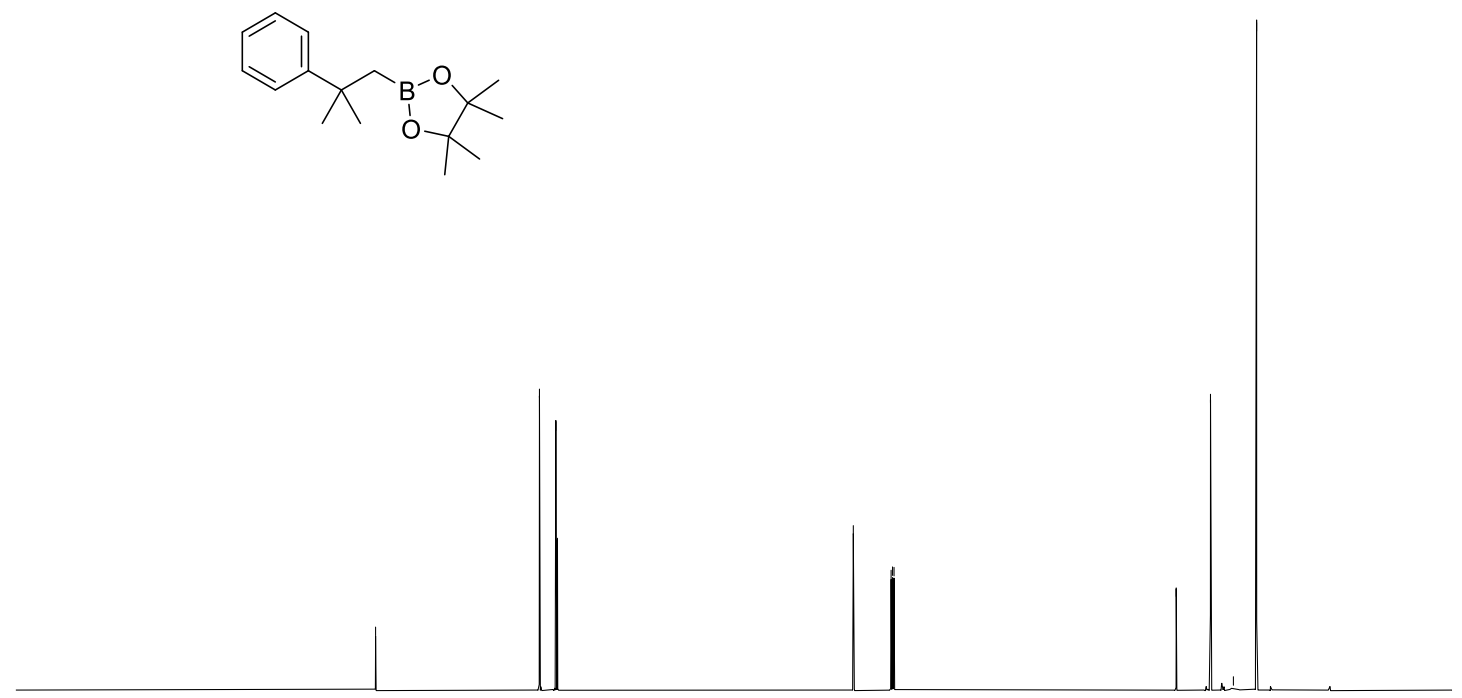

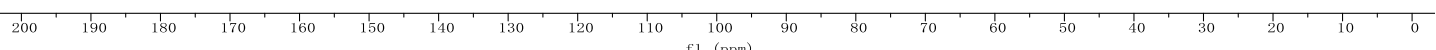
$\longrightarrow$ $\mathrm{f} 1(\mathrm{ppm})$ 


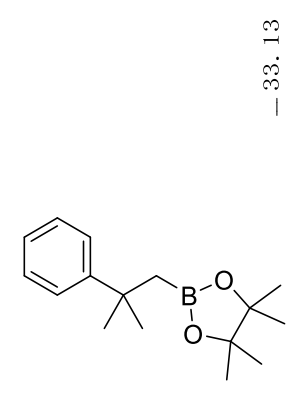

$\stackrel{m}{\stackrel{\infty}{\infty}}$
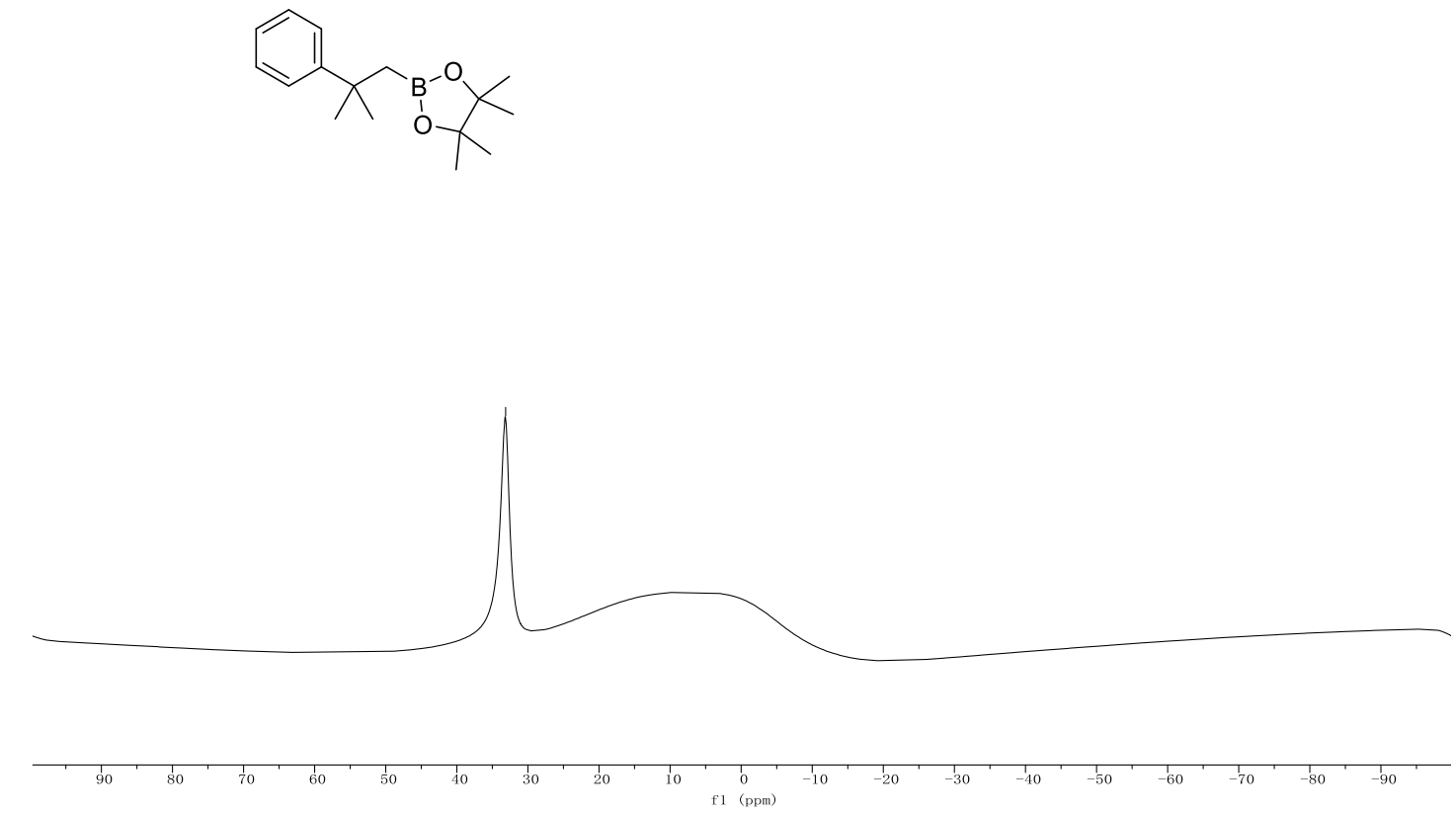

\section{Compound 58}

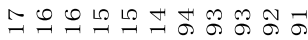

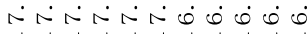

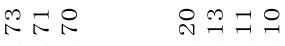

نู่

皮

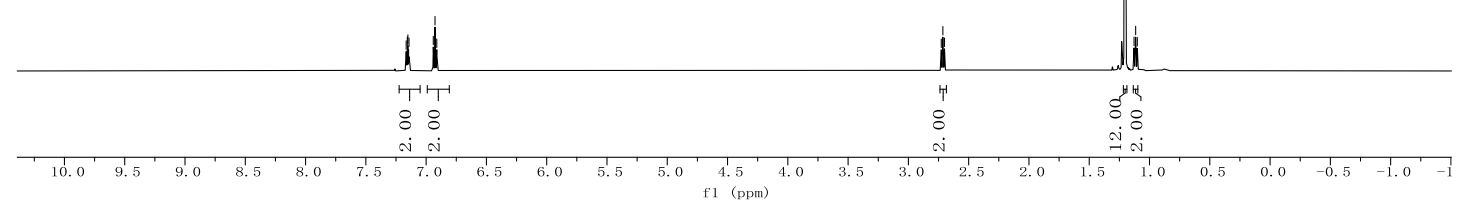




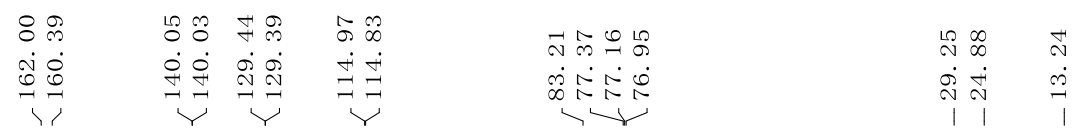<smiles>CC1(C)OB(CCc2ccc(F)cc2)OC1(C)C</smiles>
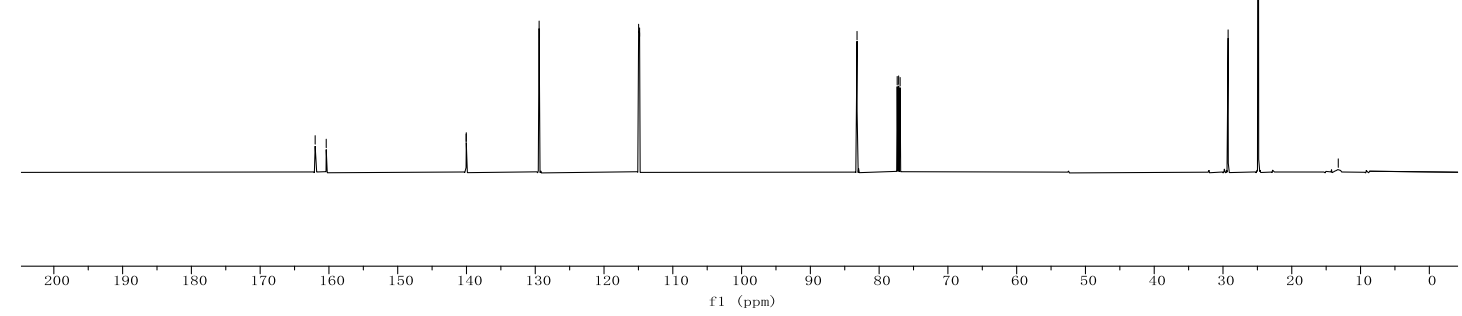

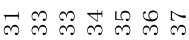

$\infty \infty_{\infty}^{\infty} \infty \infty^{\infty} \infty$
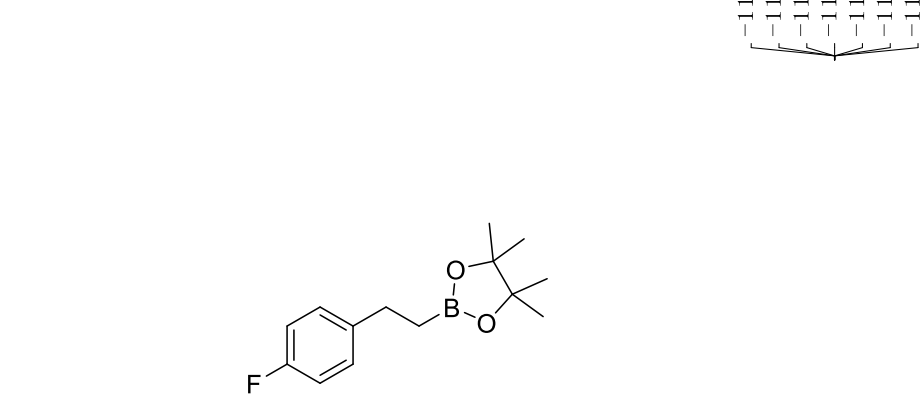

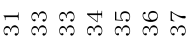

$\infty \dot{\infty} \infty \dot{\infty} \infty \dot{\infty} \dot{\infty}$

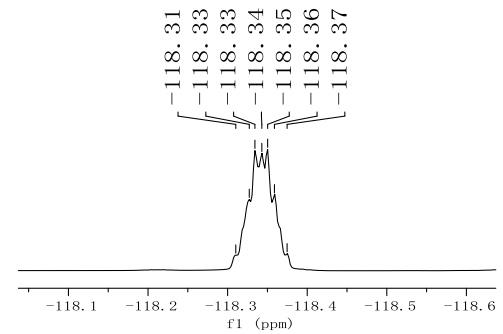

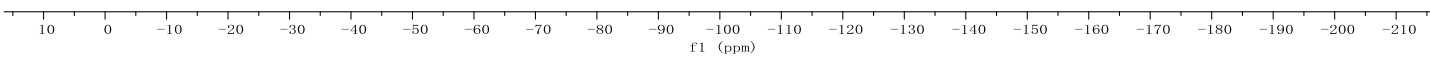



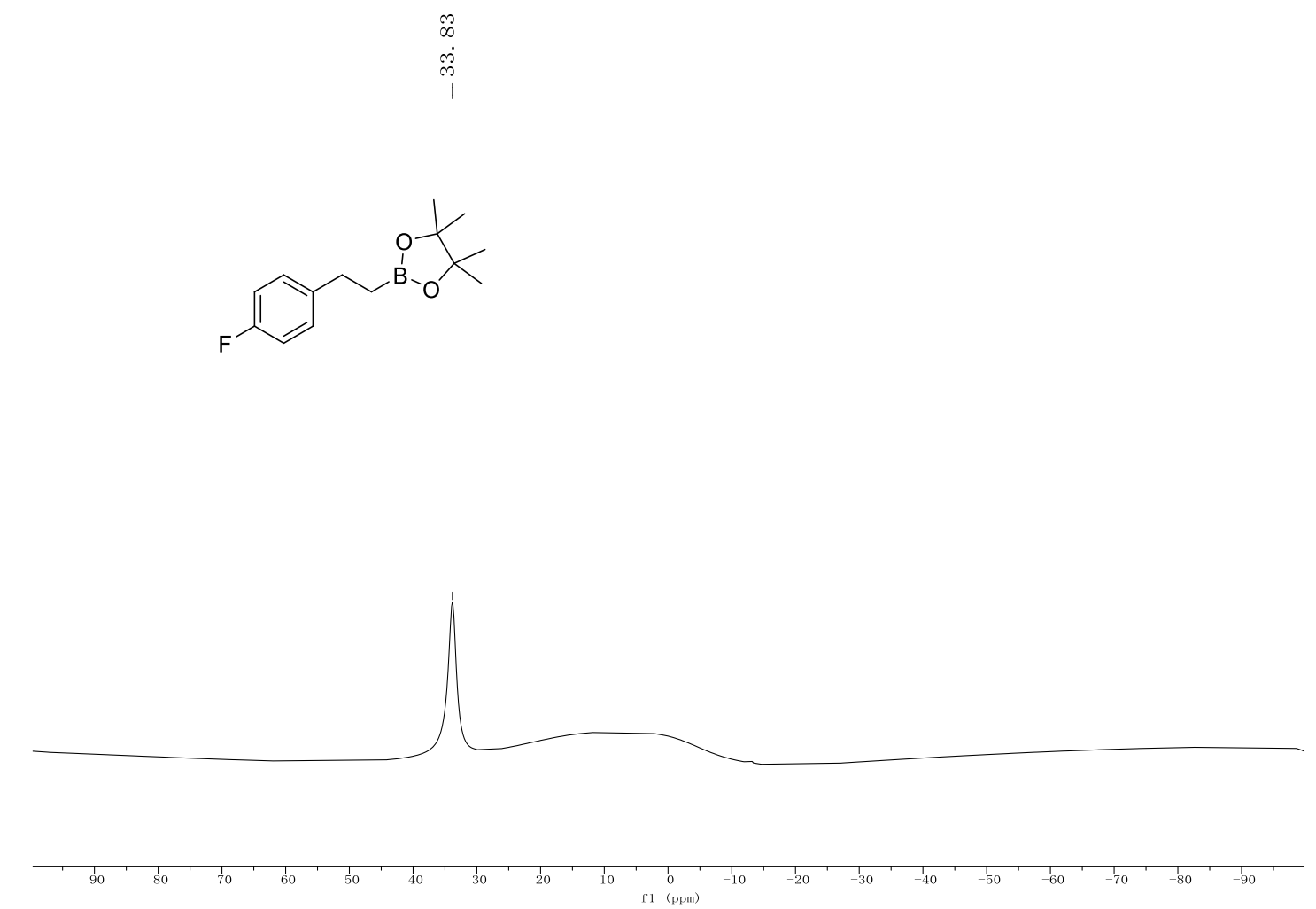

\section{Compound 59}

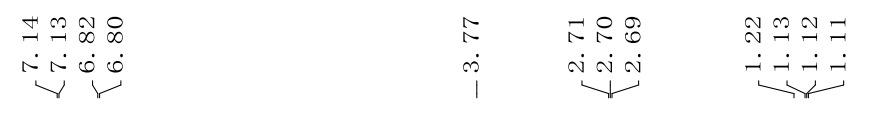

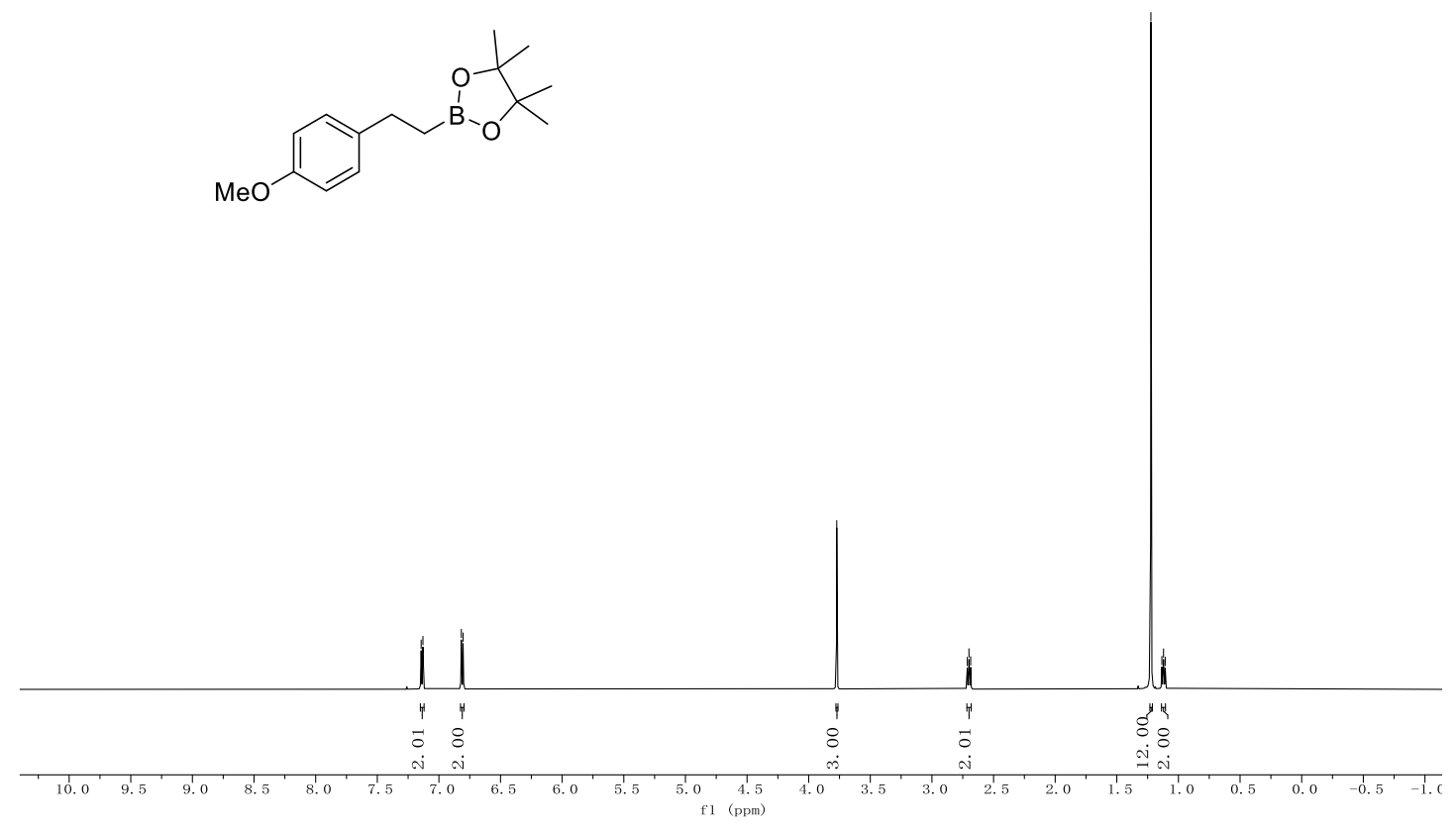




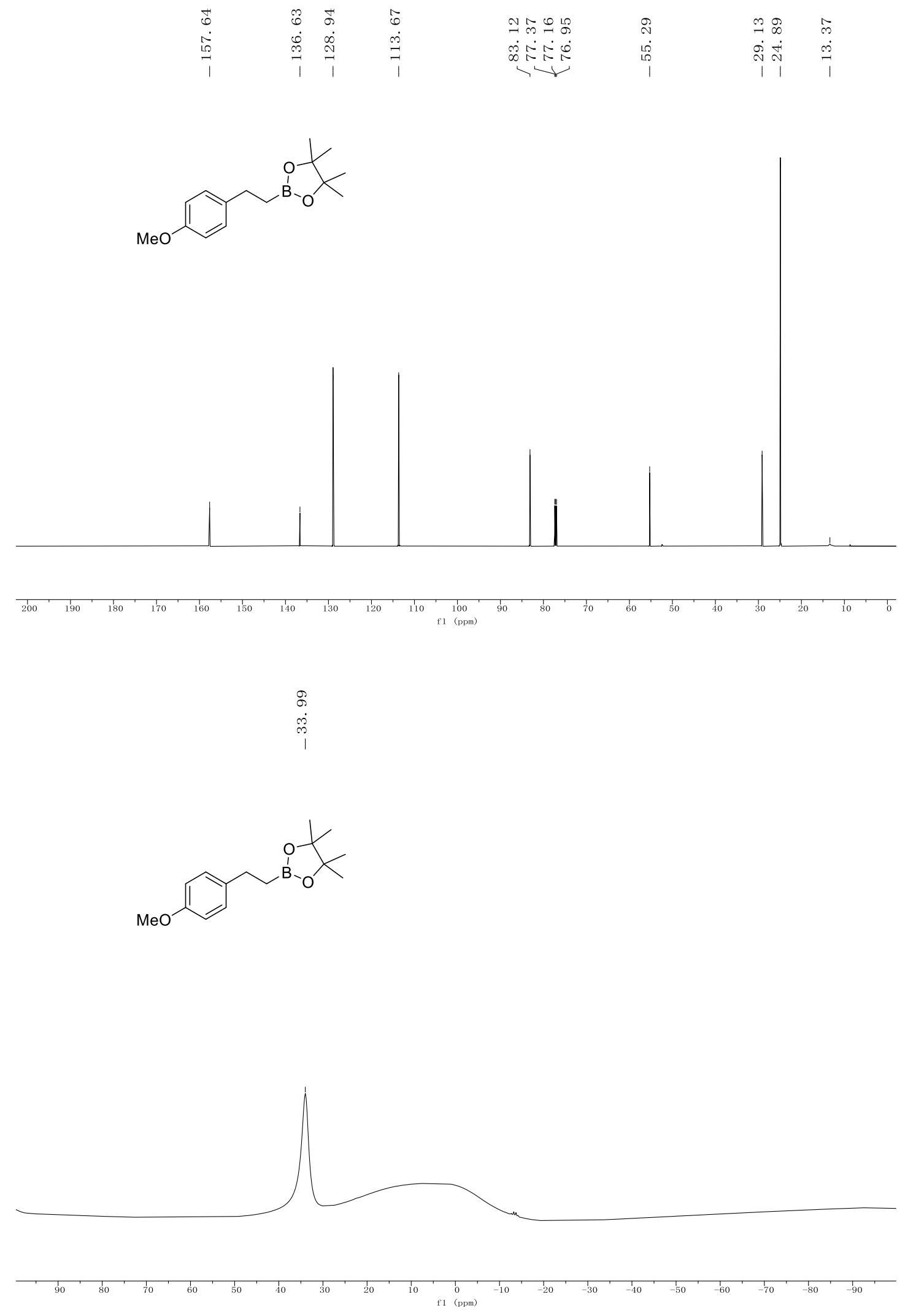




\section{Compound 60}

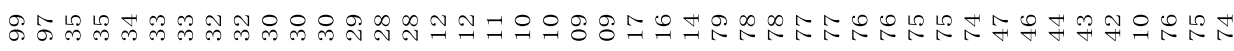

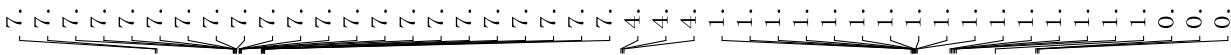

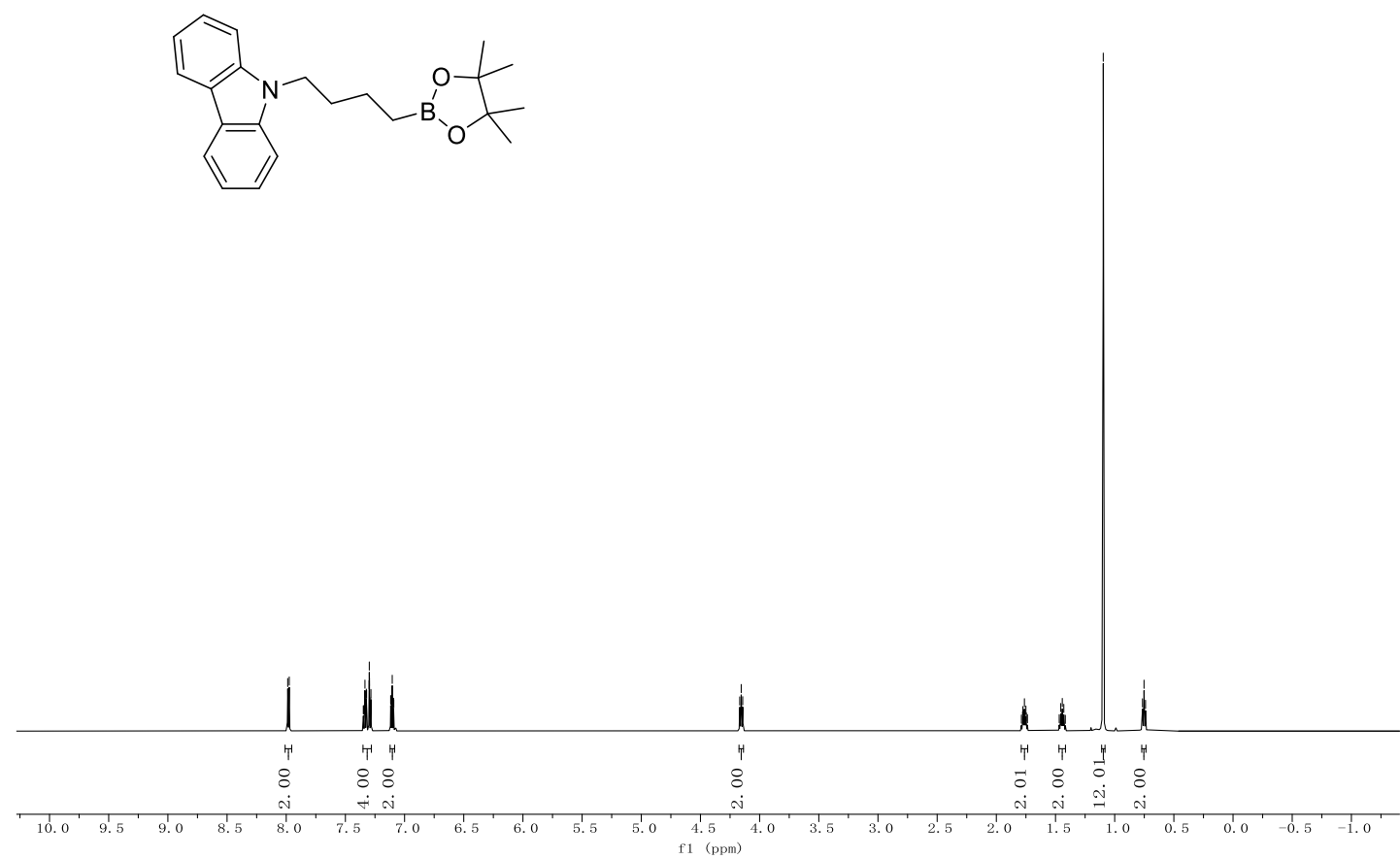

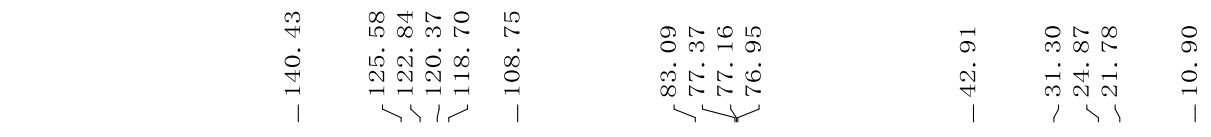
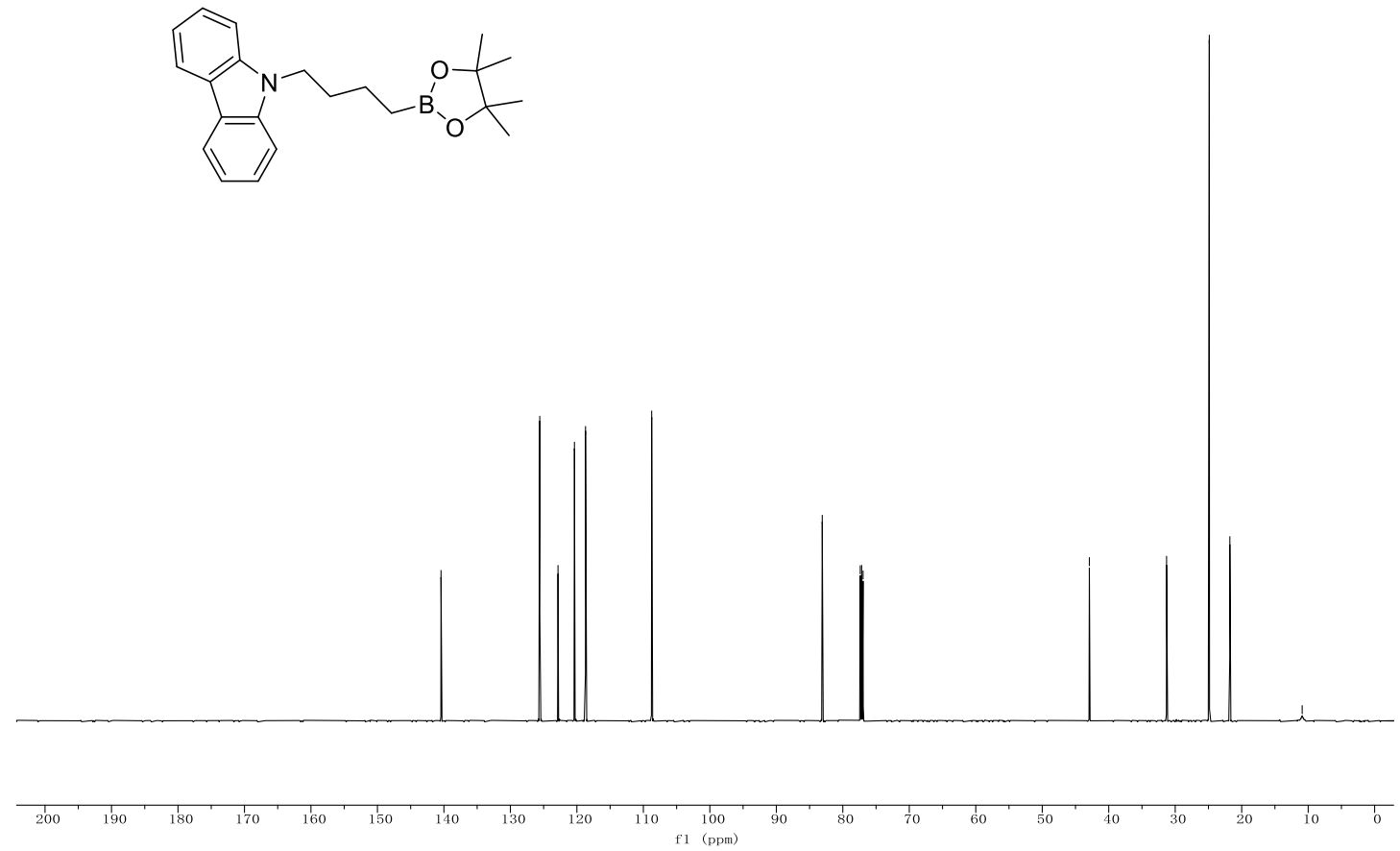

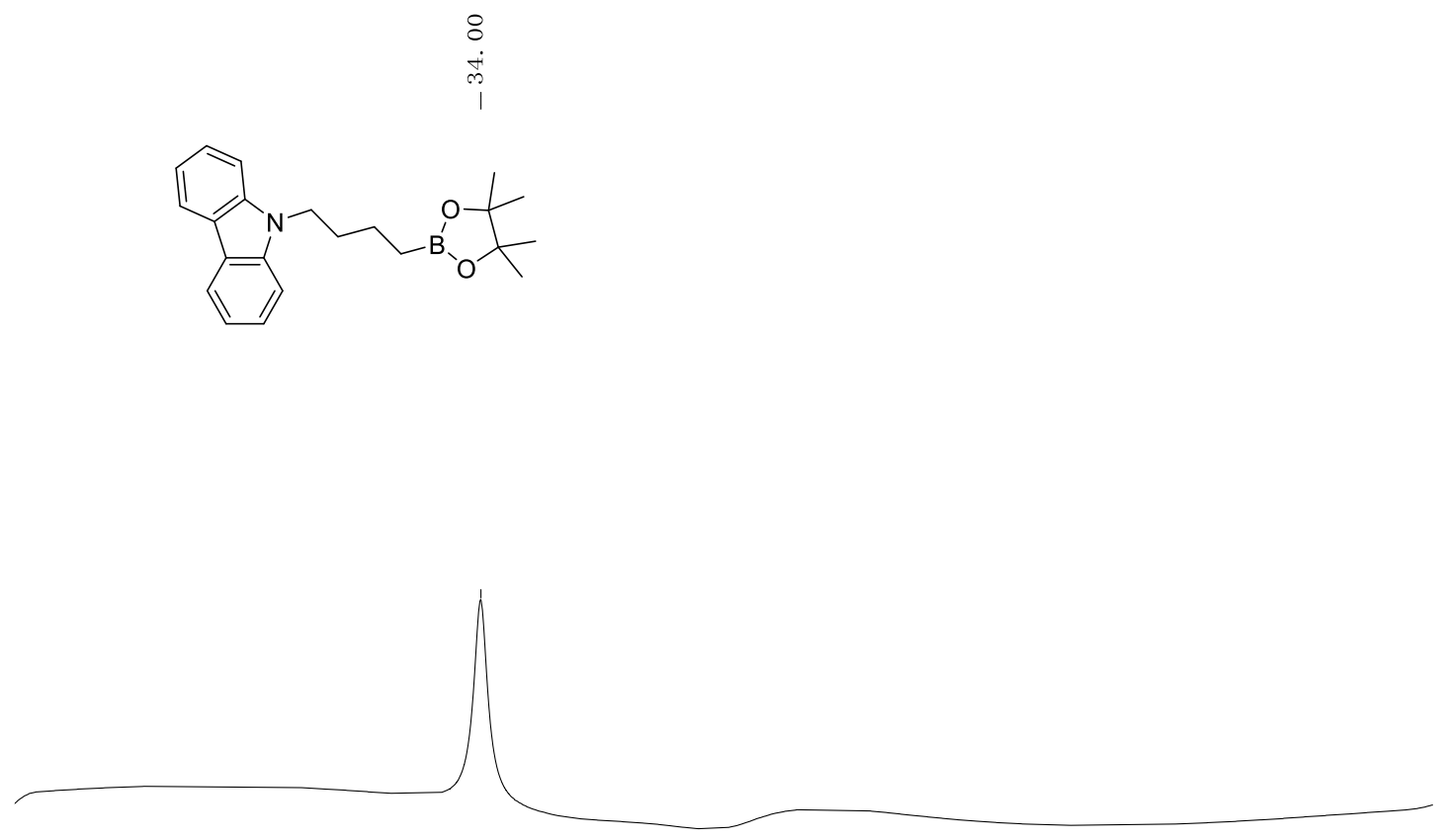

\section{Compound 61}

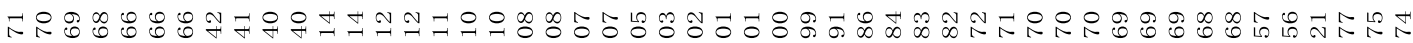

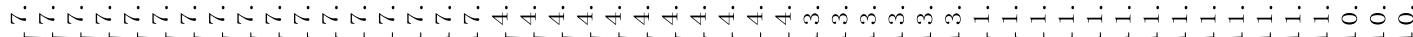

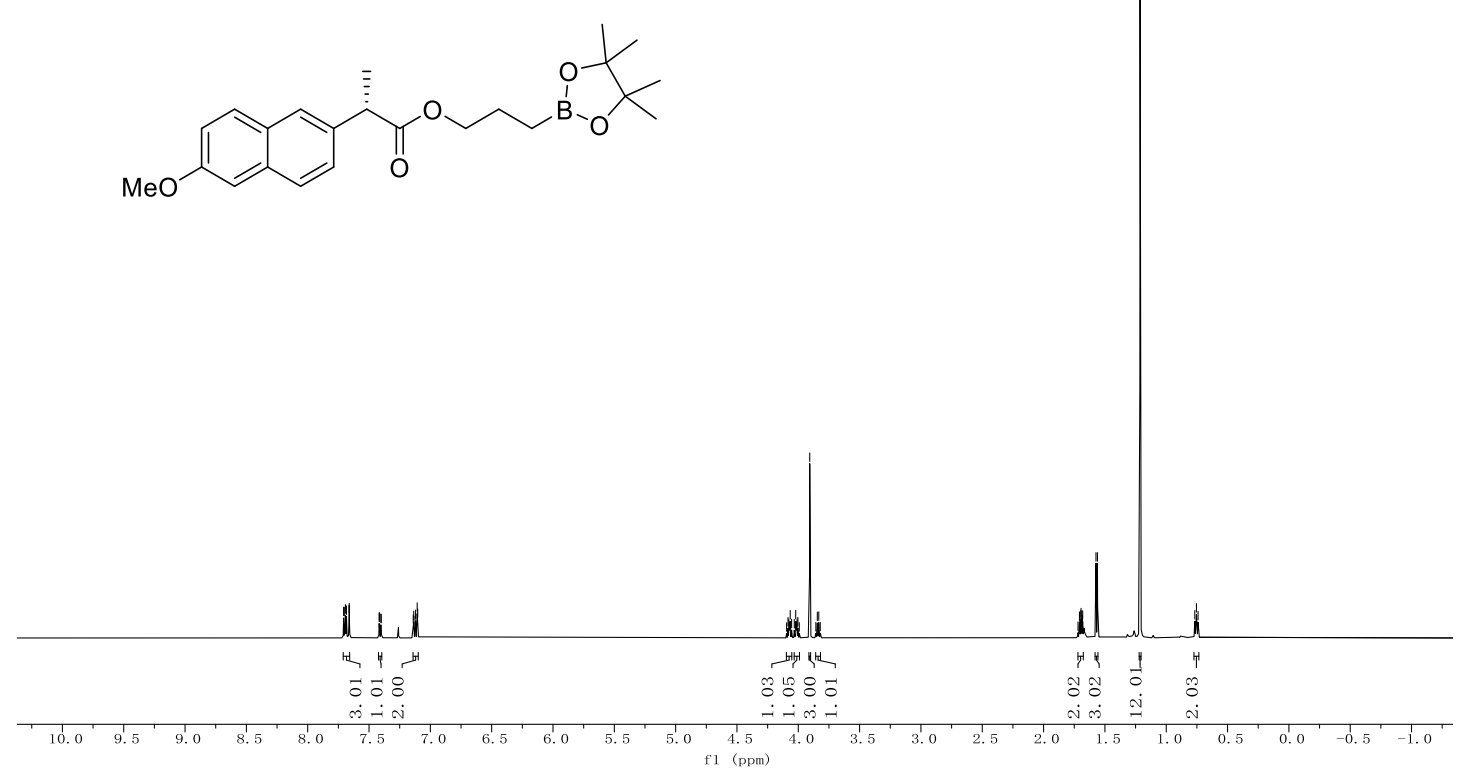



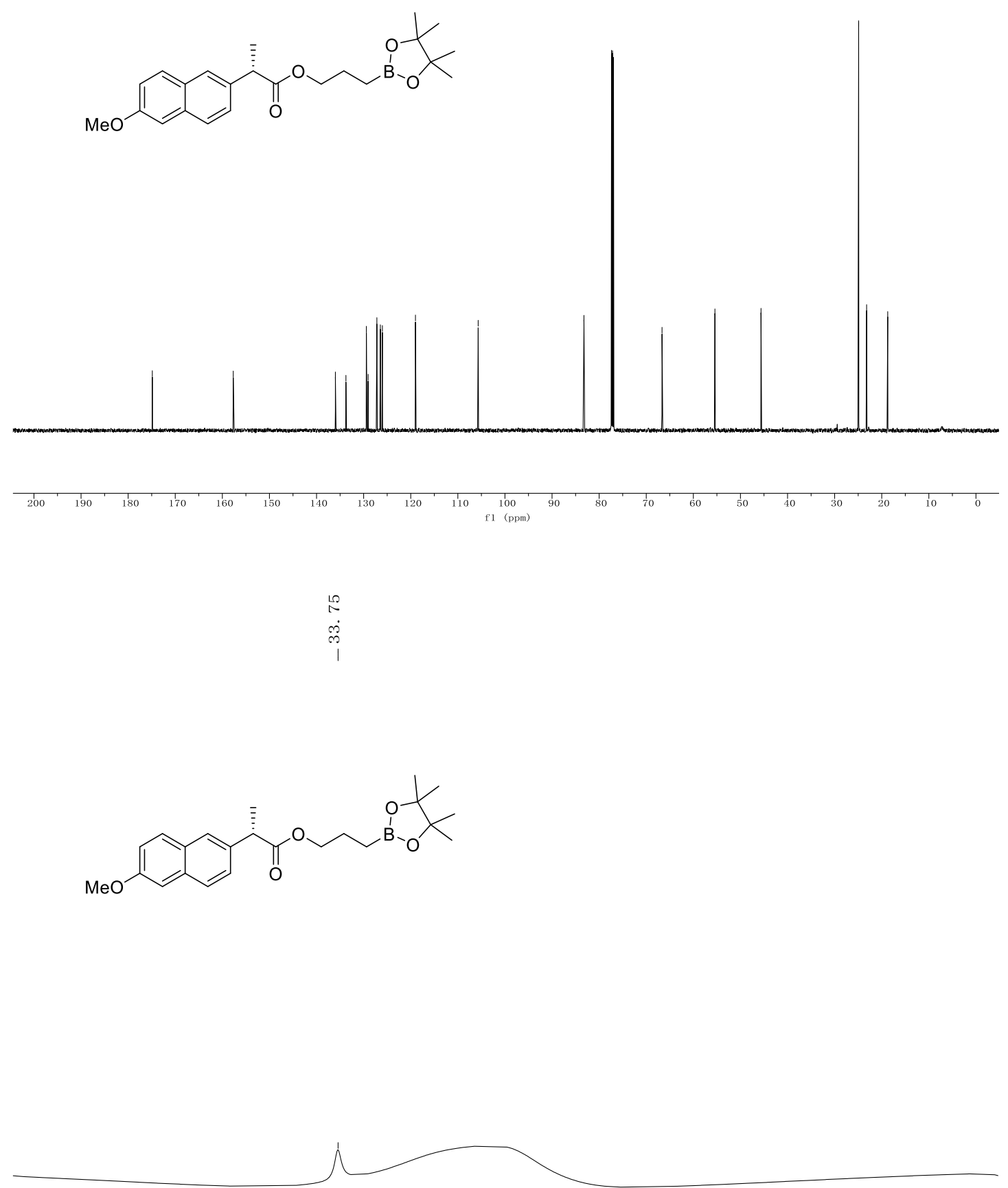


\section{Compound 62}

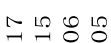

N只N

$\rightarrow$

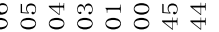

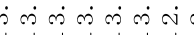

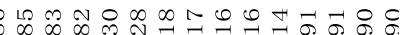

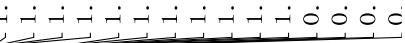

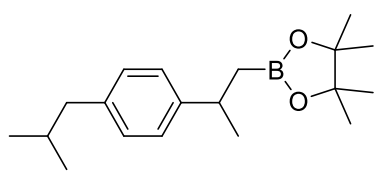

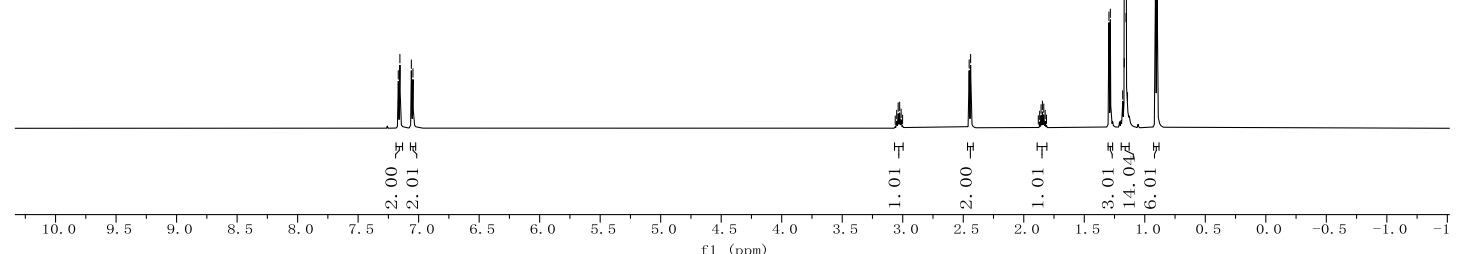
Ұ $8: 87$

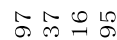

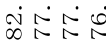

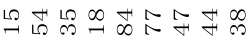
$\begin{array}{ccc}\dot{0} & \infty & \infty \\ \stackrel{0}{*} & \stackrel{0}{N} \\ 1 & 1 & 1\end{array}$

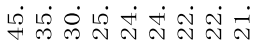
舟

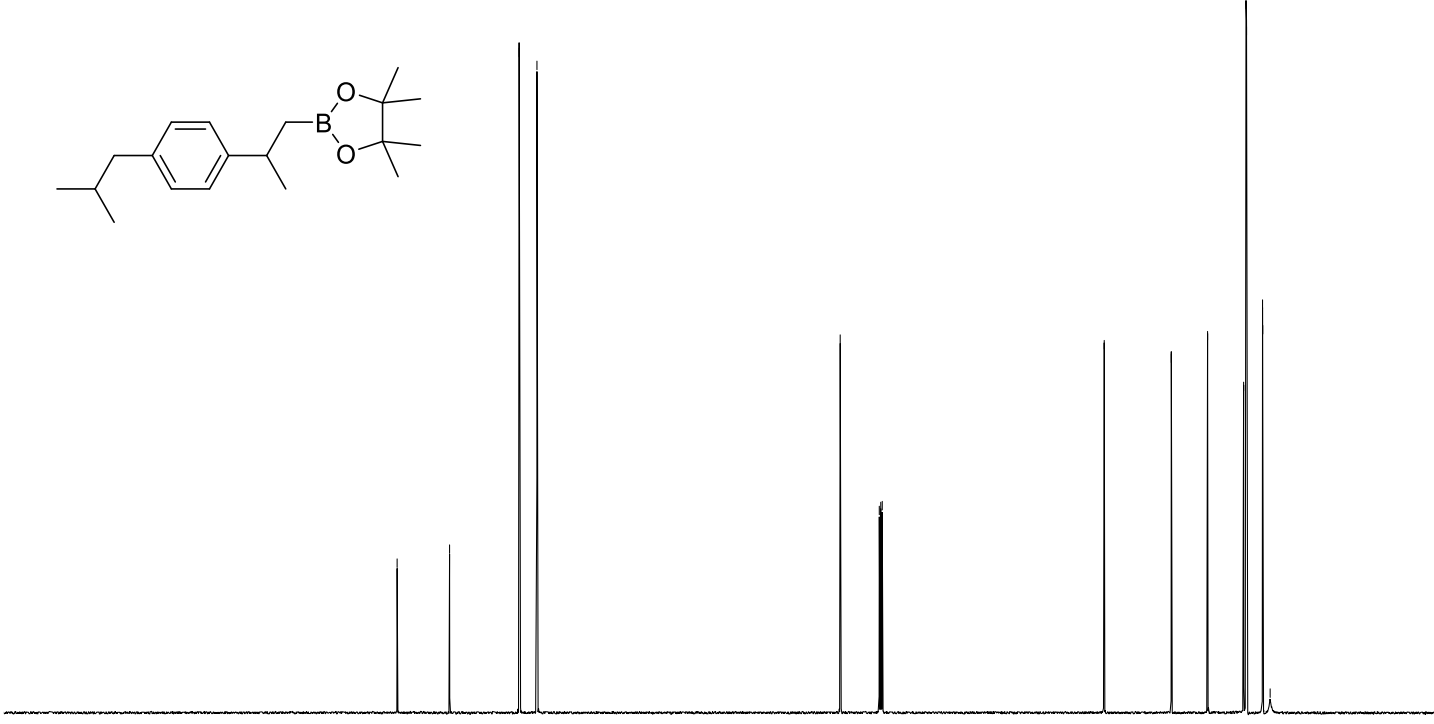

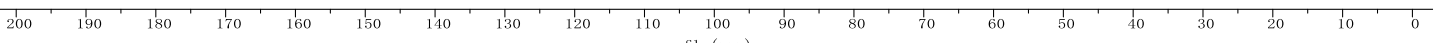
f1 (ppm) 

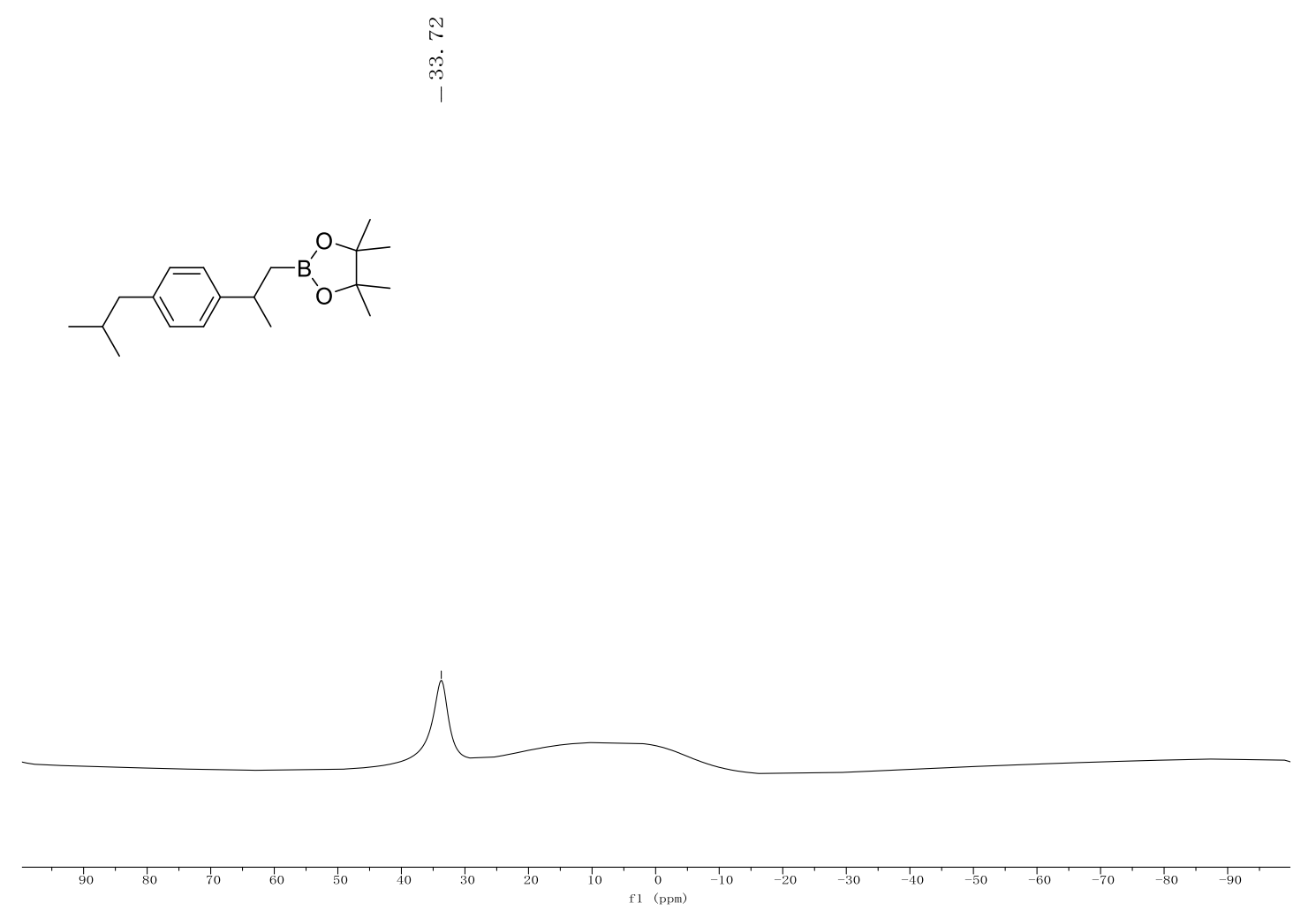

\section{Compound 63}

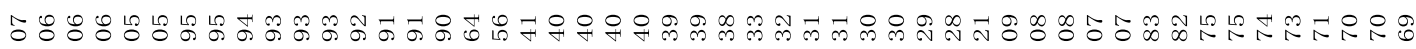
ம்

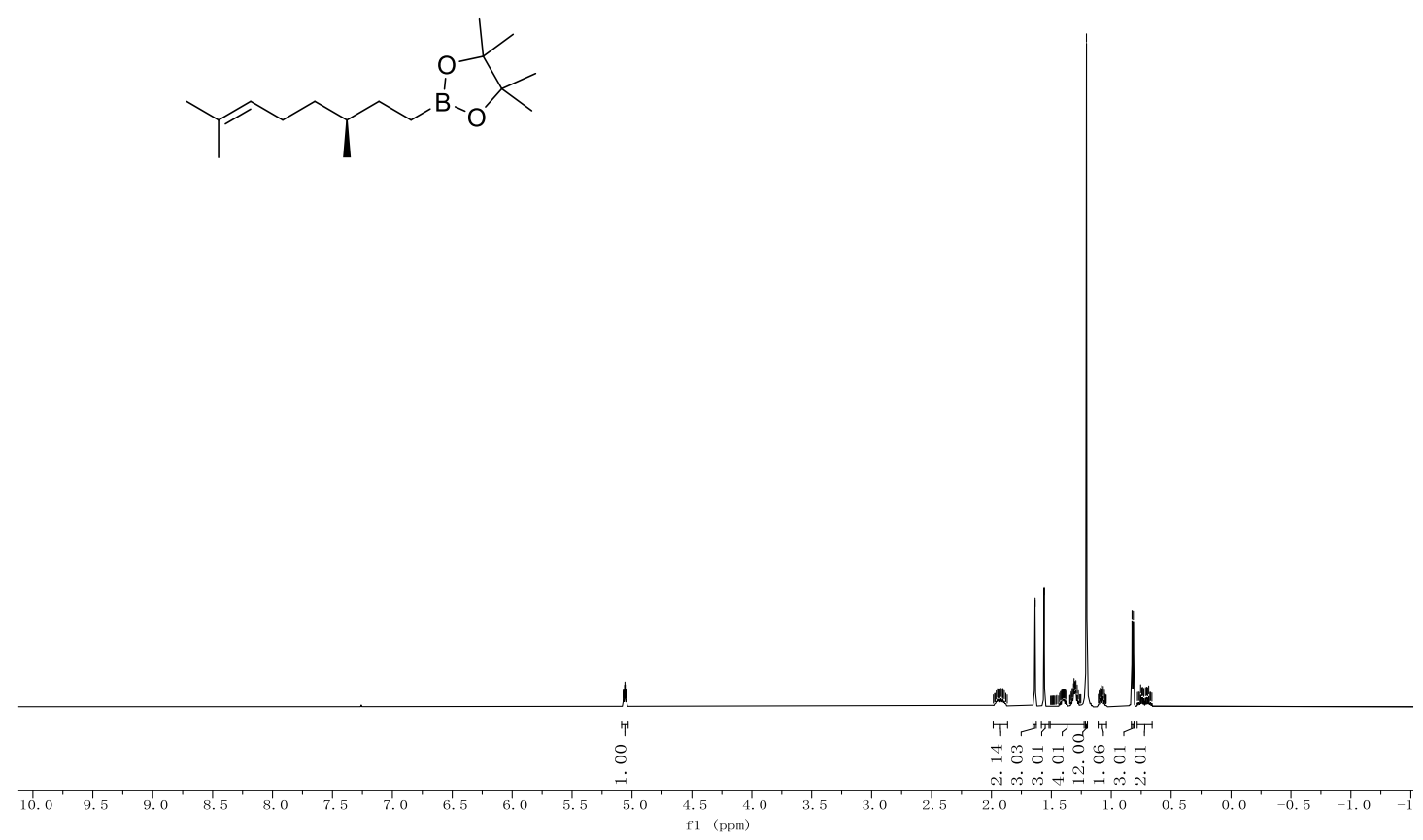




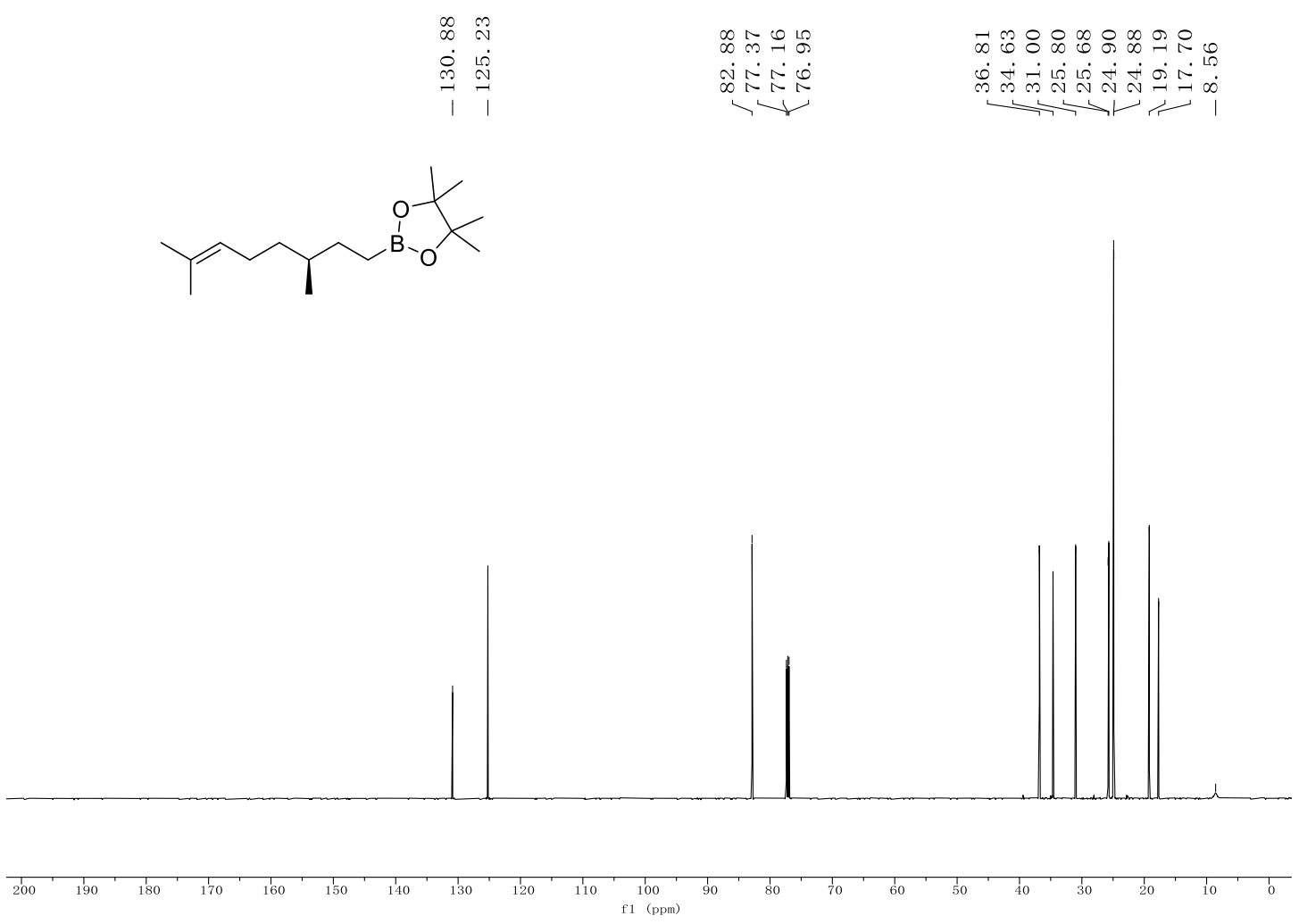

$\stackrel{\llcorner}{N}$
$\stackrel{m}{m}$
$i$
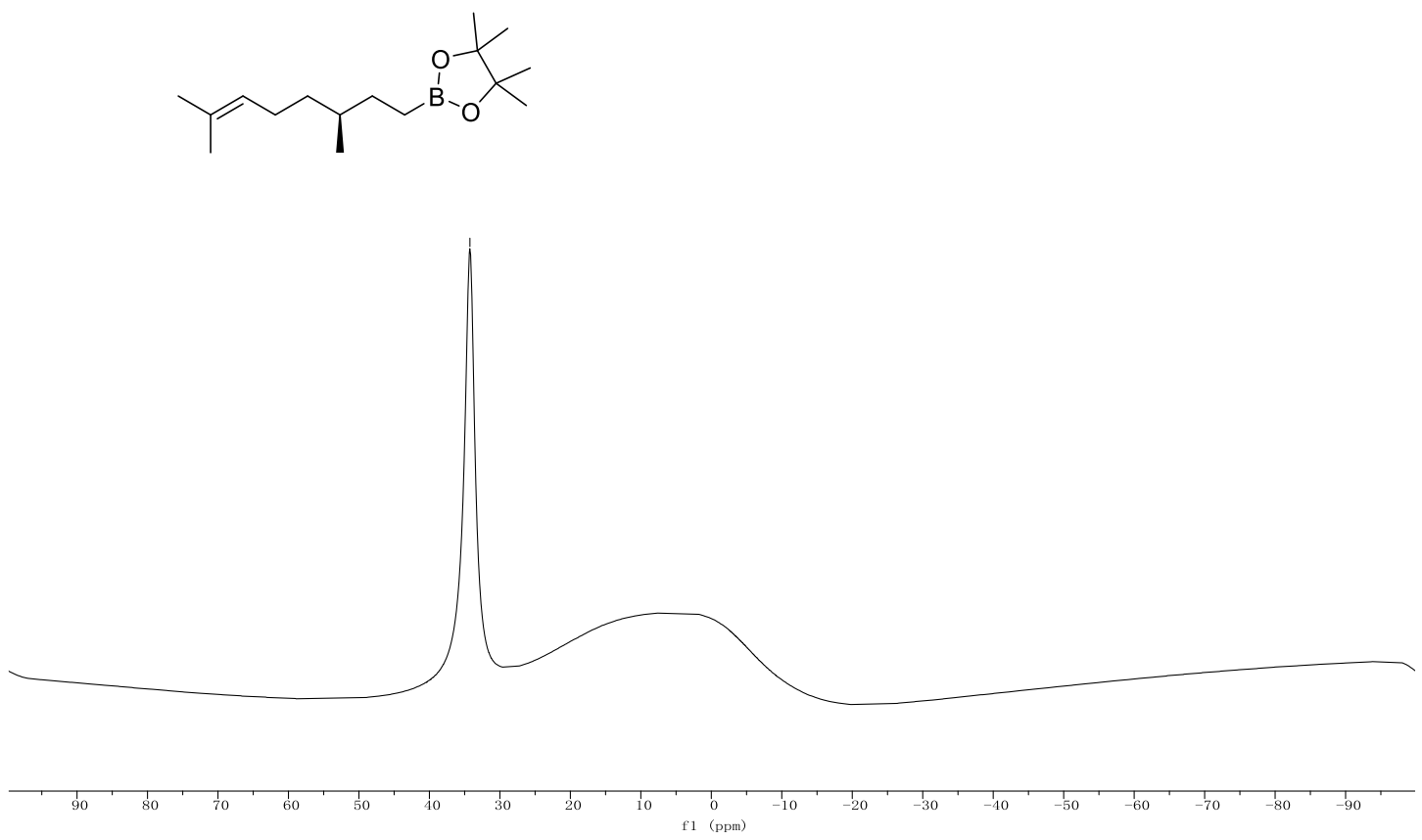

S180 


\section{Compound 64}

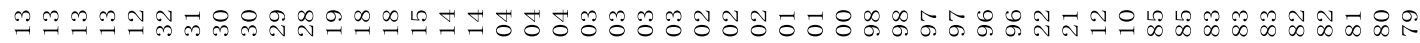
เి
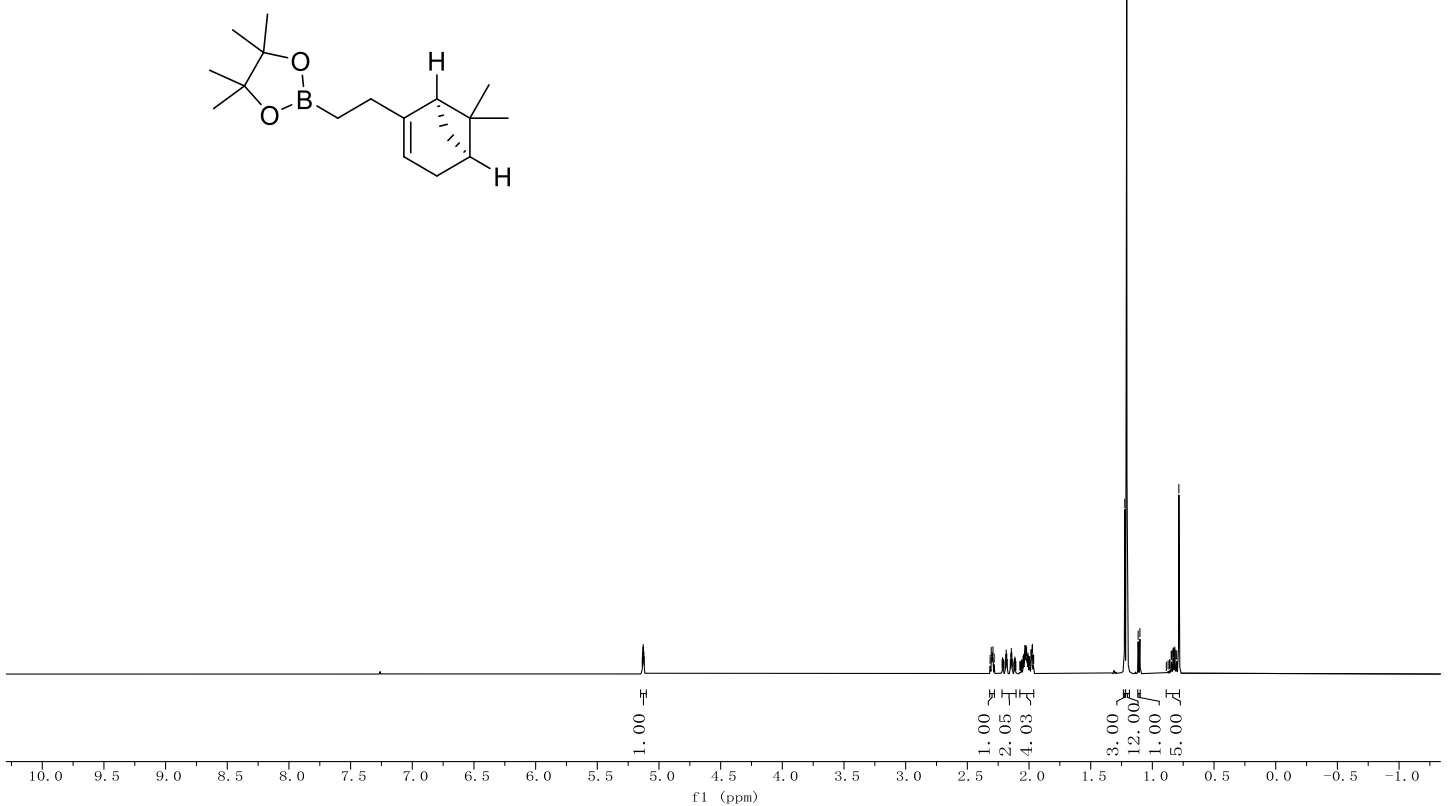

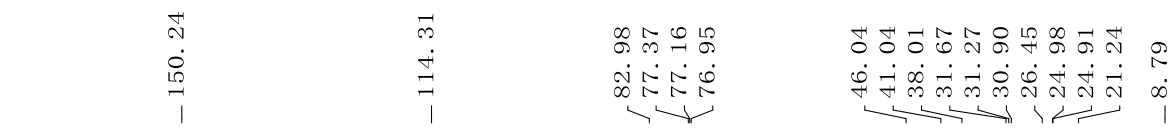
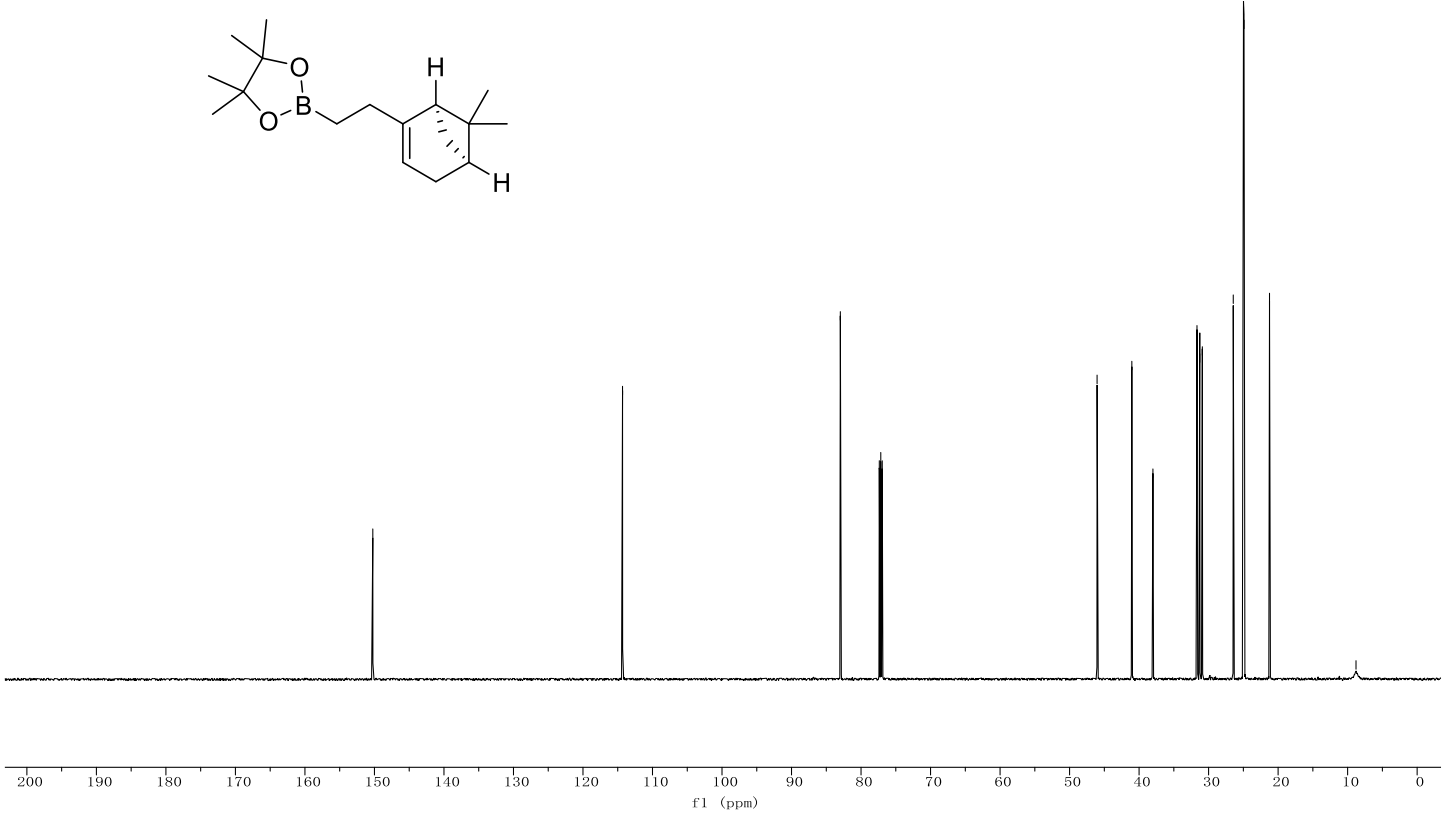

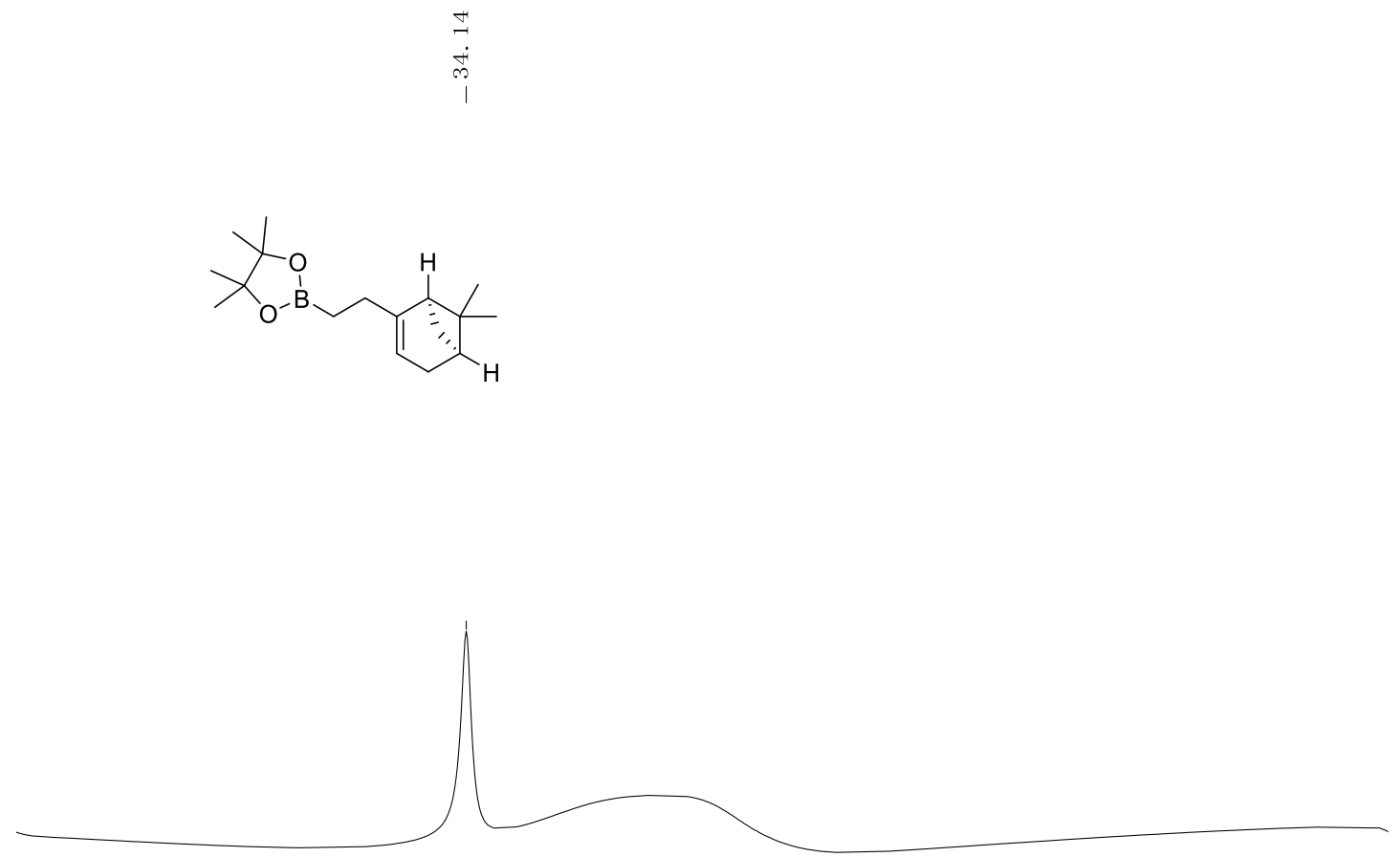

\section{Compound 65}

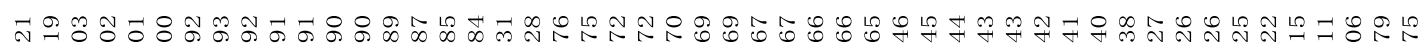
ñNূñ<smiles>CC(C)c1ccc2c(c1)CC[C@H]1[C@@](C)(CBr)CCC[C@@]21C</smiles>

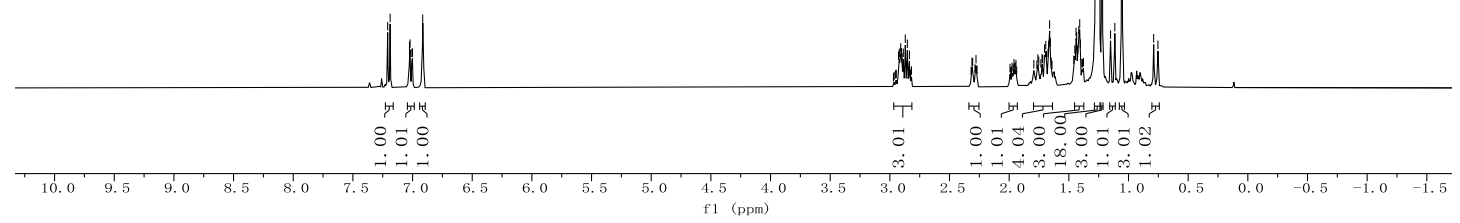




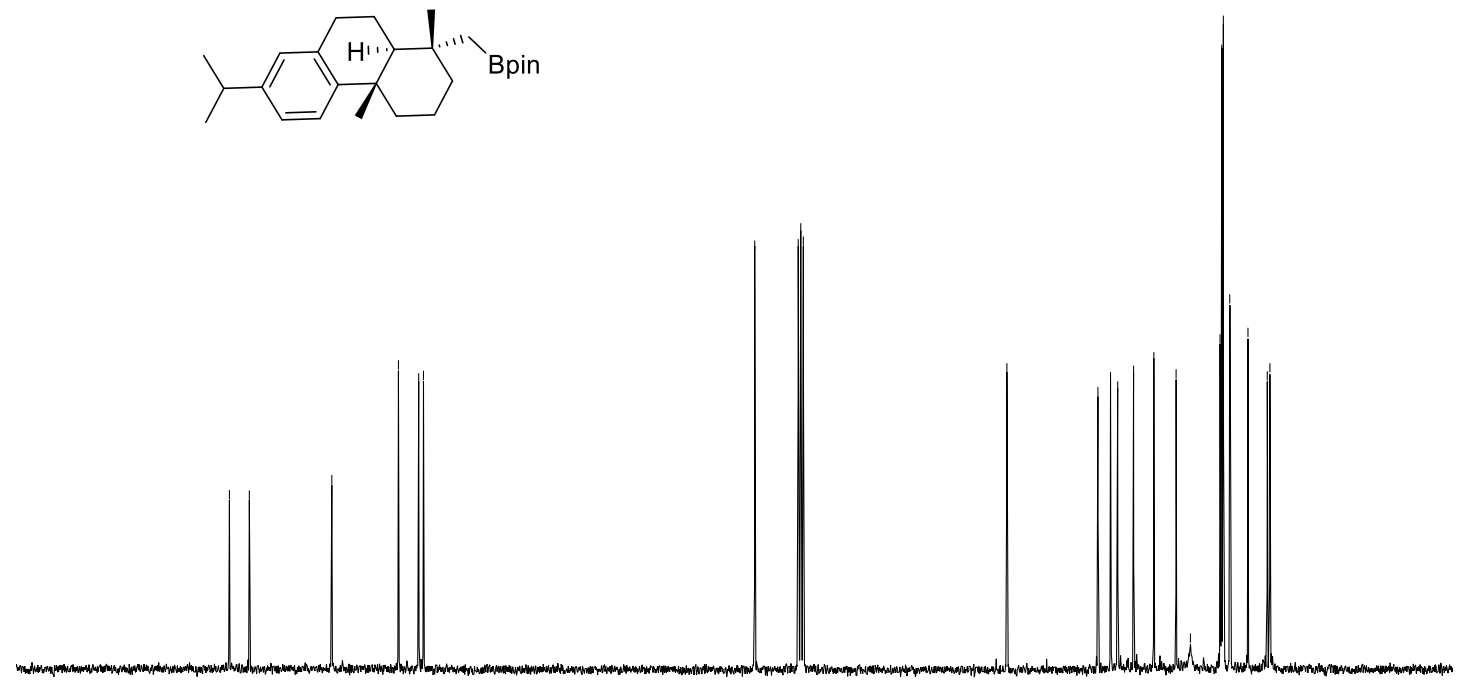

।

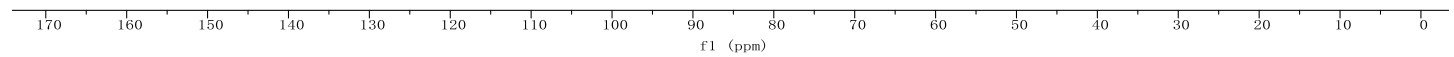

$\infty$
$\stackrel{\infty}{N}$
$\infty$
$i$
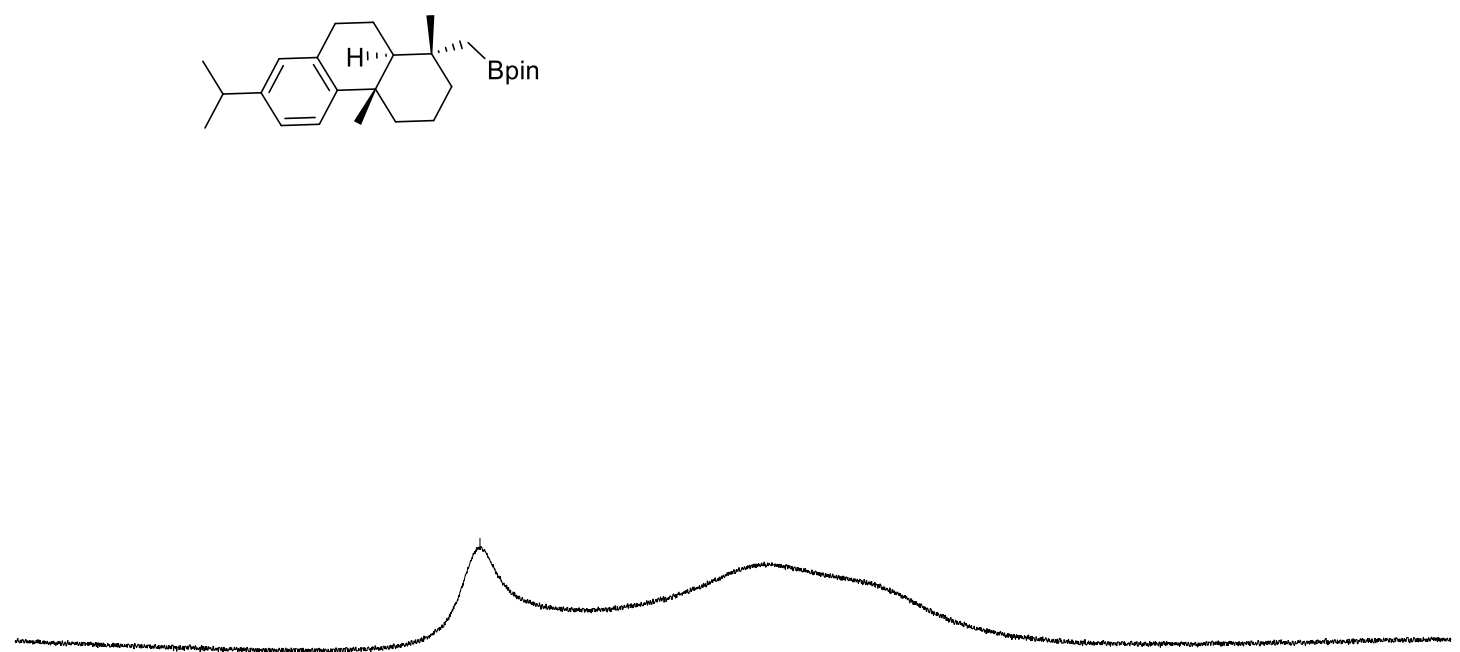


\section{Compound 66}

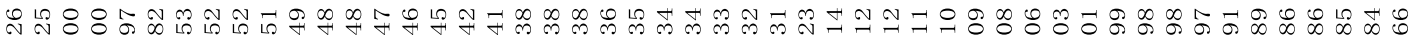
ம் ف
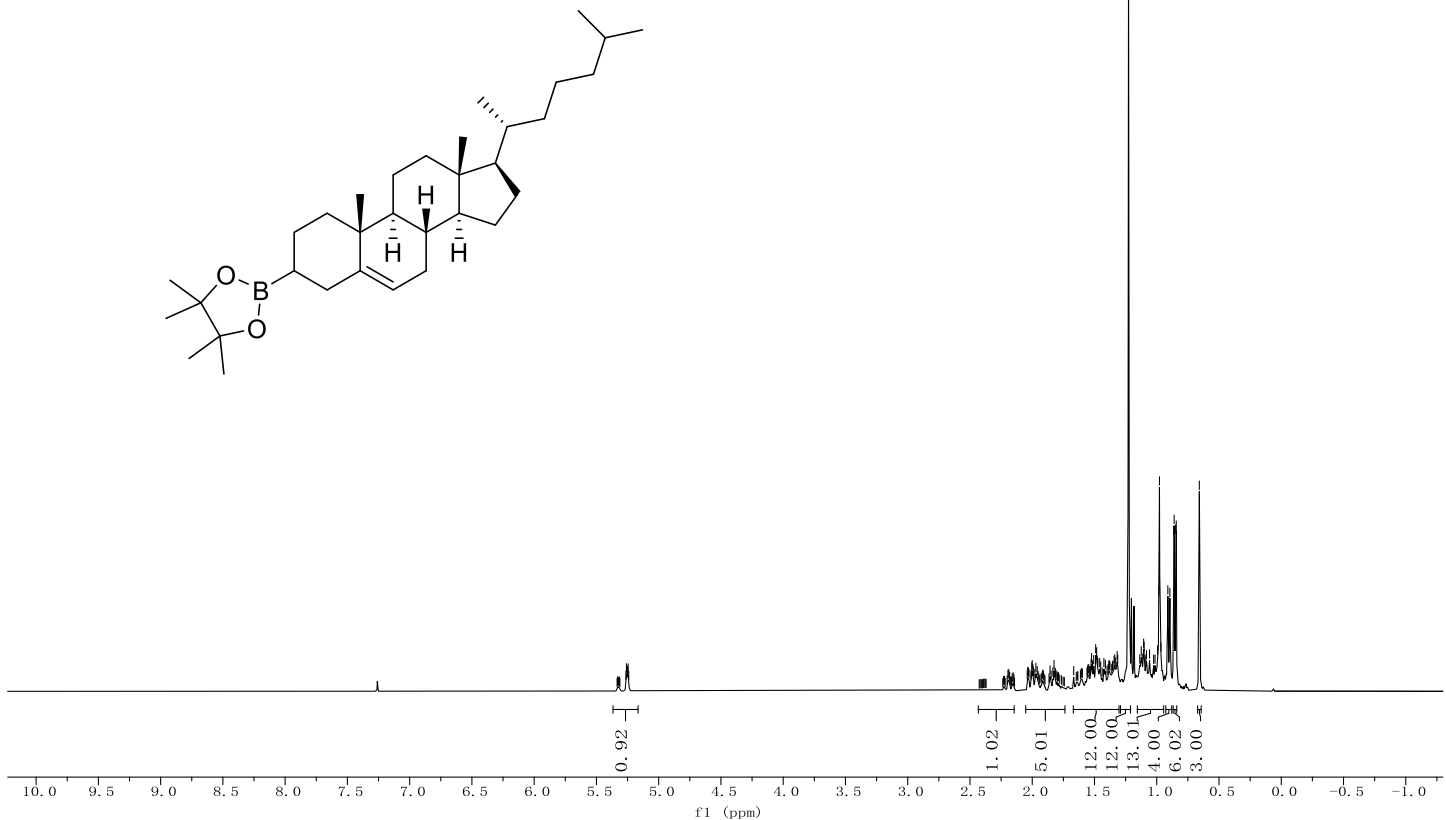

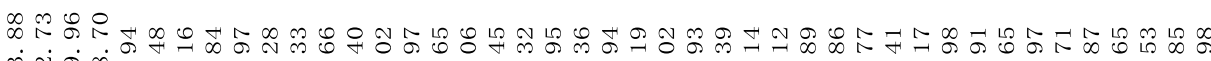

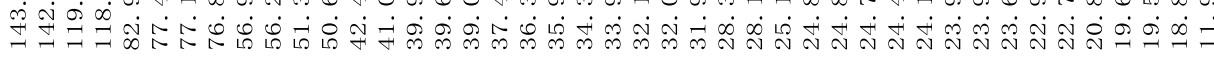
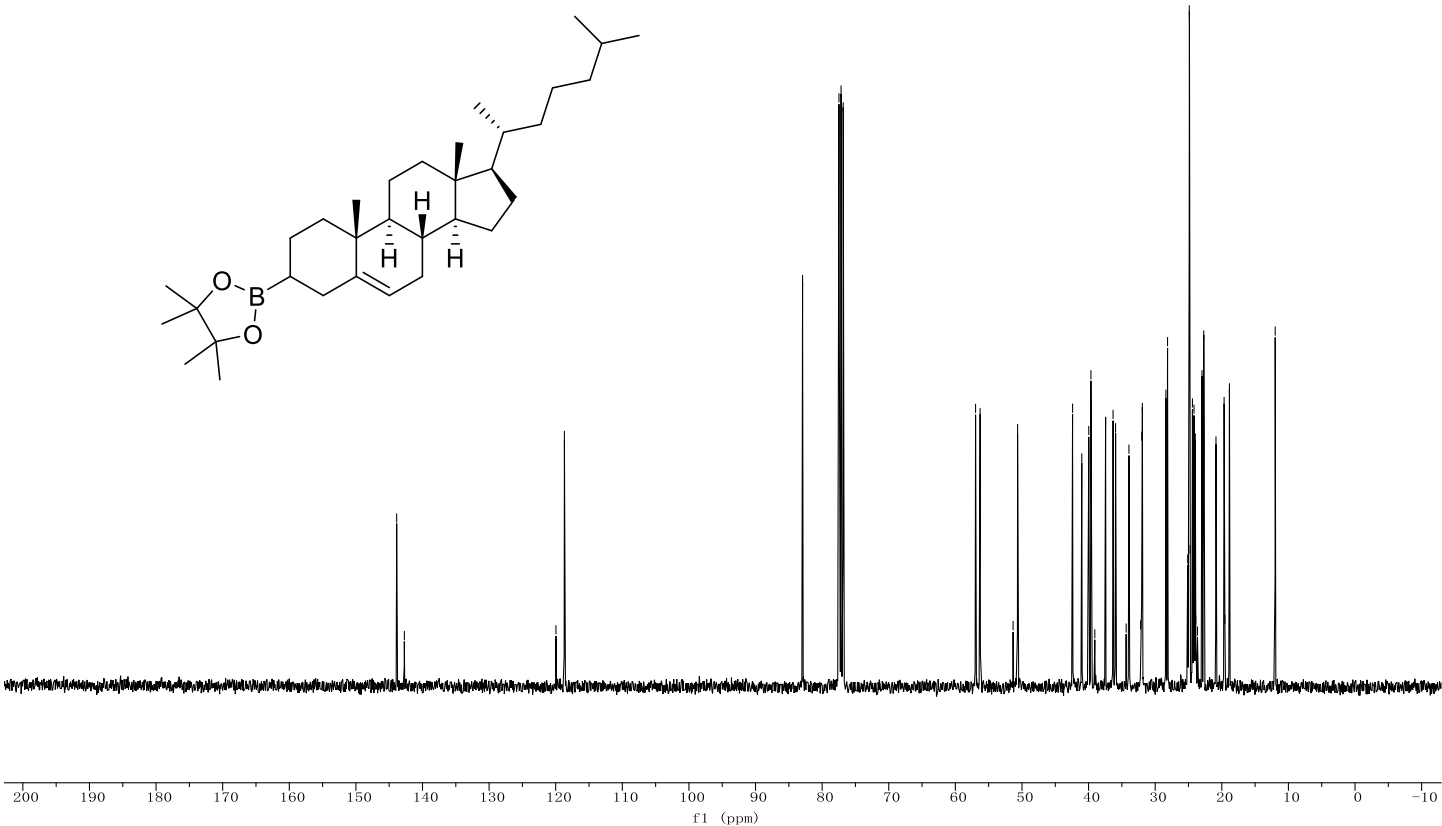

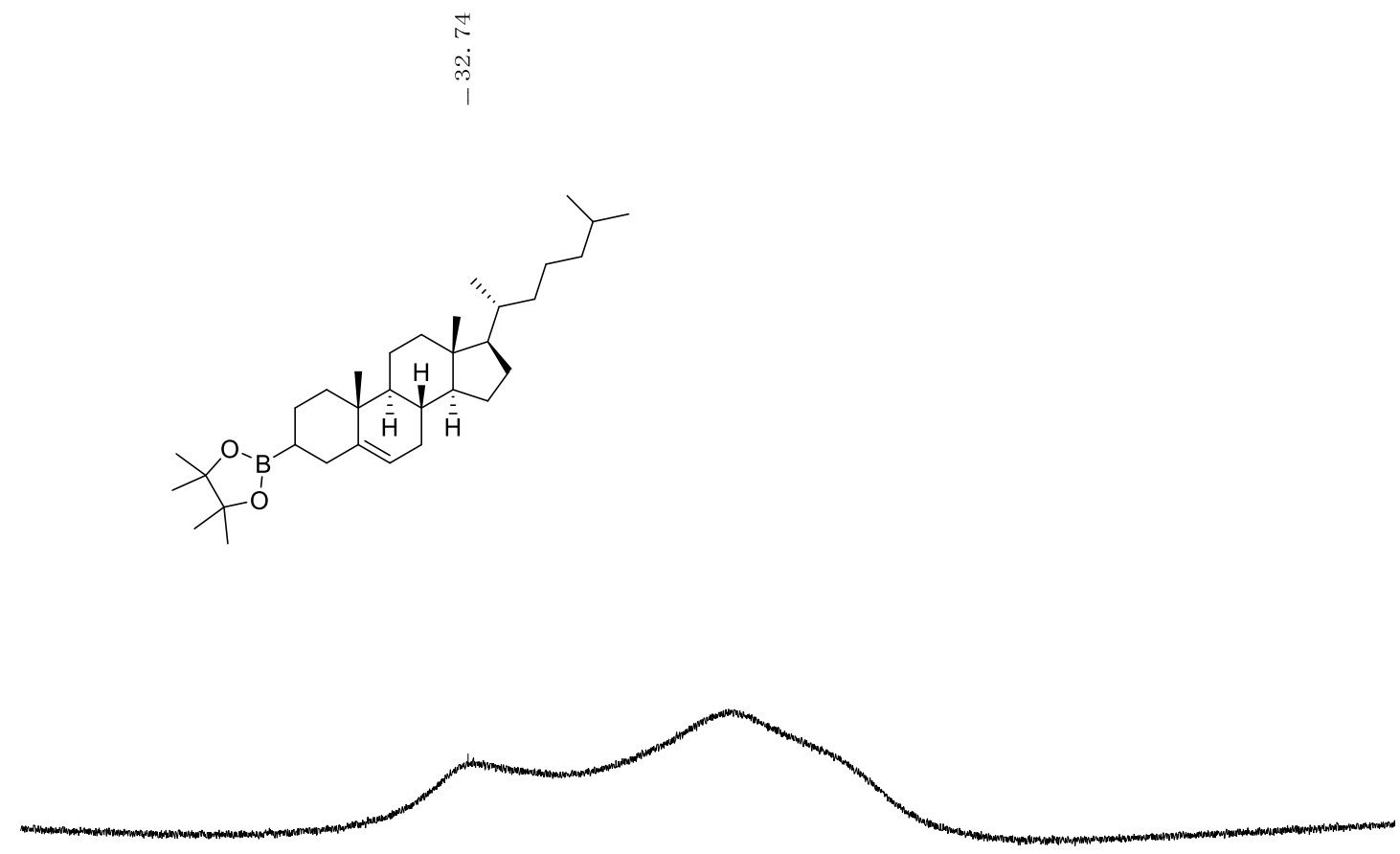

\section{Compound 67}

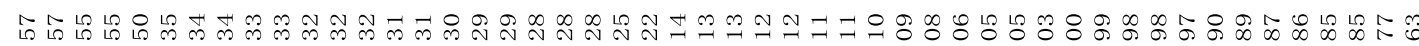

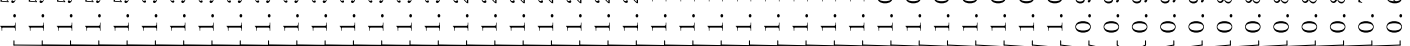

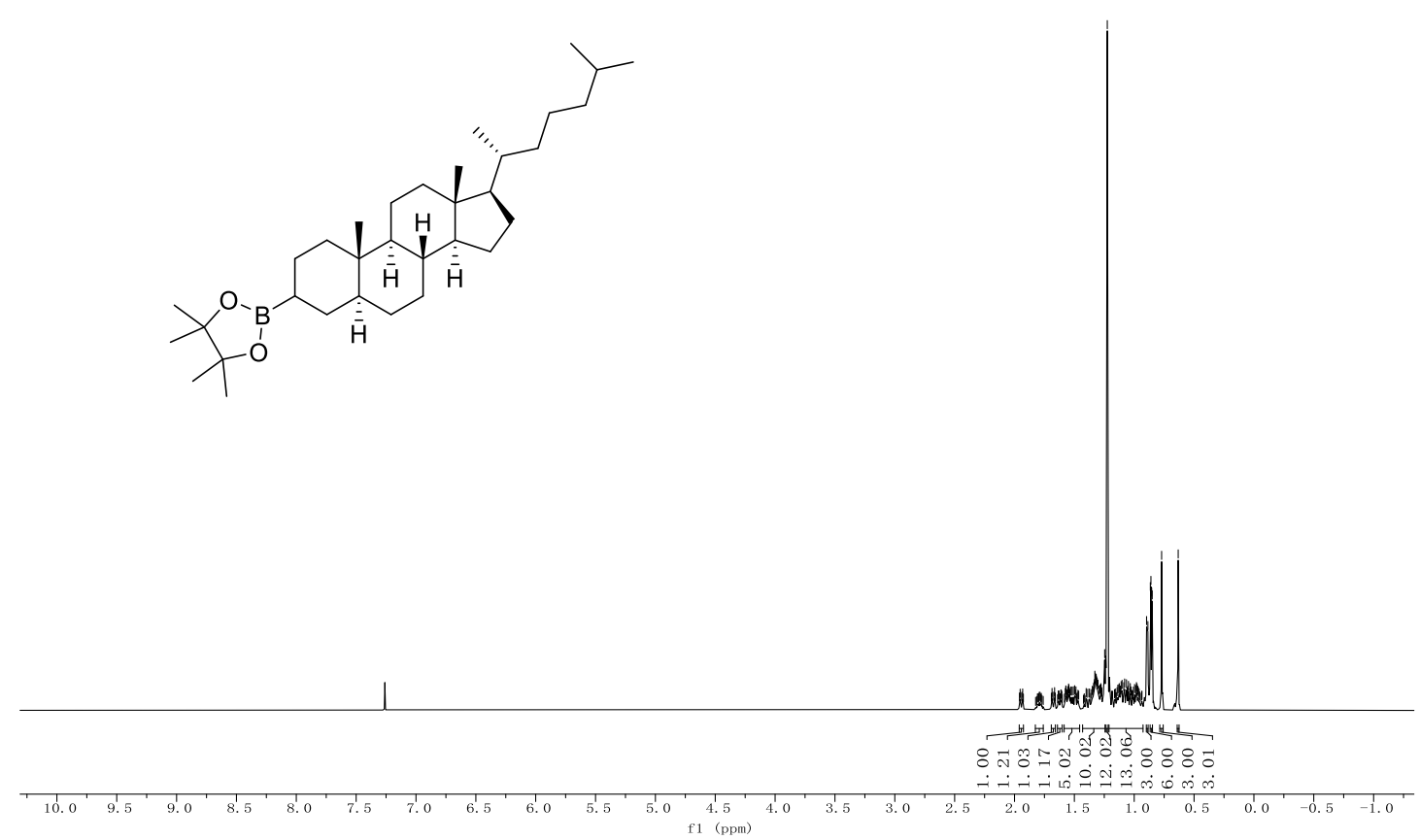



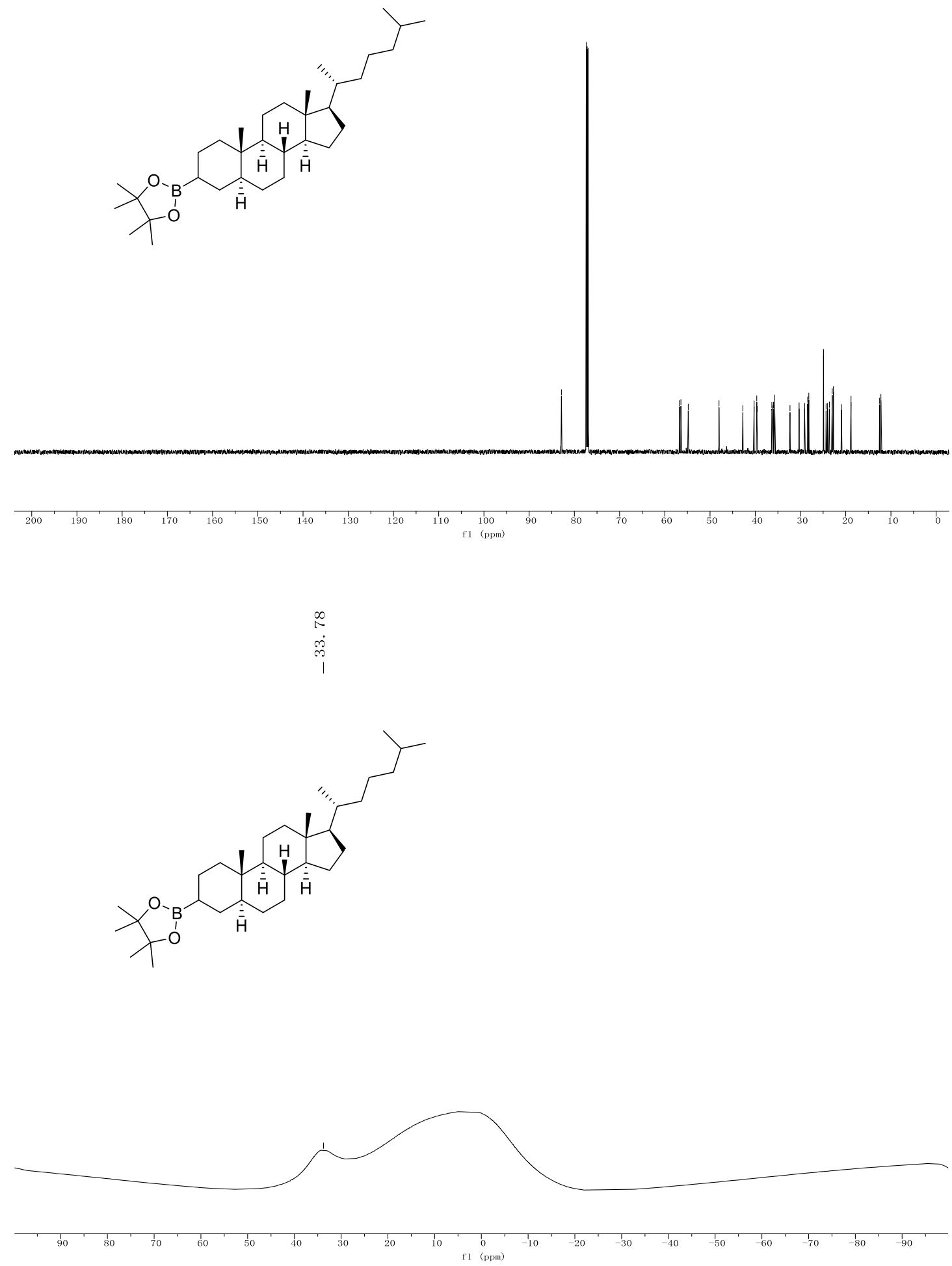